\title{
Deployment of efficient wireless sensor nodes for monitoring in rural, indoor and underwater environments
}

SANDRA SENDRA COMPTE 


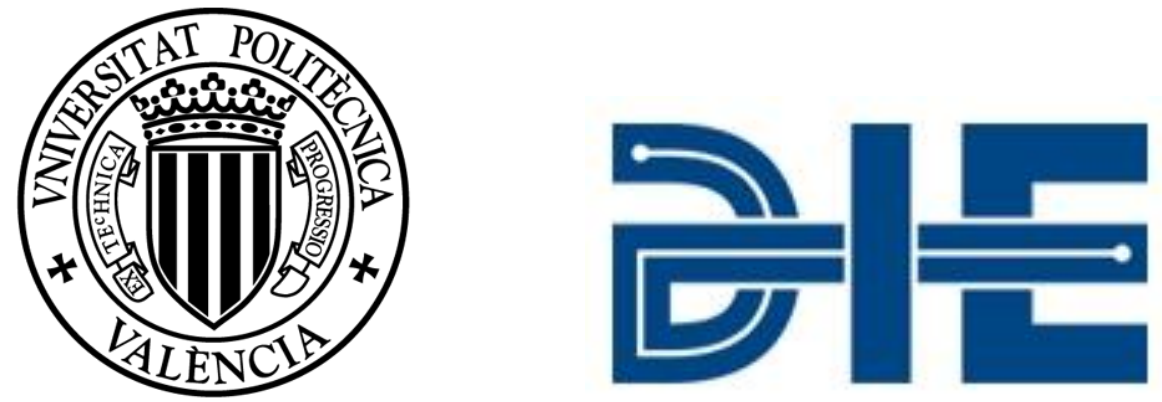

UNIVERSITAT

DEPARTAMENTO POLITĖCNICA

DE VALĖNCIA

DE INGENIERÍA

ELECTRÓNICA

\section{Deployment of Efficient Wireless Sensor Nodes for Monitoring in Rural, Indoor and \\ Underwater Environments}

by

\section{Sandra Sendra Compte}

Supervisors:

PROF. DR. JAIME LLORET MAURI

PROF. DR. MIQUEL ARDID RAMÍREZ

Tutor:

PROF. DR. JOSÉ FRANCISCO TOLEDO ALARCÓN

September 2013 
First Edition, 2013

(c) Sandra Sendra Compte

(c) of the present edition:

Editorial Universitat Politècnica de València

www.lalibreria.upv.es

ISBN: 978-84-9048-135-6 (printed version)

Publishing reference: 5669

Any unauthorized copying, distribution, marketing, editing, and in general any other exploitation, for whatever reason, of this piece of work or any part thereof, is strictly prohibited without the authors' expressed and written permission. 


\section{Abstract}

There are many works related to the design and development of sensor nodes which present several applications. Wireless sensor networks can facilitate and improve some aspects of our daily lives. It is easy to think that if this type of device is so beneficial to us and to our environment, its price should be relatively cheap. But we can see that this is not true. Why these devices are so expensive? Would it be possible to develop devices with the same capabilities and lower prices? How can I make my low-cost sensor nodes?

This dissertation answers these questions and shows some of the many applications that sensor nodes may have. In this dissertation, we propose (and implement in some cases) the development of sensor nodes for environmental monitoring, from low-cost devices. For the implementation of a sensor node and network which joins all these nodes, it is important to know the environment where they will work. Throughout this dissertation, we present the research carried out for the development of sensors in three main application areas.

In the first of these areas, we present multisensor devices developed for environmental monitoring. The application of wireless sensor networks to the environment requires a study of how signals are affected depending on the distance, vegetation, ambient humidity, etc. We focus our developments on the fire detection in rural areas and on the control of pests in vineyards where the early detection of these events generates high economic savings. We also propose the development of a sensor network which will help us to reduce and prevent wolves' attacks and theft in livestock. Finally, within this group, we present a network to detect material anomalies in building and a sensor network which allows us to monitor the elderly or disabled people who move along with a group on a tour or activity.

The second group of applications is related to the monitoring of spaces in indoor environments. For this, we analyze the behavior of wireless signals in different scenarios. These results allowed us to extract a new method for designing wireless networks in indoor environments. Our method allows defining the best location of network devices and sensor nodes indoors saving $15 \%$ of the sensors needed.

Finally, we present a study on underwater freshwater communications based on electromagnetic waves, where we analyze the dependency of underwater communications as a function of working frequency, temperature, data transfer rates and modulation.

Related to underwater environment, we present two proposals. First one refers to the implementation of a sensor network for marine farms which allows us to reduce the amount of waste deposited on the seabed and reduce the percentage of wasted food. The second proposal is the development of two oceanographic sensors which allow us to control the amount of food and feces deposited in seabed and the water turbidity control in a very simple and inexpensive way. 
All these developments and proposals have been preceded by a comprehensive study on the energy problems in wireless sensor networks. We have also presented several techniques which can be used to prolong the network lifetime and improve its stability. 


\section{Resumen}

Existen muchos trabajos relacionados con el diseño y desarrollo de nodos sensores, donde se presentan gran variedad de aplicaciones. Las redes de sensores inalámbricos pueden facilitarnos y mejorar algunos aspectos de nuestra vida diaria. Es fácil pensar que si este tipo de dispositivo es tan beneficioso para nosotros y para el entorno donde vivimos, su precio debería ser relativamente barato. Pero podemos comprobar que esto no es así. ¿Por qué estos dispositivos son tan caros? ¿Sería posible desarrollar dispositivos con las mismas capacidades y precios más económicos? ¿Cómo puedo fabricar mis nodos sensores de bajo coste?

Esta tesis responde a estas preguntas y muestra algunas de las muchas aplicaciones que los nodos sensores pueden tener. En esta tesis hemos propuesto (e implementado en algunos casos) el desarrollo de nodos sensores para la monitorización del medio, a partir de dispositivos de bajo coste. Para la implementación de un nodo sensor, y en definitiva la red que une a todos estos nodos, es importante conocer el medio donde trabajarán. A lo largo de este documento se presentan las investigaciones llevadas a cabo para el desarrollo de sensores en tres ámbitos de aplicación.

En el primero de ellos, se desarrollan dispositivos multisenores para la monitorización del medio. La aplicación de las redes de sensores inalámbricas al medio natural precisa un estudio de cómo se ven afectadas las señales en función de la distancia, vegetación, humedad del ambiente, etc. Focalizamos nuestros desarrollos en la verificación de incendios en zonas rurales y en el control de plagas en viñedos donde la detección precoz de estos eventos genera elevados ahorros económicos. También proponemos el desarrollo de una red de collares sensores para ganado domestico, que nos ayudará a reducir y prevenir en muchos casos, los ataques de lobos y hurtos de crías. Por último, dentro de este grupo, presentamos una red que permite detectar anomalías de los materiales en edificios y otra red de sensores que permite monitorizar las personas mayores o deficientes que se mueven junto con un grupo en una excursión o actividad.

El segundo grupo de aplicaciones hace referencia a la monitorización de espacios en entornos de interior. Para ello hemos analizado el comportamiento de las señales inalámbricas en diferentes escenarios. Los resultados nos han permitido extraer un nuevo método de diseño de las redes inalámbricas en interiores. Nuestro método permite definir la mejor ubicación de los dispositivos de red y nodos sensores en interiores con un ahorro en el número de sensores del $15 \%$.

Por último, se presenta el estudio sobre las comunicaciones subacuáticas basadas en las ondas electromagnéticas donde analizamos la dependencia de las comunicaciones subacuáticas en agua dulce en función de la frecuencia, temperatura, tasas de transferencia de datos y modulación.

Relacionado con el medio subacuático, presentamos 2 propuestas. La primera de ellas hace referencia a la implementación de una red de sensores para granjas marinas que nos permite reducir la cantidad de residuos depositados en el lecho marino y reducir el porcentaje de comida desperdiciada. La segunda propuesta es el desarrollo de dos sensores oceanográficos que nos 
permitirían controlar la cantidad de comida y heces depositadas en el suelo y controlar la turbidez del agua de manera simple y económica

Todos estos desarrollos y propuestas han estado precedidos por un exhaustivo estudio sobre los problemas energéticos que las redes de sensores inalámbricas presentan y las técnicas que pueden emplearse para prolongar la vida útil de la red y mejorar su estabilidad. 


\section{Resum}

Hi ha molts treballs relacionats amb el disseny i desenvolupament de nodes sensors on es presenten gran varietat d'aplicacions. Les xarxes de sensors sense fils poden facilitar i millorar alguns aspectes de la nostra vida diària. És fàcil, pensar que si aquest tipus de dispositiu és tan beneficiós per a nosaltres i per a l'entorn on vivim, el preu hauria de ser relativament barat. Però podem comprovar que això no és així. ¿Per què aquests dispositius són tan cars? Seria possible desenvolupar dispositius amb les mateixes capacitats i preus més econòmics? Com puc fabricar els meus nodes sensors?

Aquesta tesi respon a aquestes preguntes i mostra algunes de les moltes aplicacions que els nodes sensors poden tenir. En aquesta tesi hem proposat (i implementat en alguns casos) el desenvolupament de nodes sensors per a la monitorització del medi a partir de dispositius de baix cost. Per a la implementació d'un node sensor, i en definitiva la xarxa que uneix a tots aquests nodes, és important conèixer el medi on treballaran. Al llarg d'aquest document es presenten les investigacions dutes a terme per al desenvolupament de sensors en tres àmbits d'aplicació.

En el primer d'ells, es desenvolupen dispositius multisensors per a la monitorització del medi. L'aplicació de les xarxes de sensors sense fils al medi natural necessita un estudi de com es veuen afectades les senyals en funció de la distància, vegetació, humitat de l'ambient, etc. Focalitzem els nostres desenvolupaments en la verificació d'incendis en zones rurals i en el control de plagues en vinyes on la detecció precoç d'aquests esdeveniments genera elevats estalvis econòmics. També proposem el desenvolupament d'una xarxa de collarets sensors per a bestiar domèstic, que ens ajudarà a reduir i previndre en molts casos, els atacs de llops i furts de cries. Finalment, dins d'aquest grup, presentem una xarxa que permet detectar anomalies dels materials en edificis i una altra xarxa de sensors que ens permet monitoritzar les persones grans o deficients, que es mouen juntament amb un grup, en una excursió o activitat.

El segon grup d'aplicacions, fa referència a la monitorització d'espais en entorns d'interior. Per a això hem analitzat el comportament dels senyals sense fils en diferents escenaris. Els resultats ens han permés extraure un nou mètode de disseny de les xarxes sense fils en interiors. El nostre mètode permet definir la millor ubicació dels dispositius de la xarxa i nodes sensors en interiors amb un estalvi, en el nombre de sensors, del $15 \%$.

Finalment, es presenta l'estudi sobre les comunicacions subaquàtiques basades en les ones electromagnètiques on analitzem la dependència de les comunicacions subaquàtiques en aigua dolça en funció de la freqüència, temperatura, taxes de transferència de dades i modulacions.

Relacionat amb el medi subaquàtic, presentem 2 propostes. La primera d'elles fa referència a la implementació d'una xarxa de sensors per a granges marines que ens permet reduir la quantitat de residus dipositats al fons marí i reduir el percentatge de menjar desaprofitat. La segona proposta és el desenvolupament de dos sensors oceanogràfics que ens permetrien controlar la quantitat de 
menjar i excrements dipositada a terra i controlar la terbolesa de l'aigua de manera simple $\mathrm{i}$ econòmica

Tots aquests desenvolupaments i propostes han estat precedits per un exhaustiu estudi sobre els problemes energètics que les xarxes de sensors sense fils presenten i les tècniques que poden emprar-se per a allargar la vida útil de la xarxa i millorar la seva estabilitat. 


\section{Table of Contents}

Abstract..............................................................................

Resumen....................................................................... $\quad \mathrm{v}$

Resum........................................................................... vii

List of Figures...................................................................

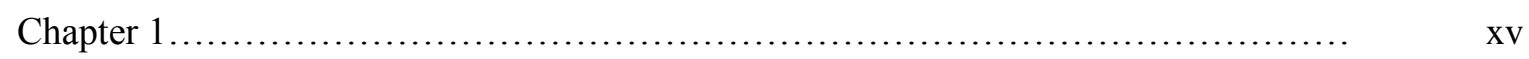

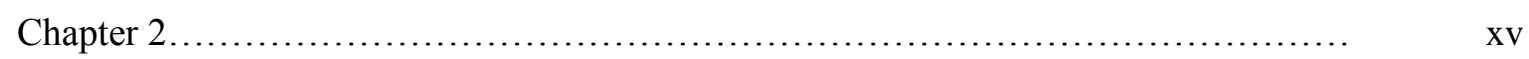

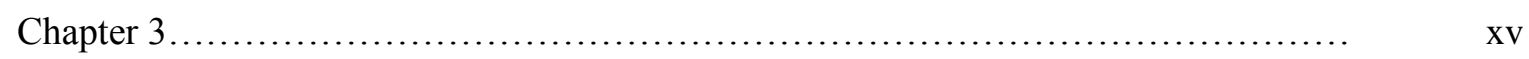

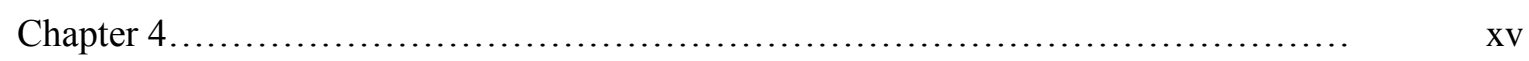

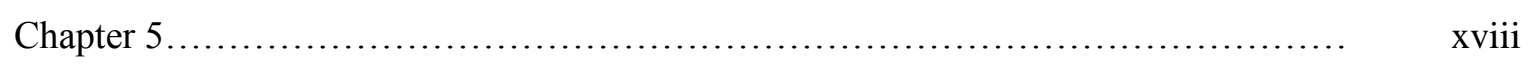

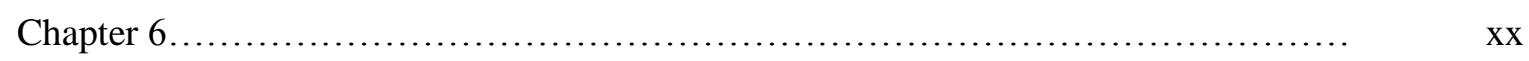

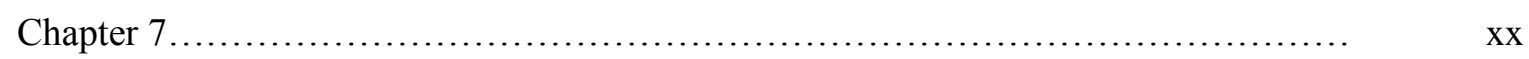

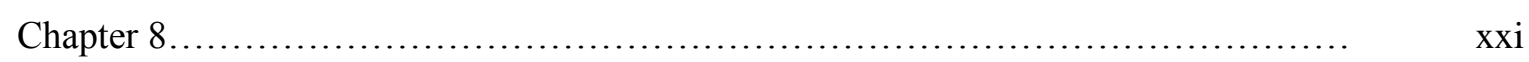

List of Tables......................................................................... xxii

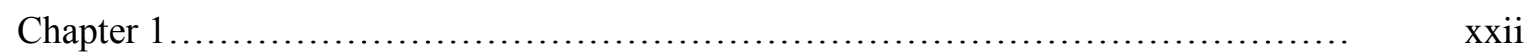

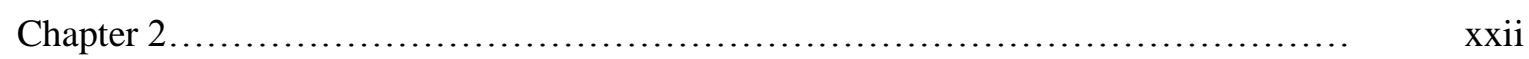

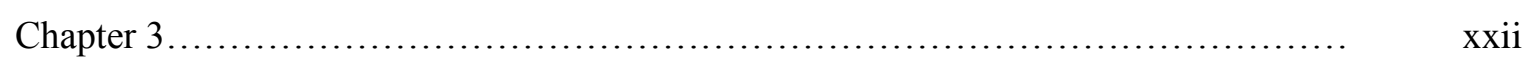

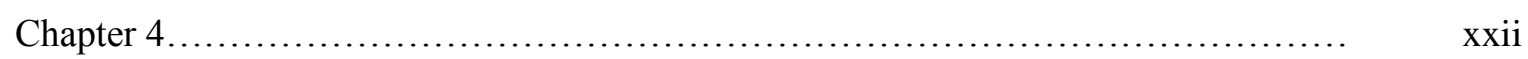

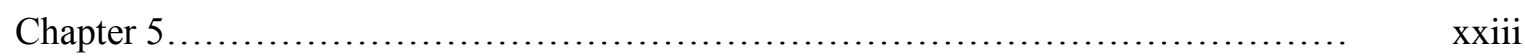

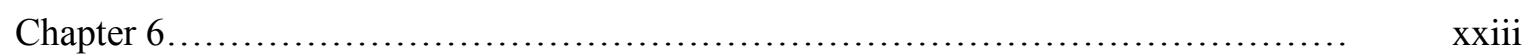

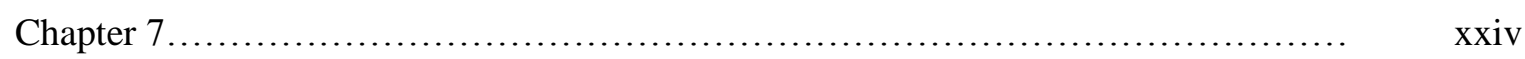

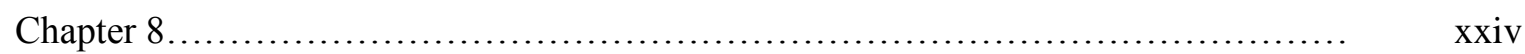

Acknowledgments............................................................... $\quad$ xxv

Chapter 1:Introduction.......................................................... 1

1.1 Introduction....................................................... 1

1.2 Research goals......................................................... 5

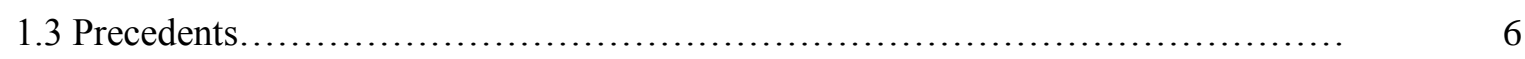

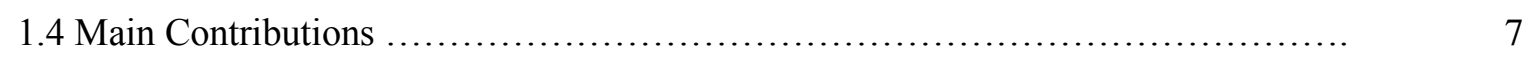

1.5 Structure of the dissertation............................................. 9

Chapter 2: State of the art in Wireless Sensor Networks.............................. 11 


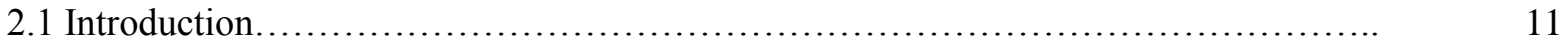

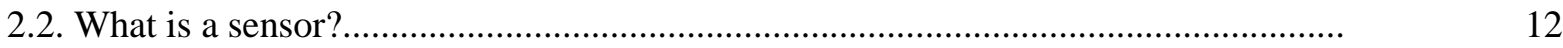

2.2.1 Types of sensors................................................... 13

2.3. State of the art in energy issues............................................ 19

2.3.1 Analysis of energy status and saving energy strategies based on applications..... 19

2.3.2 Analysis and comparatives of energy consumption of different wireless

technologies.......................................................... 22

2.3.3 Power-saving mechanisms in WLANs from the protocols perspective............. 23

2.4. State of the art in monitoring of natural environments.......................... 25

2.4.1. Fire detection and verification......................................... 25

2.4.2 Animal monitoring to prevent theft and attacks by wild animals............... 27

2.4.3 WSN for Vineyard Monitoring ........................................ 27

2.4.4 WSNs for detecting deficiencies in construction........................... 30

2.4.5 WSN for disabled and elderly people................................... 31

2.5. State of the art of WSN in indoor environments.............................. 33

2.5.1 Related Work of indoor coverage measurements........................... 33

2.5.2 Related Work of interference measurements in indoor environment............... 35

2.5.3 Related Work of wireless sensor positioning in indoor environments............. 36

2.6. State of the art in underwater wireless communications......................... 37

2.6.1 Underwater communications based on acoustic waves........................ 38

2.6.2 Underwater communications based on optical waves......................... 41

6.2.3 Underwater communications based on electromagnetic waves................. 43

2.6.3.1 EM waves in freshwater......................................... 43

2.6.3.2 EM Waves in seawater............................................ 44

2.6.4. Miscellaneous of existing measurements of underwater wireless communications technologies.............................................. 46

2.6.5. Existing measurements of underwater wireless communications based on

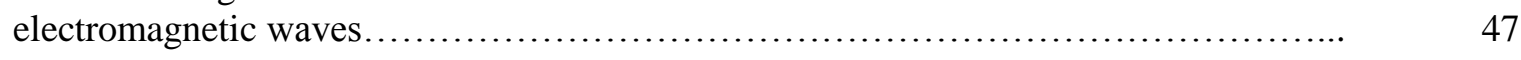

2.7 State of the art of WSN for Underwater Applications............................. 47

2.7.1 Problems in Marine Fish Farms............................................ 48

2.7.2 Simulators for Marine Fish Farms........................................ 48

2.7.3 Current Methods to Measure the Water Turbidity........................... 50

2.8 Conclusion................................................................ 51

Chapter 3: Energy issues in Wireless Sensor Networks............................. 53

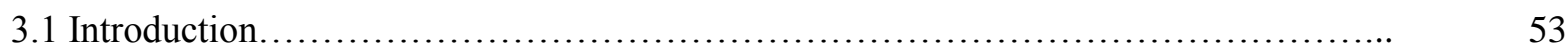

3.2 Energy Issues in Hardware ............................................ 54

3.2.1 Wireless Senor Node Hardware Structure................................. 54 
3.2.2 Characteristics and Requirements of a Wireless Sensor Node.................. 56

3.2.3 Hardware Availability................................................. 56

3.2.4 Energy Consumption in Transmission and Initial Consideration for

Environmental Applications.............................................. 59

3.3 Saving Energy in WSN from the Point of View of Protocols and Topology

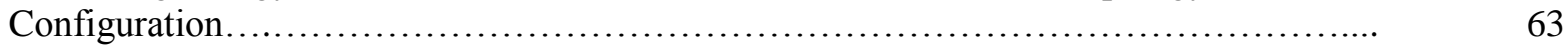

3.3.1 Group-based Wireless Sensor Networks................................ 64

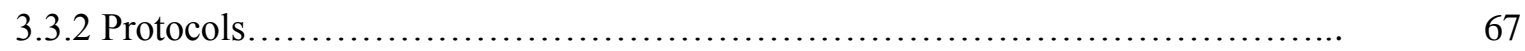

3.3.2.1 Efficient MAC Protocols............................................... $\quad 67$

3.3.2.2 Efficient Routing Protocols........................................ $\quad 75$

3.4. Optimizing Configurations in Network Devices. Measurements in actual devices..... $\quad 86$

3.4.1 Measurements in Wireless Access Points ................................ 86

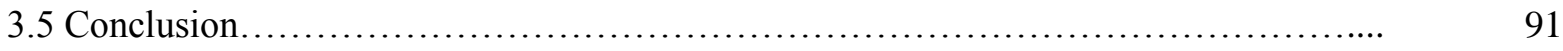

Chapter 4: Wireless Sensor Nodes for Environmental Monitoring.................. 93

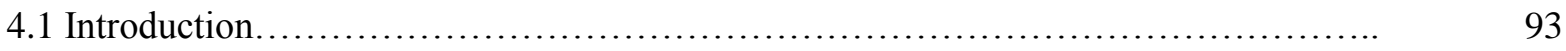

4.2 Sensor Deployment for Natural Environmental Monitoring........................ 94

4.2.1 Wireless Sensor Network Deployment for Rural and Forest Fire Detection and

Verification............................................................. 94

4.2.1.1. Radio Design, Number of Devices Needed and Channel Distribution Plan.. $\quad 96$

4.2.1.2 Hardware Deployment........................................... 99

4.2.1.3. System Design and Operation Mode................................... 102

4.2.1.4. User Interface ................................................. 104

4.2.1.5. Performance Test................................................. 105

4.3 A Wireless Sensor Network for Vineyard Monitoring............................. 112

4.3.1. Wireless Sensor Network and Wireless Sensor Node Deployment.............. 114

4.3.1.1. Analytical Network Model for Node Placement ......................... $\quad 115$

4.3.1.2. Wireless Sensor Node............................................ 120

4.3.1.3. Camera ........................................................... $\quad 124$

4.3.1.4. Rotation System............................................... $\quad 125$

4.3.2. Sensor Network Traffic Measurement..................................... 127

4.4 Intelligent Wireless Sensor Network to Detection and Protection of the Attacks to the

Sheep and Goats ...................................................... 129

4.4.1 Sensor Network.................................................... $\quad 129$

4.4.1.1 Monitoring system of heart rate and body temperature..................... 130

4.4.1.2 Placement of sensors on the animals................................ 134

4.4.1.3 Design of the network topology..................................... 134

4.4.1.4 System algorithms............................................ $\quad 136$

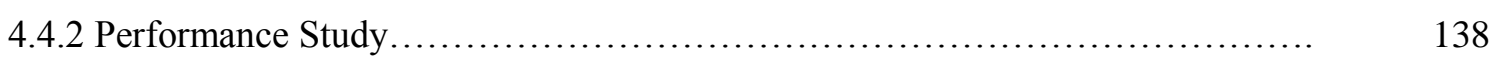


4.5 Deployment of Wireless Sensor Nodes to Detect the Cement Degeneration in

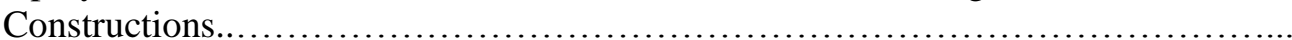

4.5.1 Node Description................................................. 143

4.5.1.1 Sensor description.............................................. 145

4.5.1.2 Temperature sensor............................................... $\quad 145$

4.5.1.3 Vibration sensor............................................... $\quad 146$

4.5.1.4 Humidity sensor................................................ $\quad 147$

4.5.1.5 Gyroscope sensor................................................. 148

4.5.1.6 Electric power system................................................ $\quad 149$

4.5.1.7 Multisensor Node.................................................. $\quad 150$

4.5.2 Adequacy of the Sensor Nodes to Protect them from the Environment............ 150

4.5.3 Decision Algorithm..................................................... 151

4.5.4 Position of the Sensors in the Structure.................................. 152

4.5.5 Wireless Sensor Network.............................................. 154

4.5.5.1 Developed topology............................................. 154

4.5.5.2 Network operation and its performance.............................. 155

4.5.5.3 Web interface for data query....................................... 158

4.6 Development of Wireless Sensor Nodes for Taking Care of Disabled and Elderly

People...................................................................... 158

4.6.1 System Operation.................................................. $\quad 162$

4.6.2 Performance Test......................................................... 165

4.6.2.1 System operation performance........................................ 165

4.6.2.2 Alarms.......................................................... $\quad 170$

4.6.2.3 Decision rules by mixing alarms.................................. 171

4.6.2.4 Ad hoc Network routing protocol.................................. 176

4.7 Conclusion.................................................................. 179

Chapter 5: Wireless Sensor Development for Monitoring in Indoor Environments.... 181

5.1 Introduction............................................................... 181

5.2 Tools Used in the Measurement Process....................................... 183

5.2.1 Software Used in the Test Bench........................................ 183

5.2.2 Hardware Used in the Test Bench........................................ 183

5.2.3 Measurements in Scenario 1: CRAI...................................... 184

5.2.3.1 The building ................................................... 184

5.2.3.2 Description of UPV wireless network............................... 186

5.2.3.3 Coverage measurements.......................................... $\quad 186$

5.2.3.4 Comparative study of three wireless signals.......................... 191

5.2.4 Measurements in Scenario 2: Garage.................................... 199 
5.2.4.1 Coverage measurements for garage................................. 200

5.2.4.2 Testbench description for interference measurements..................... 208

5.2.5 Measurements Comparison for Both Scenarios and Sensor Placement in Indoor Environments.

5.2.5.1 Method for estimating the best position of wireless sensors in indoor environments.

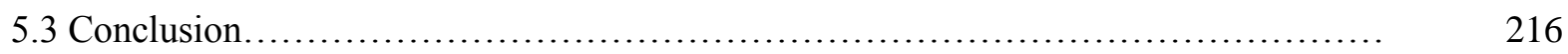

Chapter 6: Underwater Wireless Communications based on Electromagnetic Waves. 219

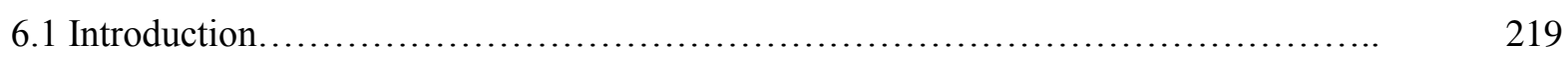

6.2 Signal behavior of EM waves in fresh water................................. 221

6.2.1 Experimental setup................................................... 221

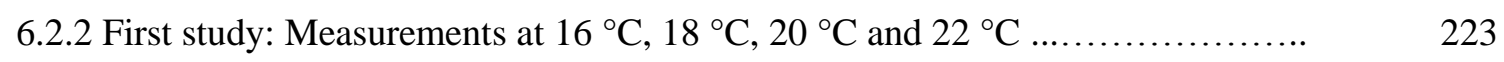

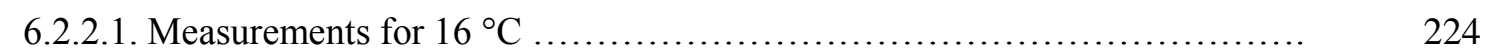

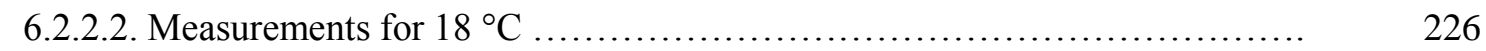

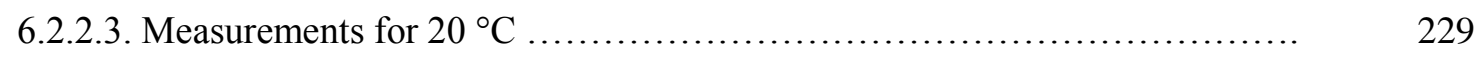

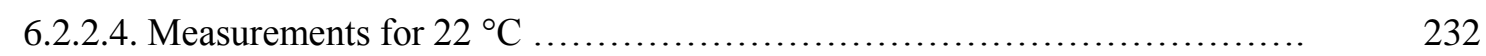

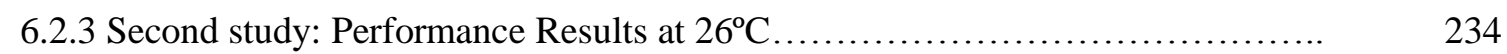

6.2.3.1Performance of BPSK modulation and analytical study for 1Mbps data 234

6.2.3.2 Performance of QPSK modulation and analytical study for 2 Mbps data 236

6.2.3.3 Performance of CCK modulation and analytical study for 5.5 Mbps data $\quad 237$ transfer rate.................................................. 237

6.2.3.4 Performance of CCK modulation and analytical study for 11 Mbps data 238

6.3 Summary of Best Results and Comparison with Other Studies......................... 239

6.4 Mathematical Model 243

6.5 Conclusion............................................................. 246

Chapter 7: Application of WSN in marine environment................................. 247

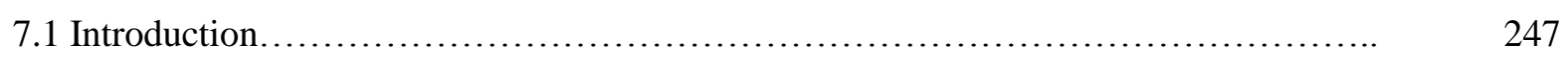

7.2. Problem Description...................................................... 249

7.3. Seabed Depositions Estimation .......................................... 252

7.4. Wireless Sensor Node for Depositions Monitoring............................ 254

7.4.1. Sensor Nodes Operation.......................................... 254

7.4.2 Sensor Mobility Model................................................ $\quad 255$

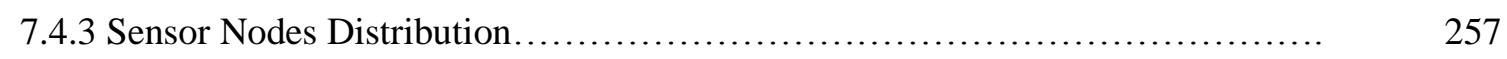

7.5. Low Cost Turbidity Sensor................................................ 259

7.5 .1 Our Proposal...................................................... $\quad 260$ 
7.5.2 Cost of OurTturbidity Sensor................................................. 261

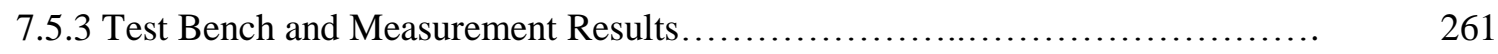

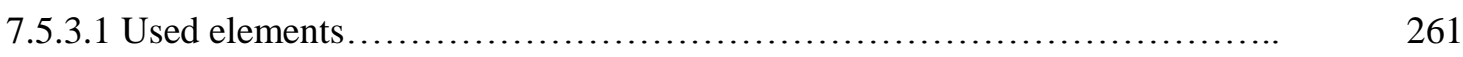

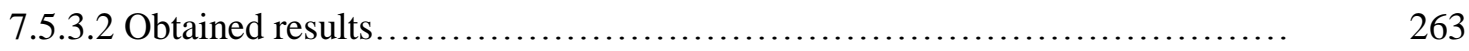

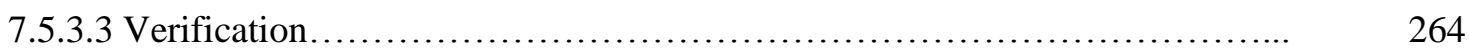

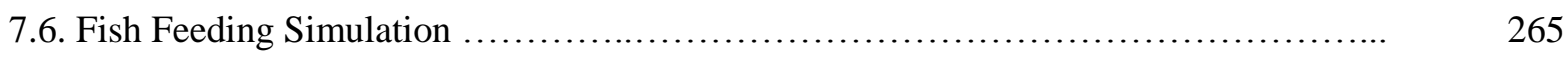

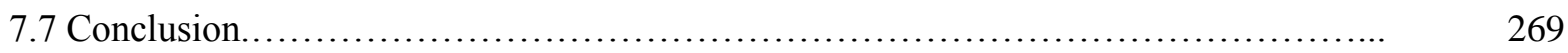

Chapter 8: Conclusion................................................................. 270

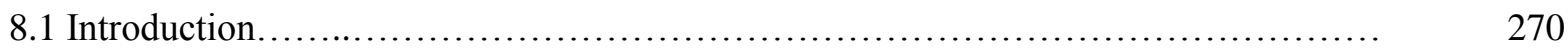

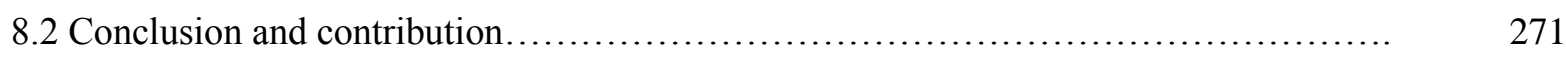

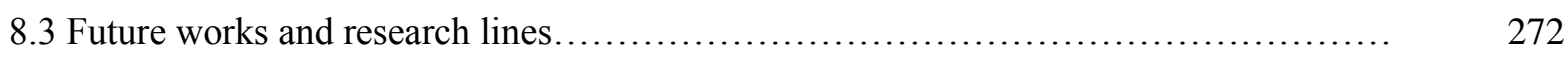

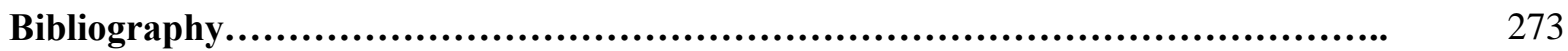




\section{List of Figures}

\section{Chapter 1}

There are not figures in this chapter.

\section{Chapter 2}

Figure 2.1. Block Diagram of sensor........................................ 13

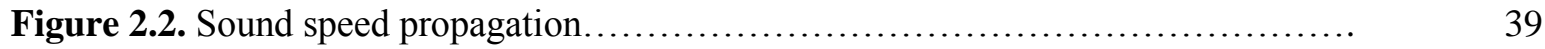

Figure 2.3. Scattering effect................................................. 42

Figure 2.4. Speed propagation............................................ 45

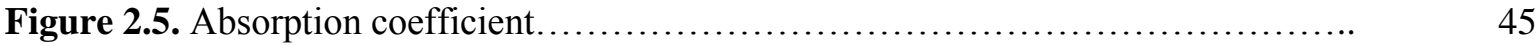

\section{Chapter 3}

Figure 3.1. Block diagram of a typical wireless sensor node........................ 55

Figure 3.2. Radio energy consumption model.................................. 59

Figure 3.3. Power consumption comparison.................................... 62

Figure 3.4. Number of sensors needed by each technology to cover several surface areas $\quad 62$

Figure 3.5. Regular network............................................... 65

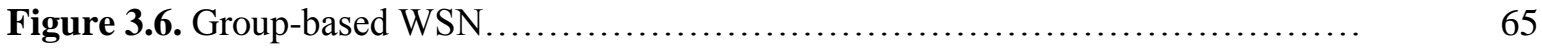

Figure 3.7. Topologies used to study the wireless APs............................ 87

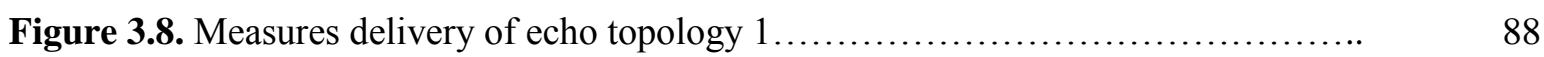

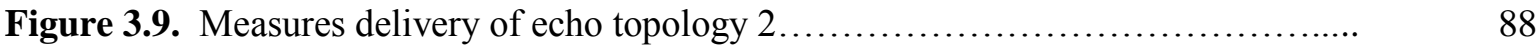

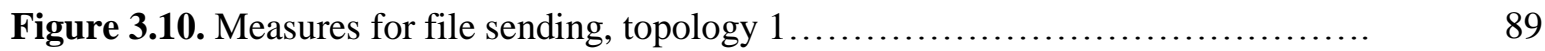

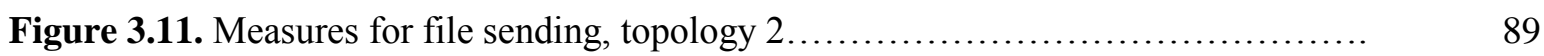

\section{Chapter 4}

Figure 4.1. The rural area where the test bench has been performed................... 95

Figure 4.2. Fire detection and verification design proposal........................ 96

Figure 4.3. Coverage distance vs. meters of vegetation............................. 98 
Figure 4.4. Number of devices needed per area that is wanted to be covered............. 99

Figure 4.5. Number of devices needed per area that is wanted to be covered............. 100

Figure 4.6. Hardware distribution on board........................................ 100

Figure 4.7. Integrated Circuit used to provide two serial ports....................... 101

Figure 4.8. System Mode Operation Flowchart................................... 103

Figure 4.9. Messages when there is an alarm..................................... 103

Figure 4.10. Main web page................................................... 104

Figure 4.11. A Single Camera visualization web page............................... 105

Figure 4.12. Octets per second obtained by the four situations....................... 106

Figure 4.13. Packets per second obtained by the four situations..................... 106

Figure 4.14. Bytes per second when there are four cameras........................ 107

Figure 4.1.5 Packets per second when there are four cameras...................... 107

Figure 4.16. Number of bytes per second....................................... 108

Figure 4.17. Number of packets per second.................................. 109

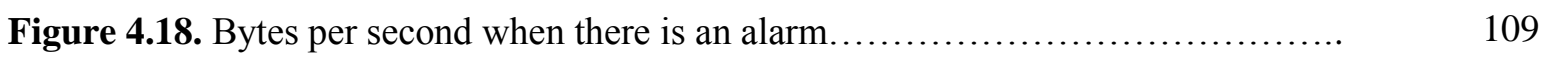

Figure 4.19. Packets per second when there is an alarm........................... 109

Figure 4.20. Power consumption............................................... 111

Figure 4.21. Power consumption in the network because of the packets transmitted and received.

Figure 4.22. Vineyard monitored by cameras.................................. 115

Figure 4.23. Radio coverage for several typical antenna gains...................... 117

Figure 4.24. Sensing coverage of a wireless sensor camera......................... 118

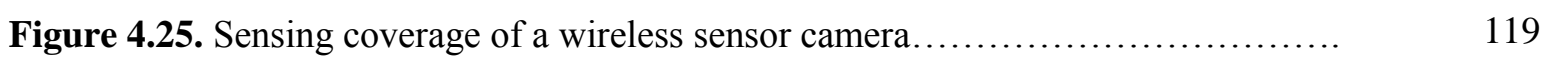

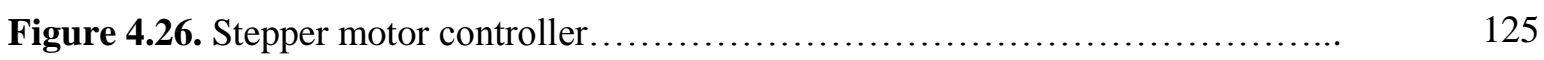

Figure 4.27. Program diagram................................................ 126

Figure 4.28. Sensor node for vineyard monitoring.............................. 127

Figure 4.29. Bytes comparison when there is $4 \mathrm{Mbps}$ video stream, $512 \mathrm{kbps}$ video stream and our system.

Figure 4.30. Packets comparison when there is $4 \mathrm{Mbps}$ video stream, $512 \mathrm{kbps}$ video stream and our system.

Figure 4.31. Structure of the wireless network.............................. 130

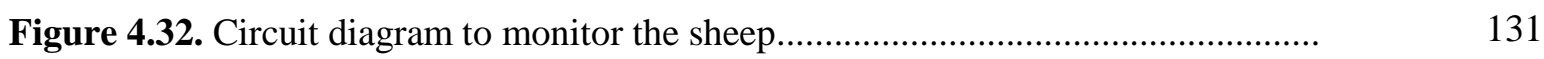

Figura 4.33. Belt whit sensors............................................. 132

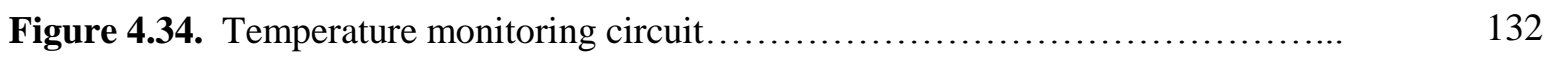


Figure 4.35. Circuit for cardiac frequency monitoring.....

Figure 4.36. ON/OFF circuit to control the wireless node.

Figure 4.37. Placement of sensors on the sheep.....

Figure 4.38. Waspmote node and its battery......

Figure 4.39. Decision algorithm

Figure 4.40. Algorithm to control the energy regulator.

Figure 4.41. Communication Protocol..

Figure 4.42. Simulation of the decision algorithm.

Figure 4.43. Consumed bandwidth.

Figure 4.44. Bytes sent by the node.

Figure 4.45. Bytes sent by the AP.

Figure 4.46. FlyPort module with USB Nest programmer.

Figure 4.47. FlyPort current consumption.

Figure 4.48. Schematic and image of the temperature sensor.

Figure 4.49. Current consumption vs. Temperature for temperature sensor.

Figure 4.50. Current consumption, depending on the sensor resistance value.

Figure 4.51. Schematic and actual image of the vibration sensor.

Figure 4.52. Schematic and actual image of the humidity sensor.....

Figure 4.53. Current consumption vs. RH in \% for humidity sensor.

Figure 4.54. Schematic of the gyroscope sensor.

Figure 4.55. Power system image and its schematic.

Figure 4.56. Total current consumption for sensor node.

Figure 4.57. Image of a multisensor node with its physical sensors.

Figure 4.58. Decision Algorithm if a building is suffering a collapse of some element of its structure.

Figure 4.59. Multisensor node locations in a structure

Figure 4.60. Network topology.

Figure 4.61. Sensor node registration protocol.

Figure 4.62. Bandwidth consumed by the network when a connection is performed.....

Figure 4.63. Http packets in the network when sending the sensed data

Figure 4.64. Bytes in the network during the 10 seconds.

Figure 4.65. Web Interface for each sensor node................................ 158

Figure 4.66. Sensors embeded in a smart device................................ 159

Figure 4.67. Network Topology ............................................ 163 
Figure 4.68. Alarm levels and propagation as a function of time as a function of the time.

Figure 4.69. Message flow between devices.

Figure 4.70. Collaborative decision algorithm.

Figure 4.71. Compass measurements for 4 devices.

Figure 4.72. Sound levels for 4 devices.

Figure 4.73. GPS measurements for 4 devices.

Figure 4.74. Light intensity measurements for 4 devices.

Figure 4.75. Gyroscope measurements in $\mathrm{X}$ axis for 4 devices.

Figure 4.76. Gyroscope measurements in $\mathrm{Y}$ axis for 4 devices.

Figure 4.77. Gyroscope measurements in $\mathrm{Z}$ axis for 4 devices.

Figure 4.78. Accelerometer measurements in $X$ axis for 4 devices.

Figure 4.79. Accelerometer measurements in $Y$ axis for 4 devices.

Figure 4.80. Accelerometer measurements in $\mathrm{Z}$ axis for 4 devices.

Figure 4.81. State of compass alarm.

Figure 4.82. State of microphone alarm

Figure 4.83. State of GPS alarm.

Figure 4.84. State of light alarm.

Figure 4.85. State of gyroscope Alarm

Figure 4.86. State of Accelerometer alarm.

Figure 4.87. Combined alarm for GPS and Gyroscope sensors

Figure 4.88. Combined alarm for GPS and Accelerometer sensors.................... 175

Figure 4.89. Combined alarm for compass and GPS sensors......................... 176

Figure 4.90. Combined alarm for GPS and microphone sensors..................... 176

Figure 4.91. Topology used in our simulations................................ 177

Figure 4.92. Volume of packets in the network, for the four protocols as a function of the time

Figure 4.93. Volume of kilobits in the network, for the four protocols as a function of the time.

\section{Chapter 5}

Figure 5.1. Map of Higher Polytechnic School of Gandia

Figure 5.2. Ground floor of the CRAI bulilding. 
Figure 5.3 First floor of the CRAI building.................................... 185

Figure 5.4 Second floor of the CRAI building................................. 185

Figure 5.5. Radio coverage map of the ground floor for UPVNET ................... 187

Figure 5.6. Radio coverage map of the ground floor for UPVNET2G............... 187

Figure 5.7. Radio coverage map of the ground floor for EDUROAM .................. 188

Figure 5.8. Radio coverage map of the first floor for UPVNET ...................... 188

Figure 5.9. Radio coverage map of the first floor for UPVNET2G.................. 189

Figure 5.10. Radio coverage map of the first floor for EDUROAM.................... 189

Figure 5.11. Radio coverage map of the second floor for UPVNET ................... 190

Figure 5.12. Radio coverage map of the second floor for UPVNET2G................. 190

Figure 5.13. Signal strength on the ground floor................................... 191

Figure 5.14. Signal strength on the first floor................................... 191

Figure 5.15. Signal strength on the second floor................................... 192

Figure 5.16. Radio coverage map of the second floor for EDUROAM.................. 192

Figure 5.17. Average signal strength on the ground floor........................... 193

Figure 5.18. Average signal strength on the first floor................................. 194

Figure 5.19. Average signal strength on the second floor.............................. 194

Figure 5.20. Relocation of APs on the ground floor................................... 195

Figure 5.21. Estimated signal strength on the ground floor for the proposed AP

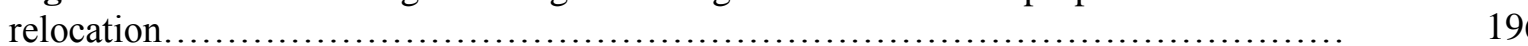

Figure 5.22. Relocation of APs on the first floor...................................... 196

Figure 5.23. Estimated signal strength on the first floor for the proposed AP relocation

Figure 5.24. Relocation of APs on the second floor.................................. 197

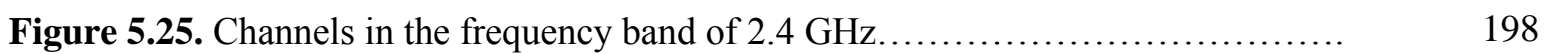

Figure 5.26. Scenario and APs placement........................................ 200

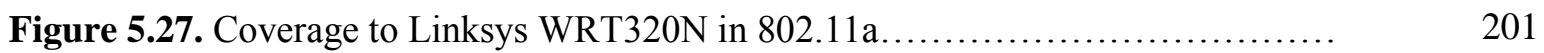

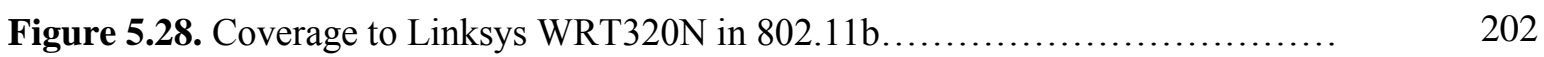

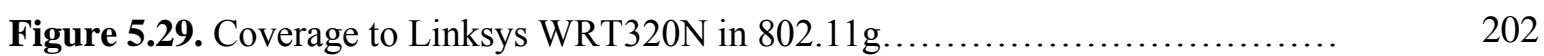

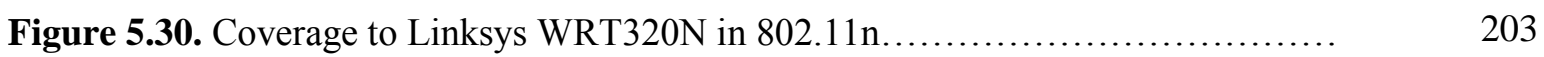

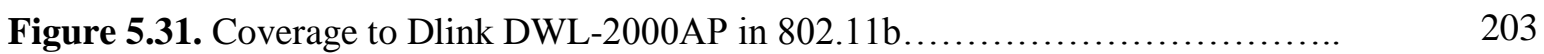

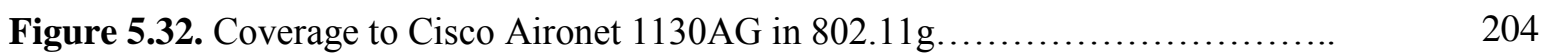

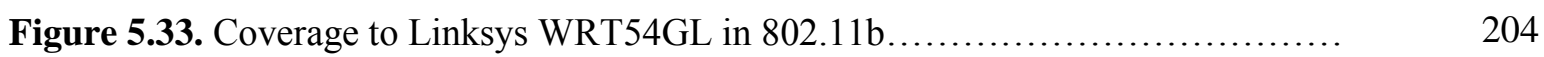

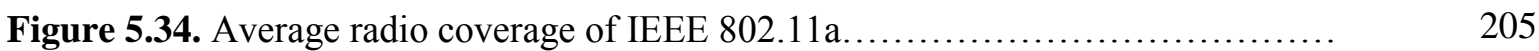




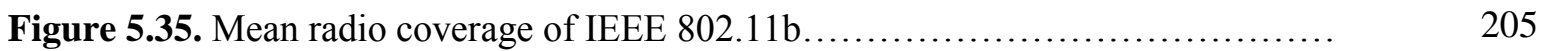

Figure 5.36. Average radio coverage of IEEE 802.11g........................... 206

Figure 5.37. Mean radio coverage of IEEE 802.11n................................. 206

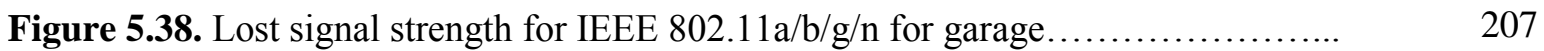

Figure 5.39. Network topology for interference measurements....................... 208

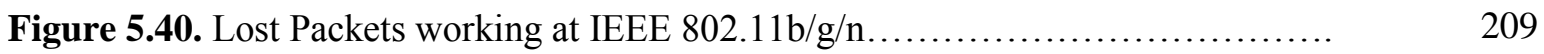

Figure 5.41. Lost Packets working at IEEE 802.11a................................ 210

Figure 5.42. Bandwidth Consumption for all devices............................ 210

Figure 5.43. Throughput in $\%$ for all devices.................................. 211

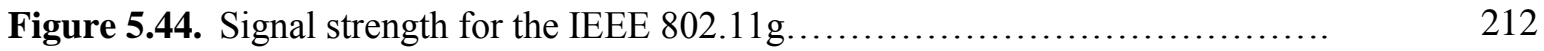

Figure 5.45. Behavior for four variants in CRAI................................ 212

Figure 5.46. Sensor distribution in a building and the estimated values of signal strength

\section{Chapter 6}

Figure 6.1. Swimming pool where measures have been taken........................ 222

Figure 6.2. Packet flow diagram............................................. 222

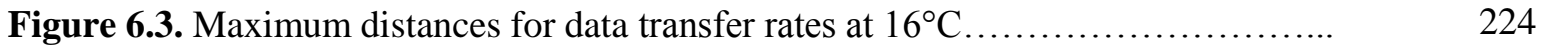

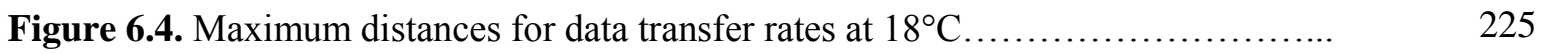

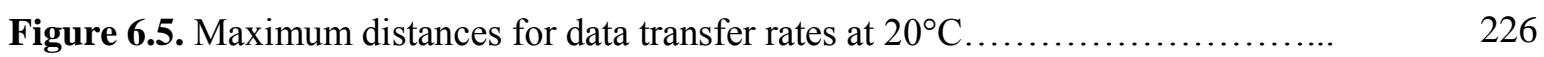

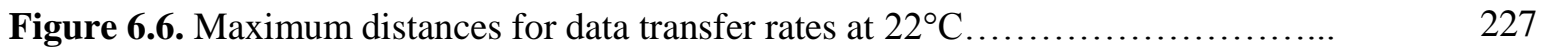

Figure 6.7. Average RTT and maximum distance for $1 \mathrm{Mbps}$ at $26^{\circ} \mathrm{C} \ldots \ldots \ldots \ldots \ldots \ldots . \ldots . \ldots$

Figure 6.8. Average RTT and maximum distance for $2 \mathrm{Mbps}$ at $26^{\circ} \mathrm{C} \ldots \ldots \ldots \ldots \ldots \ldots . . \ldots$

Figure 6.9. Average RTT and maximum distance for $5.5 \mathrm{Mbps}$ at $26^{\circ} \mathrm{C} \mathrm{\ldots .............} 230$

Figure 6.10. Average RTT and maximum distance for $11 \mathrm{Mbps}$ at $26^{\circ} \mathrm{C} \ldots \ldots \ldots \ldots \ldots . . . . . . .231$

Figure 6.11. Estimated maximum distances for BPSK modulation........................ 236

Figure 6.12. Estimated maximum distances for QPSK modulation................... 236

Figure 6.13. Estimated maximum distances for OFDM modulation.................... 237

Figure 6.14. Estimated maximum distances for CCK transmission scheme............... 237

\section{Chapter 7}


Figure 7.1. Total production of fish from aquaculture in the world.................. 248

Figure 7.2. European total production of fish from aquaculture in $2010 \ldots \ldots \ldots \ldots \ldots \ldots . \ldots$

Figure 7.3. Marine fish cage with a sensor network for monitoring the fishes activity... 251

Figure 7.4. Carbon emision from the cage................................... 253

Figure 7.5. Sedimentation for $\delta T=15$, in a fish farm with 6 cages. ................... 253

Figure 7.6. Operation diagram of an ultrasonic sensor............................. 254

Figure 7.7. Radiation diagram for UM18-X111X sensor series..................... 255

Figure 7.8. Maximun sensed area by one sensor................................. 255

Figure 7.9. Wireless Sensor node movement....................................... 256

Figure 7.11. Movement in the vertical plane..................................... 257

Figure 7.12. Movement in the Horizontal plane..................................... 257

Figure 7.13. Sensor density as a function of distance to the center of the cage........... 258

Figure 7.13. Esquematic of our low cost turbidity sensor........................... 260

Figure 7.14. Value of dissolution turbidity as a function of the dissolution

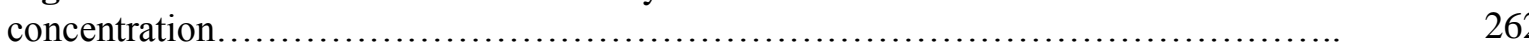

Figure 7.15. Value of dissolution concentration as a function of the amount of solute..

Figure 7.16. Output voltage as a function of the turbidity value and our mathematical

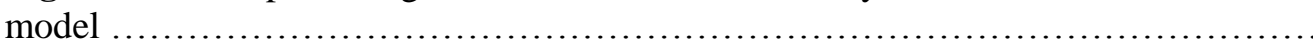

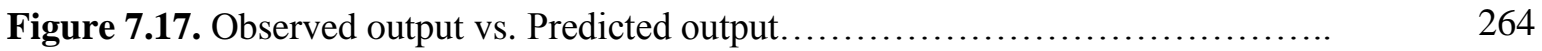

Figure 7.18. Output voltage value for the verification samples...................... 265

Figure 7.19. System performance for regular procedure in winter and summer.......... 266

Figure 7.20. System performance for our system in winter and summer............... 267

Figure 7.21. Pellets in the seabed for a regular procedure in winter and summer........ 268

Figure 7.22. Pellets in the seabed for our system in winter and summer................ 268

\section{Chapter 8}

There are not figures in this chapter. 


\section{List of Tables}

\section{Chapter 1}

Table 1.1. Comparison of wireless technologies

\section{Chapter 2}

Table 2.1. Sensor Classification........................................... 13

Table 2.2. Constant Value.......................................................... 39

Table 2.3. Measures and correlation between disc and nephelometer.................. 50

\section{Chapter 3}

Table 3.1. Devices consumption comparison. ................................. 58

Table 3.2. Considerations in WSNs........................................... 63

Table 3.3. Differences between cluster-based and group-based networks................. 66

Table 3.4. MAC Protocols....................................................... 74

Table 3.5. Routing Protocols.............................................. 85

Table 3.6. Hardware features for all wireless access points. ........................ 87

\section{Chapter 4}

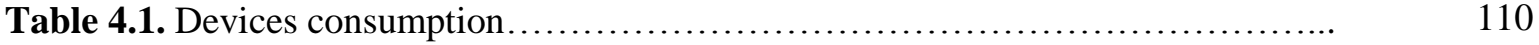

Table 4.2. Available hardware comparison for sensor nodes......................... 121

Table 4.3. Energy consumption comparison................................... 123

Table 4.4. Weight and energy requirements comparison........................... 123

Table 4.5. Cameras comparison ............................................... 124

Table 4.6. Image sizes comparison ........................................ 125

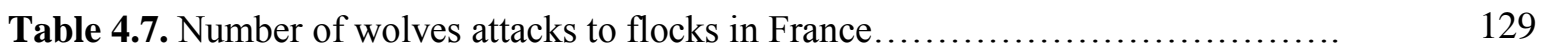

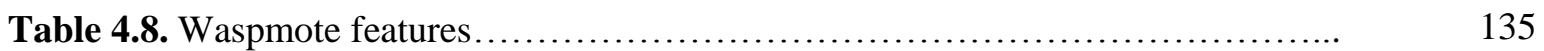

Table 4.9. Features of wireless modules.......................................... 143 
Table 4.10. Relative humidity and its resistant value

Table 4.11. Errors and thresholds of the sensors

Table 4.12. Decision rules................................................ 174

Table 4.13. Comparison of the routing protocol's principal features

\section{Chapter 5}

Table 5.1. APs used for our tests.......................................... 184

Table 5.2. Channel distribution for the ground floor.............................. 198

Table 5.3. Channel distribution for the first floor................................... 199

Table 5.4. Channel distribution for the second floor.............................. 199

Table 5.5. Equations that estimate the initial value in our studied technologies.......... 214

Table 5.6. Equations estimating the signal strength of the first stage value............... 214

Table 5.7. Equations estimating the signal strength of the second stage................ 215

\section{Chapter 6}

Table 6.1. Comparison of different wireless standards............................... 221

Table 6.2. Modulations of IEEE 802.11b/g standard used in our tests.................... 223

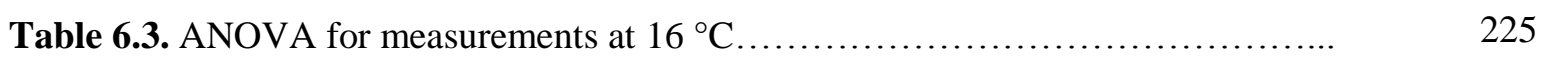

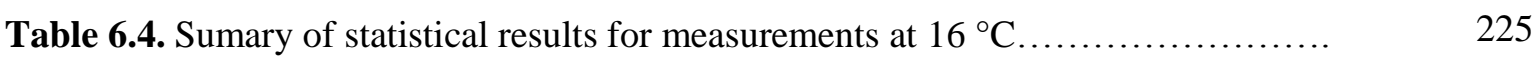

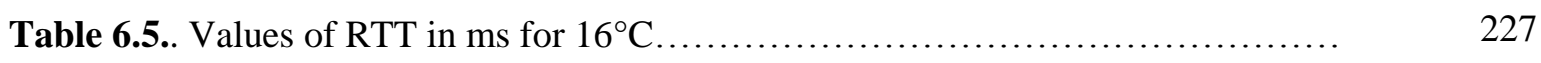

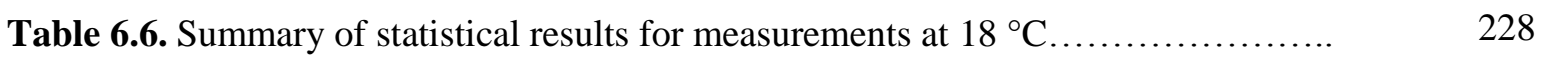

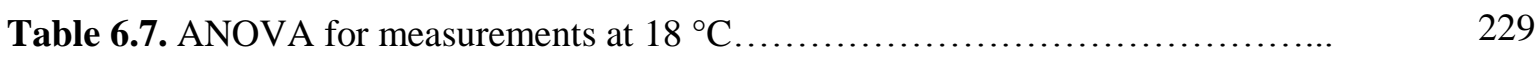

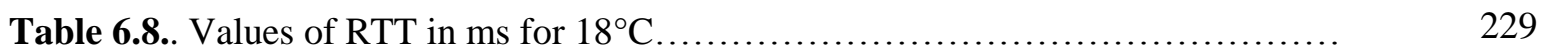

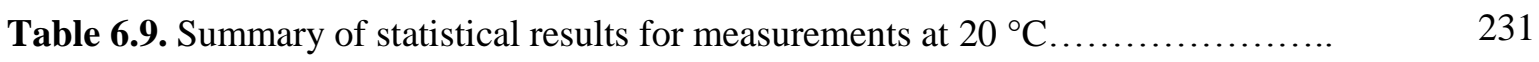

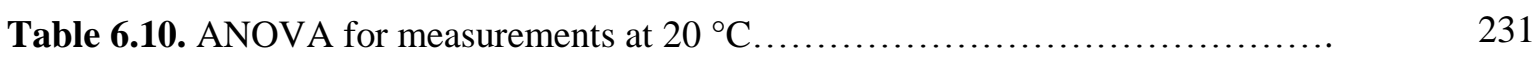

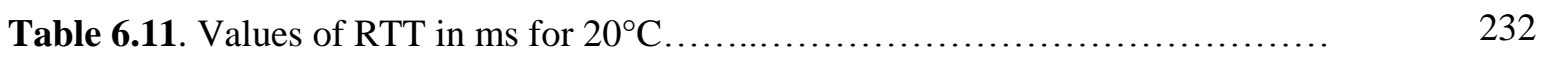

Table 6.12. Summary of statistical results for measurements at $22{ }^{\circ} \mathrm{C} \ldots \ldots \ldots \ldots \ldots \ldots . . \ldots \ldots$

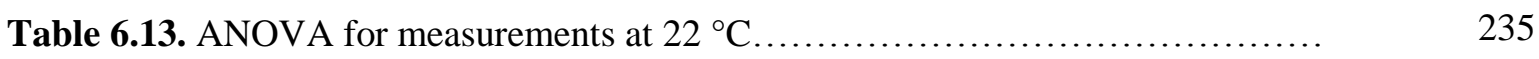

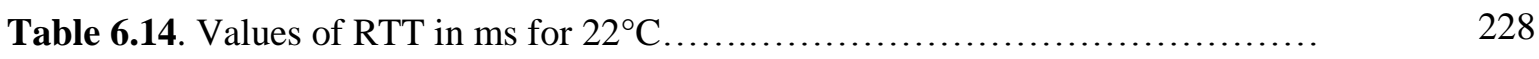

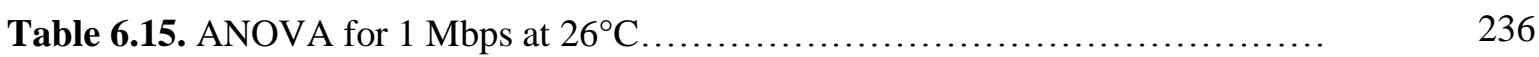

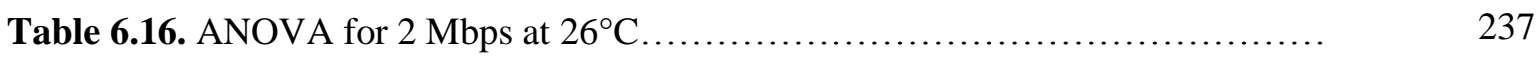

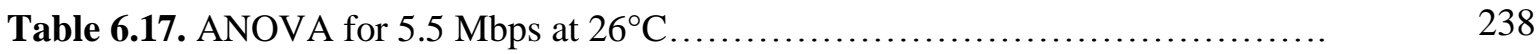




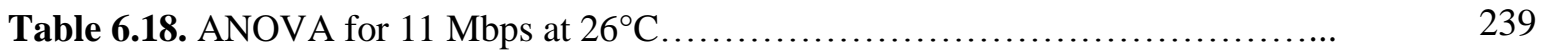

Table 6.19. Summary of best results........................................ 240

Table 6.20. Comparison of best results in several studies......................... 241

\section{Chapter 7}

Table 7.1. Density of sensors in each area...................................... 250

Table 7.2. Price of main components......................................... 253

Table 7.3. Samples, their concentration and turbidity............................ 254

Table 7.4. Unknown samples............................................. 257

Table 7.5. Verification results.................................................... 257

\section{Chapter 8}

There are not tables in this chapter. 


\section{Acknowledgments}

I think that in a Thesis, it is not only important our capabilities. To carry out a Thesis, it is necessary to have good support from your friends, family and supervisors. For this reason, I would like to thank all those who have been helping me along this time.

Firstly, I would like to thank to my parents Jose Maria and Maria Jose and my brother Josep for all their unconditional support, patience and advices given to me over the years.

I appreciate the important tasks that my directors Jaime Lloret, Miquel Ardid and José Francisco Toledo, have done with their support and advices.

Thank to all my colleagues at the Communications \& Remote Sensing research laboratory and at the "Escuela Politécnica Superior de Gandia - UPV", for their ideas on research topics, and for the personal and technical support.

Thanks Carlos D. Llorens for all your tips about electronic.

Finally, I would like to make special mention to Jaime Lloret who besides being my advisor and supervisor on several occasions, he has become a good friend and colleague. Thank you very much to open me the door to this experience and invite me to see the world of research. Thank you so much for believing in me and in my work when others did not do it. I will never forget all you have done for me.

\section{Agradecimientos}

Creo que en una Tesis, no solo son importantes nuestras capacidades. Para realizar una tesis, es necesario tener el soporte de familiares, amigos y directores. Por esta razón, me gustaría agradecérselo todos aquellos que de un modo u otro me han estado ayudando todo este tiempo.

En primer lugar, me gustaría dar las gracias a mis padres, José María y María José y mi hermano Josep todo su incondicional apoyo, la paciencia y los consejos que me han dado en los últimos años.

Aprecio mucho las importantes labores que mis directores Jaime Lloret, Miquel Ardid y José Francisco Toledo, con su apoyo y consejos.

Gracias a mis compañeros de la línea de investigación de "Comunicaciones y Teledetección” en la "Escuela Politécnica Superior de Gandia - UPV", por sus ideas sobre temas de investigación, y por el apoyo personal y técnico.

Gracias Carlos D. Llorens por todos sus consejos sobre electrónica.

Por último, me gustaría hacer una mención especial a Jaime Lloret que además de ser mi tutor y director en varias ocasiones, se ha convertido en un buen amigo y compañero. Muchas gracias, por abrirme la puerta a esta experiencia e invitarme a conocer el mundo de la investigación. Muchas gracias por creer en mí y en mi trabajo cuando otros no lo hicieron. Nunca olvidaré todo lo que has hecho por mí. 



\section{Chapter 1}

\section{Introduction}

\subsection{Introduction}

A Wireless Sensor Network (WSN) can be defined as a network of small embedded devices, called sensors, which communicate wirelessly following an ad hoc configuration. They are located strategically inside a physical medium and are able to interact with it in order to measure physical parameters from the environment and provide the sensed information [1][2].

The nodes should be small size devices in order avoid causing any visual impact to the ecosystem and they should not consume too much energy. We should also keep in mind that the nodes have limited their data process and storage capacity. We cannot forget that WSNs should implement a security system in order to avoid intrusions, as any other network. The typical structure of a WSN usually has sensor nodes. They are radio processors that take the sensor data (physical magnitude values of the medium) and send the information to the base station. The base station is the data gatherer which is usually a common computer or embedded system with higher process capacity. The gateway is the interconnection between the sensor network and the data network.

It is important to define the amount of sensors and the best position of them for sensing the environment or tanking measurements from the surrounding areas. Sensor nodes need to communicate between them. For this reason, it is also essential to know the behavior of signal in each medium, their scalability [3] and their connection strategy for communication [4].

WSNs are considered spontaneous networks because they are formed dynamically depending on the connectivity between nodes, their position and nodes drop due to failures. These kinds of 
networks are characterized as being easy to be deployed and self-configuring. We can highlight the following features in WSNs:

- Dynamic topologies: In a wireless sensor network, the topology is always changing because nodes can fail or new nodes can join the network. These changes affect the communication between sensors.

- Variability channel: The radio channel is highly variable. There are several phenomena, such as the attenuation, fast fainting, slow fainting and interference that can cause data errors.

- Ad hoc networks: Generally, sensor networks do not have a wired network infrastructure. All motes or nodes are transceivers and routers simultaneously. However, the concept of sink node is important; this node collects the information and sends it to a central computer capable of processing these data.

- Failure tolerance: A sensor node should be able to continue operating despite of the existence of errors in the system.

- Multi-hop or broadcast communications: This type of networks use any routing protocol to enable communications multi-hop, although it is also very common the use of messages sent in broadcast.

- Power saving: It is one of the most important features in these networks. Currently, the motes have limited energy. A sensor node should have an ultra low consumption processor and transceiver radio. It is one of the most restrictive features.

- Limited hardware: In order to get an adjusted consumption, the hardware should be simple; this brings a limited process capacity.

- Production costs: Sensor networks are formed by high number of nodes. Motes must be economic to create a reliable network.

Despite the great evolution of these networks, it is always possible to improve the efficiency of these networks, their operation and the interaction with the environment.

Sensors nodes are able to gather data from the environment. We only need to choose the adequate transducer. Sensor networks can be useful in many application areas, such as habitat monitoring, fire detection, personal tracking, animal monitoring, reservoir water controlling, or intruders controlling, among others.

This dissertation focuses the deployments in four main areas. Each application area presents particular characteristics:

\section{Rural and natural monitoring:}

- Application where a scientist wants to collect measurements of inaccessible and hostile environment over a period of time to detect changes, trends, etc.

- Energy constrains.

- It is needed a large number of nodes by measuring and transmitting synchronized periodically.

- It is necessary to have high life time of the WSN.

- The coordination of information needs synchronization in WSN.

- It usually presents a relatively stable physical topology. 
- No strict latency requirements.

- This type of networks does not need frequent network reconfiguration.

\section{Building monitoring:}

- This kind of applications needs a continuous sensing.

- But nodes do not send continuous data.

- Low energy consumption.

- Importance of the "status" of a node.

- Real-time requirements: the importance of communication latency.

\section{Terrestrial animal monitoring and tracking systems:}

- Application to control animals that are labeled with sensor nodes in a given region.

- Despite of the rest of applications, the topology of these networks is very dynamic, due to the continuous movement of the sensor nodes.

- Energy constrains.

- The WSN should be able to discover new nodes and form new topologies.

\section{WSN for underwater environments:}

- Underwater communications can be based on the use of optical signals, electromagnetic signals and the propagation of acoustic and ultrasonic signals

- Great reliance water parameters.

- Energy constrains.

- High attenuation due to the conductivity of the water.

- The performance of these networks depends on the technology used.

In many occasions, the sensor deployment can be very expensive, such as oceanographic sensors. Due to the big quantity on sensors which can be necessary to monitor a zone, the price of a WSN can be very high.

It is possible to use several wireless technologies to implement the communications between nodes. The main wireless technologies used in WSN are the following.

Bluetooth is an open specification for wireless networks based on radiofrequency that operates in the Industrial Scientific Medical (ISM) frequency band $(2.4 \mathrm{GHz})$ forming wireless personal area networks (WPAN) [5]. It has low energy consumption and its cost is quite low. Bluetooth controls its interference and susceptibility to interference by using spread spectrum modulation. It uses a frequency hopping spread spectrum signalling method (FHSS). Bluetooth was formalized in IEEE 802.15.1 standard in its version 1.2. Bluetooth technology uses a small area network without infrastructure (piconets). Nodes share a physical channel with a clock and a unique sequence of jumps in the same piconet. In Bluetooth different channels can coexist. While a master can belong only to one piconet, any other device can belong at the same time to several piconets. This overlap is denominated scatternet (dispersed network), although there are not defined routing capacities among them. This technology is developed to be used for the devices interconnection like computers, mp3, PDAs, etc. at a distance of about $10 \mathrm{~m}$, although lasts products in the market 
achieve $100 \mathrm{~m}$. of coverage radius. Nevertheless, this coverage range can be greater having higher antenna gain and bigger distances can be gotten using signal repeaters.

ZigBee is an alliance, without spirit of lucre, of more than 100 companies, where most of them are semiconductors makers. ZigBee and IEEE 802.15.4 are standards-based protocols that provide the network infrastructure required for wireless sensor network applications [6]. 802.15.4 defines the physical and MAC layers, and ZigBee defines the network and application layers. This technology is focused to create low-rate personal area networks (LR-WPAN). Their objectives are to develop and to implant a wireless technology of low energy consumption and low cost. It is presented as a very economic solution for Wireless Sensor Networks. It is also destined towards energy management, automation and remote control applications. ZigBee products work in a band of frequencies that includes $2.4 \mathrm{Ghz}$, using the modulation OR-QPSK with expansion DSSS, and $868 \mathrm{Mhz}$ in Europe or $915 \mathrm{Mhz}$ in North America with the modulation BPSK with expansion DSSS. Its energy consumption is very low; it consumes 20 times less energy than IEEE 802.11 standard and their implementation is relatively simple. On the contrary, their typical coverage range is around $50 \mathrm{~m}$, although this value could increase, depending on the environment.

Inside the IEEE 802.11 standard [7], standard variants like IEEE 802.11a, IEEE 802.11b, IEEE 802.11g and IEEE 802.11n are included among others. The original standard also defines the CSMA/CA protocol as the access method. CSMA/CA is a carrier sense and multiple access method used to avoid collisions among the data frames. The $2.4000-2.4835 \mathrm{GHz}$ band is divided into 13 channels each of width $22 \mathrm{MHz}$ but spaced only $5 \mathrm{MHz}$ apart, with channel 1 centred on $2.412 \mathrm{GHz}$ and 13 on $2.472 \mathrm{GHz}$ to which Japan adds a 14th channel $12 \mathrm{MHz}$ above channel 13. The availability of channels is regulated by country, constrained in part by the allocated radio spectrum to various services. IEEE $802.11 \mathrm{~b}$ data is encoded using direct sequence spread spectrum signaling (DSSS) technology. It uses CCK (Complementary Code Keying) modulated with the QPSK technology to achieve a maximum raw data rate of $11 \mathrm{Mbit} / \mathrm{s}$. IEEE $802.11 \mathrm{a} / \mathrm{g}$ use orthogonal frequency division multiplexing (OFDM) methods with maximum 52 sub-carriers, achieving a maximum raw data rate of $54 \mathrm{Mbps}$. IEEE 802.11n is a new standard for wireless communications that presents a significant increase in the maximum raw to a maximum theoretical value of 600 Mbit/s. The current state of the art supports a PHY rate of $450 \mathrm{Mbit} / \mathrm{s}$, with the use of 3 spatial streams at a channel width of $40 \mathrm{MHz}$. Furthermore, IEEE 802.11n uses MIMO (multiple-input multiple-output); this is the utilization of multiple transmitter and receiver antennas to improve the system performance. MIMO technology provides Spatial Division Multiplexing (SDM) where multiple independent data streams are transferred simultaneously. This causes that data rate and coverage area increases, in contrast with the other variants. Basically, 802.11 can work in two ways; the ad-hoc mode, when the stations communicate to each other directly, and Infrastructure mode, in which the stations access to the network through one or several access points. Its coverage can reach more than 300 meters. The effective overall range of $802.11 \mathrm{a}$ is less than that of $802.11 \mathrm{~b} / \mathrm{g}$. In theory, 802.11a signals are absorbed more readily by walls and other solid objects in their path due to their smaller wavelength and, as a result, cannot penetrate as far as those of $802.11 \mathrm{~b}$.

The main problem of Bluetooth and IEEE 802.11 technology in order to become a wearable technology at level of sensors network is the complexity of the protocol and the topology rigidity of the networks, as well as the high consumption of power of the devices. Depending on the case, in our implementation we could need high coverage range than the one offered by Bluetooth or 
Zigbee, while very small energy consumption is required. Although many wireless sensor networks developers have adopted Bluetooth and Zigbee technologies for sensors communication, we usually prefer to use the IEEE 802.11 standard. We can see their differences in Table 1.1.

Table 1.1. Comparison of wireless technologies

\begin{tabular}{|c|c|c|c|}
\hline & WLAN (IEEE 802.11) & Bluetooth (802.15.1) & Zigbee (802.15.4) \\
\hline $\begin{array}{l}\text { Frequency } \\
\text { Bands }\end{array}$ & $\begin{array}{c}5 \mathrm{GHz}(\mathrm{a} / \mathrm{n}) \\
2.4 \mathrm{GHz}(\mathrm{b} / \mathrm{g} / \mathrm{n})\end{array}$ & $2.4 \mathrm{GHz}$ & $\begin{array}{c}2.4 \mathrm{GHz} \\
868 / 915 \mathrm{MHz}\end{array}$ \\
\hline $\begin{array}{l}\text { Raw Data } \\
\text { Rate }\end{array}$ & $\begin{array}{c}11 \mathrm{Mbps}(2.4 \mathrm{GHz} \text { in } \mathrm{b}) \\
54 \mathrm{Mbps}(2.4 \mathrm{GHz} \text { in } \mathrm{g} \text { or } 5 \mathrm{GHz} \text { in a) } \\
500 \mathrm{Mbps}(2.4 \mathrm{GHz} \text { and } 5 \mathrm{GHz} \text { in } \mathrm{n})\end{array}$ & $1 \mathrm{Mbps}$ & $\begin{array}{l}250 \mathrm{Kbps}(2.4 \mathrm{GHz}) \\
40 \mathrm{Kbps}(915 \mathrm{MHz}) \\
20 \mathrm{Kbps}(868 \mathrm{MHz})\end{array}$ \\
\hline $\begin{array}{l}\text { Number of } \\
\text { channels }\end{array}$ & $\begin{array}{l}\text { 11-14 (depends on the country in b/g/n), } \\
\text { working at } 2.4 \mathrm{GHz} \text {. } \\
24 \text { non-overlapping } 20 \mathrm{MHz} \text { channels } \\
\text { and up to } 12 \text { non-overlapping } 40 \mathrm{MHz} \\
\text { channels, working at } 5 \mathrm{GHz} \text { in } \mathrm{n} . \\
52 \text { channels in a }\end{array}$ & 79 & $\begin{array}{c}16(2.4 \mathrm{GHz}) \\
10(915 \mathrm{MHz}) \\
1(868 \mathrm{MHz})\end{array}$ \\
\hline Data types & Digital & Digital, Audio & $\begin{array}{c}\text { Digital, Key-Value } \\
\text { Pairs }\end{array}$ \\
\hline $\begin{array}{l}\text { Coverage } \\
\text { Range }\end{array}$ & $100 \mathrm{~m}-300 \mathrm{~m}-900 \mathrm{~m}$ & $10 \mathrm{~m}-100 \mathrm{~m}$ & $30 m-50 m$ \\
\hline $\begin{array}{l}\text { Battery } \\
\text { Autonomy }\end{array}$ & Hours & Days & Years \\
\hline Architecture & Star & Star & Star, tree, cluster \\
\hline $\begin{array}{c}\text { Best } \\
\text { Applications }\end{array}$ & Internet Inside building & $\begin{array}{l}\text { Computer and phone } \\
\text { peripherals }\end{array}$ & $\begin{array}{l}\text { Low-cost control } \\
\text { and monitoring }\end{array}$ \\
\hline
\end{tabular}

For all of these reasons, the main motivation of this Thesis is to create low cost sensor nodes for monitoring different environments. The use of these wireless sensor nodes will facilitate us the interaction with the environment and in the case of people monitoring, WSN will reduce the reaction time, if someone have suffered some mishap. To achieve our goals, we decide to use commercial components and the most appropriate wireless technology for each case.

\subsection{Research goals}

In this subsection, we present the objectives and the main steps followed to perform our proposals which are to implement energy efficient sensor nodes and sensor networks for monitoring different environments.

We think that the use of WSN can improve the operation of current systems. In order to develop our idea and improve the existing systems, we must carry out the following tasks:

1. Analyzing the need of improving the existing systems for environmental monitoring.

- Studying current monitoring systems. 
- Analyzing the deficiencies in current monitoring systems.

2. Defining the features that a wireless sensor node should present.

- Studying the hardware characteristics that nodes must have.

- Defining energy constraints that nodes may have from different perspectives.

- Simulating and establishing the energy consumption of a node depending on the amount of information transmitted, protocol type, mode of operations and hardware features.

3. Deploying our sensor node.

- Analyzing the type of sensor that can be found and how it interacts with the medium.

- Designing the proposed systems.

- Developing and programming the algorithms for the correct processing of measurable parameters.

4. Improving the network design and sensor positioning techniques.

- Analyzing the current systems for sensor positioning.

- Studying the performance of our devices in real environments.

- Defining the new process of positioning sensors.

- Comparing our results with existing systems to demonstrate the feasibility of our method.

5. Adapting our system in order to be used in real environments.

- Adapting sensor nodes for using them in the medium.

- Simulating the network's behavior when it is being used in the real environment.

- Verification of operation for developed prototypes.

\subsection{Precedents and motivation.}

The idea to create sensor nodes to monitor different environments is not entirely new. We can find several examples about this. However, most applications which are proposed here and the conclusions drawn from each study are very interesting and novelty.

The starting point of this Thesis was my Master's Thesis and an agreement with the company "La maquinista Valenciana S.A." which we proposed the development of a multi-sensor node capable of processing information of sensors placed on buoys and sending it over the wireless network. After seeing the results of this prototype, we decided to extend the application areas of these networks.

While this Thesis was being done, one of my colleagues in my research group was developing another Thesis which proposed a group-based architecture and protocol for wireless sensor networks [8]. In a sensor network, it is important how to communicate each device, but is also very important the device characteristics and the way they interact with the sensor. 
With this proposal, we could communicate our devices. For this reason, we started to investigate the physical principles in which sensors are based and the main needs which can be solved with this.

The main applications can be found in the rural and natural environment where the integration of technology can help us to improve the sustainability of the economy in marine farms, agriculture and livestock.

The investigations related to underwater environment have been partially supported by four research projects:

- Project: "Red Cognitiva Basada en Grupos de Sensores Colaborativos para el Sensado y Monitorización del Entorno Acuático", "Ministerio de Ciencia e Innovación", through the "Plan Nacional de I+D+i 2008-2011" in the "Subprograma de Proyectos de Investigación Fundamental". Project reference: TEC2011-27516.

- Project: "Desarrollo de un Sensor Subacuático Modular Multipropósito con Altas Tasas de Transferencia", "Universidad Politécnica de Valencia", through the PAID-05-11 multidisciplinary projects.

- Project: "Desarrollo de Sensores Subacuáticos para la Monitorización del Comportamiento de los Peces con el Objetivo de Mejorar la Productividad y Sostenibilidad de una Granja Marina”, "Universidad Politécnica de Valencia”, through the PAID-05-12 multidisciplinary projects.

- Project: "Participación del IGIC en los Telescopios de Neutrinos de Antares y KM3NET", "Ministerio de Ciencia e Innovación", through the "Plan Nacional de I+D+i 2010-2012" in the "Subprograma de Proyectos de Investigación Fundamental no orientada". Project reference: FPA2009-13983-C02-02.

We have also found unsolved needs in the human life where the use of WSNs implies an improvement in the quality of life of some persons.

Finally, to develop efficient WSNs, it is also interesting to know how many sensors would need to cover an area and where to place them. We think that technology should be available to everyone. We believe that it is interesting to investigate about the development of sensor nodes which take into account the limitations of energy which can be found in natural environments.

There are several works related to this Thesis but all of them have been used to research new concepts about sensors deployment and its new applications in real environments. For this reason, these works will be referenced in their corresponding chapters in order to introduce ideas or solution in each chapter.

\subsection{Main Contributions}

In this section we are going to show a summary about every contribution performed on behalf this Doctoral Thesis.

This Thesis provides the following scientific contributions:

\section{Identification and analysis of main energy problems that wireless sensor networks present.}


- Detailed analysis of energy problems that nodes may occur.

- Mathematical modelling of energy consumption in nodes depending on the hardware and the amount of information transmitted on the network.

- Analysis of energy consumption of a network as a function of topology, protocol type, network device type and characteristics of network devices.

\section{Contribution to the development of low cost sensor nodes for environmental monitoring.}

- Analysis of needs to integrate new technologies with the environment and the benefits that they entail.

- Customizing the hardware to the application needs, reducing the energy consumption to increase network lifetime and improve its stability.

- Design and development of low cost systems.

- Adding intelligence to the nodes for improving their performance.

Methodology for designing wireless networks and sensor positioning in indoor environments.

- Analysis of the actual behavior of wireless signals (for each standard) in indoor environments.

- Actual interference analysis of IEEE 802.11 in indoor environments.

- Proposal of a new method of wireless network design and sensor positioning which reduces by $15 \%$ the number of needed sensors.

Contribution to the evaluation of underwater communications based on electromagnetic waves.

- Analysis of underwater freshwater communications depending on the type of modulation, type of data transfer rate and working frequency.

- Determining the dependency of underwater freshwater communications with the temperature.

- Comparison and verification of actual measurements with existing theoretical models.

- Mathematical modelling of underwater freshwater communications based on measured parameters.

Contribution to the design and development of low-cost oceanographic sensors for improving sustainability in marine fish farms.

- Developing a deposition monitoring sensor and a low cost turbidity sensor for monitoring the parameters of the water in fish farms.

- Determining the density of sensors needed from uneaten and feces depositions in seabed.

- Verification by simulation of the differences between the amount of food thrown into the cage with traditional systems and with our food dispensing proposal based on fish behavior monitoring. 


\subsection{Structure of the dissertation}

Considering the problems that currently exist in the field of environment monitoring which could be solved by WSN, the issues in the implementation of sensor nodes and goals of this dissertation, the rest of document is structured as follows.

In Chapter 2, we present the state of the art of the environmental monitoring by using WSN. First of all, we define what a sensor node is and what kind of sensors/transducers we can find. Because energy limitations are important, this chapter presents some of the main widely used energy conservation techniques. These can be divided depending on the routing or MAC-protocols, the operation mode of the nodes and the radio system. We have also investigated the use of WSN in rural monitoring and studies about measurements and wireless sensor positioning in indoor environments. Finally, we show the main investigations about underwater wireless communications and the applications of WSNs in marine environment.

Chapter 3 analyzes the most important aspects to be considered when we want to implement a sensor node. In this chapter, we present the main features and requirements of a wireless sensor node and a selection of the most used commercial wireless nodes. In addition, we present the main issue which can be found in nodes, considering only the hardware features. From the characteristics of these nodes, we define the analytical expression of energy consumption in wireless nodes as a function of time. After that, we compare the features of several routing and MAC protocols which implement saving energy techniques. Finally, we carry out a practical study about the power consumption for wireless access points from different manufacturers with different topologies and configurations. All of these analysis and studies allow us to define the optimal parameters to achieve a reduction in energy consumption in these devices to develop energy efficient communications networks.

Sensors and wireless networks can be used in several application fields. For this reason, from this point, each chapter presents the study of wireless communications in an environment and the development, the network implementation and verification of our system in various applications.

In Chapter 4, we present several wireless sensor deployments for monitoring a medium. It presents two groups of applications. The first group includes an optimized Wireless IP multisensor Network to detect and locate the focus of the fire. The interesting part of this system it that it is able to verify the existence of fire by means of images. The system can be used to monitor fires in wide extension fields of rural, agricultural and forest using the Wireless Local Area Network (WLAN) technology. The second deployment is a WSN that uses an image processing system in each wireless node to distinguish if a vine has bad leaves or not. Finally, we use the IEEE 802.15.4 technology to deploy a WSN to detect wolves' attacks in flocks. To process the data from network, the system uses a smart algorithm which decides what kind of alarm has to be generated. The second group of applications presents a non-invasive technique based on a WSN for the detection of cement degeneration in constructions and a WSN to take care of disabled and elderly people.

In Chapter 5, we show the development of propagation models which describe the signal behavior for IEEE $802.11 \mathrm{a} / \mathrm{b} / \mathrm{g} / \mathrm{n}$ networks in indoor environments. To achieve the goal of this Chapter, we analyze the signal strength level in two buildings of different size. The first scenario is the library of the "Escuela Politécnica Superior de Gandia", the surface of which is approximately $2000 \mathrm{~m}^{2}$. The second scenario is a garage which is approximately $100 \mathrm{~m}^{2}$. We also perform a study of the interference that these wireless devices can suffer and network performance which is 
obtained in depending on the working channel. The measures are analyzed as a function of the type of device and the standard type. Finally, from the results of both scenarios, we propose a new method for wireless sensor nodes positioning which reduces in $15 \%$ the number of nodes required to cover an area.

One of the main problems in underwater communications is the low data rate available due to the use of low frequencies. Moreover, there are many problems inherent to the medium such as reflections, refraction, energy dispersion, etc., that greatly degrade communication between devices. We can use acoustic waves, optical waves and Electromagnetic (EM) waves to implement an underwater link. Each technique has its own characteristics with benefits and drawbacks, mainly due to the chemical characteristics and physical constraints of the medium. But due to the conducting nature of water, the underwater communications based on EM waves in freshwater are not being investigated. Due to this, we decided to investigate this kind of underwater communication.

In Chapter 6, we perform a study of electromagnetic waves behavior depending on the temperature at $2.4 \mathrm{GHz}$ in the underwater freshwater environments. In our study case, we fix the water conditions and we measure the EM transmission characteristics as a function of several network parameters such as working frequency, data transfer rates modulations and water temperature. We want to demonstrate that some combinations of temperature and working frequency generate better results than others. We also compare our results with statements from works performed at higher temperatures and other works.

To finish, in Chapter 7, we present the application of WSNs in underwater environment. First, we show what the main problems are in the aquaculture sector and in existing marine farms. We analyze the seabed deposition estimation which can be generated by a fish cage and we propose the use of an optical sensor for monitoring the deposition in seabed. Furthermore, we perform a study of the number of sensors and the density of sensors we should use to achieve accurate measurements of this parameter. This sensor is part of a complex network composed by several groups of sensors used to optimize the process of fish feeding. For this reason, we also simulate the amount of food used in a regular procedure of fish feeding and with our proposal. Consequently, the amount of food is related to the uneaten food and depositions. Finally, we present a low cost turbidity sensor which helps us to control the quality of water. The design of this sensor includes the processes of implementation, measurements and verification.

Finally, in Chapter 8 we show a summary of conclusions viewed in each previous chapter, because every chapter has its own conclusions. Moreover, in this chapter we describe some future works related to sensors deployment and their applications. 


\section{Chapter 2}

\section{State of the Art in Wireless Sensor Networks}

\subsection{Introduction}

Wireless sensor networks have become increasingly popular due to their wide range of applications.

Many wireless sensor networks developers have adopted Bluetooth and Zigbee technologies for sensors communication. But these technologies have a big problem: the coverage radio is very short; so many sensors are needed to cover wide areas and sensors are expensive. They also lack of a high bandwidth. IEEE $802.11 \mathrm{a} / \mathrm{b} / \mathrm{g} / \mathrm{n}$ technologies have higher coverage areas and higher bandwidth but their energy consumption is quite higher.

WSNs are typically used for remote environment monitoring in areas where providing electrical power is difficult. Therefore, the devices need to be powered by batteries and alternative energy sources. Energy consumption is one of the biggest constraints of the wireless sensor node and this limitation combined with a typical deployment of large number of nodes has added many challenges to the design and management of wireless sensor networks.

When we decide to deploy a sensor node or a WSN, we should consider several aspects. First of all, it is important to define our application and environment where the network will be installed. The second factor to keep in mind is the wireless technology we want to use. Each technology offers a set of features and a behavior as a function of the environment and the possible loses for refractions or reflections in objects. The energy consumption is also one of the most important 
issues to consider in sensors deployment. Finally, it is important to check the correct operation of the system because its response will inform us about the state of the environment.

In this chapter, we are going to see the state of the art of WSNs. Firstly, we will see the main investigations which are being performed in regards to energy limitations. We will group these works from the point of view of analysis and comparatives of energy consumption of different wireless technologies. We will also see the main works about power saving mechanisms in WSNs from the MAC and routing protocols perspective.

One of the main objectives of this Thesis is the deployment of sensors for environmental monitoring. For this reason, the first step is to define what a sensor node is and what kind of sensors/transducers we can find. In Section 3, we present some of the most important works in the field of rural and animal monitoring. We have also included the implementation of WSN for people monitoring. Section 4 shows investigations in indoor communications where we have included previous works about indoor coverage measurements, interference measurements in indoor environments and wireless sensor positioning in indoor environments. Finally, Sections 5 and 6 show the main investigations about underwater wireless communications and the applications of WSNs in marine environment. Section 7 shows the conclusion of this chapter.

\subsection{What is a sensor?}

A sensor is a device, formed by sensitive cells, that transforms physical or chemical magnitudes (instrumentation variables) in useful signals to measure or control systems (electrical magnitude). The instrumentation variables depend on the type of sensor and they can be temperature, distance, acceleration, inclination, displacement, pressure, humidity, $\mathrm{pH}$, etc. The electric magnitude can be a value of electric resistance, or an electric capacity, or a voltage, or an electric current, etc.

A transducer and a sensor are different devices and they should be differentiated. A transducer is a device that transforms a physical magnitude into a different one (such as mechanical energy into electric energy). A transducer can be a microphone that converts an audio wave into an electric signal. On the other hand, a sensor is a device that provides an output signal given a specific physical quantity. The input value is called measure and it causes an answer in the sensor. An example is a Linear Variable Differential Transformer (LVDT), which converts a position into electric signal [2]. Therefore a sensor can be considered a transducer and vice versa, a transducer can be considered a sensor. All sensors can be classified and included inside one of the groups shown in Table 2.1 [9].

Frequently, the signals obtained from the sensors are not useful to be processed and it is necessary additional circuits, such as a Wheatstone bridge, or signals conditioner circuits based on amplifiers, in order to adapt the voltage levels from the rest of circuit. 
Table 2.1. Sensor Classification.

\begin{tabular}{|l|l|l|}
\hline \multicolumn{1}{|c|}{ Criterion } & \multicolumn{1}{c|}{ Type } & \multicolumn{1}{c|}{ Description } \\
\hline \multirow{2}{*}{$\begin{array}{l}\text { Energy } \\
\text { contribution }\end{array}$} & Modulator & The output energy comes from an external source \\
\cline { 2 - 3 } & Generator & The output energy is given by the input \\
\hline \multirow{2}{*}{ Output signal } & Analogical & The output varies in a continuous mode \\
\cline { 2 - 3 } & Digital & The exit varies discontinuously \\
\hline Operation mode & Defection & $\begin{array}{l}\text { The measured magnitude causes a physical effect in some parts of } \\
\text { the device }\end{array}$ \\
\cline { 2 - 3 } & Comparison & $\begin{array}{l}\text { It tries to stay the defection to zero, applying opposed effects to the } \\
\text { one generated by the magnitude }\end{array}$ \\
\hline
\end{tabular}

Most of these sensors offer us analog output signals and in many cases, we need these signals to work with digital control devices like a PC. In these cases, it will be necessary to use an analog to digital converter. Figure 2.1 shows an example with all circuit blocks needed.

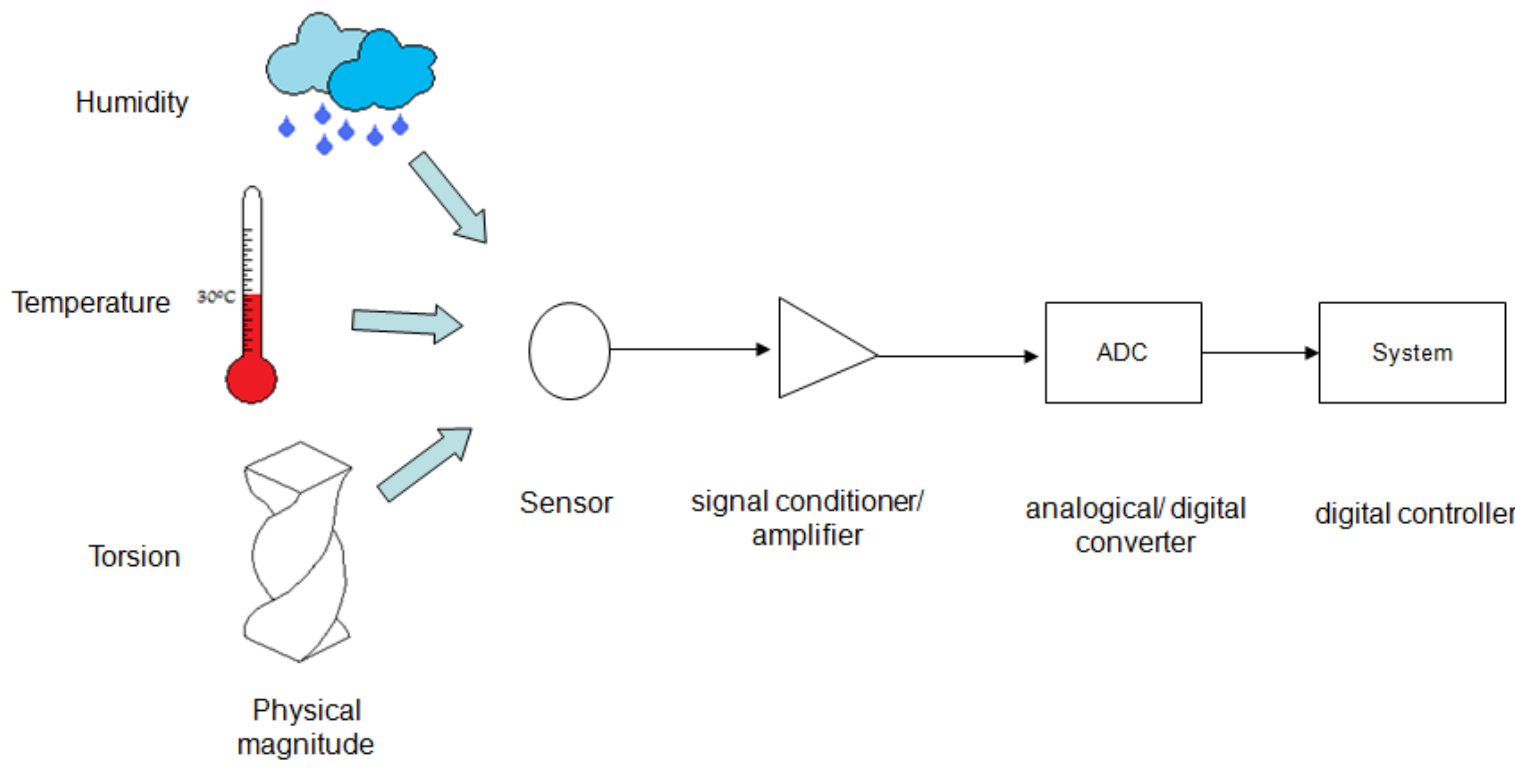

Figure 2.1. Block Diagram of sensor

\subsubsection{Types of sensors}

A sensor converts a magnitude without electric characteristic into electric signals, due to its physical effect. The physical effect can be: resistance, capacity, inductance, magnetism, charges and electric field, piezoelectric effect and Hall Effect [10]. The sensors can be classified according to the magnitude sensed or controlled [10][11]. In this section, we are going to see the most used sensors used for environmental monitoring. 


\section{Sensors of gravity based on pendulous sensors (Inclinometer)}

The inclination can be measured using the sensor ADXL202 manufactured by Analog Devices. It is an accelerometer of two axes, low consumption, digital output and it is integrated in a monolithic chip. An accelerometer is a sensor that let us to measure the movement and the vibrations, in its dynamic measurement mode, and the inclination (with regard to the gravity), in its static mode. This sensor has many advantages, due to its small size, solid integration and easy connection with the microcontroller.

The market offers devices with several mechanical solutions, many of them are based on a free weight on a viscous medium. There are sensors based on the viscous liquid movement and an electric conductive inside a cavity. The mobile parts in many cases are submerged in oil in order to avoid oscillatory movements of the pendulum. The sensors can be based on capacitive, electrolytic, piezoelectric, magnetic effect and resistive variation.

\section{Sensors of gravity based mercury contacts}

They are used to measure the inclination, although it is not possible to obtain intermediate values. It indicates an open or closed contact. The mercury contacts use to be a glass cylinder which contains two contacts. These sensors can be employed as warning system (e.g. the case of a person that falls on the floor). The sensor can be connected to a mobile phone or PDA that can call a programmed number.

\section{Sensors of pressure, force and displacement based on strain gauges}

The Strain gauges are based on the electric resistance variation according to the deformation, in a conductive thread calibrated, or in resistances built with semiconductor tracks. They are also used, combined with springy or deformable pieces, to detect indirect efforts.

In gauges of thread, the resistance is formed by a thread prepared in zigzag form on an elastic support, with a preferable direction. When the gauge is deformed in the preferable direction, a lengthening of the thread and a decrease of their section, is generated and therefore, it is registered a variation in their resistance. To measure enough resistance variations, the gauge should have a high resistance (between 100 and $1000 \mathrm{ohms}$ ), and it have to work with a very low consumption, to avoid the Joule effect. The measure of deformations requires a laborious calibration, and the amplification is usually carried out using differential methods with three threads. The semiconductor gauges have more sensibility than the thread gauges because the phenomenon of resistance variation given by the deformation is joined to a piezoelectric effect. But, this phenomenon depends on the temperature extremely and it always forces to compensate the results thermally. This hinders the calibration.

\section{Force sensors}

This type of sensor bases its operation on strain gauges. These devices transform a force, traction or a compression, in variations of electric resistance. It let us measure the force applied on an object. 


\section{Pressure sensors}

There is a broad range of pressure sensors in the industry. Mostly of them are aimed to measure the fluid pressure on a membrane. Motorola MPX2100 series devices are a small size piezoresistance that are made of pressure-sensitive silicon. They provide a voltage change directly proportional to the pressure applied to them. The sensor consists of a monolithic silicon diaphragm to measure the effort and a thin film resistor network integrated on a chip. The chip is set, calibrated and temperature compensated by laser.

In electronic sensors, the pressure acts on an elastic membrane and it measures the bending. In order to detect it, various physical principles can be used. It could be inductive, capacitive, piezoresistive, optical, monolithic (with extremely small electronic modules, fully connected), ohmic (through strain gauges) or Hall Effect, where a permanent magnet attached to the membrane causes a change on the Hall potential.

These sensors are useful for prosthetic systems for picking up objects.

\section{Sensors of proximity}

Proximity sensors are necessary when you need to determine the position of an object, without any contact with it. They may be linear (displacement sensors) or switch, where the switching between two states indicates a particular position. Proximity sensors commonly used in industry are the inductive and the capacitive ones.

Inductive proximity sensors are based on the damping phenomenon that occurs in a magnetic field due to induced currents (Foucault currents) nearby materials, but this material should be metallic.

The capacitive sensors detect changes in parasitic capacitance between the detector and the object whose distance was measured. They are used to measure distances to metallic and nonmetallic objects, such as wood, liquid and plastic materials.

\section{Ultrasonic sensors}

The sensor emits ultrasonic pulses that are inaudible to the human ears. The impulses travel at the speed of sound and reach an object, then the waves are reflected and captured again by the ultrasonic receiver. A controller is built to deliver a burst of pulses and then start counting the time it takes to get the echo. This time is proportional to the distance to the object. These devices are capable of detecting transparent objects like glass and plastics. However, in very turbulent air environments, with high noise, cannot be used because of its dependence on the medium.

\section{Optical}

The photocells are sensing elements. The same device can hold the emitter and the detector system of the light beam, which is reflected on the object. They usually work with frequencies in the infrared band. The advantages of such detectors are immunity to electromagnetic interference, large detection distances, high response speed, color identification and detection of small objects. There is an improvement of the optical sensors that uses optical fiber. The main disadvantage is that it cannot detect transparent objects. 
When a proximity detector is needed, several factors must be considered. The detections of short distances (less than $50 \mathrm{~mm}$ ) can be accomplished with inductive or capacitive sensors. The election depends on the metallic nature of the material. For long distances (more than $50 \mathrm{~mm}$ ), it is necessary to use ultrasonic or optical sensors. Proximity sensors can be installed in wheelchairs or blind systems in order to alert the users of the proximity of an object.

\section{Sensors of humidity}

There are several types of humidity sensors. Resistive humidity sensors consist of a thin sheet of a polymer capable of absorbing water, on which two contacts are printed in a conductive material, woven metal or coal. When the water content of the polymer changes, the electrical resistance through the polymer varies and this parameter is measured.

We can also find capacitive humidity sensors, where the permittivity of the dielectric changes when it is in contact with other values of humidity.

\section{Sensors of acidity}

An ISFET (Ion Sensitive Field Effect Transistor) is an electronic-chemical sensor that reacts to changes in the $\mathrm{pH}$. When the ion concentration changes (the basic $\mathrm{pH}$ or acidic $\mathrm{pH}$ properties change), the quantity of ions that passes through the membrane and is deposited on the transistor gate, varies. This occurs when the sensor is in contact with the substance to be tested and varies the current flowing through the transistor. This device is based on MOSFET transistors and it is formed by the following elements:

- A gate or membrane usually made of $\mathrm{Si}_{3} \mathrm{~N}_{4}, \mathrm{Al}_{2} \mathrm{O}_{3}$ and $\mathrm{Ta}_{2} \mathrm{O}_{5}$.

- $\quad$ Source and drain areas for the substance.

This type of sensor is often used in chemical, food and ecology industry. Both humidity sensors could be used as warning system for urine detection.

\section{Temperature sensors based on NTC and PTC thermistor}

A thermistor is a resistor whose value varies with the temperature. There are two types of thermistors: NTC (Negative Temperature Coefficient), which resistive value decreases when the temperature increases and PTC (Positive Temperature Coefficient), whose electric resistance value increases when the temperature increases.

In electronics, it is important to know the temperature of the devices and systems used for sensing. It is important to protect the circuits from overheating and avoid reaching dangerous levels of warming.

\section{Temperature sensors based on RTD}

RTD (Resistance Temperature Detector) sensors are based on the join of a conductive of platinum and other metals. They are used to measure the temperatures by contact or immersion. It is used where semiconductors or other sensitive materials cannot be used. Its operation is based on the 
dilation phenomenon. That is, when the temperature rises, the metal expands and increases the electrical resistance.

\section{Temperature sensors based on bimetal}

If an object is formed by two or more metals joined, it is called bi-metallic object. It is not a mixture or alloy of two metals. It is a device consisting of several layers of different metals. Trimetal and tetrametal are objects made for three and four separate metals respectively. Bimetal thermostats convert a temperature change in a mechanical movement. They are formed by two layers of metals with different thermal expansion coefficients and by temperature variations tend to bend toward the side of lower coefficient of expansion.

This property is used in power circuit breakers, where the current through the bimetal heats it. It opens the circuit to limit the peak current.

\section{Integrate circuits for temperature}

There are a wide variety of integrated circuit temperature sensors. These sensors are grouped into four main categories: output voltage, output current, output resistance and digital output. In voltage we find LM35 (in ${ }^{\circ} \mathrm{C}$ ) and LM34 (in K) from National Semiconductor. In current, one of the most well known is the AD590 from Analog Devices. The resistance output sensors are less common (there are some from Phillips and Siemens).

\section{Pirosensors (remote flame sensors)}

There are sensors that can determine the presence of a fire at a certain distance. It is based on the detection of a range of ultraviolet light (very sensitive in the range of wavelengths from 185 to 260 $\mathrm{nm}$ ). E.g. a sensor built with UVTron bulb manufactured by Hamamatsu Photonics can detect a match into a sunny room 5 meters away. A wide variety of remote flame sensors can be found. Some of them detect ultraviolet light. Others detect infrared light.

This type of sensor is often used to detect gas-fuelled flames and liquid fuel as the main component of fire alarms or monitors combustion burners, etc...

\section{Light sensors based on photodiode}

The photodiode is a semiconductor diode formed by a PN junction where the semiconductor is exposed to a light through a crystal cover (with a lens form). It is especially sensitive to the incidence of visible light or infrared. All semiconductors are sensitive to light, but the photodiodes are designed to achieve the maximum sensitivity.

In a PN junction, the current flows from the $\mathrm{P}$ crystal to the $\mathrm{N}$ crystal. It is called direct polarization. There is not any current flowing in the opposite direction. In the photodiode, the current that varies with the changes of the light, is circulating in opposite direction to the one allowed by the PN junction. That is, the photodiode should be inversely polarized in order to work properly and it should record an increment of the current when the diode is excited by the light. 
The silicon photodiodes are built to be sensitive to the visible light (wavelength up to $1.1 \mu \mathrm{m}$ ) or germanium to be sensitive to the infrared light (wavelength up to approximately $1.8 \mu \mathrm{m}$ ), although there are devices with other semiconductor materials.

\section{Sensors of light based on photoresistance}

A photoresistance or LDR (light-dependent resistor) is an electronic component whose resistance decreases when increases the incident light intensity.

The electrical resistance value of an LDR is low when light strikes and very high when it is dark ( $1 \mathrm{M} \Omega$ or more in the dark and $100 \Omega$ in bright light). A LDR is a high resistance semiconductor such as cadmium sulfide (CdS). If the light has high frequency, photons are absorbed by the semiconductor and give enough energy to the bound electrons to jump into the conduction band. The resulting free electron, and its hole, conducts electricity, thereby the resistance decreases.

The resistance values vary with the delay (tenths of seconds). It is different going from dark to bright than from bright to dark. For this reason, the LDR is not usually used in applications where the light signal varies rapidly.

LDRs are manufactured in various types and they can be found in many consumer items such as cameras, security alarms or on/off street lighting systems.

\section{Sensors of light based on phototransistor}

The phototransistors are very similar to the regular transistors. They are composed by a semiconductor material, forming two PN junctions. They have three external connections: collector, base and emitter. Its packaging usually contains a small opening to allow the light penetration in order to produce the photoelectric effect. Its collector current flow can be controlled by the base current. The phototransistor current conduction is controlled by the current given when the light enters through the small opening.

Phototransistors have very short response time and it can respond quickly to very quick light changes. Because there is an amplification factor involved, the phototransistor delivers quite much electric current variations in response to the changes of the light intensity. The phototransistors are more sensitive to the light than the LDRs.

\section{Sensors of light based on photocells}

A photocell performs a direct conversion of light energy into electrical energy. This phenomenon is called photovoltaic generation. Some materials can absorb and emit photons of light electrons; it is the photoelectric effect. The result of this effect is an electric current that can be used as energy to power circuits.

Photovoltaic cells or solar cells are composed by the same semiconductor materials that are used in the microelectronics industry. A thin semiconductor wafer, with a specific treatment, generates an electric field that is positive on one side and negative on the other. When light energy falls into it, the electrons are beaten and torn from the atoms of the semiconductor material. The electrons uprooted form an electric flow that provides energy. 
Photovoltaic cells are often used to obtain solar energy and to charge batteries. A sensor based on a photoelectric cell that is capable of activates a circuit with its own energy in presence of the light. Moreover, due to their size, fragility and price, the photoelectric cells are not considered the most desirable option to be used as a detector of light signals.

\section{Sensors of light based on CCD cells}

CCD devices (Charge-Coupled Device) are integrated circuits that have an array of light sensitive cells. They are a large number of sensitive items that are packaged in a small area. They handle a large amount of image information.

The integrated circuit comprises a set of capacitor electrically coupled that are aligned each other. It can transfer their charge using methods based on electric fields. The light-sensitive elements are the capacitors, which are loaded or unloaded depending on the light that crash to them. These features make the CCD a key element for the development of existing video cameras and photographic extremely miniaturized with high image quality. They are also used for some types of sensors that read visual information such as scanners, readers, faxes, classifiers letters, binders of documents, etc.

\subsection{State of the art in energy issues}

The power management schemes of wireless sensor networks have attracted high attention in recent years. Much published research has addressed all kinds of issues related to them.

We can find several works related to energy conservation techniques. They tend to focus on comparative routing protocols or MAC-protocols. Some of them show techniques related to the operation mode of the nodes and its radio system. In this section we will see some of these works, and we will provide some of the key conclusions presented in these papers.

\subsubsection{Analysis of energy status and saving energy strategies based on applications}

Some authors have focused their analysis on the energy consumption from the point of view of the development of software tools. These tools aim to help in the control of the consumption of our networks as it is the case of those programmed applications for estimating power consumption of network devices such as routers, switches, etc. The report published in November of 2011 [12] shows a comparative of applications that estimate the power consumption of devices. However, the document indicates that the results provided by this software are not very accurate (they compare their values with the ones gathered from the real power consumption). Therefore, we must understand these tools as approximation tools, that can help us to do an initial design of our system, but they can never replace actual measurements over real devices.

Due to the recent emergence of new network services and applications, networks are growing, regarding to the number of devices. The increase of the number of network devices also implies an 
increment of the power consumption. In 2009, M. Kakemizu et al. [13] discuss the possibility of keeping the energy consumption in 2025 at the same level as nowadays by reducing the number of equipments and network devices. To do this, authors propose the development of new technologies based on the flow of information going through the network. They propose two mechanisms that allow efficient use of resources. These are called ECO switching and ECO routing. The first one is based on a new model of switching packets that eliminates the packet buffers and routing tables. The second mechanism includes some paths to the routers when the traffic volume is low. This permits them to wait in sleep mode while they do not receive/transmit anything. When a router needs transmit/receive something, it is activated.

Finally, A. P. Bianzino et al. [14] surveyed several strategies to bring the network to the concept of "Green Network" and explained why it is important to close networks to this concept. Authors explain several strategies that would achieve the objectives pursued by a green network. The authors argue that the design strategies should be based on the simplification and unification of the network to avoid excessive network devices. They also propose the implementation of mechanism of selective connection, where routers can turn-off / turn-on depending on their use, and the group of multiple processes and services using virtualization in the same hardware. This is proposed because a single device working at full capacity, consumes less power than several devices running process less complex.

V. Raghunathan et al. [15] review several techniques to address the energy consumption challenge. This work also describes recent advances in energy-aware platforms for information processing and communication protocols for sensor collaboration. The article looks at emerging and hitherto largely unexplored techniques such as the use of environmental energy harvesting and the optimization of the energy consumed during sensing. The paper presents some promising research directions for alleviating the energy problem in WSNs, including hierarchical architectures, ultralow-power MAC protocols, environmental energy harvesting, and energy aware sensing. The authors explain and present an architecture of sensor node in order to be considered energy efficient. At the same time, they present a wireless sensor module, a heliomote, which is used in different tests in order to show that it is possible to provide energy to the nodes from alternative sources instead of from a battery, which has a limited life time. They also compare three MAC protocols. These are B-MAC, STEM-T, and WiseMAC, which are characterized by low power consumption in the media access process.

Another significant work is presented by N. A. Pantazis et al. in [16]. The authors focus their explanations on the fundamental concepts of energy management, including the need of power management in the wireless sensor network, and discuss the side effects of power management in terms of cost. They say that the cost of power management must always be borne in mind when speaking about a power control system. The cost of power management is important for evaluating the performance of a power control system, no matter what the specific objectives may be. Throughout the document, they describe different types of power management systems and different approaches and goals they may have. The authors divide the power conservation mechanisms into two main categories based on their primary objectives. On the one hand, Passive PCMs are divided into three sub-categories: Physical Layer, Fine-Grain, and Coarse-Grain PCMs. In the implementation of the Coarse-Grain PCMs, two basic approaches were distinguished: Distributed and Backbone-based. On the other hand, the classification of the Active PCMs is based 
on the layer (MAC, Network, and Transport). Various algorithms were studied for each classification. Each power management scheme is discussed in terms of objective, mechanism, performance, and application scenario. The similarities and differences between schemes of the same clustering category are also presented. The authors conclude the paper by stating that although the performance of the presented power management schemes is promising, further research would be necessary to address other issues, such as quality of service (QoS). Energy-aware QoS in wireless sensor networks will certainly ensure guaranteed bandwidth, or delay, through the duration of a connection as well as provide the most energy-efficient path.

S. Saxena et al. review the main approaches for energy conservation in wireless sensor networks in [17]. They presented a systematic and comprehensive classification of the solutions related to save energy. This involves characterizing the interactions between different protocols and exploiting cross-layer interactions. They also made a protocol classification and explain each part. The authors comment that most of the solutions presented by other authors are based on the assumption that the radio energy consumption is much higher than data sampling or processing consumption, while many real applications have greater power consumption in data sampling/processing than in radio transmission. Furthermore, they observed that the data acquisition research field of has not been fully explored in terms of energy conservation. Finally, the authors come to the conclusion that there is an increasing interest towards MAC protocols used for time synchronization and energy conservation in the recent years. They also made a reference to the node's mobility, which is yet another challenging task in energy optimization.

Another survey related to protocols and energy-saving techniques is [18]. It was presented by K. Akkaya et al. This paper surveys several routing protocols for sensor networks and presents a classification of various pursued approaches. The classification is focused on three main categories: data-centric, hierarchical and location-based. This work analyzes several protocols that use contemporary methodologies such as network flow and quality of service modeling. From this work, several conclusions can be extracted. On the one hand, many protocols base their functions in some attributes such as data and query in order to avoid overhead-forming clusters, the use of specialized nodes, etc. However, in some cases, where queries can be more complex schemes, such attribute-value pairs may not be enough. On the other hand, routing protocols based on cluster are carried out by group sensor nodes to efficiently relay the sensed data to the sink. The cluster heads are specialized nodes that are sometimes chosen as a function of their available energy. A clusterhead performs the data aggregation and sends it to the sink. The authors show a table which summarizes the classification of the protocols covered in this survey. They also included in the table whether the protocol is utilizing data aggregation or not, since it is an important consideration for routing protocols in terms of energy saving and traffic optimization. The factors that affect cluster formation, cluster-head communication and how to form clusters in order to improve energy consumption and contemporary communication metrics, such as latency, are possible future research topics.

Finally, C. E. Jones et al. present in [19] a study on power saving techniques in WSN. This paper addresses the incorporation of energy conservation considerations on all layers of the wireless network protocol stack for mobile devices. Therefore, throughout the document, the authors cover the protocol stack and gradually introduce various energy saving modes (starting from low-power design within the physical layer). They show different sources of power consumption within mobile 
terminals and general guidelines for reducing the power consumed. They also show energy efficient protocols within the wireless networks' MAC layer, power saving protocols within the LLC layer and power aware protocols within the network layer. Finally, they provide some considerations that should be taken into account as regards battery power.

\subsubsection{Analysis and comparatives of energy consumption of different wireless technologies}

Several papers have been published related with the analysis of energy consumption of different wireless technologies and related with power-saving mechanisms in WLANs from the MAC level perspective

In [20], a comparative study of the Bluetooth (IEEE 802.15) and IEEE 802.11 technologies is shown. This paper makes an exhaustive analysis of the main characteristics of both technologies and in particular it analyzes three integrated models which are employed in devices that use these communication technologies. These are, Bluecore01 and Bluecore02, manufactured by CSR (Cambridge Silicon radio) and used by Bluetooth and Prism, mounted in IEEE 802.11 devices. The paper compares the devices taking into account several parameters such as the spectrum used, the modulation characteristics and interference problems, power requirements, characteristics of the network topology, the ability to create an efficient network, the creation speed of the networks, the characteristics of the links among the devices of a single basic cell and the maximum attainable throughput; security systems and the ability to offer a given QoS. As we can see in this study, each device has been manufactured for a purpose; the first ones are developed to work inside a room, therefore it does not need so much transmission/reception power and it consumes less; while the IEEE 802.11 system has higher transmission/reception power, and its consumption, consequently is higher.

In [21], L. M. Feeney et al. present an exhaustive study of Lucent WaveLAN IEEE 802.11 device. Their paper shows a series of experiments giving detailed measurements of the energy consumption of this device operating in an ad hoc networking environment. The data is presented as a small mathematical development that models, by means of a collection of linear equations, the energy consumption in sending, receiving and discarding broadcast and point-to-point data packets of various sizes. Their results suggest that energy consumption and bandwidth utilization are not related, because it is necessary to consider the cost of transmitting, receiving and discarding packets. But it should be also considered the proportions of broadcast and point-to-point traffic used by the protocol. Furthermore, the number of hops required in a multi-hop routing environment usually increases due to the reduced transmission range associated with higher data rates and due to the high fixed overhead.

In [22], the authors propose three asynchronous power-saving protocols which are directly applicable to IEEE 802.11-based MANETs. These protocols are named dominating-awake-interval, periodically-fully-awake-interval, and quorum-based protocols. They focus their study from two points of view: The first one enforce the hosts to send more beacon packets than the original IEEE 802.11 standard does and the second one establishes a wake-up and sleep patterns for the hosts. Their protocols are designed according to the following rules: They seek to send higher number of beacons in order to prevent the inaccurate neighbor problem, a mobile host in power save mode 
should insist more on sending beacons. Their protocol does not count on clock synchronization and the wake-up patterns of two hosts must overlap with each other no matter how much time their clocks drift away. Finally, in order to predict the wake-up moment, if a host hears another host's beacon, it should be able to derive that host's wake-up pattern based on their time difference. They demonstrated with simulations that their power-saving protocols can save much energy with reasonable route establishment probability.

\subsubsection{Power-saving mechanisms in WLANs from the protocols perspective}

G. Razzano et al. [23] describe a new control architecture solution that is adopted for a Wireless Local Area Network (WLAN) developed in the framework of the WIND-FLEX (Wireless Indoor Flexible High Bit rate Modem Architecture) project. It considers the power consumption due to the wireless modem and present a novel control entity based in fuzzy logic which has been already applied for the determination of the transmission power in Code-Division Multiple-Access (CDMA) scheme. The consumed power is greatly influenced by the choice of the bit rate used for the packet transmission and the reduction of power consumption is obtained by defining the most appropriate bit rate for data transmission, with respect to the traffic situation.

The paper in [24] describes several techniques to reduce the dynamic power consumption in WLAN systems to help to increase the battery life in mobile systems. They suggest techniques that can be used to reduce dynamic power consumption for the 802.11n system level and for the baseband. They propose to reduce the devices consumption is its initial design, because a small device consumes less energy. But the reduction of the manufacture technology could suppose a considerable rise in the price on the final product. The intention of reducing the devices consumption should not suppose an accused ascent of the final product price.

In [25], we can see a paper focused on the design and evaluation of an enhancement of the distributed Wireless LAN power saving mechanisms defined in the IEEE 802.11 and 802.11e standards. The main objective of the No Data Acknowledgment (NDACK) mechanism is to increase the power saving by reducing the duration of the awake periods when the stations have no pending data stored at the AP. They use this method to increase the battery lifetime of Wireless LAN mobile devices while providing the required QoS. Their results demonstrate that the power consumption due to the channel congestion can be reduced with NDAck and QoS.

E.-S. Jung et al. Present in [26] an optimization of the power saving mechanism in the Distributed Coordination Function (DCF) in IEEE 802.11 standard, which is divided into so-called beacon intervals. At the start of each beacon interval, each node in the power saving mode periodically wakes up for duration called the ATIM Window. During this time period, the nodes exchange control packets to determine whether they need to stay awake for the rest of the beacon interval. The energy saving and throughput achieved by the nodes are directly affected by the size of the ATIM window. They propose an adaptive mechanism to dynamically choose a suitable ATIM window size according to observed network conditions. Their new protocol has been called Dynamic Power Saving Mechanism (DPSM) and it allows that a node also can power off its wireless network interface whenever it finishes packet transmission for the announced packets. Initially, each node begins with ATIM window size equal to ATIMmin. A node will increase its ATIM window size to the next higher value, if any of the above rules are satisfied, at the beginning 
of the next beacon interval. Finally we can see that the proposed scheme can improve energy consumption without degrading throughput.

Because the traditional ad hoc wireless MAC protocols are designed without considering the battery state, in [27] a paper is presented by S. Jayashree et al. and they show an energy efficient battery aware MAC protocol, the main aims of which are minimal power consumption, longer battery life, fairness, and higher throughput. A battery consists of an array of electro-chemical cells. BAMAC models the battery behaviour, using a stochastic process named Markov Chain. In it, each node maintains a battery table which contains information about the remaining battery charge of each of its one-hop neighbours. The entries in the table are arranged in the non-increasing order of the remaining battery charges. The RTS, CTS, Data, and ACK packets carry the remaining battery charge of the node that originated the packet. A node listening to these packets makes a corresponding entry in its battery table. Their proposed protocol is implemented using GloMoSim simulator and it has been compared with DWOP protocol. In the simulation, we can see that the life of the batteries is extended around a $70 \%$ and it reduces the consumption of the nominal capacity of the battery for package transmission in $21 \%$, compared with IEEE 802.11 and the DWOP MAC protocols.

Other works propose new protocols based on existing ones. They do not make distinction of the type of technology used in the background. We can point out [28], where their authors propose new protocols that can be applied to wireless sensor networks. The paper proposes a new energyefficient approach for clustering nodes in ad hoc sensor networks. HEED (Hybrid Energy-Efficient Distributed clustering) periodically selects cluster heads according to a hybrid of their residual energy and a secondary parameter. HEED also seeks prolonging network lifetime by distributing energy consumption. It tries to reduce the high control on the network. The paper shows the clustering process and determines that the cluster head position can be uniform. HEED is compared with LEACH, WCA and GC protocols, among others. HEED optimizes resource usage according to the network density and application requirements.

Finally, in [29], G.P. Halkes et al. compared S-MAC and T-MAC, which try to save energy by introducing a duty-cycle to mitigate idle listening time, with CSMA/CA. This choice was taken because it is the most important cause of energy consumption in typical sensor network scenarios where the communications between nodes is not continuous. They show the effects of low-power listening, a physical layer optimization, in combination with these MAC protocols. The results show that using a low-power listening is very effective at mitigating idle listening. The absolute lowest energy consumption is reached in combination with T-MAC, while the results about S-MAC show that this protocol suffers from over-provisioning. Since its duty cycle is fixed for all nodes, often a rather large value must be selected to avoid dropping messages under peak loads, which causes SMAC's idle-listening to deteriorate for increasing traffic loads. Although S-MAC achieves acceptable results, they are not as good as those of T-MAC with low-power listening. T-MAC presents an aggressive time-out policy that allows it to adapt seamlessly to variations in traffic induced by typical sensor network applications at the expense of a reduction in peak throughput. TMAC performs slightly better for variations over time (events) than for variations in location. 


\subsection{State of the art in monitoring of natural environment}

\subsubsection{Fire detection and verification}

Several technological solutions based on wireless networks have been proposed to detect and monitor a fire. The related literature shows systems based on satellites, infrared cameras, wireless cameras and sensor networks. Some of these wireless systems are implemented alone, but there are some that mix several technologies. Moreover, there are other types of technologies, such as a GPS system, which can be added to improve their performance.

There is an important system for forest fire detection based on satellite imagery: MODIS [30]. It studies the images taken from satellites. But, weather conditions are an important problem in these systems. Clouds and rain absorb parts of the frequency spectrum and reduce spectral resolution of satellite imagery. So, the performance of this system changes very much. Satellites can monitor a large area, but the resolution of satellite imagery is low. A fire is detected when it has grown quite a lot, so real time detection cannot be provided. Moreover, these systems are very expensive.

$\mathrm{Li}$ et al presented an algorithm based on satellite remote sensing to detect fire across the Canadian boreal forest zone [31]. The authors use images provided by the Advanced Very High Resolution Radiometer (AVHRR). The paper shows the analysis and how their algorithm works in order to detect a fire by using several graphics. The system presents several advantages: automatic operation, consistent data quality, cost-effective use, and rapid response, but not in real-time.

Thierry Antoine-Santoni et al. [32] designed a system, called Firesensorsock, to protect every sensor node (mote) of a wireless sensor network in order to avoid these devices being damaged or destroyed when they are sending data, detecting or controlling a fire. Firesensorsock is a special protection dedicated to the thermal insulation of the sensors that leave intact their ability to sense thermal data. Thus, the objective of this work is to have a wireless sensor network that is able to resist being burnt. The sensors will continue transmitting data flow to the final user. Results show a significant change of the temperature and humidity inside the protection, which determines the presence of a fire. Besides, the authors point out that a WSN protected with Firesensorsock is capable of sensing thermal data in the open air. They are able to detect a fire and track the fire spread during its spatial and temporal evolution.

Nowadays, wireless sensor networks are widely used to monitor and to detect a fire, and there is a fair amount of literature on it. An example is the FireBug system. In [33], the authors present a system based on a wireless sensor network for forest fire monitoring. The design is performed with MICA motes using GPS attached. Its objective is to gather environment parameters like temperature, relative humidity and barometric pressure when there is an active fire. Motes communicate with a base station and data are stored in a database server. In order to access to this server, a web browser based on a web application, or any other application capable of communicating with the database server, is necessary. This system uses the Crossbow MICA2 mote and TinyOS programmed in the nesC language. This software is specifically developed for embedded devices. This architecture was tested using 10 motes in two prescribed burns in California on the 16th and 30th of September 2004. Results were satisfactory and motes were capable of reporting data correctly before they were burned.

A proposal for fire rescue applications is described in [34]. First, the authors show the requirements that have to be considered for this kind of network, including accountability of 
firefighters, real-time monitoring, intelligent scheduling and resource allocation, and web-enabled service and integration. According to these requirements the authors propose FireNet. It is a wireless sensor network architecture where sensors are distributed in the vehicles, forming a selforganized heterogeneous network with the fire fighters. Finally, according to the requirements abovementioned and the characteristics of wireless sensor networks, the authors present several research challenges from the point of view of new protocols, hardware and software for WSNs. FireNet architecture is considered to be very useful in fire rescue processes.

The Forest-fires Surveillance System (FFSS) has been developed to survey the mountains of South Korea [35]. Son et al. propose architecture composed of WSNs, a transceiver, middleware and a Web-application. The nodes of this network gather measurements of temperature, humidity and illumination from the environment. These data are concentrated in one node of the WSN called sink-node. This node sends the data to the transceiver (gateway) connected to Internet. Then, a middleware program determines the forest-fire risk-level by a formula from the Forestry Office. If a fire is detected, FFSS automatically activates an alarm to facilitate an early extinguishing of the fire. In this work, the nodes use TinyOs as an operating system. Besides, the WSN use a minimum cost path forwarding (MCF) to send their data to a sink-node.

Hefeeda and Bagheri [36] presented a WSN for forest fire detection based on the FireWeather Index (FWI) System, which is one of the most comprehensive forest fire danger rating systems in North America. This system determines the risk of propagation of a fire according to several index parameters. So, weather data is collected by the sensor nodes and it is analyzed in this work to calculate these indexes. Another aspect analyzed is the number of measurements taken from different sensors to minimize error estimation. They present and simulate a distributed algorithm to solve this problem. Finally, the authors compare their algorithm against others in the literature and they conclude that the proposed algorithm extends the network lifetime and can provide higher detection accuracy in some areas.

The objective of FireWxNet [37] is to determine the behavior of fire rather than its detection. It consists of a WSN that is used to measure weather conditions around an active fire. Webcams are used to get visual data of burned area and a base station which is capable of providing long distance communication. Every half an hour, the system measures temperature, relative humidity, wind speed and direction. In contrast, cameras provide images continuously about the current state of the active fire. The developed system uses five, long-distance wireless links, three sensor networks, and two web-cameras. The results of the system are very good and they show that it is very useful to analyze fire behavior.

To conclude, Garcia et al. have presented some papers about sensor networks for fire fighting. In one of them they propose a simulation environment called Equipment Destined for Orientation and Security (EIDOS) [38]. This platform analyzes and combines the geographical information of the area (topography, combustible...) and the data sensed by network nodes (temperature, humidity, wind direction and speed) to create a model of the fire. All these data are sent directly to the firefighters' handheld devices to help them with the forest fire fighting. This paper describes and simulates the proposed system, but it is not implemented in a real environment. The same authors proposed a wireless sensor network to gather environment data in real time [39]. The difference with the other papers is that these data are sent from the wireless sensor network to a base station and they are used to feedback a fire simulator. The approximations calculated by the simulator are 
more precise and they can be used to compute better predictions about the fire evolution and its behavior.

Systems based on satellite images are not widely used because they do not provide real time fire detection and they are high cost. Nowadays, wireless sensor networks are fashionable in firefighting, but although it is the technology most used to detect fires, there are very few implementations published in the literature. Almost all the works published about Wireless Sensor Networks on Rural Fire detection are only theoretical or talk about their possible use, but very few of them present a deployment. On the other hand, in most cases, sensor networks only recollect data about the environment in order to detect and analyze the fire, its behavior and evolution. They do not verify their fire detection.

\subsubsection{Animal monitoring to prevent theft and attacks by wild animals}

Currently, sensor networks are widely used in livestock for health monitoring and for animal identification [40]. In intensive farming, sensor systems are mainly used to control several environmental variables, such as temperature and humidity [41]. In extensive cattle, sensor networks are also used to monitor remotely the flock position in the area of livestock grazing [42].

Another important application of sensor networks is a system for detecting the collective stress in livestock. The stress in animals is usually related with variations of corporal temperature, heart rate and respiratory rate [43]. For this type of monitoring, it can be used under skin sensors [44][45] or outer sensors [46]. The monitoring of these parameters allows us the modeling and detection of collective stress episodes in sheep and goats flock caused by wolf attacks. So, if we are able to detect any sign of attack (by the animal behavior), we will able to generate acoustic and visual alarms in order to scare the wild animals, and avoid the attack.

In [47], authors show an experiment where a sheep, in a regular situation, has heart rates between 60 to 80 beats per minute. When the animal is being attacked or stressed by other causes, it can register heart frequencies up to 225 beats per minute. Finally the biologist and inventor of this prototype Jean-Marc Landry, aims to study a system that strews a chemical repellent on the wolf to generate its flee.

\subsubsection{WSN for Vineyard Monitoring}

A basic machine vision system consists of a camera, a computer equipped with an image acquisition card, and a lighting system. In addition, the adequate software should be considered in order to convey the correct signals to electronic equipment in order to perform successfully the acquisition operation, the storage and the image processing process [48]. There are very few technical works about image processing and pattern recognition in precision agriculture. In this section we show some of these related works.

In paper [49], T. Baidyk et al. present two systems for image recognition. They show the structure and the recognition algorithms and the limited receptive area (LIRA) neural classifier. Then, they show several applications for their proposal. The first application is an image 
recognition system based on adaptive control system for micromechanics where the LIRA neural classifier is proposed for texture recognition of mechanically treated metal surfaces. Based on this first application, and taking into account that agriculture tends to over-use pesticides to kill insects and pests, the authors propose to use a web-camera based computer vision system in order to automate the task of recognition of larvae. The system is able of recognize the difference between the textures corresponding to the larvae and the real world background. Thus, if the system locates the position of the insects and larvae, the use of pesticides will be local and chemical products consumption will be lower. The proposed system consists of a series of neural classifiers, held together and organized into layers. Each classifier decides based on the captured image, whether the searched parameter is present or not. Based on this decision, this node is labeled as ON or OFF and transmits its result to the next layer. This new layer has a smaller number of classifiers and collects the responses from the previous layer. The answers are transmitted to an upper layer and each layer has a top most comprehensive filters, which will define if the generated image is part of an insect or not. Once the image is formed, it is processed and validated by different combinations of horizontal, vertical and diagonal distortion of the image. Because the recognition of larvae and worms of different sizes, shapes, colors and positions is not a trivial task, the system needs an initial training stage based on the display of different images. With this pre-processing technique, the authors train the system to be able to recognize and extract the larvae of any image with different backgrounds.

Sometimes it is difficult to use machine vision to distinguish the weeds from the main crop in real time, due to the large processing capacity and resources needed for this purpose. Artificial neural networks (ANNs) have evolved and, actually, solve some of these difficulties by interpreting images so quick and effectively. A work based on the same type of system than in the previous work is presented by C.C. Yang et al. in [50]. The aim of this study is to develop a backpropagation ANN model that could distinguish young corn plants from weeds. In order to perform the various tests, the authors use a series of cameras, which are adjusted to obtain images with sufficient clarity. Each image is preprocessed in bitmap format with the Image Processing Toolbox v2.0 for MATLAB v5.0 and is converted to indexed images based on a red-green-blue (RGB) color system. Each pixel of an image is classified in one category, represented by an integer in the range from 0 (black) to 255 (white). Finally, each assigned color index number serves as an ANN input. After data pass through different layers of the neural network nodes, the system obtains as an output a value of 0 or 1 . This value depends on whether the system has been considered that the image has been taken of a useful plant or a weed. The same tests are performed for different types of neural networks (multiple outputs). The authors remark that although it may take approximately 20 hours to properly train the network, if this training is done correctly, the system needs only a second to process each image. This processing and image recognition system could be used in conjunction with an herbicide spraying system in order to improve efficiency and reduce the amount of applied herbicide.

Another work related to the detection of weeds in agriculture is presented by C.C. Yang et al. in [51]. In this case, the authors present the initial stages of developing a system to capture, and an image processing technique to detect weeds. Using a fuzzy logic system, the authors are able to create a weed map, in order to let the system take decisions on the exact location and the amount of herbicide that can be applied to the field. In order to perform various tests, the authors used a commercial digital camera and a personal computer. The image colors are compared with the 
intensities of RGB color system. Depending on the color intensity values, they obtain a binary matrix (obtained through the fuzzy logic), which provides information on the green zone of weed cover, and the distribution of weeds. The authors were able to perform such functions with common cameras without the need to use complex devices. In addition, their system is able to control the amount of herbicide that is thrown into the field, and to reduce the pollution soil and water, getting significant cost savings.

In [52], D. G. Sena et al. used machine vision for detecting a type of worm (Spodoptera frugiperda) that affects to maize plantings. This control is generally only performed by pesticides. The authors show the flow diagram and the evaluation results of their algorithm for the identification of maize plants damaged by a worm using color digital images. The proposed algorithm has two stages. In the first stage, the images are taken and converted to gray scale images. Then, they are processed to create binary images using an iterative algorithm. During this process, the leaves are segmented and divided into pixels. In the second stage, the images are divided into blocks and those blocks, which leaf surface is exceeding 5\%, are selected. On each selected block, any anomaly is identified and is marked as damaged or undamaged by using the number of objects found in each block. The authors conclude their work performing an efficiency assessment of their algorithm. The studied insect has eight stages of maturation and the efficiency of the proposed algorithm is higher in the first two phases, obtaining $94.72 \%$ in the percentage of right answers (considering as affected blocks, those who have at least 10 objects).

P. E. Cruvinel et al. present in [53] an automatic method based on image processing for measure drop size distribution of agricultural sprinklers. In this paper, their authors seek to determine the exact size of the sprinklers drops, because if they are too small, the droplets could be subject to wind drift and could distort the pattern of irrigation application, but if the drops are too big, they acquire more kinetic energy and could lead problems concerning to soil erosion, aggregated breakdown, surface sealing, and infiltration. To achieve this goal, the authors present a technique based on image processing, using properties of Fourier analysis and correlation in the frequency domain. This direct measurement on the sprinkler drops allows studying the effects of pressure and nozzle size on the distribution of water. With this system, farmers would be able to regulate the size of the drops, to adjust the amount of water to rural needs, and take into account factors such as wind.

Antonia Macedo-Cruz et al. presented an assessing method to classify the land covered with oat crop, and to quantify planting density affected (and not affected) by very low temperatures (frost) in [54]. They use a combination of three thresholding strategies (the Otsu's method, the Isodata algorithm, and the Fuzzy thresholding) in order to quantifying the damage. They state that this merging gives better results than taking each of them separately. The used classification strategy involves three main procedures: an automatic thresholding, the fusion of thresholds and classification using the merged value, the validation of the classifier by computing the fuzzy error matrix to measure the accuracy. Finally, they show the accuracy of the classifier.

Moreover, the use of cameras can provide numerous benefits over traditional homogeneous sensor networks. An example is provided by Purushottam Kulkarni, et al. in [55]. They propose a camera sensor network for object detection, recognition and tracking. Their main contributions are the novel mechanisms introduced for low-power low-latency detection, low-latency wakeups, efficient recognition and tracking. But, their proposal use light image processing techniques to 
detect the targets, thus it would be a weak system to be used for detecting any type of deficiency, pest, disease or other harmful agents.

Liang Liu et al. investigate the coverage of camera sensor networks for target detection [56]. They propose a localization-oriented sensing model based on the perspective projection of the camera sensors. Then, they analyze how the probability of the localization-oriented coverage changes with the number of sensors and the parameters of the proposed model.

Finally, in [57], Codruta Istin et al. propose a method for real-time distributed image acquisition through wireless networks for traffic monitoring. They also present a collaborative online algorithm, which identifies an optimized set of cameras that must be turned on to cover the field of view loss.

On one hand, we see that the first works presented in this section are only focused on image processing, without taking care in depth how should be the devices used for image detection, how can be they placed to take images and how many of them should be in the field to cover an area. Moreover, all of them develop the work without giving details about the movement of the camera (if is fixed or mobile) or if it could change its direction of view. The second ones are only focused on target detection and tracking, but they do not take much care on the image processing. On the other hand, none of the previous works show the sensor network design, the wireless sensor deployment, how the node processes the images for vineyard monitoring. Moreover, the distributed wireless sensor network proposed in this work permits low bandwidth consumption, allowing higher scalability than when all video streams are transmitted through the network, in order to cover larger cultivated areas.

\subsubsection{WSNs for detecting deficiencies in construction}

There are few works related to the study of deficiencies in construction materials and the application of WSNs in the construction sector.

We can find a new design based on ZigBee networks, named as Automated Material TRACKing (AMTRACK) in [58]. This proposal is based on the combination of a ZigBee network and an ultrasound system to improve the positioning accuracy for automated materials tracking in construction process.

Another important work is presented by N. Kurata et al. in [59]. The paper provides an introduction of WSN technology for ubiquitous structural monitoring (USM), and reports a research activity on the development of a sensor module for USM. In addition, they present a sensor board equipped with a MEMS (Micro-ElectroMechanical Systems) acceleration sensor.

In 2006, a WSN was designed, deployed and tested in the Golden Gate Bridge of San Francisco with the objective to monitor its structural condition [60]. Sixty-four motes were distributed over the main span and southern tower, comprising a large wireless vibration sensor network. The spatially dense array resulted in an effective signal-to-noise ratio compared to single, isolated, sensors. The array also allowed the values obtained from both torsion and vertical, to be analyzed easily and accurately.

J. Matthew et al. [61] presented a WSN consisting of fifty-six accelerometers accommodated by twenty-eight local nodes, which facilitate simultaneous, real-time and high-rate acquisition of the 
vibrations throughout the bridge structure. Authors show a laboratory model and damage scenarios, with several simulation results, for different testbeds of bridge movements and vibrations.

G. Sun et al. [62] use a WSN to deliver the data of the corrosion of reinforcing concrete steel in structures. The novelty of this proposal is that the sensors of this corrosion monitoring system are powered by the electrochemical energy of the corrosion process of the reinforcing concrete structure. This is an example of energy-harvesting sensor platform.

Finally, C. Kim et al. [63] present a system for monitoring construction materials in large-scale construction projects where there is no data communication network. The system is based on a radio frequency identification system (RFID) to identify a variety of construction materials, and a wireless network based on ZigBee technology (IEEE 802.15.4) that it is used to transfer the collected information to the end users. Authors confirmed the viability of their system using RFID and ZigBee technologies in an indoor scenario. Their experiments, demonstrated that the integration of RFID and ZigBee modules could improve existing management processes for construction resources in large and complex construction sites.

We can see that there are several attempts to use WSNs in construction, but there are few implemented systems.

\subsubsection{WSN for disabled and elderly people}

This section reviews the related literature that takes profit of the smartphones' embedded sensors and shows some real life applications. We have also added other works that describe useful implementations to improve the disabled and elderly people quality of life.

Because millions of people carry mobile phones nowadays, many developers have unconsciously deployed a strong infrastructure which can help us to implement new proposals, based on the sensing capabilities of these devices. Following this idea, Nicholas D. Lane et al. [64] discuss the state of the art of mobile phone sensing. This work analyzes some aspects in many different disciplines such as business, healthcare, social networks, environmental monitoring, and transportation. In addition, they review the main sensors embedded in mobile phones and discusses which possibilities could open this technology to the society.

Because the processing capabilities of mobile devices are growing more and more, the incorporation of several sensors and increase the complexity of the applications needed to control all these sensors is possible. In this line, C. Muldoon et al. [65] propose the use of a Multi-Agent System called "Collaborative agent tuning" to optimize the system operation in devices with limited computing capacities. This solution permits developers to manage and control all available sensors in mobiles devices and smartphones.

When we talk about wireless sensor networks, we think on networks formed by geographically dispersed sensors that work together to monitor the environment. The increased use of smartphones suggests developing large sensor networks using these mobile devices as sensor nodes [66]. In [67], Kansal et al. present a first attempt towards the understanding and implementation of a programmable sensor network using the infrastructure created by the different mobile devices. The proposed system uses the collected samples of the phone sensors and shares the data with other users publishing it in a data repository. 
Demographic forecasts indicate that elderly population will increase exponentially in the future in developed countries, mainly because of the new advances in medicine and prevention illness systems. This trend makes the development and implementation of new supporting technologies imperative in the daily environment. In [68], Immaculada Plaza et al. outline the opportunities that mobile applications offer to improve the quality of life of the elderly people. An example of this idea is the TeleCARE project, presented by L. M. Camarinha-Matos and H. Afsarmanesh [69]. The main goal of this project is to develop a system for tele-supervision following a multi-agent approach, which will facilitate independent lifestyles for elderly people. The infrastructure includes both fixed terminals and mobile phones and allows giving independence and better quality of life to elderly people and their families.

The advances of wireless network technology and the development of body sensors are helping to improve the care of patients with reduced mobility at hospitals and health centers [70]. Accidental falls in the elderly and disabled people is considered one of the major health problems, in terms of primary care costs, of the public and private health systems [71]. We found several approaches that use smartphones for elderly people falls detection. In [72], authors detect falls using the sensors of an Android smartphone. P. Martín et al. present in [73] a multi-agent system for mobile devices that can detect the falls of people using the sensors embedded in the device. If a fall is detected, the system generates a message with the GPS position coordinates that is sent via SMS and at the same time the device can also make emergency calls. The presented proposal has low computational cost, which implies less power battery consumption. In addition, the traffic generated into the network is acceptable. It has a fast mechanism to reduce the impact of the falls for elderly people, while permits an automatic and quick response to the potential incidents.

Fall detection must be lightweight to mobile phone resources consumption. To achieve this goal we can use learning techniques like the semi-supervised algorithm used by Ali Fahmi et al. in [74]. Each user moves differently, for this reason, the system should have adjusted the classification parameters to the particular movements of the user. In [75], the authors propose an adaptive algorithm that allows the personalization of the patient profile.

The care of elderly people that live alone and the extension of their independence are the goals of the activities tracking system described by Muhammad Fahim et al. in [76]. Moreover, Boštjan Kaluža et al. presented in [77] a system with the same goals by using a multi-agent approach. It is able to send an alert when there is an emergency or unusual behavior.

A. Costa et al. [78] presented a system that allows health professionals knowing the vital parameters of the patients in their care. This system is developed for PDA or smartphones, and it is able to send information about a monitored patient which is provided by a set of sensors. It permits a remote diagnosis, avoiding critical situations in unsupervised environments which may lead to the death of the patient.

In [79], Reza Olfati-Saber et al., analyze the consensus and cooperation algorithms in networked multi-agent systems. This work presents several algorithms that provide rapid agreement between all mobile devices allowing effective task performance by self-organizing networked systems.

Finally, A. Arcelus et al. provided an overview of the smart home technology in [80]. Authors describe how a smart system could be implemented in a house in order to help elderly and disabled people. This system is similar to the one presented in our paper. But, on one hand, it can only be 
used indoors, and, in the other hand, the authors only provide some ideas and they do not show in detail the operation of their system.

So, as far as we know there is not any smart collaborative system like the one presented in chapter 4 which uses the information of the embedded sensors and takes decisions after knowing the information of the embedded sensors in its neighbor's devices.

\subsection{State of the art of WSN in indoor environments.}

\subsubsection{Related Work of indoor coverage measurements}

There have been many studies of indoor coverage for single-transmitter and single-receiver protocols, like IEEE 802.11a, IEEE 802.11b and IEEE 802.11g [81]. Others use the measurements obtained from the signal level of a group of access points to perform the channel planning while avoiding interference [82]. There are some that locate clients by using the signal strength received by several access points [83]. IEEE 802.11 infrastructure has the advantage of being available in numerous indoor environments, and is deployed in densities that allow for the possibility of positioning with meter level accuracy.

As IEEE 802.11 networks are widely deployed, there has been a significant amount of work about planning IEEE 802.11n wireless networks. In such networks, the use of Multiple-Input Multiple-Output (MIMO) transmission scheme changes the expected behavior of signal level due to its multiple antenna use, exploiting physical phenomena such as multipath propagation to increase the transmission rate and reduce the error rate.

Foschini [84] derives theoretically that for the same SNR a 2x2 MIMO channel can hold twice the amount of bandwidth than using a single transmission and receiving antenna. As shown in [85] even further gain can be expected by the use of larger arrays of antennas in both reception and transmission.

Most of the recently published papers have modeled the MIMO channel matrix with independent and identically distributed Gaussian entries, which is an idealistic assumption, especially for indoor scenarios. More realistic MIMO channel models can be generally divided into three classes: raytracing, scattering and correlation models [86]. Anyway, for indoors need very large simulation time and complexity for trying to provide a good prediction of the channel behavior. On the other hand, correlation-based models do not provide detailed information about coverage, which could be needed for applications like indoor positioning [87].

There are still a few indoor IEEE 802.11n channel measurements reported in the literature. Simulation methods of these channels based on direct measurements are even fewer. At the $5 \mathrm{GHz}$ band, for example, the publicly available IEEE TGn models [88] are the most convenient tools for MIMO channel simulations. However they have their own limitations; e.g., they are based on single-input single-output (SISO) channel models presented in [89][90] which do not reflect accurately the multipath propagation channel.

Another work that studies the coverage is presented by E. Amaldi et al. in [90]. This paper describes the optimization models with hyperbolic and quadratic objective functions. The authors propose heuristic methods that combine greedy and local search phases, and show the need of 
appropriate planning models and procedures that are specific to WLANs. The authors suppose that the system affects to the coverage planning process, and the incidence of overlapping regions should be taken into account in the planning procedure (beside of all the other optimization parameters). The computational results show that their heuristics provide near-optimal solutions within a reasonable amount of time.

Some other researchers have based their methods in empirical [91] and analytical [92] studies. On the one hand, A.R. Sandeep et al. [91] suggest an indoor empirical propagation model (IEPM) in order to predict the signal strength of an indoor Wi-Fi network. It is a predictive model based on the Wall Effect Factor (Wef) and the Wall Attenuation Factor (Waf). From this model, authors can calculate the RF coverage area before installing a WLAN and so that calculating the number of access points needed. Therefore, this model means low cost and development time. On the other hand, Eisenbl et al. [92] perform an analytical study about the best location for APs and channel assignment in order to improve the performance of a WLAN. Up to now, these features have been analyzed independently, but according to this paper the greatest optimization is achieved from studying these two features simultaneously by mathematical programming. Authors propose an integrated model in order to reach a balance between both features and to optimize the indoor design of WLANs.

Expanding analyzing studies, M. Kamenetskyt et al. [93] analyze different methods for obtaining the most optimum location for the WLAN's access points. In order to evaluate the performance of these methods, they use an objective function which maximizes the coverage area and signal quality. Then different approaches to coverage planning for WLAN systems are reviewed and the most suitable for numerical evaluation are selected. From this evaluation, authors propose a new optimization scheme based on the combination of two approaches: using pruning in order to set initial locations for access points and refining these by using either neighborhood search or simulated annealing.

Then, E. Amaldi et al. [94] present a new modeling approach taking into account the effect of the IEEE802.11 access mechanism. It influences on radio coverage due to the coverage overlap between APs and its impact on the system capacity. So, they explain and discuss novel mathematical programming models based on quadratic and hyperbolic objective functions considering this. Finally, some initial results on synthetic instances are shown.

Some abovementioned authors improve its initial approach (published in [94]) because it is difficult to tackle even for small instances. So, they propose and analyze effective heuristics in [95] to tackle hyperbolic and quadratic formulations in order to maximize the overall network capacity. It is based on a combined greedy and local search algorithms turn out to provide near-optimal solutions in a reasonable amount of time.

Following with empirical papers, Kaemarungsi and Krishnamurthy [96] study the performance of the received signal strength (RSS) from IEEE 802.11b wireless network interface cards in order to improve the indoor location systems based on location fingerprints. Moreover, they point out the influence of the users' presence on the RSS, both the proximity of the human body to antenna and its orientation. These features affect the mean value and the spread of the average RSS values. So, if the position system is deployed in an environment with people, it is essential to take it into consideration while collecting RSS values for the fingerprint. In contrast, for applications that make use of sensors without human presence, this influence shouldn't be considered. 
J. Lloret et al. [97][98] show studies about an empirical coverage radio model for indoor wireless LAN design. This model has been tested on a vast number of buildings of a great extension area with over 400 wireless APs in order to get quick successful results. The objective of the model is to facilitate the design of a wireless local area network WLAN using simple calculations, because the use of statistical methods takes too much time and it is difficult to implement in most situations. The proposed analytical model is based on a derivation of the field equation of free propagation, and takes into account the structure of the building and its materials.

J. N. Davies et al. [99] measured the IEEE 802.11n signal level in a real building, but they do not make any comparison with IEEE 802.11a or IEEE 802.11g.

In [100], authors propose a new WLAN design strategy called capacity based WLAN design. The method guarantee radio coverage to the target service area and provide a specified data rate capacity to carry the traffic demand from each user in the service area. The methodology proposed determines the number of APs, frequency channels, power level and the placement of the APs that ensure the constraints as data rate density requirements, radio propagation conditions and physical limitations, related with receiver sensitivity. Authors performed several design experiments, which show the benefits of their method over the traditional coverage - based design.

Moreover, I. Broustis et al [101], suggest that when we perform large wireless sensor network deployments, it is possible to detect large amount of interference, because their small capacities. They tell us that to improve the overall capacity of the network; we can base our proposals in the intelligent frequency allocation across APs, in the load balancing of user affiliations across APs and in an adaptive power control for each AP. In their work, they search interdependencies between the three functions in order to understand when and how to apply them to the network design. The authors performed the measures following a study based on the quantification of the effects of three optimization schemes proposed in many different scenarios. From the results, we can see that applying simultaneously the three optimization schemes is not always preferable, because it can sometimes degrade the performance by up to $24 \%$ compared to using only two of the schemes.

\subsubsection{Related work of interference measurements in indoor environments}

From the IEEE 802.11 WLAN interference side, Nicolescu [102] proposed a model for interference in dense wireless networks that enables a low complexity procedure to collect the interference map and can be used to predict the damage from several simultaneous interferers. Unfortunately measurement of the interference map faces asymmetries in the card and channel behavior, which make the complexity still prohibitive for dense multiple card networks, requiring direct measurements in indoor deployments. Also, Fuxjäger et al. [103] show that the assumption of perfect independence between non-overlapping channels does not always hold in practice, by means of simple experiments with commercially available hardware, and found that the level of interference varies with physical distance, concurrent link-load, modulation rate, frame size, transmission power, receiver sensitivity and design, antenna patterns, etc., calling also for more direct measurements.

J. Padhye et al. present in [104] an interference measurement-based study between links in a static, IEEE 802.11, multi-hop wireless network. Then, the authors propose a simple empirical 
estimation methodology that can predict pair wise interference using only measurements. These tests are based on heuristics methods where the wireless links are defined by their packet loss rate. They state that this methodology could be applicable to any wireless network that uses omnidirectional antennas.

Related to interference and throughput measures in WLAN, J. Jun et al. present in [105] an accurate formula to estimate the throughput in IEEE 802.11 networks, for several variants (802.11, $802.11 \mathrm{~b}, 802.11 \mathrm{a}$ ), in the absence of transmission errors and for various physical layers, data rates and packet sizes. The authors cite some applications where it is very important to know the maximum throughput in order to design them correctly. Theoretical Maximum Throughput can be used to facilitate optimal network provisioning, for example, in multimedia applications. It can influence the topological distribution of the nodes in the case of ad-hoc networks.

Although analytical studies and network simulations may provide valuable insights of the WLANs' operation, they cannot predict the actual performance of practical implementations with high accuracy. Moreover, measurements obtained from file transfer operations are also limited by the need to specify the processor type, processor speed and the network operating system. B. Bing presents in [106], an experimental study to characterize the behavior in terms of throughput and response time of two commercial AP under different degrees of network load. They are WavePOINT, from Lucent Technologies, and Spectrum24, from Symbol Technologies. The tests showed important characteristics such as throughput and response time under various network loads. The author also shows that the length of a data frame and the wireless bit rate also affects to the WLAN's transmission capabilities. But, the performance of an IEEE 802.11 WLAN is generally unaffected by the type of frame and the use of reservation frames such as RTS and CTS.

\subsubsection{Related Work of wireless sensor positioning in indoor environments}

Because when we are working with wireless sensors its distribution may lead to significant differences in coverage estimation, it is important to know their behavior and the possible the impacts of devices on the network coverage [107]. There are several papers and surveys about wireless sensor network coverage studies where authors explain the main aspects to be considered in the implementation of WSNs [108]. Most of these papers are focused on knowing the exact location of the sensors, based on algorithms location, GPS location, etc. We have found some few works that analyze the sensor placement, trying to minimize the number of devices needed to cover an area, while optimizing the way to gather of information, but none of them are like ours.

The first of them is the work presented by S. Martinez and F. Bullo [109]. They propose motion coordination algorithms aimed at mobile sensor networks. They study the optimal positioning of a sensor in motion and coordination strategies in mobile sensor networks. In their work, they show a mathematical development, which defines a total cost function called "sensitivity performance measure" and find the best design to their algorithms in order to maximize this function. The purpose of this paper is mainly the design of distributed movement algorithms to improve the coordination and the data collection in an environment.

Related to IEEE 802.15.4 or Zigbee technology and sensor tracking, A. Redondi et al. [110] present a Personal Localization and Tracking System (PLTS) functional module, which leverages wearable devices mounted on patients and the network architecture to implement the 
localization/tracking service. To locate the devices, authors use a localization algorithm based on the Received Signal Strength (RSS), called LAURA, which requires a minimal setup cost, and is able to track the position of moving patients with an average accuracy below 2 meters. This system implements localization, tracking and monitoring of patients hosted at nursing institutes by exploiting a wireless sensor network based on the IEEE 801.15.4 (Zigbee) standard.

E. Coca et al. also present a location system made of wireless sensor nodes build around the Chipcon CC2431 System and its performance [111]. The main idea of this proposal is to put the reference nodes in fixed positions and to construct a table with RSSI values measured in a virtual grid. Their measurements (in indoor environments) were conducted to see if the influence of the external factors, such as the electromagnetic interfering RF emission, affects the results. Authors have compared the results obtained with similar systems in almost the same conditions, and investigate the possibility of using the location and time information to determine the movement speed of mobile nodes. They have used the Green Peak Technologies WSN evaluation kit and Ti Location Engine to perform their tests. Authors conclude that the single difference between the two systems is the algorithms used for filtering the raw location information.

$\mathrm{X}$. Li et al [112] present an algorithm that takes in consideration the value of the received signal strength indication (RSSI) to locate the sensors in an ad hoc sensor network. The proposal uses adaptive quantization scheme for each node in order to determine the range level used in the quantization process. As results show, this algorithm improves the range estimation accuracy when distance information is not available or too erroneous.

Finally, we found the paper authored by S. S. Dhillon and K. Chakrabarty [113]. They present two efficient algorithms for the placement of sensors in a field. The authors also raise the problem of optimizing the sensor placement and the minimum number of devices that should be used to provide sufficient coverage. They argue that its implementation must take into account the features of the place. For example if the network is deployed outdoors it must be taken into account obstacles such as buildings and trees, irregular elevations of surfaces or mountainous terrain and the power required to transmit information between sensors. These algorithms are designed to calculate the average coverage and to maximize coverage of the most vulnerable points within the coverage area. Finally to demonstrate the efficiency of each algorithm, authors present different case studies and simulate their behavior.

None of these studies specified which strategies should be used for positioning sensors in indoor environment. In our proposal, we have used some studies and tests performed to determine the best placement of a sensor, taking into account factors like the coverage offered by an AP, the indoor multipath effects caused by signals reflections on the walls and the interferences generated by the signal of adjacent channels.

\subsection{State of art in underwater wireless communications}

Current systems for underwater communication essentially use three methods for transmitting information. These methods are based on sound waves, in the Electromagnetic waves (EM) and optical signals. Each one of these techniques has advantages and drawbacks, mainly due to the physical constraints [114][115]. 
The signal propagation can be explained by the physicochemical properties of the water and the physical properties of the light.

- The physicochemical factors that influence the light properties are the transparency, i.e. the amount of light transmitted in the seawater; the absorption, the amount of radiation is retained and turbidity, which reduce water clarity by the presence of suspended matter.

- The physical properties of the light are: Reflection, Refraction and Extinction, which measures the degree of the light that can penetrate into the marine environment. In addition, the two most important factors in seawater are the temperature and salinity, which determine the density of the water. In the ocean, the density tends to increase with depth, so the upper water layers have always higher density. All these factors are very important to know how to spread the light and sound in the ocean.

The most widely used waves for underwater communications are the acoustic waves. There is a huge variety of articles, which describe the propagation of such waves. An example of Path Loss analysis give by the reflection and reflection is provided in [116]. Moreover, we can see in [117] the effects of depth and temperature in this type of waves. For systems based on optical communications, we can find too a variety of studies of their propagation and losses [118]. There is not too much documentation about high frequency in underwater communications because most of the works are designed for low frequencies in order to achieve large communication distances, preventing the power losses generated in high frequencies.

This section will show some of the main expressions of different types of waves in underwater environment and the factors that may cause difficulties in communication operation.

\subsubsection{Underwater communications based on acoustic waves}

The sound is produced by a vibration of the molecules in an elastic substance. Mechanical energy propagation of sound is absorbed in the medium by which it is spread. In water, the sound propagates faster and has lower energy losses that in the air. Sounds and ultrasonic sound waves are transmitted into the sea at a speed between 1400 and $1600 \mathrm{~m} / \mathrm{s}$, while in the air the speed propagation is $340 \mathrm{~m} / \mathrm{s}$. This is because seawater is not compressed, i.e. it cannot be reduced to a lower volume, so that the absorption of sound waves is minimal, contrary to happens in the atmosphere, where sounds are absorbed at very short distances.

There are several factors that influence the distance that sound can travel underwater. On the one hand, particles of seawater can reflect, scatter and absorb certain frequencies of sound as well as certain particles in the atmosphere can reflect, scatter and absorb certain wavelengths of light. Seawater absorbs 30 times the amount of sound absorbed by distilled water, attenuating certain frequencies of sound. Low frequency sounds, and pass over tiny particles tend to travel farther without causing any loss by absorption or scattering. Studies have shown that factors such as salinity, temperature and pressure changes cause the speed of sound propagation under water.

In general we can say that the ocean is divided into horizontal layers in which the speed of sound depends greatly on the temperature in the upper regions and the pressure in the lower regions. The top layer is heated by the sun whose temperature varies depending on the season. In middle latitudes, the water is perfectly mixed by the action of waves and currents. But there is also a 
transitional layer called the thermocline, where temperatures drop continuously with depth as the temperature drops, the speed of sound decreases. However, there is a point, which ranges from 600 meters to $1 \mathrm{Km}$ below the surface, from which temperature changes are slight. In this case, the main factor influencing the speed of sound is increasing pressure causes the sound speed increases.

The expression 2.1 relating the value of the sound speed propagation in water, taking into account the temperature $(\mathrm{T})$ in degrees Celsius, salinity $(\mathrm{S})$ expressed in parts per thousand and the depth (z) in $\mathrm{m}$ [119]. Table 2.3 shows the values of constants.

$$
c(T, S, z)=a_{1}+a_{2} T+a_{3} T^{2}+a_{4} T^{3}+a_{5}(S-35)+a_{6} z+a_{7} z^{2}+a_{8} T(S-35)+a_{9} T z^{3}
$$

Where $a_{1} . . a_{9}$ represent constants whose value are expressed in Table 2.2.

Table 2.2. Constant Value

\begin{tabular}{|l|l|l|l|l|l|}
\hline Constant & Value & Constant & Value & Constant & Value \\
\hline$a_{1}$ & 1448.96 & $a_{4}$ & $2.374 \times 10^{-4}$ & $a_{7}$ & $1.675 \times 10^{-7}$ \\
\hline$a_{2}$ & 4.591 & $a_{5}$ & 1.340 & $a_{8}$ & $-1.025 \times 10^{-2}$ \\
\hline$a_{3}$ & $-5.304 \times 10^{-2}$ & $a_{6}$ & $1.630 \times 10^{-2}$ & $a_{9}$ & $-7.139 \times 10^{-13}$ \\
\hline
\end{tabular}

If we take the expression 2.1 and represent it, we obtain Figure 2.2, which shows the values acquired by the speed of sound propagation, taking into account the effect of temperature and pressure variation according to depth.

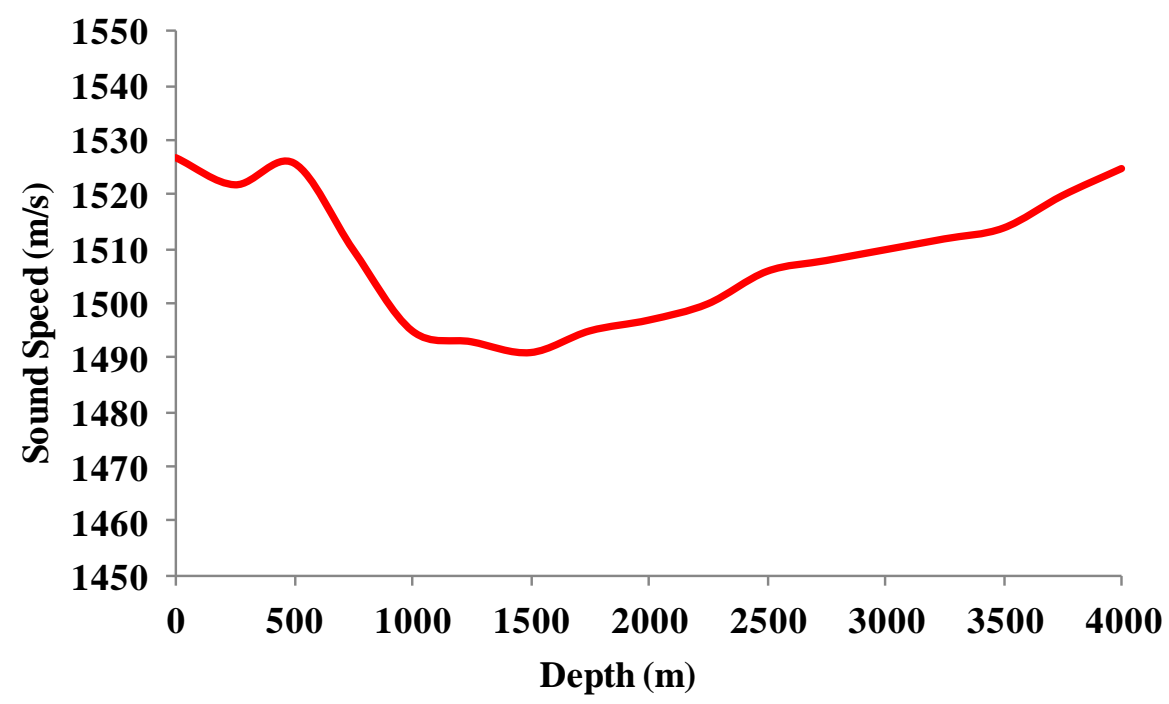

Figure 2.2. Sound speed propagation.

Underwater acoustic communications are mainly influenced by the physical properties of the medium's own sound wave and by path loss, noise, multi-path, Doppler spread, and high and variable propagation delay.

- Propagation speed: The propagation speed of sound through water is much smaller than 
the propagation of electromagnetic signals. The speed of sound in water depends on the water properties as temperature, salinity and pressure, as seen in Figure 2.2. A typical speed of sound in water near the ocean surface is about $1525 \mathrm{~m} / \mathrm{s}$, which is about 4 times faster than the speed of sound in air, but 5 orders of magnitude slower than the speed of light.In approximate terms, the sound speed increases $4.0 \mathrm{~m} / \mathrm{s}$ for every degree Celsius increase in temperature of the water. When salinity increases 1 practical salinity unit (PSU), the sound speed in water increases $1.4 \mathrm{~m} / \mathrm{s}$ when the pressure of water (each additional $1 \mathrm{~km}$ deep), the speed of sound increases about $17 \mathrm{~m} / \mathrm{s}$.

- Absorption: When there is swelling at sea, it is converted into energy that is absorbed by the medium, due to its intrinsic characteristics. When the acoustic signals propagate through the seawater, suffer the same effect, reducing its initial energy. The absorption phenomenon is more pronounced in a range of frequencies than the other, the high frequencies (above $100 \mathrm{kHz}$ ) are more sensitive, so we can ensure that it is frequency dependent.

We can express this behavior by the expression 2.2, which has the contribution of the main salts in seawater [120]:

$$
\alpha=0,106 \frac{f_{1} f^{2}}{f_{1}{ }^{2}+f^{2}} e^{\frac{(p H-8)}{0,56}}+0,52\left(1+\frac{T}{43}\right)\left(\frac{S}{35}\right) \frac{f_{2} f^{2}}{f_{2}{ }^{2}+f^{2}} e^{-z / 6}+0,00049 f^{2} e^{-\left(T / 27^{+Z} / 17\right)}
$$

Where $\alpha$ is the attenuation in $\mathrm{dB} / \mathrm{km}, \mathrm{f}$ the frequency in $\mathrm{kHz}, \mathrm{z}$ the depth in $\mathrm{km}, \mathrm{T}$ the temperature in ${ }^{\circ} \mathrm{C}$, and $\mathrm{S}$ the salinity in ppt. The first term is the contribution from boric acid, the second term is the contribution of magnesium sulphate, and the last term is the pure water contribution.

When a signal is transmitted through a medium that can generate losses must be taken into account several factors. The main mechanisms for energy losses are the geometric spreading, absorption losses and scattering losses.

- Attenuation. It is caused mainly by absorption, when the acoustic energy is converted into heat energy. The attenuation is caused by scattering and reverberation (a deep), refraction and dispersion (surface). The attenuation also increases with distance and frequency.

- Geometric expansion. Geometric expansion is the loss of energy in the acoustic wave is spreading, due to conservation of energy. This refers to the diffusion of sound energy as a result of the expansion of the wave fronts, i.e. when a pulse of an acoustic wave propagates, as the wave front moves away from the origin, covers a larger area. Therefore, the wave energy per unit area becomes smaller. This phenomenon is independent of the frequency; we can distinguish two geometric expansion:

○ Spherical, where the source spreads out omnidirectional and it is characteristic of deep-water communications. The energy losses, caused by the geometric expansion, are proportional to the square of the distance.

○ Cylindrical, which takes into account the horizontal radiation only and characterizes the communications in shallow water. The energy losses caused are proportional to the distance.

Other factors such as ambient noise, dispersion or multipath effect also may hinder communication underwater.

- Noise. Acoustic communications noise is defined as any unwanted sound that interferes 
with the communication between communication systems. Basically we can distinguish two types of noise:

○ Generated noise. Is the noise caused by machinery such as engines and natural activity of the medium, as animal life.

- Ambient noise. It is related to the movements of water, tides and currents, storm or water bubbles when it hits the coast, etc., which can generate losses up to $26 \mathrm{~dB} /$ $\mathrm{m}$. Also seismic and biological phenomena can be considered. All of these phenomena are known as hydrodynamic [121].

- Delay and delay variation. Due to the nature of the medium, the variation of signal delay can be very high and it may turn out to be very detrimental to the efficient design of a protocol and would complicate the estimation of the Round Trip Time (RTT), an important parameter for many common communication protocols.

- Doppler spread and Scattering. Scattering is the phenomenon whereby a set of particles moving in one direction can bounce with the particles on the environment, and its effect can deviate from a predefined straight line movement [121]. Moreover, if the sea is calm, the signals near the surface, are reflected almost perfectly, whit the only strain that can generate phase difference. However, when the sea is rough, the waves can move the point of reflection of the acoustic wave and causes the dispersion of energy. These two phenomena can cause losses of signal strength due to multipath effect.

- Multi-Path. Multi-path propagation can cause degradation of the acoustic communication signal, mainly due to the generation of interference intersymbol (ISI) and the destruction of information. The generation of multiple paths between transmitter and receiver depends on the link configuration. Communications where you create vertical channels do not suffer a very high scattering loss, due mainly the signal does not found obstacles to reflect, while the horizontal channels, due to the effect of the surface, may experience greater losses. The level of diffusion is a function of depth and distance between the transmitter and receiver.

\subsubsection{Underwater communications based on optical waves}

The light is a radiation mix of different frequencies. The optical signals used for wireless communications are generally limited to short distances because the water has a fairly high absorption factor in the optical waveband.

Not all frequencies within the spectrum of light are affected the same way. Each color is a particular frequency radiation. For this reason, the reds colors are the first to disappear, while the latter are the blue and green colors. The wavelengths of blue-green colors, offers a good performance for broadband communication (10 Mbps to $150 \mathrm{Mbps}$ ) to moderate distances between 10 and 100 meters. In theory, by using optical signals for communication under water, could reach speeds of 1 Gbps.

However, the optical signals have two main disadvantages. In the first factor, the suspended particles cause light dispersion and the second factor, due to the physical properties of water, the optical signals are absorbed quickly. 
The light propagation depends on the medium traversed. For this reason, the light does not travel at the same speed in air than in water. When light propagates in an aqueous medium, its intensity decreases exponentially due mainly to the attenuation that is produced by two main causes:

- Absorption: of light energy is converted into another type of energy, usually heat or chemical energy. This absorption is produced by:

- Algae, which use light as an energy source.

- Organic and inorganic particulate matter in suspension.

- Inorganic compounds dissolved.

$\circ$ The water itself.

- Scattering: This phenomenon is the result of the collision of the beam with particles in suspension, causing multiple reflections. More turbidity in water cause higher scattering effect, making difficult the light penetration. Figure 2.3 shows a diagram about the scattering effect in a beam, when they incident on the particles in suspension.

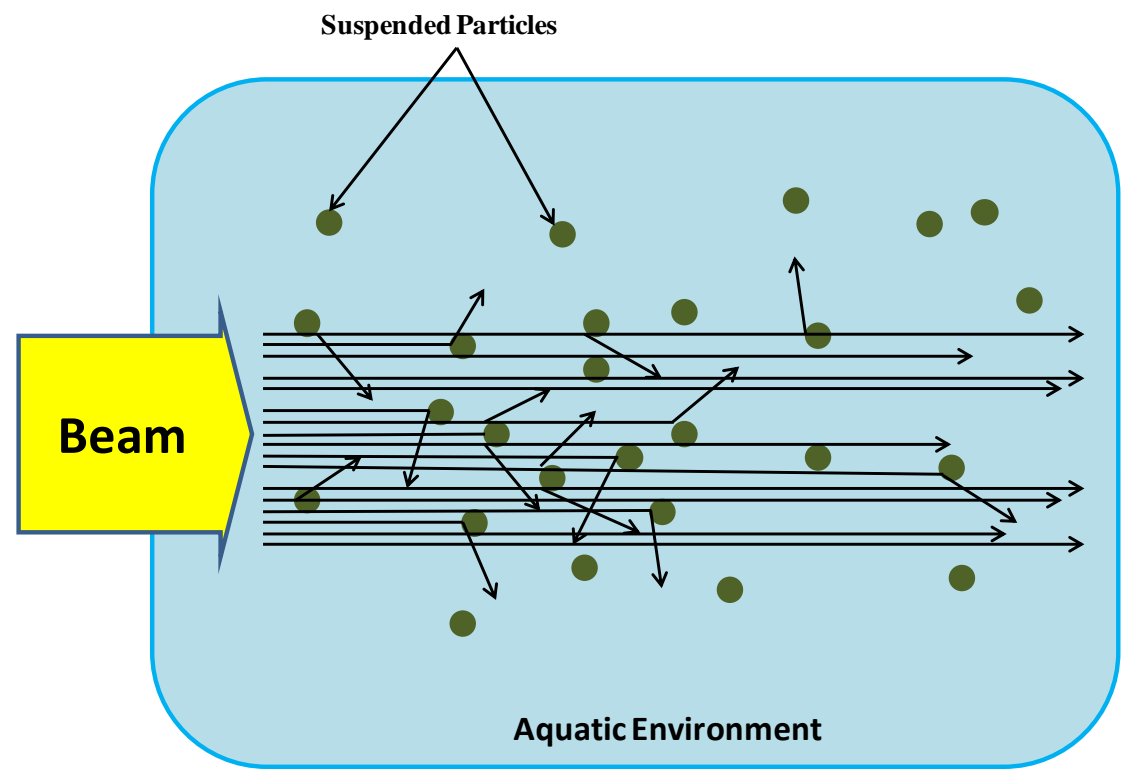

Figure 2.3. Scattering effect

As Figure 2.3 shows, the light in the ocean suffers the diffuse scattering effect. This means that the light does not always follow the shortest way to highlight an object, but many reflected rays arrive and diverted from its original path. The amount of organic matter in suspension contained in seawater cause the light intensity diminish in the propagation direction, because it is absorbed by these particles, this parameter is called absorption coefficient or extinction factor of light, and provides the value of transparency of the water.

It is possible to calculate the total energy scattered by a particle through extremely complicated calculations [122], and using the expressions of the Mie scattering theory [116], which is valid for all possible relationships of particle diameter and wavelength of the signal. According to the Mie theory when the wavelength of light is similar to the diameter of the particle, light interacts with particles in a cross-sectional area larger than the geometric cross section of the particle. This allows us to express the total energy scattered by a particle in all directions (see Equation 2.3) [123]. 
Where $I_{s c a}$ is the scattered light intensity, $I_{0}$ is the incident light intensity; $r$ is the radius of the particle and $\sin \phi d \phi d \theta$ represents the solid angle for the calculation the entire surface area of the sphere. With this expression would be able to calculate the amount of light beam energy is lost due to beam collisions with particles suspended seen in the water. A much larger particles or water grade of turbidity was, it will register the more absorption of light beam in the medium.

\subsubsection{Underwater communications based on electromagnetic waves}

The use of EM waves to transmit signals in the water is characterized to be a fast and efficient communication between network nodes.

Moreover, the use of EM waves in the radio frequency band has several advantages over the acoustic waves, mainly is faster and can be used in higher work frequencies (which results in a higher bandwidth). Furthermore, there are several factors that limit the use of EM waves in the water. EM waves are propagated in very different ways depending on the type of water where the system is implemented.

\subsubsection{EM waves in freshwater}

EM waves have several advantages over acoustic waves when used to transmit signals in water. They provide fast and efficient communication between network nodes, and, because they use higher work frequencies, they provide higher data rates. There are several factors that limit the use of EM waves in the water. EM waves are propagated in very different ways depending on the type of water where the communication system is implemented.

Freshwater is a medium that has low loss. The propagation speed of the signals $\mathrm{c}$ can be expressed by following the approximation shown in Equation 2.4 [124].

Where $\chi_{e}$ the electric susceptibility of the medium and $\mu_{r}$ is the magnetic permeability of medium (in this case, it is the water).

The absorption coefficient $\alpha$ for the propagation of EM in freshwater can be approximated by Expression 2.5 [124]:

Where $\sigma$ is the electrical conductivity, $\chi_{e}$ the electric susceptibility of the medium and $\mu_{r}$ is the magnetic permeability of medium (in this case, it is the water). Expressions 2.15 and 2.16 show that 
the wave propagation and absorption coefficients in freshwater are independent of the working frequency of the transmitted signals.

\subsubsection{EM Waves in seawater}

Unlike freshwater, seawater is a medium that has large losses. The conductivity of seawater is mainly due to the concentration of Total Dissolved Solids (TDS) in water. The average salinity of seawater in the oceans is about 34 parts per thousand (ppt).

The electrical conductivity in seawater is about two orders of magnitude higher than in the freshwater. The electrical conductivity of seawater is a function of salt content (salinity) and temperature. At frequencies below $1 \mathrm{GHz}$ its value is given by the Expression 2.6:

Where $C$ is the salt content in parts per thousand and $\mathrm{T}$ is the temperature in degrees Celsius.

At $20^{\circ} \mathrm{C}$, seawater has an average value of $5 \mathrm{~S} / \mathrm{m}$, while freshwater features around the 0.005 to $0.05 \mathrm{~S} / \mathrm{m}$.

The dielectric permittivity of seawater is also a function of salinity and temperature. It is often used a value of 80 for the relative permittivity of sea water at $20^{\circ} \mathrm{C}$, although the real value at low frequency is about 70. However, at frequencies below about $100 \mathrm{MHz}, \varepsilon_{\mathrm{r}}$ is much lower than 60 .

In mediums with high conductivity used for the signals transmission, the speed of propagation and absorption losses of EM waves are directly proportional to the carrier frequency. The speed propagation of EM wave in seawater can be expressed by the approximation 2.7 [124]:

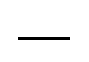

Where $\mu$ is the magnetic permittivity of the material, $\mathrm{f}$ is the frequency of the carrier signal and $\sigma$ represents the material conductivity. Moreover, the absorption losses in sea water can be approximated by (see equation 2.8) [124]:

Where $\mu$ is the magnetic permittivity of the material, $f$ the frequency of the carrier signal and $\sigma$ represents the electrical conductivity of the material.

Figure 2.4 and Figure 2.5 show the evolution of speed propagation and absorption coefficient in the case of freshwater and seawater, depending on the frequency. 


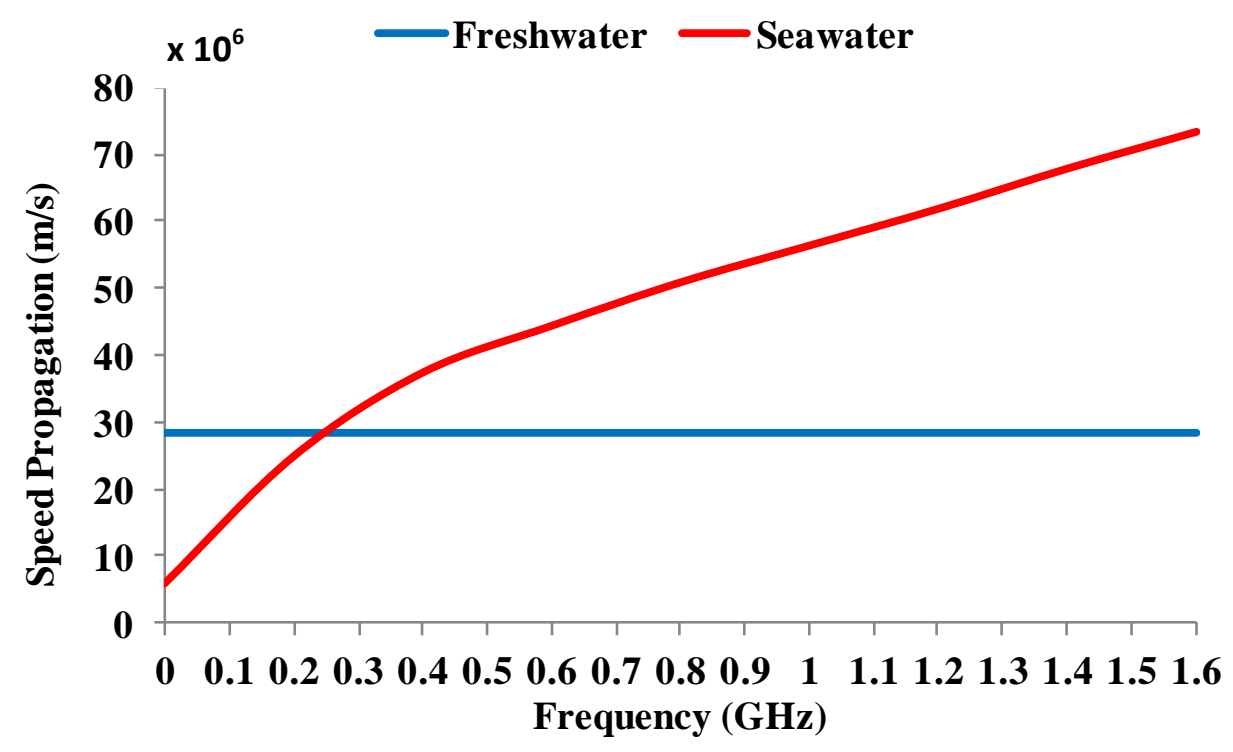

Figure 2.4. Speed propagation

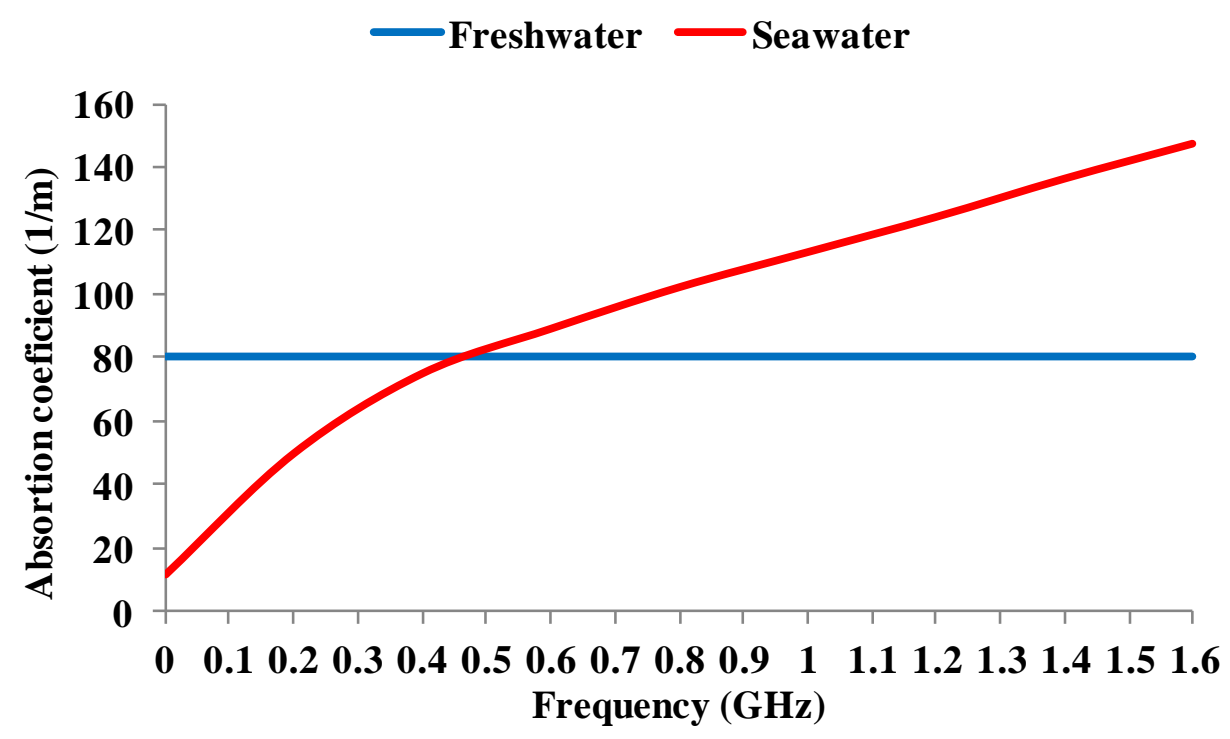

Figure 2.5. Absorption coefficient

As we can see in these graphs, in high frequencies, there is a greater coefficient of absorption; therefore it would be better to work at low frequencies, while the higher frequencies show a faster rate. However, due to high absorption of sea water, hardly EM wave can propagate in the sea water. This is the main motivation for working a lower frequency in mediums of high conductivity. Seawater is a good example of this type of medium. Therefore, communication in seawater is impractical using classical approaches based on wave propagation. 


\subsubsection{Miscellaneous of existing measurements of underwater wireless communications technologies}

In this Section, we are going to show a miscellaneous of works related to underwater communications where authors use the three most used methods.

In RF communications, researchers work with Very Low Frequency (VLF), decreasing the frequency in order to have a more effective range of communication. Concretely, some researchers of the Swansea Metropolitan University, U.K., performed their simulations at $3 \mathrm{KHz}$ and distances between nodes of about 40 meters [125].

In [126], Frater et al. compared RF and acoustic communications. They measured the maximum distances for RF. The paper shows the maximum distances for several frequencies (approximately 6 $\mathrm{m}$ at $100 \mathrm{kHz}, 16 \mathrm{~m}$ at $10 \mathrm{kHz}$, and $22 \mathrm{~m}$ at $1 \mathrm{kHz}$ ). They concluded that RF communication offers higher performance than acoustic communication in certain ranges.

Anguita et al. dismissed the RF method for underwater communication because they reported that it is strongly attenuated [127]. Thus they took as invalid the $2.4 \mathrm{GHz}$ frequency.

However EM signals offer higher throughputs than acoustic signals by up to an order of magnitude. For example, Nowsheen et al. [128] [129] developed an FPGA-based modem, that used frequencies ranging from $100 \mathrm{kHz}$ to $1 \mathrm{MHz}$ and BPSK modulation, with shipments of data packets with a duration of $1 \mathrm{~ms}$ with a wait time of $20 \mathrm{~ms}$. They stated that it is an appropriate interval to avoid the effects of reflections in the tank.

In [130], S. Jiang and S. Georgakopoulos conducted a study of the EM wave propagation in fresh water for frequencies between $23 \mathrm{kHz}$ and $1 \mathrm{GHz}$. This work presents two analyses on electromagnetic waves. On one hand, the authors measured the transmission loss given by the reflection at the air-water interface and, on the other hand, they analyzed the propagation loss inside the water due to its physical properties. They conclude that the propagation loss increases slowly for frequencies up to $1 \mathrm{MHz}$, while it remains constant between $1 \mathrm{MHz}$ to $100 \mathrm{MHz}$ and then increases dramatically for higher frequencies. In addition, the total loss in the 3 - $100 \mathrm{MHz}$ frequency range, for a depth of $1 \mathrm{~m}$, is from $10 \mathrm{~dB}$ to $45 \mathrm{~dB}$ smaller than the loss in lower and higher frequencies. Finally, they tested a half-wavelength loop antenna operating at $100 \mathrm{MHz}$ inside fresh water and they concluded that a diameter of only $5.3 \mathrm{~cm}$ is needed for the antenna.

T. Melodia et al present in [131] a survey about the advances in underwater acoustic networks. Authors briefly describe the typical communication architecture of an underwater network followed by a discussion on the basics on underwater acoustic propagation and the state of the art in acoustic communication techniques at the physical layer. They also provided an overview of the recent advances in protocol design at the medium access control, network layers and in cross-layer design. We can also see in this paper, several comparatives of proposal of different researchers where it is analyzed the underwater acoustic communications as a function of data transfer rate, working frequency and maximum range. As paper shows, the DPSK modulations has been tested in frequency bands of 2 to $50 \mathrm{KHz}$ with data rates up to $20 \mathrm{kbits} / \mathrm{s}$. the maximum distances were around $4.8 \mathrm{~km}$ (value dependent to the deep and shallow). Using coherent modulations, it is possible to achieve $60 \mathrm{~m}$ in highest frequency (band of $125-1000 \mathrm{KHz}$ ). And finally, for modulations as QPSK of 16-QAM it is possible to achieve distances up to $2.5 \mathrm{~km}$, working at $24 \mathrm{KHz}$ with a data transfer 
rates of 30Kbit/s. Finally, authors provided a detailed discussion of the existing underwater acoustic platforms for experimental evaluation of underwater acoustic networks.

\subsubsection{Existing measurements of underwater wireless communications based on electromagnetic waves}

Focusing the related works in underwater wireless communications based EM waves, we can find the following works.

We can find several surveys and previous works where authors analyzed the theory of this kind of communication method. In this work, J. Partan et al. highlighted [132] a number of important practical issues to consider in the deployment of underwater wireless networks. They provided a classification scheme for underwater networks including key factors such as link-layer range, node density, and geographic coverage of nodes, for determining the type of deployed network. Authors also included an economical discussion comparing the main underwater communication methods.

X. Che et al. [133] present an overview of the acoustic, RF, and optical waves to implement underwater wireless networks. Authors compare the three technologies and explain their main benefits and limitations. Following their study, X. Che et al. have performed a set of tests with a small-scale underwater wireless sensor network designed to monitor coastal erosions. The sensors are equipped with a magnetic coupled loop antenna and the EM waves are used in the physical layer because the tests are carried in shallow water coastal environments. From their results, authors conclude that EM communication, coupled with digital technology and signal compression techniques, in underwater environments could be both feasible and effective for a specific set of applications.

A. A. Abdou et al [134] analyzed whether it is feasible to use EM waves in an underwater communication system using the unlicensed (ISM) frequency bands $(6.7 \mathrm{MHz}, 433 \mathrm{MHz}$ and $2.4 \mathrm{GHz}$ ) or not. To perform their tests, authors use the Ez430RF2500 kit and compare the signal propagation travelling in air and in water. As their results show, the signal losses registered in water are bigger than the signal losses registered in air. In addition, authors say that the maximum distance between devices, working at $2.4 \mathrm{GHz}$, is about $20 \mathrm{~cm}$. From the results shown in this paper, authors concluded that $6.7 \mathrm{MHz}$ was the best frequency to be used in wireless sensor networks for environmental monitoring. As we will see in our work, it is possible to achieve larger distances even with higher frequencies, so we are able to have higher bitrates..

As far as we know, there is not any study on underwater communications based on EM waves where authors analyze the communication from the point of view of temperature variation and its effects on performance. This is the perspective which we want to follow in our study in Chapter 6 .

\subsection{State of the art of WSN for underwater applications}

Currently, one of the main problems that marine fish farms present is the residual accumulation on the seabed because of the lost food when the fish are fed. Moreover, the wasted food is translated into important economic losses. This section shows the works that we have found in the related 
literature where the authors study the sediment depositions caused by marine fish farms and the systems used for automatic monitoring and controlling these sediments. We have also included the current methods used to measure the water turbidity.

\subsubsection{Problems in marine fish farms.}

When a company wants to set up a marine fish farm, the first issue to be studied is the proper situation of the fish cages. In [135], O. M. Perez et al. say that site selection is a key factor in any aquaculture operation. For this reason, in this paper the authors present a study using Geographic Information System (GIS) and related technology to build a spatial database. They took water quality variables which were considered to have an influence in developing marine fish cages of seabass and seabream in Tenerife (Spain). The variables were grouped in a logical model and combined to generate outputs showing the most suitable areas for sitting the cages. All Tenerife coastlines were considered suitable for sitting a fish farm.

Urs Neumeier et al., in [136], examine the effects of detritus fish pellets on the stability of fine sediments in laboratory flume experiments. They create artificial beds subjected to erosion experiments for different periods of time ranging from 1 to 10 days. They varied the quantities of fish pellets incorporated both in the sediment matrix surface and lying on the sediment surface. A bacterial biofilm was developed at the sediment-water interface after few days. Erosion experiments showed that the addition of fish pellets reduced the surface erosion threshold by more than $50 \%$. Finally, they propose a formula for the effective shear stress experienced by the bed.

In [137], S. Porrello et al. analyze the environmental impact of the marine cages on the seabed of a western mediterranean marine fish farm. They evaluate the sensitivity of environmental hydrodynamic and geochemical variables and measure their efficiency over increasing distances. They provided the most efficient, robustness, practical and sensitive parameters. They observed organic enrichment effects just beneath the cages, moderate impact in the area $50 \mathrm{~m}$ around the cages, and no disturbance further away from this area.

A similar study is shown in [138]. E. Mantzavrakos et al. made a qualitative and quantitative assessment of the impacts on the physicochemical characteristics of the water column and the sediment caused by an intensive marine fish farm located in Greece. They analyzed the increased nutrients and organics concentrations, at the stations closest to the farm, both in the water column and the sediment. Finally, the authors viewed that there were differences between seasons. E.g. the peak values in most of the parameters measured were obtained during summer or spring. This conclusion helps researchers to introduce or activate more sensor nodes is these seasons.

\subsubsection{Simulators for marine fish farms.}

Some other researchers are working on several applications for a more rigorous control of these parameters. These works simulate the deposition and the biological effects of wasted solids from marine cage farms. 
One of these applications of optimizing the strategies of fish feeding raised in marine farms, is the mathematical model presented by M. J. Añón [139]. Allometry makes it possible to relate the magnitude of certain parts of the body regarding the size of the animal study object (relative growth). This system is developed from images captured by a photographic and a videotape device located in the installation and it classifies the fish depending on its potential growth. Its application avoids extracting the fish from the water to weigh them or to carry out periodic samplings (which can cause immunological weaknesses in the animals). This system allows an optimization of the feed supply whilst avoiding weighing the fish for its classification during the cultivation.

Another application is DEPOMOD [140]. It is able to give us a better predictive capability of the impact given by large marine cage fish farms on the benthos. DEPOMOD predicts the residual accumulation on the seabed generated by marine farm activities. With this method, we can evaluate the impact of a farm and the biomass increment.

In [141], J. G. Ferreira et al. describe a model for assessment of coastal and offshore shellfish aquaculture on a fish farm called Farm Aquaculture Resource Management (FARM). This model is mainly directed to the farmer and regulator in order to perform the following activities: species selection depending on the culture location based in these prospective analyses; timing and sizes for seeding and harvesting, in order to carry out an ecological and economic optimisation of culture practice; densities and spatial distributions, and environmental assessment of farm-related eutrophication effects. The system combines physical and biogeochemical models, bivalve growth models and screening models for determining shellfish production and for eutrophication assessment.

The model presented by O. M. Pérez et al. in [142] uses GIS (Geographical Information Systems), combined with a spreadsheet, as a Tool to Aid Modelling of Particulate Waste Distribution at Marine Fish Cage Sites. This system incorporates functions to calculate feed loading for all the cages within a pontoon independently and it uses known distribution algorithms. This model can be used to measure levels of sediment carbon and to establish a significant relationship between predicted and real sediment loading values. Some time later, R.A. Corner et al. presented in [143], an integrated model of GIS with feed loss. This system can also be used for many Marine Environmental Information Systems [144].

W.D. McCausland et al. developed a simulation model in [145] to simulate biological interactions generated to traditional fishing, aquaculture, the physical marine environment and coastal activities market interactions.

G. Lloret and J. Lloret, authors of this paper, presented in [146] a new simulator software model for marine fish farm sustainability. Their proposal takes into account many simulation models and methods in existence for sustainability in marine fish farms and they introduced them in an application with the possibility of choosing the method for modelling.

All these systems try to model the effects produced by feed loss in the marine fish cages and its environmental impact. To the extent of our knowledge, there is no work published where its main purpose is to reduce this wastage. 


\subsubsection{Current methods to measure the water turbidity}

Turbidity can be directly and quantitatively measured using several methods. In this Section, we are going to present the main ones.

E. A. Steel and S. Neuhausser [147] performed a study in Skagit River (western of Washington State) where the turbidity was measured with 3 discs: horizontal black disc, horizontal Secchi disc and vertical Secchi disc. They also measured the clarity of the river with a nephelometer. Finally, they compared the results obtained by the nephelometer and the distances of the disc. These results are shown in Table 2.3.

D.G. Smith et al [148] measured in several rivers of New Zeland the value of the water clarity with a black disc and the turbidity with a nephelometer. They also correlated both series of data and they obtained a good adjustment. Their obtained values appear in Table 2.3.

Table 2.3. Measures and correlation between disc and nephelometer

\begin{tabular}{|c|c|c|c|c|}
\hline \multirow{2}{*}{ Type of disc } & \multicolumn{4}{|c|}{ Results } \\
\cline { 2 - 5 } & Authors & Depth & NTU & Value of r \\
\hline Horizontal black disc & $\begin{array}{c}\text { E. A. Steel and S. Neuhausser } \\
\text { [148] }\end{array}$ & $3.5-5(\mathrm{~cm})$ & $1-4$ & $\mathrm{r}=-0.86$ \\
\hline Horizontal Secchi disc & $\begin{array}{c}\text { E. A. Steel and S. Neuhausser } \\
{[148]}\end{array}$ & $4-5(\mathrm{~cm})$ & $1-4$ & $\mathrm{r}=-0.85$ \\
\hline Vertical Secchi disc & $\begin{array}{c}\text { E. A. Steel and S. Neuhausser } \\
{[148]}\end{array}$ & $3-5.5(\mathrm{~cm})$ & $1-4$ & $\mathrm{r}=-0.86$ \\
\hline Horizontal black disc & D. G. Smith et al. [149] & $0.1-10(\mathrm{~m})$ & $0.1-100$ & $\mathrm{r}=0.97$ \\
\hline
\end{tabular}

An optical turbidimeter bases the turbidity measure on the light detection at different angles. The name of the instruments changes according to the angle used to perform the measurements. If the angle is $90^{\circ}$, it is called nephelometer. If the angle is $0^{\circ}$, it is called absorbimeters. Because the turbidity is a value which depends on the quantity of suspended solids in water, the solids intercept the line of light causing scattering and absorption, so not all light arrives to the detector [149].

When the turbidity is low, the light scattered away from its original direction is lower. This is one of the most important problems when we try to measure low turbidity levels with a turbidimeter. To improve these measures, M. J. Saladar proposed the use of light detector in different angles, from 90 to $180^{\circ}$. This is called backscattering [150]. Other problems that can have optical sensors are the fouling, the high variability of the size and the reflectivity of particles [151].

Laser sensors are being investigated as an alternative method to the nephelometers. T. S. Melis et al. [152] developed a study of turbidity in Colorado River. They used Laser In-Situ Scattering and Transmissometry to measure the sand and thinner material. They measured the sensor output voltage in $\mathrm{mV}$ and the concentration of the sediments in $\mathrm{mg} / \mathrm{l}$. Their results showed the correlation equation Equation 2.9.

$$
\text { Sensor output }(m V)=[\text { Sediments }]\left(\frac{m g}{l}\right) \cdot 0.971-1.942
$$


The interval of sediments concentration measured was from $10 \mathrm{mg} / \mathrm{l}$ to $160 \mathrm{mg} / \mathrm{L}$, and the recorded voltages were from $10 \mathrm{mV}$ to $150 \mathrm{mV}$. Their results show that LISST-100-B is suitable for providing data of suspended solids of the Colorado's River, The measurement results presented a high confidence bound. It was around $95 \%$.

J. R. Gray et al. in 2003 [153] took continuous in-situ turbidity measurements with the purpose of estimating the sediment concentrations in the Kansas River. They found a correlation of $\mathrm{R}=0.93$, and the formula that relates the suspended sediment concentration (SSC) with the water turbidity was (See Equation 2.10).

$$
\operatorname{SSC}\left(\frac{m g}{l}\right) \cdot 797 \cdot N T U \cdot 0.905
$$

The range of values of their experience was between $10 \mathrm{mg} / \mathrm{l}$ to $4000 \mathrm{mg} / \mathrm{l}$ and between $10 \mathrm{NTU}$ to 4000 NTU.

Other method to measure the turbidity is the use of an Acoustic Dolpper Velocimeter (ADV) with backscattering. H. Chanson et al. [154] performed an experiment where the correlation between acoustic backscatter intensity and the sediment concentration showed a monotonic increase instead of the linear relationship. It is similar than the one that we can see between turbidity and suspended sediments [154]. They prepared some experiences in the laboratory and other ones in the estuarine zone of Eprapah Creek with low sediments concentration (Eastern Australia). Their results showed that the quality of water and the composition of sediments affect to the calibration curves. For this reason, each sensor must be calibrated with the water and soil material of the study area.

There are several papers where authors use satellite images (LANDSAT) to measure the turbidity in water bodies [155][156]. However, this methodology is not interesting for our purposes because we need a continuous measurement process. In addition, we want to have values in a short period of time. Satellite systems cannot provide us short temporal scale (every hour), enough spatial resolution (few meters) and low economic prices.

The pressure-differential principle can also be used to measure the turbidity. This method is promising to be used in highly concentrated stream flows [153]. But, we cannot use this method because our measures are performed in the sea where the concentration of sediments is usually low.

\subsection{Conclusion}

The main problem in sensor node implementation is the energy constrains and the adapting of a prototype to the medium.

We have shown a detailed state of the art of wireless sensor networks. Firstly, we have defined what a sensor is and the main kind of sensors which can find. The energy limitations have been shown from the point of saving energy strategies based on applications. We have also analyzed and compared the energy consumption of different wireless technologies and finally, we have seen most important power-saving mechanisms in WLANs from the protocols perspective.

Because one of our objectives is the application of WSN for monitoring several parameters in rural, indoor and underwater environments, we have explained several related work about this. We have also presented studies about the behavior of signals in these mediums. 
Finally, work presented in this chapter has been published in the following references: [157], [158], [159], [160], [161], [162], [163], [164], [165], [166], [167], [168], [169], [170], [171], [172], [173], [174], [175], [176], [177], [178], [179], [265], [266] and [341]. 


\section{Chapter 3}

\section{Energy Issues in Wireless Sensor Networks}

\subsection{Introduction}

In a WSN, the nodes mainly use a broadcast communication and the network topology can change constantly due, for example, to the fact that nodes are prone to fail [1]. Because of this, we should keep in mind that nodes should be autonomous and, frequently, they will be disregarded. This kind of device has limited power, low computational capabilities and limited memory. One of the main issues that should be studied in WSNs is their scalability feature [3], their connection strategy for communication [4] and the limited energy to supply the device.

The desire to advance in research and development of WSN was initially motivated by military applications such as surveillance of threats on the battlefield, mainly because WSN can replace single high-cost sensor assets with large arrays of distributed sensors. There are other interesting fields like home control, building automation and medical applications. A number of hospitals and medical centers are exploring the use of WSN technology in a wide range of applications, including pre-hospital and in-hospital patient monitoring and rehabilitation and disaster response. WSNs can also be found in environmental monitoring applications such as marine fish farms [178] and fire detection in forest and rural areas [163].

As we already mentioned, sensor nodes in WSNs are usually battery powered but nodes are typically unattended because of their deployment in hazardous, hostile or remote environments. A number of power-saving techniques must be used both in the design of electronic transceiver circuits and in network protocols. The first step towards reduced power consumption is a sound 
electronic design [181], selecting the right components and applying appropriate design techniques to each case.

One of the major causes of energy loss in the WSN node is the idle mode consumption, when the node is not transmitting/receiving any information but listening and waiting for information from other nodes. There is also an energy loss due to packet collision, as all packets involved in the collision are discarded and must be retransmitted. A third cause of energy loss is the reception of packets not addressed to the node. The fourth major source of wasted energy is the transmission and possible retransmission of control packets, as these can be seen as protocol overhead.

There are several studies that present different aspects related to power saving techniques, but all of them are focused in a single way to improve the energy consumption and save power in WSNs.

In this chapter, we are going to analyze the most important aspects we should consider to implement a WSN. Firstly, we will see the main issue which can be found in nodes considering only the hardware features. This section also shows the main characteristics and requirements of a wireless sensor node and a selection of the most used commercial wireless nodes. To end this section, we will define the analytical expression of energy consumption in wireless nodes and we will compare the energy consumption of nodes as a function of the time. After that, we are going to analyze the best way to save energy in WSN from the point of view of Protocols and topology configuration. Finally, we are going to perform the measures of power consumption for wireless access points from different manufacturers with different topologies and configurations. Last Section shows the conclusion of this chapter.

\subsection{Energy issues in hardware}

In order to work with WSN, we need to know the main functional blocks of a sensor node. Each of these blocks has intrinsic energy losses, mainly due to its function. This Section also shows ones of the most used wireless nodes and the estimation of energy consumption for all of them.

\subsubsection{Wireless senor node hardware structure}

A sensor node is an electronic device that is used as an interface between the physical magnitudes that can be sensed from the medium and a data wireless network [182][183].

A node is made up of four main parts: (1) a power unit, consisting of a battery and a number of DC/DC converters, (2) a processing unit -which usually consists of a small processor and memory, (3) the physical sensors and (4) the transceiver circuit (radio system that should be formed by a transmitter and a receiver). Figure 3.1 shows the block diagram of a typical wireless sensor node. 


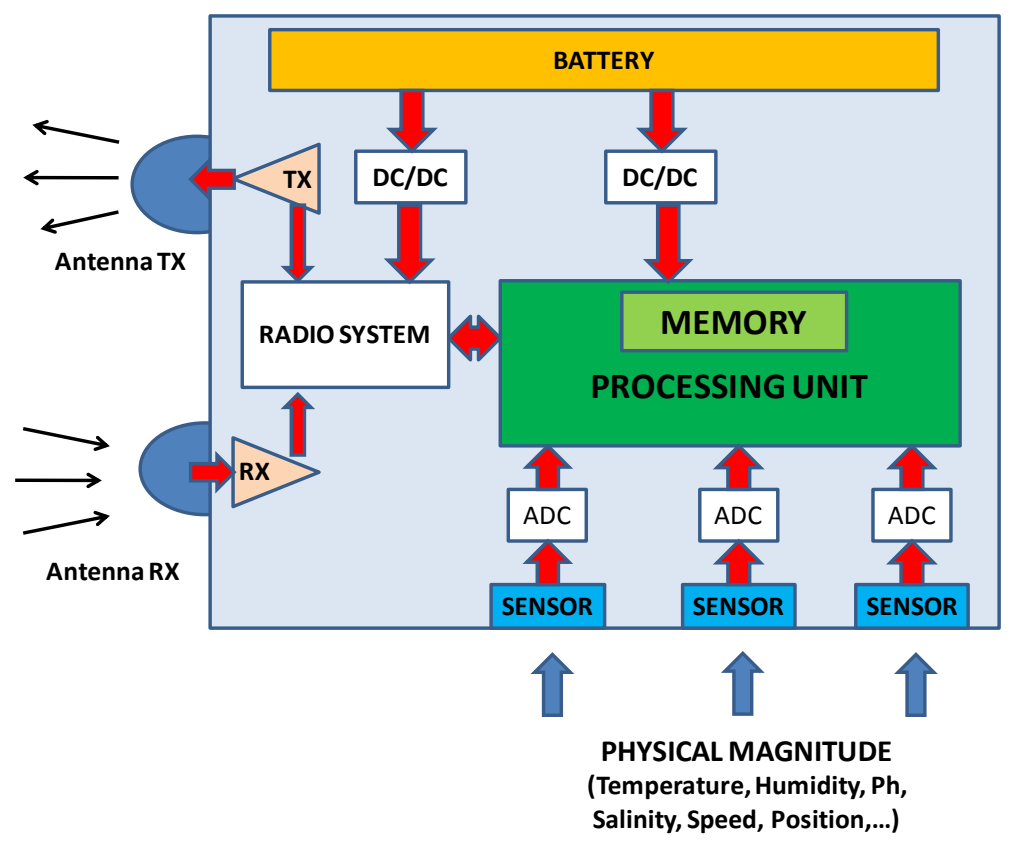

Figure 3.1. Block diagram of a typical wireless sensor node.

- $\quad$ The Processing Unit (PU) is responsible for reading out the physical sensors, extracting relevant information from the digitized data and implementing the network protocols. The PU of a wireless sensor node determines both the energy and the computing capabilities of a sensor node.

- The radio system allows wireless communication between the nodes in the network and to the outside world. Factors such as modulation scheme, data throughput in the network, transmission power and duty cycle can directly affect the energy consumption characteristics of the global system. In general, a node can work basically in three different operating modes: active (either transmit or receive), idle and sleep. Some studies on WSN and routing protocols show that contrary to popular belief, power consumption in idle mode is considerably high, comparable to the energy consumed in active mode [184]. For this reason, it is recommended to completely shut down the radio transceiver when it is not going to be used. Moreover, some important issues must be considered, e.g., a change in the system state and the related transient effects in the transceiver generate a significant increase in the amount of energy dissipated.

- Sensors transform a physical or chemical magnitude (instrumentation variable) into a useful electrical signal. Several examples of instrumentation variables are temperature, distance, acceleration, inclination, displacement, pressure, humidity and $\mathrm{pH}$. The electrical signal can be derived from a change (like resistance or capacity) in the sensor. [9].

- Batteries are complex elements whose operation depends on many factors including the size of the battery, the electrode material and the rate of diffusion of the active materials in the electrolyte. There are many types of rechargeable and non-rechargeable batteries that can be used in WSN applications. According to their electrochemical composition, which determines the energy efficiency, we can distinguish between Ni-Cd, Ni-Zn, Ag-Zn, Ni-MH and lithium ion batteries, among others. There are some important effects to consider, like 
the relaxation effect and the rated capacity effect [182], which largely determine the battery life.

- $\mathrm{DC} / \mathrm{DC}$ converters (also called voltage regulators) are responsible for providing appropriate voltages to the different circuits in the sensor node. Linear regulators have larger energy losses (approximately the output current multiplied by the voltage drop across the device) than switched regulators (typical energy loss in the range 5-15\%). Thus, the design of the voltage regulator section has a large impact on the node power consumption.

It is possible to recharge the sensor node battery with energy extracted from the environment, like light, wind, vibration [185] and electromagnetic fields.

\subsubsection{Characteristics and requirements of a wireless sensor node}

When a WSN is being implemented, particular sensor nodes features must be taken into account. In this sub-section we discuss some of the characteristics and requirements that are sought in the design and development of a wireless sensor node [182][183]. These are the following:

- High energy efficiency, in order to increase the node autonomy.

- Low cost, as a network that covers a large area can consist of hundreds or thousands of nodes. An estimation of the number of the nodes that are required to cover a given area is presented in [158].

- Distributed Sensing, in order to cover a large area despite the obstacles in the environment.

- Wireless communication, as it is the only choice for nodes deployed in remote areas or where no cabling infrastructure is available.

- Multi-hop networking. Depending on the radio parameters [186], it can be more efficient to reach a distant node or a base station using two or more wireless hops than a single large distance hop.

- Local data processing in the node, like zero suppression, data compression and parameter extraction can reduce the transmitted payload, and, thus, the power consumption.

\subsubsection{Hardware availability}

We can find in the market several embedded systems that incorporate the IEEE 802.11 standard. They are low energy consumption solutions. At the same time, these devices integrate great variety of resources and functions. Because their sizes are quite small, they can be included inside portable devices. And due to their low power consumption, they can also be fed by batteries. All these modules are also designed to enable the communication between wireless devices and a serial connection, so a sensor can be connected to the serial interface. In this sub-section we will analyze only the chipsets that have their power consumption specifications published. There are other IEEE 802.11 compliant chipsets in the market but they have not embedded serial interfaces.

Lantronix ${ }^{\circledR}$, Inc. presents three dedicated products to the IEEE 802.11 Wireless communication. The first of them is the WiPort [187]. It is a compact, integrated hardware and software module that enables to build wireless networking into any electronic device with serial or Ethernet capability. 
MatchPort b/g [188] and MatchPort b/g Pro [189] are dedicated co-processor modules that manage wireless and network activity. They use a dual processor design; one of them converts wired serial data into TCP/IP packets and providing web server capabilities, the other is an $802.11 \mathrm{~b} / \mathrm{g}$ baseband and radio chipset. MatchPort b/g and MatchPort b/g Pro enable $802.11 \mathrm{~b} / \mathrm{g}$ wireless connectivity and web services on any device with a serial interface on its microcontroller.

RF Solutions Ltd. is another manufacturer that works on embedded devices. It presents a device called S103 WLAN Compact Serial Module [190]. This module is compatible with 802.11b standard protocol. It supports 64/128-bits WEP security and can operate in ad-hoc or Infrastructure mode. It is compact and has small size $(40 \times 60 \mathrm{~mm})$ and low power consumption.

ConnectOne Ltd. has two embedded devices Nano WiReach [191] and Mini Socket iWiFi [192]. Both devices are a secure serial-to-Wireless LAN device server module and they can act as a bridge to connect serial devices to $802.11 \mathrm{~b} / \mathrm{g}$ Wireless LANs. Mini Socket iWiFi includes the iChip CO2128 IP Communication Controller chip, while Nano WiReach includes the iChip CO2144 IP Communication Controller chip. Both use a Marvell 88W8686 WiFi chipset.

Roving Networks Inc. has several products. The first of them is the RN-134 "SuRF" board [193]. It enables the connection directly from a Wireless LAN to a standard RS232 interface or through the TTL UART interface. This product is compatible whit IEEE $802.11 \mathrm{~b} / \mathrm{g}$ and it is prepared to control the remote and industrials sensors, home Automation and Telemetry measurements. It also has others products such as WiFly GSX (RN-131G) [194] and WiFly (RN111B) module [195]. They have a small form factor and extremely low power consumption. They are indicated for mobile wireless applications such as asset monitoring, GPS tracking and battery sensors. Furthermore, the WiFly (RN-111B) allows the RS485 connection.

Another manufacturer is RABBIT ${ }^{\mathrm{TM}}$. Their embedded system, called RCM4400W RabbitCore module [196] adds Wi-Fi/802.11b functionality and it also allows creating systems based in Wi-Fi technology in order to be used in Industrial Control, Remote Terminal Unit (RTU) or building Automation.

Finally, we present some products of Digi International®. It has, among others products, Connect Wi-ME [197] and ConnectCore Wi-9C [198]. These three products are fully customizable and secure IEEE 802.11 wireless embedded modules. They also can be used to create a fully webenabled and intelligent data station, in order to gather some information coming to an industrial sensor.

Table 3.1 shows a comparative of the devices previously described. The typical values of consumed current, the operating voltage and their standard wireless specification have been gathered from each device datasheet. In some devices we have also specified the CPU type that is integrated in the system. There are some parameters that are not provided by the manufacturer (we have written them in the table as not available, n/a). In the table we can see that the ones that consume less current in standby mode are Nano WiReach and Mini Socket iWiFi, but the one that consumes less current transmitting and receiving is "WiFly GSX" 802.11G Module (RN-131G).

For a regulated 3.3 volt power line (almost all of them use this voltage value), the LTC3440 Synchronous Bock-Boost DC/DC Converter can be used. It is rated to supply up to $600 \mathrm{~mA}$ of current [199], which will be enough to support even the worst case current draw of the any of that devices. 
Table 3.1. Devices consumption comparison.

\begin{tabular}{|c|c|c|c|c|c|c|c|}
\hline Device & Manufacturer & \begin{tabular}{|c|} 
Current \\
consumption \\
in TX
\end{tabular} & $\begin{array}{c}\text { Current } \\
\text { consumption } \\
\text { in } \mathbf{R x}\end{array}$ & $\begin{array}{c}\text { Current } \\
\text { consumption in } \\
\text { Standby }\end{array}$ & Core CPU & $\begin{array}{c}\text { Operating } \\
\text { Voltage }\end{array}$ & \begin{tabular}{|c|} 
IEEE \\
Specifications
\end{tabular} \\
\hline Nano WiReach & Connect One Ltd & $250 \mathrm{~mA}$ & $190 \mathrm{~mA}$ & $8 \mathrm{~mA}$ & \begin{tabular}{|c|} 
32-bit RISC \\
ARM7TDMI, low- \\
leakage, 0.13 micron, \\
running at $48 \mathrm{MHz}$
\end{tabular} & $3.3 \mathrm{~V}$ & $\begin{array}{l}802.11 \mathrm{~b} \\
802.11 \mathrm{~g}\end{array}$ \\
\hline Mini Socket iWiFi & Connect One Ltd & $250 \mathrm{~mA}$ & $190 \mathrm{~mA}$ & $8 \mathrm{~mA}$ & \begin{tabular}{|c|} 
32-bit RISC \\
ARM7TDMI, low- \\
leakage, 0.13 micron, \\
running at $48 \mathrm{MHz}$
\end{tabular} & $3.3 \mathrm{~V}$ & $\begin{array}{l}802.11 \mathrm{~b} \\
802.11 \mathrm{~g}\end{array}$ \\
\hline $\begin{array}{c}\text { S103 WLAN } \\
\text { Compact } \\
\text { Serial Module }\end{array}$ & Rf solutions & $325 \mathrm{~mA}$ & $210 \mathrm{~mA}$ & $80 \mathrm{~mA}$ & $\mathrm{n} / \mathrm{a}$ & $5 \mathrm{~V}$ & $802.11 \mathrm{~b}$ \\
\hline $\begin{array}{c}\text { "WiFly" 802.11B } \\
\text { Module (RN- } \\
\text { 111B) }\end{array}$ & Roving networks & $110 \mathrm{~mA}$ & $40 \mathrm{~mA}$ & $35 \mathrm{~mA}$ & $\mathrm{n} / \mathrm{a}$ & $3.3 \mathrm{~V}$ & $802.11 \mathrm{~b}$ \\
\hline \begin{tabular}{|c|} 
"WiFly GSX" \\
802.11G Module \\
(RN-131G)
\end{tabular} & Roving networks & $210 \mathrm{~mA}$ & $40 \mathrm{~mA}$ & $15 \mathrm{~mA}$ & $\mathrm{n} / \mathrm{a}$ & $3.3 \mathrm{~V}$ & $\begin{array}{l}802.11 \mathrm{~b} \\
802.11 \mathrm{~g}\end{array}$ \\
\hline \begin{tabular}{|c|} 
WiFly GSX Super \\
Module \\
"SuRF Board" \\
(RN-134)
\end{tabular} & Roving networks & $212 \mathrm{~mA}$ & $50 \mathrm{~mA}$ & $15 \mathrm{~mA}$ & $\mathrm{n} / \mathrm{a}$ & $3.3 \mathrm{~V}$ & $\begin{array}{l}802.11 \mathrm{~b} \\
802.11 \mathrm{~g}\end{array}$ \\
\hline MatchPort b/g & Lantronix & $360 \mathrm{~mA}$ & $225 \mathrm{~mA}$ & $76 \mathrm{~mA}$ & $\begin{array}{c}\text { Lantronix DSTni-EX } \\
\text { x86 CPU, on-chip 256 } \\
\text { KB zero wait static } \\
\text { SRAM, 2,048 KB } \\
\text { Flash, 16 KB Boot } \\
\text { ROM, } 8 \text { GPIO }\end{array}$ & $3.3 \mathrm{~V}$ & $\begin{array}{l}802.11 \mathrm{~b} \\
802.11 \mathrm{~g}\end{array}$ \\
\hline WiPort & Lantronix & $650 \mathrm{~mA}$ & $395 \mathrm{~mA}$ & $91 \mathrm{~mA}$ & \begin{tabular}{|c|} 
Lantronix DSTni-EX \\
186 CPU, on-chip 256 \\
KB zero wait static \\
SRAM, 2,048 KB \\
Flash, 16 KB Boot \\
ROM, \\
\end{tabular} & $3.3 \mathrm{~V}$ & $\begin{array}{l}802.11 \mathrm{~b} \\
802.11 \mathrm{~g}\end{array}$ \\
\hline $\begin{array}{c}\text { MatchPort }{ }^{\circledR} \mathrm{b} / \mathrm{g} \\
\text { Pro }\end{array}$ & Lantronix & $350 \mathrm{~mA}$ & $260 \mathrm{~mA}$ & $160 \mathrm{~mA}$ & \begin{tabular}{|c|} 
Lantronix $32-$ bit \\
processor, $166 \mathrm{Mhz}$ \\
159 MIPS - Dhryston \\
2.1)
\end{tabular} & $3.3 \mathrm{~V}$ & $\begin{array}{l}802.11 \mathrm{~b} \\
802.11 \mathrm{~g}\end{array}$ \\
\hline $\begin{array}{l}\text { RCM4400W } \\
\text { RabbitCore }\end{array}$ & RABBIT & $450 \mathrm{~mA}$ & $450 \mathrm{~mA}$ & $80 \mathrm{~mA}$ & $\begin{array}{c}\text { Rabbit } 4000 \\
\text { Microprocessors } \\
(60 \mathrm{MHz})\end{array}$ & $3.3 \mathrm{~V}$ & $802.11 \mathrm{~b}$ \\
\hline Connect Wi-ME & DigiBoard & $346 \mathrm{~mA}$ & $186 \mathrm{~mA}$ & $34 \mathrm{~mA}$ & $\begin{array}{c}\text { 32-bit, } 55 \mathrm{MHz} \\
\text { NS7520 processor } \\
\text { ARM7TDMI } \\
\text { ARM core }\end{array}$ & $3.3 \mathrm{~V}$ & $802.11 \mathrm{~b}$ \\
\hline $\begin{array}{c}\text { ConnectCore } \\
\text { Wi-9C }\end{array}$ & DigiBoard & $900 \mathrm{~mA}$ & $700 \mathrm{~mA}$ & $10 \mathrm{~mA}$ & $\begin{array}{c}\text { 32-bit, } 155 \mathrm{MHz} \\
\text { NS9360 processor } \\
\text { ARM926EJ-S } \\
\text { ARM core }\end{array}$ & $3.3 \mathrm{~V}$ & $\begin{array}{l}802.11 \mathrm{~b} \\
802.11 \mathrm{~g}\end{array}$ \\
\hline
\end{tabular}




\subsubsection{Energy consumption in transmission and initial consideration for environmental applications}

The sensor output is usually an analog signal. After signal conditioning (amplification, filtering) and digitization, data are processed locally in the node. As a result, a packet is eventually sent to the network using a transmitter circuit. The signal level needs to be amplified before reaching the antenna and propagated through a dispersive medium, such as water [174] or air (which does not generate as many losses as water) [163]. This guarantees an acceptable signal level at the receiver input. In addition, adequate modulation techniques should be implemented in order to minimize the loss of information.

The inverse system is implemented at the receiver node. The first stage is an amplifier or attenuator that sets the input level at the receiver circuit. The receiver applies the appropriate demodulation to obtain the original bit sequences, which are interpreted by the node.

Each of these stages involves electronic circuits that generate a considerable level of energy consumption. The distance between transmitter and receiver must also be taken into account in order to calculate the overall power dissipation [200]. Figure 3.2 shows the described block diagram.

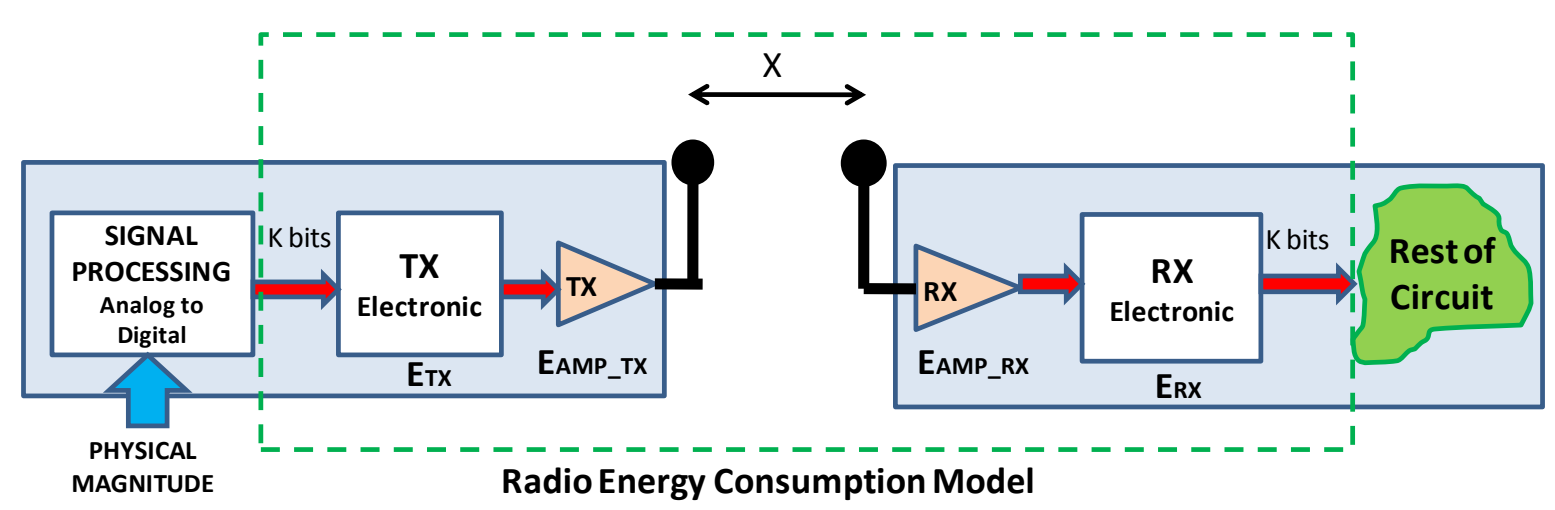

Figure 3.2. Radio energy consumption model.

Denote by $E(t)$ the energy consumption for the sensor. $E(t)$ can be broken down into five main components: the circuit energy consumption $E_{c}(t)$, the data processing energy consumption $E_{p}(t)$, the energy when it is reading and writing in the memory, the energy consumed by the physical sensor and the IEEE 802.11 chip energy consumption $E_{\text {chip }}(t)$. $E_{\text {chip }}(t)$ includes the energy consumed by the transmitter and receiver amplifier because it is included in the chip in all cases shown in table 3.1. The energy consumption is shown in Equation 3.1.

$$
E(t)=E(t)_{s}+E_{p}(t)+E_{m}(t)+E_{c}(t)+E_{c h i p}(t)
$$

The energy consumed because of data processing $\left(E_{p}(t)\right)$ is given by the expression provided by A. Wang et al in [201]. It depends of two main parts: the active energy dissipation because of the switching and the energy dissipated by the processor because of the leakage current. It can be seen in Equation 3.2. 


$$
E_{p}(t)=b N \int_{0}^{t}\left(C v^{2}(t)+\frac{I_{0}}{K} \cdot \frac{v(t) \cdot e^{\frac{v(t)}{n \cdot V_{T}}}}{(v(t)-g)}\right) d t
$$

Where $b$ is the number of bits processed, $K$ and $g$ are processor dependent variables, $N$ is the number of clock cycles per task and $C$ is the average capacitance switched per cycle and $\mathrm{I}_{0}$ is the current consumed.

M. N. Halgamuge et al propose in [202] that sensor logging energy consumption $E_{m}(t)$ depends of the current consumed reading the memory and writing in the memory. It can be formulated as is shown in Equation 3.3.

$$
E_{m}(t)=\frac{b \cdot v_{d d}}{8}\left(\int_{0}^{t_{\text {read }}} i_{\text {read }}(t) d t+\int_{0}^{t_{\text {wrie }}} i_{\text {write }}(t) d t\right)
$$

Physical sensors are usually formed by A/D circuits. We will be able to measure the energy consumed by this part of the circuit knowing the equivalent impedance of the sensor $\left(\mathrm{Z}_{\mathrm{th}}\right)$ and the voltage consumed during the interval as Equation 3.4 shows.

$$
E_{s}(t)=Z_{t h} \int_{0}^{t} v^{2}(t) d t
$$

$E_{c}$ depends on the number of resistances, condensers, coils and on the integrated circuits used in the circuit. It can be expressed as is shown in Equation 3.5.

$$
E_{c}(t)=\frac{1}{Z_{i n}} \int_{0}^{t} i^{2}(t) d t
$$

Where $Z_{\text {in }}$ is the entrance impedance of the circuit, $i(t)$ is the current consumed by the circuit and $t$ is the time when we want to finish to measure the circuit.

The chip could be in standby mode, transmitting frames or receiving frames. If we group all the time that has been in standby mode from $\mathrm{t}=0$ to $\mathrm{t}=\mathrm{t}_{0}$, all the time that has been transmitting from $\mathrm{t}=\mathrm{t}_{0}$ to $t=t_{1}$ and all the time that has been transmitting from $t=t_{1}$ to $t=t_{2}$, the energy consumption of the chip can be measured as is shown in Equation 3.6.

$$
E_{\text {chip }}(t)=\int_{0}^{t_{0}} E_{s m}(t) d t+\int_{t_{0}}^{t_{1}} E_{t}(t) d t+\int_{t_{1}}^{t_{2}} E_{r}(t) d t
$$

Where $E_{s m}(\mathrm{t})$ is the energy consumption in standby mode, $E_{t}(t)$ is the energy consumption when the chip is sending packets and $E_{r}(t)$ is the energy consumption when the chip is receiving packets.

Finally, we can define the energy consumed per bit in the transmitter-receiver model depicted in Figure 3.2 (See Equation 3.7) [203]. Several energy saving methods are discussed in [204]. 


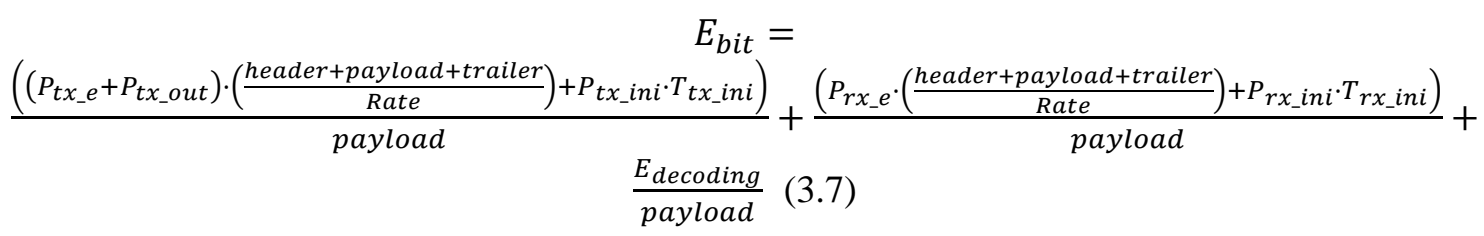

Where each parameter represents:

$\mathbf{E}_{\text {bit }}$ : energy consumed per bit

$\mathbf{P}_{\text {tx_e}_{-}}$: Power consumption in electronic transmission.

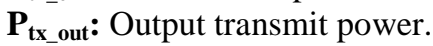

$\mathbf{P}_{\mathbf{r x}_{-} \mathrm{e}}$ : Power consumption in electronic reception

$\mathbf{P}_{\text {tx_ini: }}$ : Start-up power consumed in Transmission.

$\mathbf{T}_{\text {tx_ini: }}$ Transmitter start-up time.
$\mathbf{P}_{\text {rx ini }}$ : Start-up power consumed in reception.

$\mathbf{T}_{\mathbf{r x} \text { ini: }}$ Receiver start-up time.

Header: Length of packet header

Payload: Length of packet payload

Trailer: Length of packet trailer

$\mathbf{E}_{\text {decoding: }}$ : Decoding energy per packet.

A first obvious measure consists of adjusting the transmission power to the characteristics of the propagation path, like attenuation and range. Other more sophisticated techniques can be used, like preventing the duplication of packets in the network by using specialized routing protocols.

A frequently used approach is to control the node activity, switching the operation mode between active, idle and sleep modes. The processor consumes the most amount of energy in the active mode. In this mode the device can receive and send data and control packets and can perform data processing. Equation 3.7 represents this energy value as Ptx_e and Prx_e. In sleep mode, a device consumes the least amount of energy as the transmitter is turned off, the frequency of the main processor may be reduced and it is not possible to realize any processing operation. A considerable amount of time is required to enter and exit this mode. An intermediate state for a node, between active and sleep, is the idle state. In this mode, a device consumes less energy than in the active mode, as no data processing can take place. The device can quickly enter and exit this mode.

A scheme for dynamic power management in WSNs is described in [205]. This work proposes five different operation modes and the rules to switch between them.

A technique called Sparse Topology and Energy Management (STEM) is described in [206]. It reduces the energy consumption in the monitoring state to a bare minimum while ensuring satisfactory latency for transitioning to the transfer state by efficiently waking up nodes from a deep sleep state. The designers have full flexibility in trading latency, density, and energy versus each other.

The work presented in [207] describes several techniques to reduce dynamic power consumption in mobile battery-powered 802.11 WLAN systems. The authors propose to reduce the device energy consumption from its initial design, bearing in mind that, usually, small devices consume less power. But, the price of the final product is considerably increased when reduced manufacturing technology is used.

To complete this analysis of energy consumption, we are going to compare the energy consumed by the chipsets shown in table 4 . We will consider that the circuit used to regulate the battery needed for all the chips will consume the same energy and it will be quite low compared with the energy consumed by the chips. The energy consumed by the sensor will also be negligible compared with the energy wasted by the chips. Taking into account measurements taken in [208], we will suppose that a wireless link spends 70 percent of the time idle (in standby mode), 20 percent of the time receiving, and 10 percent of the time transmitting. Figure 3.3 shows the 
comparison of the energy consumed over the time by the chipsets shown in table 3.1. They have been ordered and numbered in the figure from the most energy consumption to the lowest one. The Chip that consumes less energy is "WiFly" 802.11B Module (RN-111B) while the chip that consumes most energy ( 6 times more than the lowest one) is ConnectCore Wi-9C.

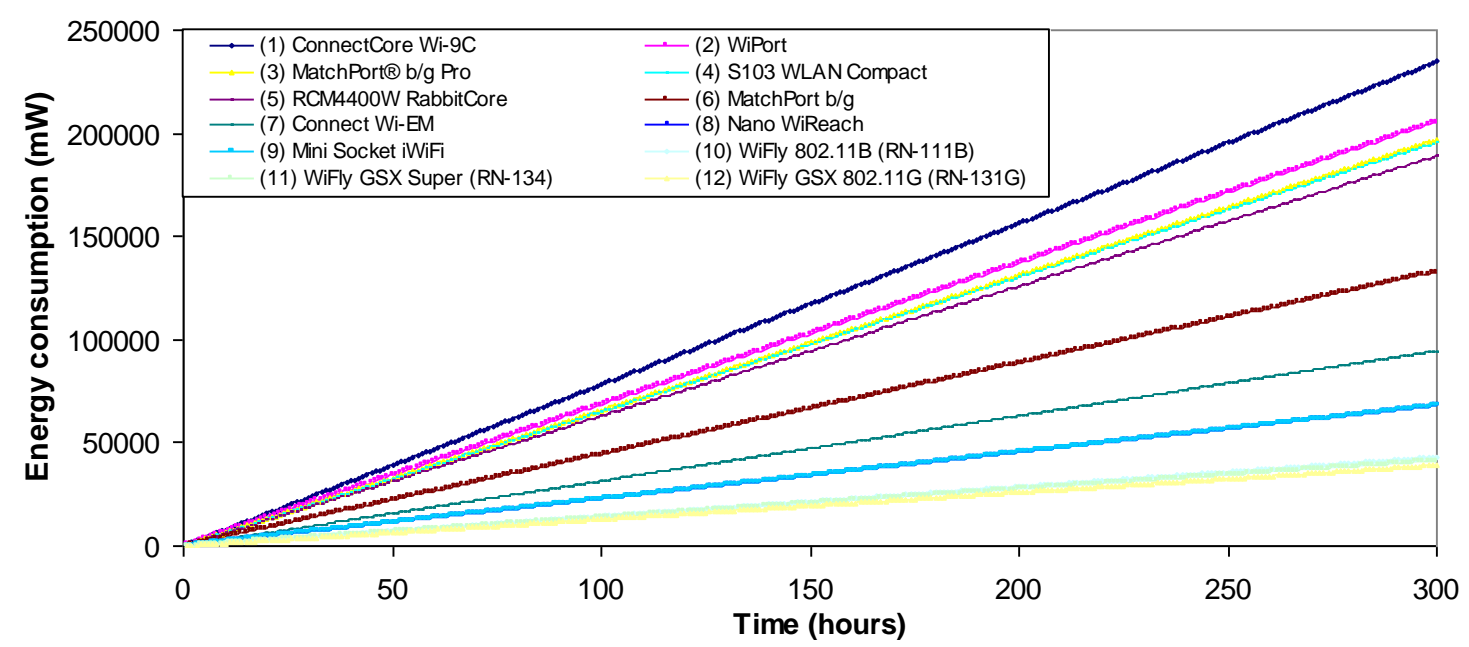

Figure 3.3. Power consumption comparison.

It is well known the wide range of applications that WSNs offer. One of them is the application in natural environments. With WSNs we can monitor small areas as a building or bigger such as rural/agricultural area. For this reason it is important to choose the best wireless technology. Of course, when the monitored area has several square kilometres, IEEE 802.11 standard will be the technology which will satisfy our requirements. Figure 3.4 shows the number of sensors needed for cover different areas according to the coverage radius of the used sensors for each technology studied. We can observe that the number of sensor using Zigbee technology will be too much when large areas have to be covered. The best technologies for these types of environments are IEEE 802.b/g and IEEE 802.11n. 


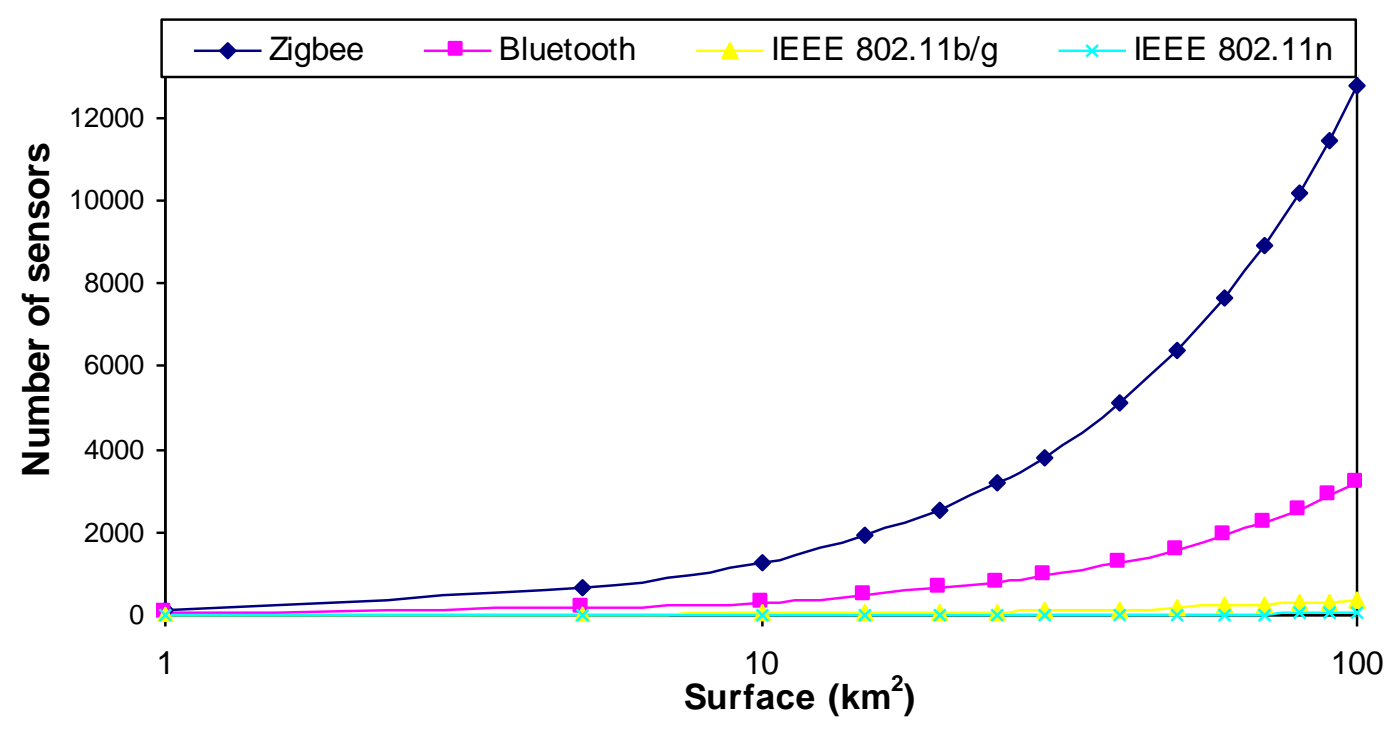

Figure 3.4. Number of sensors needed by each technology to cover several surface areas.

Taking into account the values shown in Figure 3.2, we have to achieve a balance between the power consumed by the sensor, the number of sensors needed to cover the zone and the prize of the sensors. Although Zigbee is the cheapest technology (followed by Bluethooth), this technology is not useful for large areas.

Taking into account the economic issues, IEEE 802.11n technology is more expensive than $802.11 \mathrm{~b} / \mathrm{g}$, so, nowadays, it is better to choose $802.11 \mathrm{~b} / \mathrm{g}$.

\subsection{Saving energy in WSN from the point of view of Protocols and topology configuration}

Despite the limited bandwidth of the wireless links, limited processing power and limited energy supply in the wireless nodes, many network designs are focused on taking advantage of the network in order to mitigate these limitations. One of the main pursued objectives of the WSN design is to prolong the network lifetime and prevent information degradation and loss.

In order to provide the right communication between the wireless sensor nodes some design factors and considerations that depend on the type of required application should be taken into account [209][210][211]. Table 3.2 shows some of the major considered items and their descriptions.

Table 3.2. Considerations in WSNs.

\begin{tabular}{|l|l|}
\hline \multicolumn{1}{|c|}{ Item } & \multicolumn{1}{c|}{ Description } \\
\hline Connectivity & $\begin{array}{l}\text { The connectivity in a WSN depends on the random distribution of nodes, mainly due to node } \\
\text { failures that may cause the network topology and network size to change. However, the complete } \\
\text { interconnection of nodes is desired. }\end{array}$ \\
\hline Coverage & $\begin{array}{l}\text { Radio coverage is an important design parameter in WSNs. A sensor can only monitor a limited } \\
\text { area, but it should be connected with other nodes in order to transmit the sensed information. The }\end{array}$ \\
\hline
\end{tabular}




\begin{tabular}{|c|c|}
\hline & $\begin{array}{l}\text { limit is set by the wireless technology, the accuracy of the transmission and the data rate (lower } \\
\text { data rate in larger distances). }\end{array}$ \\
\hline Data aggregation & $\begin{array}{l}\text { Data aggregation is the combination of data from different sources according to a specific } \\
\text { aggregation function, e.g., duplicate suppression, minimum, maximum and average. In a network, } \\
\text { nodes may generate duplicate packets. Therefore, it is important to reduce the number of duplicate } \\
\text { packets in the network in order to reduce the energy consumption and latency in communications. }\end{array}$ \\
\hline QoS & $\begin{array}{l}\text { The latency in a circuit sets the delivery time data from the transmitter to the receiver. However, } \\
\text { sometimes power consumption is more important than complete data accuracy. Therefore, routing } \\
\text { protocols should be aware of the quality of service and adapt to each situation. }\end{array}$ \\
\hline Node deployment & $\begin{array}{l}\text { The node Implementation in the WSN depends on the type of application and directly affects the } \\
\text { performance of the routing protocol. On the one hand, it can be a deterministic distribution, where } \\
\text { sensors are placed manually and the data is routed through default routes. On the other hand, it } \\
\text { can be a random distribution, where the resulting distribution of the nodes is not uniform. It } \\
\text { always has to find the optimal clustering that allows the best connectivity. Sometimes it has to } \\
\text { assume that the network has an energy-efficient behavior. Because the communication between } \\
\text { nodes is usually limited in bandwidth and the packet's delivery time, the most probable routes can } \\
\text { be formed by multi-hop wireless paths. }\end{array}$ \\
\hline $\begin{array}{l}\text { Energy } \\
\text { consumption }\end{array}$ & $\begin{array}{l}\text { Sensor nodes often use limited energy sources such as batteries. Therefore, the implementation of } \\
\text { energy saving techniques is needed. }\end{array}$ \\
\hline Fault tolerance & $\begin{array}{l}\text { Some sensor nodes may fail and stop the data transmission due to power shortage, physical } \\
\text { damage or environmental interference. Node failures should not interfere with the purpose of the } \\
\text { network. Therefore, MAC layer protocols and routing protocols must adapt to the formation of } \\
\text { new links and routes. The network should remain functional and should continue data } \\
\text { transmission. Sometimes, if there are many node failures to implement redundancy techniques at } \\
\text { various levels may be necessary to ensure a good level of fault tolerance }\end{array}$ \\
\hline Network Dynamics & $\begin{array}{l}\text { Many network architectures have stationary sensor nodes. However, the mobility of the nodes is } \\
\text { necessary in many applications. The routing of messages between mobile devices is more difficult } \\
\text { as the path stability, the bandwidth, energy, etc, becomes a more important consideration. } \\
\text { Moreover, the position of sensor can be detected by the network either dynamically or statically } \\
\text { using a periodic monitoring. }\end{array}$ \\
\hline $\begin{array}{l}\text { Transmission } \\
\text { Media }\end{array}$ & $\begin{array}{l}\text { In a multi-hop sensor network, the nodes involved in the communication process are connected by } \\
\text { a wireless medium. The traditional problems associated with a wireless channel (for example, the } \\
\text { losses by vegetation or rain attenuation, the error in height, etc. [163]) may also affect the } \\
\text { operation of the sensor network. Good Medium Access Control (MAC) should be used in order to } \\
\text { save energy. }\end{array}$ \\
\hline Scalability & $\begin{array}{l}\text { The number of sensor nodes that can form a network could be of the order of hundreds, thousands } \\
\text { or more. Therefore, the network and routing systems must be able to handle large number of } \\
\text { sensor nodes. Moreover, the administrator should assume that the network could grow. }\end{array}$ \\
\hline $\begin{array}{l}\text { Data sensing and } \\
\text { reporting model }\end{array}$ & $\begin{array}{l}\text { Data sensing and reporting as well as the data speed in wireless sensor networks depends on the } \\
\text { type of application. Data reporting can be categorized as either time-driven (continuous), event- } \\
\text { driven, query-driven, and hybrid [174]. The use of a reporting model depends on the type of } \\
\text { required monitoring in the system. However, it is possible to use mixed models, which bring } \\
\text { together the advantages of various types of reporting models. The routing protocol also plays an } \\
\text { important role in this item, because its performance is greatly influenced by the model of data } \\
\text { presentation, and this fact is related to energy consumption and reliability of the chosen route. }\end{array}$ \\
\hline
\end{tabular}

\subsubsection{Group-based wireless sensor networks}

In a regular WSN, when a node registers an alarm (node in green in Figure3.5), it transmits the alarm to all its neighbors, these neighbors transmits the alarm to their neighbors and so on. It spreads the warning to the entire WSN without control. In this case, a node could receive the alarm 
packet several times from different neighbors (See Figure 3.5). This situation leads to excessive energy consumption.

Group-based topologies in are based on the formation of groups of sensors instead of clusters of sensors. Moreover, there is cooperation between nodes in order to minimize the energy consumed in the network. Cluster-based networks are a subset of the group-based networks. A collaborative group-based WSN has a group of sensors and is built based on defined areas or as a function of the nodes' features [212]. Moreover, each group is formed by nodes that interact to share resources or to acquire data to produce joint results [213]. In a group-based network, each group could be using different type of routing protocol. A cluster-based network is made of a Cluster Head $(\mathrm{CH})$, Gateways and Cluster Members (CM). CH nodes fully control the cluster. The size of the cluster is usually about 1 or 2 hops from the $\mathrm{CH}$ node. When a sensor detects a new event, this sensor sends the information to all the members of the group and, depending on the case, the groups could share this information in order to reach all sensors of the WSN or just some groups. Only the closest sensors to the edge of the group will transmit the information to the sensors of other groups (see Figure 3.5). This considerably avoids raising the global energy consumption of the WSN, which is very important to prolong the lifetime of the WSN.

In the group-based network shown in Figure 3.6, the central node only performs the creation and maintenance of the boundary of the group without any other responsibility. In cluster-based networks, the gateways could be located in the boundary or in an overlapping region between clusters and manage communication with adjacent cluster, while the border nodes in a group-based network are in the boundary and only belong to one group. A summary of the differences between cluster-based and group-based networks can be seen in the Table 3.3.

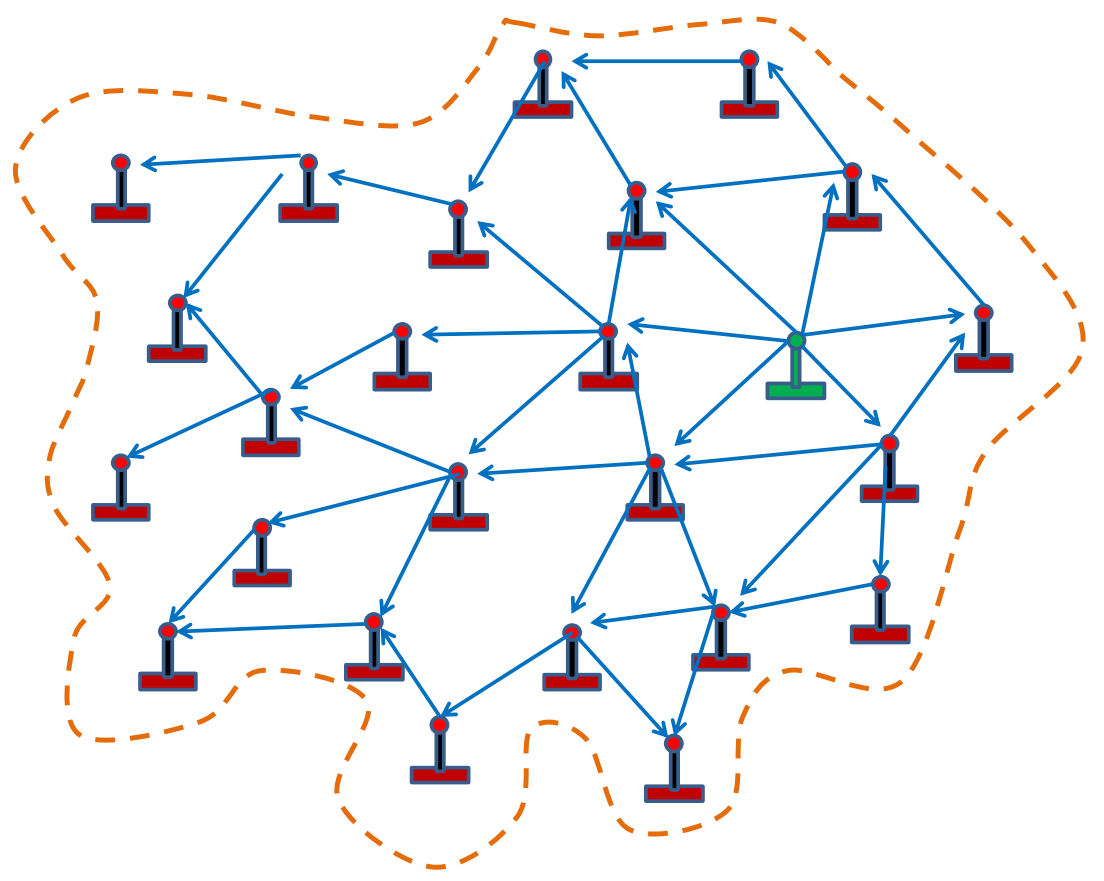

Figure 3.5. Regular network 


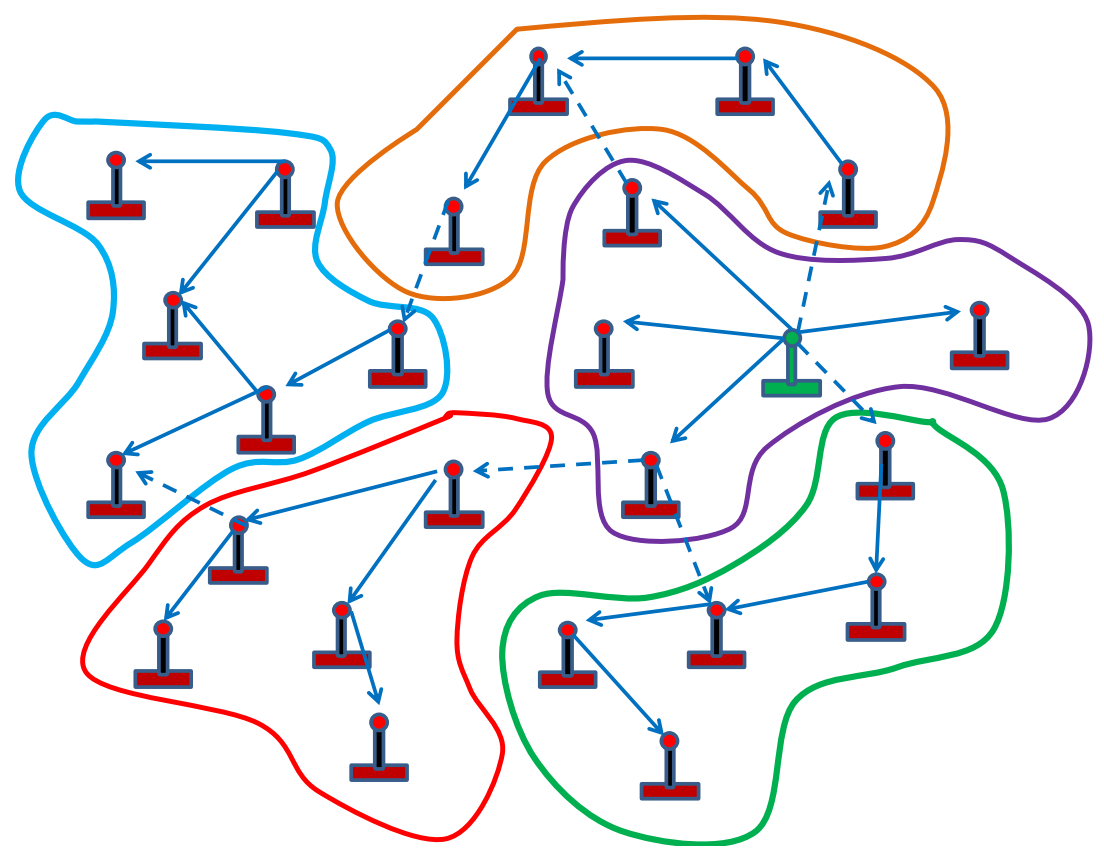

Figure 3.6. Group-based WSN

Table 3.3. Differences between cluster-based and group-based networks.

\begin{tabular}{|l|l|l|}
\cline { 2 - 3 } \multicolumn{1}{l|}{} & Cluster-based network & Group-based network \\
\hline Type of nodes & $\begin{array}{l}\text { - CH } \\
\text { - Gateways } \\
- \text { CM }\end{array}$ & $\begin{array}{l}\text { - Central node } \\
\text { - Intermediate node } \\
\text { - Border node }\end{array}$ \\
\hline $\begin{array}{l}\text { Hops between the main } \\
\text { node and farthest node }\end{array}$ & 1 or 2 & Minimum 1 \\
\hline $\begin{array}{l}\text { Topology inside each } \\
\text { cluster/group }\end{array}$ & All the same & Different in each group \\
\hline $\begin{array}{l}\text { Can the main node be a } \\
\text { gateway? }\end{array}$ & Yes & No \\
\hline $\begin{array}{l}\text { Functions of the main } \\
\text { node }\end{array}$ & Total control over its cluster & $\begin{array}{l}\text { Create and manage the } \\
\text { boundary of the group }\end{array}$ \\
\hline Type of architecture & - One-hop & Planar \\
\hline $\begin{array}{l}\text { Allows different routing } \\
\text { protocols }\end{array}$ & - Hierarchical & No \\
\hline $\begin{array}{l}\text { Group/ cluster size } \\
\text { determined by }\end{array}$ & Hops & Yeseral metrics \\
\hline
\end{tabular}




\subsubsection{Protocols}

A network protocol refers to the set of rules governing the exchange of information through a computer network.

\subsubsection{Efficient MAC Protocols}

The MAC (acronym for medium access control) sub-layer is responsible for regulating the access to a physical medium shared by several devices. MAC protocols must avoid collisions due to simultaneous transmissions and must perform other important functions like addressing, error checking and delivery notification. An efficient MAC protocol should possess many characteristics. The most important are:

- Predictability of delay

- Adaptability

- Energy efficiency

- Reliability

- Scalability

This section, a number of works that propose MAC protocols concerned with energy efficiency are briefly described.

\section{Energy and Rate based MAC Protocol - ER-MAC}

The first work that can be cited is the one presented by R. Kannan et al. [214]. This work presents the Energy and Rate based MAC Protocol (ER-MAC). It is based on TDMA and aims at avoiding energy waste. Packet loss due to collisions is absent because two nodes do not transmit in the same time slot. Although packet loss may occur due to other reasons like interference and loss of signal strength, there is no need to use a contention mechanism because the slots are pre-assigned to each node. ER-MAC uses the concept of periodic listen and sleep. Each node is assigned two TDMA slots for transmission and nodes know the transmission slots of its neighbors. Nodes periodically share information about their power levels and determine whether to use one or two slots for transmission. The proposed protocol is simulated in a scenario of 100 nodes. The results show that ER-MAC achieves a significant increase in energy savings compared to other existing MAC layer protocols.

\section{Dominating-awake-interval protocol, Periodically-fully-awake-interval protocol and Quorum-based protocol.}

In [215], the authors propose three asynchronous protocols that are directly applicable to MANET technology based on IEEE 802.11. These protocols are named as dominating-awakeinterval protocol, periodically-fully-awake-interval protocol and quorum-based protocol. The authors state that devices really send more beacon packets than original IEEE 802.11 standard and they study the best manners to wake-up the devices. Designed protocols use the following rules: (1) send the highest number of beacons in order to prevent the problem of false neighbors and (2) mobile hosts in power saving mode must put more emphasis on sending beacons. Their protocol does not use a clock synchronization system. Moreover, the patterns to wake-up two devices 
overlap as a function of the difference of time between them. Finally, in order to predict the exact timing to wake-up, when a host hears a beacon from another host, it must wake-up based on another time pattern. The simulations of their protocol show that it is efficient, saves energy and establishes the most likely route.

\section{Distributed Wireless Ordering Protocol - DWOP}

V. Kanodia et al. present the design and the analysis of the Distributed Wireless Ordering Protocol (DWOP) [216]. It is a distributed scheduling algorithm and media access protocol for wireless ad-hoc networks. It exploits overheard information from other nodes to estimate channel contention. The design of DWOP is based on a graph-theoretic problem formulation. It allows wellcharacterized deviations from the reference order in more complex topologies and achieves the exact reference ordering in fully connected graphs. DWOP enables QoS differentiation as well as fairness when combined with TCP. A theoretical model indicates that the scheme provides rapid convergence for newly arriving nodes, and extensive simulations indicate that nearly exact reference ordering can be achieved, even in complex asymmetric and perceived-collision topologies. The authors use the piggybacking head-of-line packet priorities in IEEE 802.11 control messages. This allows the nodes to assess the relative priority of their own queued packets. Moreover, the authors propose a distributed stale entry detection method that enables a quick recovery to the steady state.

\section{Battery Aware Medium Access Control - BAMAC}

Usually, MAC protocols for ad-hoc wireless networks are designed without taking into account the state of the node battery. In [217], S. Jayashree presents a MAC protocol in which each node contains a table that contains information about the battery charge level for each of its neighbors (close nodes that can be accessed). RTS, CTS, Data and ACK packets carry information regarding the battery level of the node that originated the packet. Any listening node fills its table with the information of the load levels of each neighboring nodes. This protocol uses a back-off mechanism, in order to determine which node should receive the packet. The goal of the back-off mechanism is to provide a near round-robin scheduling of the nodes which is based on some temporal parameters like the longest possible time required to transmit a packet successfully, including the RTS-CTSData-ACK handshake and the Short and DCF inter-frame spacing durations used in IEEE 802.11. Factors such as the minimum size of the contention window and rank are also considered. The nodes are scheduled based on their remaining battery capacities, that is, the higher the remaining battery capacity, the lower the back-off period. The algorithm allows a node to send the packet to a neighboring node with a higher level of battery. In this way, a uniform rate of battery discharge is guaranteed across all the nodes and consequently, the network lifetime will be longer, because the fall of nodes will be later. The proposed protocol is implemented using GloMoSim simulator and it is compared with the DWOP protocol. The simulations show that the battery life lasts around 70\% and reduces battery consumption to nominal packet transmission by $21 \%$ compared with IEEE 802.11 and MAC DWOP protocol. 


\section{Wireless Indoor Flexible High Bit rate Modem Architecture - WIND-FLEX}

G. Razzano [218] describes a control architecture that is widely used in local area networks on the structure of WIND-FLEX (Wireless Indoor Flexible High Bit rate Modem Architecture). The author studies the energy consumption in a wireless modem and presents a control method based on fuzzy logic, which has already been applied for determining the transmission power in CDMA (Code-Division Multiple-Access). Power consumption depends on the data rate and thus a reduction in the energy consumption is possible selecting the bit data rate as a function of the traffic situation.

\section{Distributed Coordination Function - DCF}

In [219], E.-S. Jung and N. H. Vaidya present a mechanism for optimizing the energy saving mechanism in the Distributed Coordination Function (DCF) of IEEE 802.11 networks. In DCF, the time is divided into beacon intervals. At the beginning of each beacon interval, each node in power saving mode periodically wakes up in a period of time called ATIM window. During this period, nodes exchange packets to determine whether the node needs to stay in active mode during the remaining time of the beacon. The energy saving and performance achieved by the nodes is directly proportional to the size of the ATIM window. The authors propose a dynamic adaptation mechanism where a suitable size of ATIM window can be chosen according to network conditions. The protocol has been called Dynamic Power Saving Mechanism (DPSM). A node is able to turn off the wireless network interface every time it finishes the packet transmission process. Initially, each node starts with a minimum ATIM window size, which is increased if they meet some rules. The authors show that the proposed system improves energy consumption without degrading the network performance.

\section{Multiple Access with Collision Avoidance}

Multiple Access Collision Avoidance MACA [220] protocol makes use of RTS, CTS, DATA and ACK sequences to operate. MACA proceeds as follows:

1) Before sending a message, the transmitter sends a RTS control message ("ready to send"), containing the length of the upcoming data message. The time taken to transmit a control message ( $\sim 30$ bytes) is called a slot.

2) If the receiver hears the RTS and is not currently "deferring," it replies with a CTS control message ("clear to send"), which includes a copy of the length field from the RTS.

3) Any station that hears a CTS defers any transmissions for long enough to allow someone to send a data message of the specified length. This avoids colliding with the CTS sender (the receiver of the upcoming data message).

4) Any station that hears an RTS defers any transmissions for a single slot (long enough for the reply CTS to be received, but not long enough for the actual data message to be sent, because contention is receiver-local).

5) Backoff: if no CTS response is received for an RTS, the sender must retransmit the RTS. It waits for an integer number of slots before retransmitting. The integer is chosen randomly between 1 and BO (backoff counter). BO is doubled for every retransmit, and reduced to 1 for every successful RTS-CTS pair. 


\section{Multiple Access with Collision Avoidance for Wireless - MACAW}

MACAW (MACA for Wireless) [221] presents a series of improvements to the basic MACA algorithm. First, the authors suggest a less aggressive backoff algorithm because the exponential increase/reset to 1 policy of MACA leads to large oscillations in the retransmission interval. They propose to increase the backoff by 1.5 after a timeout, and decrease it by 1 after a successful RTSCTS pair. Moreover, they arrange values for the backoff counter between clients in order to let the clients with lower backoff counter access the media. They also changed the backoff counter to be per-destination, rather than a single counter. The second proposal is that receivers should send an ACK to the sender after successfully receiving a data message. This is suggested because the minimum TCP retransmission timeout is relatively long $(0.5$ seconds $)$, so it takes a long time to recover from lost or corrupted messages. A link layer timeout can be more aggressive, because it can take advantage of the knowledge of the individual link latency (rather than the end-to-end timeout in TCP). Thirdly, they propose two related techniques for allowing transmitters to more effectively avoid contention:

- A DS (Data Sending) packet should be sent after a successful RTS-CTS exchange, just before the data message itself. The idea here is to explicitly announce that the RTS-CTS succeeded, so that if a pad can hear an RTS but not the CTS response, it does not attempt to transmit a message during the subsequent data transfer period. The reasoning here is subtle: as noted before, contention is only at the receiver, so one wouldn't think that a node that can hear the RTS but not the CTS should avoid transmitting. However, sending a message requires that the sender hear the CTS response (as well as the eventual ACK); therefore, if another node within range is sending, it would be pointless to also try to transmit.

- Suppose that two devices, A and B, in different cells/cluster are competing for the channel, if one of them "wins", it will effectively monopolize the channel. The authors propose to fix it by the following manner: when a receiver hears an RTS while it is deferring a transmission, at the end of the deferral period it replies with an RRTS ("ready for RTS") packet, prompting the sender to resend the RTS.

Note that the best-case performance of MACAW is actually lower than that of MACA, because the additional ACK and DS messages sent by MACAW incur overhead. However, MACAW is much more resistant to interference, and ensures much fairer allocation of the medium among different transmitters.

\section{Power Aware Multi-Access protocol with Signaling- PAMAS}

In [210], the authors develop a multi-access protocol for ad hoc radio networks. The protocol is based on the original MACA protocol [222], with the addition of a separate signaling channel. The protocol conserves the battery power of the nodes by intelligently powering off the nodes that are not actively transmitting or receiving packets. This protocol uses 6 operational modes for the node. It is based on RTS-CTS Schemes and achieves these power savings without affecting the delay or throughput behavior of the basic protocol. The RTS-CTS message exchange takes place over a signaling channel that is separate from the channel used for packet transmissions. This separate signaling channel enables the nodes to determine when and how long they can be powered off. In order to characterize the energy conserving behavior of PAMAS protocol, the authors provide several simulations where they compared the energy used by PAMAS without power conservation 
and PAMAS with power conservation. The results show that this improvement over the MACA protocol, produce energy savings of approximately $10 \%$.

\section{Sensor MAC - S-MAC}

S-MAC [223] is an improvement of PAMAS. It reduces further wastage from idle listening by making idle nodes shut off their radios. S-MAC reduces the waste of energy and self-configures. It adopts a contention-based scheme in order to have collision avoidance and good scalability. Overhearing makes contention-based protocols less efficient, so each node chooses a schedule, stores the schedule table, and exchanges it with its neighbors before starting its periodic listen and sleep modes. Their schedules are broadcasted to all their immediate neighbors, thus the time interval for listening and sleeping can be selected according to different application scenarios. In order to demonstrate the effectiveness and measure the performance of S-MAC, the authors implemented it on a wireless sensor network test bed. Motes were developed by some researchers of the University of California, Berkeley. These devices were based on an 8-bit Atmel AT90LS8535 microcontroller running at $4 \mathrm{MHz}$. However, S-MAC does not avoid collisions between two RTS or CTS messages (like PAMAS), which is a significant wastage of energy. Moreover, the sleep time interval is the same for each node, which is unfair for the nodes with less energy. To make weaker nodes sleep more can increase efficiency. Furthermore, S-MAC assigns sleep schedules without taking into account the criticality of a node.

\section{Floor Acquisition Multiple Access - FAMA}

FAMA is another MACA-based scheme that requires every transmitting station to acquire the control of the floor (i.e., the wireless channel) before it actually sends any data packet [224]. Unlike MACA or MACAW, FAMA requires that collision avoidance should be performed both at the transmitter as well as at the receiver.

In order to "acquire the floor", the transmitter node sends a RTS using either non-persistent packet sensing (NPS) or non-persistent carrier sensing (NCS), and the receiver replies with a CTS packet, which contains the address of the source node. Any station that hears the CTS packet will know which station has acquired the floor. CTS packets are repeated long enough for the benefit of any hidden sender. Authors recommend NCS variant for ad hoc networks since it addresses the hidden terminal problem effectively.

\section{Interleaved Carrier Sense Multiple Access - ICSMA}

The presence of exposed terminals is a significant problem in ad hoc wireless networks. S. Jagadeesan et al. propose a new MAC protocol called Interleaved Carrier Sense Multiple Access (ICSMA) Protocol for Ad hoc wireless networks to solve this problem in [225]. ICSMA reduces the number of exposed terminals and tries to maximize the number of simultaneous sessions in ad hoc networks. ICSMA access mechanism is based on the RTS-CTS-DATA-ACK access mechanism of the IEEE 802.11 DCF. It bases its operation in the use of two identical channels, where its handshaking process is interleaved between them. A node can originate the transmission in either channel 1 or channel 2 depending on the channel availability. In the ICSMA access mechanism, 
node A sends a RTS packet over channel 1 to Node B after waiting for a time period of DCF Inter Frame Spacing (IFS). Node B verifies the E-NAV in order to find out the availability of free time slots. If there are free available slots, then it responds a CTS packet over the channel 2 within Short Inter Frame Space (SIFS) time. If the CTS packet is not received by Node A successfully, it assumes a collision of the RTS packet or unavailability of Node B and it tries to send the RTS again after a back-off time (it uses a back-off mechanism similar to that used in IEEE 802.11 DCF). When Node A receives the CTS packet over channel 2, it transmits a DATA packet over channel 1, within SIFS time and expects the ACK through channel 2. If the ACK does not arrive within SIFS time, then Node A assumes a collision and attempts a re-transmission after back-off time. The simulations show that this protocol performed better than IEEE 802.11 when they are compared in terms of throughput, channel access delay, throughput fairness, and delay fairness.

\section{Multiple Access with Collision Avoidance by Invitation - MACA-BI}

Another MAC protocol based on MACA is MACA-BI. It was presented by F. Talucci et al. in [226]. MACA-BI eliminates the need of RTS packets, thus reducing the overhead given by each packet transmission and simplifying the implementation, while preserving the data collision free property of MACA. MACA-BI is less vulnerable to control packet corruption than MACA. In addition, the "receiver driven" mechanism of MACA-BI automatically provides traffic regulation, flow control and congestion control. This protocol is more robust to failures such as hidden terminal collision, direct collision or noise corruption and it also is less sensitive to the TX-RX turn-around time. In order to test the performance of this protocol the authors developed an analytical model in a single-hop configuration and a simulation model to evaluate it in multihop environments (all of them operating at $1 \mathrm{Mbps}$ ). The results show the efficiency of MACA-BI in wireless networks where improving the steady (predictable) traffic at higher channel speeds plays a key role. Collisions between control packets, and between control and data packets, may exist because of carrier sense failure due to non-zero propagation delays or due to the hidden terminal transmission. The authors conclude by letting us know that the probability of collisions among data packets is not possible in MACA-BI. The best manner to recover from this kind of data loss is only by using explicit ACKs.

\section{Multiple Access with Reduced Handshake - MARCH}

Multiple Access with Reduced Handshake (MARCH) protocol, presented by C.-K.Toh et al. in [227], improves communication throughput in wireless multihop ad hoc networks by reducing the amount of control overhead. It combines the advantages of both sender and receiver-initiated protocols. Unlike other receiver-initiated protocols, MARCH operates without resorting to any traffic prediction. This protocol reduces the number of handshakes required to transmit a data packet, so it outperforms other sender-initiated protocols. The novelty of this approach is that a mobile host $(\mathrm{MH})$ has knowledge of the data packet arrival of its neighboring $\mathrm{MH}$ from the overheard CTS packets. The simulation results show that MARCH outperforms MACA in several issues. MARCH protocol has a lower probability of control packet collision. Therefore its control overhead is much lower than MACA at all traffic loads. Furthermore, because it exploits the fact 
that control messages are overheard by the neighbors, this protocol is more deterministic and does not resort to network prediction, unlike most receiver-initiated protocols.

\section{Hop-Reservation Multiple Access - HRMA}

In [228], Z. Yang et al. describe a multichannel MAC protocol for ad-hoc networks operating with simple FHSS radios on ISM bands. HRMA is based on a common hopping sequence for the entire network and requires half-duplex slow frequency-hopping radios with no carrier sensing to operate. In HRMA the time is slotted. The protocol can be viewed as a time-slot reservation protocol in which a time slot is also assigned a separate frequency channel. Each slot consists of one synchronizing period, one HR period, one RTS period and one CTS period, each of which is used to exclusively send or receive the synchronizing packet, the HR packet, the RTS packet, and the CTS packet, respectively. For synchronization purposes, a special slot, called synchronizing slot, is defined. It has the same size as the regular slot. Each slot is assigned to a frequency hop. All the nodes that are not transmitting or receiving data packets are called idle nodes. They must hop to the synchronizing frequency and exchange synchronizing messages during the synchronizing period of each slot. During the HR, RTS and CTS periods of each slot, all idle nodes must dwell on the common frequency hop assigned to each slot. HRMA dynamically allocates frequency bands to nodes using a common frequency-hopping pattern. In this way, the data and acknowledgements are transmitted without hidden-terminal interference, which allows merging systems and permits nodes to join existing systems. The simulation results show that HRMA's throughput performance is significantly better than the slotted ALOHA. HRMA can achieve a maximum throughput that is comparable to the theoretical maximum value, especially when data packets are large compared to the slot size used for frequency hopping. This high throughput is obtained through a very simple reservation mechanism without the need of complex code assignment.

\section{Receiver-Based AutoRate (RBAR) protocol - RBAR}

Receiver-Based AutoRate (RBAR) protocol is a rate adaptive MAC protocol [229]. G. Holland et al. base their design on different assumptions. The first one is that the rate selection can be improved by providing timelier and more complete channel quality information. The second one is that the channel quality information is best acquired at the receiver and, finally, the third one is that the transmitting channel quality information to the sender can be costly, both in terms of the resources consumed in transmitting the quantity of information needed as well as the potential loss in timeliness of the information due to transmission delays. The novelty of RBAR is that it allows the channel quality estimation mechanism to directly access all of the information made available to it by the receiving hardware, for more accurate rate selection. The rate selection is performed on a per-packet basis during the RTS/CTS exchange, just prior to data packet transmission. Simulations show that this protocol results in a more efficient channel quality estimation which is then respected in a higher overall throughput. RBAR can be implemented inside IEEE 802.11 without significant changes.

There are some other works proposing other MAC protocols. Some of them are new proposals and others are schemes based on existing protocols. However, we have presented in this paper the most important ones. Table 3.4 shows the classification of the explained protocols. It summarizes 
the main characteristics of each one. A dash (-) means that the information is not provided by the authors or it cannot be correctly ascertained from their paper.

Table 3.4. MAC Protocols.

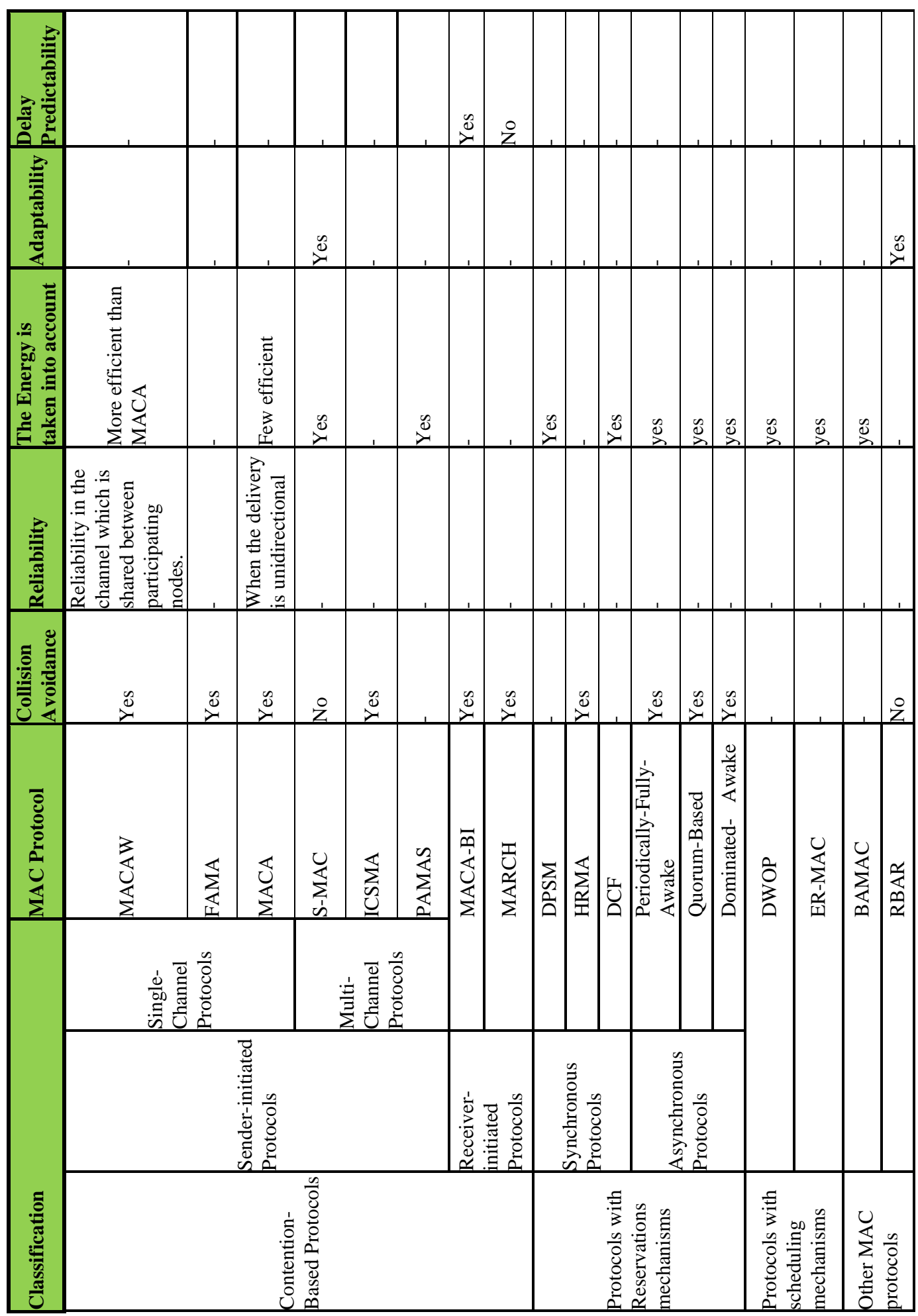




\subsubsection{Efficient Routing Protocols}

Routing protocols provide different mechanisms to develop and maintain the routing tables of the nodes of the network and find a path between all nodes of the network. Routing protocols must be adaptable to any type of topology to allow reaching any remote host in any network. Initially, a metric used for measurement must be defined in the routing protocol in order to find the best route. A routing protocol must be designed looking for very specific main objectives. Among the functions that it should have, here we highlight the following

- Maintain a reasonably small routing table.

- Choose the best route to a given destination. This would imply be the fastest, most reliable, highest capacity or the least cost route.

- Maintain a regular basis to update the routing table when nodes change their position appear in the network.

- Have a small number of messages in order to waste low bandwidth and save energy.

- Require little time to converge in order to provide the most updated network.

In this section, we will review the most well known routing protocols for WSNs that are related to energy saving techniques.

\section{Energy Aware Routing protocol - EAR}

Energy aware routing protocol [230] is a reactive protocol that aims to increase the lifetime of the network. This protocol seeks to maintain a set of paths instead of maintaining or enforcing one optimal path at higher rates, although the behavior of this protocol is similar to directed diffusion protocols. These routes are selected and maintained by a probability factor. The value of this probability depends on the lowest level of energy achieved in each path. Because the system has several ways to establish a route, the energy of a path cannot be determined easily. Network survivability is the main metric of this protocol. The protocol assumes that each node is addressable through a class-based addressing scheme which includes the location and the type of nodes. When the protocol starts, there is a process of flooding, which is used to discover all the routes between various source/destination pairs and their costs. This will allow creating routing tables, where highcost paths are discarded. By using these tables, data is sent to its destination with a probability that is inversely proportional to the cost of the node. The destination node performs a localized flooding in order to maintain the paths that are still operative. Compared to other protocols, the energy aware routing protocol provides an overall improvement of $21.5 \%$ in energy savings and increases the network life by about $44 \%$. However, having to collect location information, and the establishment of the steering mechanism for nodes, complicates the path settings.

\section{Low Energy Adaptive Clustering Hierarchy - LEACH}

Heinzelman et al. presented in [231] a hierarchical clustering algorithm for sensor networks called Low Energy Adaptive Clustering Hierarchy (LEACH). It is a clustering based protocol that includes the formation of distributed groups. It randomly selects a few nodes as cluster heads (CHs) and rotates this role to evenly distribute the energy load among the nodes of the network. In LEACH, $\mathrm{CH}$ nodes compress the data arriving from the nodes in their respective groups, and send summary packets to the base station. This reduces the amount of information transmitted to the base station. Data collection is centralized and is carried out periodically. Therefore, this protocol is 
appropriate when constant monitoring of the WSN is needed. The operation of LEACH is separated into two phases, the setup phase and the steady-state phase. In the setup phase the groups are organized and certain fraction of nodes are elected as CHs. In the steady-state phase, data transfer to the base station occurs. All elected CHs announce to the other nodes of the network, through a broadcast message, that they are the new $\mathrm{CHs}$. All non- $\mathrm{CH}$ nodes, after receiving this notice, choose the group they want to belong to. This decision is based on the intensity of the warning signal. Non$\mathrm{CH}$ nodes inform the appropriate $\mathrm{CHs}$ that it is a member of their group. After receiving all messages from the nodes that wish to be included in the cluster, the $\mathrm{CH}$ node creates a TDMA program and assigns to each node a time slot to transmit data. This program is broadcasted to all nodes in the cluster. During the steady state, the sensor nodes can sense and transmit data to the $\mathrm{CHs}$. The $\mathrm{CH}$ node, after receiving all data, adds its information and sends it to the base station. After some time, which is determined a priori, the network returns to the setup phase again and starts another round of new CHs election. Each group communicates using different CDMA codes in order to reduce interference with nodes that belong to other groups. Although LEACH is able to increase the network lifetime, there are still a number of questions about the assumptions used in this protocol. LEACH assumes that all nodes have enough transmission power to reach the base station and each node has the computational power to support different MAC protocols. Therefore, it is not applicable to networks deployed in large regions. It also assumes that nodes always have data to send, and nodes that are located close to each other have correlated data. It is unclear how the $\mathrm{CHs}$ are uniformly distributed over the network. Therefore, it may happen that the elected CHs are concentrated in one part of the network, so some nodes may not have a $\mathrm{CH}$ in their surroundings. Moreover, the idea of dynamic clustering can result in an extra overhead that can increase the energy consumption. Finally, the protocol assumes that all nodes start with the same amount of energy in each round of election, and assumes that a $\mathrm{CH}$ consumes approximately the same amount of energy.

\section{Hybrid Energy-Efficient Distributed clustering- HEED}

The paper in [232] proposes a method of saving energy for clusters of nodes in WSNs. HEED (Hybrid Energy-Efficient Distributed clustering) periodically selects the main nodes in the cluster according to a set of parameters such as residual energy and a secondary endpoint. It also seeks to extend the network lifetime by distributing energy consumption. It also tries to reduce high control on the network. The authors explain the grouping process and the determination of the responsible node. The system does not take care of the type of technology used. This work compares HEED protocol with others. HEED optimizes the use of resources according to the network density and the application requirements.

\section{Hierarchical Power-Aware Routing (HPAR)}

Another example of hierarchical protocol is presented by Q. Li et al. in [233]. The Hierarchical Power-Aware Routing (HPAR) protocol bases its operation on the division of the network into groups of sensors. Each group is formed by geographically close sensors covering a zone. Each zone is treated as an entity. In order to perform the routing between nodes, each zone is allowed to decide how a message is routed through the other areas, so maximizing the battery life of the nodes. Messages are routed along the path that has the maximum value on all the remaining minimum 
power values. This route is called max-min path. In order to send a message through an area, the route through the area and the sensors involved in estimating the power level of the area should be found. Each message is routed through the areas with the information about the estimation. The role of area management for message routing is assigned to a node. This protocol is based on the idea that the use of high residual energy nodes can be more expensive than the path with minimum energy consumption. The protocol seeks a balance between minimizing the total power consumption (using Dijkstra algorithm to find the path with least power consumption) and maximizing the minimal residual power of the network.

\section{Power-Efficient Gathering in Sensor Information Systems - PEGASIS}

In [234], an enhancement over LEACH protocol was proposed. The protocol, called PowerEfficient Gathering in Sensor Information Systems (PEGASIS), is a near optimal chain-based protocol. The basic foundation of this protocol is that nodes need only to communicate with their nearest neighbors, taking turns to communicate with the base station. When all nodes have established a connection with the base station, a new round will start and so on. This type of communication between nodes reduces the power required to transmit data through a path and ensures power distribution in all nodes. Therefore, PEGASIS has two main objectives. On the one hand, PEGASIS increases the lifetime of each node using collaboration techniques and, as a result, the network lifetime is extended. Moreover, the protocol allows only local coordination among close nodes, so the bandwidth consumed in communication is reduced. In addition, PEGASIS assumes that all nodes maintain a comprehensive database of the location of other nodes. To set the distance that each node has to its neighbor, the protocol uses the received signal strength to subsequently adjust the intensity of the signal in order to hear just one node. By contrast, PEGASIS requires adjustments to dynamic topologies in order to know where to find the destination node and in order to know where to route their data. Simulation results show that PEGASIS is able to double the network lifetime in comparison to using LEACH protocol.

\section{Hierarchical-PEGASIS}

An extension and improvement of PEGASIS (called hierarchical-PEGASIS) was introduced in [235]. Its aim is to reduce the delay of the packets transmitted to the base station. This protocol bases its operation in the assumption that only those spatially separated nodes may transmit simultaneously. It is a chain-based protocol with CDMA capable nodes, which constructs a chain of nodes forming a hierarchical structure and each selected node in a particular level transmits data to the node in the upper level of the hierarchy. In the performance test, the authors simulate simultaneous data transmissions to show how it avoids collisions through approaches that incorporate signal coding and spatial transmissions. The simulation shows that the new method ensures data transmitting in parallel and reduces the delay significantly. It has also shown that the proposal improves the previous version (PEGASIS) by a factor of about 60 .

\section{Minimum Energy Communication Network - MECN}

In [236], the authors propose a protocol, called Minimum Energy Communication Network (MECN), which calculates the energy efficiency of the subnets. It uses low-power GPS system. 
MECN identifies a region for every node. The region consists of nodes in a surrounding area where the transmission through those nodes is more energy efficient than direct transmission. The enclosure of a node is created by joining all regions that the region of the node can achieve. The main idea of MECN is to find a subnet that has fewer nodes and requires less transmission power between two particular nodes. In this way, global minimum power paths are not taken into account for all the network nodes. This is done using a localized search for each node considering its region. MECN is self-reconfigurable and thus can dynamically adapt to node failures or to the deployment of new sensors.

\section{Small Minimum Energy Communication Network - SMECN}

Small Minimum Energy Communication Network (SMECN) [237] is an improvement of MECN. In their algorithm, the authors considered for MECN the possible obstacles between any pair of nodes. Simulations show that SMECN is more energy-efficient than MECN and the links cost maintenance is lower. In addition, the number of hops for transmissions is decreased. On the other hand, finding a sub-network with a smaller number of edges introduces more overhead in the algorithm.

\section{Threshold sensitive Energy Efficient sensor Network - TEEN}

Threshold sensitive Energy Efficient sensor Network protocol (TEEN) [238] was developed for reactive networks. Sensor nodes continuously detect the environment but data transmission is only carried out when a parameter reaches a threshold value. The sensed value is stored in an internal variable in the node, called the sensed value (SV). There are two thresholds, hard threshold and soft threshold. When a parameter reaches its hard threshold value, the node switches on its transmitter and sends the sensed data to the cluster head. The soft threshold is a small change in the value of the attribute that causes the node to change to transmit mode and to start the transmission process. It gives a more accurate picture of the network, even if it means higher energy consumption. Thus, the user can decide on the tradeoff between energy efficiency and data accuracy. When CHs are changed, the new values of the above parameters are broadcasted. The main drawback of this system is that if the thresholds are not received, the sensed reported is not transmitted, and the user does not get any data from the network. In the TEEN protocol, the process of data detection consumes less power than the transmission of messages, so that energy consumption in this system is less than proactive networks. In addition, if necessary, the user can modify the soft threshold and broadcast the new parameters to the other sensors.

\section{Adaptive Threshold sensitive Energy Efficient sensor Network - APTEEN}

On the other hand, Adaptive Threshold sensitive Energy Efficient sensor Network protocol (APTEEN) [239] is a hybrid protocol that changes the frequency and threshold values used in the TEEN protocol according to the user needs and the type of application. The main feature of the APTEEN scheme is that it includes a combination of proactive and reactive policies. It has the possibility of adjusting the interval timer and the threshold values so as to redress power consumption according to the type of implemented application. In APTEEN, the node continuously monitors the environment, and only the nodes that detect an attribute value above the hard threshold 
will transmit data. The nodes will also transmit when the attribute value changes are equal to or greater than the soft threshold. If a node does not send data over a period of time equal to the timer, it will have to retransmit lost data. APTEEN uses a modified TDMA scheme to implement the hybrid network. The operation is based on the performance of TDMA, where a transmission time slot is assigned to each node in the cluster. The biggest weakness is the additional complexity to implement the features of the threshold and timer. APTEEN performance is between LEACH and TEEN in terms of energy dissipation and lifetime of the network. TEEN offers better performance by decreasing the number of transmissions.

\section{ACtive QUery forwarding In sensoR nEtworks - ACQUIRE}

In [240], Sadagopan et al. proposed a technique for querying sensor networks, which was called ACtive QUery forwarding In sensoR nEtworks (ACQUIRE).This protocol is a novel mechanism for data extraction in energy-constrained sensor networks. The key features of ACQUIRE are the injection of active queries into the network with triggered local updates. ACQUIRE performs its function in an energy efficient manner compared to other approaches. The network is a distributed database where complex queries can be further divided into several sub queries. The sink node sends a query, which is then forwarded by each node. Nodes with relevant data will respond. It is not a continuously persistent query, so the flooding does not dominate the costs associated with querying. Moreover, when data aggregation is employed, duplicate responses can generate in suboptimal data collection in terms of energy costs, so, once the query is being resolved completely, it is sent back through either the reverse or shortest-path to the base station. Moreover, ACQUIRE can provide efficient query by adjusting the value of the look-ahead number of hops. When the number of hops is equal to the network diameter, ACQUIRE mechanism behaves similar to flooding mechanism. ACQUIRE protocol shows good results with optimal parameter settings that outperform all the other schemes on complex, one-shot, non-aggregate queries for replicated data. It can reduce the energy consumption of other approached by more than $60 \%$ in some cases.

\section{Information-Driven Sensor Querying and Constrained Anisotropic Diffusion Routing - IDSQ and CADR}

Two routing techniques called information-driven sensor querying (IDSQ) and constrained anisotropic diffusion routing (CADR) are presented by M. Chu et al. in [241]. The main idea of both protocols is to maximize the information gain, by choosing the best query sensors and route data, while latency and bandwidth are minimized. This is achieved by activating only the sensors that are close to a particular event, thus data routes are dynamically adjusted. There are some differences between these protocols. While CADR aims to be a general form of directed diffusion, IDSQ is based on a protocol in which the querying node could determine which node can provide the most useful information while balancing the energy cost. Moreover, in CADR, the local information/cost gradient and end-user requirements are used in order specify an information/cost objective, and routes data, for each node. In addition, CADR can diffuse its queries only to the sensor nodes that can get the data (by only activating the right ones). IDSQ can be seen as a complementary optimization procedure because it does not specifically define how the query and the information are routed between sensors and the base station. However, simulation results shows that directed diffusion techniques, where queries are diffused in an isotropic fashion and reaching 
nearest neighbors first, are less energy-efficient than these approaches. A disadvantage of both protocols is that both need too much processing in their nodes.

\section{COUGAR}

Another data-centric protocol, presented by Y. Yao et al., is COUGAR [200]. This protocol views the network as a huge distributed database system. The main idea is to use declarative queries to summarize query processing such as the election of relevant sensors, etc. COUGAR utilizes innetwork data aggregation to obtain more energy savings. In order to reduce resource usage and thus extend the lifetime of a sensor network, COUGAR uses a user query technique, where a query optimizer generates an efficient query plan for in-network query processing. Through an additional query layer that lies between the network and application layers, this protocol supports the summary. COUGAR adds an architecture for the sensor database system, where sensor nodes elect a leader node in order to perform data aggregation and transmit the data to the sink. This fact provides in-network computation ability that can provide energy efficiency in situations where the number of sensors generating and sending data to the leader is very large. In contrast, COUGAR has some drawbacks. On the one hand, the addition of a query layer on each sensor node may add an extra overhead in terms of energy consumption and memory storage. On the other hand, in order to obtain successful in-network data computation, synchronization among nodes is required before sending the data to the leader node. Finally, the leader nodes should be dynamically maintained to prevent them from being hot-spots.

\section{Geographic Adaptive Fidelity - GAF}

Geographic Adaptive Fidelity (GAF) was presented by Y. Xu et al. in [184]. It is an energyaware location-based routing algorithm designed primarily for mobile ad hoc networks, although it may also be applicable to sensor networks. This protocol divides the network area into fixed zones where nodes collaborate with each other to play different roles and form a virtual grid. The main goal of GAF is the energy conservation by turning off unnecessary nodes in the network without affecting the level of routing fidelity. There is a virtual grid formed to cover an area. Each node uses a GPS-indicated location to associate itself with a point in the virtual grid. Inside these virtual grids, the nodes associated with the same point on the grid, receive the same value in terms of the cost of packet routing. GAF defines three states. These states are: discovery, for determining the neighbors in the grid, active reflecting participation in routing, and sleep, when the radio is turned off. GAF can increase the network lifetime even increasing the number of nodes, because, some nodes located in a particular grid area can remain in sleep mode in order to reduce the global energy consumption of the network. When a node is in sleep mode, it can change its state from sleep mode to active mode in order to balance the network load. Furthermore, the parameters related to the time for the sleep mode are specified during the routing process. In addition, to handle mobility, each node in the grid estimates its transmission time in the grid and sends its data to its neighbors. The sleeping neighbors adjust their sleeping time accordingly in order to keep the routing fidelity. Simulation results show that GAF performs as well as a regular ad hoc routing protocol in terms of latency and packet loss and increases the lifetime of the network by saving energy. However, GAF can be considered as a hierarchical protocol without aggregation, and consequently it can have the same weaknesses as a hierarchical protocol. 


\section{Geographic and Energy Aware Routing - GEAR}

Geographic and Energy Aware Routing (GEAR) is a location based routing protocol too. It was presented by Y. Yu et al. in [242]. The main idea of this protocol is to restrict the number of queries in directed diffusion considering a certain region rather than sending the queries to the whole network. In GEAR, each node keeps an estimated cost and a learning cost to reach the destination through its neighbors. In order to estimate the cost as a combination of the residual energy and the distance to a destination, it uses energy aware and geographically-informed neighbor selection heuristics to route a packet towards the destination region. The learned cost is obtained as a refinement of the estimated routing cost around the holes of the network. A hole is generated when a node does not have any closer neighbor to the target region than itself. If there are no holes, the estimated cost is equal to the learned cost and it is spread one hop back every time a packet reaches the destination. We can distinguish two phases in the algorithm flow. In the first one, when a node receives a packet, it checks its neighbors to see if there is a neighbor closer to the target region. The nearest neighbor node is selected as the next hop. When the network registers a hole, one of the neighbors is picked to forward the packet based on the learning cost function. In the second phase, when a packet has reached the region, it can be diffused in that region by either recursive geographic forwarding or by restricted flooding. Restricted flooding is usually used when the sensors are not densely deployed while recursive geographic flooding is more energy efficient in high-density networks. GEAR reduces energy consumption in the route setup and it performs better than GPSR in terms of packet delivery. The simulations show that for an uneven traffic distribution, this protocol delivers from $70 \%$ to $80 \%$ more packets than GPSR. For uniform traffic pairs GEAR, it delivers from $25 \%$ to $35 \%$ more packets than GPSR.

\section{Sequential Assignment Routing - SAR}

Sequential assignment routing (SAR) [243] was the first protocol for sensor networks that includes the notion of QoS in its routing decisions. The SAR algorithm generates multiple trees where the root of each tree is a one hop neighbor from the sink. Each tree grows outward from the sink by taking into consideration the QoS metric, the energy of each path and the priority level of each packet. This algorithm selects the path based on them. When the sensor node has exclusive use of a path, the energy resources are estimated by the number of packets. As a result, each sensor node selects its path to route the data back to the sink. Simulation results show that it offers less power consumption than other network algorithms, which only focus the energy consumption of each packet without considering its priority. In contrast, SAR maintains multiple paths from nodes to the sink which generates an overhead because of the tables and states maintenance of each sensor node, especially when the number of nodes is too big.

\section{SPEED}

A real-time communication protocol for sensor networks, called SPEED, is proposed by T. Hea et al. in [244]. SPEED is specifically tailored to be a stateless-localized algorithm with minimal control overhead. This protocol provides three types of real-time communication services, called, real-time unicast, real-time area-multicast and real-time area-anycast, for ad hoc sensor networks. SPEED is an efficient and scalable protocol for sensor networks where the resources of each node are scarce. It can also provide congestion avoidance when the network is congested. In this 
protocol, each node maintains information about its neighbors and uses geographic forwarding to find the paths. Furthermore, SPEED tries to ensure a certain speed for each packet so each application can estimate the end-to-end delay for the packets by dividing the distance to the sink by the speed of the packet before making the admission decision. The beacon exchange mechanism collects information about the nodes and their location. Then, the delay estimation at each node is calculated by the elapsed time when an ACK is received from a neighbor as a response to a transmitted data packet. After that, the node, which meets the speed requirement, is selected. If it is not possible, the relay ratio of the node will be checked. The Neighborhood Feedback Loop module calculates the relay ratio by looking at the packet failure ratios of the neighbors of a node. The algorithm eliminates congestion by sending messages back to the source nodes, thus they will pursue new routes. SPEED maintains a desired delivery speed across the network through a novel combination of feedback control and non-deterministic QoS-aware geographic forwarding. The design takes into account that the end-to-end delay depends on not only single hop delay, but also on the distance a packet travels. SPEED algorithm tries to support a real-time communication service with a desired delivery speed across the wireless sensor network, so the end-to-end delay is proportional to the distance between the source and the destination. Delivery speed is always smaller than the actual speed of the packet in the network, unless the packet is routed exactly along a straight line.

\section{Directed Diffusion}

Directed diffusion is data-centric protocol where all nodes in the directed diffusion-based network are application aware. This protocol was presented by C. Intanagonwiwat et al. in [245]. Directed diffusion protocol is suitable for query applications, which does not need global network topology maintenance. In addition, it enables diffusion to achieve energy savings by selecting good paths empirically and by caching and processing the data. This protocol has several features that can be highlighted. On the one hand, directed diffusion has the potential for significant energy efficiency. It outperforms an idealized traditional data dissemination scheme like omniscient multicast, even with an un-optimized path selection. On the other hand, diffusion mechanisms are stable under certain ranges of network dynamics. By contrast, this protocol is not the most suitable for continuous monitoring of a medium, because the computational requirements needed are high, which will imply more energy consumption.

\section{Rumor Routing}

Rumor Routing [246], presented by D. Braginsky et al. is a variation of the Directed Diffusion protocol. It represents a compromise between flooding queries and flooding event notifications. This protocol was designed for contexts in which geographic routing criteria are not applicable because a coordinate system is not available or the phenomenon of interest is not geographically correlated. The protocol is based on the following assumption: when the number of events is low, compared to the number of queries, event flooding can be efficient. Rumor routing algorithm uses long-lived packets called agents, to flood events through the network. When a node detects an event, it adds such event to its local table and generates an agent. Agents travel through the network in order to propagate information about local events to distant nodes. When a node generates a query for an event, the nodes that know the route, can respond to the query by referring its event 
table, thus the cost of flooding the whole network is avoided. Simulations show that Rumor Routing algorithm is a good method for delivering queries to events in large networks under a wide range of conditions, while maintaining energy requirements lower than other alternatives. Its design is able to be adjusted to different application requirements, to support different queries to event ratios, successful delivery rates, and route repair. In addition, it is capable to handling node failures and degrade its delivery rate linearly with the number of failed nodes.

\section{Self Organizing Protocol - SOP}

In [247], Subramanian et al. proposed a genetic architecture and a self-organizing protocol that allows large number of sensors to coordinate among themselves. The main goals of the algorithm are to minimize power utilization, localize operations and tolerate node and link failures. This routing protocol is based on a hierarchical architecture where groups of nodes are formed and merge when needed. It uses the Local Markov Loops algorithm in order to support fault tolerance. SOP can consider mobile or stationary sensors. Collected data are forwarded through the nodes to the most powerful base station.

\section{Two-Phase geographic Greedy Forwarding - TPGF}

We can also find papers which present protocols for very specific applications. In [248], presented by L. Shu et al., authors propose an efficient Two-Phase geographic Greedy Forwarding (TPGF) routing algorithm for Wireless multimedia Sensor Networks (WMSNs). TPGF is different from other geographic routing algorithms, because TPGF is a pure geographic routing algorithm that does not include the face routing concept and also does not require the computation and preservation of the planar graph in WSNs. This fact allows more links to be available for TPGF to explore more node disjoint routing paths. However, TPGF does not have the well-known Local Minimum Problem. The operation of this algorithm is divided mainly in two phases. In the first of them, the algorithm should explore the possible routing path, guarantying the correct delivery through routing path while bypassing holes in WMSNs. The second phase is responsible for optimizing the found routing path with the least number of hops. TPGF can be considered an iterative algorithm due to it can be executed repeatedly to find multiple node-disjoint routing paths. The algorithm structure contemplates as inputs, the location of the current forwarding node, the location of the base station and the locations of 1-hop neighbor nodes; meanwhile, its outputs are the location of the next-hop node or successful acknowledgement or unsuccessful acknowledgement. The goals of the simulation try to demonstrate that TPGF can find more routing paths and prove that it can have shorter average path length than other algorithms like GPSR. To evaluate the TPGF routing algorithm, the authors use a sensor network simulator NetTopo. The network size in simulation is fixed as $600 \times 400$. For each fixed number of sensor nodes and transmission radius, the average number of paths and the average path length are computed from 100 simulation results using 100 random seeds for network deployment. The simulations results show that, on the one hand, TPGF can find much more number of paths than that of GPSR on both GG and RNG planar graphs. In addition, the after optimization the average path length of TPGF is much shorter than GPSR and finally, it is proved that TPGF can have shorter average path length than that of GPSR. 


\section{Energy Consumed uniformly-Connected K-Neighborhood - EC-CKN}

Related with Network lifetime, Z. Yuan et al. present a paper [249] where propose a new sleep scheduling algorithm, named EC-CKN (Energy Consumed uniformly-Connected K-Neighborhood) algorithm, to prolong the network lifetime. In this work, the authors propose a new sleep scheduling algorithm, named EC-CKN, which is proposed to balance the energy consumption and prolongs the network lifetime. This algorithm takes the nodes' residual energy information as the parameter to decide whether a node to be active or sleep and not only can achieve the k_connected neighborhoods problem, it also can assure the $\mathrm{k}$ awake neighbor nodes have more residual energy than other neighbor nodes at the current epoch. To do the simulations, the authors suppose a model of transmitter and receiver, considering the power consumption of each part of the circuit, depending on the number of bits transmitted. To these nodes, the sleep scheduling algorithm is applied. The algorithm takes an input parameter $\mathrm{K}$, the required minimum number of awake neighbors per node. In EC-CKN, a node broadcasts its current residual energy information and computes a subset of neighbors. Before the node can go to sleep it makes sure that all nodes in subset are connected by nodes with major amount of energy and each of its neighbors has at least $\mathrm{k}$ neighbors from subset. With this process, the system guarantees, that if a node has less than $\mathrm{k}$ neighbors, none of its neighbors goes to sleep and if it has more than $\mathrm{k}$ neighbors, at least $\mathrm{k}$ neighbors of them decide to remain awake. The information needed to maintain this situation is extracted by computing locally with 2-hop neighborhood information and from the information about the residual energy exchanged. To prove its energy consumption, authors show a comparison between the energy consumption and network lifetime comparison among CKN and EC-CKN algorithm. Finally they conclude that the energy consumption in EC-CKN based WSN is well balanced.

There are more works published proposing other routing protocols for WSNs. Some of them are new, while others are based on existing protocols. Table 3.5 classifies the energy-aware routing protocols described in this section. It shows their main characteristics. A dash (-) means that the information is not provided by the authors or it cannot be correctly ascertained from their paper. Fixed BS means fixed base station. 
Table 3.5. Routing Protocols.

\begin{tabular}{|c|c|c|c|c|c|c|c|c|c|c|c|c|c|c|c|c|c|c|c|c|c|c|c|c|c|}
\hline 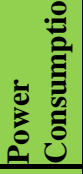 & & 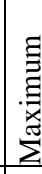 & & 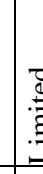 & & 总 & & & 兽 & 常 & & & 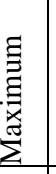 & & & 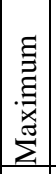 & 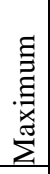 & 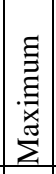 & & & 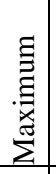 & & & 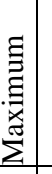 & \\
\hline 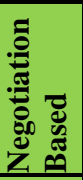 & ż & z & $z$ & 2 & \& & $\overbrace{\nu}^{\infty}$ & & $\stackrel{\circ}{z}$ & $\stackrel{\circ}{z}$ & $z$ & 2 & & $\stackrel{\circ}{Z}$ & z & z & z & z & ż & z & $\mathbb{\lambda}^{\infty}$ & ż & ż & ż & ż & \\
\hline 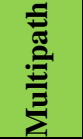 & 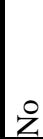 & ż & ż & $z$ & Z & $\tilde{x}^{\infty}$ & & $i_{i}^{\infty}$ & $\stackrel{\circ}{z}$ & ż & 2 & & ż & 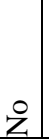 & $\dot{z}$ & $z$ & $\stackrel{0}{z}$ & ż & z & $\stackrel{0}{z}$ & $z$ & $\stackrel{0}{z}$ & z & $\dot{z}$ & \\
\hline 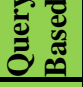 & ${ }_{\nu}^{0}$ & $\underline{z}$ & ż & 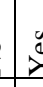 & e & $\overbrace{\nu}^{\infty}$ & 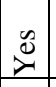 & $\stackrel{\infty}{\infty}$ & $\stackrel{\circ}{z}$ & $\underline{z}$ & 2 & & Ż & $\stackrel{0}{z}$ & ż & z & z & ż & \begin{tabular}{|l}
0 \\
$\nu$ \\
$\nu$
\end{tabular} & $\sum_{i}^{0}$ & ż & $\dot{z}$ & $\sum^{0}$ & $\dot{z}$ & $\underline{z}$ \\
\hline 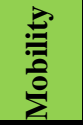 & 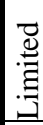 & $\begin{array}{l}\tilde{n} \\
\tilde{n} \\
\tilde{\Xi} \\
\tilde{x}\end{array}$ & $z$ & 2 & i̊ & 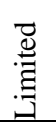 & z & 总 & 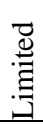 & 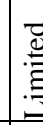 & 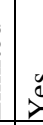 & & 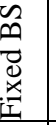 & $\stackrel{\gtrless}{z}$ & z̊ & 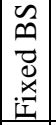 & z & 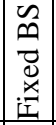 & z & $\stackrel{0}{z}$ & ż & 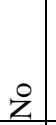 & $\underset{z}{z}$ & 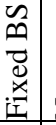 & $\stackrel{0}{z}$ \\
\hline 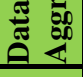 & $\stackrel{\infty}{\infty}$ & $\stackrel{\circlearrowright}{\nu}$ & 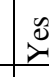 & $i$ & 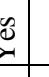 & $\stackrel{\infty}{\infty}$ & $\ddot{z}$ & $\stackrel{\circ}{z}$ & $\stackrel{\circ}{z}$ & $\underline{z}$ & $\frac{5}{2}$ & & ż & $\not 2$ & $\nu^{0}$ & 岕 & z & $\ddot{z}$ & 离 & $\tilde{\theta}^{\infty}$ & $\stackrel{0}{z}$ & ż & $z$ & $\tilde{v}^{\infty}$ & $\tilde{z}^{0}$ \\
\hline 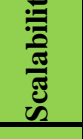 & 总 & 훙 & 窇 & 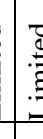 & 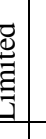 & 总 & \begin{tabular}{l}
- \\
\hdashline \\
0 \\
0
\end{tabular} & 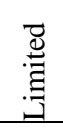 & $\begin{array}{l}-8 \\
8 \\
0\end{array}$ & 兽 & $\bar{z}$ & & 艿 & \begin{tabular}{l|} 
\\
0 \\
0 \\
0
\end{tabular} & 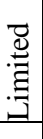 & $\begin{array}{l}- \\
8 \\
0 \\
\end{array}$ & $\begin{array}{l}3 \\
0 \\
1 \\
\end{array}$ & $\begin{array}{l}3 \\
3 \\
\vdots \\
\end{array}$ & \begin{tabular}{ll}
0 \\
\hdashline \\
0 \\
0
\end{tabular} & 总 & $\begin{array}{l}3 \\
0 \\
\\
\end{array}$ & | & 苞. & $\begin{array}{l}\overrightarrow{0} \\
\vdots \\
0 \\
\dot{0}\end{array}$ & \begin{tabular}{l}
$\overrightarrow{0}$ \\
\multirow{0}{0}{} \\
\end{tabular} \\
\hline $\begin{array}{l}\tilde{D} \\
\tilde{0} \\
\tilde{\Xi} \\
\tilde{0} \\
\tilde{0}\end{array}$ & & & & & & & 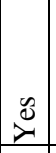 & & & & & & & & & & & & & $v^{v}$ & & & $\stackrel{0}{0}$ & & \\
\hline 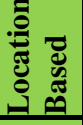 & & & & & & & & & $\stackrel{\infty}{\infty}$ & 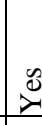 & & & & & & & & & & & & & & & $\underbrace{0}_{\lambda}$ \\
\hline 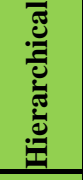 & & z & & & & & & & & & 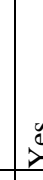 & & $\tilde{\Sigma}^{0}$ & $\sum_{\nu}^{\infty}$ & & $\stackrel{0}{0}$ & $\stackrel{0}{0}$ & $\stackrel{0}{\infty}$ & & & $\stackrel{0}{0}$ & $\sum_{\nu}^{\infty}$ & & $\stackrel{0}{\nu}$ & \\
\hline 竞 & 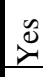 & & $\tilde{s}$ & 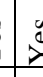 & 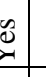 & $\stackrel{\infty}{\infty}$ & & 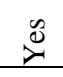 & & & & & & & $\infty$ & & & & $\begin{array}{l}0 \\
0 \\
0\end{array}$ & & & & & & \\
\hline 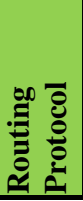 & 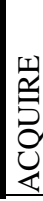 & 㞵 & 若 & 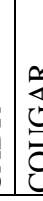 & לू. & 递 & 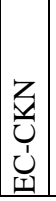 & 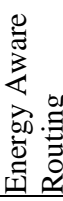 & 崩 & 选 & 囸 & 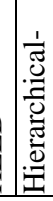 & 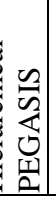 & 永 & $\begin{array}{l}0 \\
\Omega \\
\Omega\end{array}$ & 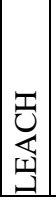 & 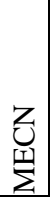 & 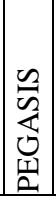 & 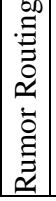 & 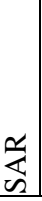 & 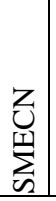 & 吕 & 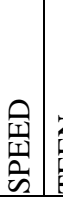 & $\begin{array}{l}Z \\
\underline{T} \\
\underline{I} \\
\underline{I} \\
\end{array}$ & 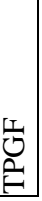 \\
\hline
\end{tabular}




\subsection{Optimizing configurations in network devices.}

Due to the development of low cost network technology and the popularization of the Internet of Things, the expansion of telecommunications networks seems limitless, allowing even providing Internet access in rural areas in developing countries. The increase in mobile devices, the advancement of new technologies and the implementation of new network services like IP telephony and IPTV [250], are generating a significant increase of network infrastructures such as routers, switches, hubs and access points (APs). This increase in network infrastructure means an increase in energy consumption in networks [251], becoming an environmental problem that needs to be addressed, and at the same time an economical problem because the price of energy is significantly increasing. For these economic and environmental reasons, the reduction of energy consumption is a critical issue for companies working in information and telecommunication technologies, especially when they are using data centers [252].

We should note that the network devices work better within a temperature range. The temperature range specified by the manufacturer is usually between $0-40^{\circ} \mathrm{C}$ [253][254], but this value varies depending the device and the task that is running. This implies the need to install a good cooling system in those rooms where host these devices, to ensure its proper functioning at peak performance. The more devices hosted in the room, the more cooling power must offer the system, what means more energy consumption in the network [255].

Currently, new energy solutions based on the introduction of IP transmission protocol to all areas of the network are being implemented. The migration of these systems to the next generation networks (NGN) saves between $30 \%$ and $40 \%$ of energy consumption [256]. Furthermore, the incorporation of routing systems and IP switching has improved the energy efficiency of data and voice transmission reducing the requirements of the network capacity between $60 \%$ and $70 \%$ [256].

With wireless networks is possible to build low-cost telecommunication networks, allowing access to the Internet in areas of difficult access, as rural areas [257]. A solution to develop networks in places where wireless APs cannot be connected directly to the grid is to place the APs connected to solar panels and batteries [258].

Energy saving techniques will increase the efficiency of the network, and thus will reduce the economic costs and environmental impact. If the network is designed with energy efficient devices and the optimal configurations, less electrical energy must be supplied from the main network distribution center.

WSNs are usually combined with wired networks. So, it is important determining the power consumption of these devices under different working conditions.

In this Section, we will see a study of the power consumption of several network devices from different manufacturers with different topologies and configurations.

\subsubsection{Measurements in wireless access points}

Table 3.6 brings together the list of wireless access points subject to study and their hardware features. All models work in the band of $2.4 \mathrm{GHz}$ except the Cisco WRT320N-EZ [259] which offer dual-band, working on $2.4 \mathrm{GHz}$ and 5.0 GHz. Cisco WRT320N-EZ is the model which 
supports more wireless protocols (IEEE $802.11 \mathrm{a} / \mathrm{b} / \mathrm{g} / \mathrm{n}$ ). It is easy to see significant differences in data transfer rates. The wireless access point Cisco WRT320N-EZ presents the highest data transfer rate with $300 \mathrm{Mbps}$ and wireless access points Avaya and Ovislink have the lowest transfer rates with values up to $11 \mathrm{Mbps}$. The wireless access point Cisco AIR-AP1121AG-EK9 [260] has more flash memory than the rest $(16 \mathrm{MB})$, but it has the same internal memory than wireless access point Cisco WRT320N-EZ (32 MB).

Table 3.6. Hardware features for all wireless access points.

\begin{tabular}{|c|c|c|c|c|c|c|}
\hline & \multicolumn{3}{|c|}{ Cisco Systems } & \multirow{2}{*}{ 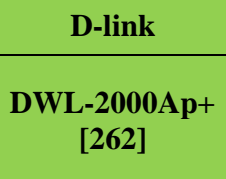 } & \multirow{2}{*}{$\begin{array}{c}\text { Avaya } \\
\text { AP-I [263] }\end{array}$} & \multirow{2}{*}{$\begin{array}{c}\text { Ovislink } \\
\text { WX-1590 } \\
{[264]}\end{array}$} \\
\hline & $\begin{array}{c}\text { AIR- } \\
\text { AP1131 } \\
\text { AG-E-K9 }\end{array}$ & $\begin{array}{c}\text { Linksys } \\
\text { WRT54GL } \\
\text { [261] }\end{array}$ & $\begin{array}{c}\text { Linksys } \\
\text { WRT320N-EZ }\end{array}$ & & & \\
\hline $\begin{array}{c}\text { Frequency } \\
\text { Band (Ghz) }\end{array}$ & 2.4 & 2.4 & $2.4 / 5.0$ & 2.4 & 2.4 & 2.4 \\
\hline $\begin{array}{c}\text { Operating } \\
\text { Temperature } \\
\left({ }^{\circ} \mathrm{C}\right)\end{array}$ & $0-40$ & $0-40$ & $0-40$ & $0-55$ & $0-40$ & $0-55$ \\
\hline $\begin{array}{c}\text { Internal } \\
\text { memory (MB) }\end{array}$ & 32 & 16 & 32 & 16 & 16 & 16 \\
\hline $\begin{array}{c}\text { Flash memory } \\
\text { (MB) }\end{array}$ & 16 & 4 & 8 & 4 & 4 & 4 \\
\hline $\begin{array}{c}\text { Max Data } \\
\text { Transfer Rate } \\
\text { (Mbps) }\end{array}$ & 108 & 54 & 300 & 54 & 11 & 11 \\
\hline $\begin{array}{l}\text { Wireless } \\
\text { protocol }\end{array}$ & $\begin{array}{c}\text { IEEE802.11 } \\
\mathrm{a} / \mathrm{b} / \mathrm{g}\end{array}$ & $\begin{array}{c}\text { IEEE802.11 } \\
\mathrm{b} / \mathrm{g}\end{array}$ & $\begin{array}{c}\text { IEEE802.11 } \\
\text { b/g/a/n }\end{array}$ & IEEE802.1 1b/g & $\begin{array}{c}\text { IEEE802.11 } \\
\text { b }\end{array}$ & $\begin{array}{c}\text { IEEE802.11 } \\
\text { b }\end{array}$ \\
\hline $\begin{array}{c}\text { Data Transm. } \\
\text { protocol }\end{array}$ & Fa. Eth. & Eth., Fa. Eth. & Eth., Fa. Eth. & Eth., Fa. Eth. & Eth. & Eth. \\
\hline
\end{tabular}

In the study of energy consumption on wireless APs, we have used two topologies formed by a wireless access point and two PCs connected to it by two types of physical interfaces as shown in Figure 3.7. In both topologies, the AP is responsible for communication between two hosts. In this part of the study, we have tried to check the impact in energy consumption, when there is a change of physical environment, (air to cable and air to air). Topology 1 uses a mixed network formed by a wireless link and a wired link. In the second case (Topology 2) both links are wireless.

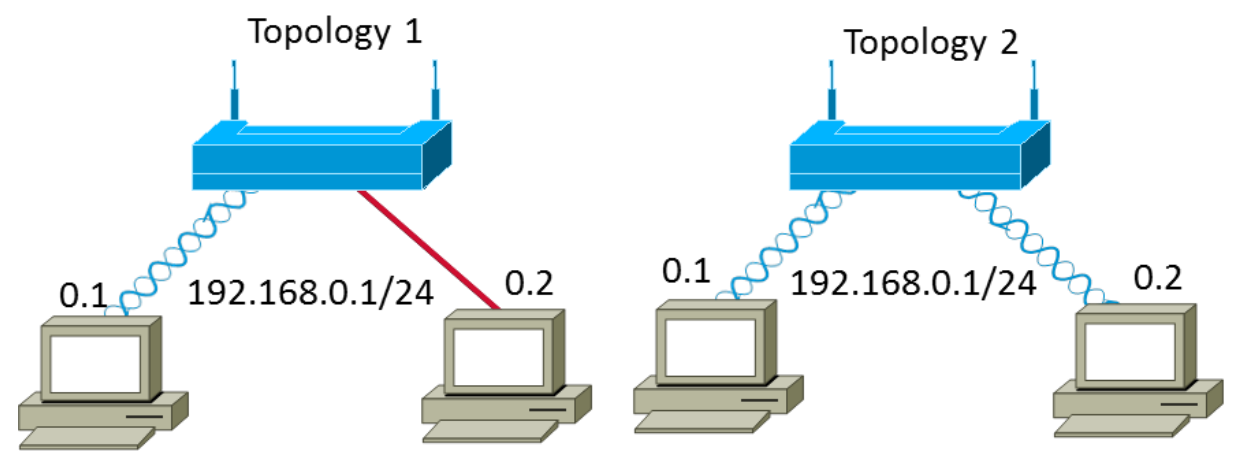

Figure 3.7. Topologies used to study the wireless APs. 
In order to determine which AP consumes less energy, we have defined two situations. In first one, we send echoes from the one computer to the other. In second case, we will send a file of a $2.45 \mathrm{~GB}$ between them. In each case, we have used all available variants of IEEE 802.11 protocol (available in each device).We have also registered the variation in energy consumption when a MAC filter is used.

Figures 3.8 and 3.9 show the values of energy consumption for the topology 1 and 2, when the echoes are sent. Figures 3.10 and 3.11 show the values of energy consumption for the topology 1 and 2 when a file is sent.

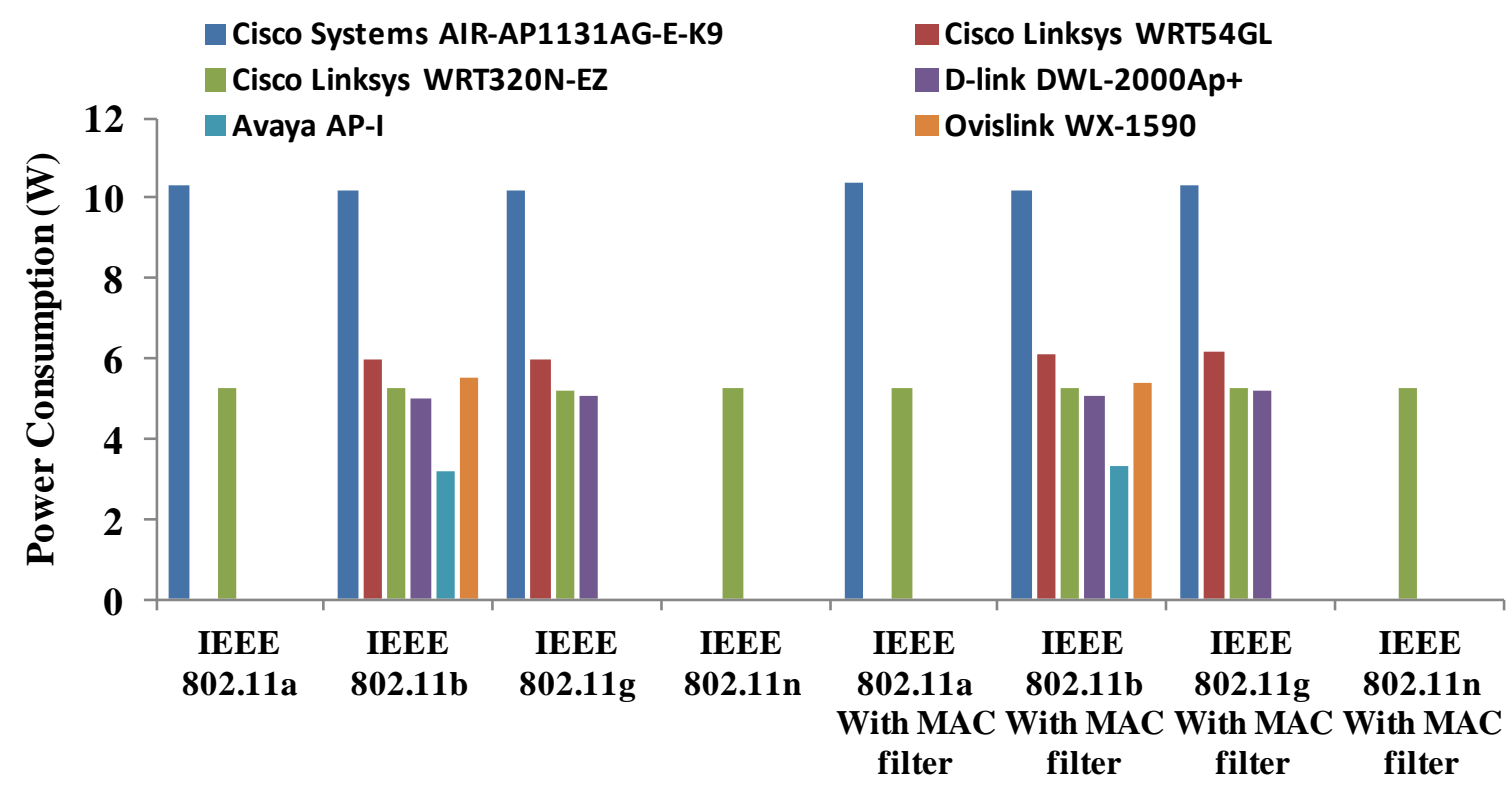

Figure 3.8. Measures delivery of echo topology 1.

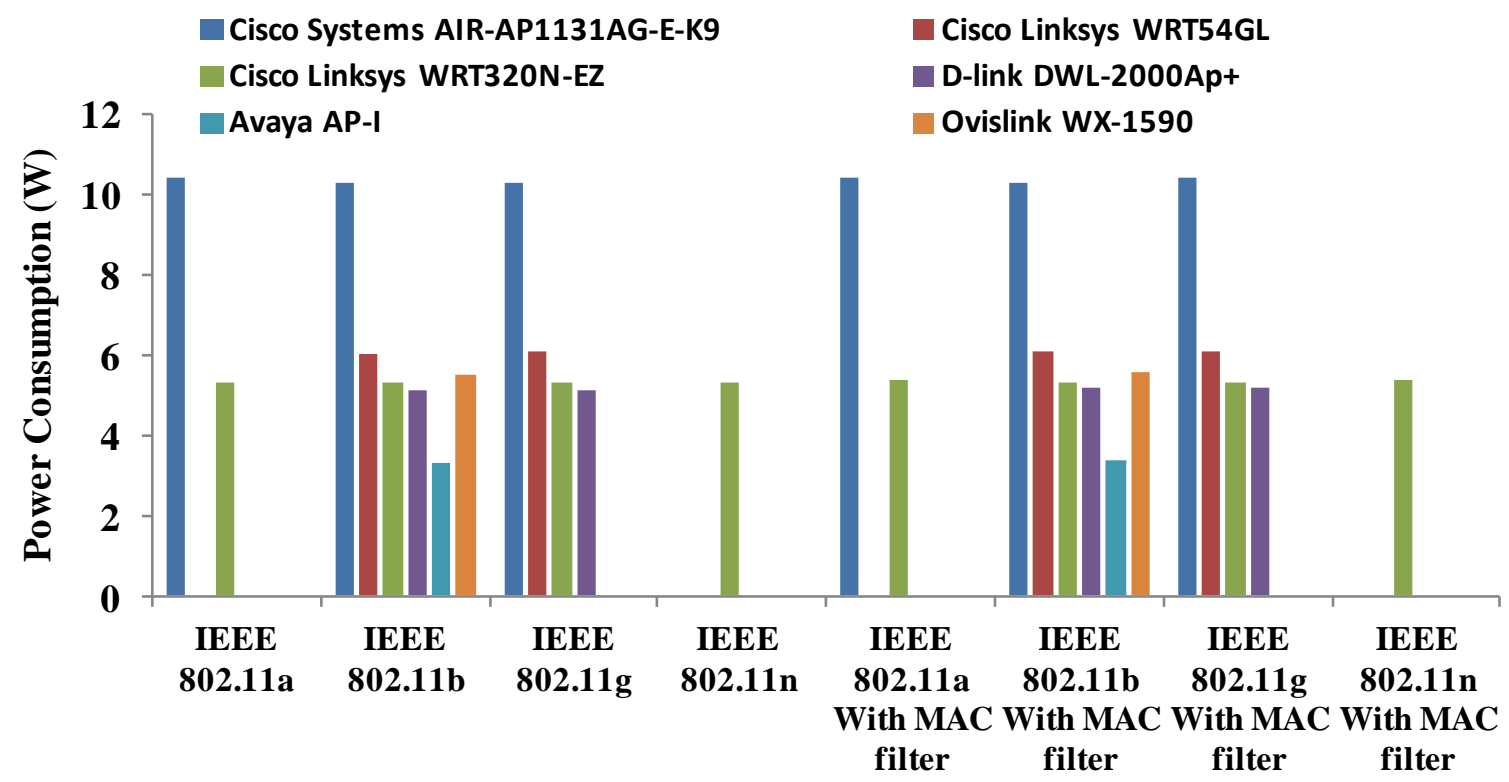

Figure 3.9. Measures delivery of echo topology 2. 


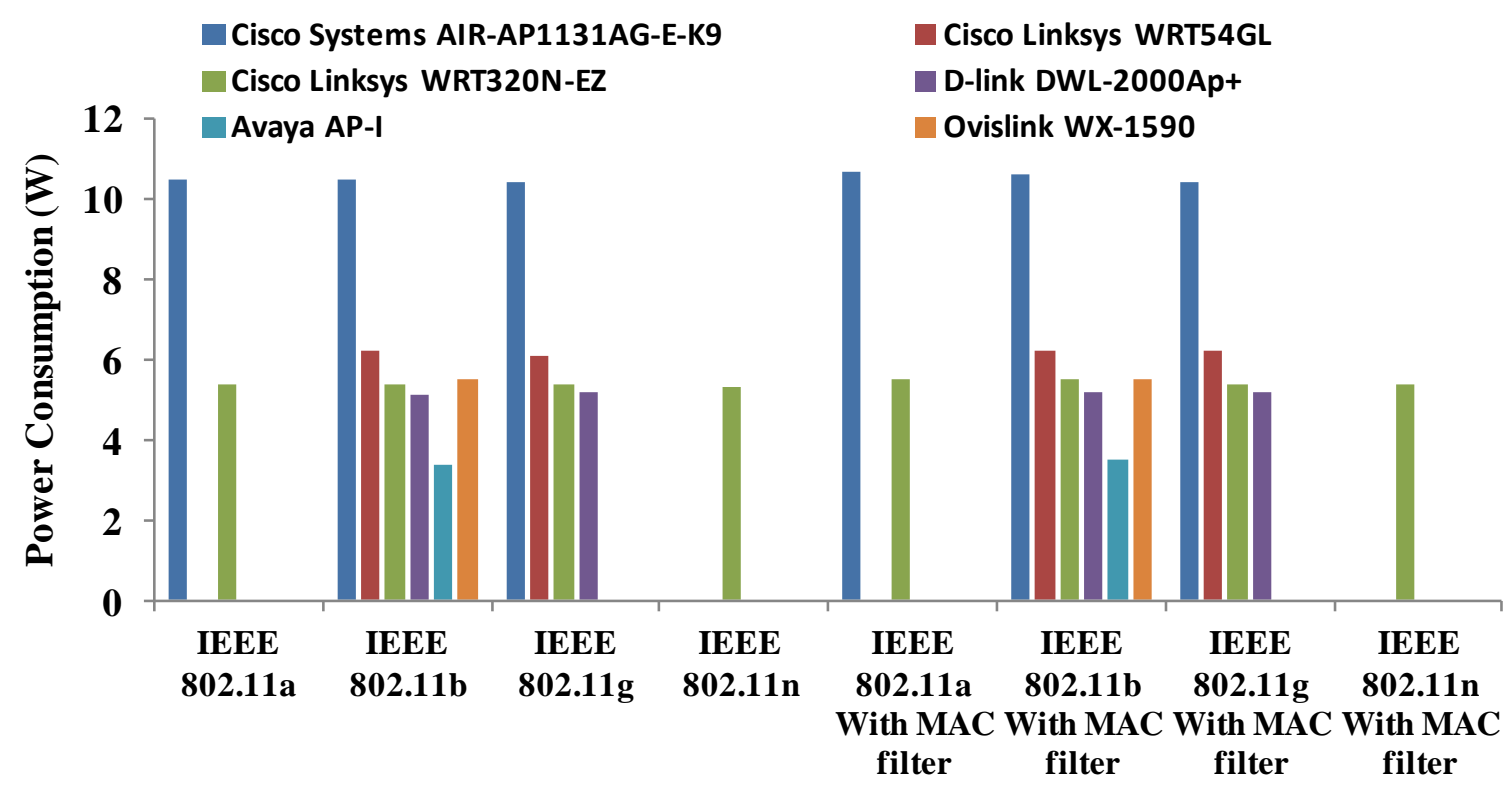

Figure 3.10. Measures for file sending, topology 1.

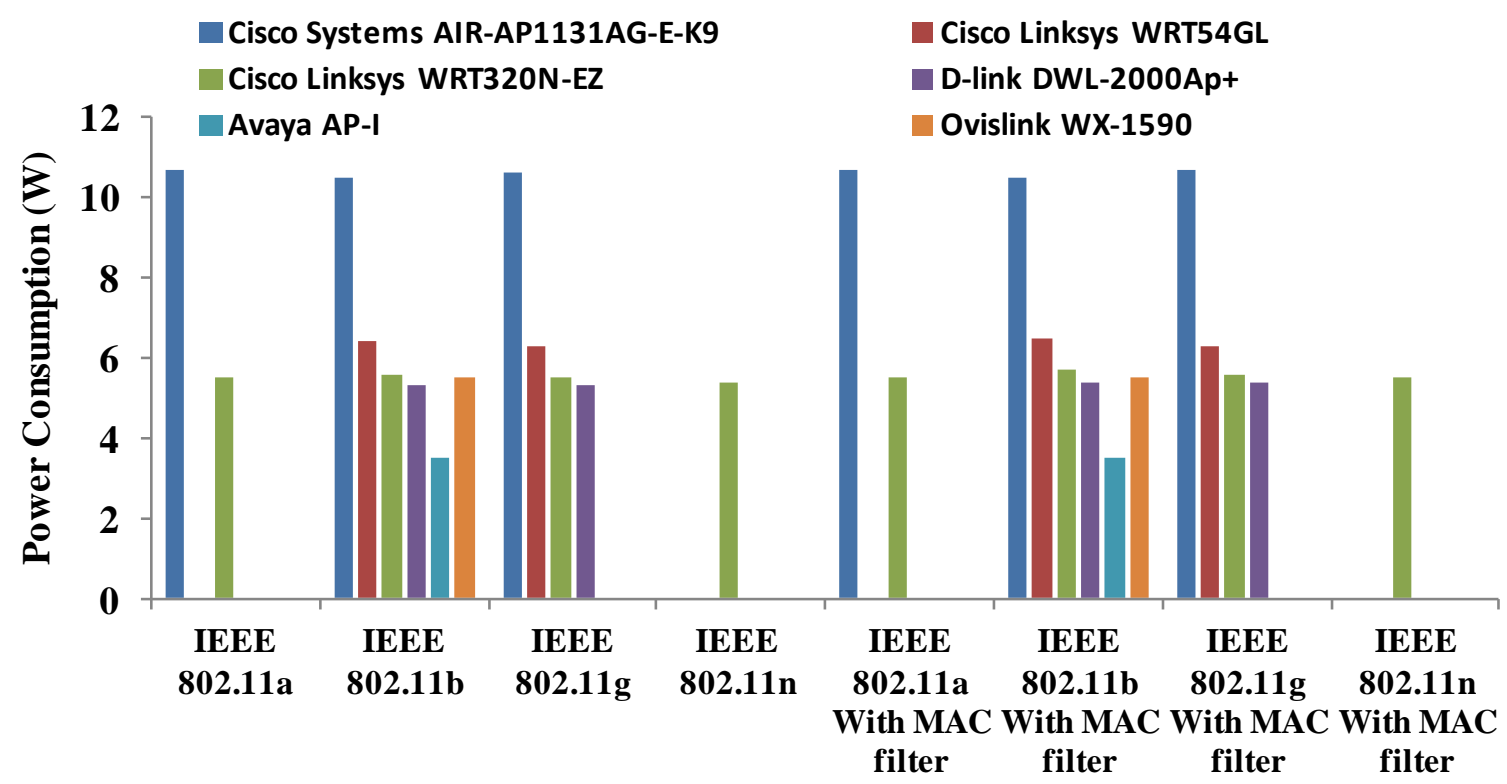

Figure 3.11. Measures for file sending, topology 2.

\section{Measurement for Cisco Systems air-AP1131AG-E-K9}

The Cisco-AP1131AG-E-K9 supports protocols IEEE 802.11a, IEEE 802.11b and IEEE $802.11 \mathrm{~g}$ and consumes $5.1 \mathrm{~W}$ in boot-up state and $5.4 \mathrm{~W}$ in idle mode. The energy consumed by this device during transmission is always between $10 \mathrm{~W}$ and $11 \mathrm{~W}$. We observed an increase in energy consumption of $2 \%$ in the topology 2 in comparison with the topology 1 . We can also see that the protocol IEEE 802.11 b consumes less energy when the AP sends the file in topology 1. As we can 
see in Figures 3.8, 3.8, 3.10 and 3.11 the MAC filter involves an increase of $2 \%$ in the energy consumption.

\section{Measures to Cisco Linksys WRT54GL}

Cisco Linksys WRT54GL allows to configure protocols IEEE $802.11 \mathrm{~b}$ and $802.11 \mathrm{~g}$. Its consumption is 5.2W during start-up mode and 5.7W in steady-state. As Figures 3.8, 3.9, 3.10 and 3.11 show, the energy consumed by this AP is considerably smaller than the consumed by the previous model (its values is approximately a $40 \%$ lower than previous model). Unlike the previous model, in this case, IEEE 802.11g protocol consumes less energy. As expected, the MAC filter for this model also introduces an increase in power consumption.

\section{Measures for Cisco Linksys WRT320N-EZ}

The Cisco Linksys WRT320N-EZ model consumes $4.5 \mathrm{~W}$ on the boot-up state and $4.8 \mathrm{~W}$ in idle mode. Figures 3.8, 3.9, 3.10 and 3.11 present the results of power consumption for this model in protocols IEEE 802.11a, IEEE 802.11b, IEEE 802.11g and IEEE 802.11n. IEEE 802.11n protocol is the one that presents lowest power consumption. Regarding to the activation of the MAC filter, both topologies register an increase in power consumption.

\section{Measurements for D-link DWL-2000Ap +}

D-Link DWL 2000AP supports the protocols IEEE 802.11b and IEEE 802.11g. Figures 3.8, 3.9, 3.10 and 3.11 show that the power consumption for this device in boot-up state is $3.4 \mathrm{~W}$ and for idle mode is $4.8 \mathrm{~W}$. In addition, this model consumes significantly less energy than the models manufactured by Cisco (analyzed in this study). In particular, it consumes approximately $16 \%$ less power than the Cisco Linksys WRT54GL which offers the same services. IEEE $802.11 \mathrm{~b}$ is the protocol which presents the lowest power consumption. The behavior of this device, in regards to the use of the MAC filter and the differences between the topologies, is very similar to previous cases.

\section{Measures for Avaya AP-I}

This AP presents more limited capacities. It only allows the protocol IEEE $802.11 \mathrm{~b}$ and as expected its power consumption is smaller than other cases. Its consumption in boot-up state is $2.8 \mathrm{~W}$ meanwhile the consumption in steady state is $2 \mathrm{~W}$. Figures $3.8,3.9,3.10$ and 3.11 show considerable differences in power consumption. The increase on power consumption introduced by the use of the MAC filter is about $3 \%$.

\section{Measures Ovislink WX-1590}

The model Ovislink WX-1590 offers similar characteristics to the previous one with limited capabilities. The main difference is that this model consumes $40 \%$ more power. Its consumption is $4.8 \mathrm{~W}$ during boot-up state and $5.4 \mathrm{~W}$ in sleep mode. This device only allows the protocol IEEE 802.11 b. For the rest of the parameters studied, we observe the same behavior observed in earlier devices. 


\subsection{Conclusion}

One of the most important aspects to consider in the WSN design is the energy limitation. In this chapter, we have presented the main causes of energy loss in wireless sensor nodes. Firstly, we discussed the energy wastage given by the electronic circuit. We have modeled the energy consumption of wireless sensor network nodes in a different manner than other works, taking into account the time wasted in each process (transmitting, receiving, processing, etc.).

We have also seen the energy consumption as a function of the wireless technology. Although Zigbee and Bluetooth are the most used because their energy consumption is quite low, they have very short coverage radio; so many sensors are needed to cover wide areas. We have shown the IEEE $802.11 \mathrm{a} / \mathrm{b} / \mathrm{g}$ chipsets that satisfy the requirements needed to be used in wireless sensor networks and can be bought in the market. Although IEEE $802.11 \mathrm{a} / \mathrm{b} / \mathrm{g}$ technologies have higher coverage areas and higher bandwidth, their energy consumption is quite higher, so we have discussed their consumption and how it can be decreased.

The amount of information generated in a cooperative group-based sensor network is different than the information generated in a regular network. In group-based sensor networks, when a sensor detects a new event, an alert is sent to its group and it is distributed to neighboring groups. The cooperation between groups is used to send messages between groups in order to obtain the right alert level. To see the comparative of energy consumption in both networks, we have simulated them and the results have proved that group-based sensor network protocols allow larger lifetime for the nodes in a WLASN than the other protocols.

Following our analysis, we have compared several MAC and routing protocols that have been designed to optimize the power consumption without compromising the data delivery in WSNs. Finally, we have performed several tests over actual network devices where we have determined which network devices from which brand and model, and which routing and wireless network protocols are most appropriate for designing a communications network energetically efficient.

To conclude, we should keep in mind that the efficient design of WSN implies a correct design from the point of view of hardware, network topology and protocols. For future works, we would like to include in our investigation the concept of energy harvesting and the possibility of include it in our prototypes.

Finally, work presented in this chapter has been published in the following references [157], [158], [265], and [266]. 



\section{Chapter 4}

\section{Wireless Sensor Development for Environmental Monitoring}

\subsection{Introduction}

Wireless sensor networks have become a research field that is constantly growing due to the variety of purposes to which it may be applied. The WSN have great potential in the field of natural environments monitoring, industrial applications or urban monitoring.

The detection of events such as forest fires, the presence of a pest in a field, missing persons or other events can be detected visually. However, it has passed a long time, the consequences of these events can become very serious.

The incorporation of new technologies in our daily activities allows us to detect these events faster. Early detection allows us to take quick solutions to prevent damage and irreversible consequences.

The application of WSN in any of these areas allows us to develop networks in places where we cannot use wired networks. Due to the small size of these devices, their installation is very simple and versatile. Moreover, the visual impact is minimal and the animal living in that environment will not upset their life. Furthermore, due to its scalability, it is very easy to change the type of topology, increasing or decreasing the number of nodes of the network.

Finally, the high demand of these wireless devices makes very low costs. 
For these reasons, in this Chapter, we are going to present several wireless sensor deployments for natural environmental monitoring.

First of all, we will see three applications for natural environment monitoring. In this Section we present an optimized Wireless IP multisensor Network to detect and locate fire. We use a system of cameras to verify it. The second deployment of this Section is a WSN that uses an image processing system in each wireless node in order to distinguish if a vine has bad leaves or not. Finally, we are going to present a WSN based on IEEE 802.15.4 technology which is capable to intelligently detect when a flock is being attacked by wild animals. The data generated by the WSN is processed by a smart algorithm which will decide what kind of alarm has to be generated and consequently, the system will warn the owner and other services such as the environmental unit of animal control.

The second application group presents a non-invasive technique based on a WSN for the detection of the cement degeneration in constructions. Nodes measure the temperature, humidity and structural movements of the construction in order to quantify the degeneration of cement. The sensor node placement is studied in depth in order to predict very soon any deterioration of the building materials by monitoring the effects of the cement degeneration.

Finally, in the third application group, we will present a WSN to take care of disabled and elderly people. This Section describes a smart system based on the embedded sensors of mobile devices in order to determine if a person that wears one of these devices has suffered an incident or has a problem, when the group of persons is doing an activity.

Last Section shows the conclusion of this Chapter.

\subsection{Sensor Deployment for Natural Environment Monitoring}

\subsubsection{Wireless Sensor Network Deployment for Rural and Forest Fire Detection and Verification}

In this section, we explain the place where we set up our deployment and the main features of a rural area in order to introduce the reader to the main issues that should be taken into account designing the wireless network. We also study the number of devices needed per coverage area.

The rural environment, where our test bench has been developed, is a $2 \mathrm{Km}$ diameter circle (see Figure 4.1). It is located in "El Encín", Alcalá de Henares, Madrid, Spain. "Explora El Encín" is a popular scientific project of the "Instituto Madrileño de Investigación y Desarrollo Rural, Agrario y Alimentario (IMIDRA)". IMIDRA is entirely dedicated to research, innovation and scientific spreading tasks. One of the main objectives is to present the surroundings closest to Madrid, its agriculture and its researches to the citizens. It is uninhabited. There are different types of cereal cultivation (wheat, barley, oats, maize, etc.), vegetables (chick-peas, lentils, peas, etc.), grapevines and other types of plants, with different production systems. The area also has different types of trees, with a great fauna and flora variety. The main focus of "Explora el Encín" is to show the natural environment to people who have some physical or sensorial disability. Current society feels more and more that this type of natural space should be protected in order to conserve many species of animals and native plants. Because of the environmental importance, and the great variety of species cultivated inside, we were required to deploy a system to detect a fire, using wireless 
multisensors, and verify it, using wireless cameras, in order to decrease the reaction time and to avoid a big disaster.

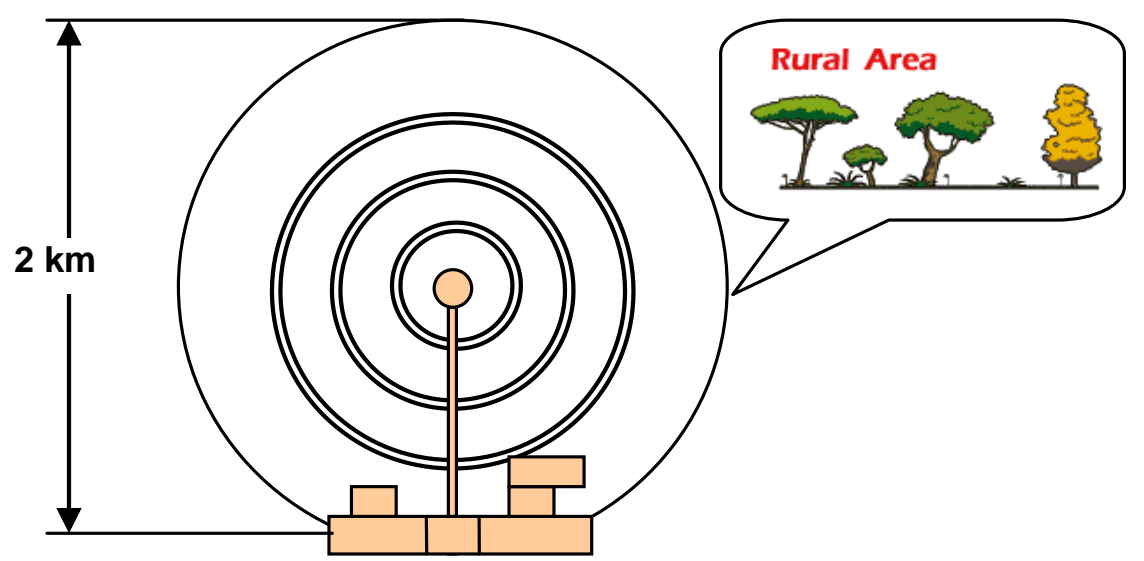

Figure 4.1. The rural area where the test bench has been performed

A rural environment video-surveillance design is very different compared to home or enterprise designs. The presence of animals and the reduction in coverage because of the vegetation has to be considered. It involves the following issues:

We have to minimize the visual impact of the data network in the rural environment. So, data wires should be avoided.

- We have to avoid the use of electric wires because it could damage animals, so the power has to be obtained using batteries and solar panels. It implies that the devices have to be low power consumption to minimize costs and visual impact (the greater the power consumption, the bigger the solar panel).

- The video camera has to be very small in order to reduce the visual impact to animals, but it should have enough quality to obtain good images.

- Enough bandwidth is needed in the wireless network to be able to stream video from different video camera devices.

- The rural area has plenty of trees, animals and vegetation. These objects diminish the received power, so we must be sure that the received signal in our wireless network has enough power.

- Nowadays an IEEE 802.11g WLAN has a maximum bandwidth rate of 54 MBps (close to 30 Mbps of effective bandwidth), so we should test how many wireless cameras and multisensors could transmit to a single access point without having a video quality reduction.

Our design uses one or several IEEE 802.11g access points (depending on the number of wireless cameras and the number of fire detectors) placed on a visible position from all parts of the rural area. We use wireless IP cameras with high gain antenna (to reach large distances). The wireless multisensors are distributed strategically around the rural or forest area, but always located inside the coverage area of the wireless access points (see Figure 4.2). Both, multisensors and cameras are under the coverage area of an access points. The access points of the network are connected wirelessly using IEEE 802.11g (if they are close enough) or using optic fiber using IEEE $802.3 \mathrm{u}$. The access points of the network allow the connectivity of all multisensors and IP cameras in the network to a central server. The frames are sent through the data link later without the need of 
any routing protocol in the network. The position of the multisensors is initially saved in a central server, but it could be changed at any time. Although in our initial implementation the multisensors are not mobile, we can implement mobile sensors that can be monitored using GPS or wireless positioning based systems [267].

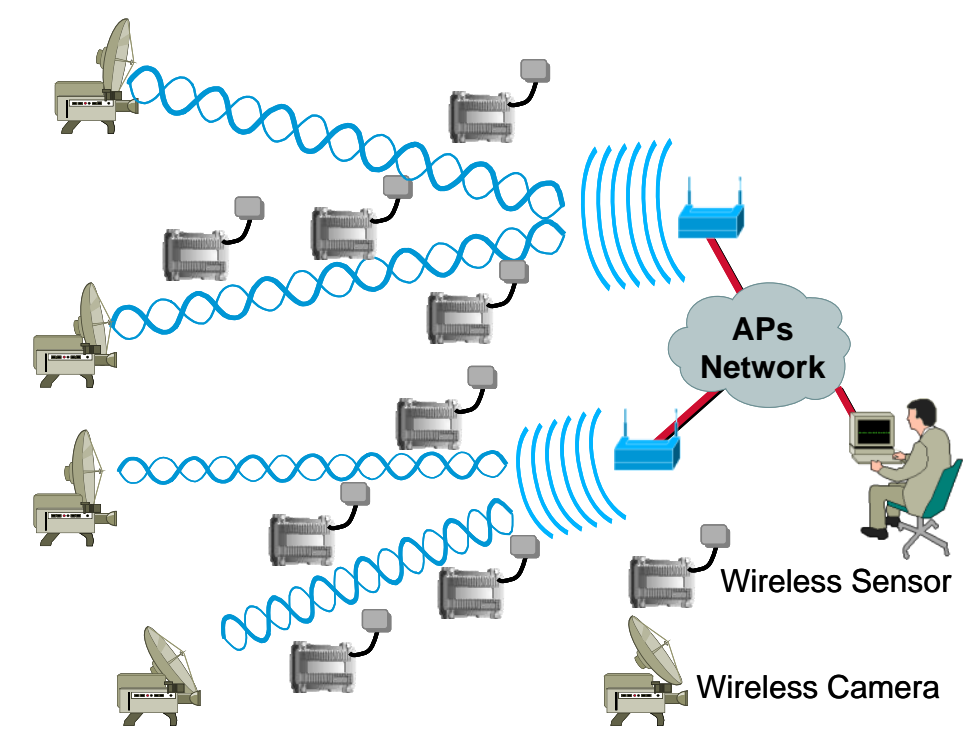

Figure 4.2. Fire detection and verification design proposal.

The wireless multisensor sends a fire alarm through the access points to a central server if the combination of its physical sensors gives that there is fire. The input variables of the multisensor are fire infrared radiation and smoke, but we are planning to add temperature (and the quick changes of temperature), humidity and $\mathrm{CO}_{2}$ [268]. The field of vision of the wireless cameras covers the whole geographical area where the multisensors are placed (the cameras can rotate $270^{\circ}$ horizontally and $90^{\circ}$ vertically). The central server has a database that relates each multisensor with a certain horizontal and vertical direction of any one of the closest wireless IP cameras. Then, the server sends a frame to the closest cameras. They move their objective toward the multisensor that detected the fire. The images are seen from all cameras placed near the affected zone to a computer placed in the firefighter control room. All devices have an IP address and these images and information could be accessed from Internet.

Because the data registered by the sensors are combined with the decisions of the firefighters after they have seen the images taken from of the cameras, this system offers full information to the firefighter squads, facilitating the extinction tasks. Moreover, the affected area can also be visualized using the controls of the camera at the time. It can also be used to improve fire control and surveillance.

\subsubsection{Radio Design, Number of Devices Needed and Channel Distribution Plan}

In order to design the wireless sensor network we have studied the signal loss during its path in a rural or forest environment. We need to know how far the Wireless IP camera and the wireless sensor could be from the access point to receive enough signal power. To calculate this parameter we use the power balance formula (given by equation 4.1). This equation states that the received 
signal power, in $\mathrm{dBm}$, is equal to the transmitted power plus the transmitter and receiver gain, minus the basic loss and minus other losses produced by objects (such as trees or humidity) [269].

$$
P_{r x}(d B m)=P_{t x}(d B m)+G_{t x}(d B)+G_{r x}(d B)-10 \cdot n \cdot \log d-L_{r a i n}(d B)-L_{\text {vegetation }}(d B)
$$

Where $n$ is the attenuation variation index. $n=2$ for air medium and $d$ is the distance between the transmitter and the receiver. We have considered rain loss, which depends on the place where the wireless system is installed, and vegetation loss that depends on the number of trees closer to the signal path between the transmitter and the receiver. None of the works that we have found in the literature about WLAN design have taken into account all the parameters that we have considered in our study [270] [271] [272]. The value of these losses can be obtained from references [273] and [274]. So, in our environment, the coverage distance is given by equation 4.2. More details about the steps followed are given in a previous work from the same authors [275]

$$
d=10 \frac{P_{t x}+G_{t x}+G_{r x}-L_{\text {rain }}-L_{\text {vegetation }}-P_{r x}}{20}
$$

In order to calculate the distance between devices, but bearing in mind the vegetation, we are going to fix some parameters. On the one hand, theoretical transmitted power is $-40.2 \mathrm{dBm}$ for an IEEE 802.11g WLAN device at 1 meter, and we estimate $-80 \mathrm{dBm}$ threshold power for the far-away IP camera and sensor to have enough quality of signal, so our received power must be greater than or equal to this mark. Let's use a $20 \mathrm{dBi}$ omnidirectional antenna for the access point (Gtx), $12 \mathrm{dBi}$ directional yagi antennas for all wireless IP cameras $\left(\right.$ Grx $\left._{\text {camera }}\right)$ and $7 \mathrm{dBi}$ onmidirectional antennas for the sensors $\left(G r x_{\text {sensor }}\right)$. On the other hand, this study has been done in Spain, which has two main hydrometric areas: the $\mathrm{H}$ area and the $\mathrm{K}$ area [276], so losses due to rain, in the worst case, have a value of $0,026 \mathrm{~dB}$ for two kilometers. In order to know the losses because of the vegetation, we have used the recommendation given in reference [277], so we can assume a loss of $1.2 \mathrm{~dB} / \mathrm{m}$. Equation 4.3 shows the formula needed to design our WLAN:

$$
d=10^{\frac{59.77+G r x-1.2 \bullet m}{20}}
$$

Where $m$ is the number of meters of vegetation and Grx is the gain of the antenna of the device (the IP camera or the sensor). Figure 4.3 shows the coverage distance (the distance between access points and the wireless IP cameras and the sensors) as a function of the meters of vegetation through the light of sight path. We can see that around 34 meters of vegetation is allowed in the case of an IP camera and approximately 30 meters of vegetation for the sensors. 


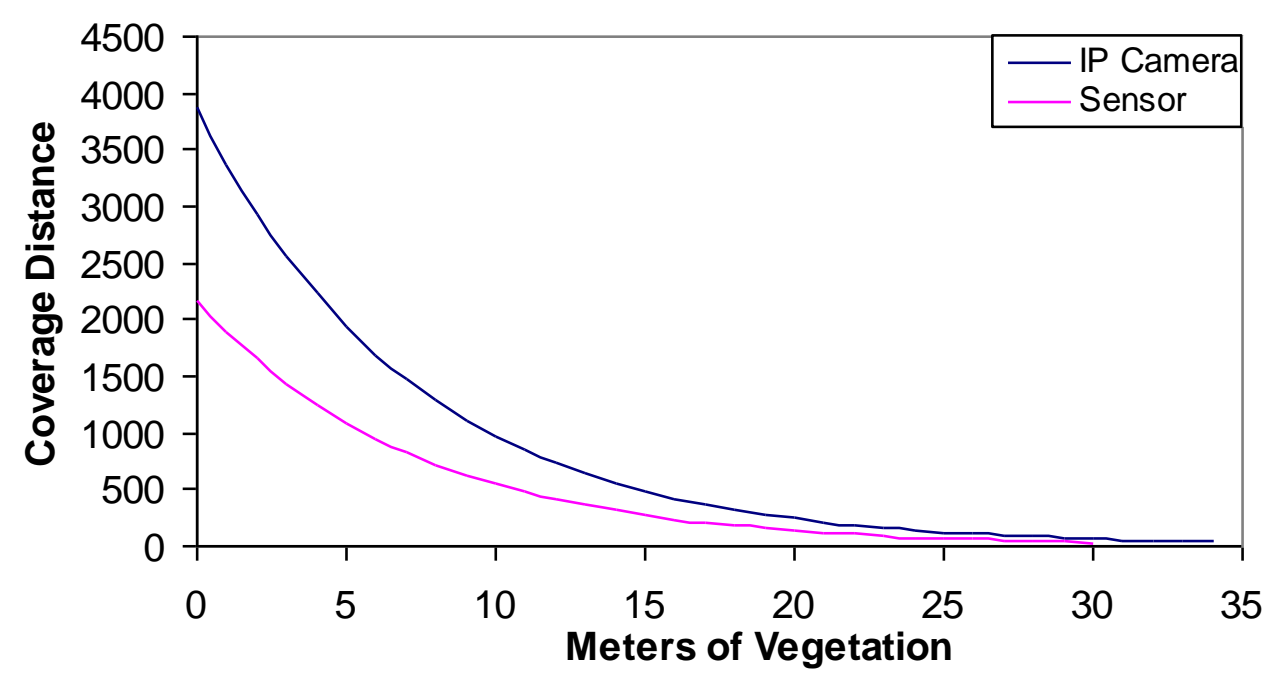

Figure 4.3. Coverage distance vs. meters of vegetation.

The radio coverage of the devices depends on the leafiness of the forest. In order to know the number of devices needed for a given area, we have studied the mean coverage area of every device used in our system. The forest density can vary from 40.000 to 200.000 trees per square kilometer (it means a tree every 50 meters in the first case and a tree every 5 meters in the second case). On the one hand, let us suppose that we are measuring a forest where the trees have an average diameter of 3 meters at a height of 3 meters from the ground (the place where the sensors are placed). We also suppose that a regular forest in Spain has a tree every 10 meters. On the other hand, let us suppose that there are no more than 3 meters of vegetation from the IP camera and the Access Point (because the IP cameras have to be placed very high to acquire a good view from the forest and the access points should be placed strategically in the line of sight of the sensors and the IP cameras), so every camera covers a radius of 2,940 meters approximately.

Taking into account the measurements provided in Figure 4.3, if we have 24 meters of vegetation, there could be a distance of 80 meters approximately (a tree every 10 meters with a diameter 3 meters) from the sensor to the access point. Figure 4.4 shows the number of devices that can be placed in our system as a function to the area that is wanted to be covered. We have supposed the worst case: an access point every 6 sensors in order to maximize the area covered.

Access points are non-root bridge with clients that let the multisensor connect to the access point while it can be connected with root access points. There are access points acting and root bridges that let non-root bridges connect to the infrastructure. The access points that let us configure this type of infrastructure are Cisco Aironet (C) 350 Series Wireless Bridges [278].

Figure 4.5 shows an example of the topology. There are more sensors than access points and fewer cameras than the other devices (the cameras are the most expensive devices). There is an access point configured as a root and three access points configured as a non-root access points. 


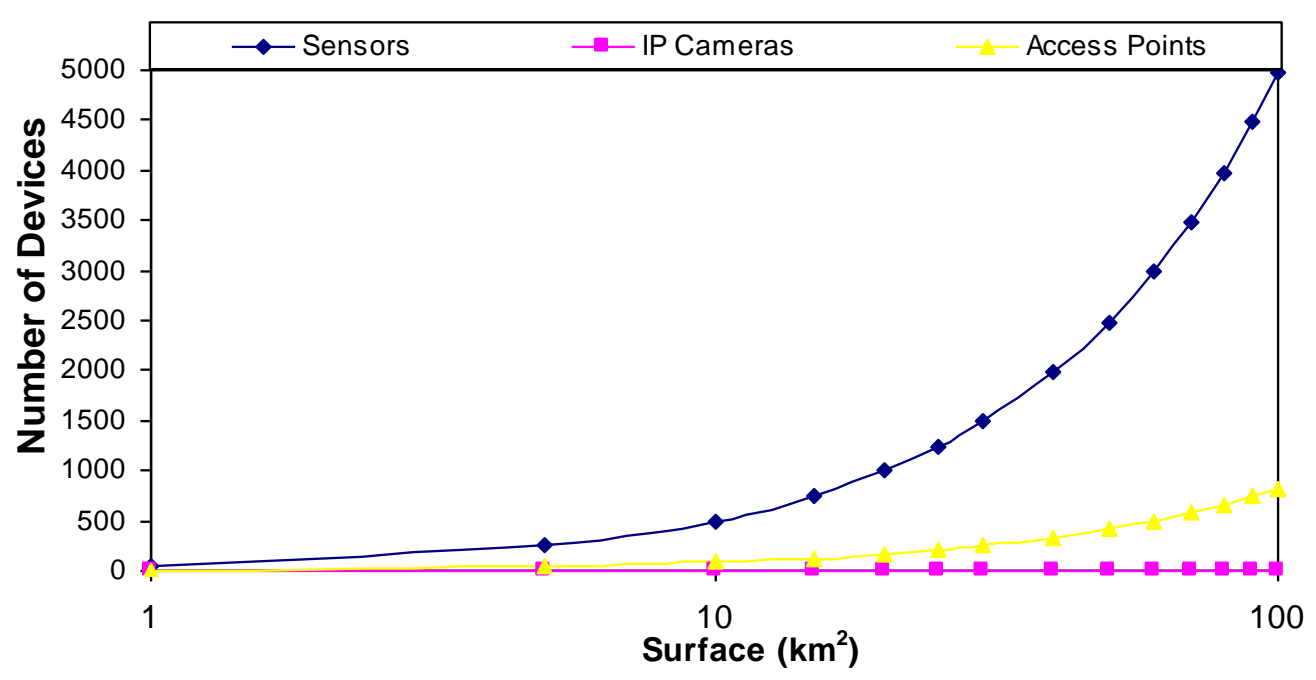

Figure 4.4. Number of devices needed per area that is wanted to be covered.

\subsubsection{Hardware Deployment}

In this section we show a sensor node that is able to sense several parameters from the same place while it is able to form an IP network of multisensors. In order to achieve our aim, we looked for a device with a control unit. This control unit manages and controls all sensors connected to the device. On the other hand, the electronic circuit must have several input interfaces in order to connect several physical sensors. Several of the main aspects taken into account were circuit costs, the operative system used and the possibility of adding several physical sensors to the device, in order to enable optimum choice.

\section{Wireless Sensor}

Our proposal is based on the use of the Linksys WRT54GL router, from Cisco Systems inc., as the core controller [279]. It is an embedded system that has a wireless IEEE $802.11 \mathrm{~b} / \mathrm{g}$ interface, a FastEthernet interface in its board, so it meets our pre-requisites. In addition, Linksys WRT54GL offers internally General Purpose Input/Output (GPIO), UART (JP2) and ETAJ (JP1) ports. Some extensions can be made to the router by using these ports. Figure 4.6 shows the embedded board and its hardware distribution.

One of the main features that have caused the use of the Linksys WRT54GL as a sensor node was the possibility of installing a Linux Kernel 2.4. On one hand, it is a known operative system, so we did not need to learn a new operative system and, on the other hand, we know all the possibilities that a Linux is able to provide us. So, at software level, this model is based on open source software, causing the development of different specific software applications for it and expanding the factory default capabilities. 


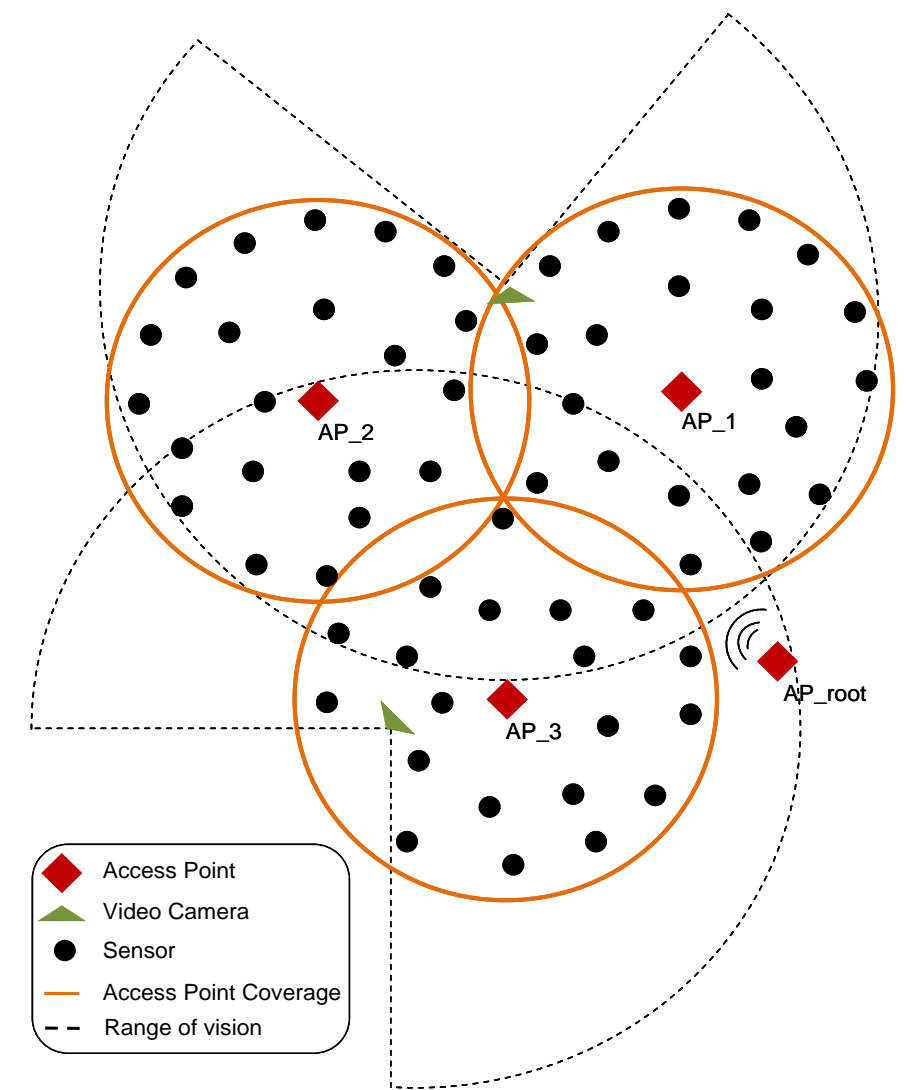

Figure 4.5. Number of devices needed per area that is wanted to be covered.

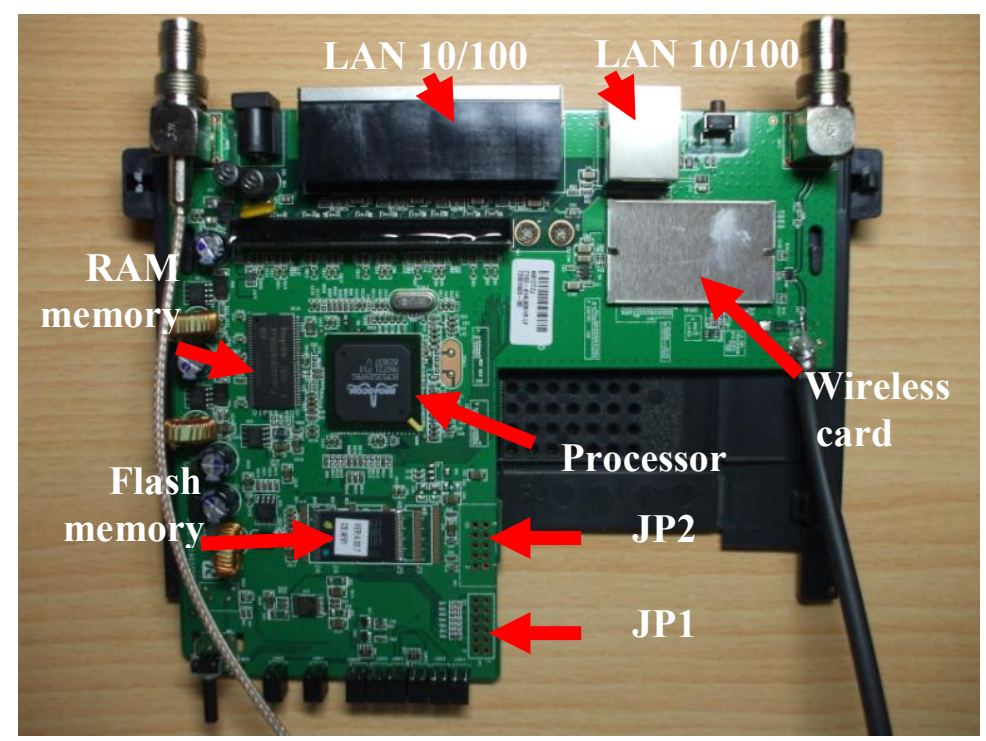

Figure 4.6. Hardware distribution on board.

In order to connect two sensors directly to the board, we made an extension using the GPIO of the Linksys WRT54GL router. It provided us two serial ports through the JP2 port. Figure 4.7 shows serial ports connected on board by welding pins on JP2. Then we added two DB9 Female DCE ports because we wanted flexibility in order to change the type of sensor connected to our 
device. To be able to connect a device with RS232 connection, it is needed a logical levels adapter based on MAX233 Integrated Circuit. The Linksys WRT54GL router has TTL family logical levels so we had to adapt them to the RS232 family logical levels. Figure 4.7 shows the integrated circuit used to provide two serial ports. A RS232 line converter is needed to go from $+3.3 \mathrm{~V}$ to $+5 \mathrm{~V}$.

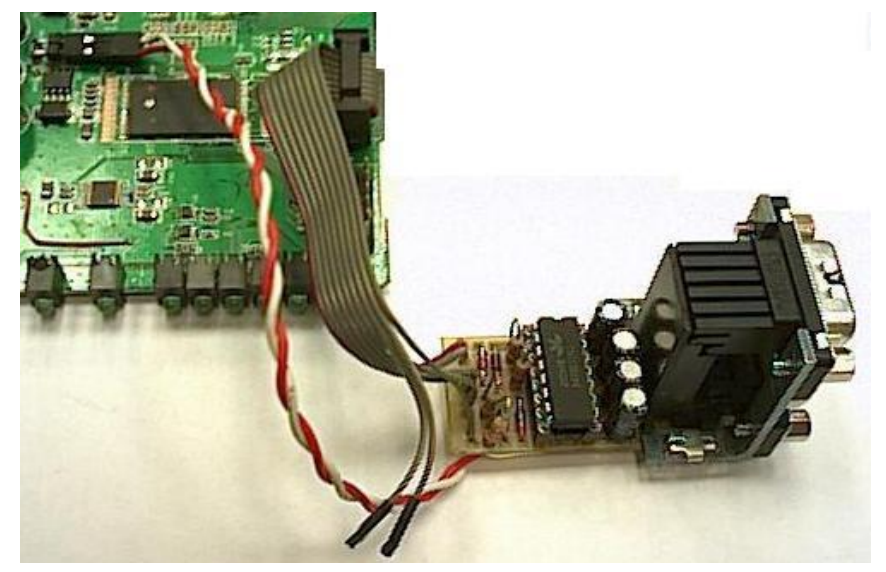

Figure 4.7. Integrated Circuit used to provide two serial ports.

Also, we can connect an SD card reader to some of the GPIO pins found inside the Linksys WRT54GL router and with the help of a little driver we can use as a block device from Linux. The SD card reader has been tested for a $1 \mathrm{~GB}$ SD card. It allows us to install applications for signal processing and store and manage acquired data from both sensors. Now, the sensor node is able to store and process data without the need of sending the measurements taken continuously. We developed a process that is running in the Linksys WRT54GL router. It checks the values obtained by both serial ports. In one port, we connected a smoke detector and in the other port we connected a fire infrared detector. The input variables of the multisensor are fire infrared radiation and smoke, but we are planning to add temperature (and the quick changes of temperature), humidity and $\mathrm{CO}_{2}$. We programmed a software application that only gives a positive value if both sensors have values higher than a threshold; otherwise it is a false alarm. But this decision can be changed as desired using a combination of the measurements gathered. The system is able to gather data, process it internally, and send only alarms or statistical data spontaneously, saving energy.

\section{Wireless IP Camera}

The wireless cameras selected transmit MPEG-4 standard video compression, which has higher compression and quality compared to other standards. It also consumes low bandwidth. MPEG-4 is commonly used in video streaming over IP environments. The video is streamed using the HTTP protocol (we have chosen this protocol to facilitate the video visualization) with very good results. Chosen cameras are able to stream video with a resolution of $320 \times 240$ using 25 fps (PAL system) and they are able to transmit audio in both directions (from the camera and to the camera). Their working temperature is between $-5.5^{\circ} \mathrm{C}$ and $75^{\circ} \mathrm{C}$.

Wireless camera video streaming is transmitted from the camera to the server directly. The person sited in the server (a fire fighter) can choose by software the camera to be watched and the software opens a connection with that camera and receives the video streams sent via the camera. 


\section{Photovoltaic system}

The photovoltaic system is formed by a photovoltaic panel, the battery, the load regulator and an inverter. There are 3 basic types of photovoltaic panels, all of them use silicon: monocrystalline cells, polycrystalline cells and amorphous cells. We have used the polycrystalline cells because they have higher performance than the amorphous cells (between $11 \%$ and 13\%) and they are cheaper than the monocrystalline cells. There are many types of batteries that can be used in a photovoltaic system: Lead-acid battery, VRLA battery, AGM battery, Gel battery, Nickel-cadmium battery, etc.

First we studied the solar radiation map of Spain. Taking into account the values provided by references [280] and [281], Spain has high solar radiation values. Concretely, Madrid, the place where we deployed our test bench, has 1,560 hours of sunlight per year, which means a mean value of 4.27 hours of sunlight per day. The battery of the sensor has to be able to provide power during 20 hours at least.

In our case, the sensor consumes $12 \mathrm{~V}$ and $100 \mathrm{~mA}$ approximately, so the power consumed by the sensor is $1.2 \mathrm{~W}$. Taking into account that a polycrystalline cell has a mean performance of $12 \%$, the power needed to supply the sensor and to charge the battery simultaneously is $60 \mathrm{~W}$. Let us suppose that we use have a battery of 24VAh fully charged. The sensor will discharge the battery in 240 hours if there is not sunlight. Such number of hours without sunlight is very difficult to happen in Spain.

\subsubsection{System Design and Operation Mode}

First, we placed the wireless IP cameras in strategic places to watch interesting zones. Then, we placed wireless IP sensors in some critical points with more risk. Both, sensors and cameras are under the coverage area of the access points.

The mode of operation is as follows. All cameras have been recorded with the coordinates where they have to move and focus for each sensor placed in their visual coverage. The server has a database with the position of the sensors and the name of the cameras placed in the rural area close to every sensor (they are stored by name: sensor_1, sensor_2, etc.). When a sensor detects a fire, it sends an alarm directly to the server. This alarm message has the name of the sensor. When the server receives this message, it searches in its database the closest wireless cameras to that sensor and sends them a message with the name of the sensor that has sent the alarm and the position they must move to in order to watch the image of that zone. Finally, the cameras move their objective to the coordinates of the sensor. The camera will show what is happening in that zone and the fire fighter can corroborate if there is a fire or not.

When there is an alarm, a firefighter sees the video streams from all the wireless IP cameras of the affected zone. On the other hand, the images could also be watched by other users when they request it. Figure 4.8 shows the flowchart with the mode operation from the point of view of the system. 


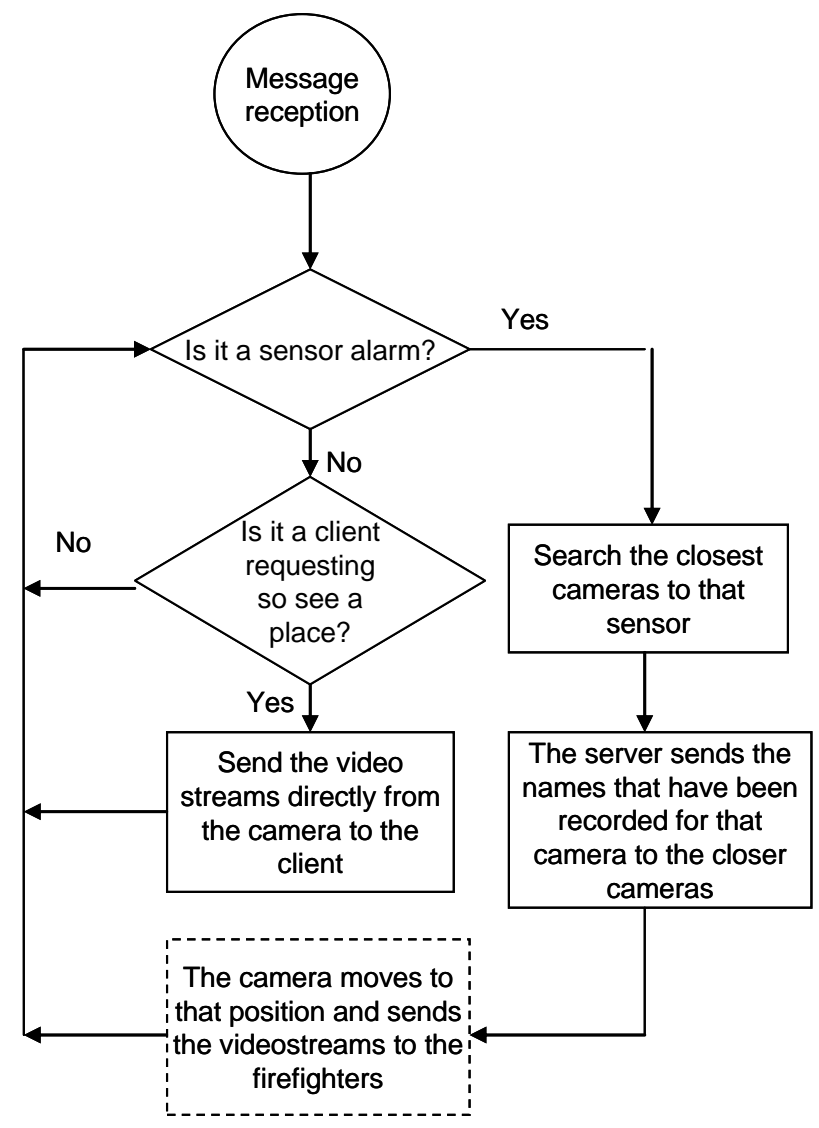

Figure 4.8. System Mode Operation Flowchart.

The system is highly scalable because a camera can cover as many sensors as positions can be recorded. On one hand, if it is placed at the top of a mountain, more area can be viewed by the camera. On the other hand, the database of the server can have many entries. There has to be one entry for each sensor. In our design plan we have considered that every sensor has to be seen by two wireless IP cameras at least. We have programmed all these instructions over http protocol to be easily implemented in other systems. Figure 4.9 shows the messages sent when there is an alarm.

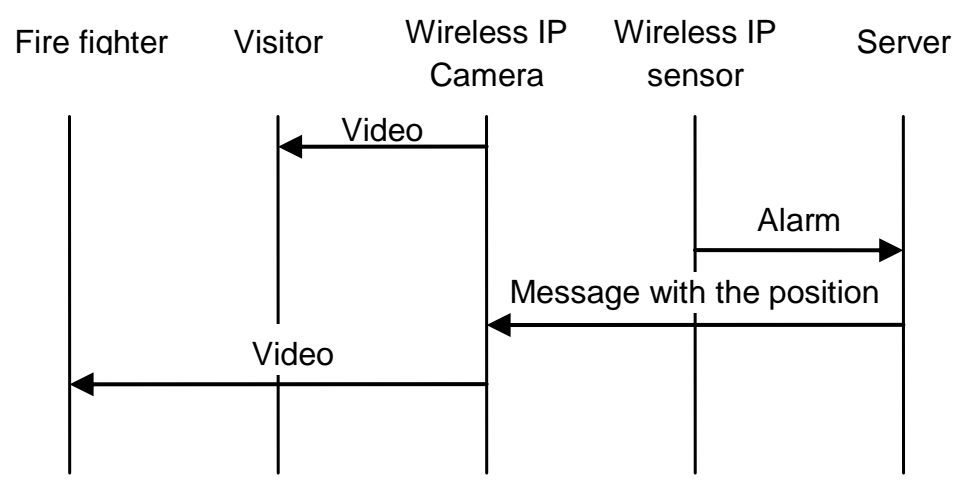

Figure 4.9. Messages when there is an alarm. 


\subsubsection{User Interface}

We have developed a web page that shows the video streams received from several Wireless IP cameras in real time. Images are shown without jumps and there is not any quality images reduction. Figure 4.10 shows the developed web page.

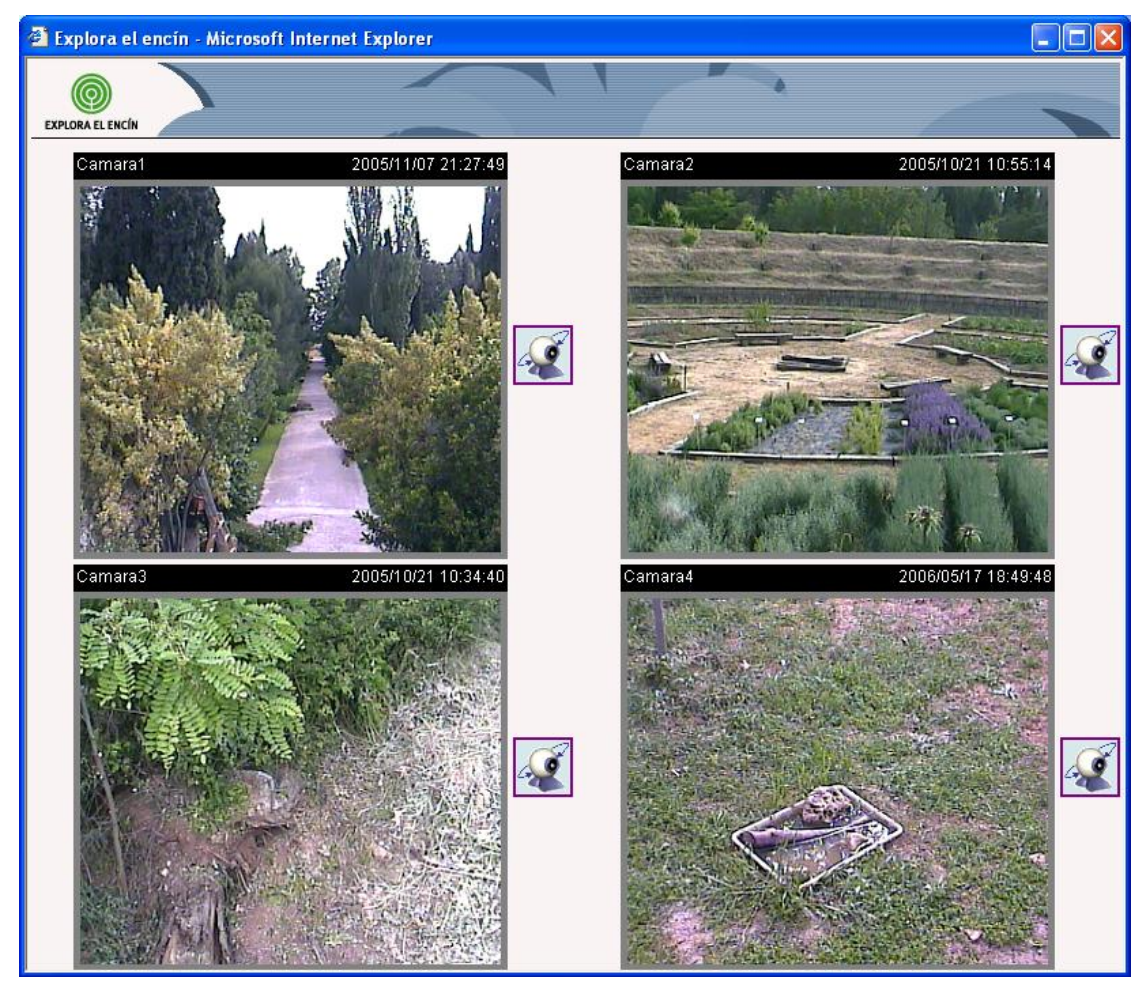

Figure 4.10. Main web page.

Clicking the icons placed on the right of every video image, we can access the control web page for each camera. The web page for each camera (see Figure 4.11) shows a greater image with the same quality and the user is able to change its vertical (till 90 degrees) and horizontal (till 270 degrees) orientation.

We can also vary the zoom lens to a $10 \mathrm{x}$ value and focus the video image (it can be done automatically). We can vary the Iris lens to obtain a better visualization and we have enabled two buttons called "Auto pan" and "Auto patrol" that moves the camera automatically to have a panoramic view of the place. We have also enabled a button to pick up photographs.

All cameras can be accessed independently and their control is independent, so users or the fire fighter can access to a camera and control it without any disturbance. 


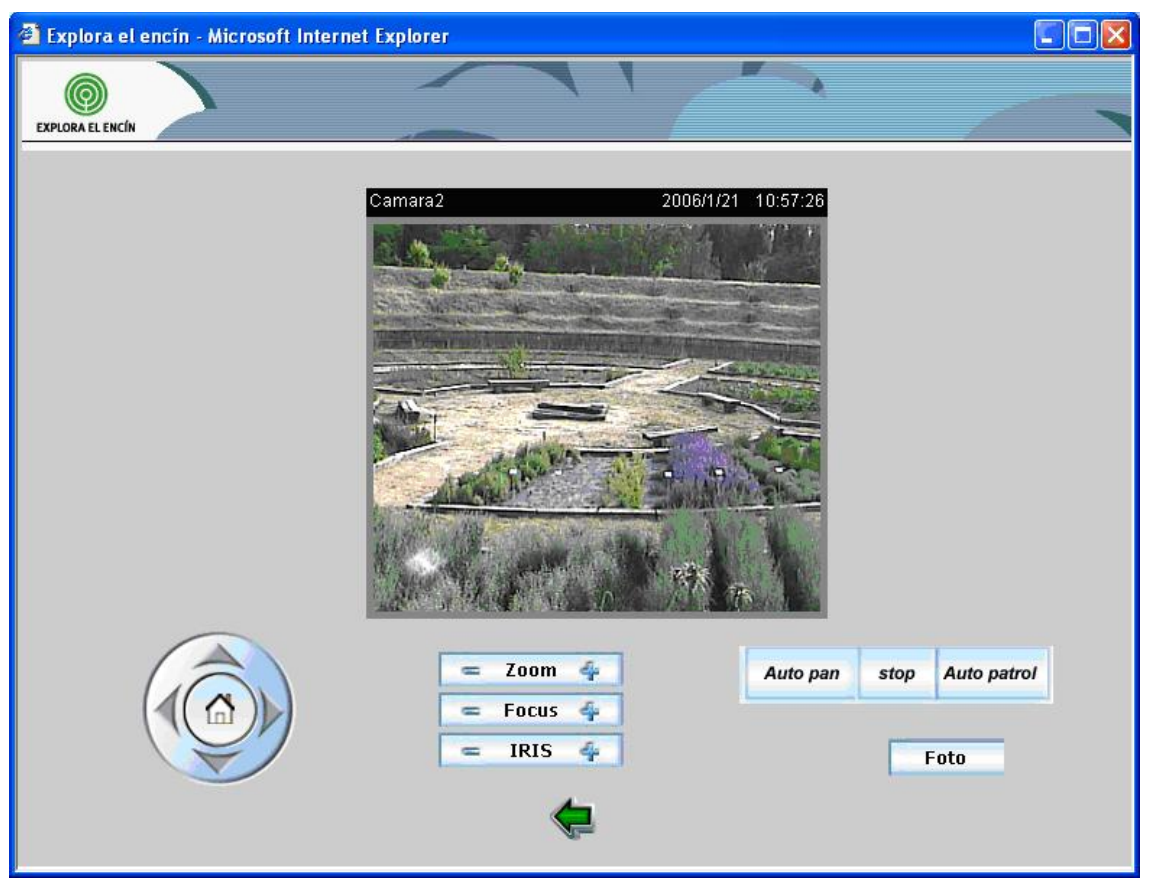

Figure 4.11. A Single Camera visualization web page.

\subsubsection{Performance Test}

This section shows several test benches that have been set up in order to show the performance of the implementation.

\section{IP Camera Bandwidth Consumption Comparison}

We have measured a wireless IP camera placed in the rural area transmitting MPEG-4 codec compressed video over http protocol in order to test the number of bytes per second and the number of messages per second in the network. It lets us know the camera bandwidth consumption. The video resolution was $320 \times 240$ at 25 fps. The wireless IP camera also transmitted audio at $24 \mathrm{Kbps}$ from the camera to the web page. We have measured, for 2 minutes and 30 seconds, the following situations:

- 1st Situation: The camera is acquiring video from a fixed place where there is not any motion, and the camera is not moving.

- 2nd Situation: The camera is acquiring video from a fixed place where there is motion, but the camera is not moving.

- 3rd Situation: The camera is acquiring video while it is moving.

- 4th Situation: There are 4 cameras acquiring video from fixed places where there is not any motion and the cameras are not moving. We show how much bandwidth just one camera needs.

Then, we have measured the network for 15 minutes in order to know how the network performs for longer times. 
Figure 4.12 shows the number of bytes per second for the 1st, 2nd, 3rd and 4th situations. We can observe that the worst case is when the video acquired has motion. On the other hand, we think that the moving camera situation is rampant because of the bandwidth used to control the camera. There is no case with more than 140,000 bytes per second (1.12 Mbps). Then, we have divided the number of bytes per second obtained by the number of cameras. The average number of bytes is around 109.2 KBytes (873 Kbits), while the average number of bytes per second when there is just one camera is $63.6 \mathrm{KBytes}(509 \mathrm{Kbits})$. So the system allows 34 cameras in each access point.

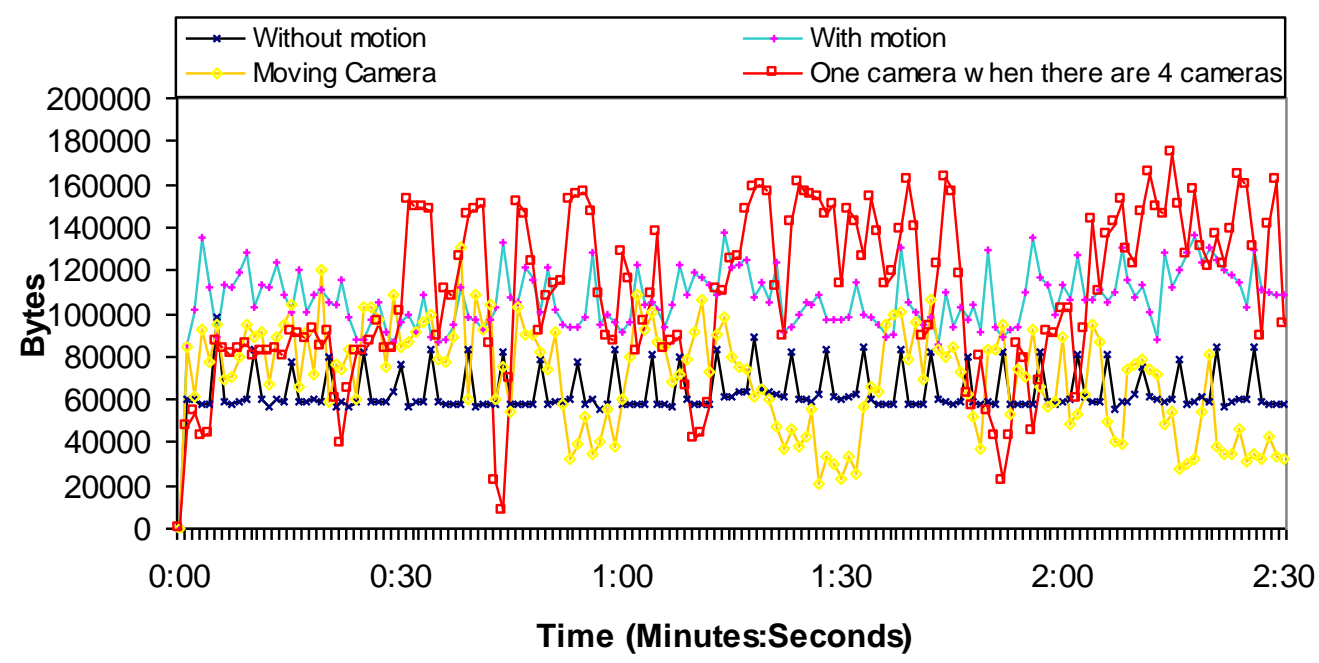

Figure 4.12. Octets per second obtained by the four situations.

Figure 4.13 shows the number of packets per second for the 1st, 2nd, 3rd and 4th situations. We can observe that the worst case is when the video acquired has motion. The moving camera sends messages in a rampant manner without any rule. The average number of packets per second, due to one camera, when there are four cameras is around 167 packets/s, while the average number of packets per second when there is just one camera is 180 packets/s. So the system allows many cameras.

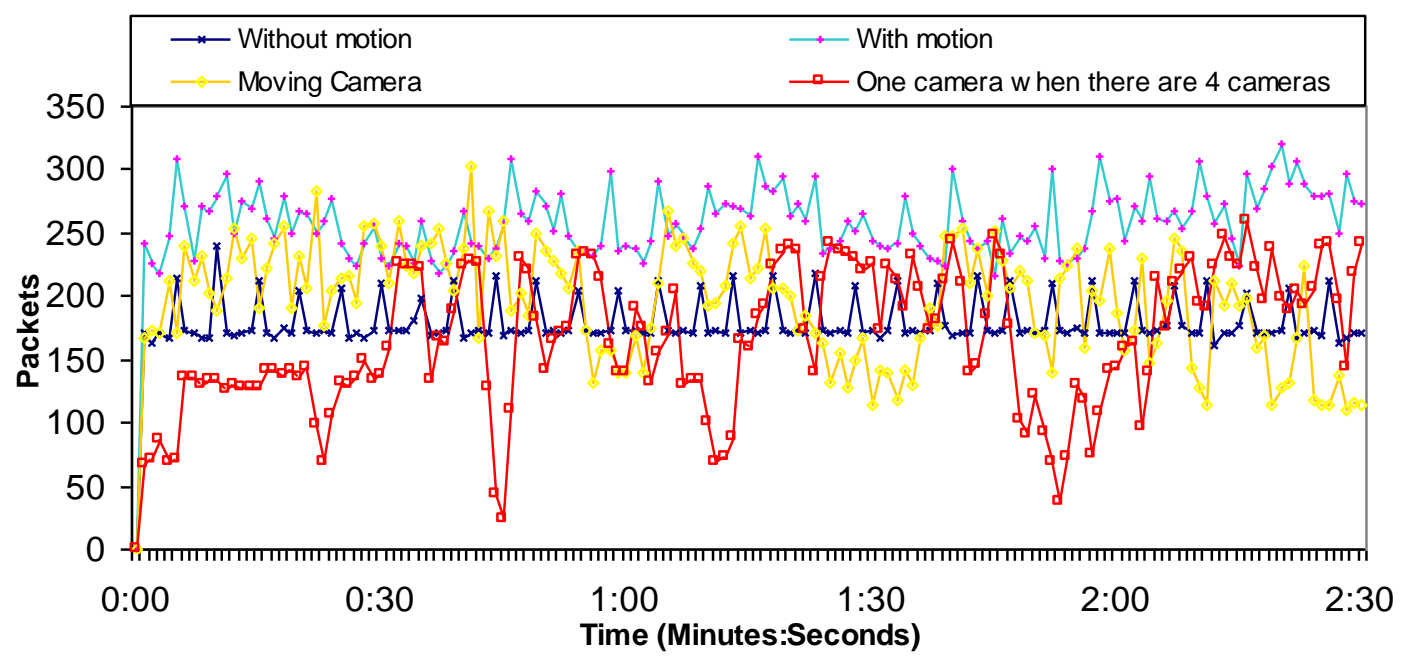

Figure 4.13. Packets per second obtained by the four situations. 
Figure 4.14 shows the number of bytes per second when there are four cameras (in the $4^{\text {th }}$ situation, but the measurements of all cameras). We can observe that there is not more than 700.000 bytes per second (5.6 Mbps) in the wireless network. Measurements show us that the implemented system can support video streaming from four wireless IP cameras without problems.

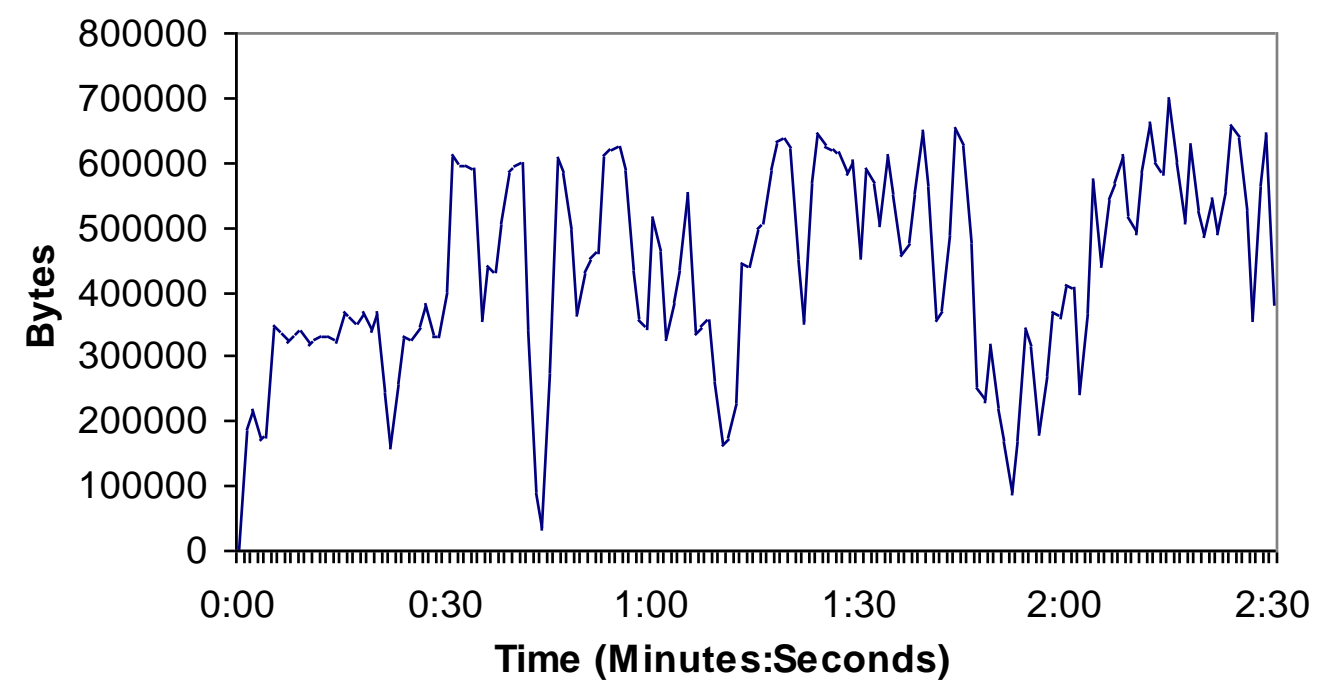

Figure 4.14. Bytes per second when there are four cameras.

Figure 4.15 shows the number of packets per second in the $4^{\text {th }}$ situation, but showing the measurements of all cameras. We can observe that it is not more than 1000 packets per second in the wireless network, so there are not so many messages through the medium at the same time. As the number of packets sent through the network is proportional to the number of cameras in the wireless network, 34 cameras will send less than 34,000 packets per second through the network.

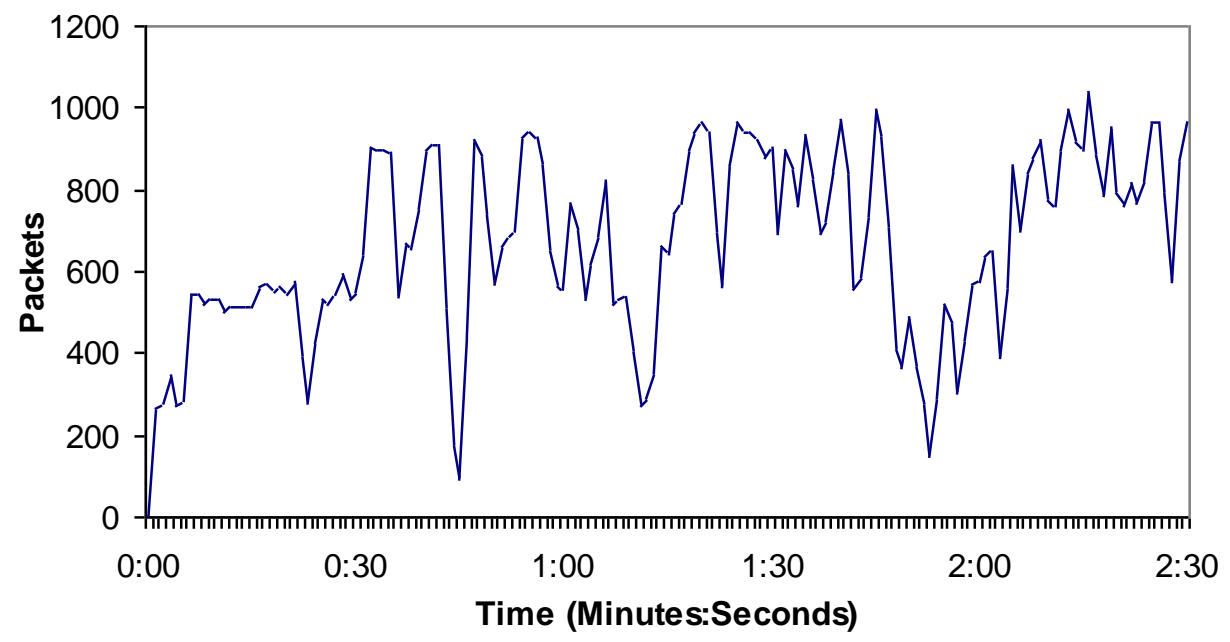

Figure 4.1.5 Packets per second when there are four cameras. 


\section{IP Camera Bandwidth Consumption Performance and Stability}

We have measured all four wireless IP cameras transmitting MPEG-4 codec compressed video over HTTP protocol in order to test the number of bytes per second and the number of messages per second through the network during 15 minutes in order to see the performance of the network and its stability. The video resolution was $320 \times 240$ at $25 \mathrm{fps}$. The wireless IP camera also transmitted audio at $24 \mathrm{Kbps}$ from the camera to the web page.

Figure 4.16 shows the number of bytes per second. There are not more than 800,000 bytes in the wireless network and the mean value was 514,013 bytes. The measurements show that the implemented system can support video streaming from all four Wireless IP cameras without problems because there is not so much bandwidth consumed and the system is stable.

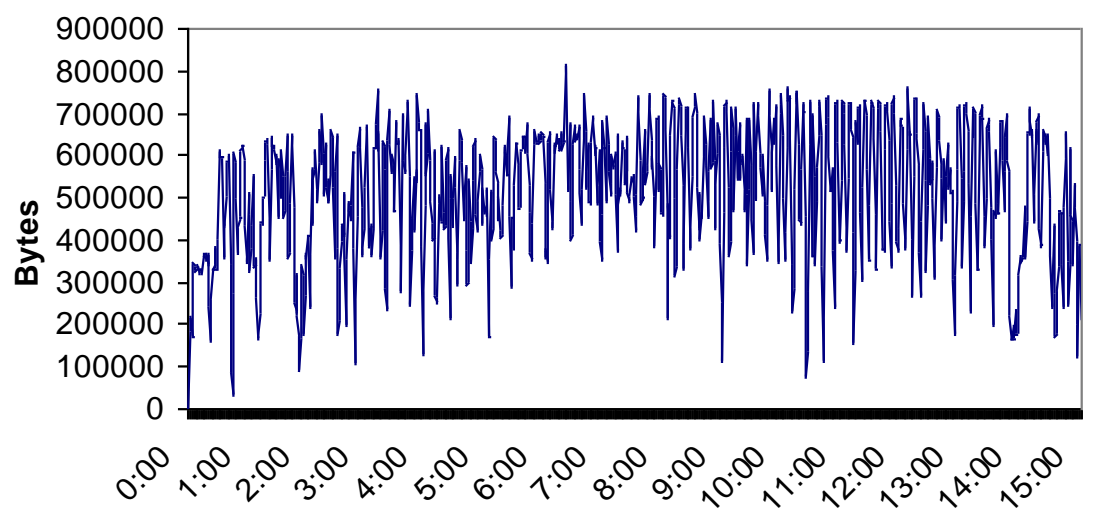

Time (Minutes:Seconds)

Figure 4.16. Number of bytes per second.

Figure 4.17 shows the number of packets per second in this case. No packet peak reaches 1,200 packets in the wireless network and the mean value was 798 packets. There are not too many messages through the medium at the same time. As the number of packets sent through the network is proportional to the number of cameras in the wireless network, 34 cameras will send around 40,800 packets per second through the network in this case. On the other hand, we could add one more access point. Taking into account the area covered by the cameras (few cameras are needed to cover large distances) and the number of sensors per each access point (see the research study made in Figure 4.4), the designed system is scalable with the only limitation of the bandwidth of the backbone technology used to join the access points.

\section{Multisensor Bandwidth Consumption}

Figure 4.18 shows the number of bytes per second when a multisensor sends an alarm to the server. Peaks value is 1850 bytes, and it is due to the network traffic. The alarm was sent between the 60 and the 70 seconds, so it consumes very low bandwidth. There is an average value of 100.35 bytes per second because of the multisensors.

Figure 4.19 shows the number of packets per second when there is an alarm. It shows that the maximum number of packets per second in the network is seven. We have shown that an alarm 
makes peaks of 3 packets as is shown between the 60th and the 70th second. There is an average value of 0.68 packets per second.

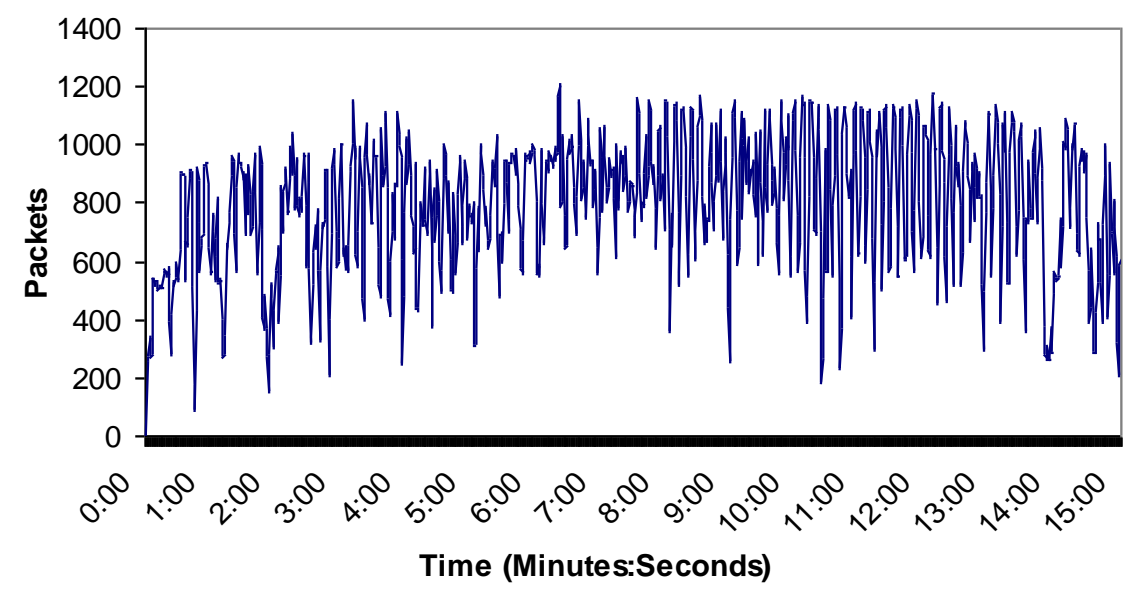

Figure 4.17. Number of packets per second.

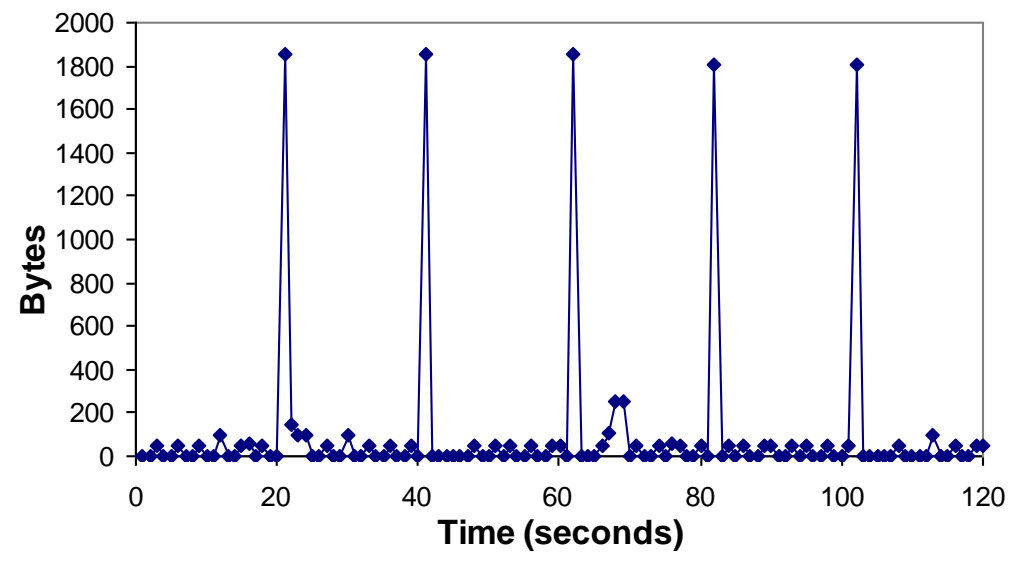

Figure 4.18. Bytes per second when there is an alarm.

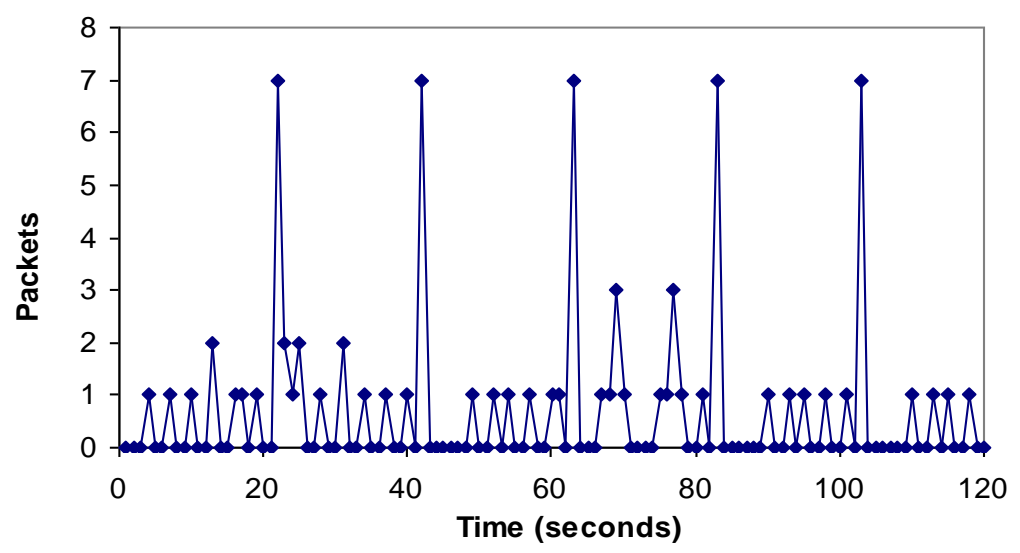

Figure 4.19. Packets per second when there is an alarm. 


\section{Power Consumption}

Although we have provided every device with batteries and solar panels that allow charging the batteries when the sun is shining, we have studied the energy consumption of every device. In this section we provide the power consumption for all devices used in our deployment.

Table 4.1 shows the power consumption for each device depending on its state. The device that consumes more power is the Cisco Aironet 350 Bridge and the one that consumes less power (but if it is not moving) is the Wireless IP Camera (D-Link DCS-5220). On the other hand, we can observe that the wireless IP Camera does not have "Idle mode", so it will be sending images all the time. So it has higher power consumption.

Table 4.1. Devices consumption.

\begin{tabular}{|c|c|c|c|}
\hline Device & Idle Mode & Wireless ON & Transmitting or Receiving \\
\hline $\begin{array}{l}\text { Linksys } \\
\text { WRT54GL v1.1 }\end{array}$ & $2,400 \mathrm{~mW}$ & $3,240 \mathrm{~mW}$ & $3,360 \mathrm{~mW}$ \\
\hline $\begin{array}{l}\text { Cisco Aironet } \\
\text { 350 Bridge }\end{array}$ & $4,320 \mathrm{~mW}$ & $5,760 \mathrm{~mW}$ & $6,240 \mathrm{~mW}$ \\
\hline $\begin{array}{l}\text { D-Link } \\
\text { DCS-5220 }\end{array}$ & - & $\begin{array}{l}\text { 2,640 mW (video: } 176 \times 144) \\
\text { 2,760 mW (video: } 320 \times 240 \text { ) } \\
\text { 3,000 mW (video: } 640 \times 480 \text { ) }\end{array}$ & $\begin{array}{l}\text { 2,640 mW (video: } 176 \times 144 \text { ) } \\
\text { 2,760 mW (video: 320x240) } \\
\text { 3,000 mW (video: 640x480) } \\
\text { 5,280 mW (moving the camera) }\end{array}$ \\
\hline
\end{tabular}

In order to show the power consumption of the devices shown in Table 4.1 over time, we consider that the circuit used to regulate the battery needed for all the devices consumes the same energy and it is quite low compared with the energy consumed by the devices. The energy consumed by the sensor is also negligible compared with the energy wasted by the chips. Taking into account measurements taken in [282], we suppose that a wireless link spends 70 percent of the time idle (in standby mode), 20 percent of the time receiving, and 10 percent of the time transmitting. Figure 4.20 shows the power consumption over time by the devices shown in Table 3.1. The device that consumes more power in our system is the Cisco Aironet (C) 350 Bridge, which is used as Non-root Access Points and as root Access Points. The worst case for the IP Camera is when it is always moving. It does not happen too much. The best cases are when the multisensor and the IP Camera transmit with a video quality of $176 \times 144$ at $25 \mathrm{fps}$.

In order to simulate the total amount of time that a multisensor will be able to be transmitting and receiving with a totally charged battery, we take into account the considerations and values provided in [231] by Heinzelman et al. The transmission and the reception energy are given by the expressions 4.4 and 4.5 :

$$
\begin{gathered}
E_{T x}(k, r)=\left(E_{T x}+E_{a m p} r^{2}\right) \cdot k \\
E_{R x}(k)=E_{R x} \cdot k
\end{gathered}
$$

where $k$ is the number of bits sent in the packet, $E_{T x}=E_{R x}=50 \mathrm{~nJ} / \mathrm{bit}, E_{a m p}=100 \mathrm{pJ} / \mathrm{b} / \mathrm{m}^{2}$. 


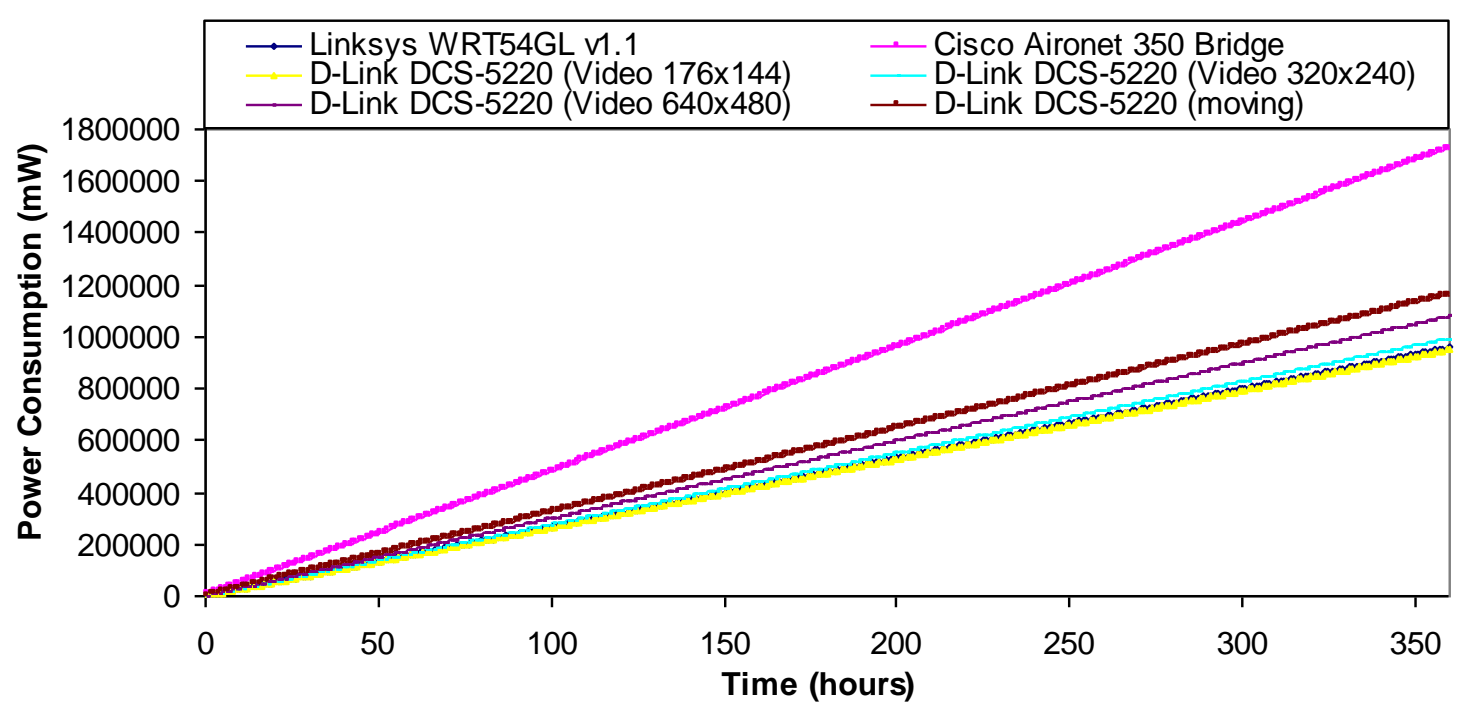

Figure 4.20. Power consumption.

Taking into account the measurements taken in Subsection 2.1.5.3., the multisensors send an average value of 1,175 bits per packet. Now, we can estimate the power consumption because of the packets transmitted and received by all multisensors over time. The multisensor is supplied by a $12 \mathrm{~V}$ - 24Ah battery with a solar panel, but we switch off the solar panel (no sun shinning for many days) in order to know its lifetime. We have considered the circuit energy consumption, the data processing energy consumption, the energy, when it is reading and writing in the memory, and the energy consumed by the physical sensor as being close to zero because the energy consumed is very little compared with that consumed by the nodes due to all the packets sent and received. Figure 4.21 shows the energy consumption of a multisensor in the worst case (always transmitting or receiving without any time being in the idle mode). The multisensor will be able to be in this hard mode for more than 16 hours. Then, the battery has to be charged using the solar panel

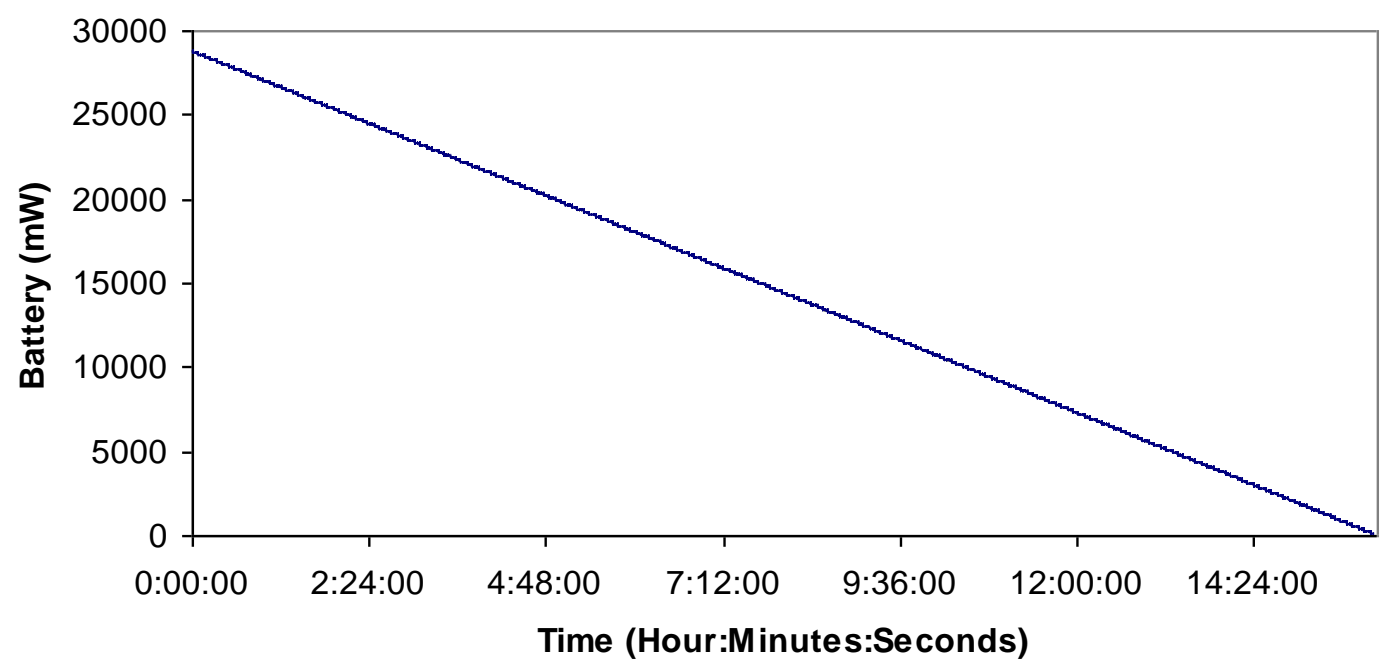

Figure 4.21. Power consumption in the network because of the packets transmitted and received. 


\subsection{A Wireless Sensor Network for Vineyard Monitoring}

The vine is one of the oldest crops. It is believed that the Vitis vinifera cultivation has its beginnings in an area between the Black Sea and Caspian Sea. The first evidence of vine cultivation in Spain dates from $520 \mathrm{BC}$ and the grape growing and winemaking had its spread throughout the peninsula during Roman's time.

Spain is the first country in the world in land devoted to wine production, featuring a total of approximately 1.1 million hectares. It is followed by other countries like France and Italy, according to the balance on the global wine situation made by the International Organization of Vine and Wine (OIV) in 2009 [283]. When we talk about wine production, Spain generates approximately $13.1 \%$ of total world wine. With these indicators, it is not difficult to conclude that this type of cultivation is very important for some of the European countries and they need to keep it in good conditions in order to ensure, in each harvest, the grapes and wine in good performance and quality [284].

There are some European regulations that cite the economic relevance of diseases in grapevine, regarding some pathogens. For example, the directive 68/193/CEE of the European council [285] cites some virus disease: Grapevine Fanleaf Virus (GFLV), Arabis mosaic virus (ArMV), Grapevine leafroll-associated virus 1 (GLRV-1), Grapevine leafrollassociated virus 3 (GLRV-3) and Grapevine fleck virus $(\mathrm{GFkV})$. This text has been revised several times. The last one is the directive of the European Parliament and of the Council COM (2010)359 [286]. There are very few regulation and directives about vine deficiencies. The oldest law we have found is from 1901 [287]. It is an Act to consolidate the Acts relating to a certain vine disease and to vegetation diseases. New Zealand Legislation has some laws about pest, deficiencies, and diseases such as the one presented in [288] about Bisosecurity that includes grape vine.

Nonetheless, it is known that some diseases, such as diamine putrescine [289] or Leafroll [290], can cause visible damage to the grape (hindering their sales and production), as well as to the vine. They can cause the reduction of grape production, with consequent economic losses. But this looses could be caused by other reasons. An example of a non-parasitic apoplexy was recorded in Lanzarote during the harvest of 1994, where economic losses became up to $60 \%$ in some regions [291]. It is important to remember that because the plants do not assimilate the nutrient treatments immediately, they can also have losses on the following restoration years of the vineyards after the infection. This phenomenon occurs because most diseases and deficiencies often affect the most young branches and shoots, which hinders the proper growth of the plant [292].

Generally, a deficiency or excess of some nutrient, the presence of a virus, pest or a parasite on a vine, produces a physical change on the plant, creating anomalous pigmentation especially in the leaves. For this reason, it is quite important the foliar analysis [293].

We can distinguish different types of harmful agents in the vine. These are the following ones [294]:

- Physiological or disorders in vine that are not caused by pests and diseases: lack or excess irrigation, extreme conditions due to meteorological factors, natural aging, deficiencies or excesses of nutrients in the vine, bad soil, irrigation with saline water, damage to roots by tilling, treatment plant performed poorly, improper pruning. 
- Fungus: Most of them attack leafs, grapes and stems. They can even grow well in both wet and dry regions. Some well known funguses are powdery mildew, downy mildew, and bunch rot.

- Vine pests and insects: The problem can be evaluated as it develops. Insects feed on buds, leaves, and grapes. The most damaging are those that feed on grapes because resulting rot can spread throughout the entire cluster. Some of they are next ones: tetranychus urticae, meloidogyne, Heterodera, Ditylenchus, lobesia botrana, colomerus viti, eriophyes vitis, phylloxera vastatrix, haltica ampelphaga, byctiscus betulae, sparganothis pilleriana, among others.

- Vine diseases generated by viruses and bacteria: Virus and virus-like diseases and other infectious diseases of grapevines are induced by intracellular pathogens of various nature. For example grapevine degeneration, grapevine decline, leafroll complex, rugose wood complex, yellow mottle, line pattern, Yellow dwarf, stun, Ajinashika, Fleck, Roditis leaf discoloration, Yellow speckle, among others.

There are several ways and methods to diagnose and find out what harmful agent or nutritional deficiency has the vine. These are the following ones [295]:

- Visual inspection of the crop to check for deficiencies signs: Only critical deficiencies are noted, but the damage symptoms observed sometimes can be unreliable.

- Soil analysis: Qualify the soil nutrient levels and other characteristics.

- Analysis of the plant tissue: measurements of nutrient levels in plant tissues. It can detect weaknesses that could not be found in the soil test.

- Bioassays: These are methods to diagnose nutrient deficiencies which combine the techniques of tissue analysis and testing in pots.

- Field Test: This test is the oldest and most reliable method to diagnose nutrient deficiencies, but it is an expensive procedure.

These methods are used for a first step in the vine exploration, because the field tests are quite expensive and difficult to manage. Besides, usually, it is not possible to perform it on the plant, only in a laboratory.

Over the years, the society has included technological advances in agriculture. They may be classified according to their nature. We find biochemical products, such as pesticides and fertilizers, farm machinery, such as tractors or irrigation timed systems, and the ground nutrients, among others. From the mechanical and the information technology application perspective, the technological improvements should not disturb the environment. Some examples of information technology applied to the agriculture, rural, and forest areas are applications based on the detection of natural disasters, monitoring and control of agriculture, ecosystems and geophysical measurement systems, flood detection, precision agriculture, biological complexity of environmental mapping, and detection of forest fires [171] [173]. Moreover, it is easy to find some deployed sensor systems for monitoring all meteorological parameters of the environment such as temperature, humidity, wind, etc. [296] or distributed monitoring systems based on Wireless Sensor Network (WSN) technology [297]. This technology cannot be used to obtain a new product for the agriculture; but to improve existing techniques and working conditions of the people in charge of the fields, and to improve the diseases diagnosis in order to ensure the final product quality. 
Frequently, these technological advances require a high initial financial contribution, but over the years, this is outweighed by the benefits obtained from the field [298].

In this chapter, we propose a WSN that uses an image processing system in each wireless node in order to distinguish if a vine has bad leaves or not. This bad leaves could be caused by a deficiency, pest, disease or other harmful agent. When the wireless sensor detects any unusual status in the vine leaves, it generates an alarm that will be routed to the sink node in order to warn the farmer. The benefits of this deployment are huge and the farmer will recoup the investments of this implementation very fast (the time will depend on where the vineyard is planted, the probability of having deficiencies, pests or diseases, and depending, on how far is living the farmer and on how many time he/she usually spends observing the vineyard looking for any deficiency, pest or disease). The main contributions of this paper are the following:

- We provide a detailed design of the WSN where we discuss the radio coverage distance and the field of view of the cameras used to detect the deficiency, pest, disease or other harmful agents.

- We also present an innovative way to estimate the number of nodes needed to sense an area.

- While other works show the design of new sensor devices, we use a cost effective sensor which is based on existing IP routers that can be found in the market, and we adapt them to be used for our purpose.

- The image processing technique explained in this paper let us distinguish between the land and the good leaves from the bad leaves in order to detect any symptom caused by a deficiency, pest, disease or other harmful agent.

\subsubsection{Wireless Sensor Network and Wireless Sensor Node Deployment}

The proposed wireless sensor network is based on a set of nodes capable of capturing images, process them internally in each node and take an output response based on its decision (if it has found a bad leaf in the field or not). This design is a distributed system where each node takes its own decisions, thus avoiding sending too many messages through the network to a central server in order to take those decisions. Based on our previous studies, we know that each camera, when using a MPEG-4 codec compressed video over HTTP protocol, will require a traffic flow bandwidth of $800 \mathrm{Kbps}$ for a video resolution of $320 \times 240$ at $25 \mathrm{fps}$. It implies a maximum of 30 cameras in IEEE 802.11g network [299], which is not scalable and useful for large fields. This pushed us to make a distributed design process when image processing is needed in the WSN, thus avoiding the limitation of the number of cameras because of the bandwidth requirements. So, on a vineyard, we deployed a WSN where wireless sensor nodes should be strategically placed in order to maximize the sensed area to be covered by the nodes while minimizing the number of nodes to be placed in the field (that is maximizing the wireless radio coverage). Figure 4.22 shows the designed WSN topology. In this work we will make our design and deployment over planar field or over smooth hills (which usually happens in almost all vineyards in Spain), but in case of uneven fields, sensor nodes should be placed at the top of the hills or the mountains. 


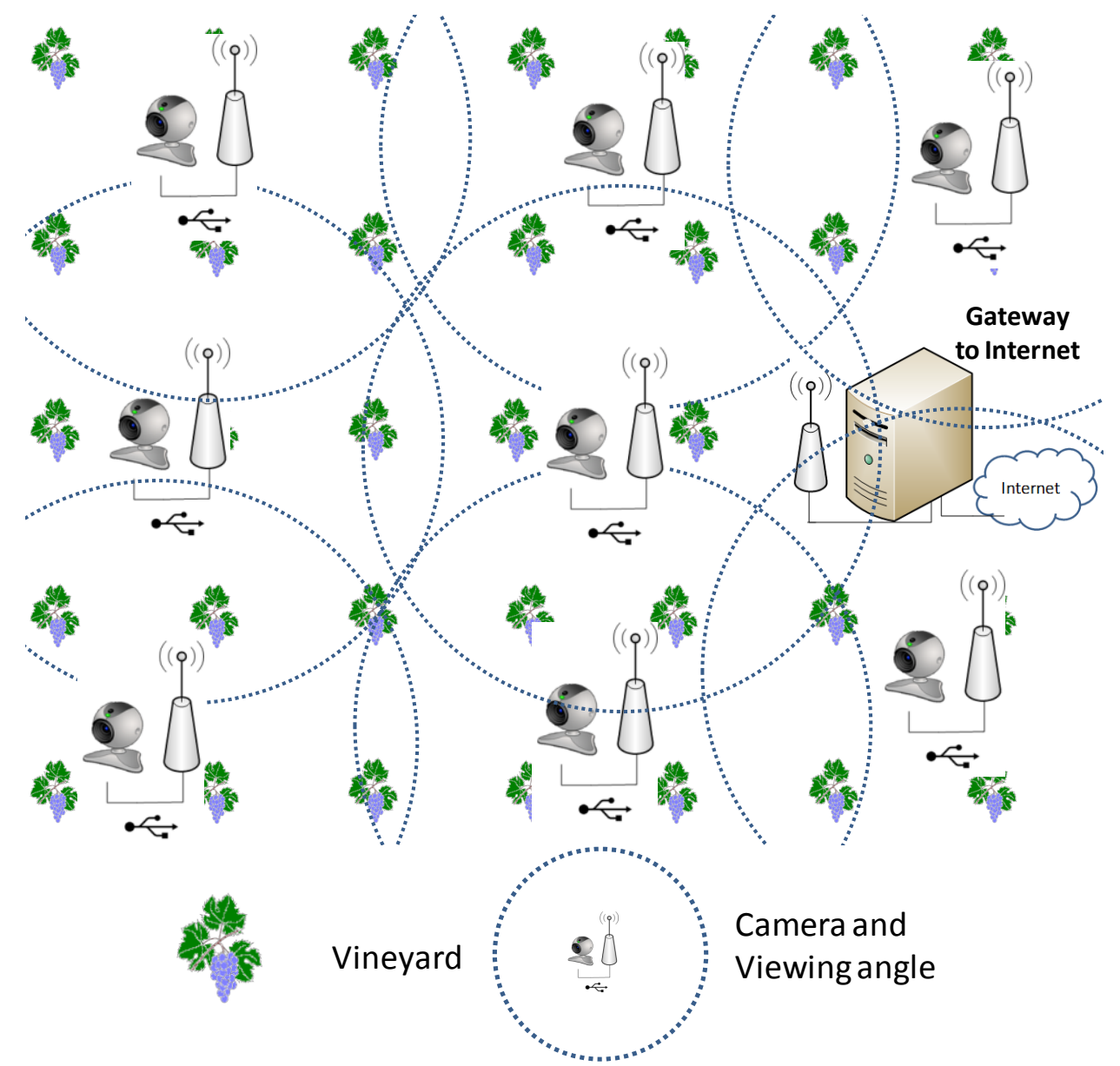

Figure 4.22. Vineyard monitored by cameras.

The fact of using IEEE 802.11 technology imply to have more power consumption than when using other technologies, such as Bluetooth or ZigBee. But we used it in order to cover larger areas because the sensors could be quite far. It let us have lower deployment cost because less devices have to be deployed. However, we should take into account the energy consumption, so we took into account the recommendations published by the some of the authors of this paper for WLANs in [168]. In order to select the best routing WSN protocol for our purposes, we took into account the work presented by some of the authors of this paper in [300]. Thus, we selected Optimized Link State Routing (OLSR) protocol as the routing protocol because it is the one that has the best performance for our case.

In our design we use a single sink node which is placed in the vineyard house.

Although our initial design is based on the aforementioned statements, we can make use of group-based protocols in order to cover bigger areas [3].

\subsubsection{Analytical Network Model for Node Placement}

One of the first issues to take into account is the placement of the wireless sensor node. The position of each sensor is determined by two different coverage areas. The fist one is the radio coverage area, which allows the sensors communicate. The second one is the sensing coverage area. 
Usually, the radio coverage area of a sensor node is quite different from the sensing area. Moreover, generally, there is not any relationship between them. Both types of coverage areas could be affected by the field where the WSN is deployed, but it could be affected just one of them. The factors that affect the sensing area are different from the factors that affect the radio coverage area. The goal is to have the physical space within the sensing range of at least one wireless sensor. The number of wireless sensors deployed is usually higher than optimum required for the physical space due to the lack of precise sensor placement.

In a rural and agricultural zone, the radio coverage area for a wireless sensor mainly depends on the type of antenna, and its gain, used by the wireless sensor, its high respect to the top of the vineyard plants and the humidity of the place. In our model we take placement of the wireless sensor at the top of a post of 6 meters high (vineyard plants do not use to have more than 2-2.5 meters high), so we will assume that the vineyard plants do not affect to the radio coverage because of the difference in height. In order to estimate the radio coverage we use the power balance formula (given by equation 4.6). This equation states that the received signal power, in $\mathrm{dBm}$, is equal to the transmitted power plus the transmitter and receiver gain, minus the basic loss and minus other losses produced by other issues (such as vegetation or humidity), but in this case we will only take into account the humidity. The value of humidity losses can be obtained from reference [301].

$$
P_{r x}(d B m)=P_{t x}(d B m)+G_{t x}(d B)+G_{r x}(d B)-10 \cdot n \cdot \log d-L_{\text {humidity }}(d B)
$$

Here $n$ is the attenuation variation index. $n=2$ for air medium and $d$ is the distance between the transmitter and the receiver. So, solving equation 1 in $d$, we obtain the coverage distance in equation 4.7.

$$
d=10^{\frac{P_{t x}+G_{t x}+G_{r x}-L_{\text {humidity }}-P_{r x}}{20}}
$$

In order to estimate the distance between devices we fix some parameters. The theoretical transmitted power is $-40.2 \mathrm{dBm}$ for an IEEE 802.11g WLAN device at 1 meter $\left(P_{t x}\right)$ and at $2.44 \mathrm{Ghz}$ (mean value in the $2.4 \mathrm{GHz}$ WLAN range). We fixed to $-80 \mathrm{dBm}$ the threshold power in order to have enough quality of signal $\left(P_{r x}\right)$. So, the received power must be greater than or equal to this mark, otherwise it is not in the radio coverage area. We will consider omnidirectional antennas for both the transmitter and the receiver, so we will call Gain. Moreover, this study has been done in Spain, which has two main hydrometric areas: the $\mathrm{H}$ area and the $\mathrm{K}$ area [302], so losses due to rain, in the worst case, have a value of $0,026 \mathrm{~dB}$ for two kilometers. Equation 4.8 shows the formula needed to design the radio coverage of each wireless sensor.

$$
d=10^{\frac{39.77+2 \cdot \text { Gain }}{20}}
$$

Figure 4.23 shows the radio coverage for several typical antenna gains of $2.4 \mathrm{GHz}$. 


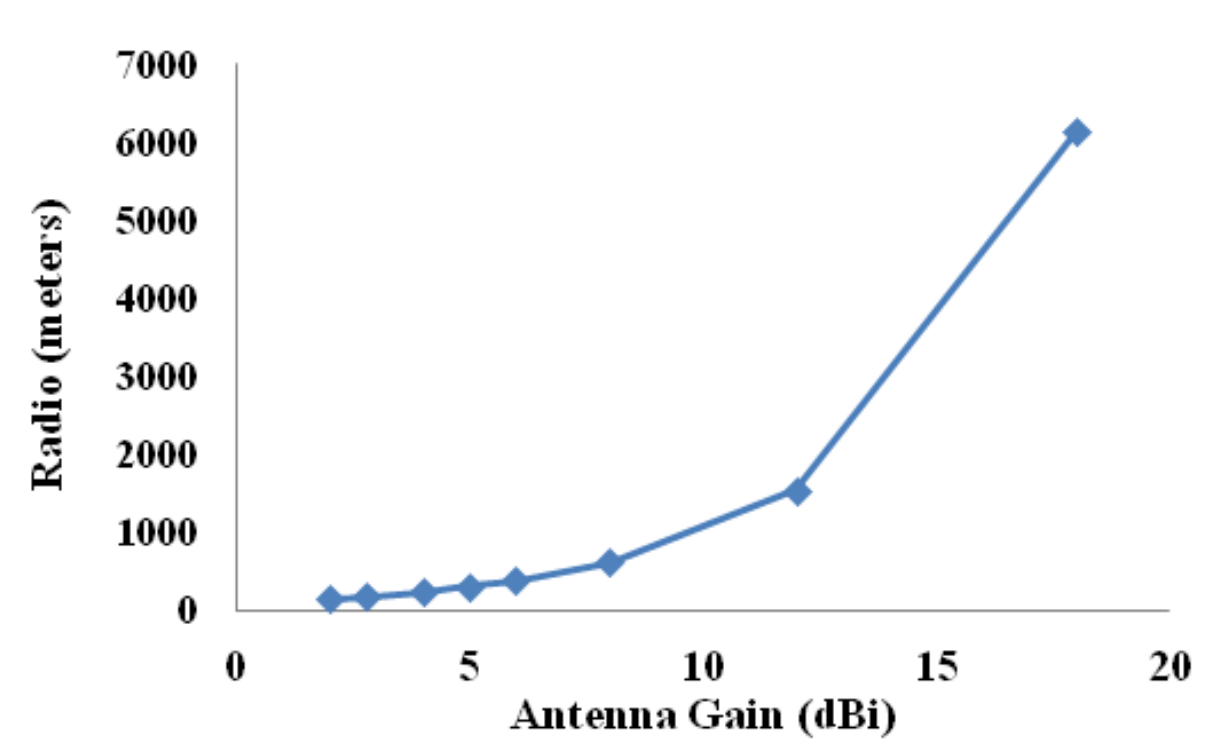

Figure 4.23. Radio coverage for several typical antenna gains.

The sensing coverage area of a node is also called sensing field (or region) of the node. The characteristics of sensing equipment are completely different and rely on the application requirements. The sensing region of an isotropic sensor in the ideal environment is circular (or elliptical). But a directional sensor has a finite angle of view and thus cannot sense the whole circular area, so its sensing region would be a sector in a two-dimensional plane or a 3D zone.

The most commonly used model for sensing coverage is the disk model, which assumes that the sensing region for a sensor is a circular region centered on it [303]. Any point placed in the sensing radius of the sensor could be sensed. So, any stem, grapes or leaves will be covered if is closer than the maximal sensing distance $\left(d_{\max }\right)$. When the camera only covers a region (not the whole circle), the target must be located inside the angle of the rotatory movement of the camera. Based on the perspective projection model of a camera sensor, we propose a partial circle model. The area inside a partial circle bounded by a radius $r$ and an arc $\alpha$ is provided by Equation4. 9 .

$$
\text { Area }=\frac{1}{2} d_{\max }^{2} \cdot\left(\pi \cdot \frac{\alpha}{180}-\sin \alpha\right)
$$

Here $r$ is the circle radius and $\alpha$ is the angle (in degrees). We also define a minimal sensing distance because the focal length of the camera could be very small, so $d_{\min }$ will be the minimal distance of effective imaging. Figure 4.24 shows the partial circle area covered by a wireless sensor camera. $r$ is the distance between the camera and the target.

The field of view (FoV) of a camera (if the image system is rectilinear) depends on the collimator focal length and the target size (assuming the sensed image includes the whole target). It can be estimated by Equation 4.10

$$
F o V=2 \cdot \frac{D}{d} \arctan \left(\frac{d}{2 \cdot f_{c}}\right)
$$

Here $D$ is the dimension of the full image, $d$ is the dimension of the target and $f_{c}$ is the focal length of collimator. 


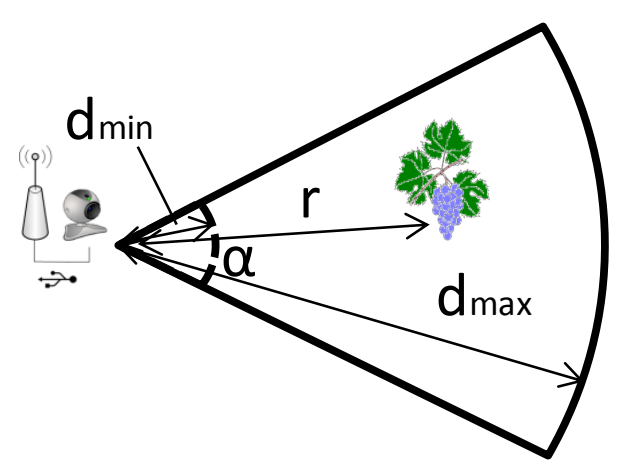

Figure 4.24. Sensing coverage of a wireless sensor camera.

Let us assume that all nodes have same radio coverage radio (thus the same radio coverage area) and the same sensing area, but the radio coverage area and the sensing areas are different. Moreover, we assume that all nodes have the same computation capabilities.

Let a network of wireless sensor nodes be $G=(V, E)$, here $V$ is the set of wireless sensors and $E$ is the set of connections between them. Let us suppose $n=|V|$ (the number of sensors of $V$ ) to cover a field, which we refer to as the sensing field. The sensing coverage area of a wireless sensor is denoted by $S$ while the radio coverage area is denoted by $C$. The sensing area is different to the radio coverage area $(S \neq C)$. For simplicity, we assume a sensing area of a node $i$ being a disk centered at sensor $i$ with radius $s$. Likewise, a sensor $i$ covers a target $q$ if and only if target $q$ is in the sensing disk of $i$. That is, if $d_{\min } \leq d(L c, L q) \leq d_{\max }$, here $d(L c, L t)$ is the Euclidean distance between $L c$ (camera location) and $L q$ (target location), $d_{\max }$ the maximal sensing distance and $d_{\min }$ the minimal sensing distance. It is desired a non-overlapping sensing areas in order to maximize the performance of the network. Two sensors can communicate directly with each other if and only if each sensor is inside the radio coverage area of the other. That is, if $d\left(L c_{1}, L c_{2}\right) \leq C$, here $d\left(L c_{1}\right.$, $L c_{2}$ ) is the Euclidean distance between the camera 1 and the camera 2 and $C$ is the radio coverage radius of the wireless sensors (we have assumed that all wireless sensors have the same radio coverage radio). A node that can directly send messages to its neighbors, we call it 1-hop communication.

The position of the wireless sensors on the network coverage area (also called as Region Of Interest, ROI), should be those ones which coverage percentage is maximized and coverage holes is minimized. There are three main strategies:

- Visual forced algorithm [304]: the sensors and the objects to be sensed in the ROI make the placement of sensors away from the objects and also from each other, so that their sensing areas are not overlapping and a full coverage is achieved.

- Grid based sensor networks divide the ROI into square grids and the sensors are placed at the center of each square [305]. Generally, the bigger the grid size with respect to the sensing range the higher accuracy sensing the ROI or the higher probability to object tracking.

- Computational geometry approach is frequently used in WSN coverage optimization. The most commonly used computational ones are Voronoi diagram and Delaunay triangulation [306]. 
In our case, the vineyards are usually placed in a planar field where there not use to be high drops. Moreover, both the radio coverage area and the sensing area are circular areas. So the easiest deployment system is the grid-based method applied to the sensing coverage.

The sensing region is usually divided into two sub-regions: the uncovered region (which points cannot be sensed) and the covered region (which points can be sensed). In our deployment we try to have an uncovered region as close to zero as possible.

In order to deploy the wireless sensor cameras we will assume that the radio coverage radius is equal or higher than two times the sensing coverage radius (that is, using previous nomenclature, $c$ $\geq 2 \cdot s$ ). This will allow us to have full coverage of the ROI while maintaining radio connectivity between wireless sensors. Figure 4.25 shows eight wireless sensor cameras (in red). The sensing area of each sensor is drawn by blue circles and the radio coverage area (two times the sensing coverage area) is drawn in yellow. This system is also called hexagonal grid. It allows covering all the sensing field while maintains each sensor connected with at least 2 or 3 neighbors. Bearing in mind that $\mathrm{c}$ is the radio coverage radius and $\mathrm{s}$ the sensing coverage radius, the distance between sensors to avoid holes without sensing field is $\sqrt{\mathbf{2}} \cdot \boldsymbol{s}$.

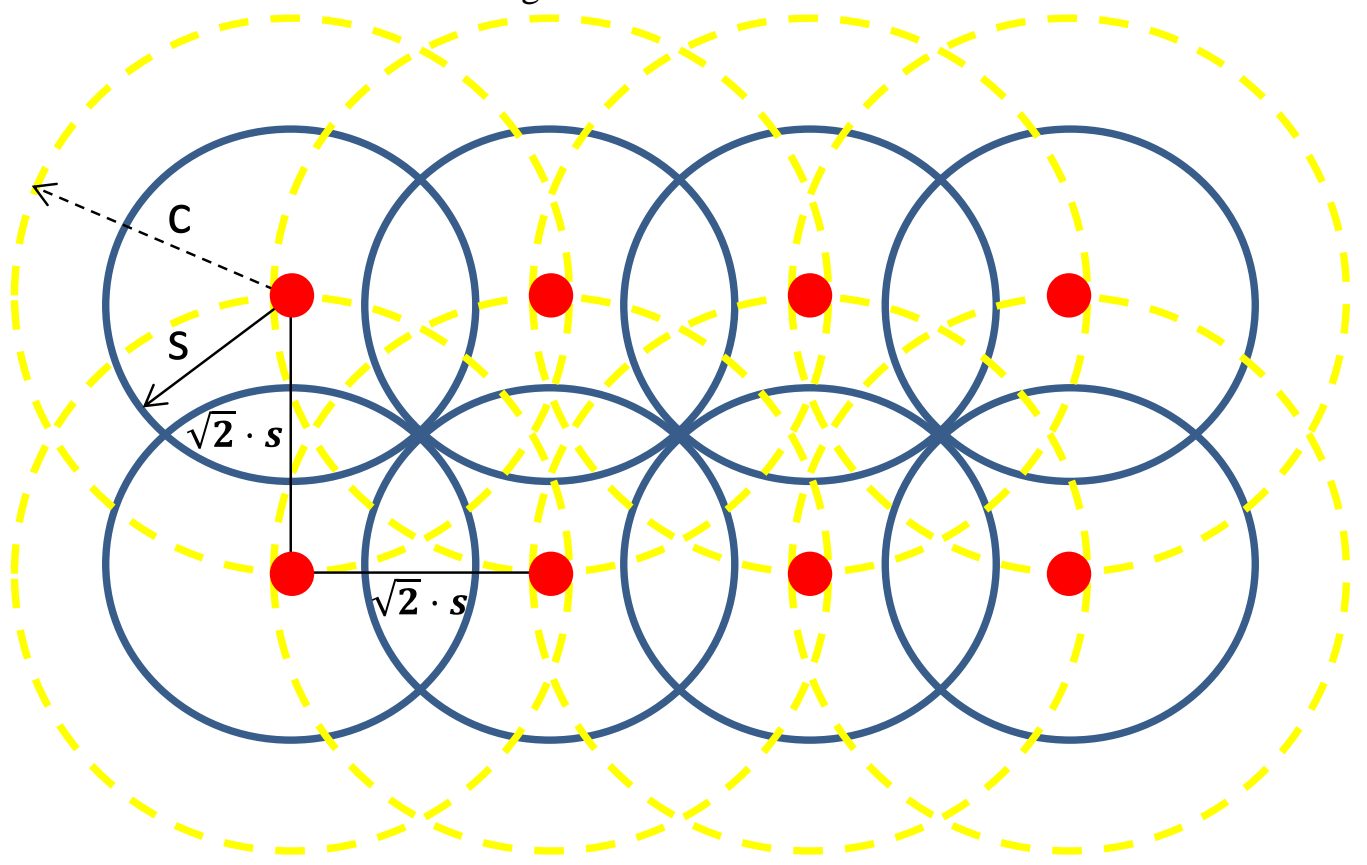

Figure 4.25. Sensing coverage of a wireless sensor camera.

Now, we can estimate the overlapping sensing area (see A in Figure 4.25) taking into account the circular segment equation [307]. This is given by Equation 4.11.

$$
A=0.864 \cdot s^{2}
$$

Hexagonal grid is the worst among all since it has the biggest overlapping area [308], but it is the one that covers the entire sensing field.

The area covered by all the WSN as a function of the number of nodes $n$ when $n \geq 2$ in the WSN is expressed by Equation 4.12.

$$
A=n \cdot \pi \cdot s^{2}-0.864 \cdot a_{n}
$$


Here $a_{n}=\sum_{i=1}^{n}\left(a_{n-1}+(-1)^{n}\right), \mathrm{n} \geq 2$ and $\mathrm{a}_{1}=1$.

\subsubsection{Wireless Sensor Node}

In order to design our sensor node, several factors must be considered. On the one hand, the node must be able to capture the image with enough quality and the image processing must be computed inside the node in order to detect any unusual status of the leaves that could be caused by a deficiency, pest, disease or other harmful agents. When the symptom is observed in a leaf, the node must send an alarm to the gateway node.

We decided to use any available access point, or wireless router, in the market in order to reduce costs of node deployment and take advantage of existing hardware. The main features of the needed hardware are that it must use IEEE $802.11 \mathrm{a} / \mathrm{b} / \mathrm{g} / \mathrm{n}$ technology and it must also have at least one USB port in order to add a camera. The device should have the best hardware features because it must be able to process images. Moreover, the selected device for image capture, such as a video camera or webcam, must have USB and must adapt its hardware characteristics to the hardware characteristics of the access point or wireless router. Finally, the camera should be able to rotate, with the aim of having a larger viewing area and therefore reduce the number of required sensor nodes.

In general, the original firmware of the access points and the wireless routers in the market does not allow some operations. Therefore, we should customize the device firmware in order to enable these additional features.

One of the most used custom firmware is OpenWRT [309]. It is a distribution based on GNU/Linux used for embedded devices such as routers and access points. It was driven initially by the GPL license and its intention was to allow manufacturers and users to modify and improve the code of your devices. OpenWRT provides a fully writable file system with package management. It is a very basic operating system that offers the possibility of complement it, with additional functionalities, using packages that allow users full customization ability to particularize the use of the device to the desired application.

There are many devices that can operate under the OpenWRT firmware. In order to design our system, we need a wireless device that has to be able to work on IEEE 802.11 standard, and must have at least one USB 2.0 port. The USB port will be needed to connect a camera and acquire high quality images from the vineyard. It is important to choose the best hardware features in order to facilitate the tasks of image processing. Table 4.2 shows only those devices that meet our requirements [310]. It displays information about the type of hardware of the devices, CPU Speed, RAM and flash memory, number of USB ports and the version, the type of wireless card used and the wireless technology used, among other features. 
Table 4.2. Available hardware comparison for sensor nodes.

\begin{tabular}{|c|c|c|c|c|c|c|c|c|c|c|c|}
\hline 苞 & 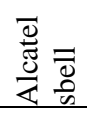 & $\frac{9}{8}$ & $\frac{0}{\underline{z}}$ & 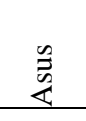 & $\frac{0}{\underline{z}}$ & $\frac{n}{3}$ & $\frac{0}{2}$ & 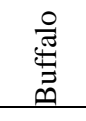 & 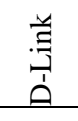 & 弟 & 䖟 \\
\hline ङ্ّ & 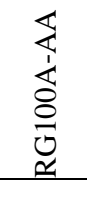 & 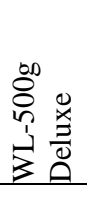 & 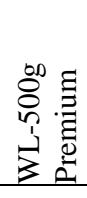 & 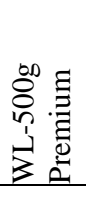 & 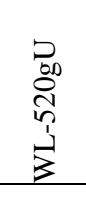 & $\begin{array}{l}n \\
\hat{⿵} \\
\hat{\hat{O}} \\
\dot{3} \\
\vdots\end{array}$ & $\begin{array}{l}80 \\
8 \\
0 \\
\dot{1} \\
3\end{array}$ & 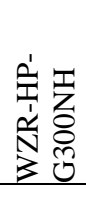 & 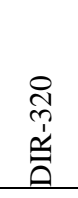 & 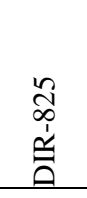 & 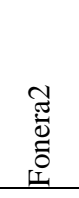 \\
\hline 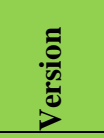 & 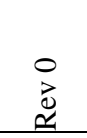 & & 5 & $s$ & $\therefore$. & a. & a. & 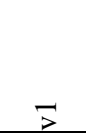 & $\overline{4}$ & $\begin{array}{l}\stackrel{\infty}{\infty} \\
\vec{\infty}\end{array}$ & 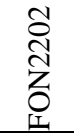 \\
\hline 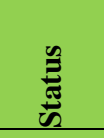 & 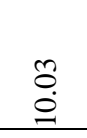 & $\begin{array}{l}\hat{a}_{\infty} \\
\infty\end{array}$ & $\begin{array}{l}{ }_{\infty} \\
\infty\end{array}$ & $\begin{array}{l}\sigma_{\infty} \\
\infty\end{array}$ & $\begin{array}{l}\underset{\infty}{\infty} \\
\infty\end{array}$ & $\stackrel{\varrho}{\stackrel{0}{\varrho}}$ & 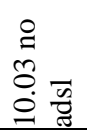 & 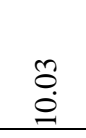 & $\stackrel{8}{\stackrel{\leftrightarrow}{\circ}}$ & 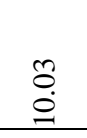 & $\stackrel{\leftrightarrow}{\stackrel{\leftrightarrow}{9}}$ \\
\hline 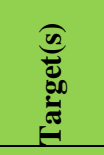 & 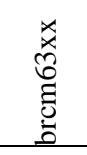 & 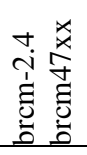 & 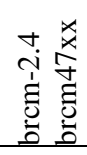 & 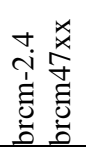 & 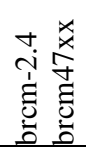 & 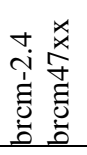 & 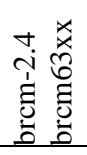 & 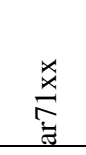 & 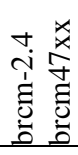 & 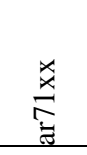 & 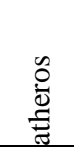 \\
\hline 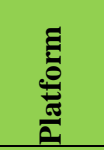 & 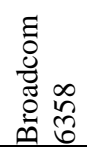 & 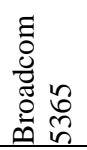 & 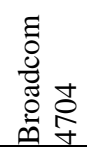 & 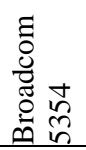 & 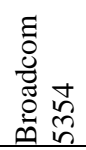 & 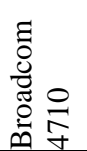 & 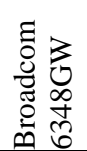 & 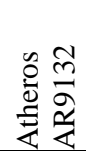 & 吾 & 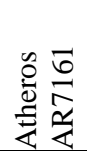 & 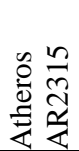 \\
\hline 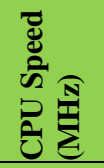 & 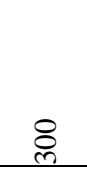 & ڤ్రి & 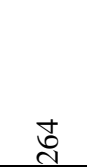 & g & $\overbrace{0}^{\circ}$ & さี & బొ & \& & g & $\stackrel{\infty}{\infty}$ & $\infty$ \\
\hline 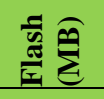 & $\stackrel{ }{2}$ & $\theta$ & $\infty$ & $\infty$ & $\theta$ & $\theta$ & $\theta$ & ले & $\theta$ & $\infty$ & $\infty$ \\
\hline 产金 & ले & ల్ల & है & స్ల & 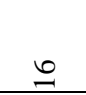 & & 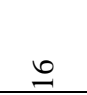 & t & $\approx$ & t & $\approx$ \\
\hline 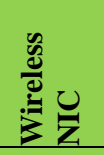 & 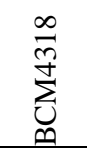 & $\begin{array}{l}0 \\
\sum_{0}^{+} \\
\sum_{\infty}^{+} n\end{array}$ & 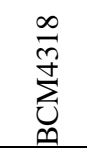 & $\begin{array}{l}\text { 吕 } \\
\sum_{0}^{n} \\
\sum_{\infty}^{n}\end{array}$ & 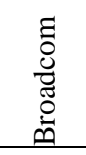 & $\begin{array}{l}\stackrel{8}{\circ} \\
\sum_{0}^{+} \\
\end{array}$ & $\sum_{\substack{f\\
}}^{\infty}$ & $\begin{array}{l}\frac{3}{2} \\
\frac{8}{4}\end{array}$ & 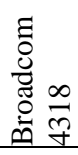 & 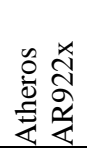 & 总 \\
\hline 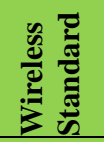 & 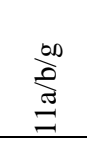 & $\stackrel{\text { bo }}{ٍ}$ & $\stackrel{\infty}{D_{0}}$ & $\stackrel{\text { bo }}{\varrho}$ & $\stackrel{\infty}{D_{0}^{\circ}}$ & $\stackrel{\text { bo }}{\varrho}$ & $\stackrel{\text { bo }}{\stackrel{\text { D }}{=}}$ & $\stackrel{\text { s. }}{\stackrel{\text { s. }}{ٍ}}$ & 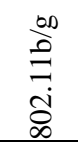 & 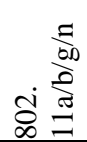 & $\stackrel{\text { bo }}{ٍ}$ \\
\hline 跤 & 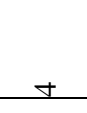 & & & & & & + & $\begin{array}{l}\frac{11}{b 0} \\
\frac{b 0}{60}\end{array}$ & $n$ & 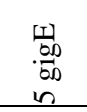 & \\
\hline 粕 & - & $\begin{array}{l}\stackrel{i}{\mathrm{i}} \\
\underset{i}{\mathrm{i}}\end{array}$ & $\begin{array}{l}\stackrel{0}{i} \\
\underset{d}{d} \\
\end{array}$ & $\begin{array}{l}\underset{j}{d} \\
\underset{j}{d}\end{array}$ & $\exists \underline{\bar{E}}$ & $\exists$ & $\begin{array}{l}\stackrel{0}{\mathrm{j}} \\
\underset{\mathrm{d}}{\mathrm{N}}\end{array}$ & $\stackrel{\infty}{\infty}$ & 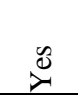 & ¿્ર & $\begin{array}{l}\underset{\mathrm{i}}{\check{x}} \\
\end{array}$ \\
\hline
\end{tabular}




\begin{tabular}{|c|c|c|c|c|c|c|c|c|c|c|c|c|}
\hline טֶ: & 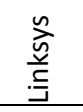 & 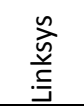 & 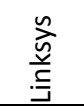 & $\begin{array}{l}\text { 忩 } \\
\text { 点 } \\
\end{array}$ & 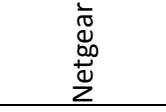 & 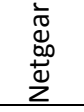 & 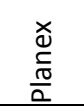 & 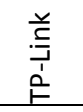 & $\begin{array}{l}\text {.丷 } \\
\stackrel{.}{I} \\
\stackrel{1}{1}\end{array}$ & $\begin{array}{l}\text { 兰 } \\
\stackrel{\vec{J}}{1} \\
\underline{\underline{1}}\end{array}$ & 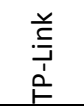 & 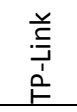 \\
\hline$\sum_{\bar{g}}^{\bar{g}}$ & 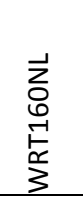 & 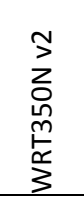 & 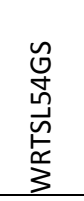 & 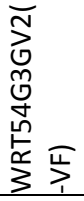 & 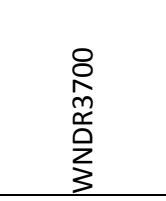 & $\begin{array}{l}5 \\
\stackrel{5}{0} \\
\infty \\
0 \\
0 \\
\square\end{array}$ & 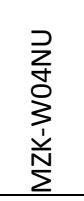 & 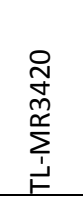 & 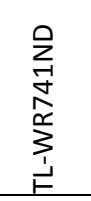 & 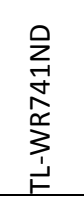 & 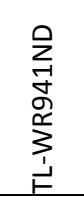 & 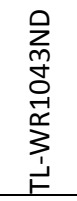 \\
\hline 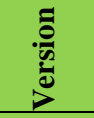 & -1 & $\begin{array}{l}\vec{i} \\
\dot{i} \\
\dot{\sim}\end{array}$ & 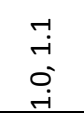 & -1 & c. & م. & م. & -1 & -1 & $\underset{+}{\rightarrow}$ & $\sim$ & $\begin{array}{l}0 \\
\stackrel{i}{I} \\
\stackrel{H}{1}\end{array}$ \\
\hline$\sum_{\tilde{E}}^{\mathscr{E}}$ & $\begin{array}{l}m \\
\text { O. } \\
\text { Oे }\end{array}$ & \begin{tabular}{l}
$m$ \\
0 \\
0 \\
\hdashline
\end{tabular} & g̊ & $\begin{array}{l}m \\
0 \\
0\end{array}$ & $\begin{array}{l}m \\
0 \\
0\end{array}$ & 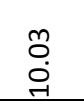 & $\begin{array}{l}m \\
0 \\
0\end{array}$ & 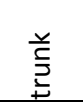 & $\begin{array}{l}m \\
0 \\
0 \\
\end{array}$ & 羙 & $\begin{array}{l}m \\
0 \\
0\end{array}$ & $\begin{array}{l}m \\
0 \\
0\end{array}$ \\
\hline 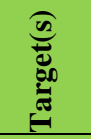 & 交 & 음 & 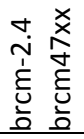 & 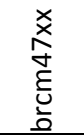 & 希 & 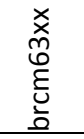 & 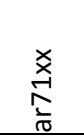 & 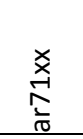 & 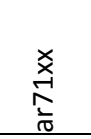 & 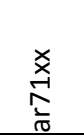 & 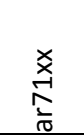 & 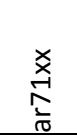 \\
\hline$\frac{\Xi}{\underline{0}}$ & 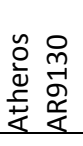 & 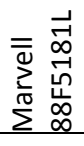 & 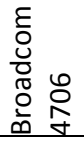 & 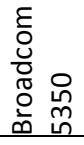 & 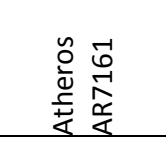 & 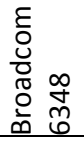 & 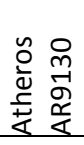 & 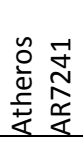 & 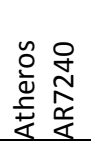 & 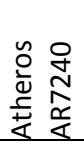 & 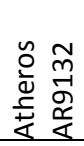 & 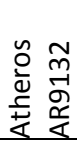 \\
\hline 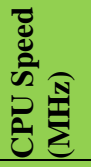 & ষ্ণ & ৪ & $\underset{\sim}{\mathbb{~}}$ & $\underset{N}{\mathbb{U}}$ & $\begin{array}{l}8 \\
0 \\
0\end{array}$ & $\stackrel{\text { ٌ }}{\sim}$ & প্ণ & প্ & ৪ & ষ্ণ & প & প্ \\
\hline 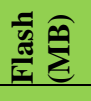 & $\infty$ & $\infty$ & $\infty$ & $\underset{-1}{0}$ & $\infty$ & $\theta$ & $\infty$ & $\theta$ & $\theta$ & $\theta$ & $\theta$ & $\infty$ \\
\hline$\sum_{\underline{\Sigma}} \hat{\theta}$ & $\tilde{m}$ & $\tilde{m}$ & $\tilde{m}$ & $\tilde{m}$ & ఫ & $\stackrel{0}{-1}$ & $\tilde{m}$ & $\tilde{m}$ & $\tilde{m}$ & $\tilde{m}$ & $\tilde{m}$ & $\tilde{m}$ \\
\hline 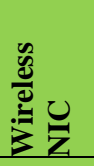 & 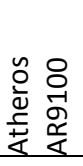 & 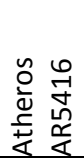 & $\begin{array}{l}\varepsilon \\
0 \\
0 \\
0 \\
0 \\
\frac{0}{0} \\
\end{array}$ & $\begin{array}{l}\varepsilon \\
0 \\
0 \\
0 \\
0 \\
\frac{0}{0}\end{array}$ & 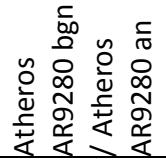 & 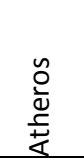 & 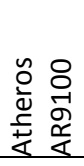 & 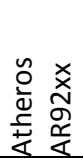 & 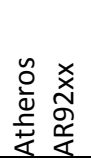 & 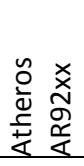 & 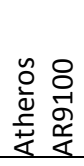 & 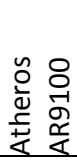 \\
\hline 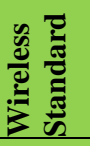 & 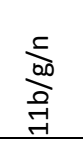 & $\begin{array}{l}\frac{5}{50} \\
\text { and } \\
\text { a } \\
\end{array}$ & $\begin{array}{l}\frac{10}{0} \\
\frac{a}{7} \\
-1 \\
ت\end{array}$ & $\stackrel{\infty}{a}$ & 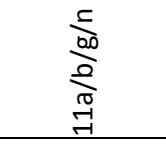 & $\begin{array}{l}\frac{\infty}{0} \\
\frac{a}{a} \\
\vec{y}\end{array}$ & $\begin{array}{l}\frac{F}{00} \\
\frac{0}{0} \\
\frac{1}{-1}\end{array}$ & 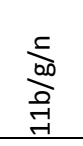 & $\begin{array}{l}\frac{F}{0} \\
\frac{0}{0} \\
\frac{7}{7}\end{array}$ & $\begin{array}{l}\frac{5}{00} \\
\frac{0}{\partial} \\
\frac{7}{7}\end{array}$ & $\begin{array}{l}\frac{c}{b 0} \\
\frac{0}{\partial} \\
\vec{y}\end{array}$ & $\begin{array}{l}\frac{5}{00} \\
\substack{0 \\
\frac{\partial}{7} \\
-1}\end{array}$ \\
\hline 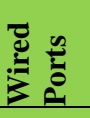 & Ln & ᄂn & Ln & n & 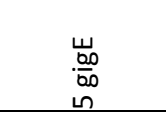 & $\begin{array}{l}\vec{U} \\
\stackrel{\alpha}{\alpha} \\
+ \\
+ \\
+\end{array}$ & ㄴ & n & n & in & n & 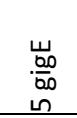 \\
\hline $\begin{array}{l}\text { है } \\
\text { อ }\end{array}$ & $\stackrel{y}{x}$ & $\sum_{x}^{\tilde{y}}$ & 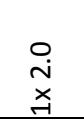 & $\begin{array}{l}\stackrel{0}{i} \\
\underset{m}{m} \\
\end{array}$ & 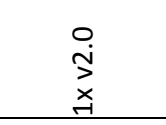 & $\begin{array}{l}-1 \\
\stackrel{-}{\lambda} \\
\underset{-}{x} \\
\end{array}$ & 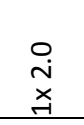 & 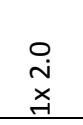 & 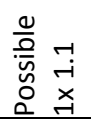 & 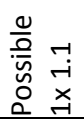 & 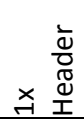 & 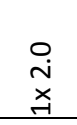 \\
\hline
\end{tabular}


We selected the routers with higher processing capacity because we need to process images. The models with higher speed processors are Netgear WNDR3700 and D-Link DIR-825. Both work with Atheros AR7161 processor, (32 bit MIPS 24K processor core) [311], with a CPU Speed of $680 \mathrm{MHz}$ and $64 \mathrm{MB}$ of RAM memory.

Moreover, we should keep in mind that our system will be mounted on a field, where it is difficult to get energy from the electrical network in many cases. In these situations, it is common to use batteries and electrical power generation systems from alternative sources. Therefore, another factor that was taken into account was the energy consumption of the device. Table 4.3 shows the energy consumption in active mode, idle mode and standby of some devices (we have included in this table only the ones of which some consumption information can be found in their technical specifications and the ones of which consumption information is published in a research paper). Active mode occurs when the device is transmitting or receiving and processing, Idle mode occurs when the device is neither transmitting nor receiving or processing, but it is able to receive and process, and Sleep mode (or Standby mode) occurs when it is able to process or receive, but not to transmit. We have written a dash "_." when the information was not found. Some values have been provided using the mean values of the measurements taken during a period of time.

Table 4.3. Energy consumption comparison.

\begin{tabular}{|c|c|c|c|c|}
\hline Brand & Model & $\begin{array}{c}\text { Active Mode } \\
\text { (Watts) }\end{array}$ & $\begin{array}{c}\text { Idle Mode } \\
\text { (Watts) }\end{array}$ & $\begin{array}{c}\text { Sleep mode or Standby } \\
\text { mode (Watts) }\end{array}$ \\
\hline Alcatel-sbell & RG100A-AA & 7 & 5 & 3 \\
\hline Asus & WL-HDD2.5 & 4,56 & 3,12 & - \\
\hline Buffalo & WZR-HP-G300NH & 5 & - & - \\
\hline D-Link & DIR-825 & 7 & - & 2,15 \\
\hline Fon & Fonera2 FON2202 & 4,8 & 4,15 & 2,57 \\
\hline Netgear & WNDR3700 & 6 & 4,77 & 4 \\
\hline Netgear & DG834GT & 5,19 & 4,92 & - \\
\hline TP-Link & TL-MR3420 & 8 & - & - \\
\hline TP-Link & TL-WR741ND & 4 & - & - \\
\hline TP-Link & TL-WR1043ND & 9 & 6,9 & - \\
\hline
\end{tabular}

Focusing only to the ones with higher processing capacity (which is the purpose of our research), Table 4.4 shows a comparison in terms of weight and energy requirements. Even its size and/or its weight can be important for the node design.

Table 4.4. Weight and energy requirements comparison.

\begin{tabular}{|c|c|c|c|}
\hline Brand & Model & $\begin{array}{c}\text { Power } \\
\text { Requirements }\end{array}$ & weight \\
\hline Netgear & WNDR3700 & $12 \mathrm{~V} / 1 \mathrm{~A}$ & $500 \mathrm{~g}$ \\
\hline D-Link & DIR-825 & $12 \mathrm{~V} / 2 \mathrm{~A}$ & $900 \mathrm{~g}$ \\
\hline
\end{tabular}


Finally, we chose the Netgear WNDR3700 device. This router is capable of operating at frequencies of $2.4 \mathrm{GHz}$ and $5 \mathrm{GHz}$, with transfer speeds up to $300 \mathrm{Mbps}$. It also incorporates mechanisms to implement QoS and security systems to ensure the wireless network integrity as a double firewall, DoS, IDS, WPA, WPA2, and WEP.

\subsubsection{Camera}

Another important element in the sensor node is the device for image capture. We analyzed different cameras and webcams available on the market. The first limitation was given by the hardware requirements forced on by the router or AP. Table 4.5 shows cameras that meet these requirements.

Table 4.5. Cameras comparison.

\begin{tabular}{|c|c|c|c|c|}
\hline Camera Model & Sensor & $\begin{array}{c}\text { Resolution } \\
\text { (pixels) }\end{array}$ & $\begin{array}{c}\text { System } \\
\text { Requeriments }\end{array}$ & USB Version \\
\hline $\begin{array}{l}\text { Hercules Classic } \\
\text { Webcam[312] }\end{array}$ & VGA & $1280 \times 960$ & $\begin{array}{l}\text { CPU Processor } 500 \\
\text { MHz, } 64 \text { MB RAM } \\
\text { Memory. }\end{array}$ & $\begin{array}{l}\text { USB } 1.1 \text { and } \\
\text { USB } 2.0\end{array}$ \\
\hline ClickSmart 420 [313] & $\begin{array}{l}\text { CMOS } \\
\text { VGA }\end{array}$ & $\begin{array}{c}640 \times 480 \\
\text { (video) } \\
1.280 \times 960 \\
\text { (photography) }\end{array}$ & $\begin{array}{l}\text { CPU Processor } 500 \\
\text { MHz, } 64 \text { MB RAM } \\
\text { Memory. }\end{array}$ & $\begin{array}{l}\text { USB } 1.1 \text { and } \\
\text { USB } 2.0\end{array}$ \\
\hline QuickCam Cordless [313] & CMOS & $510 \times 492$ & $\begin{array}{l}\text { CPU Processor } 400 \\
\text { MHz, } 64 \text { MB RAM } \\
\text { Memory. }\end{array}$ & $\begin{array}{l}\text { USB } 1.1 \text { and } \\
\text { USB } 2.0\end{array}$ \\
\hline $\begin{array}{l}\text { QuickCam for Notebooks } \\
\text { Pro [313] }\end{array}$ & $\begin{array}{l}\text { CCD } \\
\text { VGA }\end{array}$ & $640 \times 480$ & $\begin{array}{c}\text { CPU Processor } 400 \\
\text { MHz, } 64 \text { MB RAM } \\
\text { Memory. }\end{array}$ & $\begin{array}{l}\text { USB } 1.1 \text { and } \\
\text { USB } 2.0\end{array}$ \\
\hline $\begin{array}{c}\text { Creative WebCam NX Pro } \\
{[314]}\end{array}$ & $\begin{array}{l}\text { CMOS } \\
\text { VGA }\end{array}$ & $1024 \times 768$ & $\begin{array}{c}\text { CPU Processor } 233 \\
\text { MHz, } 32 \text { MB RAM } \\
\text { Memory. }\end{array}$ & $\begin{array}{l}\text { USB } 1.1 \text { and } \\
\text { USB } 2.0\end{array}$ \\
\hline $\begin{array}{c}\text { Creative WebCam Instant } \\
\text { [315] }\end{array}$ & $\begin{array}{l}\text { CMOS } \\
\text { VGA }\end{array}$ & $\begin{array}{c}352 \times 288 \\
\text { (video) } \\
640 \times 480 \\
\text { (photography) }\end{array}$ & $\begin{array}{l}\text { CPU Processor } 266 \\
\text { MHz, } 64 \text { MB RAM } \\
\text { Memory. }\end{array}$ & USB 1.1 \\
\hline A4tech PKS-635K [316] & $\begin{array}{l}\text { CMOS } \\
\text { VGA }\end{array}$ & $640 \times 480$ & $\begin{array}{l}\text { CPU Processor } 166 \\
\text { MHz, } 32 \text { MB RAM } \\
\text { Memory. }\end{array}$ & $\begin{array}{l}\text { USB } 1.1 \text { and } \\
\text { USB } 2.0\end{array}$ \\
\hline
\end{tabular}

Between of all of them, we chose the Hercules Webcam Classic, because it is the camera with better features. It is a small camera with a resolution of 1.3-Mpixel (1290x960) and it can work with both USB 1.1 and USB 2.0. This resolution let us obtain different image sizes as a function of the desired quality of image. Table 4.6 shows the sizes that can be obtained, depending on its quality in dots per inch (dpi). A picture quality between 250 and 300 dpi is considered a good quality image. Chosen cameras obey that the sensing coverage area is approximately a half of the radio coverage radius. 
Table 4.6. Image sizes comparison.

\begin{tabular}{|c|c|c|c|c|c|c|c|}
\hline $\begin{array}{c}\text { Size } \\
\text { (pixels) }\end{array}$ & $\begin{array}{c}\text { 80dpi ( average } \\
\text { monitor } \\
\text { quality) }\end{array}$ & $\begin{array}{c}\mathbf{1 3 3} \mathbf{~ d p i} \\
\text { (in cm) }\end{array}$ & $\begin{array}{c}\mathbf{1 5 5} \mathbf{~ d p i} \\
\text { (in cm) }\end{array}$ & $\begin{array}{c}\mathbf{1 7 5} \mathbf{d p i} \\
\text { (in cm) }\end{array}$ & $\begin{array}{c}\mathbf{2 0 0} \mathbf{d p i} \\
\text { (in cm) }\end{array}$ & $\begin{array}{c}\mathbf{2 5 0} \text { dpi } \\
\text { (in cm) }\end{array}$ & $\begin{array}{c}\text { 300dpi } \\
\text { (in cm) }\end{array}$ \\
\hline $1280 \times 960$ & $30.4 \times 40.6$ & $18.3 \times 24.4$ & $16.3 \times 21.7$ & $\begin{array}{c}13.9 \times \\
18.6\end{array}$ & $\begin{array}{c}12.2 \times \\
16.3\end{array}$ & $9.8 \times 13.0$ & $8.1 \times 10.8$ \\
\hline
\end{tabular}

\subsubsection{Rotation System}

Finally, we provided mobility to the node with the aim of increasing the viewing angle of the camera. It allows us to reduce the number of nodes required to monitor the area.

There are different ways of implementing this system. However, we bet to implement an economical and simple low energy cost option, but $100 \%$ functional. Our system is based on the control of camera movement, using a stepper motor, which is controlled by a PIC that has a small program inside. Stepper motors are commonly used for the construction mechanisms in applications that require precise movements.

The system operation is very simple. The sensor node rotates on itself and it periodically captures images and processes them in order to determine if there is any unusual status of the leaves or not. The number of degrees, that the camera is able to rotate at every turn, can be as small as $0.72^{\circ}$, and up to $90^{\circ}$, depending on selected engine. The number of times that the camera has moved (respect to its initial position) can be stored and sent when this information is required. As we can see in Figure 4.26, the information about the position of rotation is sent via RS-232 to the router, which is equipped with an RS-232 interface. The router is responsible for sending the alarm signal with this value.

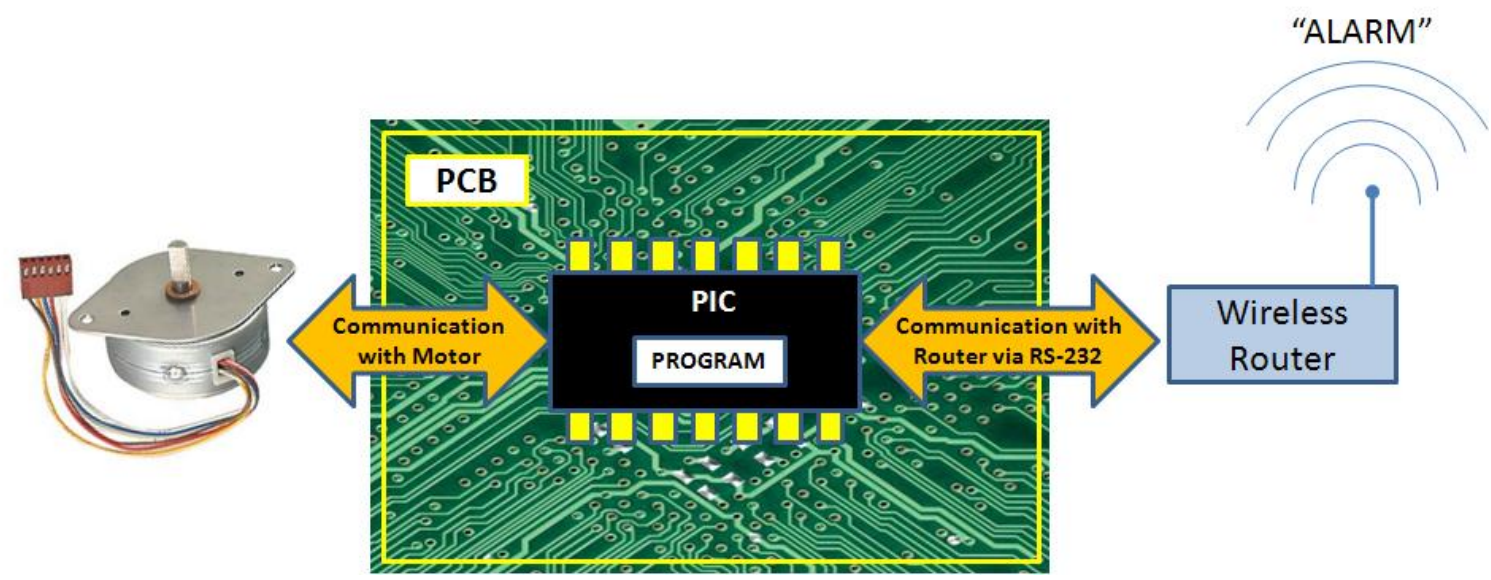

Figure 4.26. Stepper motor controller.

When the node detects a symptom in the processed image, it sends the position value jointly with the alarm signal. Thus, knowing which node has generated the alarm and the position of the camera, it is possible determine exactly, what field area is sick. Figure 4.27 shows a simplified program diagram that would perform this operation. 


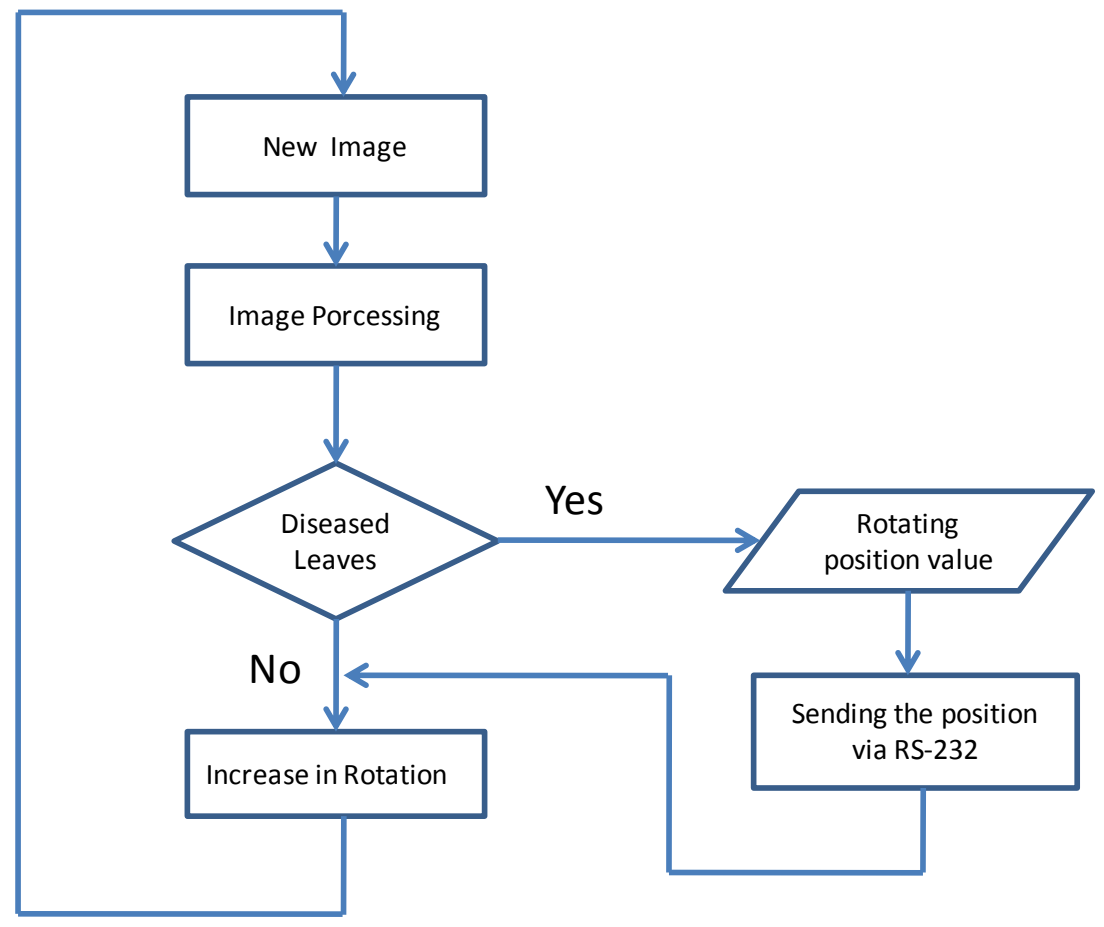

Figure 4.27. Program diagram.

In order to encode the position value, we must consider, first the type of engine that will be used. For our application we use a stepper motor, with rotation increments of $1.8^{\circ}$. This means that we could go through 360 degrees in 200 steps of $1.8^{\circ}$. Moreover, we note that signals that are sent through the serial port have a data field of 8 bits (without taking into account, start bit, stop bit, parity bit, etc). Therefore, we can identify each motor step with an 8-bit binary value, being "00000000" $0^{\circ}$ and "11000111" would be $358.2^{\circ}$.

Moreover, if we analyze the mechanic part of the development, we can make the system capable of rotating 360 degrees, using a mobile brush for electric power transmission to the router and the engine. Figure 4.28 shows a cross section of the node once assembled. This is a shell composed of a fixed base that holds the battery and another moving part that protects the camera, router, and the stepper motor. The union between the two sides is made by epicyclical or planetary gearing and an annulus gearing, in order to transmit rotary motion from one place to another.

The use of devices capable of performing turns of $360^{\circ}$ reduces the number of devices to be installed along the area being monitored. 


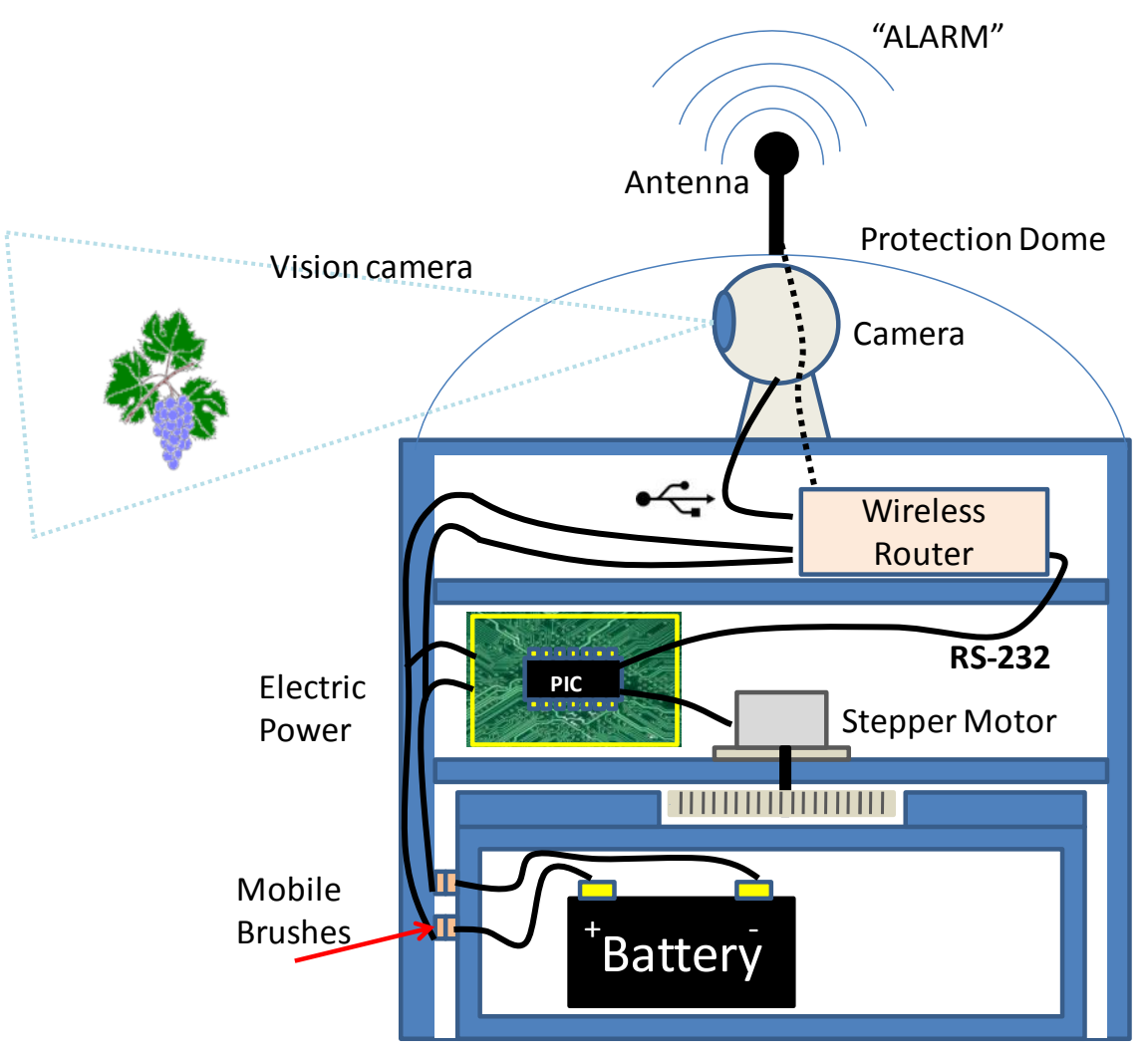

Figure 4.28. Sensor node for vineyard monitoring.

\subsubsection{Sensor Network Traffic Measurement}

In order to show the benefits of the proposed system, in this section we compare the traffic measurement provided by a single sensor node that takes images of a $360^{\circ}$ of view from the vineyard field and transmits them to a central server, in order to analyze the images, with our sensor node that only sends one image (when it founds a bad leaf while going around $360^{\circ}$ ). In order to have enough image quality, the images had a resolution of $640 \times 480$ pixels and a frame rate of 30 fps. No audio was transmitted. Figure 4.29 shows a comparison of two video bit rates (4 Mbps and $512 \mathrm{kbps}$ ) with our system, which sent a picture of 640x480 pixels. The camera needed 120 seconds to make a round of $360^{\circ}$. We can see that there are more bytes sent to the network for the $4 \mathrm{Mbps}$ video stream. There is a mean value of 146130.64 bytes per second. When the $512 \mathrm{kbps}$ is sent there is a mean value of 74785.76 bytes per second. In our case, the picture was sent in the $20^{\text {th }}$ second. There are other packets in the network because of the regular procedure of the WSN. In our case there is a mean value of 4259.86 bits per second. Now we can see that, in terms of bytes transmitted, there is a decrease of $97.09 \%$ from the $4 \mathrm{Mpbs}$ to our system and a decrease of $94.30 \%$ from the $512 \mathrm{kbps}$ to our system. Bearing in mind that the most power consumption issue in a sensor to transmit bytes through the wireless interface [241], we can state that our system saves energy. 


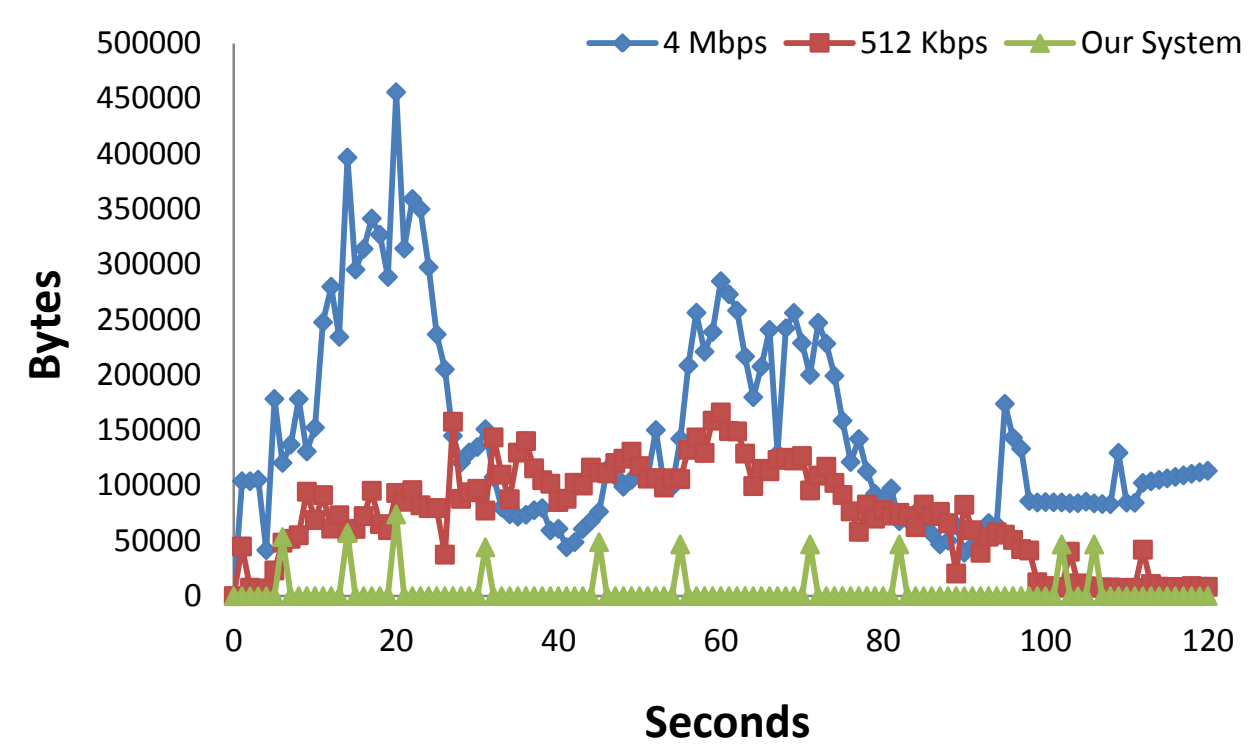

Figure 4.29. Bytes comparison when there is 4 Mbps video stream, 512 kbps video stream and our system.

Now, in Figure 4.30, we compare the number of packets sent to the network for the $4 \mathrm{Mbps}$ and the $512 \mathrm{kbps}$ video streams with our system. We can see that there are quite less packets sent to the wireless in our system. Only the forwarded messages and the image sent in the $20^{\text {th }}$ second can be seen in the network. 4 Mbps video stream is the one that sends more packets.

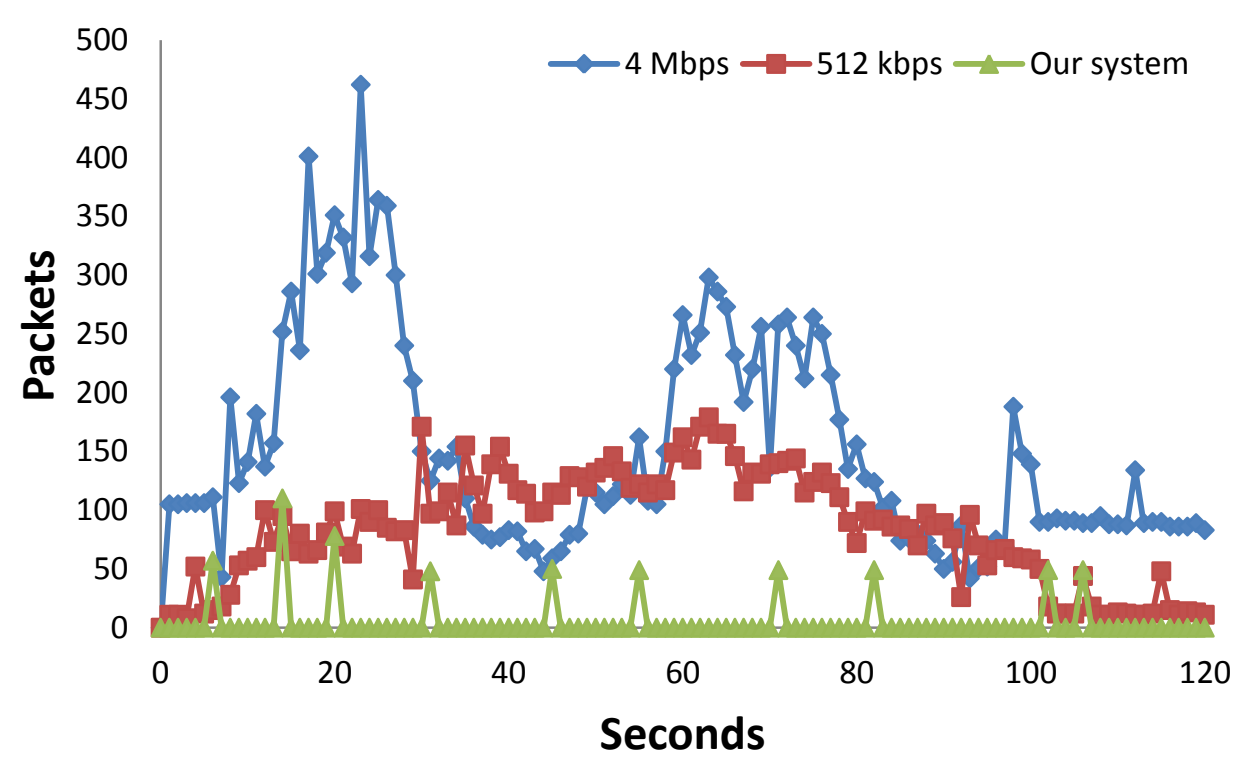

Figure 4.30. Packets comparison when there is $4 \mathrm{Mbps}$ video stream, $512 \mathrm{kbps}$ video stream and our system.

These measurements show that our system saves energy because there are fewer packets and fewer bytes transmitted to the wireless medium. 


\subsection{Intelligent WSN to detection and protection of the attacks to the sheep and goats}

WSNs can be used in several activities in the agricultural sector where the combination of new technologies and the knowledge of some existing problems can provide many benefits. In the livestock sector, a WSN could be used for controlling the animals and other environmental parameters, such as attempts of animals' theft or flock attacks by wild animals, among others.

In 2011, the "Asociación Agraria de Jovenes Agricultores" (ASAJA) [317], announced that the number of wolf attacks (Canis lupus) in Castilla y Leon (Spain) had increased up to $42 \%$. In the same year, the Principado de Asturias' (Spain) government announced that the number of wolfs attacks had stabilized at an average of 3,500 attacks per year (after an increase of $24 \%$ of these attacks during 2008 and 2009) [318].

Wolf attacks are also continuously growing in France, reaching values of 4,290 victims in 2011. As Table 4.7 shows, these livestock losses were translated into economic losses of 1,548,052 Euros to the French government [319]. These attacks are registered mainly in the north of Spain and in most of the pastoral areas of the rest of Europe. Massive wolf attacks cause the illegal hunting of these predators and the incompatibility of the wolf recovery (because this species is considered endangered). Furthermore, the financial resources designed for rural areas are also negatively affected.

Table 4.7. Number of wolves attacks to flocks in France

\begin{tabular}{|c|c|c|}
\hline Year & Number of Victims & Economical Compensation \\
\hline 2004 & 3.188 & $494.255 €$ \\
\hline 2005 & 3.762 & $1.016 .105 €$ \\
\hline 2006 & 2.552 & $702.861 €$ \\
\hline 2007 & 2.994 & $912.930 €$ \\
\hline 2008 & 2.747 & $794.577 €$ \\
\hline 2009 & 3.263 & $1.039 .293 €$ \\
\hline 2010 & 4.180 & $1.157 .408 €$ \\
\hline 2011 & 4.290 & $1.548 .052 €$ \\
\hline
\end{tabular}

The aim of this Section is to show the development of a WSN capable to intelligently detect when a flock is being attacked by wild animals. The data generated by the WSN is processed by a smart algorithm which will decide what kind of alarm has to be generated and consequently, the system will warn the owner and other services such as the environmental unit of animal control.

\subsubsection{Sensor Network}

In order to repel wolf attacks, we propose to use a WSN connected to an access point (AP) forming a wireless network infrastructure. Each animal (or some percentage of animals) wears a 
wireless node provided with a temperature sensor and an ECG circuit. This section presents the wireless node of our system. Figure 4.31 shows the structure of the proposed network.

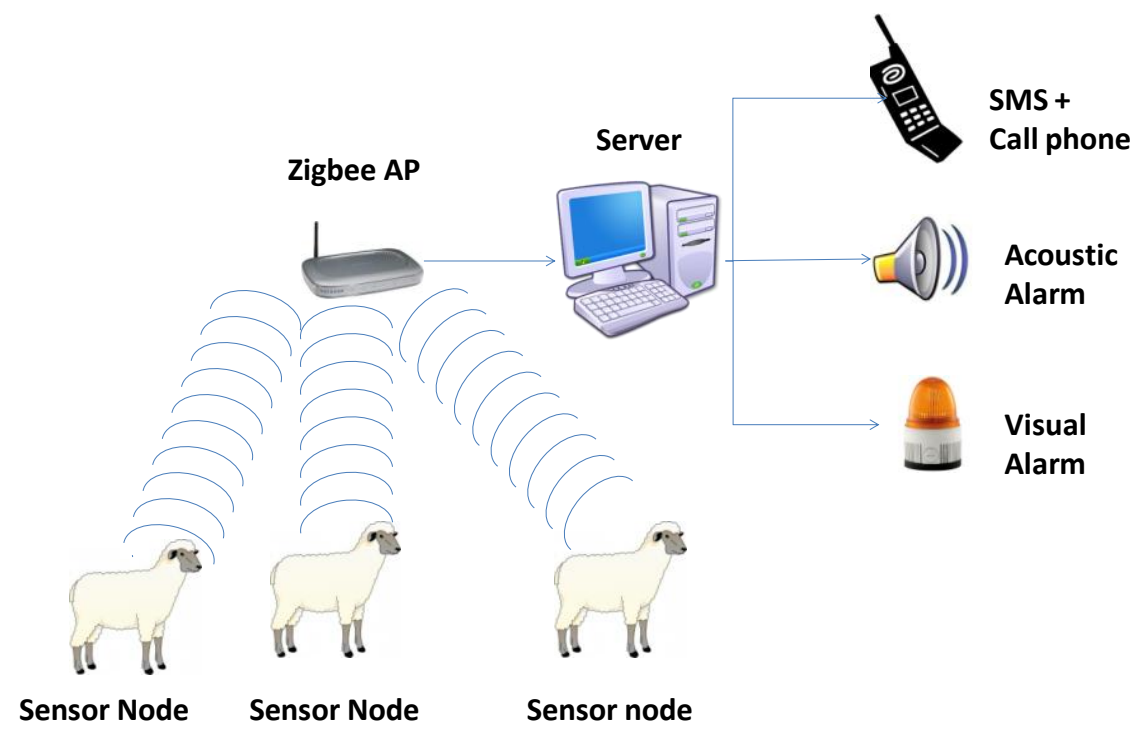

Figure 4.31. Structure of the wireless network

Our system determines that there is a situation of collective stress, when it detects 5 alarms, from 5 animals, within a maximum period of 5 minutes. When an alarm is generated, the AP communicates the situation to the server. The server is the responsible to activate the acoustic and visual alarm. The first stage is intended to frighten and scare off potential predators or intruders. Moreover, the server sends a text message, warning of the time of the alarm, followed by an automatic call, to the farm owner.

\subsubsection{Monitoring system of heart rate and body temperature.}

In the presence of a threat as wolves or unknown persons, the sheep undergoes an elevation of heart rate and body temperature. At rest, the temperature of a sheep is around $38.5-39.5{ }^{\circ} \mathrm{C}$. Sheep and goats have a resting heart rate around 70-80 beats per minute. In a stressful situation, this value can be tripled. Sheep, as well as other mammals, have different points where the heart rate can be measured. To determine heart rate, we must measure it as a differential measurement of the values recorded in 2 or 3 different points of the body. The connections arrangement of each electrode group is called bypass. In our proposal, we use the limb leads and we are going to employ two measuring points on the body of the animal.

This section will show the proposed circuit for our belt monitoring system. For this measurement, it is proposed the use of an instrumentation amplifier followed by selective filters, to capture the signals correctly. Finally, we present the measurement system for the animal and the ON/OFF circuit to control the node.

With the aim of monitoring the sheep body temperature and its heart rate, we propose to use a platinum temperature sensor, which is in contact with the animal body. To measure heart rate, we will implement an ECG circuit using an instrumentation amplifier able to take differential measures. The electrodes will be connected to the front legs of the animal. Thus, it will determine the heart 
rate of the animal using DI limb bypass. DI is a connection between electrodes on the front left leg and the front right leg. When the front left leg is in a positive force field over the front right leg, in DI is detected a positive upward deflection.

Figure 4.32 shows the block diagram of the circuit. The entire circuit is located in a small box on the animal's collar. Each of these boxes has a wireless node based on IEEE 82.15.4 technology. The node is powered by a rechargeable battery. The measures from both the temperature sensor and ECG circuit will be processed by the node, which will establish a connection with the Zigbee AP. Recharging the battery is done through a flexible solar panel to be integrated into sheep collar. The regulator will be in charge to determine at what time the battery must be recharged or not. Finally, because the wolves attack occur at night, our node will monitor the animal during the hours of darkness. The rest of time, the node will remain off. Figure 4.33 shows the belt with sensors.

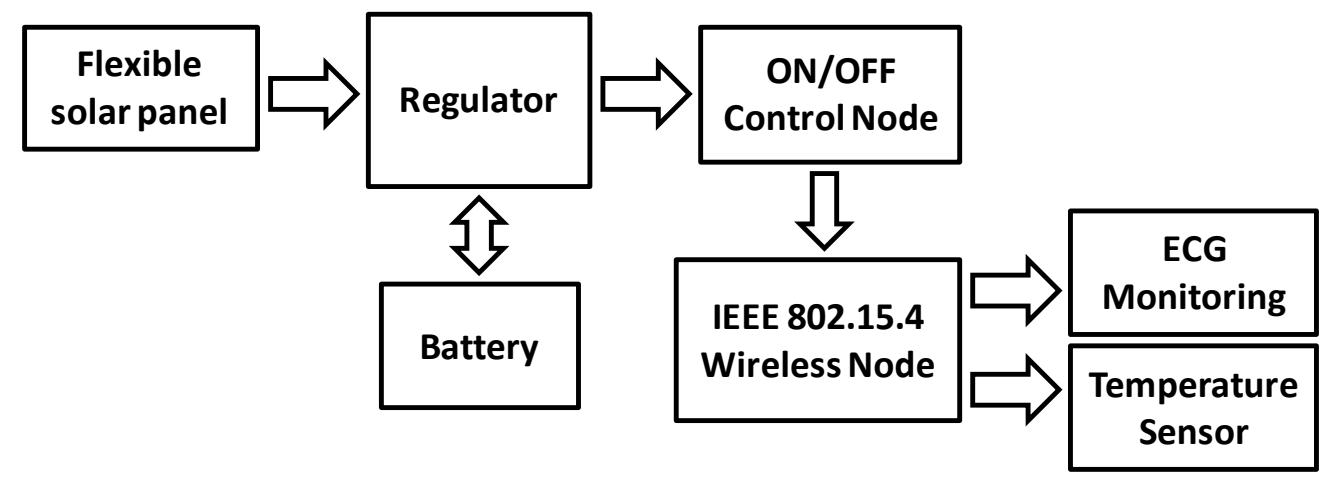

Figure 4.32. Circuit diagram to monitor the sheep.

\section{Body temperature sensor}

To implement the sensor body temperature, we use a RTD (P0K1.516.7WB010) mounted within a Wheatstone bridge. This platinum sensor is able to measure the temperature in a range between $200{ }^{\circ} \mathrm{C}$ and $+750{ }^{\circ} \mathrm{C}$ with high accuracy and stability. It has a metal chassis which allows a quick response (max. 7.5 seconds for air) by temperature changes. The Wheatstone bridge output is connected to an operational aplificador in differential configuration. Figure 4.34 shows the circuit diagram for monitoring the body temperature. The output voltage and body temperature are related by equation 4.13 .

$$
\text { Vout }=\frac{R_{5}}{R_{4}} \cdot \frac{\frac{R_{1}}{R_{2}}-\frac{R_{0}(1+\alpha(t-273))}{R_{3}}}{\left(1+\frac{R_{1}}{R_{4}}\right)\left(1+\frac{R_{0}(1+\alpha(t-273))}{R_{3}}\right)}
$$

\section{Cardiac frequency sensor.}

The ECG is a physical test widely used to assess, in a noninvasive way, the condition of the heart. This test can assess the state of the conduction system of the heart, its muscle, and indirectly, the condition of this organ. For the measurement of the sheep heart rate, we use a circuit based on three operational amplifiers of two stages. Figure 4.35 shows the schematic of our ECG circuit. Equation 4.14 shows the relationship between the output voltage (value to be processed) and voltage values taken in the right leg (V1) and the left leg (V2). The voltage values V1 and V2 are related to the frequency rate. 


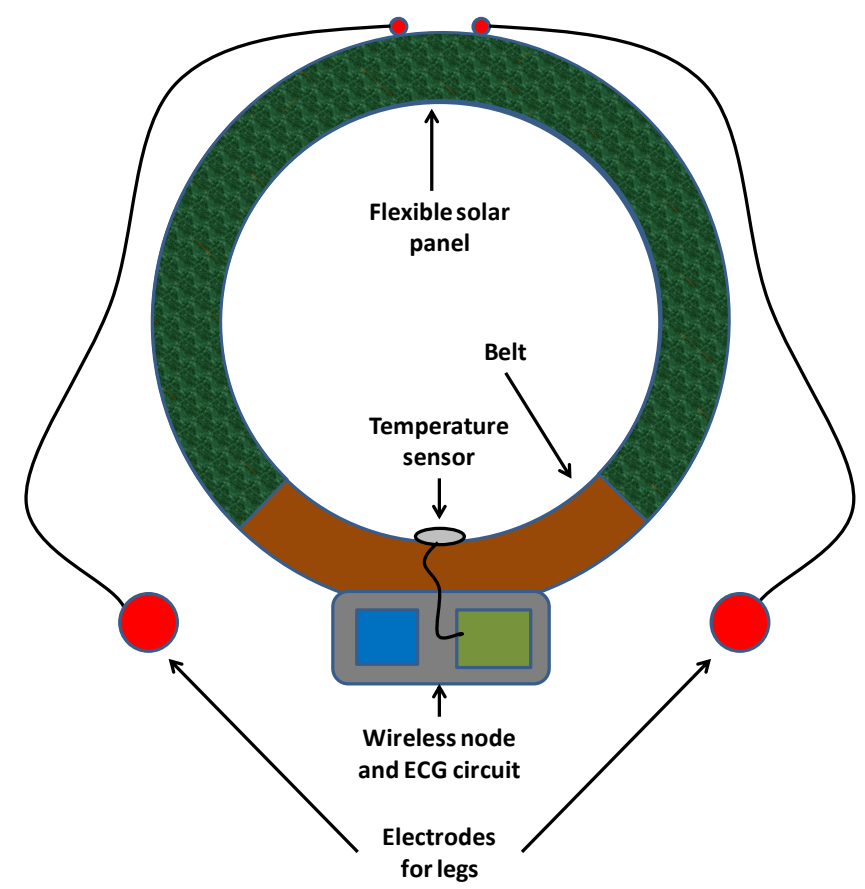

Figure 4.33. Belt whit sensors.

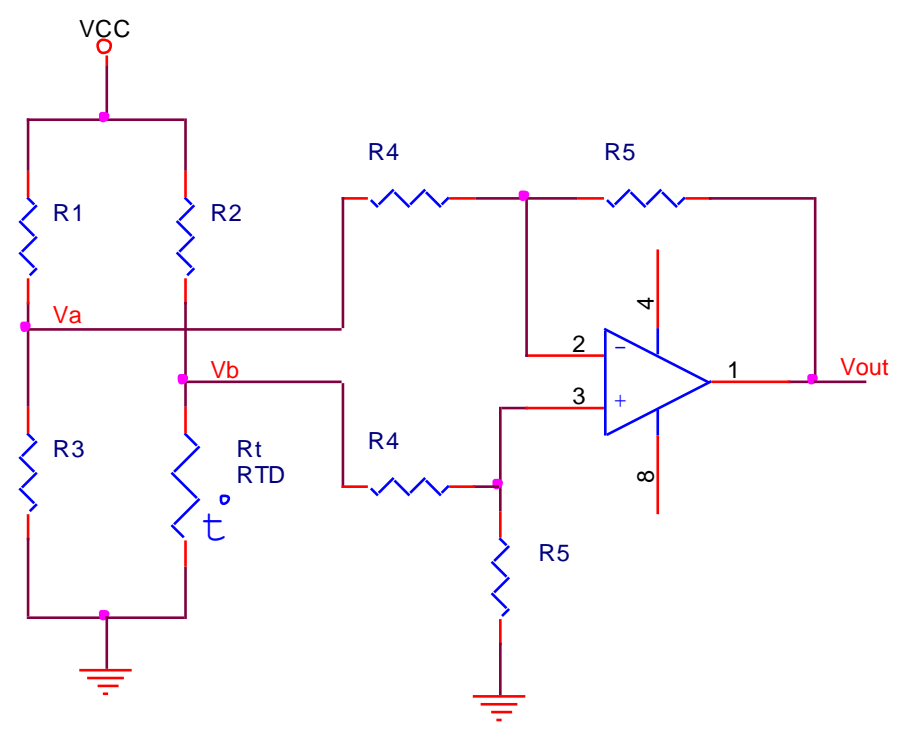

Figure 4.34. Temperature monitoring circuit

$$
\text { Vout }=\frac{\left(V_{A}-V_{B}\right)}{2} \cdot\left[\frac{1-\frac{R_{2} R_{3}}{R_{1} R_{4}}}{1-\frac{R_{3}}{R_{4}}}\right]-\left(V_{A}-V_{B}\right) \cdot\left[\frac{1-\frac{R_{2}}{R_{1}}}{1-\frac{R_{3}}{R_{4}}} \cdot\left(\frac{1}{2}+\frac{R_{b}}{R_{g}}\right)+\frac{R_{2}}{R_{1}} \cdot\left(\frac{1}{2}+\frac{R_{a}}{R_{g}}\right)\right]
$$

This circuit can be implemented with individual operational amplifiers or as an integrated designed for this purpose. In our case we will use the instrumentation operational amplifier INA 125 able to work in single supply. The amplifier gain can be adjusted using Equation 4.15.

$$
G=4+\frac{60 K \Omega}{R g}
$$




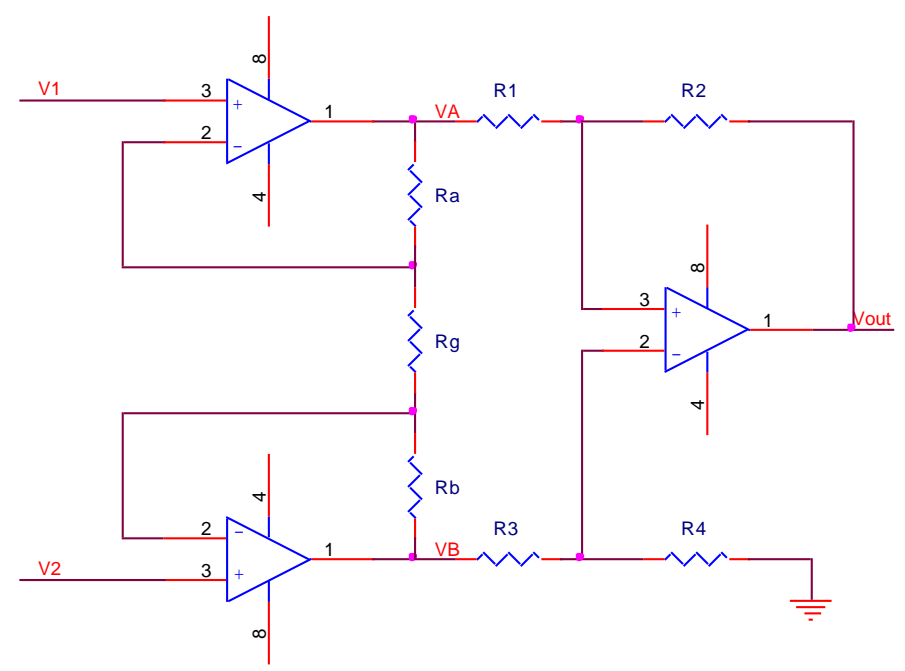

Figure 4.35. Circuit for cardiac frequency monitoring

\section{ON/OFF circuit to control the wireless node}

Wolves are animals of nocturnal habit. For this reason, our wireless nodes and consequently the monitoring of vital signs of sheep should operate overnight. To this end, we will employ a light control circuit which will act between the battery connection and the node. As a light sensor, we will use a light dependent resistor (LDR). The circuit operates as an electronic switch where the activation/deactivation of the relay and consequently the wireless node, depends solely on the bias of transistor Q1. The circuit is a simple bias network where the LDR controls the flow of current through the transistor base. Figure 4.36 shows the control circuit for wireless node where the relay will be activated in the presence of the dark.

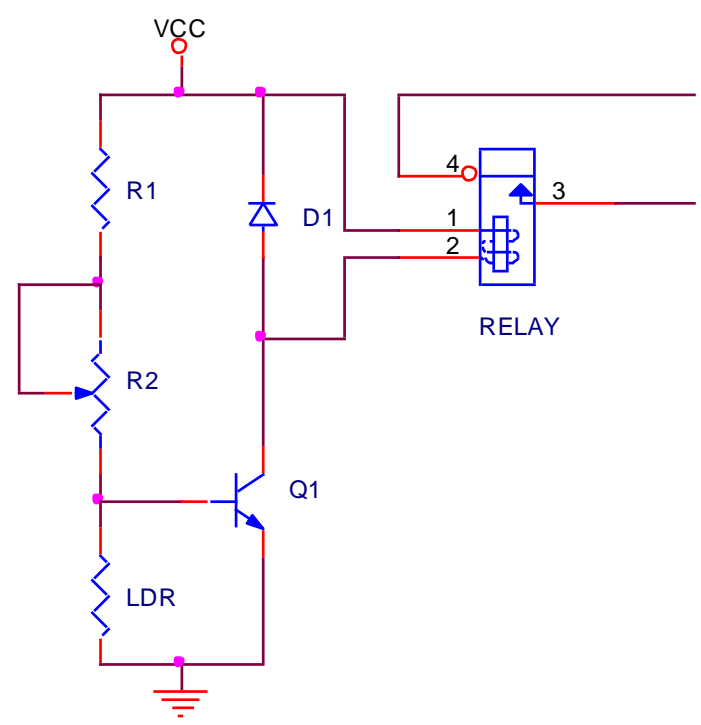

Figura 4.36. ON/OFF circuit to control the wireless node 


\subsubsection{Placement of sensors on the animals.}

Figure 4.37 shows the sensors placement on the animal. On the one hand, the sheep wears two electrodes attached with a wrist band to the front legs (the same procedure can be used for a goat). The connection with the belt is made by a wire protected by a metal mesh that will prevent that the animal can gnaw the cord. On the other hand, the temperature sensor is located in the inner part of the belt. The metallic encapsulated is in contact with the skin of the animal. All circuits are protected by a small box which is attached in the belt.

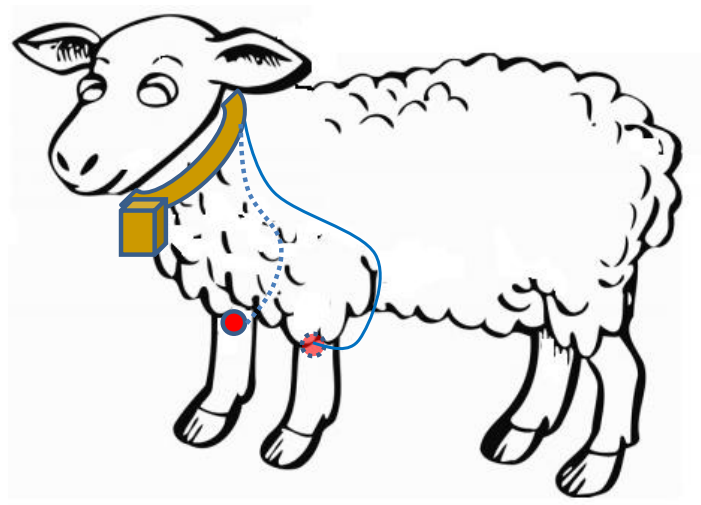

Figure 4.37. Placement of sensors on the sheep

\subsubsection{Design of the network topology}

Our design uses ZigBee as a wireless communication protocol. This communication standard has been designed by ZigBee Alliance. It is a standardized group of solutions that can be implemented by any manufacturer. ZigBee is based on the IEEE 802.15.4 standard for wireless personal area networks (WPAN). Its low power consumption feature make this protocol ideal for high that require secure communications, but with low data rates at short distances that maximizes the network lifetime [320].

The sensor node is made using Waspmote node. It is designed by Libelium Company. It is able to combine several sensors and actuators by using its programming environment. The programming language is $\mathrm{C}++$ simplified restricted it to the API provided by the manufacturers [321]. Waspmote nodes can be connected by star topology, tree topology or mesh network topology. We have implemented a network in star topology. Figure 4.38 shows a Waspmote node and its battery. The main characteristics of these nodes are shown in Table 4.8 [322]. 


\section{Chapter 4: Wireless Sensor Development for Environmental Monitoring}

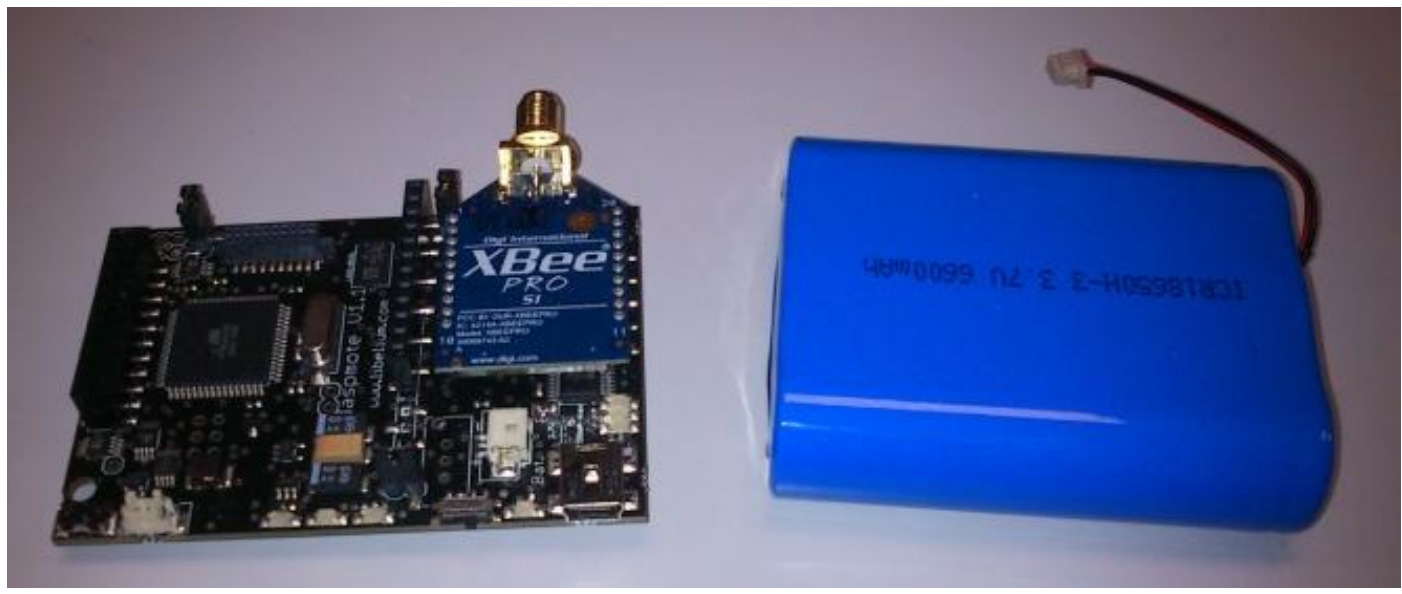

Figure 4.38. Waspmote node and its battery

Table 4.8. Waspmote features.

\begin{tabular}{|l|l|}
\hline \multicolumn{1}{|c|}{ Component } & \multicolumn{1}{c|}{ Features } \\
\hline Microcontroller & A Tmega 1281 \\
\hline Frequency & $8 \mathrm{MHz}$ \\
\hline SRAM & $8 \mathrm{~KB}$ \\
\hline EEPROM & $4 \mathrm{~KB}$ \\
\hline FLASH & $128 \mathrm{~KB}$ \\
\hline SD Card & $2 \mathrm{~GB}$ \\
\hline Weight & $20 \mathrm{gr}$ \\
\hline Dimensions & $73.5 \times 51 \times 13 \mathrm{~mm}$ \\
\hline
\end{tabular}

We used ezpot ZigBee 101 [323] as the access point. It acts as the gateway between the Zigbee WSN and server that process the data and takes the smart decisions. It has a ZigBee port which allows the WSN connection and a Fast Ethernet port which is connected to the server. Its characteristics are the following ones:

- DIN rail or panel mount.

- Support 10/100 Mbps Ethernet.

- Compatible with RS-232, RS-422 and RS-485 serial interface

- Compatible with communications LAN and WAN.

- In Server mode supports individual customer sessions (enhances the security).

- Management password protected.

- Virtual COM drivers for Windows NT/2000/XP/2003/Vista/Win7.

AP specifications explain that the AP can be used for distances up to 100 meters, which is enough to cover the area of a stable. 


\subsubsection{System algorithms}

This section shows the communication protocol and the algorithms designed to take decisions based on the information received from the sensor nodes. In addition, we are going show the procedure to generate alarms when a situation of collective stress is detected.

The operation of our wireless sensor network is based mainly on a smart decision algorithm based on the signals received from the flock of sheep or goats. The algorithm goal is to assess whether it is producing a collective stress situation by the presence of a predator. Because our nodes only work during the night hours (during the day, sheep and goats are with the shepherd, the energy regulator is the responsible of determining when the node will be working, and when the battery must be recharged.

This section shows the three necessary algorithms for the operation of the wireless sensor network. They help us to determine if the flock is being attacked. We are also going to explain the smart algorithm that analyzes the received data. Using a previous learning phase, the system will be able to define the type of alarm and the type of alert that should be generated.

\section{Decision algorithm based on a smart tagging system.}

An animal can register an increase of their vital signs due to multiple causes. However, this does not mean that the animal is being attacked. But, generally, when a wolf or an intruder is near the flock, several animals are altered.

Our system tries to control this behavior by following next procedure. When an animal registers elevated body temperature and heart rate, the system process the event as an individual alarm signal, which is sent to the AP. The AP will send this event to the server responsible of the control of the area and it will generate a message. At this time, a 5 minutes timer is activated. If there are 5 or more messages before the account expires, the system will consider that it is being produced a collective stress situation. The detection of a collective stress situation activates the acoustic and luminous alarms. Simultaneously, the server will send a text message to the farm owner or other people depending of the type of case, which is decided by a smart algorithm.

The system can detect several types of cases. Each situation will generate a different type of alarm, which will advise different persons.

Firstly, a sheep or goat may be altered by natural causes, such as the presence of a female in mating season (alarm level 1). But the animals may be frightened by the presence of thieves (alarm level 2), by the presence of wild animals (alarm level 3) or by the presence of some kind of natural phenomena, such as a fire, a flood, etc (alarm level 4).

Each type of alarm (from the lowest, level 1, to the highest, level 4), must be addressed. For this reason, when a level 1 alarm is generated, the system will only notify this status to the farm owner. When an alarm of level 2 is generated, the system will warn the farm owner and the police (to try to catch the alleged thief). A level 3 alarm will generate a warning message to the farm owner and to the environmental unit for animal control. Finally, a level 4 alarm means that due to an unusual event, the entire flock is in danger. So, the alert will be communicated to the owner and to the emergency services.

Figure 4.39 shows the decision algorithm which will control the generation of alarms. 


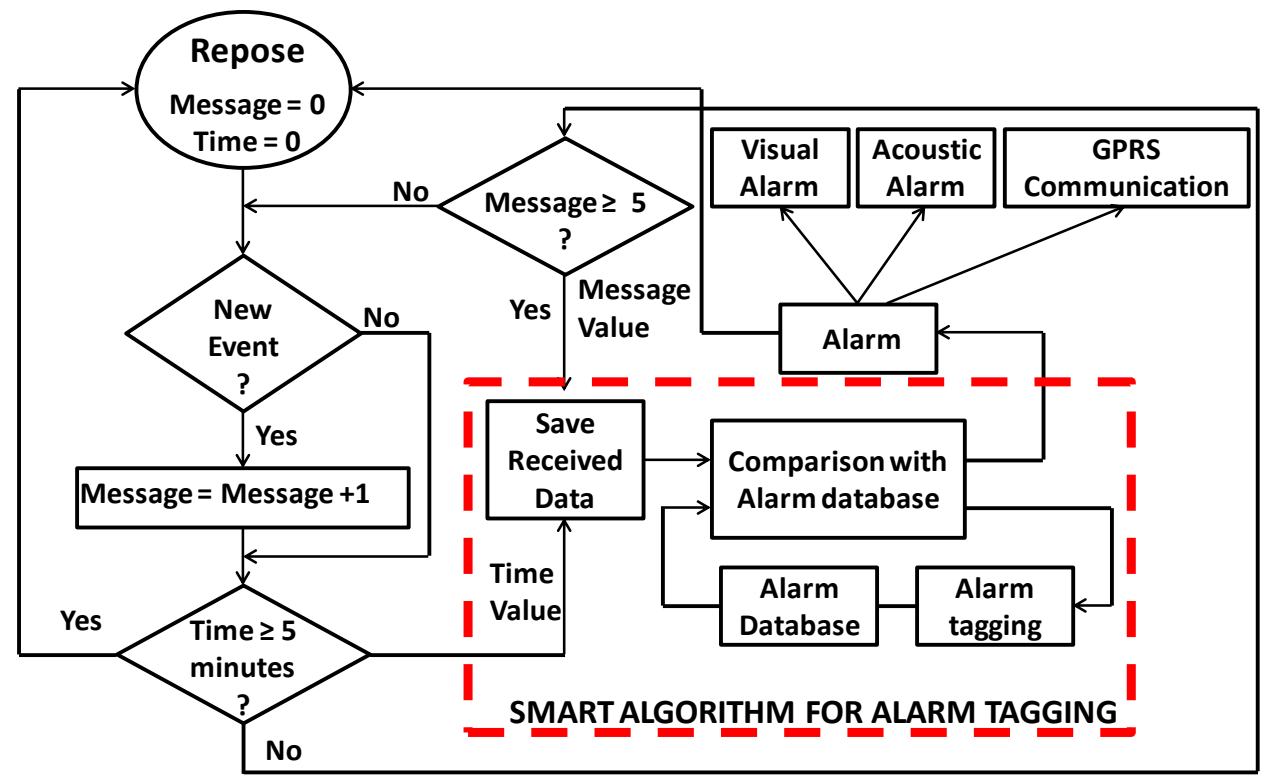

Figure 4.39. Decision algorithm

For the proper operation of our intelligent algorithm, we must perform a training system for alarm tagging. In order to achieve this goal, we need to establish a period of manual alarms tagging. This allows us to create an initial alarm database. This database will provide the flock behavior patterns.

When the system detects a collective stress situation ( 5 o more messages received in less than 5 minutes), the time values and messages are stored. Then, the algorithm will analyze each received data which will be compared with the data from the database (the initial data comes from the training phase). After determining the priority of the alarm, the system will perform, on the one hand, an alarm tagging and then, it will be save in the database, and, on the other hand, the communication and the alarm generation (Visual alarm, acoustic alarm and GPRS communication).

The intelligent algorithm, allows the WSN reporting the status of the animals. In addition, when there is a threat, the system will generate several deterrents (visual and acoustic alarms) that may scare the threats, while the owner is going to the farm.

\section{Energy regulator control Algorithm}

One of the most important issues to be considered in WSNs is to have as low as possible power consumption. This will prolong the network lifetime and facilitate to maintain more stable communications.

In order to prolong devices lifetime, we use an energy regulator algorithm which is able to control the periods when a node will work and when the battery needs to be recharged.

Figure 4.40 shows the control algorithm to control the energy regulator. First, we should detect if it is night or not. During the daytime, the system must check the battery status. If the charge level is below $100 \%$, the battery should be recharged through the solar panel. Otherwise, the system will remain in idle mode and it will turn off the node. When night falls, the energy regulator control activates the relay that controls the battery connection to the sensor node, enabling wireless link with the AP. 


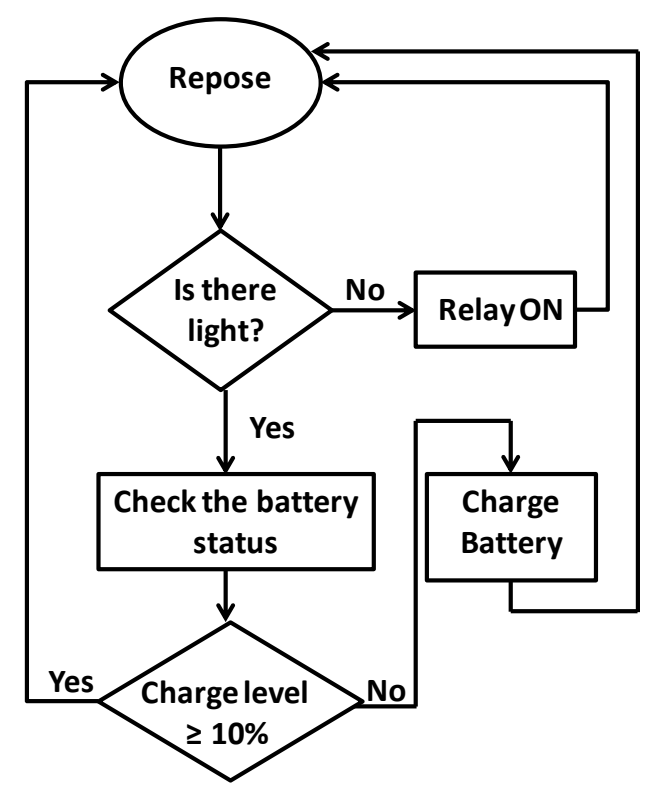

Figure 4.40. Algorithm to control the energy regulator

\section{Communication Protocol}

When a sensor node wakes up, it attempts to communicate with the AP. In order to do this, the node scans the wireless networks that are within range. The AP configured to let the access to the sensor nodes will reply with an ACK message. Each node will request the authentication to the AP which will respond with a confirmation (if the node is registered).

Finally, the node of each sheep or goat will be associated with the AP. From that moment, the AP will await the arrival of any alarm message. Figure 4.41 shows the message flow procedure between a sensor node and the AP.

\subsubsection{Performance Study}

This section simulates the decision algorithm that generates the alarms. We also show real measurements from a real environment using Zigbee wireless technology.

Figure 4.42 shows the simulation results of our decision algorithm. As we can observe, an alarm is generated individually at 50 seconds causing the first signal message. After 5 minutes no more alarms are registered and consequently, the value of the alarm signal returns to 0 . From the second 390 , different individual alarms are generated, so the message value increases. When this value is equal or higher than 5 , the alarm is activated. 


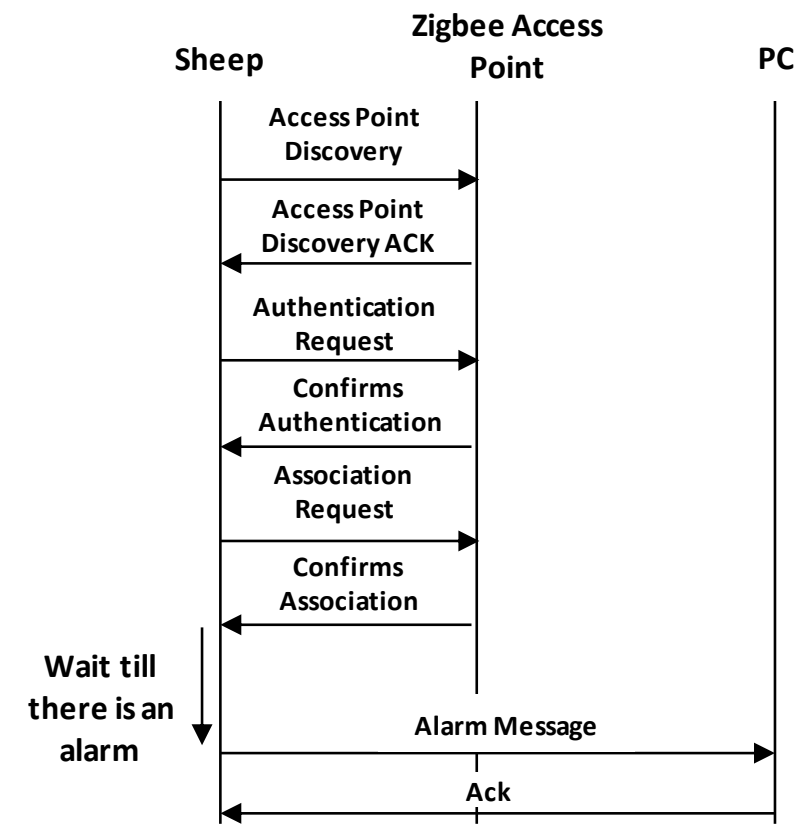

Figure 4.41. Communication Protocol

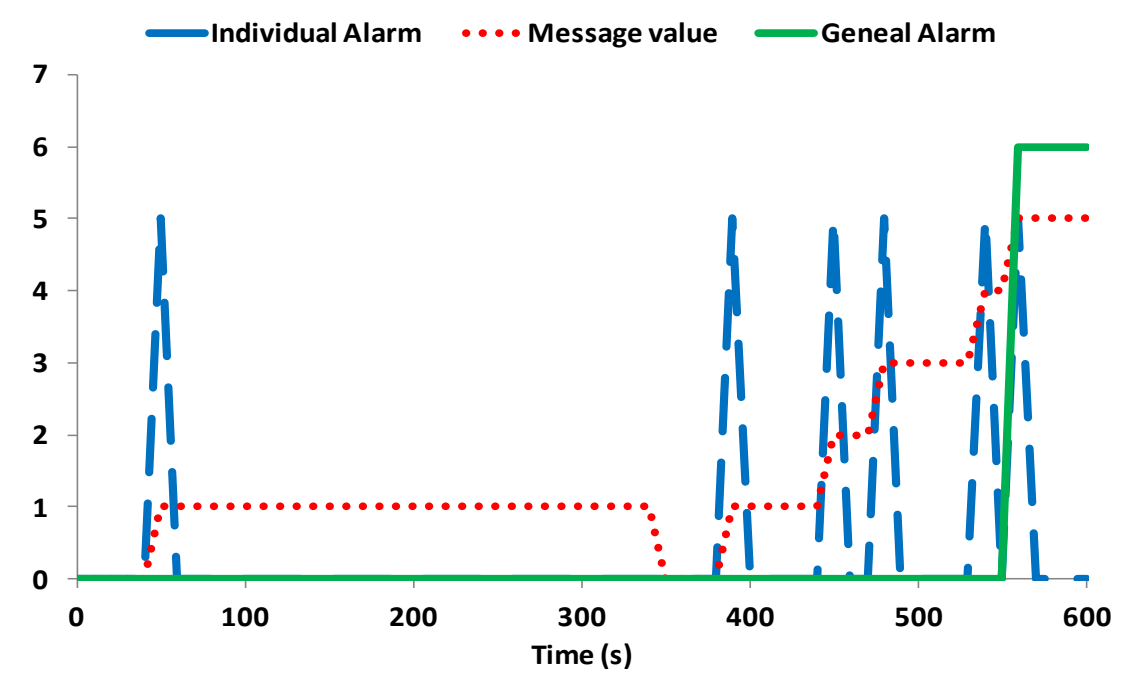

Figure 4.42. Simulation of the decision algorithm

In order to show the performance of our Zigbee network, we have connected a node Zigbee and Zigbee AP. This allows us to study the individual network performance due to the operation of a single node placed in single sheep or goat.

Figure 4.43 shows the bandwidth in bits per second consumed in the network due to the communication protocol. In $15^{\text {th }}$ second, the node sends a broadcast message to discover the reachable APs. Immediately, it receives an ACK message from the AP. After this reception, the node authenticates with the AP using the password that was previously configured. The node receives the confirmation of this authentication (which is received approximately on the $18^{\text {th }}$ 
second). Finally, the node sends an alarm message in the $31^{\text {st }}$ second and receives the ACK message.

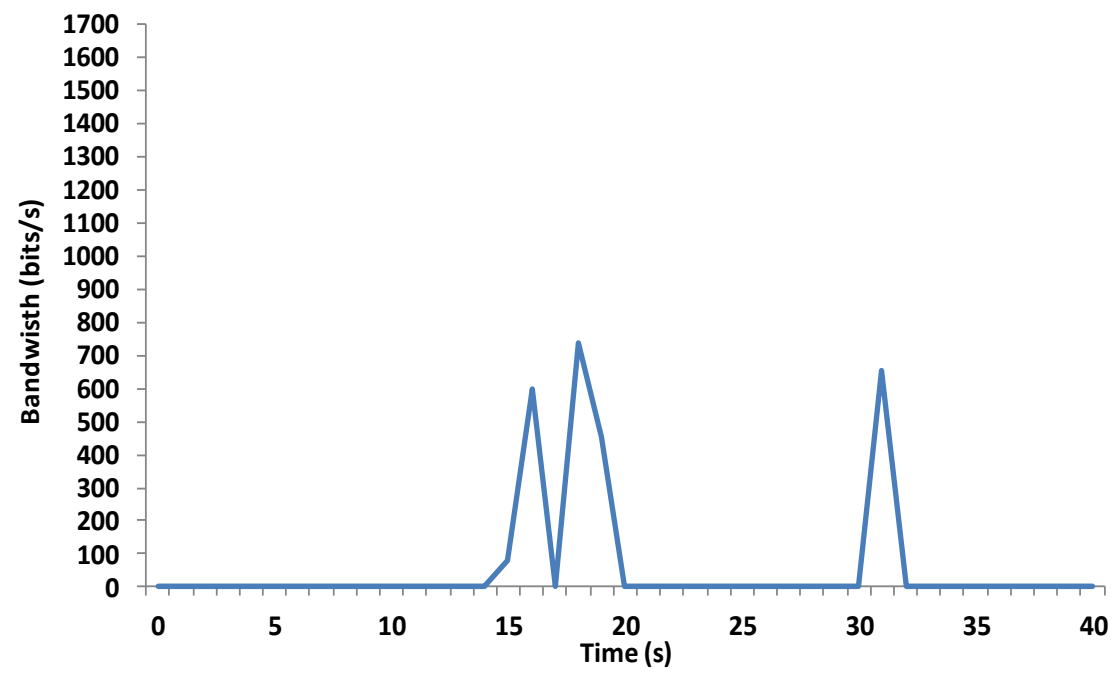

Figure 4.43. Consumed bandwidth.

Figure 4.44 shows the bytes sent by the sensor node during the above described communication process. The moment with greater registered number of bytes is when the node tries to associate with the AP. We noticed that there is another peak of number of bytes when the node sends the last alarm (last peak of the graph).

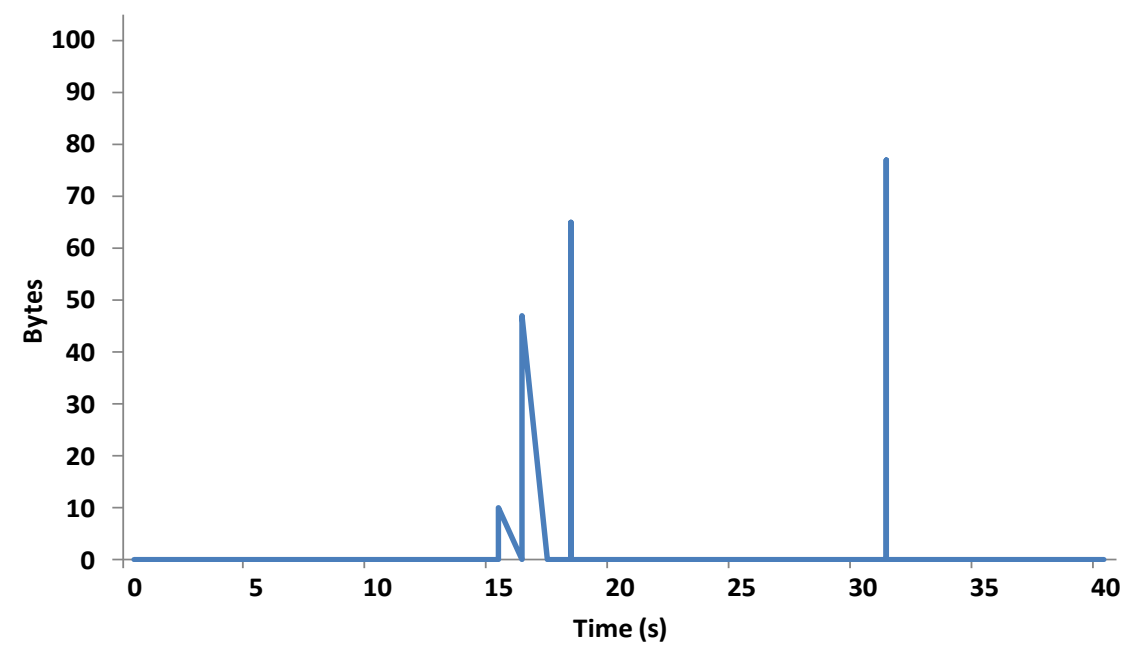

Figure 4.44. Bytes sent by the node

Figure 4.45 shows the responses of AP to messages sent by the node according to the described communication protocol. We can see that there is more number of bytes sent during the sensor nodes registration process, so the system does not consume too much bandwidth once all sensor nodes have been registered. 


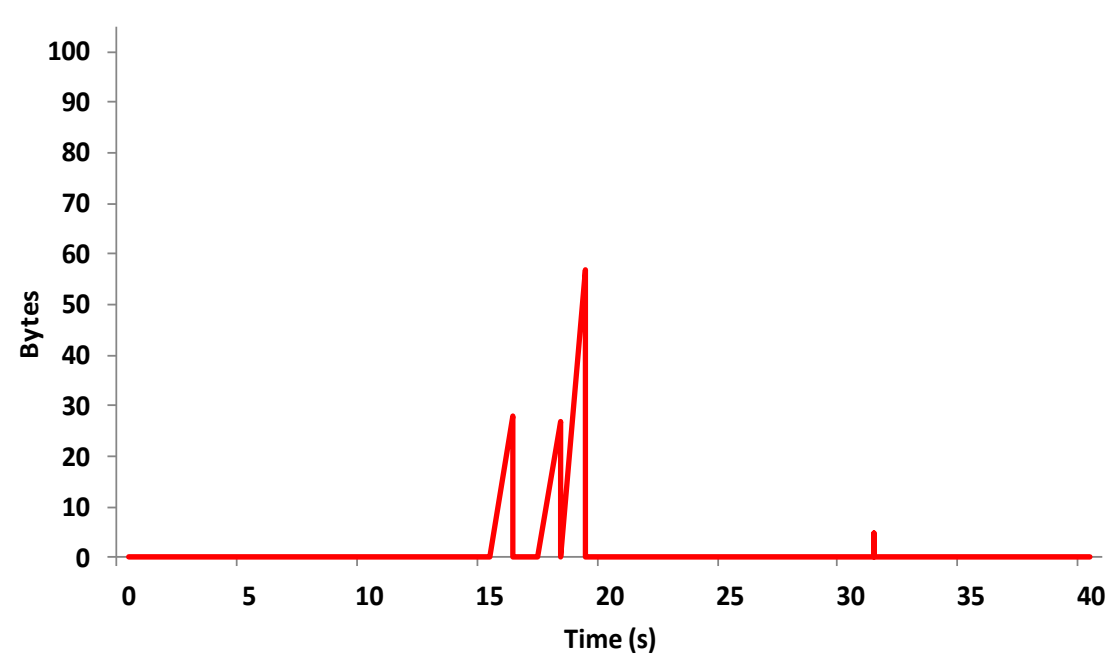

Figure 4.45. Bytes sent by the AP.

\subsection{Deployment of Wireless Sensor Nodes to Detect the Cement Degeneration in Constructions.}

Some recent news announced that several buildings from the 60's and 70's collapsed because of the deterioration of the building materials. Their common feature was that they were constructed in the same decade, so the type of cement was almost the same and all of them were affected by the humidity and salinity because they were close to the sea. Many buildings were built in that decades all around the world and when a building collapses there could be human damages.

Several studies have demonstrated that it is very difficult to predict when a building will fall down. Moreover, sometimes it is quite difficult to perform a regular monitoring of the degeneration of the cement inside the building due to the existence of difficult access areas [324]. These issues caused high impact in the economic costs in the building maintenance.

Concrete is a compact mass produced by lithification after mixing certain materials. Reinforced concrete has at least the following components: cement, fine aggregate, coarse aggregate, water and steel [325]. When the component materials of the concrete structures do not have the appropriate dosage, the structure may suffer some damage in the presence of humidity. These damages are difficult to be identified, so intrusive techniques must to be used [326].

Loss of strength of the building structure is a pathology caused by the penetration of the humidity into the concrete or steel. The mass disintegration of the resistant building components can be given by the corrosion of aluminium, carbonation or by the corrosion of the reinforcement. These are the main reasons:

- Aluminosis is given when humidity interacts with concrete structures with high presence of calcium aluminate cement. It can be detected by using chemical tests.

- Carbonation is caused by the presence of $\mathrm{CO}_{2}$ concentrations in the air with humidity and temperature. It can be detected by using a reagent. 
- Oxidation is a chemical reaction of the metal with the air. The current flow between them, i.e. electric influx, causes the oxidation of the metal. If the oxidation is porous, the metal has corrosion.

All of them are caused by the humidity. Another physical parameter to take into account in the degeneration of the cement is the temperature. It is a catalyst for chemical reactions. The temperature effect use to be linked with the humidity effect. At high temperatures and humidity the cement structure passes from hexagonal structure to cubic structure which is denser. This makes the cement particles to occupy less space and hence the overall structure acquires a greater porosity. Thus, it generates the loss of the mechanical properties of the material. When the degradation of the cement becomes critical, structural changes appear in the building. Thus, the next step is to monitor the vibration and the angular accelerations of the building.

A Wireless Sensor Network (WSN) is a set of devices that are connected wirelessly and are able to obtain information from the environment. These devices do not have wired infrastructure or centralized administration. They have low computing capacity and memory, and very low power consumption. In order to gather information from the environment they need a series of sensors capable of detecting chemical or physical magnitudes from the environment and transform them into electric variables [327][9]. There are multiple types of sensors that can measure many types of physical variables, but in our case we need to measure the humidity, temperature, vibration and angular accelerations.

There are multiple types of network topologies in WSNs. One of the most common ones, because of its energy efficiency, is the cluster-based topology. Its structure uses devices with more processing features in order to concentrate multiple leaf devices. They build a 2-layer architecture, where the leaf nodes are placed in the lowest layer and the cluster head form the highest layer allowing connections between clusters. This system increases considerably the scalability of the network. Moreover, there are many ways to connect cluster heads. One of them is using a wireless mesh network [328]. It combines the advantages of wireless technology at a minimum cost. In a mesh topology, each node is connected to many nodes. It allows building different paths from a source to a destination and it is capable of balancing the traffic load. Moreover, it supports fault tolerance, so when a node fails, the network can self-configure to find new routes.

A WSN deployment should ensure that the sensor node power consumption is low in order to prolong the network lifetime [327][161]. For this purpose, we should perform an optimal network implementation, and a correct selection of the medium access and routing protocols, with lowest power consumption [157], and an appropriate sensors design.

In this Section, we propose a non-invasive technique based on a wireless sensor network for the detection of the cement degeneration in constructions. It has 2 levels. The lowest level is formed by sensor nodes which are in charge of monitoring the environmental parameters. They measure the temperature, humidity, and structural movements of the construction in order to quantify the degeneration of cement. The highest level is formed by routing nodes that act as a cluster heads and form a wireless mesh network. The sensor node placement is studied in depth in order to predict very soon any deterioration of the building materials by monitoring the effects of the cement degeneration. 


\subsubsection{Node Description}

In order to implement our multisensor node, we can use several wireless modules [158]. These wireless modules are usually based on IEEE 802.11 or IEEE 802.15.4 standards. The main differences between them are the features such as number of ports o processor. It is possible to find modules based on open source code and modules with proprietary solutions. Table 4.9 shows some wireless modules which could be used in our proposal

Table 4.9. Features of the wireless modules.

\begin{tabular}{|c|c|c|c|c|c|}
\hline Model & Processor & \begin{tabular}{|l|} 
Wireless \\
Technology \\
\end{tabular} & \begin{tabular}{|l|} 
Power consumption \\
(From Catalogue)
\end{tabular} & Program code & Ports \\
\hline $\begin{array}{l}\text { V-Link -LXRS 8 Channel } \\
\text { Wireless Analog Sensor } \\
\text { Node } \\
\text { (MicroStrain) } \\
\text { (V-Link, 2013) } \\
\end{array}$ & $\mathrm{n} / \mathrm{a}$ & IEEE 802.15.4 & $\begin{array}{l}\text { R-T streaming: } 2.4 \mathrm{~mA} \\
\text { Datalogging: } \\
25 \mathrm{~mA} \\
\text { Sleeping: } 0.1 \mathrm{~mA}\end{array}$ & $\begin{array}{l}\text { Node } \\
\text { Commander }\end{array}$ & $\begin{array}{l}-8 \text { input channels } \\
\text {-internal } \\
\text { temperature } \\
\text { sensor }\end{array}$ \\
\hline $\begin{array}{l}\text { RN-XV } \\
\text { (Roving Networks) } \\
(\mathrm{RN}-\mathrm{XV}, 2013)\end{array}$ & $\begin{array}{l}\text { Microchip } \\
\text { processors }\end{array}$ & IEEE $802.11 \mathrm{~b} / \mathrm{g}$ & $\begin{array}{l}\text { Sleep: 4uA } \\
\text { Rx: } 40 \mathrm{~mA} \\
\text { Tx: } 180 \mathrm{~mA}\end{array}$ & $\mathrm{C} / \mathrm{C}++$ & $\begin{array}{l}-8 \text { general } \\
\text { purpose digital } \\
\mathrm{I} / \mathrm{O} \\
-3 \text { analog sensor } \\
\text { interfaces. } \\
\end{array}$ \\
\hline $\begin{array}{l}\text { PremierWave EN } \\
\text { (Lantronix) } \\
\text { (PremierWave EN, 2013) }\end{array}$ & $\begin{array}{l}\text { 32-bit ARM9 } \\
\text { Processor }\end{array}$ & $\begin{array}{l}\text { IEEE } \\
802.11 \mathrm{a} / \mathrm{b} / \mathrm{g} / \mathrm{n}\end{array}$ & $\begin{array}{l}\text { Standby: } 302 \mathrm{~mA} \\
\text { Tx: } 577 \mathrm{~mA}\end{array}$ & $\mathrm{C} / \mathrm{C}++$ & $\begin{array}{l}\text { interfaces that } \\
\text { include Ethernet, } \\
\text { Serial, USB, } \\
\text { I2C, and SPI } \\
\end{array}$ \\
\hline $\begin{array}{l}\text { RabbitCore RCM5400W } \\
\text { Series } \\
\text { (Digi International) } \\
\text { (RCM5400W,2013) }\end{array}$ & $\begin{array}{l}\text { 32-bit NS9215 } \\
\text { processor }\end{array}$ & IEEE $802.11 \mathrm{a} / \mathrm{b} / \mathrm{g}$ & $\begin{array}{l}\text { Max: } 786 \mathrm{~mA} \\
\text { Idle: } 533 \mathrm{~mA} \\
\text { Slepp: } 138 \mathrm{~mA}\end{array}$ & $\mathrm{C} / \mathrm{C}++$ & \begin{tabular}{|l}
-4 high-speed \\
UARTs \\
-12 -bit/8-channel \\
-64 GPIOs \\
-2 external IRQs \\
\end{tabular} \\
\hline $\begin{array}{l}\text { Waspmote Zigbee } \\
\text { (Libelium) } \\
\text { (Waspmote, 2013) }\end{array}$ & $\begin{array}{l}\text { Atmel ATmega } \\
1281\end{array}$ & IEEE 802.15.4 & $\begin{array}{l}\text { ON: } 15 \mathrm{~mA} \\
\text { Sleep: } 55 \mu \mathrm{A} \\
\text { Deep Sleep: } 55 \mu \mathrm{A} \\
\text { Hibernate: } 0.07 \mu \mathrm{A}\end{array}$ & $\mathrm{C}$ & $\begin{array}{l}-7 \text { Analog (I) } \\
-8 \text { Digital (I/O) } \\
-1 \text { PWM, } \\
-2 \text { UART } \\
\text {-I2C,USB,SPI } \\
\end{array}$ \\
\hline $\begin{array}{l}\begin{array}{l}\text { Waspmote } \\
\text { (Libelium) }\end{array} \\
\text { (Waspmote, 2013) }\end{array}$ & $\begin{array}{l}\text { Atmel ATmega } \\
1281\end{array}$ & IEEE $802.11 \mathrm{~b} / \mathrm{g}$ & $\begin{array}{l}\text { On: } 33 \mathrm{~mA} \\
\text { Sleep: } 4 \mathrm{uA} \\
\text { Rx: } 38 \mathrm{~mA} \\
\text { Tx: } 38 \mathrm{~mA}\end{array}$ & $\mathrm{C}$ & $\begin{array}{l}-7 \text { Analog (I) } \\
-8 \text { Digital (I/O) } \\
-1 \text { PWM, } \\
-2 \text { UART } \\
\text {-I2C,USB,SPI } \\
\end{array}$ \\
\hline $\begin{array}{l}\text { FlyPort } \\
\text { (OpenPICUS) } \\
\text { (FlyPort features, 2012). }\end{array}$ & $\begin{array}{l}\text { 16 Bit Processor } \\
\text { PIC24FJ256 }\end{array}$ & IEEE802.11 b/g/n & $\begin{array}{l}\text { Wi-Fi connected: } \\
\text { 127.5mA } \\
\text { Standby: } 38.5 \mathrm{~mA} \\
\text { Sleep: } 11 \mathrm{uA}\end{array}$ & $\mathrm{C}$ & $\begin{array}{l}-10 \text { Digitals I/O } \\
-4 \text { analog inputs } \\
-1 \text { UART. } \\
-1 \text { SPI Port. } \\
-1 \text { I2C Port. }\end{array}$ \\
\hline
\end{tabular}

Between them we have chosen Flyport because its features are enough for our proposal. Its price is the cheapest one and the programming tools are easy to get. FlyPort is a programmable compact Wi-Fi module based on the open source platform openPICUS. It can be used without the need of using an external host processor, such as a computer. It combines the power of the processor (Microchip PIC24FJ256K) with the connectivity of Microchip's transceiver with Wi-Fi certification.

This module is composed of two parts. The first one has the Wi-Fi module, formed by the transceiver and the processor, as well as different input/output, buses, etc. The second part is the programmer device, called USB Nest. Figure 4.46 shows an image of the device. 


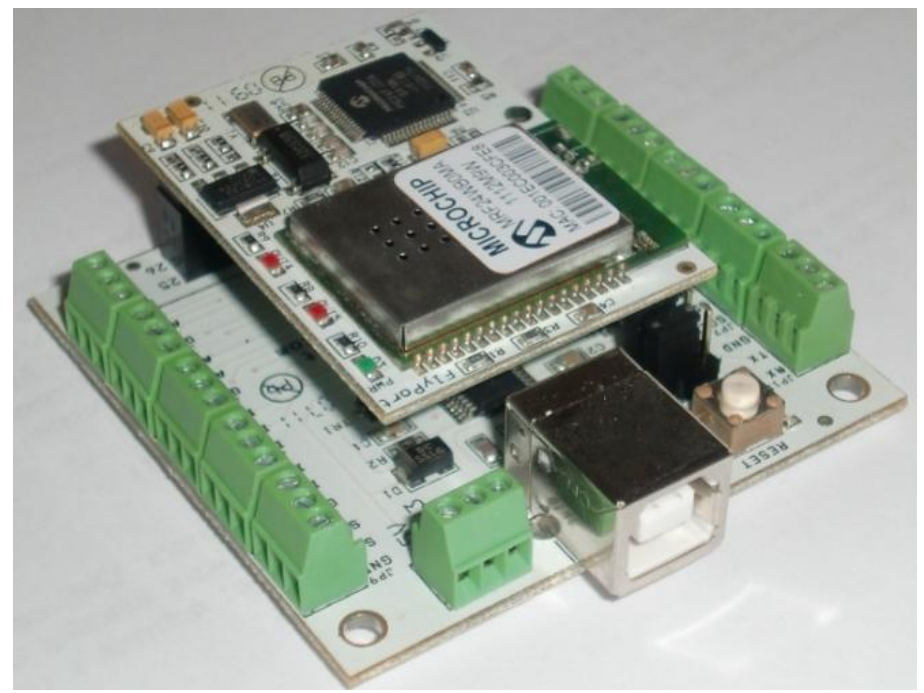

Figure 4.46. FlyPort module with USB Nest programmer

The advantage of this device, compared to some others shown in the market, is that it is modular and can be easily programmed in $\mathrm{C}$. The use of such modules facilitates the systems integration, the development of new applications based on the concept of Internet of Things (IoT), and the development of sensor nodes, capable of being integrated into more complex networks.

This device has the following features:

- 16 Bit Processor PIC24FJ256, 256K Flash, 16K Ram,16Mips@32Mhz.

- Transceiver MRF24WB0MB, 802.11b/g/n Wi-Fi certified.

- Power Supply 5V or 3.3V, integrated LDO.

- Integrated RTC 32,768 Khz quartz onboard.

- 10 Digitals I/O: remappable at Runtime, 9 of these can be used as PWM.

- 4 Analog inputs: 10 bits ADC, Vref=2.048 V.

- 1 UART.

- 1 SPI Port.

- 1 I2C Port.

- Reset (Active Low).

- Small Size: 35X48X7 mm.

- Weight: $11 \mathrm{gr}$

Figure 4.47 shows the current consumption of the device during 75 seconds. 


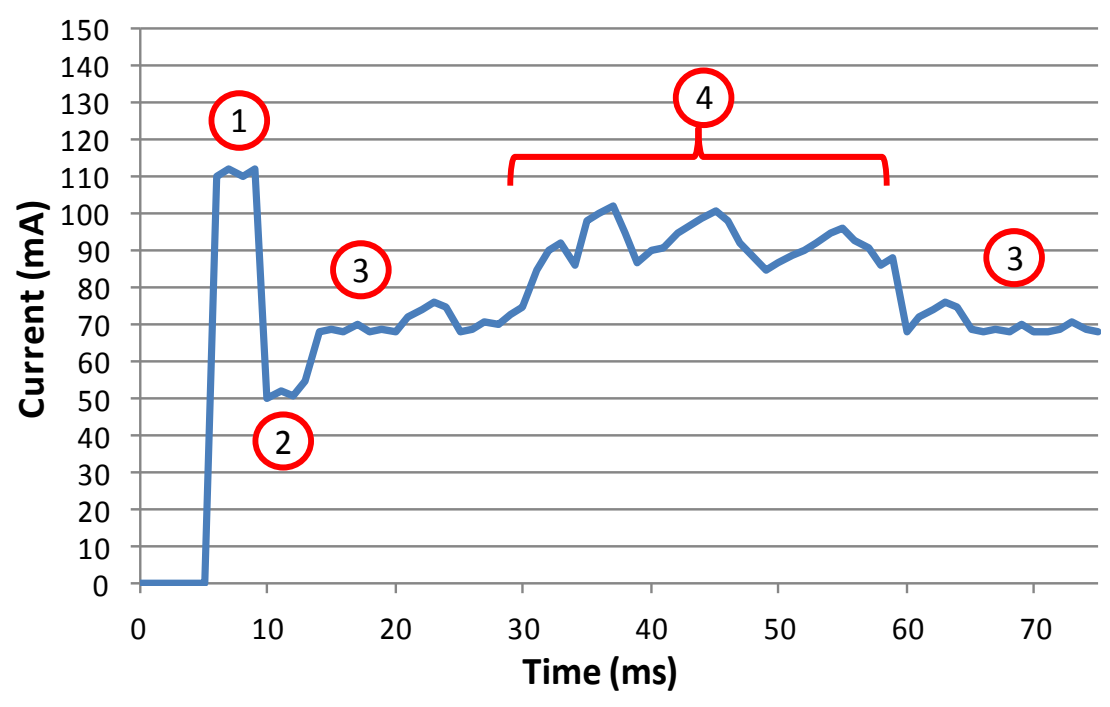

Figure 4.47. FlyPort current consumption

In order to analyze the device power consumption, we must consider that the device is being fed with $3.3 \mathrm{~V}$. Keeping in mind this, we observed that it has a current consumption of $110 \mathrm{~mA}$ in its initialization phase (phase 1). This process takes between 3 and 4 seconds. While the node is not associated with any network (phase 2), its consumption is around $50 \mathrm{~mA}$. It reaches up to $77 \mathrm{~mA}$ when the node is connected to a network (phase 3 ). When the node is transmitting and receiving data, its consumption fluctuates between $102 \mathrm{~mA}$ and $85 \mathrm{~mA}$ (phase 4).

\subsubsection{Sensor description}

In order to take the values of the environmental parameters, such as temperature, humidity, vibration or angular accelerations, the first step is to have the appropriate physical sensors in order to capture and convert the parameter value to a voltage, current or resistance value. This value is proportional to the measured variable. We also need to use a system, based on batteries, that allows us to feed the entire system (physical sensors and sensor node).

We have developed 4 sensors, which are the most appropriate to detect the aluminosis in the reinforced concrete. They are temperature sensor, humidity sensor, vibration sensor and a gyroscope sensor. In this section, we show the developed sensors and their schematic. In addition, we describe their operation and show the power consumption of each sensor.

\subsubsection{Temperature sensor}

The LM335 is a precision temperature sensor that can be calibrated easily. It operates as a 2terminal Zener diode, where the breakdown voltage is directly proportional to the absolute temperature, having increments of $10 \mathrm{mV} /{ }^{\circ} \mathrm{K}$ (see Figure 4.48). 

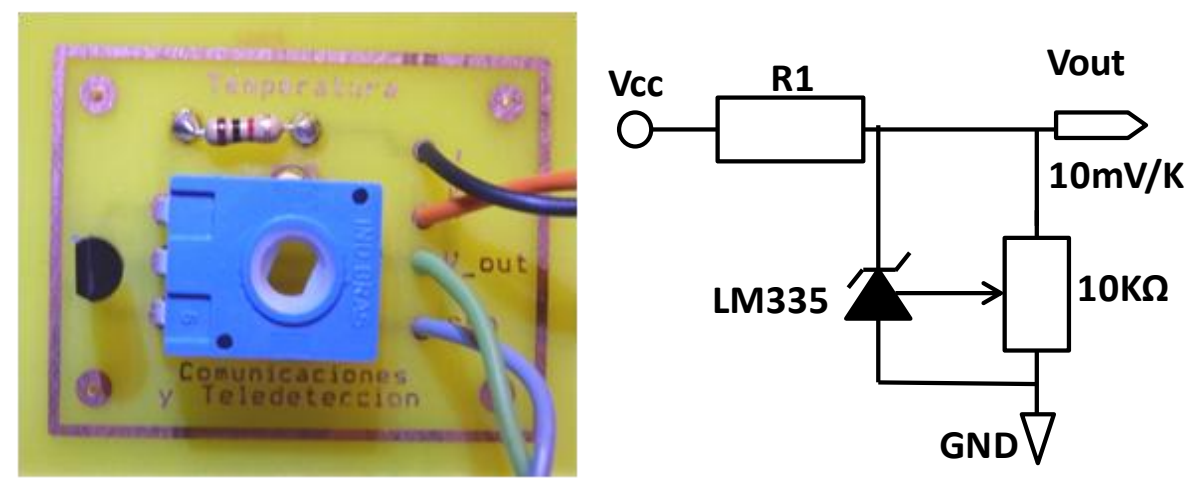

Figure 4.48. Schematic and image of the temperature sensor.

LM335 is calibrated at $25{ }^{\circ} \mathrm{C}$. It has an output voltage of $2.982 \mathrm{~V}$, introducing an error lower than $1{ }^{\circ} \mathrm{C}$ in a range of $100{ }^{\circ} \mathrm{C}$ temperature. Its output is linear, unlike other temperature sensors. In order to implement our sensor, we used a $1 \mathrm{~K} \Omega$ resistor and a $10 \mathrm{~K} \Omega$ potentiometer, which helped us to calibrate the sensor.

Equation 4.16 shows the relationship between the voltage supplied by the sensor and the measured temperature.

$$
T\left({ }^{\circ} \mathrm{C}\right)=\left(100 \cdot V_{\text {out }}\right)-273
$$

If we analyze their power consumption as a function of the temperature (see Figure 4.49), we see that it has a linear behaviour, with a consumption of $0.34 \mathrm{~mA}$ when there is $25^{\circ} \mathrm{C}$.

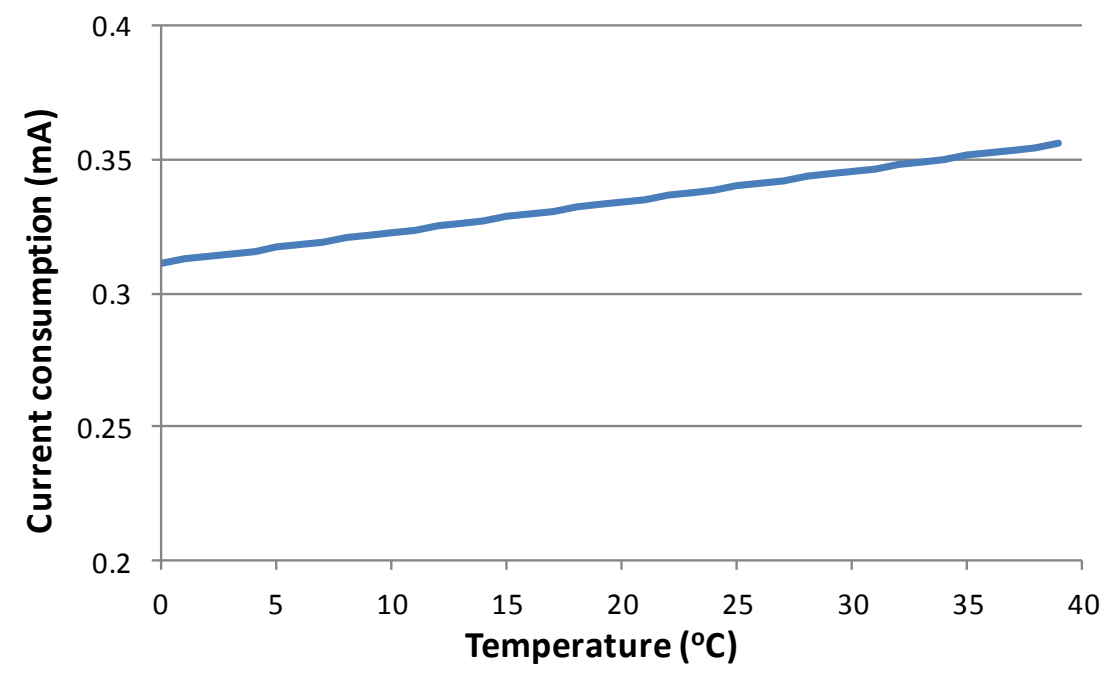

Figure 4.49. Current consumption vs. Temperature for temperature sensor.

\subsubsection{Vibration sensor}

The micro-vibration sensor MVS0608-02 is formed by a metal capsule containing a small sphere of metal. It is used to detect small movements and vibrations through the movement of the microsphere.

When the ball moves between two contacts, there is a change of the resistance between both external connection pads. It varies from several $\mathrm{M} \Omega(>30 \mathrm{M} \Omega)$ to less than $100 \Omega$. It is fully 
passive sensor. It does not require signal conditioning and can operate with very low currents (around $0.2 \mu \mathrm{A}$ ). Figure 4.50 shows the current consumption of the sensor as a function of the resistance value of the vibration sensor.

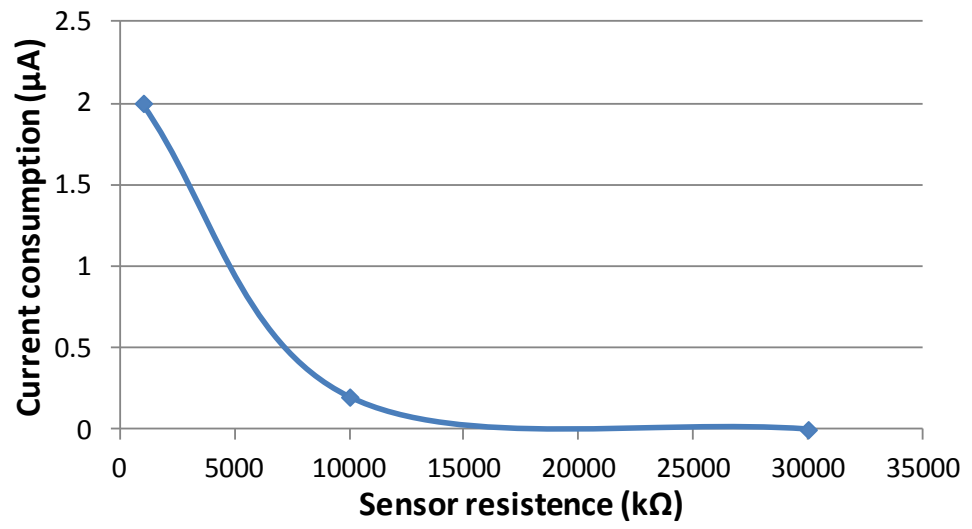

Figure 4.50. Current consumption, depending on the sensor resistance value.

The circuit for using this sensor is shown in Figure 4.51. The value of the other components are $\mathrm{R} 1=5.1 \mathrm{M} \Omega, \mathrm{R} 2=1 \mathrm{~K} \Omega$ and $\mathrm{C} 1=100 \mathrm{pF}$.
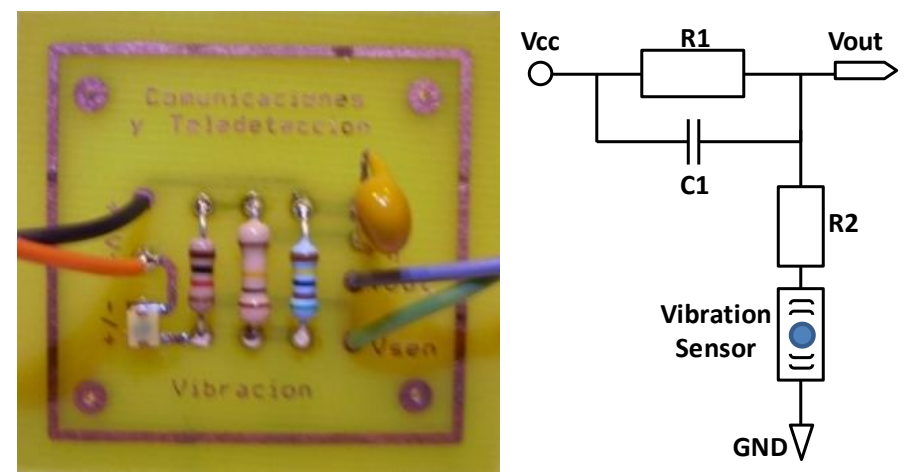

Figure 4.51. Schematic and actual image of the vibration sensor.

In order to reduce the sensitivity of the sensor and facilitate the measurement of the parameters, we can vary the value of $\mathrm{C} 1$ capacitor to a value ranging from $10 \mathrm{pF}$ to $1 \mathrm{nF}$. For higher values of $\mathrm{C} 1$, e.g. $100 \mathrm{nF}$, the output peaks can come up to an average analog value, which is easier to monitor. The average power consumption of the sensor, when it is working, is about to $0.7 \mu \mathrm{A}$. When the sensor is in repose its consumption is around $0.55 \mu \mathrm{A}$.

\subsubsection{Humidity sensor}

The type of humidity sensor selected for this node is resistive. That is, its resistance varies with the relative humidity ( $\mathrm{RH}$ in \%). This variation is shown in Table 4.10.

Table 4.10. Relative humidity and its resistant value

\begin{tabular}{|c|c|c|c|c|c|c|c|c|}
\hline \% RH & $\mathbf{2 0}$ & $\mathbf{3 0}$ & $\mathbf{4 0}$ & $\mathbf{5 0}$ & $\mathbf{6 0}$ & $\mathbf{7 0}$ & $\mathbf{8 0}$ & $\mathbf{9 0}$ \\
\hline Resistive Value $(\mathrm{K} \Omega)$ & 5000 & 920 & 220 & 66 & 23 & 9 & 4.2 & 1.9 \\
\hline
\end{tabular}


Because it is complicated to measure the resistance value of the sensor when the humidity value increases, we have designed a resistive circuit. It is fed with $3.3 \mathrm{~V}$ and our output signal is directly the drop voltage across the sensor.

The circuit used in this case is shown in Figure 4.52, where R1 =1 M $\Omega$.
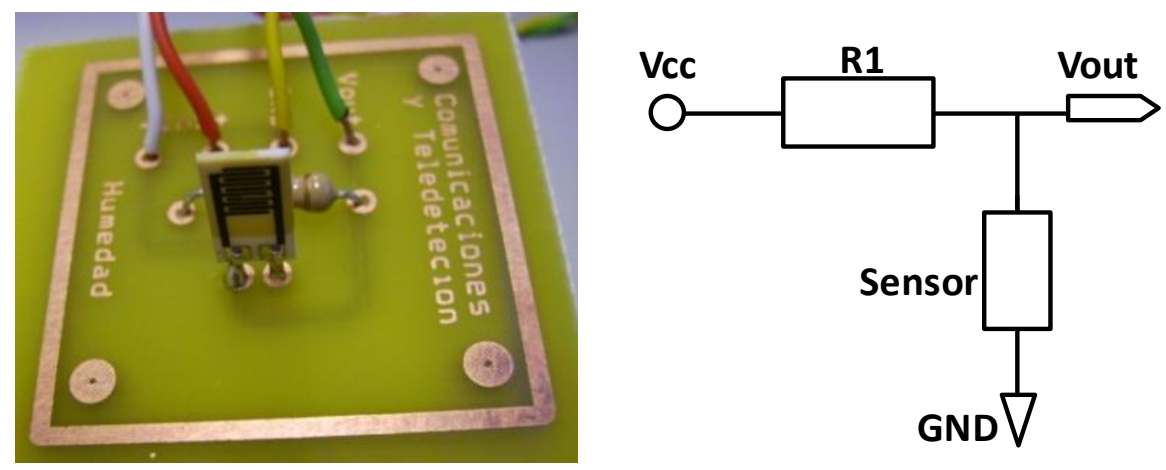

Figure 4.52. Schematic and actual image of the humidity sensor

Figure 4.53 shows the current consumption of the humidity sensor, in $\mu \mathrm{A}$, as a function of the $\mathrm{RH}$, in \%. As we can see, when RH increases, the current value decreases. It reaches 0 A when RH is above $90 \%$.

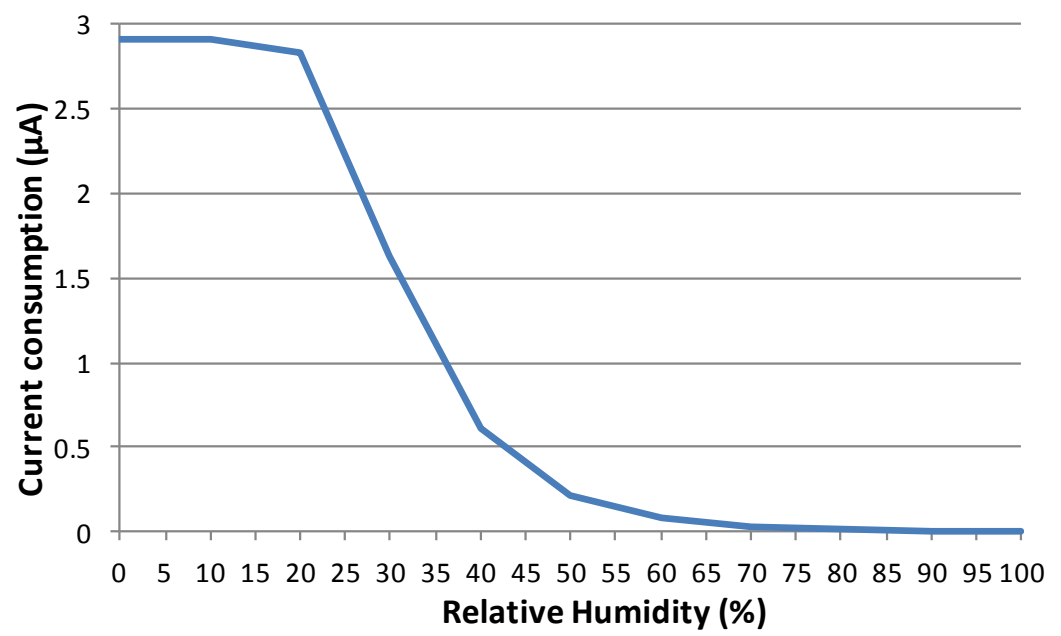

Figure 4.53. Current consumption vs. RH in \% for humidity sensor.

\subsubsection{Gyroscope sensor}

We have chosen LY330ALH sensor. Its size is around $5 \mathrm{~mm}$. It allows high levels of integration. The device includes a sensor element comprising a single driving mass, maintained in continuous oscillation and capable of reacting when an angular velocity is applied. It is based on the Coriolis principle. The sensor output provides an analog output voltage proportional to the angular velocity measurement.

The current consumption of this sensor is quite high during its working phase (4.2 mA), while in power-down mode its consumption is close to $5 \mu \mathrm{A}$. These sensors are mostly in operation mode, which requires less energy. They are activated only when a change in the measured magnitude is registered. It is fed at 3.3 V. Figure 4.54 shows the image of the circuit and its schematic. 


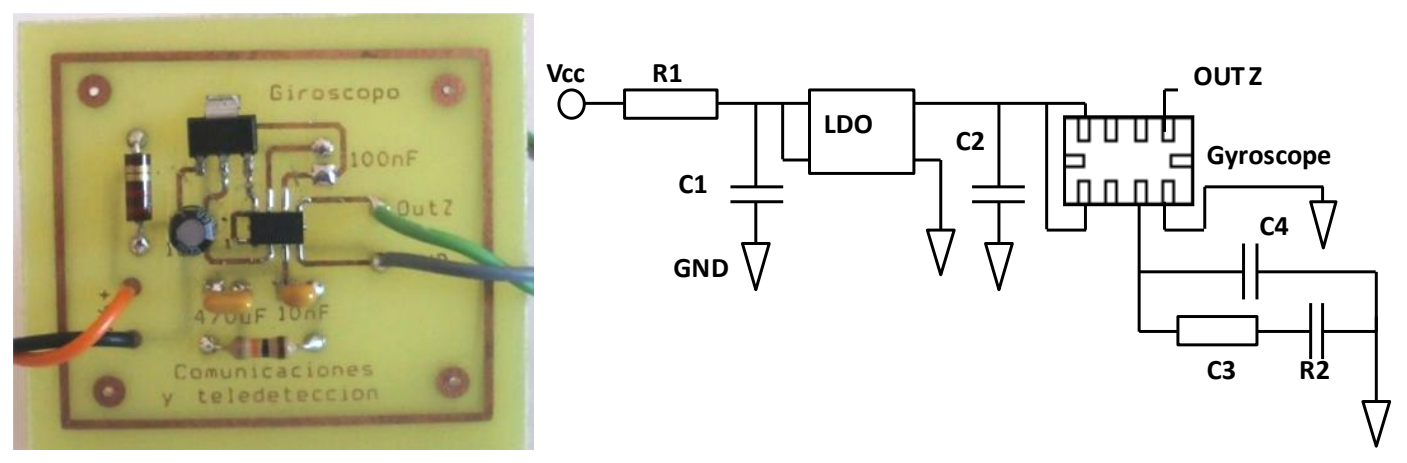

Figure 4.54. Schematic of the gyroscope sensor

\subsubsection{Electric power system}

The power system is based on the use of the NCP565 voltage regulator, which transforms the input voltage up to $18 \mathrm{~V}$ to an output voltage of $1.2 \mathrm{~V}, 1.5 \mathrm{~V}, 2.8 \mathrm{~V}, 3.0 \mathrm{~V}, 3.3 \mathrm{~V}$ or even other standard values, with a simple calculation of R1 and R2 resistor values. The circuit and its schematic are shown in Figure 4.55.
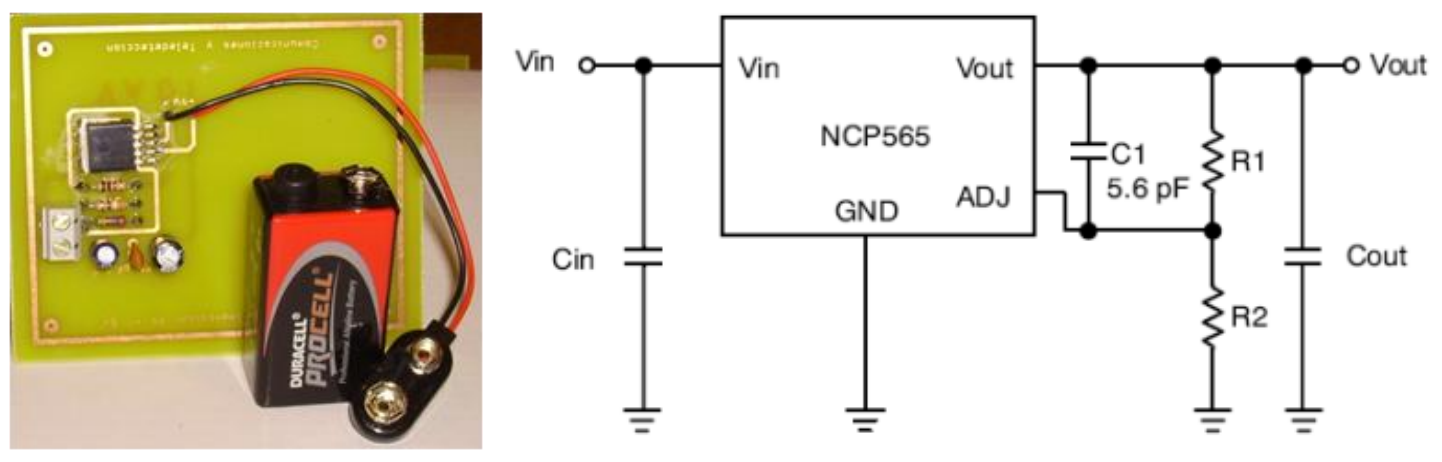

Figure 4.55. Power system image and its schematic.

Equation 4.17 shows the relationship between the output voltage and the values of the other components.

$$
V_{\text {out }}=V_{\text {ref }} \cdot\left(\frac{R_{1}+R_{2}}{R_{2}}\right)-I_{\text {Adj }} \cdot R_{2}
$$

In our case, the input voltage is provided by a $9 \mathrm{~V}$ battery and it is obtained an output voltage of $3.3 \mathrm{~V}$. In order to obtain it, the value of the resistors and capacitors are $\mathrm{R} 1=2.6 \mathrm{k} \Omega, \mathrm{R} 2=1 \mathrm{~K} \Omega$, Cin $=150 \mu \mathrm{F}$ and Cout $=1 \mathrm{uF}$.

Figure 4.56 shows the current consumption, in $\mathrm{mA}$, of the whole system when it is collecting data from 4 sensors simultaneously. As we can see, the average consumption stands at around the $1.1 \mathrm{~mA}$. 


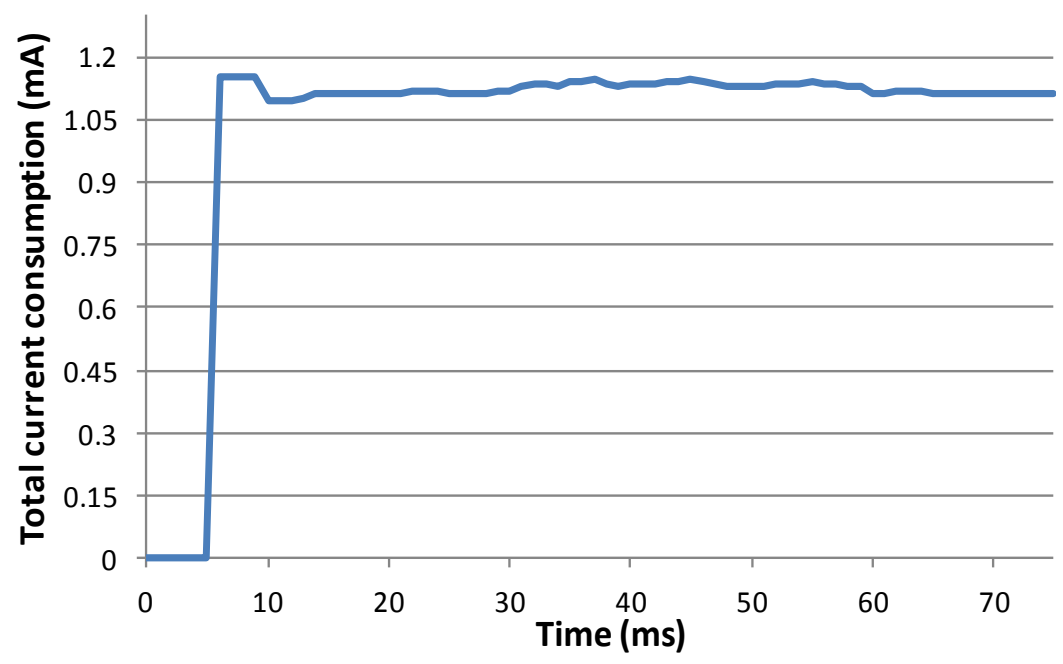

Figure 4.56. Total current consumption for sensor node.

\subsubsection{Multisensor Node.}

As we have described before, we selected Flyport for the multisensor node. It supports up to 4 analog sensors and 10 digital sensors. The power supply circuit, along with the battery, is used to feed the sensor nodes and the transducers. Figure 4.57 shows a wireless multisensor node with 3 physical sensors.

\subsubsection{Adequacy of the Sensor Nodes to Protect them from the Environment.}

Multisensor nodes (and the routers, which are described later) are placed in a hostile environment and are prone to be damaged because of the oxidation. In order to keep the devices safe and avoid unexpected failures, we protect them using a waterproof case with some kind of copolymers polypropylene or PVC with neoprene gaskets. These types of boxes have the advantage of keeping its contents, isolated from the environment, concretely from the humidity and temperature.

Our sensor node, which consists of the feeding system, 4 sensors and the Flyport module, is introduced in a sealed box. However, the integrated circuits in charge of measuring the temperature and humidity are in contact with the medium. Therefore, they are fixed on the surface of the case and they are wired to its conditioning circuit through small sealed holes.

The routers are protected from the adverse environment using the same procedure. It uses a wired power cable that leaves to the exterior of the case using sealed connectors, which are formed by a nut system and neoprene gasket. 


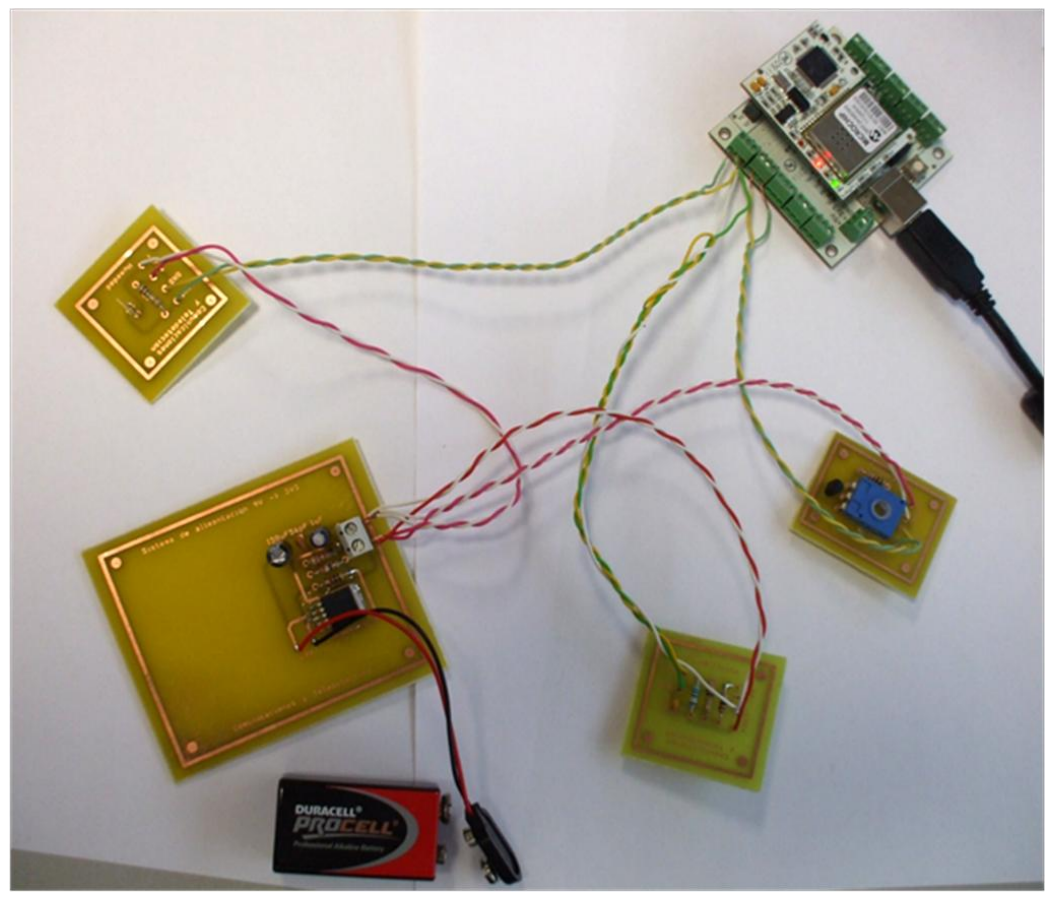

Figure 4.57. Image of a multisensor node with its physical sensors

\subsubsection{Decision Algorithm.}

In order to determine if the structure that is being monitored has some sort of movement or vibration, we should acquire and analyze the measured parameters.

The system takes the data and compares them with previous stored data. If these values maintain the same values, the system remains at rest until the timer $\mathrm{P}$ expires which specifies a new data query

The system checks if the temperature and humidity have changed. If humidity and Temperature are higher, the sample period will decrease. If humidity and Temperature are lower, the sample period will increase.

Finally, the data of sensors are saved. After that, the data are sent to the server and the system will return to the "Sensing of data sensor" state.

Figure 4.58 shows the decision algorithm used for processing our variables.

The sampling frequency will depend mainly on the geographical location of environmental parameters and the state of the building.

For normal situations where it is known that the building suffers from some type of disease, we can define a period of taking measurements once per quarter.

We will establish additional measures few weeks after a period of heavy rains, water leaks affecting the structure or overloaded of structure. In these periods, we establish intakes daily measurements until the structure recovers the correct humidity levels. Throughout the day, the best time to take the measures will be at 4:00 AM and 4:00 PM. These are the moments of day which higher temperature and humidity differences are registered. 


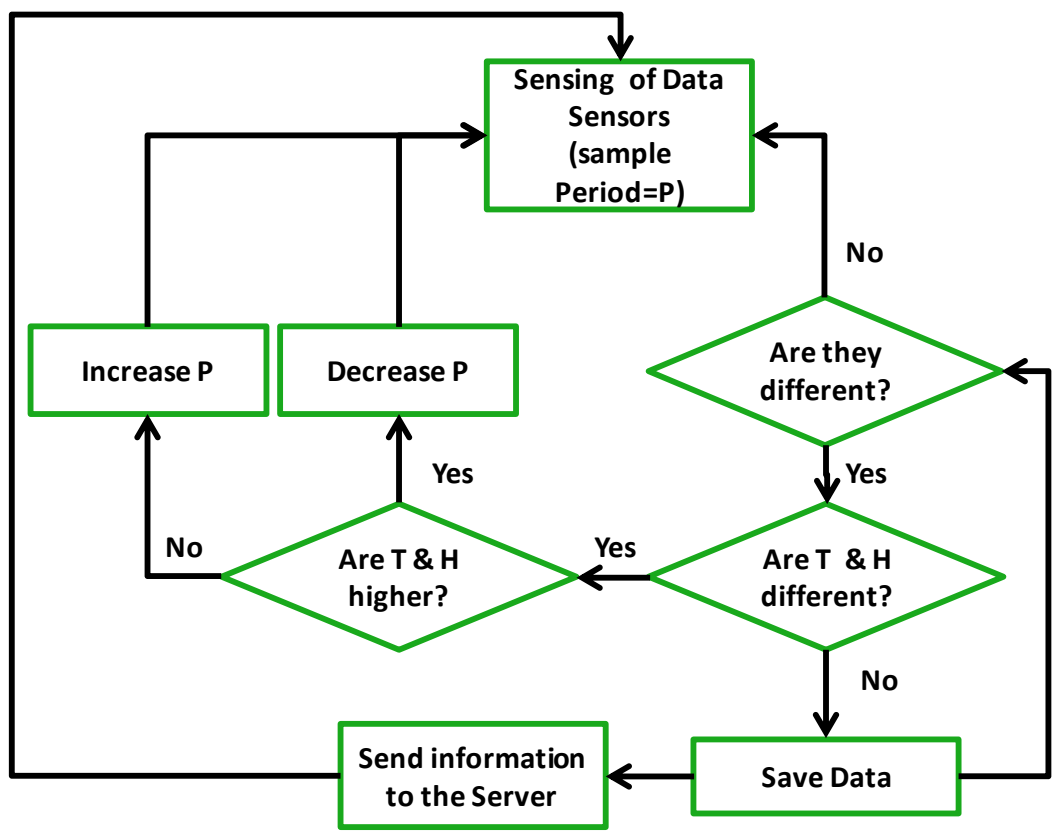

Figure 4.58. Decision Algorithm if a building is suffering a collapse of some element of its structure.

\subsubsection{Position of the Sensors in the Structure}

Pathological degeneration processes can cause concrete and resistant structural elements cracking and breaking. These processes are associated with the appearance of stains and efflorescence on the concrete surface.

The materials of the structures can undergo chemical reactions that worsen the cohesion of the material, increasing dysfunctionality of the reinforced concrete. This deterioration of the material can generate pathologies such as aluminosis, carbonation and reinforcement corrosion, causing the disintegration of the concrete mass by the lack of resistance. When the laminated steel for construction is corroded, it is able to expand up to several times its section. The overlying concrete, which may suffer its own pathologies, suffers these expansion efforts and it is unable to counteract this tension. However, there is a very important factor, which is difficult to predict, that can be generating segregation or disintegration of the concrete when the operators are pouring it. These failures can result in large voids or porosity, allowing the excessive moisture in the material, weakening the places previously described. It can also cause a failure in the armor coating, causing deprotection of the armor and, thus, facilitating the action of moisture on steel.

The most suitable place for the location of the sensors is the structural elements of the first order: support shoes, bearing walls, supports, beams and slabs. When there are diseases, these are usually caused by a failure of the main elements. For this reason, the elements that are not concrete, and have no structural function, do not need be monitored.

However, there may be other injuries that cause the same effects and give false positives. To minimize these false positives, we must know the monitored material. Thus, identifying the types of degradation, such as the consequences of corrosion in reinforced concrete that are moved to the surface of the concrete, or the lack of cohesion of the concrete, we can detect failures of both materials. 
It is useful to know that during the first months or years of the building lifetime, the structure undergoes differential movements' to settle down. After its stabilization, if there are not decompensate loads, there should not be movement (except in structural failure cases, mainly caused by the aforementioned diseases or field failures). During the first months of the structure lifetime, several false positives could be detected.

Visual effects always manifest in the weakest places. The most suitable places to detect these effects are corners and the intermediate space between them. If we perform a mechanical analysis using physical laws, we can see that the best locations to place the sensors in the structure are those where the mechanical force moment diagrams reach their maximum efforts. These places are the bending, axils and shear. They must be able to withstand the maximum loads for which they were designed. If there is a failure in these places, because of the lack of resistance, these places will be the first ones to endanger the structure. In regular conditions, we know that bending moments diagram have their maximum value in the supports and in the center of the beam. Thus, the axial diagram presents the optimum point in the bottom or the top of the pillars. These places are associated with the bending stress and shear which are placed at one third of the length of the beam

Because the main cause of these diseases is the humidity and our sensors are able to detect the moisture, we must know the different sources of moisture:

- Filtration.

- Runoff.

- Osmosis.

- Capillarity.

- Condensation by direct contact with the source of moisture and land cover (associated with attack alkali in alkali lands)

- Facilities of water (evacuation, supply, central heating, solar heating, pools, reservoirs, etc).

- Humid environments caused by different sources: lakes, snow in the mountains, tropical environments or zones near to the sea, which are associated with alkali attack described above.

Therefore, the best way to monitor these pathologies is to place the sensor node as close as possible to these sources.

Figure 4.59 shows the strategic points in a simple structure for installing the multisensor nodes. The different locations have been selected based on their proximity to a source of moisture, alkalis and sulfates.

In WSN used to monitor environments, we can find mobile nodes and fixed nodes. When the network consists of mobile nodes, it is necessary keep them localized by positioning algorithms [329]. Our network does not this problem because the nodes are in a fixed and known position. Keeping in mind these previous considerations, we should also note that due to the nature of some building materials, the location of the sensor nodes (considering the position of the AP), can be very critical [330].

In order to maintain a good level of coverage in the whole of floor, we should avoid to placing the nodes behind walls next to a toilet. The losses in these places can be up to $20 \mathrm{~dB}$. The main cause of this behaviour is the pipes embedded in the walls. So, a special treatment of these walls should be taken in the computation of coverage in the building [83]. 


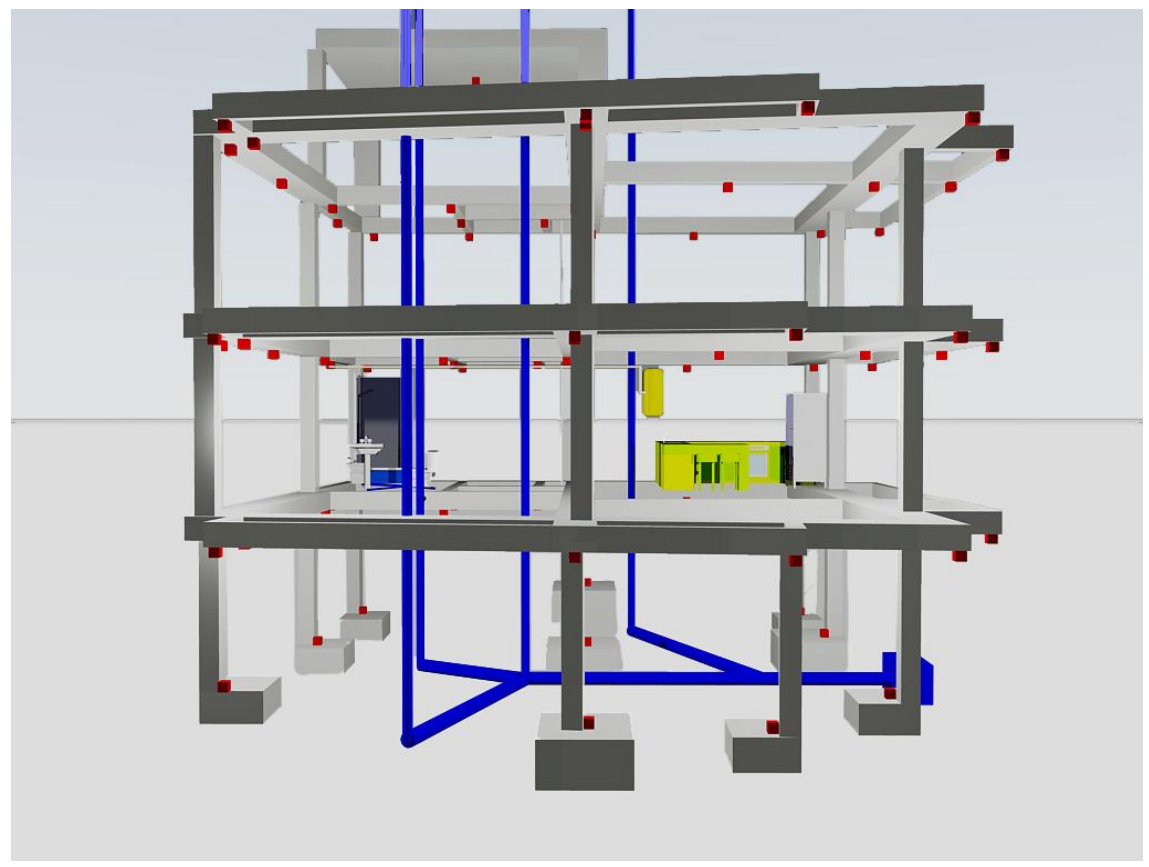

Figure 4.59. Multisensor node locations in a structure.

Furthermore, metal wrought components placed between plants acts as a shielding. In this case, a floor is considered as an infinite surface compared with the AP and the signal meanwhile, if we consider a pillar, it is only an object where signals can reflect or refract. Sensor nodes should not place exactly above the AP of the inferior plant or vice versa. Although, the distance between them is low, it is possible to receive a slight signal. In addition, the signal attenuates quickly as soon as this distance increases. A good option, if possible, is to place the APs in the crystal interior patios (crystal skylights). The signal propagation through the crystals is better than the propagation through walls and metal wrought [97].

\subsubsection{Wireless Sensor Network}

In this section we explain the designed topology and how sensor nodes are connected. In addition, we show the network operation and the volume of data flowing through it. This section also shows the web interface of the sensor nodes.

\subsubsection{Developed topology}

The Wireless Sensor Network is formed by two layers. The lowest level is formed by sensor nodes which measure the temperature, humidity, and structural movements of the construction. The highest level is formed by the routing nodes. Each router node form a cluster and acts as cluster head. Router nodes have higher computing capacity, memory and power than sensor nodes. Sensor nodes must be authenticated with the routing nodes in order to join the WSN.

Router nodes form a wireless mesh network in their level. They build the backbone of the WSN. Moreover, router nodes allow higher distances between them than with sensor nodes.

In order to deploy our network, we have chosen Linksys WRT54GL, with a customized firmware, as the router nodes. In this case, we used DD-WRT, which is free Linux-based firmware 
for several wireless routers. It allows high flexibility and control over the device. The routing protocol used to route the information through the router nodes level has been OLSR [331], which presents the best performance for the selected firmware [300]. Each wireless sensor node connects with the router node using an infrastructure type connection. Figure 4.60 shows the designed network topology.

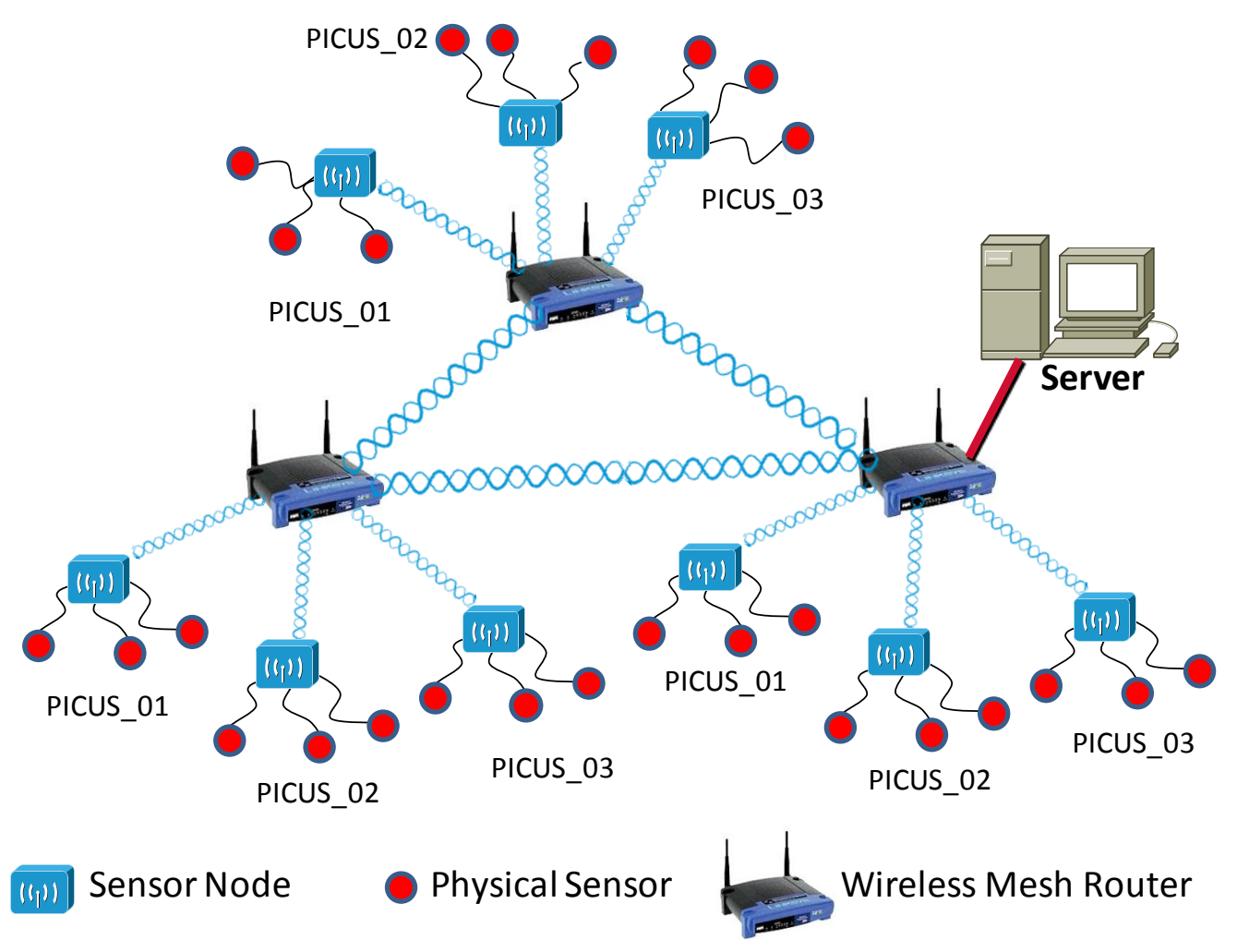

Figure 4.60. Network topology

In order to automatically let the system know where each sensor node is placed and allow a dynamic network, we used a hybrid stochastic approach for wireless sensors self-location in indoor environments. It was developed by us in [267]. It is based on a combination of deductive and inductive methods to determine their positions using WLAN technology. But, it can be replaced by any other system [108].

\subsubsection{Network operation and its performance.}

All sensor nodes must be registered with a router node. When a new sensor node appears in the network it broadcasts a probe packet. Then, it selects the best router node based on the received probe-response packets (using the radio signal strength indicator). Next, it authenticates with the router node. After it, the sensor node sends the association request to the router node. The sensor node registration procedure is shown in Figure 4.61. Once the network is formed and nodes are registered with their routers, our sensor network can start working. 


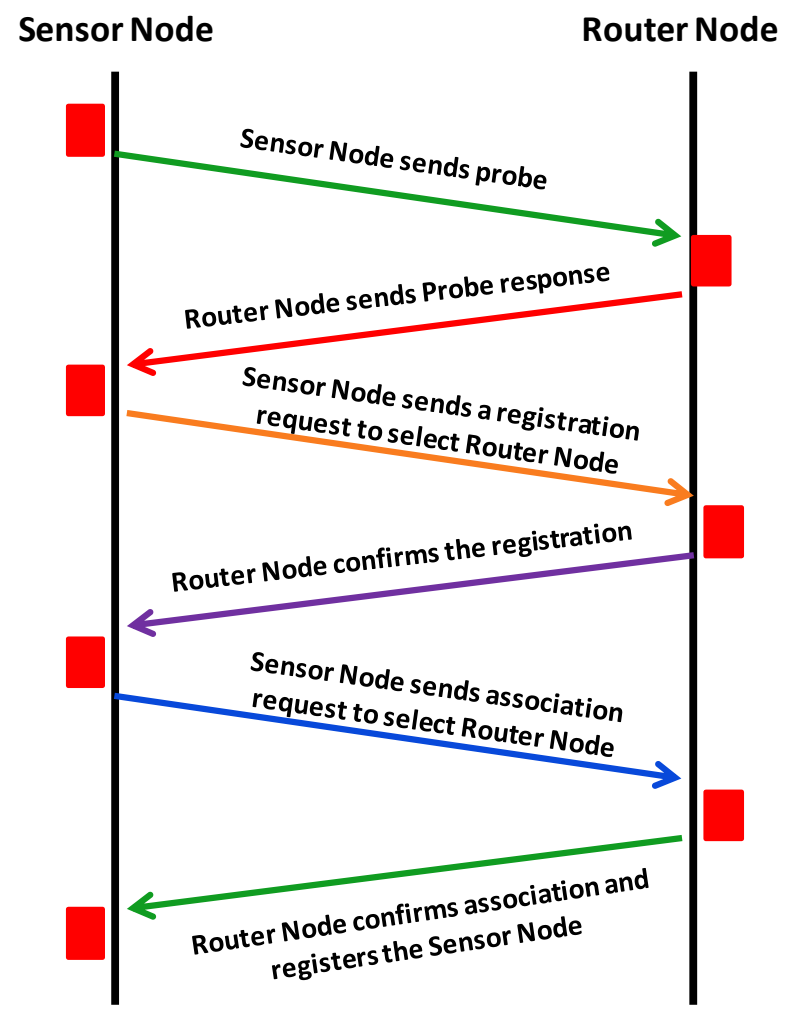

Figure 4.61. Sensor node registration protocol

Then, the node remains in sleep mode, but every hour it wakes up, senses the environment and sends the data to the router in order to forward it to the server. This procedure takes about 2 minutes. Node can also wake up when the physical sensor registers an event (building movement or vibration) or there is a new reconnection. While it does not gather any data from the environment, the node turns off its radio, reducing their energy consumption down to 25-30 mA. (a quarter of its total energy consumption). Thus, we are reducing the power consumption of each device.

Figure 4.62 shows the bandwidth consumed in the network, during a connection between the $45^{\text {th }}$ second and $100^{\text {th }}$ second. We can see that when there is a connection, it is generated a number of control packets, which are responsible to register the nodes in the router. These packages are small, having a bandwidth consumption close to $8-10 \mathrm{Kbps}$. When the node wakes up (at about the $65^{\text {th }}$ second), it senses the environment and, then, it sends the registration to the router and, after it, sends the sensed data. We can see a punctual increase of the bandwidth of the network at about the $68^{\text {th }}$ second, which reaches $244 \mathrm{kbps}$. Finally, during next 10 seconds, the node is sending data to its router. There is an average bandwidth consumption of $125 \mathrm{kbps}$.

The sensor information is displayed on the web page of each node. The data shown are updated every $50 \mathrm{~ms}$. It is also transmitted to the server. Figure 4.63 shows the http packets generated during the 10 seconds used to send the sensed data. The number of packets in the network is around 125 packets per second, taking into account the http packets and control packets sent through the network.

Finally, Figure 4.64 shows the amount of bytes needed to send the sensed data during approximately 10 seconds. The average value of bytes generated during these 10 seconds is 119.1 bytes. 


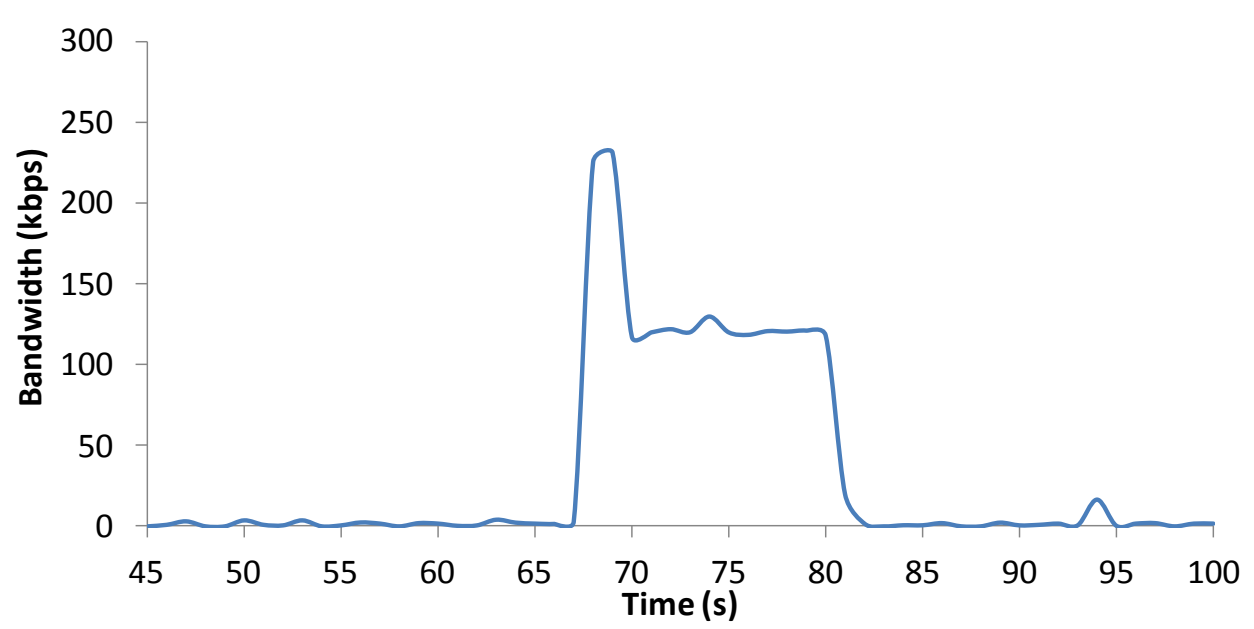

Figure 4.62. Bandwidth consumed by the network when a connection is performed

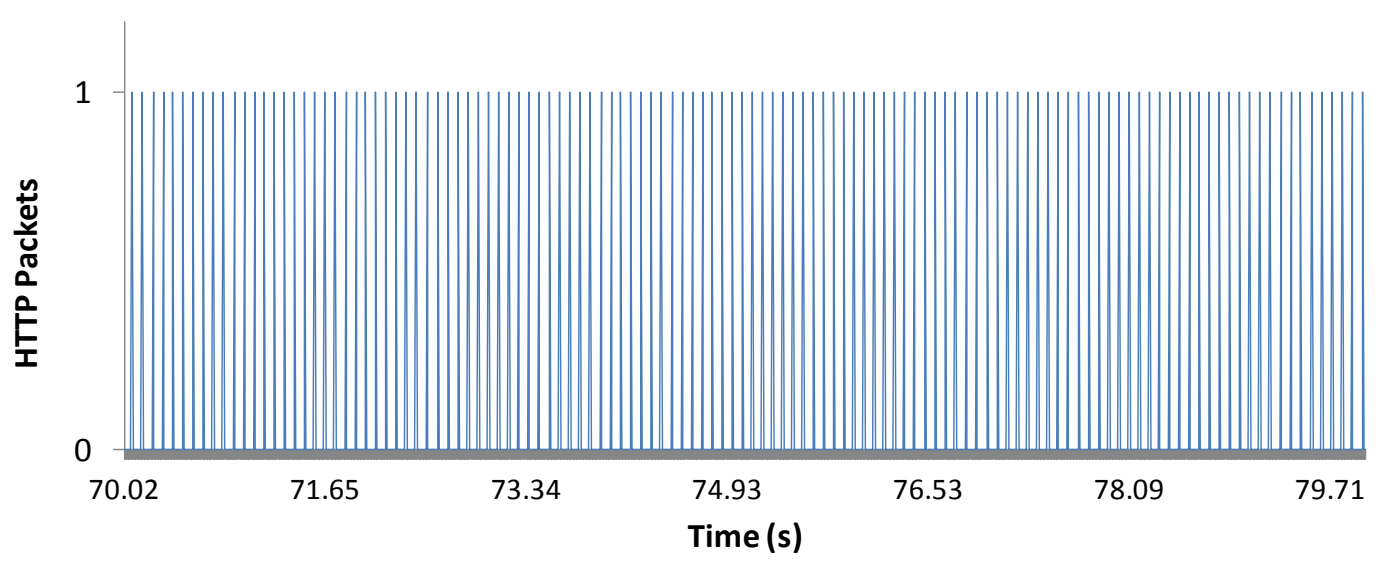

Figure 4.63. Http packets in the network when sending the sensed data

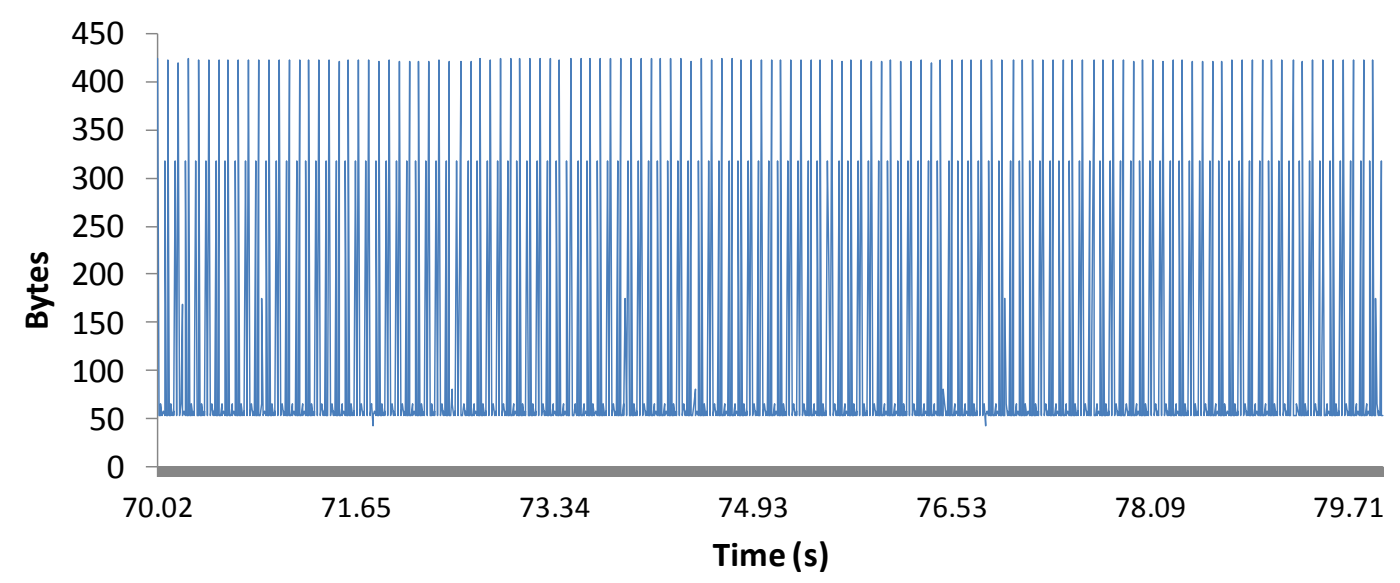

Figure 4.64. Bytes in the network during the 10 seconds 


\subsubsection{Web interface for data query}

Although each sensed data of each sensor node is stored in the central server, each sensor node is able to show its sensed values using a web server placed inside it, which is accessible from anywhere of the network. It shows the gathered data with update intervals of 0.05 seconds. Figure 4.65 shows an image of its web interface. Each node can be reached via IP address. As it is shown in Figure 4.65, the reached node is identified in the picture as "Active Node". In this case, we are querying the node PICUS 01, which has 3 sensors connected: temperature, humidity and vibration. In addition, we also see information about the activity of the digital inputs/outputs, identified as LEDs and buttons.

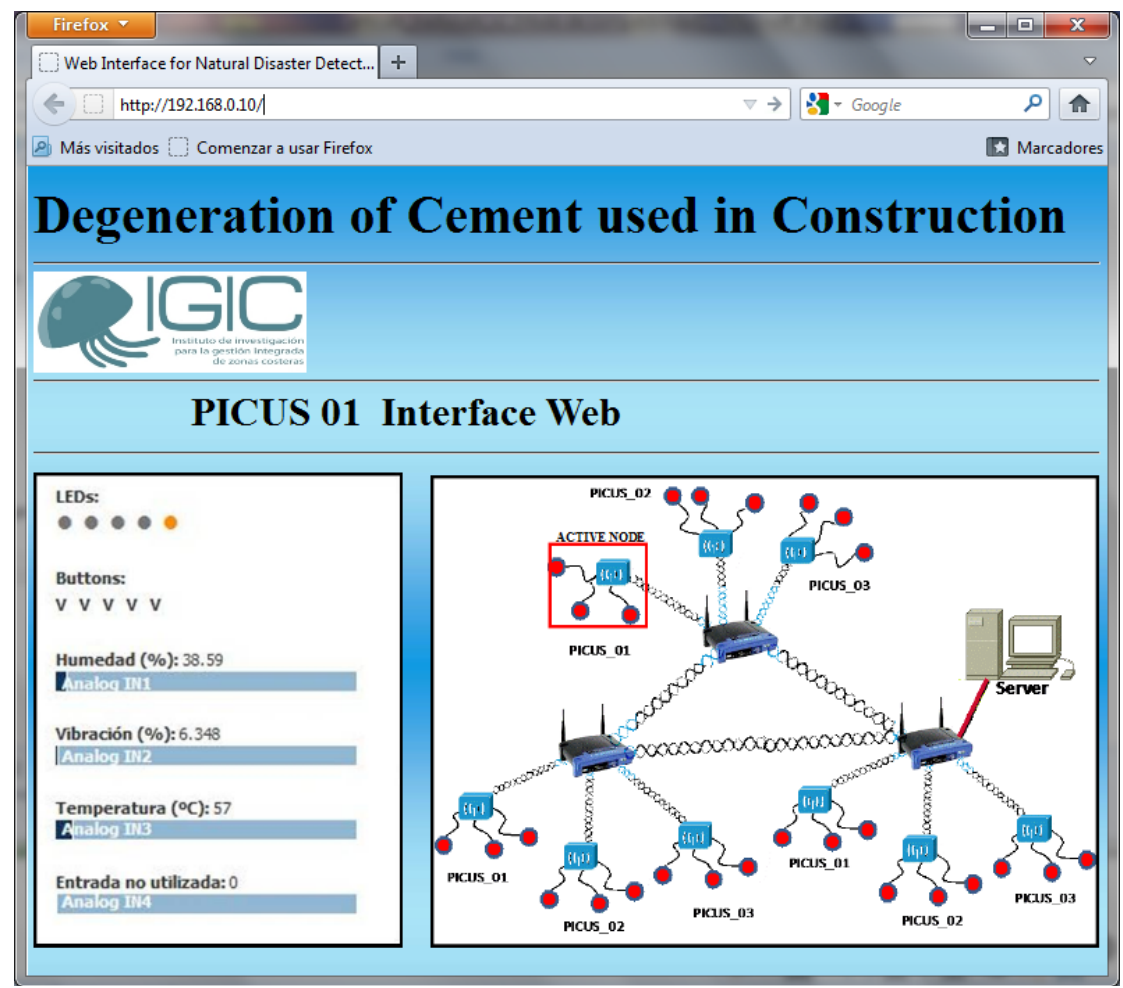

Figure 4.65. Web Interface for each sensor node

\subsection{Development of Wireless Sensor Nodes for Taking Care of Disabled and Elderly People}

The amount of sensors that a mobile device can integrate is quite high. Most of the smartphones available in the market include many of the sensors presented in this section. Although several sensors have not yet been integrated into mobile devices, we can see that the technology is already developed. Therefore, it is only a matter of time to have them embedded into the smartphones. In this section, we show the embedded sensors which could be used in our system. We also present the decision algorithm procedure included in our system.

Our purpose is to use our system in a group of disabled or elderly people when they are moving in a rural area or in a city, but it can be used indoors or in a fixed place. Moreover, the system can 
also be used to take care of children. Therefore the values of all sensors are similar (considering a tolerance). Given this fact, we can use the sensor values of our neighbor devices to compare whether the change of our sensor values are different of our neighbor sensors or not. Although it can be used in any type of environment, for design and development we will suppose the worst case: people are moving in an unknown place.

In our system each person has a smartphone that integrates several sensors and transducers, such as proximity/light sensor, magnetic sensor, acoustic sensor or microphone, compass, gyroscope, accelerometer, GPS locator or others, such as a barometer (see Figure 4.66). Our sensors do not measure physical conditions of the body. Devices only measure if a person has fallen, if he/she has changed his/her position, if there is more sun light, etc.

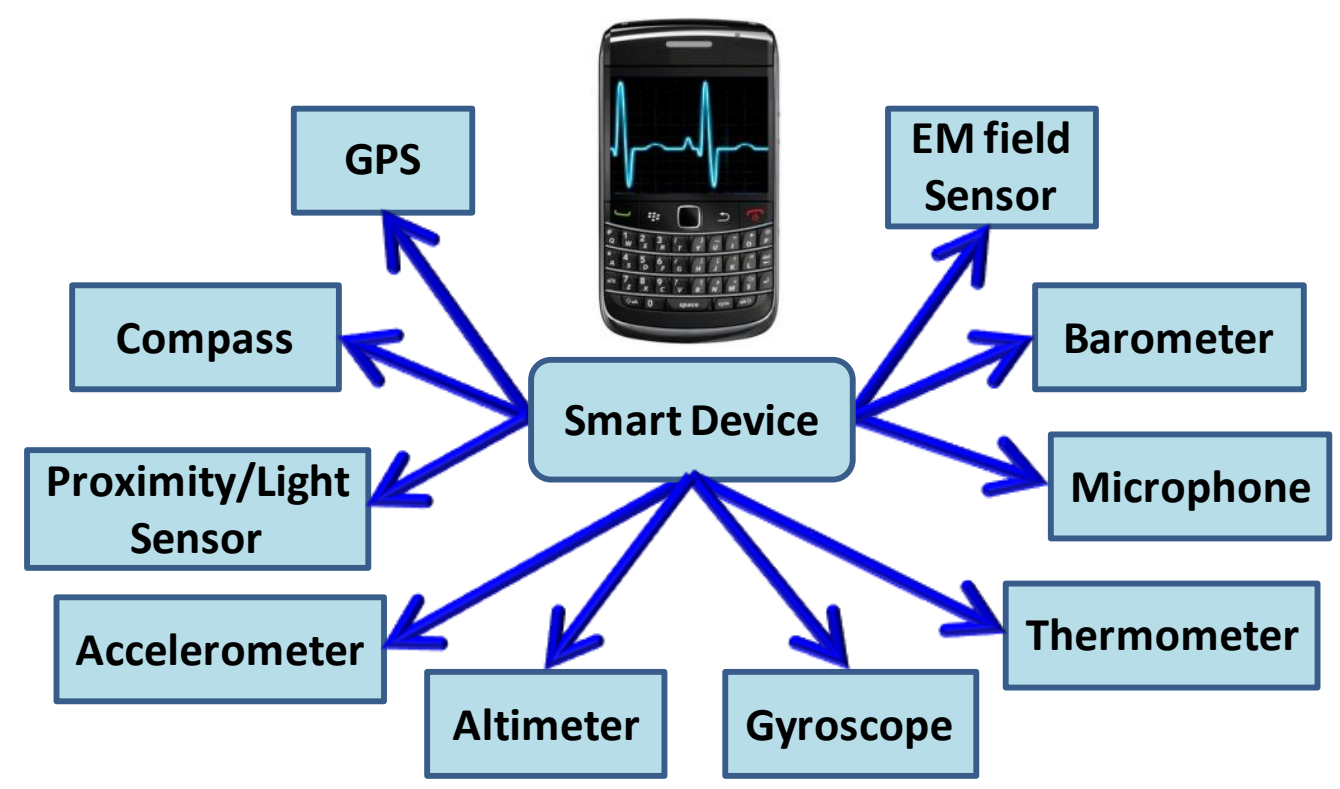

Figure 4.66. Sensors embeded in a smart device.

The most common embedded sensors in current smartphones are the following ones:

- Compass: An electronic compass is a navigational instrument that bases its operation in the terrestrial electromagnetic field. The magnetic needle indicates the direction of Earth's magnetic field, pointing to the north and south poles. With this sensor, we can determine the direction deviation from the North position in degrees. This sensor let us know in which direction group is walking. If a person is walking to another direction, the sensor will inform us.

- Accelerometer: This sensor is capable of measuring the acceleration and speed changes of an object. We can find several types of accelerometers. The first type is the Hall Effect accelerometers where a seismic mass with a magnet interacts with a Hall Effect sensor that detects the changes of the magnetic field. The second type is based on capacitors. In this case, seismic mass is placed between the plates of a capacitor. It changes the current between the capacitor plates when the mass moves. The third type of accelerometer is the three-axis accelerometer $(\mathrm{X}, \mathrm{Y}, \mathrm{Z})$. It is integrated in a single chip of silicon. The principle of operation of these accelerometers is the MEMS (Micro Electric-Mechanical System) technology which is based on heat transfer by natural convection. These devices measure internal changes in heat transfer caused by acceleration, offering significant advantages over the use of a traditional 


\section{Sandra Sendra Compte}

structure based on solid proof masses. With a simple application it is easy to determine the distance traveled by the person. With the correct processing on the three axis signals, we can determine if a person is running or is going up on a lift. Moreover, the sensor can also inform us if the person has fallen. The average speed of a walking person is set to approximately $1 \mathrm{~m} / \mathrm{s}$.

- Gyroscope: It is a mechanical device consisting of a rotationally symmetric body which rotates around its axis of symmetry. When the gyroscope is subjected to a momentum, which tends to change the orientation of its rotation axis. Its behavior is apparently paradoxical, because the axis of rotation, rather than change the direction as a none-rotating body would do; it changes its direction perpendicular to the "intuitive" one. We can find several types of gyroscopes. The first type is the piezoelectric gyroscope. This is based on the use of an oscillating crystal. This oscillation arises when the acceleration acts on it and the crystal is deformed. Therefore its electrical resistance varies. The second type is the capacitive gyroscope, which is composed of two parts: a mobile part that performs an input/output vibrating movement regarding to the axis of rotation of the gyroscope and a fixed part, which is perpendicular to this movement. These two parts form a capacitive structure that can store an electric charge (as a capacitor). Finally, the third type is the MEMS gyroscope, which is based on the vibration but using materials at molecular level. This technology allows its integration in a small silicon chip and even mobile three-dimensional parts. The system analyzes the variations (which should be stable). The Coriolis Effect is used to calculate the variables. We can use gyroscopes to measure deviations from the reference direction. We can use a gyroscope to know if a person has fallen and he/she cannot get up. We will express the spin as a proportional value, between 0 (for the position repose) and 0.99 for a full circle.

- GPS: It is a satellite global navigation system that determines worldwide the position of an object, person or vehicle with a few meters of accuracy. We can even get few centimeters of accuracy by using differential GPS. The receiver must locate at least three satellites to determine its position. It receives signals identifying and indicating the clock time of each satellite. Based on these signals, the device synchronizes the GPS clock and calculates the time that takes the signals to get to the device. With this information it determines by triangulation the distance of each satellite to the measuring point. Knowing the distance, the relative position to the three satellites is easily determined, and then the absolute position or actual coordinates of the measuring point is easy determined. We will know de position for all devices using the GPS values with an accuracy of about $2.5 \mathrm{~m}$. Furthermore; we can calculate the speed of the person with the received data. Using the GPS, we can determine the position of each person in the group and if one of them leaves the group.

- Microphone: The microphone is an electroacoustic transducer. Its function is to translate the vibrations, due to the acoustic pressure exerted to the capsule by the sound waves, into electrical energy. It allows the registration, storage, processing and transmission of audio signals. We can find different types of microphones made of carbon, piezoelectric, dynamic, and capacitive, among many others. According to some studies provided by the WHO (World Health Organization), the sound levels, in sleeps hours should be $30 \mathrm{~dB}$, and cannot exceed $45 \mathrm{~dB}$, while, the ambient noise in a city is from $65 \mathrm{~dB}$ to $70 \mathrm{~dB}$. The levels for a man and a woman crying are $85 \mathrm{~dB}$ and $80 \mathrm{~dB}$ respectively. If a person is exposed to sound levels higher than $75 \mathrm{~dB}$, it can suffer several illnesses such as hypertension and heart problems. 
- Proximity/Light sensor: A photoelectric sensor responds to the light intensity changes. There are several skin diseases, such as Lupus or photophobia, where patients cannot receive sunlight directly. In order to protect the people who suffer these diseases, we need to know the maximum light intensity for a person. 1200 lux is a low light intensity, while when there are 2800 lux, high light intensity (light outdoors) is considered. With this sensor we can detect the index of solar radiation that a person may be receiving.

- Magnetometer: It is used to detect the magnetic field strength variation. Since the magnetic field influence decays with the distance, it is important to place the magnetometer as closer as possible to the target. It can be used as a metal detector.

- Temperature sensor: A temperature sensor can be manufactured with many configurations, differing in their optical and electronic components, its technology, size and package. For the implementation of temperature sensors in mobile devices such as PDAs or smartphones, it is commonly used resistive temperature sensors or semiconductor sensors, whose operation is identical to a diode. The temperature is given as an indirect measure of the variation of electrical parameters of these electronic components.

- Altimeter/Barometer sensor: Its operation is based on the relationship between atmospheric pressure and altitude above sea level. Atmospheric pressure decreases with altitude, about $1 \mathrm{hPa}$ per 27 feet (8.2 meters) above the sea level. It is taken as baseline the sea level, but its operation is subject to weather changes. For this reason, a single device usually gives both values.

Although the smartphone used for this work does not integrate the temperature and altimeter sensors, other mobile phone models, made for sports people, may integrate them [332]. Furthermore, it has been recently developed a precision analog output temperature sensor for low current applications (STLM20 manufactured by STMicrorelectronics) [333]. These sensors will most probably be integrated into new smartphones soon. Table 4.11 shows the errors and the thresholds taken into account for our simulations.

Table 4.11. Errors and thresholds of the sensors.

\begin{tabular}{|l|l|l|}
\hline Sensor & Error & Threshold \\
\hline Compass & 1 degrees & 10 degrees \\
\hline Accelerometer & $1 \mathrm{~cm}$. & $10 \%$ \\
\hline Gyroscope & $1 \%$ & $5 \%$ \\
\hline GPS & $2.5 \mathrm{~m}$ & - \\
\hline Microphone & $1 \mathrm{~dB}$ & $10 \%$ \\
\hline Proximity/Light sensor & 0.02 lux & $2500 \mathrm{lux}$ \\
\hline Magnetometer & $1 \mu \mathrm{T}$ & $10 \%$ \\
\hline Temperature sensor & $1^{\circ} \mathrm{C}$ & $10 \%$ \\
\hline Altimeter/Barometer sensor & $1 \mathrm{~m}$ & $20 \mathrm{~m}$ \\
\hline
\end{tabular}




\subsubsection{System Operation}

With a correct signal processing of the sensor values of the state $s$ and comparing these values with the values of its neighbors, our system is able to detect some situations or events that are beyond ordinary cases, such as:

- A person that is falling.

- The distance between a person and the group is increasing.

- A person is screaming, but it is not the noise of the place.

- A person with a skin disease is receiving sunlight.

- A person is moving in a different direction to the group direction.

Moreover, this system would allow us to distinguish other types of events that could bring the system to generate alarm signals when in certainty the situation does not require it:

- A device detects high noise, so a regular system will generate an alarm thinking that the user is crying or shouting, but he/she is very close to the neighbors to which the system will ask for the value of their sensors. Because levels picked up by its neighbors are very similar, the alarm would not be sent.

- A person is walking backwards but with the group. It generates the alarm by the compass sensor because it detects a change of direction. Without a collaborative system this issue will not be advised.

- A person scampers near the group. It would generate the alarm because of the difference of the values of the sensors. However, it may not be an alarm because the person is not moving away from the group.

- One person performs reciprocating movements. When the system compares the value of the sensors with the values of the nearest neighbors, it would generate the alarm because of their differences. But this situation does not involve an emergency case.

There could be many more cases. An alarm is generated as a combination of the value sensors of the device, but after considering the received replies from the neighbors. Therefore, our system considers the following statements:

- Difference of sensor values with respect to the previous values.

- Difference of the current state with the sensor values of the neighbors.

- The combination of several alarms, to distinguish between a true emergency situation and a situation that has generated a false positive.

As Figure 4.67 shows, we consider a group of people traveling together (or in a same place). We are thinking on people that must be always monitored, which can have Alzheimer or other issues such as degenerative diseases. The system is also suitable for monitoring children. In either case, each person is carrying a smart device connected to a wireless ad hoc network using IEEE $802.11 \mathrm{~b} / \mathrm{g}$ technology, where each device maintains wireless connections with their nearest neighbors. 


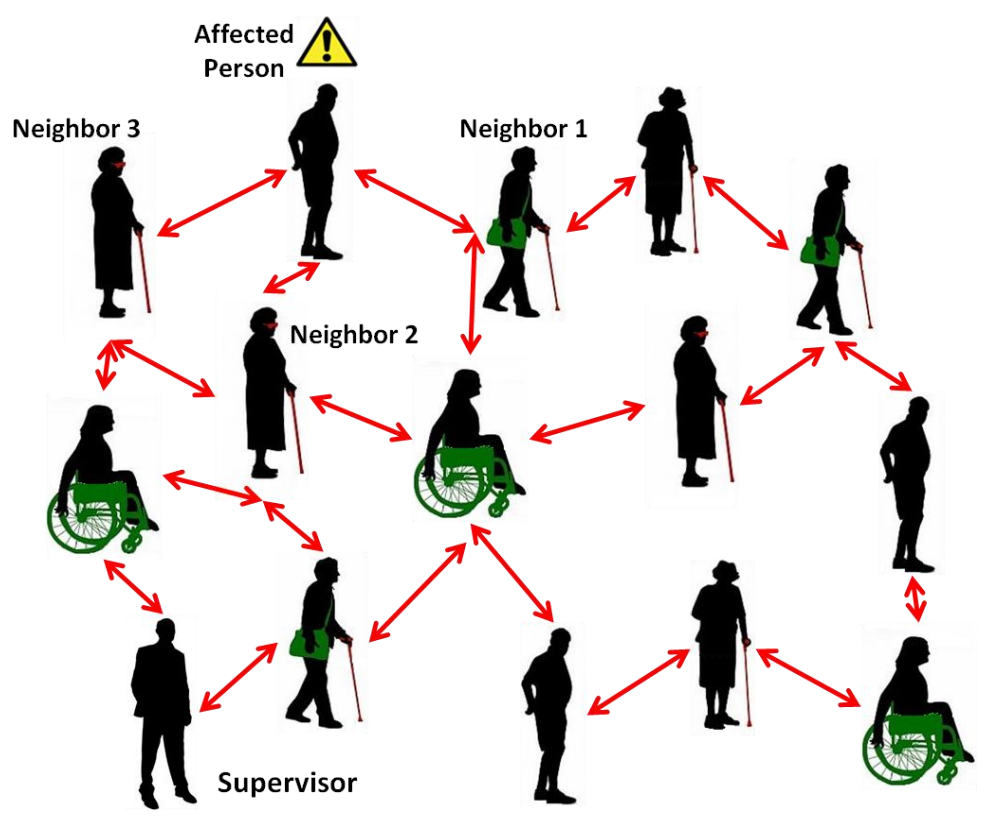

Figure 4.67. Network Topology.

Our proposal works with the same power saving approaches and energy optimization techniques as regular systems [157]. Our system will generate more messages when a threshold is exceeded. Only in this situation, there will be more exchanges of messages. So, we take profit of existing systems.

In order to determine whether an alarm must be announced or not, we have split the alarm procedure in four stages. Figure 4.68 shows the sequence of alarms as a function of the time. The procedure is as follows. The device is always monitoring the values from the sensors by using a small application that collects the value of each sensor. When the smartphone detects that a sensor has exceeded its threshold the system goes to the first alarm level. If after some time it is not disabled, it goes to the second level, where it sends a message to its closest neighbors requesting their values for this sensor.

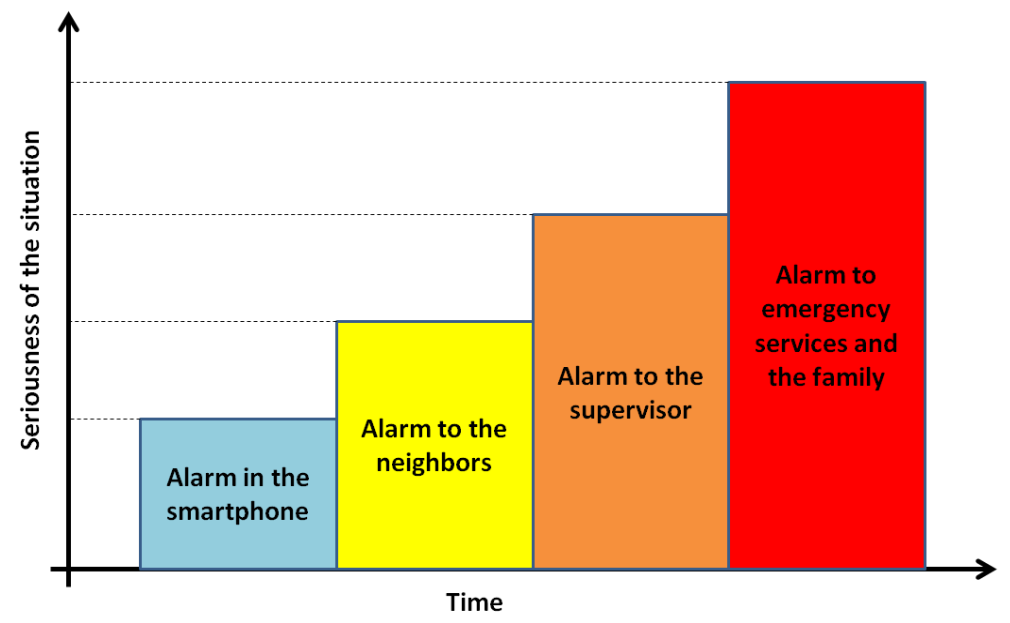

Figure 4.68. Alarm levels and propagation in function of the time. 
When the reply is received, it compares the received values with its values. If the result of the comparison of both values is very similar (considering the accuracy of each sensor), it will mean that the system has detected a false-positive, and the system will return to its resting state, monitoring the sensors activity. But, when the compared values are very different, it means that there is a real warning, generated for example, by the fall of a person, or because a person has been separated from the group. Then, it goes to the third level, where the device of the affected person sends a message with the cause of the alarm to the person in charge of the group (the guide or supervisor). If after some time the alarm is still active, because no one has stopped it, it will go to the fourth level. At this level, the smartphone will send an automatic message to emergency services and to the family. They will be able to determine the location of the person, thanks to the GPS coordinates.

When a device needs information from its neighbors, the first step is to know its neighbors. Initially, the affected person device sends a broadcast packet. Only nodes that are closer to him will reply. After assessing the situation (based on the sensor values), if the system decides to send an alarm, it will follow the four stages alarm procedure. When the alarm is sent to the supervisor, it is routed using a regular routing protocol (routing protocols are discussed in the performance test section). Supervisor will respond with an acknowledgment packet. Figure 4.69 shows the message flow between the affected person, neighboring nodes and the supervisor.

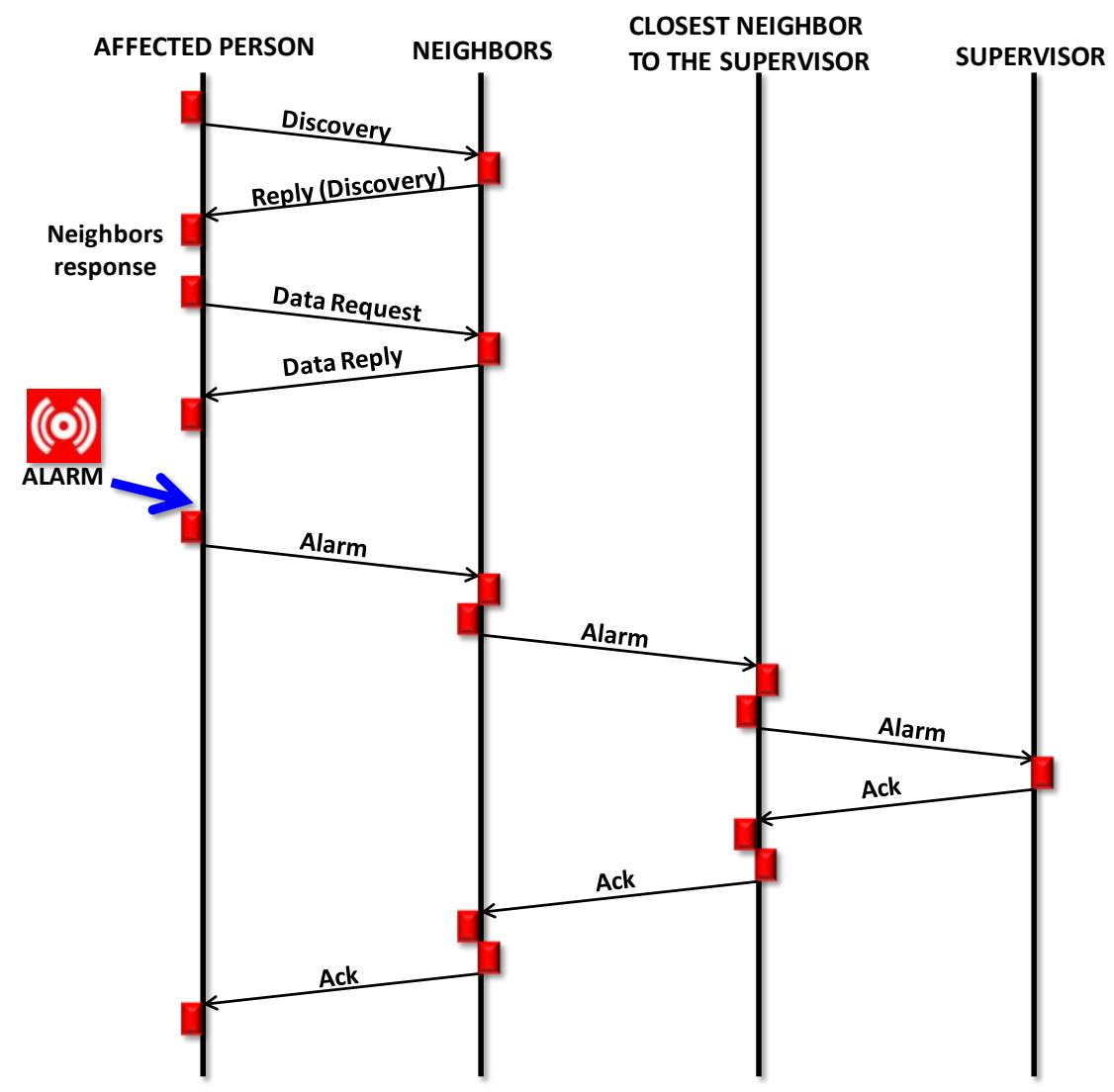

Figure 4.69. Message flow between devices 
Figure 4.70 shows the collaborative decision algorithm which is in charge of processing the data from the sensors and determine if there is an alarm and whether the detected alarm is a real one or a false positive.

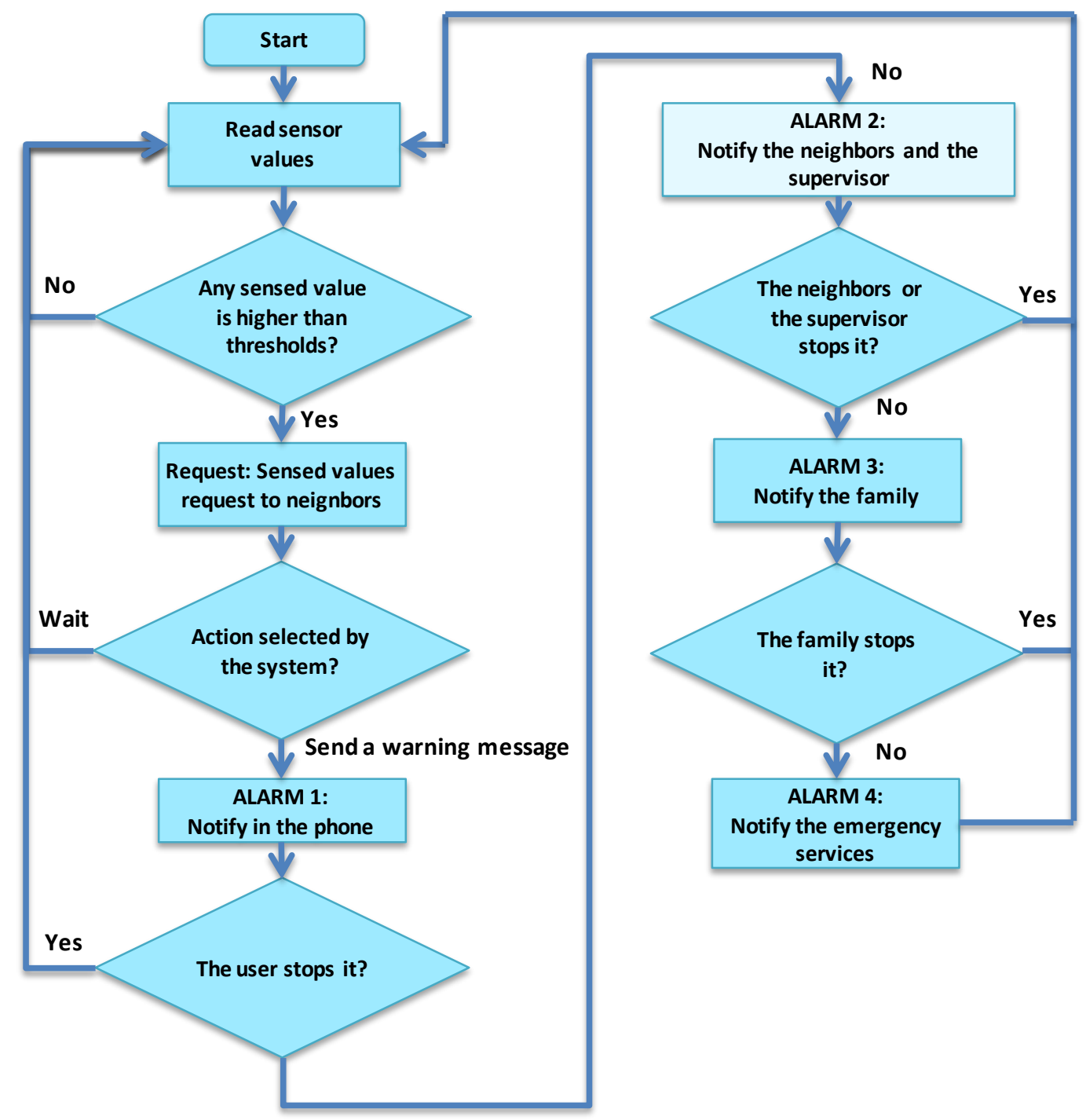

Figure 4.70. Collaborative decision algorithm.

\subsubsection{Performance Test}

\subsubsection{System operation performance}

In this subsection we simulate a scenario like the one shown in Figure 4.67. Initially, the mobile device of the affected person produces an event. Before creating a local alarm state, each device checks if its values exceed a threshold or if the parameters vary from their previous states. If one of these cases occur, the value that has generated the local alarm will be compared with its nearest neighboring nodes. Then, the affected device sends a request to its closest neighbors in order to compare its value with their values. If there is a difference higher than a threshold, the device generates a warning message to the supervisor. We have simulated our system using Matlab [334 


\section{Sandra Sendra Compte}

]. It is a high-level technical computing language and an interactive environment for algorithm development, data visualization and analysis.

In the first case the system monitors a person that has changed its direction. The group direction changes can be tracked using the compass sensor. 100 samples of the compass sensor of 4 devices are collected. From the $27^{\text {th }}$ sample, one of the devices starts to change its travel direction (the affected person) compared to its neighbors (neighbor 1, neighbor 2 and neighbor 3), which follow the same path. Figure 4.71 shows this situation.

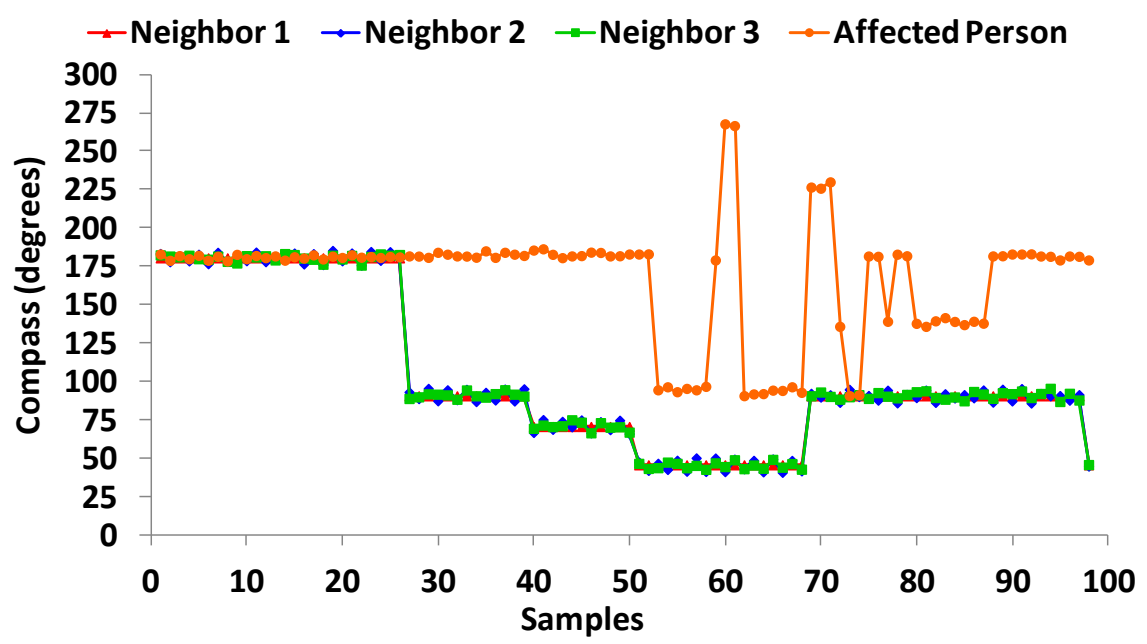

Figure 4.71. Compass measurements for 4 devices

Moreover, in this case we can also include joint sensor values. E.g. the detection of a person that although he/she is walking with the group, he/she has moved around and walked backward, changing the direction of the person but going in the same direction of the group (he/she will not increase the distance to the group.

The second case monitors a person that is screaming for help. Figure 4.72 shows the sound level that is recorded on a device on a tour of an urban area, in $\mathrm{dB}$. From the $35^{\text {th }}$ sample, the device of the affected person has values greater than $80 \mathrm{~dB}$, indicating that this person is screaming. Neighbor 1, neighbor 2 and neighbor 3 present similar values, which may be registered in urban environments.

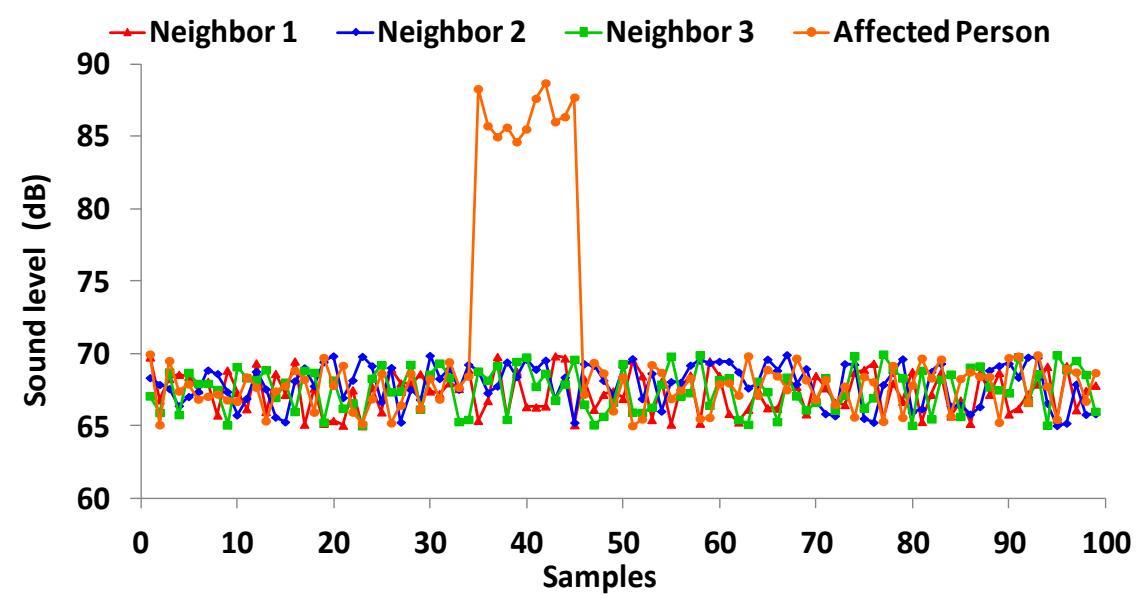

Figure 4.72. Sound levels for 4 devices. 
As we have seen, in an urban environment, noise levels means are located around the $65-70 \mathrm{~dB}$. This is the background noise we have over our measurement.

There are specific cases where to measure a person crying could be difficult. When an ambulance passes with the siren on (depends on state law), it exceeds the average noise values in urban environment. It is often placed at $80 \mathrm{~dB}$. In this case, we would not be able to determine if the person is in a danger situation, so the query to other sensors should be necessary. There is another way to detect whether the increased noise level produced by a shout or an emergency vehicle. This would be possible if the system is able to distinguish the frequency of the received sound by using a simple frequency analysis.

In the third case, the system monitors a fixed place where it is expected to not have any leaving from that place. In the following scenario we have fixed a location to relate the devices. Figure 4.73 shows the distance that each monitored node presents to the fixed place (it could be the place of the supervisor). We can see that neighbor 1, neighbor 2 and neighbor 3 are always around $5 \mathrm{~m}$. away, while the affected person starts to leave the group, from sample $40^{\text {th }}$.

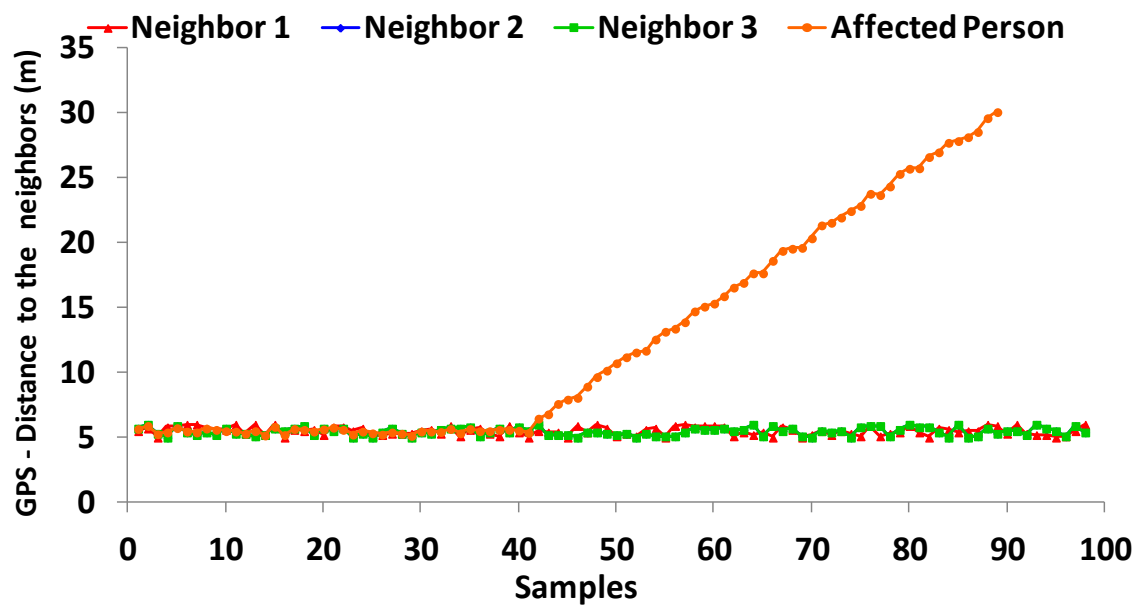

Figure 4.73. GPS measurements for 4 devices

In the forth case the system monitors if a person leaves a building or a covered place and is exposed to the sunlight. There are persons that may be affected by sunlight exposure because they suffer some sort of photophobia or hypersensitivity. Figure 4.74 shows the light intensity in lux received by 4 persons in the same place. The intensity value remains close to 2000 lux in almost all samples, except from $80^{\text {th }}$ and $95^{\text {th }}$, where there is a light intensity greater than 3000 lux. In this case, the device sends a warning message only if the person wearing the device has that disease.

In order to show the behavior (using the gyroscope) when a person has fallen, or is moving strangely, we have included the fifth case. The gyroscope sensor included in the smartphone provides the variation produced in each axis. In this case, we monitor separately the values of the 3 axes in 4 devices. Figures $4.75,4.76$ and 4.77 show the gyroscope behavior in its $\mathrm{X}, \mathrm{Y}$ and $\mathrm{Z}$ axes respectively. We can see how the system performs when a person falls down. It usually does not affect only to one axis, in this case two axes are involved, but we can see some impact in the third axis. In the three Figures, we can see that after sample $33^{\text {rd }}$ the values for the 3 neighbors remain similar, while the values for the person that has tripped are changing. 


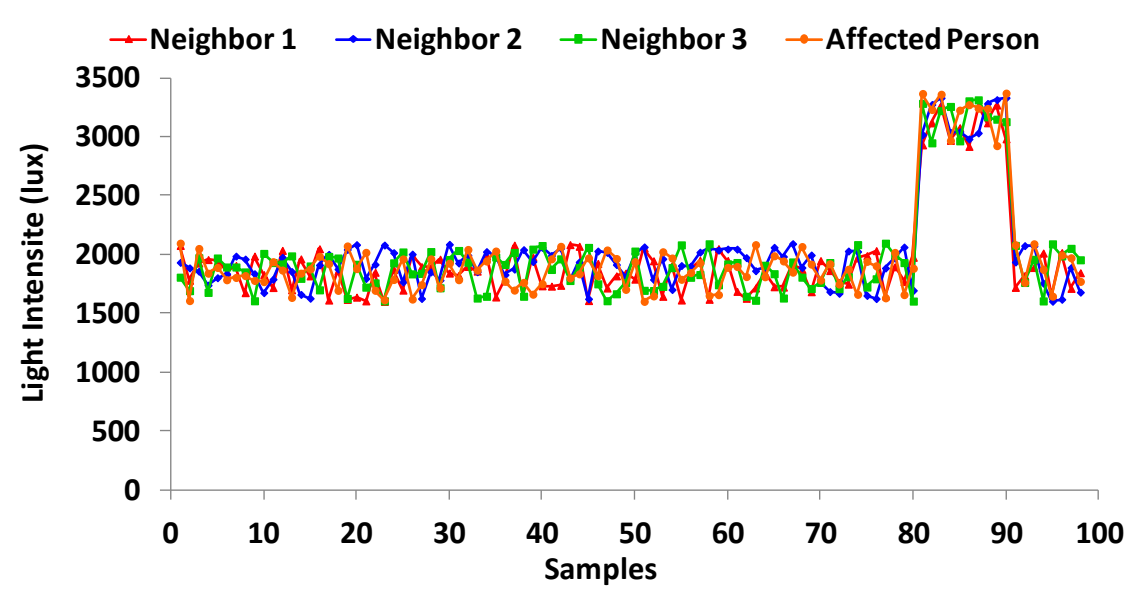

Figure 4.74. Light intensity measurements for 4 devices.

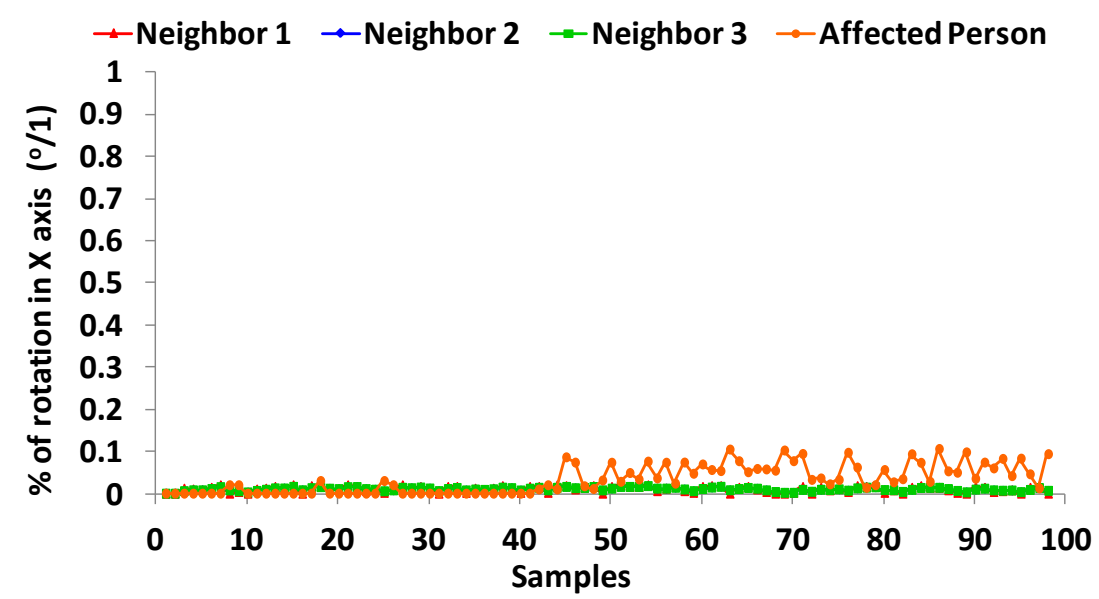

Figure 4.75. Gyroscope measurements in $\mathrm{X}$ axis for 4 devices.

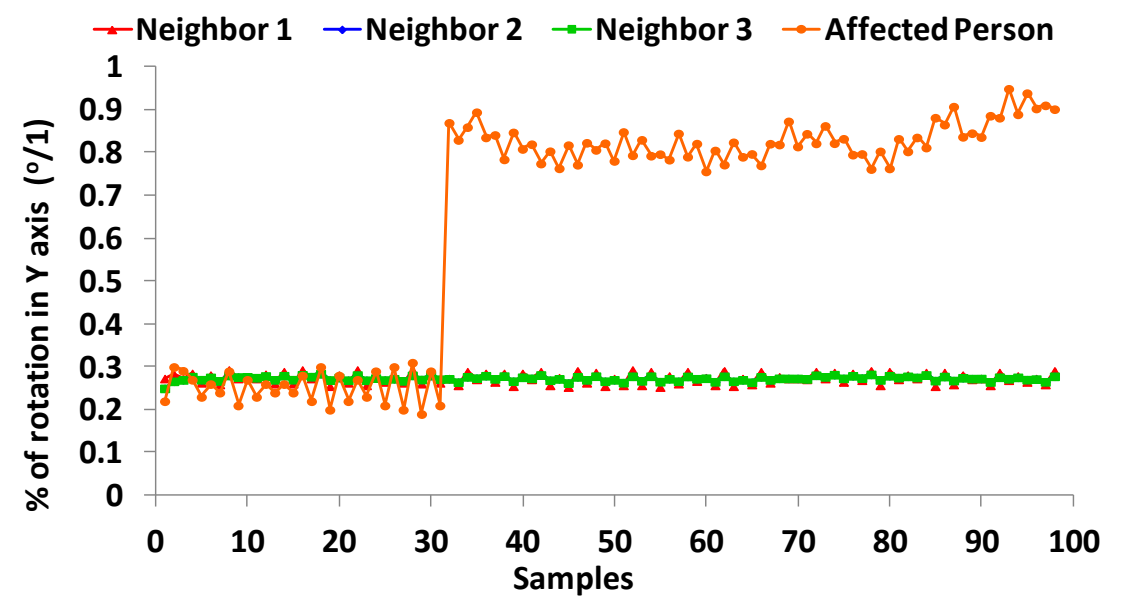

Figure 4.76. Gyroscope measurements in $\mathrm{Y}$ axis for 4 devices. 


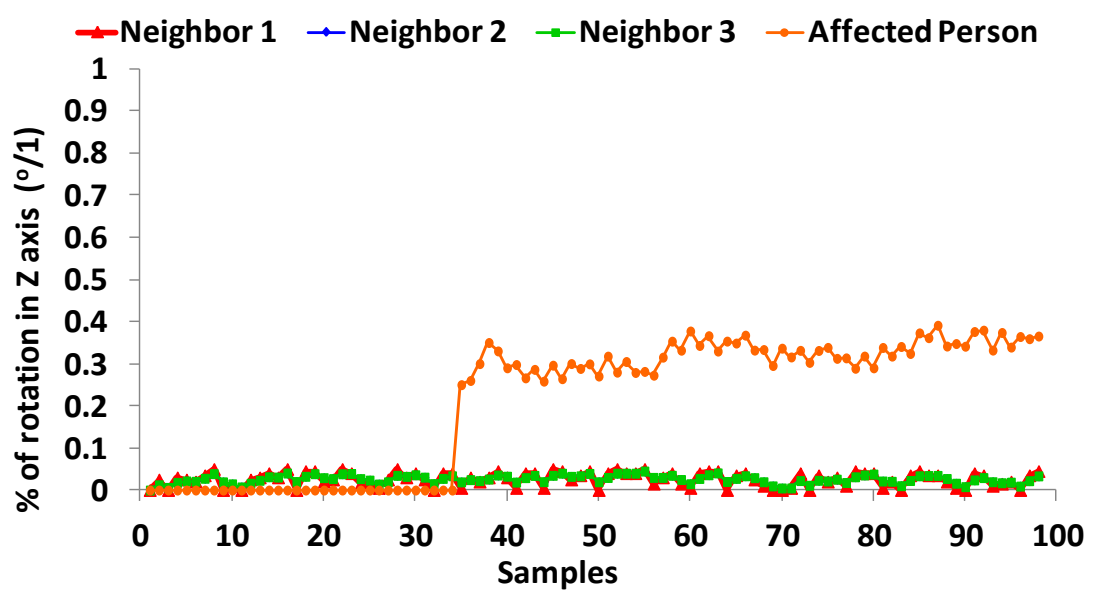

Figure 4.77. Gyroscope measurements in $\mathrm{Z}$ axis for 4 devices.

In order to show the behavior (using the accelerometer) when a person has fallen or is moving strangely, we have included the sixth case. An accelerometer is capable of measuring the same behavior in the three axes than the previous case. We have also monitored separately these values for 4 devices. Figures 4.78, 4.79 and 4.80 show the accelerometer behavior in its $\mathrm{X}, \mathrm{Y}$ and $\mathrm{Z}$ axes respectively.

We can see that the largest variations are recorded in the $\mathrm{X}$ axis, which is the direction of the movement of the group. First, we observe that between samples $13^{\text {th }}$ and $21^{\text {th }}$, all neighbors reduce their speed to $0 \mathrm{~m} / \mathrm{s}$, which means that they have stopped. Then, they continue walking. At sample $45^{\text {th }}$ we can see that something has happened to the affected person, because the smartphone detects different values in the $X$ axis than its neighbors. This is because the person has tripped in its way. We can see that, at this sample, $\mathrm{Z}$ axis also detected a variation in its values. Because neighbors 1,2 and 3 notice the fall of the affected person, they also stopped between samples $55^{\text {th }}$ and $62^{\text {th }}$. Variations on the $\mathrm{Y}$ axis are very subtle, and may be due to small movements of the person in this axis. Finally, neighbors 1, 2 and 3 continue on their way and they increase their value in the $X$ axis, while the affected person maintains its value very close to $0 \mathrm{~m} / \mathrm{s}$.

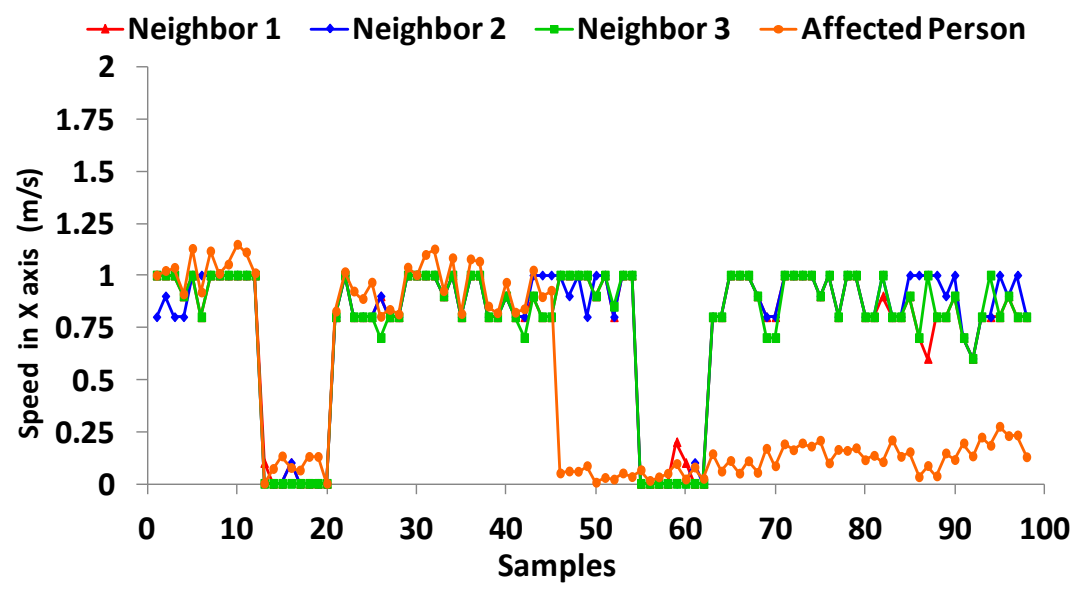

Figure 4.78. Accelerometer measurements in $\mathrm{X}$ axis for 4 devices. 


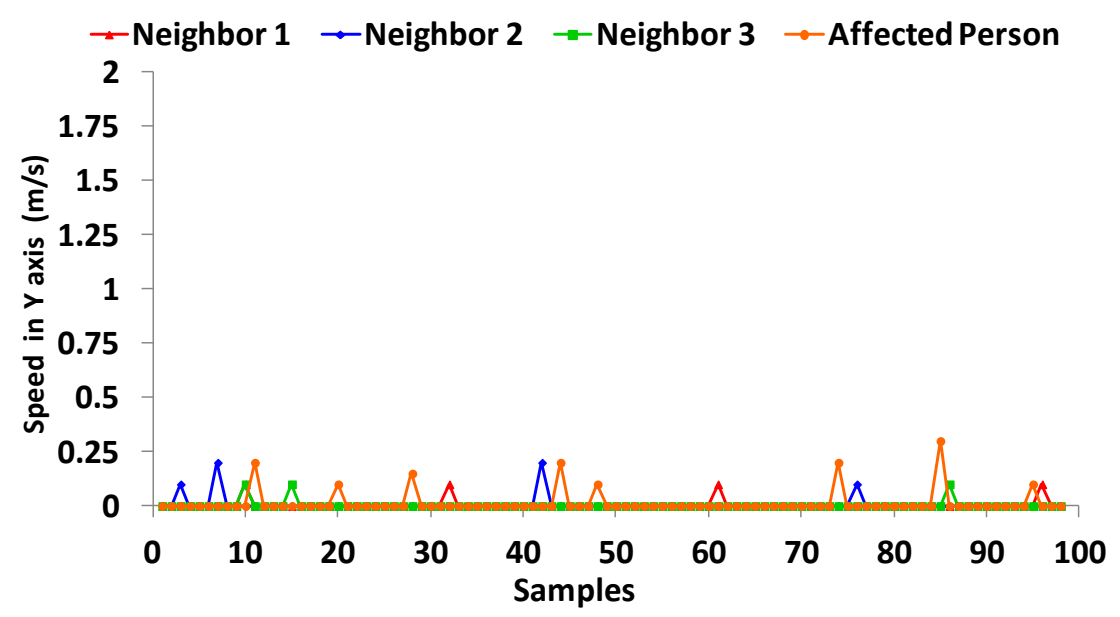

Figure 4.79. Accelerometer measurements in $\mathrm{Y}$ axis for 4 devices.

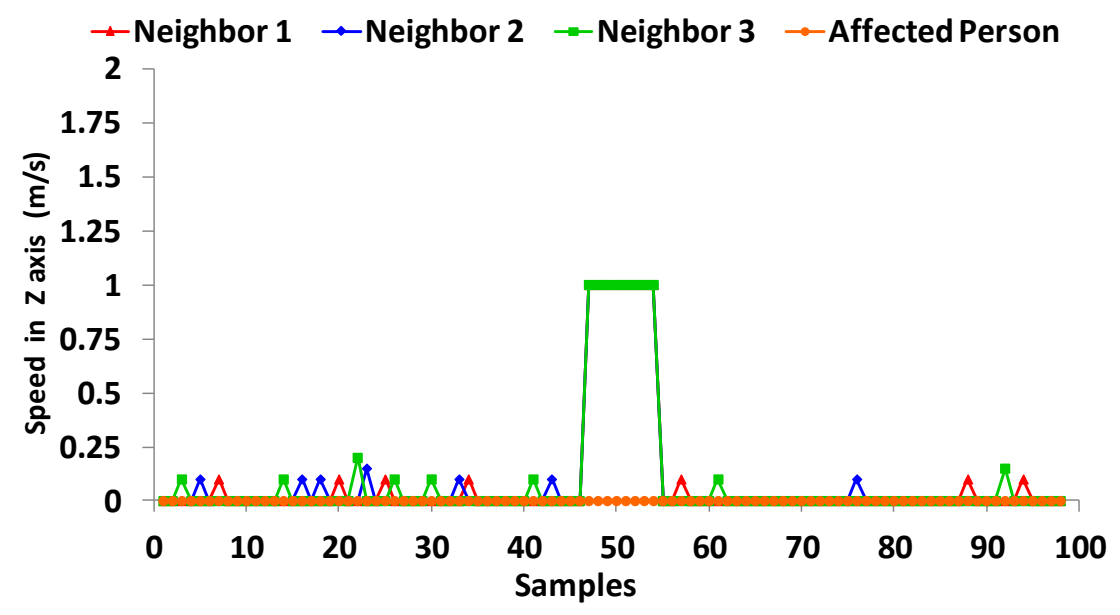

Figure 4.80. Accelerometer measurements in $\mathrm{Z}$ axis for 4 devices.

\subsubsection{Alarms}

From the graphs shown in the previous section, and taking into account the measurements captured by the sensors, the device decides whether the affected person is in a dangerous situation and if it has to transmit the alarm message. This assessment is done by the MDP, depending on the state of the system defined by its sensor values compared with the sensor values of the neighbors, and the utility value of the alarm action for this state. In this section, we will see the generating of alarms starting from the cases seen in the previous section.

Figure 4.71 (first case) shows the alarm of the compass sensor. The four signals are analyzed, using the smart decision algorithm, in Figure 4.81. 


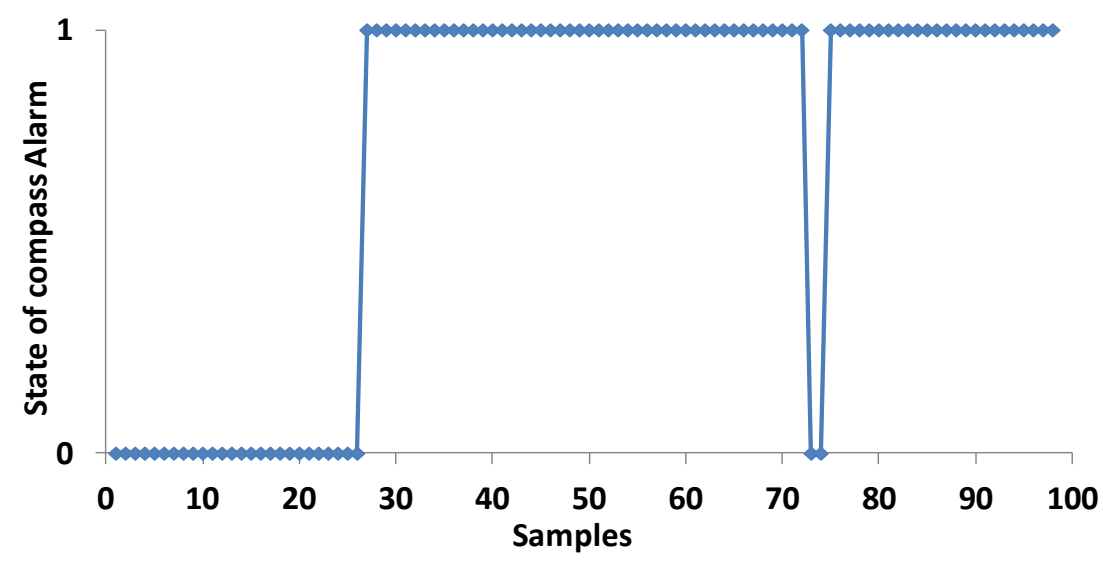

Figure 4.81. State of compass alarm

We can observe that as soon as the there is a variation between the affected person and its neighbors, the system starts to process the alarm. This occurs, starting from sample $27^{\text {th }}$. Moreover, we can see that in samples $73^{\text {rd }}-75^{\text {th }}$, the alarm is deactivated and then is reactivated. This means that although the affected person does not follow the same direction as the rest of the group during this short time period, the affected person has walked in the same direction than the group.

In the second case (Figure 4.72), we have processed signals from the microphones of 4 neighbors. Figure 4.82 shows the alarm generated in the device of the affected person. In it, we can see that from the sample $35^{\text {th }}$, a high level of alarm is established until it reaches sample $45^{\text {th }}$, where noise levels are restored and thus the alarm is disabled.

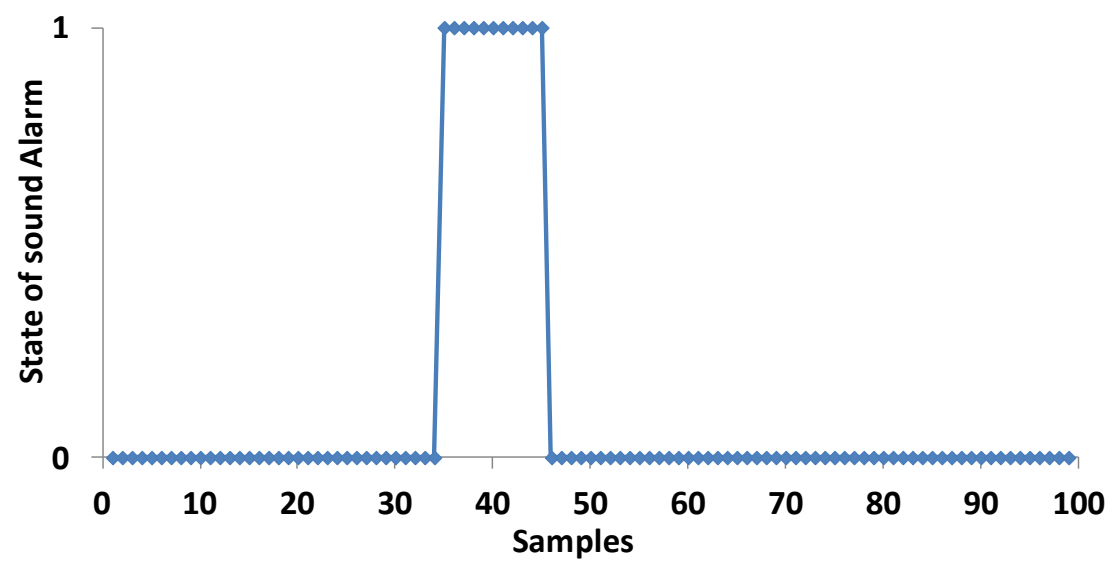

Figure 4.82. State of microphone alarm.

Another sensor that is very important for our system is the signal from the GPS sensor. We can use it to determine if the person is separated from the rest of the group (third case). Figure 4.83 shows the state of the alarm of the GPS sensors of the four neighbors. The alarm is activated from sample $46^{\text {th }}$. 


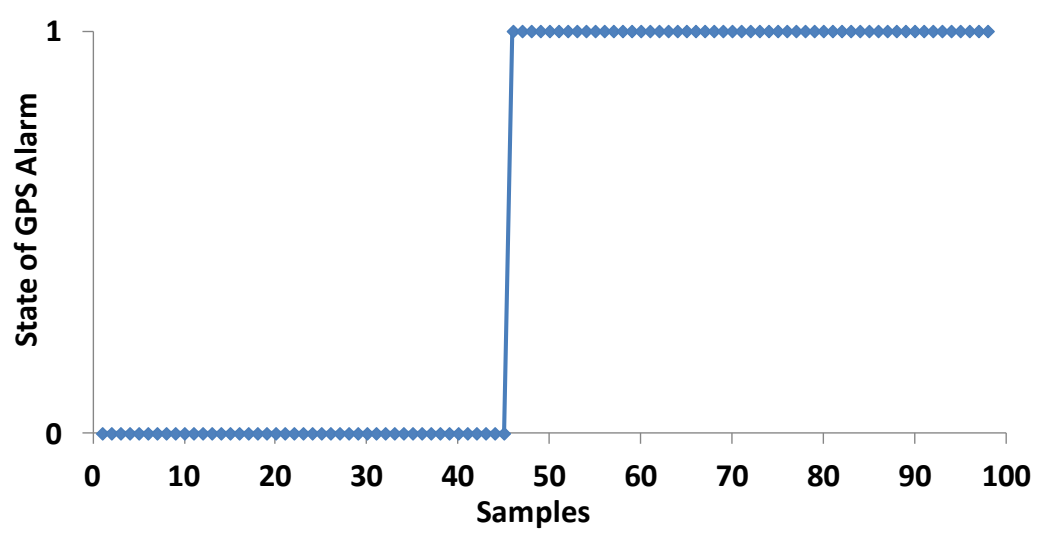

Figure 4.83. State of GPS alarm

The alarms generated by the light sensors work differently than the other sensors. The detection of high levels of light, above a threshold, might not be dangerous for a person, unless it suffers of some kind of photosensitivity (fourth case). Therefore, the algorithm is executed differently. In this case, the value of light collected by the sensor is firstly compared with the threshold value and then, it is compared with its neighbors, to check that the measured value is correct. If the comparison result is positive, the signal alarm is generated in the device of the affected person and it is propagated following the algorithm. Figure 4.84 shows the state of the light alarm. In this case, we see that the alarm is activated between samples $80^{\text {th }}$ and $91^{\text {st }}$.

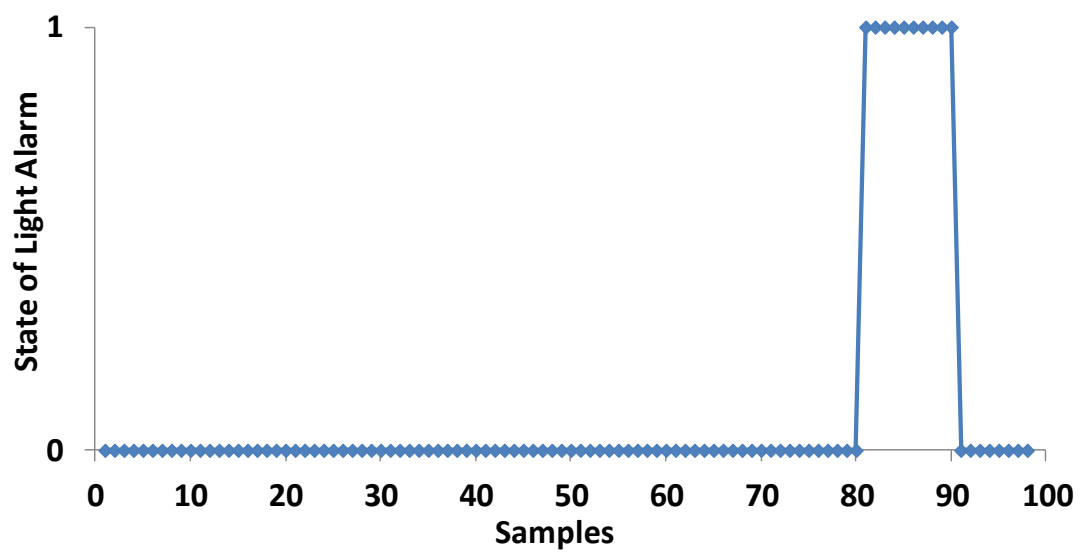

Figure 4.84. State of light alarm

The generation of the alarm for the gyroscope and accelerometer sensors (fifth and sixth case) is different from the rest. In these two cases, the alarms are calculated as a combination of the response of the $\mathrm{X}, \mathrm{Y}$ and $\mathrm{Z}$ axis, for each sensor. If we examine Figures 4.75, 4.76 and 4.77, corresponding to the behavior of the axes $\mathrm{X}, \mathrm{Y}$ and $\mathrm{Z}$ of the gyroscope sensor, we can see that the largest variations occur in the $\mathrm{Y}$ and $\mathrm{Z}$ axis. The alarm of the gyroscope sensor is obtained as a combination of the three signals. As Figure 4.85 shows, after the $31^{\text {st }}$ sample there is a series of activations/deactivations of the alarms, which could generate instability of the system. 


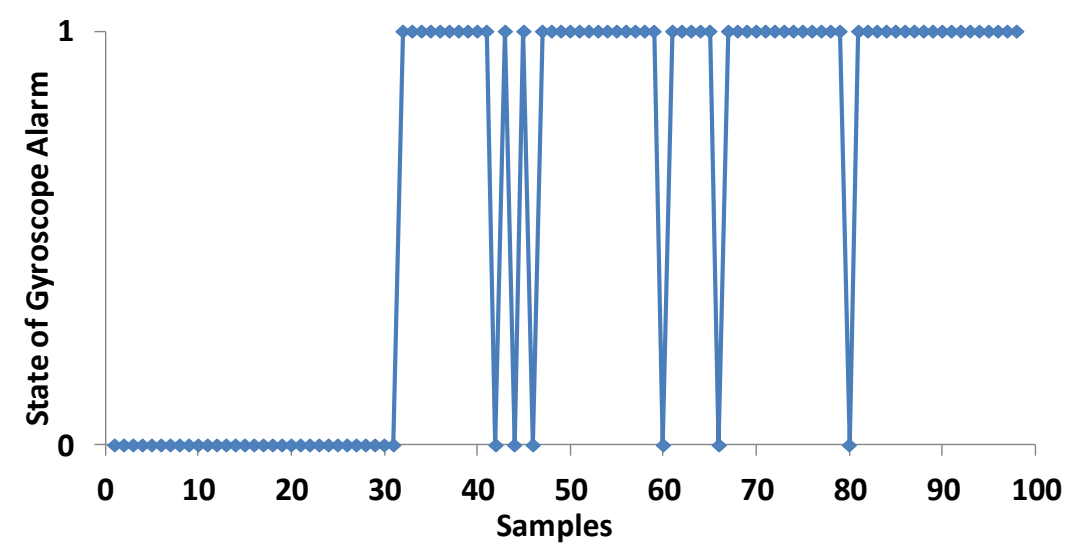

Figure 4.85. State of gyroscope Alarm

Following the same approach, the behavior of Figures 4.78, 4.79 and 4.80, have been combined. Figure 4.86 shows the alarm of the acceleration sensor. We see that between sample $58^{\text {th }}$ and $63^{\text {rd }}$, the alarm turn off. However, and for the same reasons as in the previous cases, it does not mean that the alarm situation has disappeared.

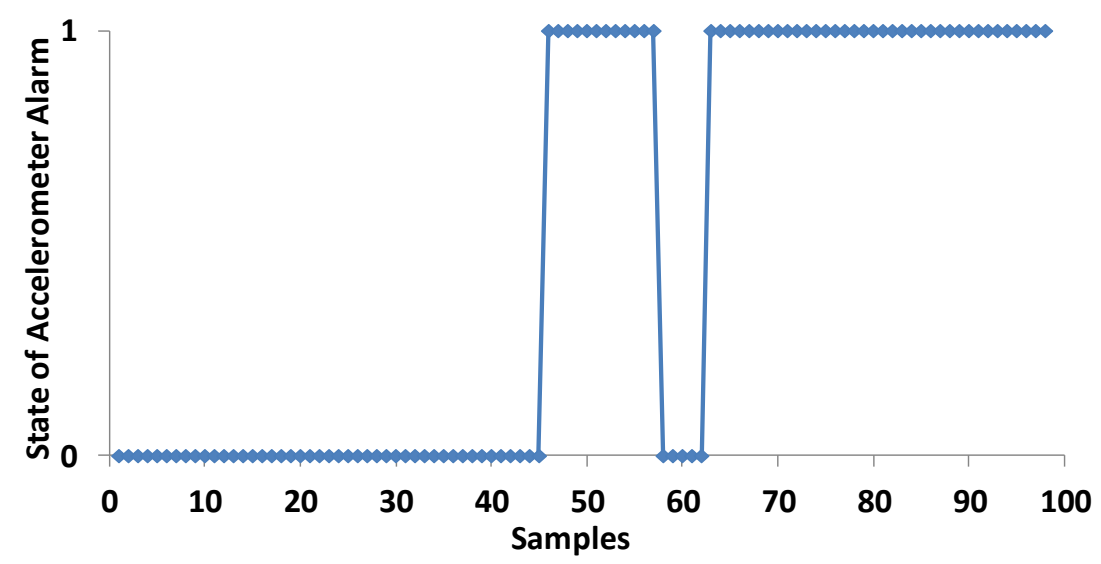

Figure 4.86. State of Accelerometer alarm

\subsubsection{Decision rules by mixing alarms}

In the previous section, we have seen several alarms that are activated depending on the data obtained by each sensor and their comparison with the values collected by its neighbors. However, if we treat the alarms for each sensor separately, we could get false positives. For this reason, we decide when to send the alarm message as a combination of alarm signals from various sensors.

Following this approach, we have several cases where the generation of an alarm, from the information collected from a sensor, could induce to an error. This fact permits us to know several states that we can always consider as not dangerous states, so we define the initial value of the alarm action for these states as -1 . Moreover, we can also define other states that can be always considered as dangerous, so we define the initial value of the alarm action for these states as 1 . For example, there could be cases where a person is playing and moving back to forth, thus it would 
activate the gyroscope alarm. However, if the person is close to the group, it would not be a reason to generate the alarm, but if it leaves the group, the alarm will be generated.

In this Section we will see the behavior of several cases where the activation of a sensor could lead to false results. In Table 4.12 the decision rules derivate from these cases and their utility values for the alarm action are shown.

Table 4.12. Decision rules.

\begin{tabular}{|l|l|l|}
\hline Rules & Q(s,wait) & Q(s, Send message alarm) \\
\hline $\begin{array}{l}\text { State } s \text { with Gyroscope alarm }=1 \& \\
\text { GPS alarm }=0\end{array}$ & 1 & -1 \\
\hline $\begin{array}{l}\text { State } s \text { with Gyroscope alarm }=1 \& \\
\text { GPS alarm }=1\end{array}$ & -1 & 1 \\
\hline $\begin{array}{l}\text { State } s \text { with Accelorometer alarm }=1 \& \\
\text { GPS alarm }=0\end{array}$ & 1 & -1 \\
\hline $\begin{array}{l}\text { State } \text { with Accelorometer alarm }=1 \& \\
\text { GPS alarm }=1\end{array}$ & -1 & 1 \\
\hline $\begin{array}{l}\text { State } \text { with } \text { Compass alarm }=1 \& \\
\text { GPS alarm }=0\end{array}$ & 1 & -1 \\
\hline $\begin{array}{l}\text { State } s \text { with } \text { Compass alarm }=1 \& \\
\text { GPS alarm }=1\end{array}$ & -1 & 1 \\
\hline $\begin{array}{l}\text { State } s \text { with Sound alarm }=1 \& \\
\text { GPS alarm }=0\end{array}$ & 1 & -1 \\
\hline $\begin{array}{l}\text { State } s \text { with } \text { Sound alarm }=1 \& \\
\text { GPS alarm }=1\end{array}$ & -1 & 1 \\
\hline
\end{tabular}

Figure 4.87 shows the case of a person that is moving back to forth, but the alarm is not generated till the person leaves the group. Here we can see that before the $32^{\text {nd }}$ sample, nothing happens. But after this value the gyroscope alarm is activated, but no alarm message is sent. However, because after $46^{\text {th }}$ sample there is a significant increase of the distance of the affected person to the group, there will be a message alarm. Combining the response of both sensors, we obtain a combined alarm, which offers greater reliability than processing only the gyroscope sensor data.

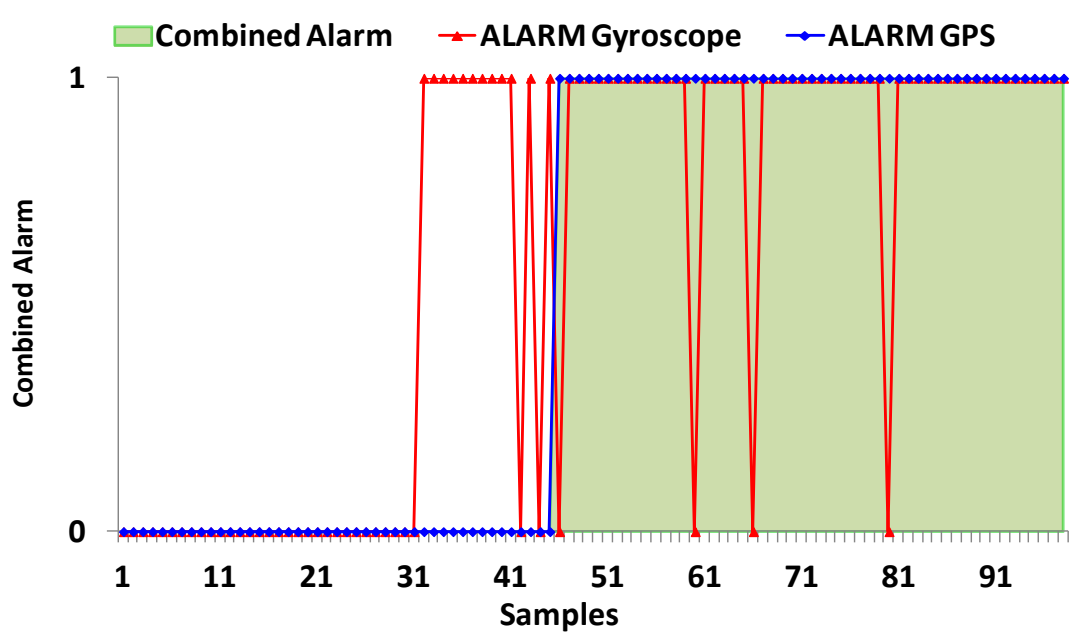

Figure 4.87. Combined alarm for GPS and Gyroscope sensors. 
Another situation where a single sensor could generate a false positive, but it could be corrected by our smart collaborative protocol, is the following one. Imagine a new situation where there is a group of young people with some kind of deficiency. In this group, one of them starts to run around the group but without departing from the group. In this case, the acceleration sensor would generate an alarm. However, while the affected person is near the group, the situation is not necessarily a hazard. If GPS activates its alarm, jointly with the accelerometer alarm, there will be an emergency situation for the affected person, so the alarm message will be sent. Figure 4.88 shows the behavior of this case. We can see that although the accelerometer alarm has been activated, only when the distance from the affected person to the group increases exceeding the limit established as safe, the combined alarm of both sensors will send the alarm message. We may also note that although the accelerometer alarm is disabled, as the distance of the affected person to the group, is high, the combined alarm will persist.

In the following situation, we have a person who begins to spin on its own, or even walk backwards, but always within the group. The compass sensor generates an alarm, because its value compared with their neighbors exceeds the specified threshold as normal, but no alarm message is generated until the person leaves the group. Figure 4.89 shows this situation where, although the compass sensor alarm is activated from the sample $25^{\text {th }}$, only when the GPS alarm is activated, the combined alarm is activated and thus the ala message is sent.

Finally, we will show a case where there is an alarm generated by the noise. It is activated because it detects an increment of the noise level. But it does not necessarily mean a dangerous situation for the person who is screaming. However, if the sound alarm is activated while the distance of the affected person to the group is also increasing, it could mean that the person has suffered an accident and cannot follow the group. Figure 4.90 describes this situation. We can see that the combined alarm is only active when the distance to the group increases.

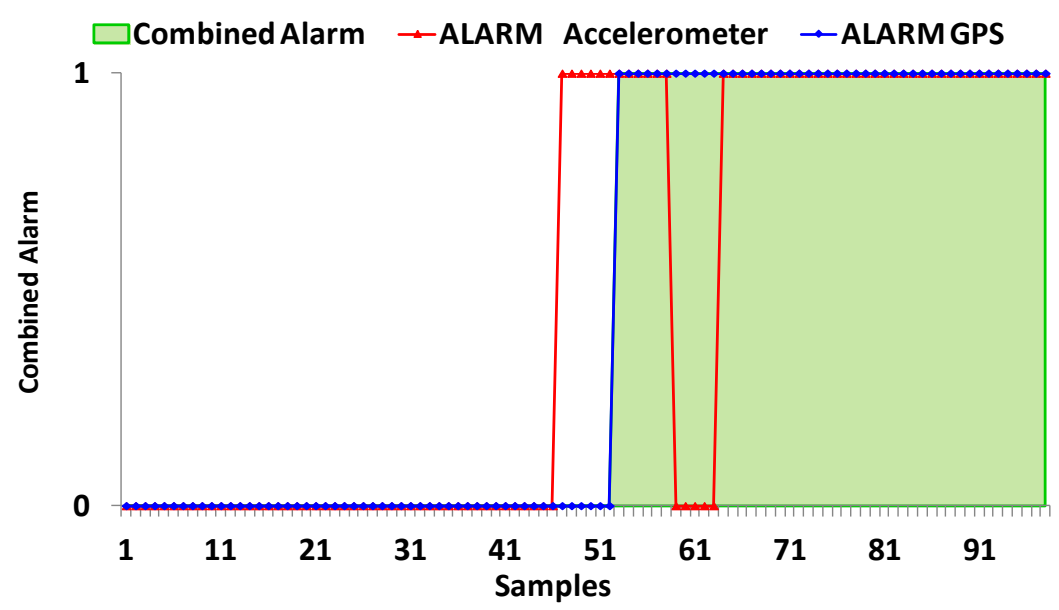

Figure 4.88. Combined alarm for GPS and Accelerometer sensors 


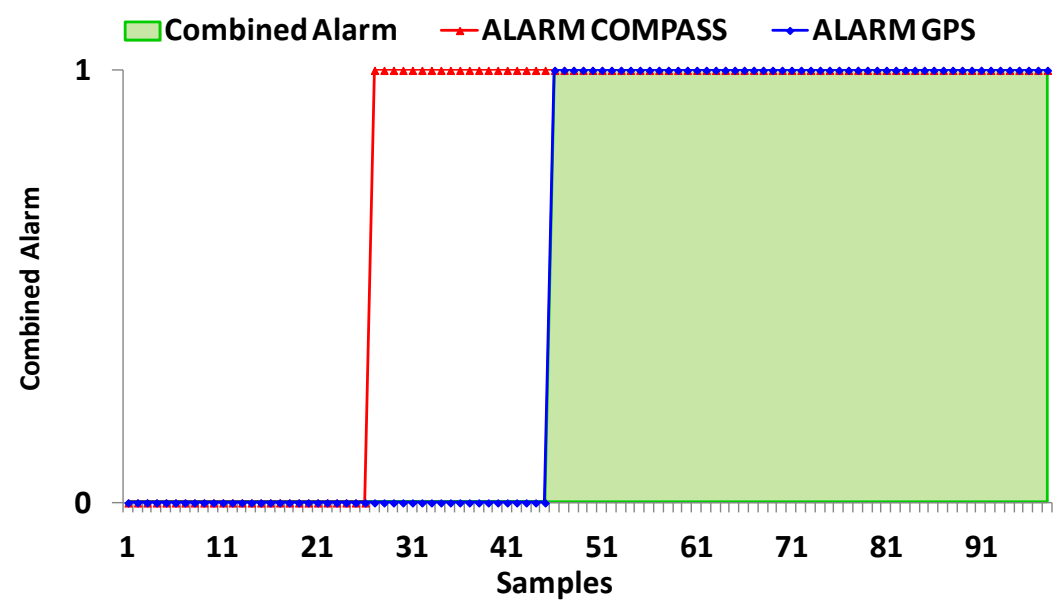

Figure 4.89. Combined alarm for compass and GPS sensors

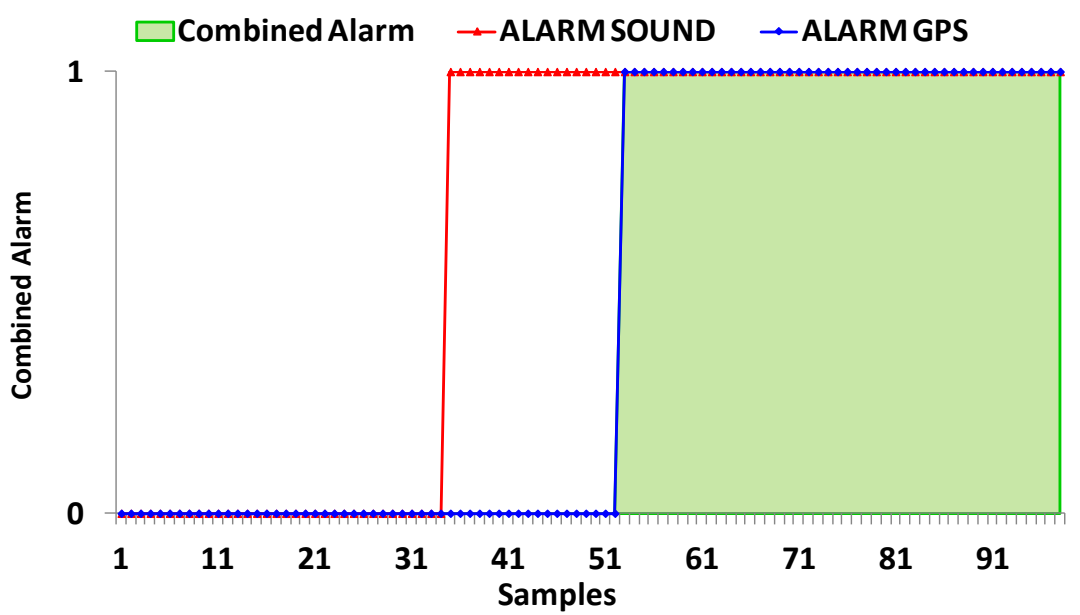

Figure 4.90. Combined alarm for GPS and microphone sensors.

\subsubsection{Ad hoc Network routing protocol}

In order to communicate all network devices, we should choose the most appropriate routing protocol for our network. In order to do this, we have simulated and compared 4 of the most used routing ad hoc protocols for a medium size group of persons. We have used the ns-2 simulator which allows generating any kind of topology. Figure 4.91 shows the topology employed for our simulations.

In Figure 4.91 we can see that node "0" represents the person suffering the accident and node "29" is the supervisor. Protocols used in this comparison are:

- Ad hoc On-Demand Distance Vector (AODV).

- Destination-Sequenced Distance-Vector Routing (DSDV).

- Dynamic Source Routing (DSR).

- Optimized Link State Routing (OLSR).

After simulating these 4 protocols, we have obtained some parameters that are very useful for our study. 
(10)

(8) (4)

(7)

(2)

(a)

Affected (3)
Person
(17)

(15)
(25) 29

(24)

(28)

(26)

(27)

(18)

(22)

(16)

(13)

(19)

(23)

Figure 4.91. Topology used in our simulations.

In Table 4.13 we present the comparison of the main parameters to evaluate routing protocols, such as: the time of reception of the first packet of the alarm message to the supervisor, the chosen route with the number of hops between nodes and the network overhead. We can see that the time needed to receive the first packet by the supervisor in proactive protocols vary between 10.19 seconds for OLSR and 72.30 seconds for DSDV, while for the reactive routing protocols, AODV and DSR, the first packet arrives at the supervisor position in less than a half of a second.

Table 4.13. Comparison of the routing protocol's principal features

\begin{tabular}{|l|l|l|l|l|}
\hline Protocol & $\begin{array}{l}\text { Time of reception of } \\
\text { the first packet }\end{array}$ & Route & $\begin{array}{l}\text { Number of } \\
\text { hops }\end{array}$ & Overhead \\
\hline DSDV & $72.30 \mathrm{~s}$ & $0-9-14-15-20-28-29$ & 6 & $22 \%$ \\
\hline AODV & $0.09 \mathrm{~s}$ & $0-9-5-12-15-24-28-29$ & 7 & $1 \%$ \\
\hline DSR & $0.13 \mathrm{~s}$ & $0-9-14-15-24-28-29$ & 6 & $29 \%$ \\
\hline OLSR & $10.19 \mathrm{~s}$ & $0-9-14-15-25-28-29$ & 6 & $67 \%$ \\
\hline
\end{tabular}

Figure 4.92 shows the number of packets in the network as a function of the time, so we can clearly see the behavior of the network in proactive routing protocols when the routing tables converge. For OLSR this convergence occurs approximately in 10 seconds and for DSDV in approximately in 70 seconds.

As we can see, proactive routing protocols like DSDV and OLSR introduce higher number of packets in the network than the others because they have to keep the routing tables updated. DSDV has a mean value of 135.18 packets per second with a standard deviation of 64.66 packets per second, and OLSR presents an average of 399.16 packets per second and a standard deviation of 164.5 packets per second. On the other hand, reactive routing protocols, AODV and DSR, introduce a smaller amount of packets into the network compared with the proactive protocol. Concretely, in AODV protocol we observed a mean value of 157.37 packets per second with a standard deviation of 16.19 packets per second and for DSR an average of 147.57 and a standard deviation of 15.75 packets per second. 


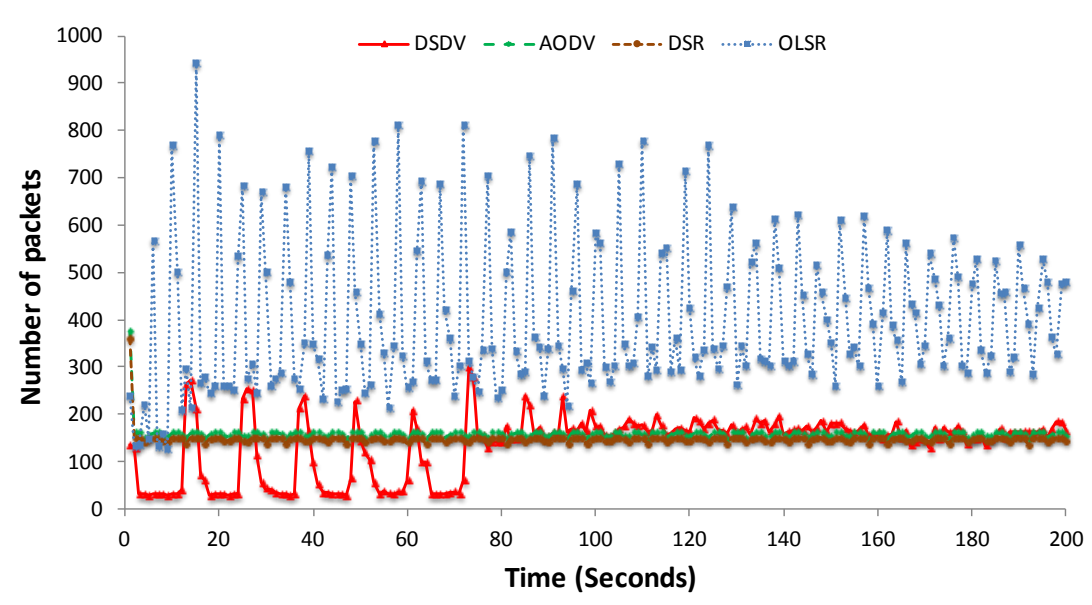

Figure 4.92. Volume of packets in the network, for the four protocols as a function of the time.

Figure 4.93 shows the amount of kb delivered through the network as a function of the time. As we know, OLSR routing protocol is one of most used ones in ad hoc networks, however, it presents the biggest number of transmitted bits. This is because OLSR is a proactive routing protocol, where all paths to all possible destinations are calculated a priori, keeping regular updates.

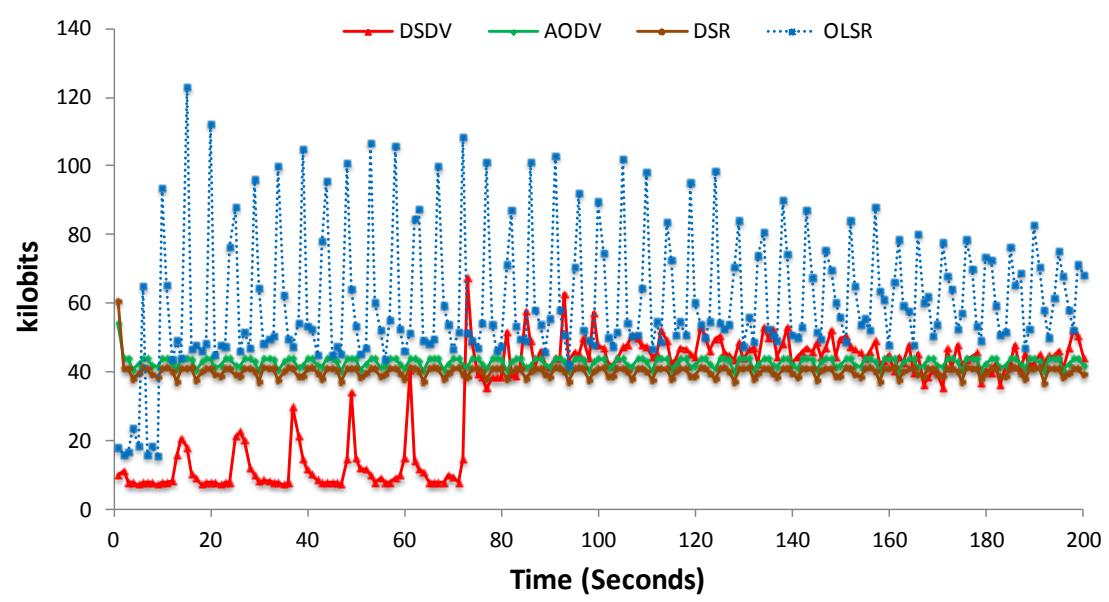

Figure 4.93. Volume of kilobits in the network, for the four protocols as a function of the time

The main disadvantage of this protocol is that it introduces overhead on the network, even on networks with few traffic flow. However, it can select valid routes almost immediately. In this case, we can estimate that OLSR shows a data flow close to $80 \mathrm{~kb}$, along the entire simulation time. Its average value of $\mathrm{kb}$ in the network is around $61 \mathrm{~kb}$. On the other hand, DSDV is a proactive routing protocol based on routing tables, where each network node maintains a routing table containing all possible destinations and the number of hops that a packet should travel to the destination. As we see, update messages must be sent continually through the network in order to maintain the consistency of the tables. However, the size of these packets is much lower than those used by OLSR. This makes to have an average amount of information in the network around $33 \mathrm{~kb}$. Lastly, we have AODV and DSR. They are reactive routing protocols, i.e., routes to find a path between senders and receivers are calculated only when they are needed to send information. For this reason, 
it has fewer network overheads. AODV has an average data volume of $42.7 \mathrm{~kb}$, while DSR presents an average value of $40 \mathrm{~kb}$.

\subsection{Conclusion}

This chapter has shown that the use on WSN in our daily life can help us in several aspects.

Firstly, we have presented three developments of WSN to monitor some facts in nature. On the one hand, we have seen a system to control and verify the existence of fire in a rural area. This system reduce the time between a fire is originated and the arrival of firefighters at the fire. We have also presented a system to detect diseases in vineyards and a WSN based on IEEE 802.15.4 standard to avoid the wolves' attacks and thefts.

Related to the buildings monitoring, we have presented a non-invasive technique based on a WSN for the detection of the cement degeneration in constructions. Our system is able to measure the temperature, humidity, and structural movements of the construction in order to quantify the degeneration of cement. In this case, our system cannot avoid the collapse of building but it can warn the inhabitants of the building, if the building registers any type of movement that can unbind in a collapse. Early detection of these parameters will prevent any personal loss

Finally, we have presented a WSN to take care of disabled and elderly people. Our smart system is based on the embedded sensors of mobile devices in order to determine if a person that wears one of these devices has suffered an incident or has a problem. As we have seen, the responses of several devices are combiner to avoid false positives. In this case, the main advantage of our system is that it is possible to detect any problem in a disabled and elderly person and the localization of this person will be faster. In addition, it is very cheap because nowadays everybody has a smartphone.

As we have seen, it is possible to integrate new technologies in several application fields where the main feature is that these systems improve the detection of the problem and consequently the response of emergency services is much faster reducing economic and personal losses (in some cases).

Finally, work presented in this chapter has been published in the following references [162], [163], [164], [165], [166] and [167]. 



\section{Chapter 5}

\section{Wireless Sensor Development for Monitoring in Indoor Environments}

\subsection{Introduction}

In recent years, wireless sensor networks (WSNs) based on the IEEE 802.11a/b/g/n [7] have experienced a spectacular market growth, due to the improvement in performance and their low cost. They are fairly easy to be installed and offer benefits such as mobility, flexibility, lower costs (in the network deployment) [335] and scalability. The location of the sensors in a WSN is usually placed randomly and, in some cases, they are just placed in an easy access location. It is very important to know the behavior of the signals within a building.

When a wireless network is designed for a specific environment, it is necessary to study the distribution of the place in order to determine the better location for each AP and the channel distribution. The goal is to provide the greatest possible coverage but avoiding overlapping among channels according to the building distribution. It is very difficult and tedious to analyze each place in detail before installing, because each building has a different distribution and with different sources of interferences. An accurate analysis of the signals generated by the access points (APs) and network devices could help us to improve network coverage within the building [336].

One of the most important things which must be considered when a wireless network is being designed is the wireless signal losses. They depend on the number of walls and obstacles crossed in its propagation path, the materials used in the building construction, the type of obstacles and the multipath effect. There are several materials such as furniture and metallic structures or wood, very used in buildings, or simply through normal walls and floors, which affect to the wireless signals 
reducing significantly their signal level [337][84]. The multipath effect and the signal attenuations caused by reflections over the walls, floors and roofs generate high impact because they enhance the scattering and diffraction. Moreover, there are other factors in indoor environments such as electromagnetic fields provided by electronic devices [338] which can affect to the data transmission and network performance. An accurate design also means a good sized network, where only the APs/routers/wireless nodes needed to cover the service area and to obtain high efficiency must be used. The number of wireless devices (and deployed networks) working under the IEEE 802.11 technology are growing and the probability of generating interference between systems is getting higher. There are some technical limitations that require a detailed study of the optimal placement of sensors to provide wireless coverage indoors. They can impede the communication by attenuating the signal between the transmitter and receiver. Interferences caused by electromagnetic waves from other systems could be reduced selecting the most appropriate frequency band for the wireless network and a good channel assignment.

There are many studies on the signal propagation [337][338] and studies on special challenges when designing indoor WLANs [97]. Some factors such as the distance between emitter and receiver affect the delay between echoes which enlarge the delay spread. So, although it is quite easy to estimate the area of the radio coverage in a free space, it is very difficult to calculate it in indoors since the building distributions are not uniform [339]. People walking in any corridor or facility close to the emitter or the receiver may also cause significant variations [97].

Regards to WSN, there are several indoor applications that require an optimum and high performance sensor placement. These sensors can be part of a larger network dedicated to security and motion detectors, anti-theft systems or alarm systems such as smoke detection systems, pollution detection systems, fire systems, etc. Therefore, it is important to know what the best place to locate a sensor is. The best way to determine where to place a sensor is doing a detailed coverage study which provides an AP working in a specific wireless technology [170]. With these tests someone can start to place the sensors in the most appropriate places, taking into account the problems due to indoor environments.

In addition to all the design parameters discussed above, it is essential to analyze and study which type of traffic and users the target WLAN goes to support, and how much and how many respectively. Depending on that, it will require more or less resources, bandwidth and performance. Finally, the physical distribution must be considered in order to select the APs locations since each AP needs a power supply and a point of connection to the wired network. Performing a correct and optimal design of an indoor wireless networks would subsequently let the network administrators include multiple services such as positioning and tracking of people and objects.

This chapter shows the developed propagation models for IEEE $802.11 \mathrm{a} / \mathrm{b} / \mathrm{g} / \mathrm{n}$ networks in indoor environments. These propagation models help us to know the best position of wireless sensor nodes in these environments. To do this, in Sections 5.2.3 and 5.2.4, we perform two practical studies of signal behavior in two buildings of different sizes. As Scenarios, we have chosen the CRAI (Learning and Research Resources Centre), a building of the Higher Polytechnic School of Gandia, of the Polytechnic University of Valencia, which surface is approximately 2000 $\mathrm{m}^{2}$ and a garage which is approximately $100 \mathrm{~m}^{2}$. In each scenario, it is determined the signal strength level registered in each point of buildings. We also analyze the interference that these wireless devices can suffer and the network performance obtained depending on the working 


\section{Chapter 5: Wireless Sensor Development for Monitoring in Indoor Environments}

channel. All of these measures are analyzed as a function of the type of device and the standard type (Section 5.2.5).

Finally, from the propagation models extracted in both scenarios, we propose a new method for wireless nodes positioning which reduces to $15 \%$ the number of nodes required to cover an area.

Last Section shows the conclusions of this chapter.

\subsection{Tools Used in the Measurement Process}

This section shows the tools used to carry out our tests and studies in order to characterize the behavior of the wireless signals in indoor environments. We also show a detailed description of the place and the strategies for taking the measurements.

\subsubsection{Software Used in the Test Bench}

In order to perform our study and tests, we have used 3 software tools which are usually used for testing the network.

- InSSIDer is an open-source program that graphically displays all visible networks in an area. This program can detect the wireless networks and manage, in a graphic mode, the intensity of these signals. It lists their SSID, MAC address, channel, Radio Signal Strength Indicator (RSSI), type of network, security, speed and signal intensity and allows monitoring the signal quality via a chart using the received RSSI. It is able to work in $2.4 \mathrm{GHz}$ and $5 \mathrm{GHz}$ and it is capable to record the signal strength over a five-minute period. [340].

- Microsoft Windows commands. They are a set of programs that allow manipulating the information in the hard disc and coordinate the microprocessor operation. There are some utilities and commands, which allow checking the status of the network connection.

- Net Meter monitors network traffic and shows the bandwidth rate used by a network device. It monitors the network traffic through all network connections and displays real-time graphical and numerical downloading and uploading speeds. The software program can display transfer rates of multiple network connections at the same time. [341].

\subsubsection{Hardware Used in the Test Bench}

We have selected four APs from different brands and models to perform our tests. All of them are capable of working in different IEEE 802.11 variants $(\mathrm{a} / \mathrm{b} / \mathrm{g} / \mathrm{n}$, depending on the model). The used models are described in Table 5.1. In addition, we have used three computers to take the coverage measurements: one laptop with dual core processor at $1.67 \mathrm{GHz}$ per core and 1 GByte of RAM, and in order to take the interference measurements, we have used two desktop PCs with an AMD $1700 \mathrm{MHz}$ CPU and 1 GByte of RAM memory.

For measurements performed in the first scenario, we have used Cisco Aironet access points 1130 A, which are those installed on the CRAI. In measurements performed in the garage, all APs have been used. 
Table 5.1. APs used for our tests.

\begin{tabular}{|l|l|l|l|}
\hline Device & Wireless technology & $\begin{array}{l}\text { Working } \\
\text { Frequency }\end{array}$ & Power output \\
\hline Linksys WRT320N & IEEE $802.11 \mathrm{a} / \mathrm{b} / \mathrm{g} / \mathrm{n}$ & $2.4 \mathrm{GHz}$ and $5 \mathrm{GHz}$ & $17 \mathrm{dBm}$ \\
\hline Dlink DWL-2000AP+ & IEEE $802.11 \mathrm{~b} / \mathrm{g}$ & $2.4 \mathrm{GHz}$ & $16 \mathrm{dBm}$ \\
\hline Cisco Aironet $1130 \mathrm{AG}$ & $\begin{array}{l}\text { IEEE } 802.11 \mathrm{a} / \mathrm{g} \\
\text { Compatible with IEEE802.11b }\end{array}$ & $2.4 \mathrm{GHz}$ and $5 \mathrm{GHz}$ & $\begin{array}{l}\text { IEEE } 802.11 \mathrm{a}: 17 \mathrm{dBm} \\
\text { IEEE } 802.11 \mathrm{~g}: 20 \mathrm{dBm}\end{array}$ \\
\hline Linksys WRT54GL & IEEE $802.11 \mathrm{~b} / \mathrm{g}$ & $2.4 \mathrm{GHz}$ & $18 \mathrm{dBm}$ \\
\hline Linksys WUSB600N & IEEE $802.11 \mathrm{a} / \mathrm{b} / \mathrm{g} / \mathrm{n}$ & $2.4 \mathrm{GHz}$ and $5 \mathrm{GHz}$ & $\begin{array}{l}16 \mathrm{dBm} \\
\text { Sensitivity:-91dBm. }\end{array}$ \\
\hline
\end{tabular}

\subsubsection{Measurements in Scenario 1: CRAI}

The CRAI building was built in 2007. It is composed of 3 floors where different services for the students are offered. It contains the library, computer labs and open access classrooms. Figure 5.1 shows the map of this space. It is the $\mathrm{H}$ building of this university campus.

Now we are going to describe the scenario where the measurements have been taken from the wireless networks and the type of hardware and software used to perform our research.

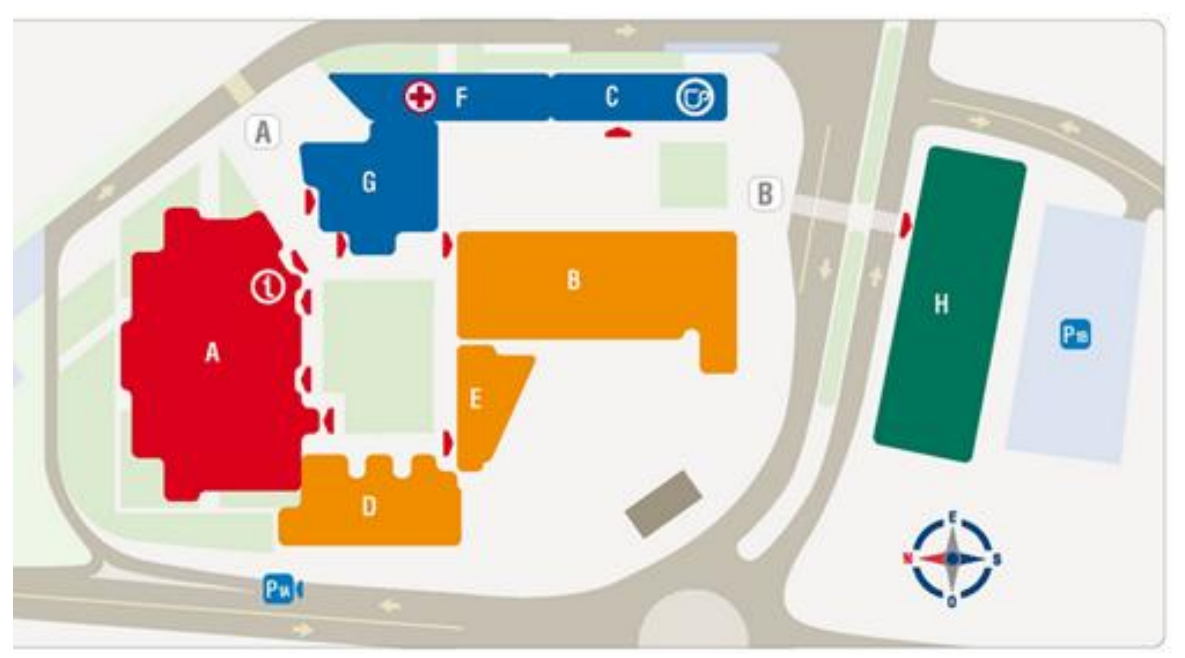

Figure 5.1. Map of Higher Polytechnic School of Gandia.

\subsubsection{The building}

The ground floor (see Figure 5.2) contains an information desk, several staff offices, a library and a large study room with a consultation area and several group study rooms. Finally, there is a multipurpose room where events and exhibitions are sometimes held.

On the first floor (see Figure 5.3) we can find several computer labs, some classrooms to perform Final Degree Projects, and others group and individual study rooms. 
On second floor (see Figure 5.4), there are a large library with magazines, journals, books and audiovisual resources, and some computer labs and professor offices.

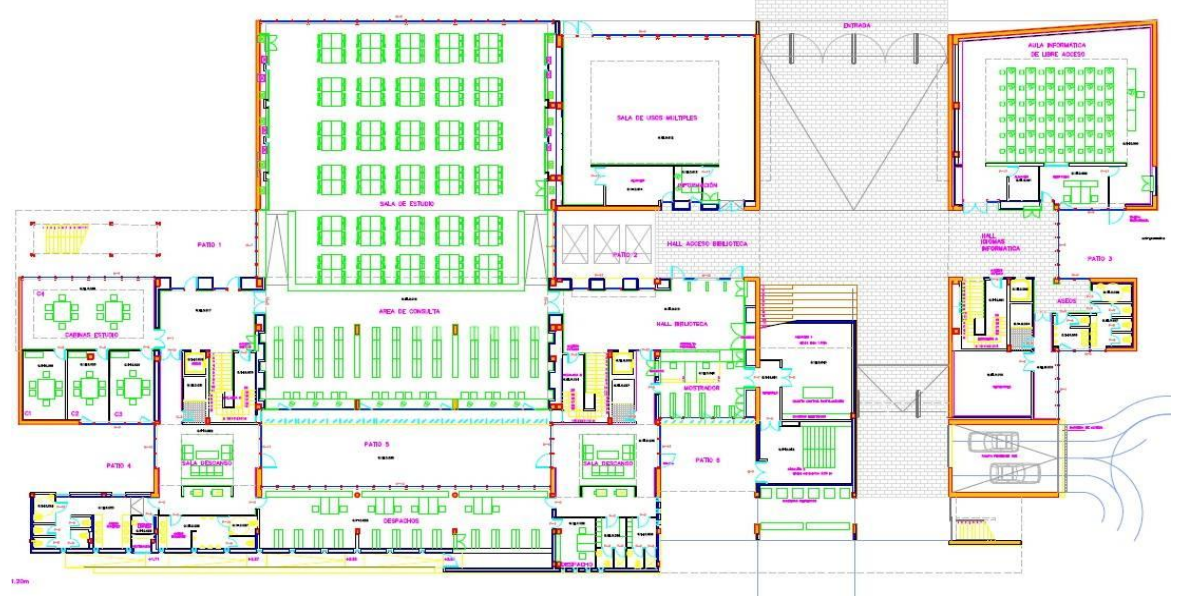

Figure 5.2. Ground floor of the CRAI bulilding.

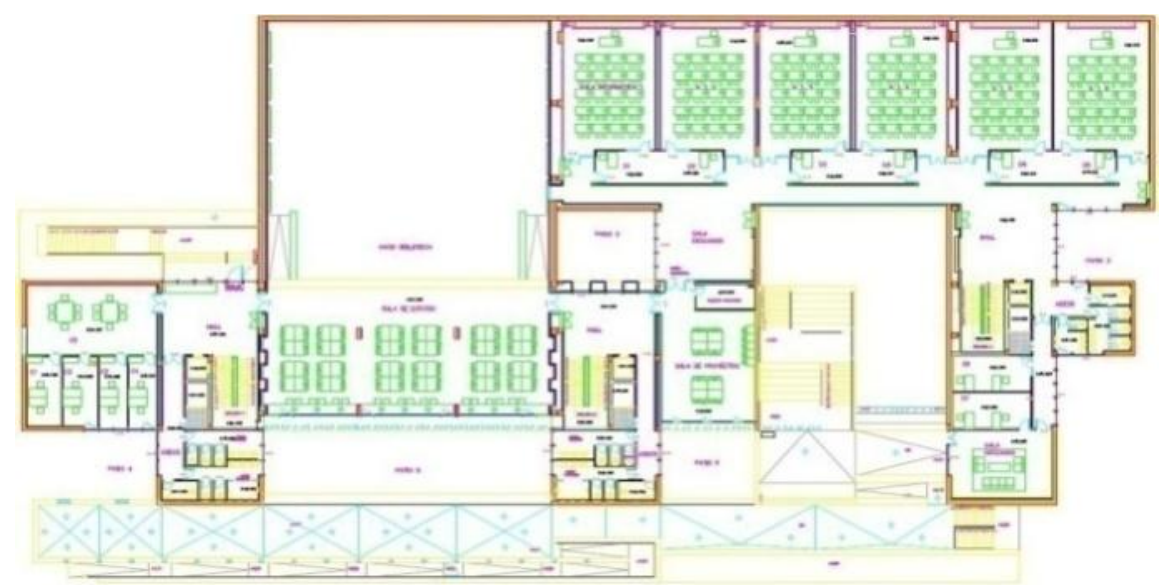

Figure 5.3 First floor of the CRAI building

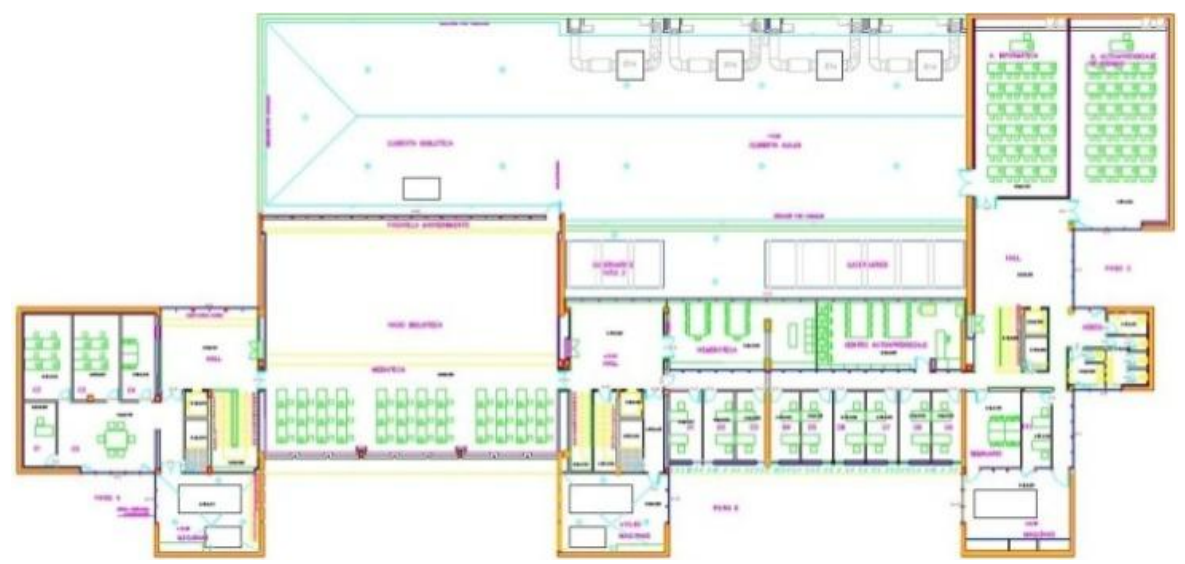

Figure 5.4 Second floor of the CRAI building 


\subsubsection{Description of UPV wireless network}

Higher Polytechnic School of Gandia is a campus of the UPV and shares four wireless networks with the main campus, their SSIDs are EDUROAM, UPVNET2G, UPVNET and UPV-INFO. Each one of these allows university users to access to the Internet and the university resources. Their main features are:

- UPVNET: a wireless network with direct connection to all the resources of the UPV. It requires a wireless card with configured with WPA/WPA2 security.

- UPVNET2G: a direct network connection to all resources of the UPV and the Internet. It requires a wireless card configured with WPA/WPA2 security.

- EDUROAM: this wireless network is widely deployed in universities and research centers in Europe. It provides Internet access for all their members. Users only need a username and a password from their home institution. It requires a wireless card configured with WPA/WPA2 security. This network only provides Internet access.

- UPV-INFO: this wireless network works as a consultation area. It only provides all information about how the wireless network cards must be configured in the users' devices in order to connect them to some of the abovementioned networks. It uses private IP addressing and it does not allow users to access to the Internet. A second connection is needed to access to the Internet and the UPV resources. This second connection can be a Virtual Private Networking (VPN). It should only be used by very old computers that do not support WPA encryption.

We have analyzed three of these networks (UPVNET, UPVNET2G, EDUROAM), because these are the only ones that allow users to access to the Internet.

\subsubsection{Coverage measurements}

We only have considered the walking area from which users typically connect to the wireless network. So, bathrooms, exterior stairways, storage rooms, etc. have been excluded. In order to perform this coverage analysis, a grid of 4 meters $\mathrm{x} 4$ meters has been drawn in each floor. This allows us to take measurements for the different networks from the same places. The laptop in charge of taking measurements was located at a height of $100 \mathrm{~cm}$ above the ground.

\section{Ground floor}

This subsection shows the coverage study on the ground floor. There are 5 APs to cover the entire plant. There are four places with the highest coverage level (the values are higher than $-50 \mathrm{dBm}$ ). We highlight 2 rooms, Room A, the multipurpose room, and Room B, the computer room (see Figure 5.5, 5.6 and 5.7). The AP located outside the wall of the computer room provides coverage levels below $-70 \mathrm{dBm}$ inside the classroom for all three cases.

Figure 5.5 shows the coverage area and levels of UPVNET wireless network on the ground floor. Room A presents signal strength of $-90 \mathrm{dBm}$ due to the signal attenuation suffered by the wireless signals when they cross some walls. 


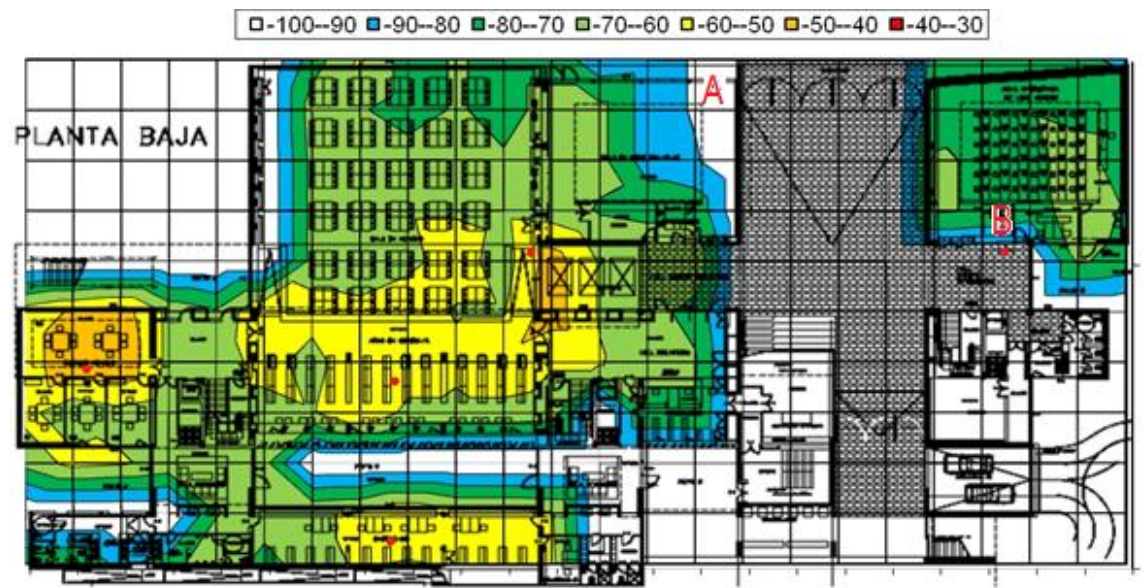

Figure 5.5. Radio coverage map of the ground floor for UPVNET

Figure 5.6 shows the coverage area for the UPVNET2G wireless network on the ground floor. We find three places where signal strengths are higher than $-50 \mathrm{dBm}$. These places are just those ones where the APs are located currently. The multipurpose room has a very low coverage on the left side because the signal is greatly attenuated by several walls.

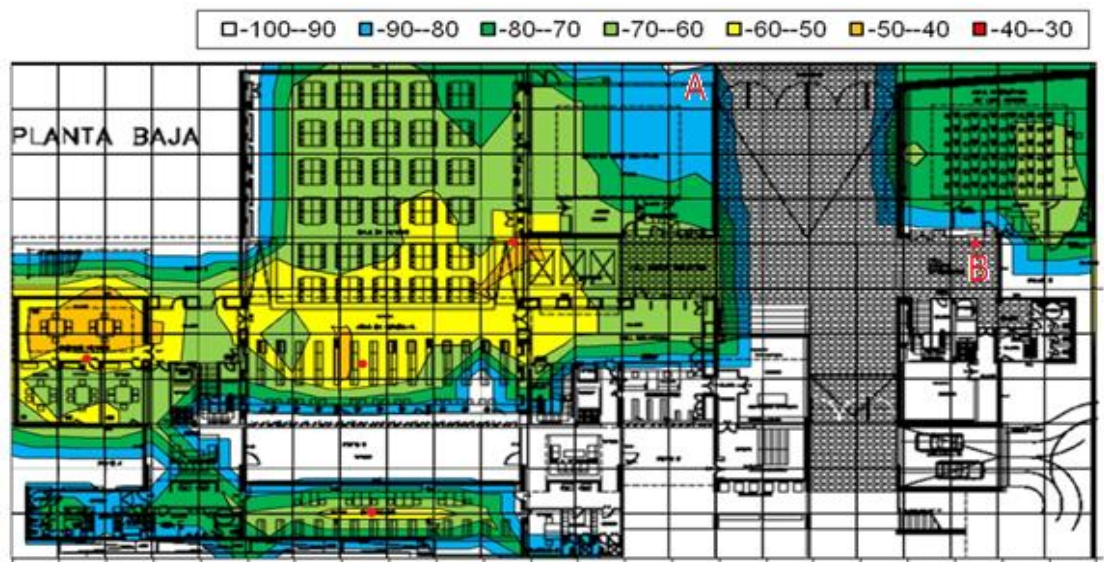

Figure 5.6. Radio coverage map of the ground floor for UPVNET2G

Figure 5.7 shows the value of signal strength for EDUROAM wireless network on the ground floor. Again, there are three places with signal strengths higher than $-50 \mathrm{dBm}$, which correspond to the current location of the APs. In this case, more than half of the room B has signal strength levels below $-70 \mathrm{dBm}$. 


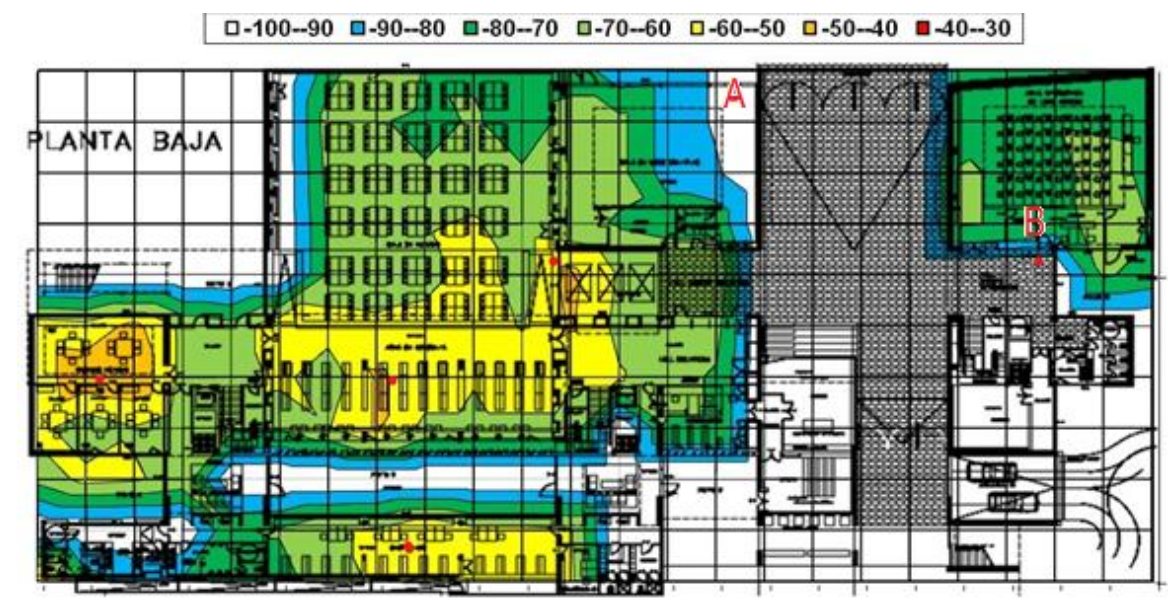

Figure 5.7. Radio coverage map of the ground floor for EDUROAM

\section{First floor}

This subsection shows the signal strengths measured on the first floor. In this case there are 4 APs to cover the entire plant. There are 4 places with the highest signal strengths (higher than $50 \mathrm{dBm}$ ).

Figure 5.8 shows the signal strength for UPVNET wireless network on the first floor. The rooms at the left side have low radio coverage because the AP is not located in the correct place. The offices at the right side have also very poor signal strength because they are very close to the stairs and they suffer important signal attenuation.

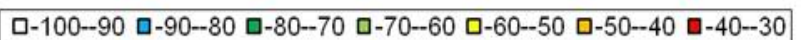

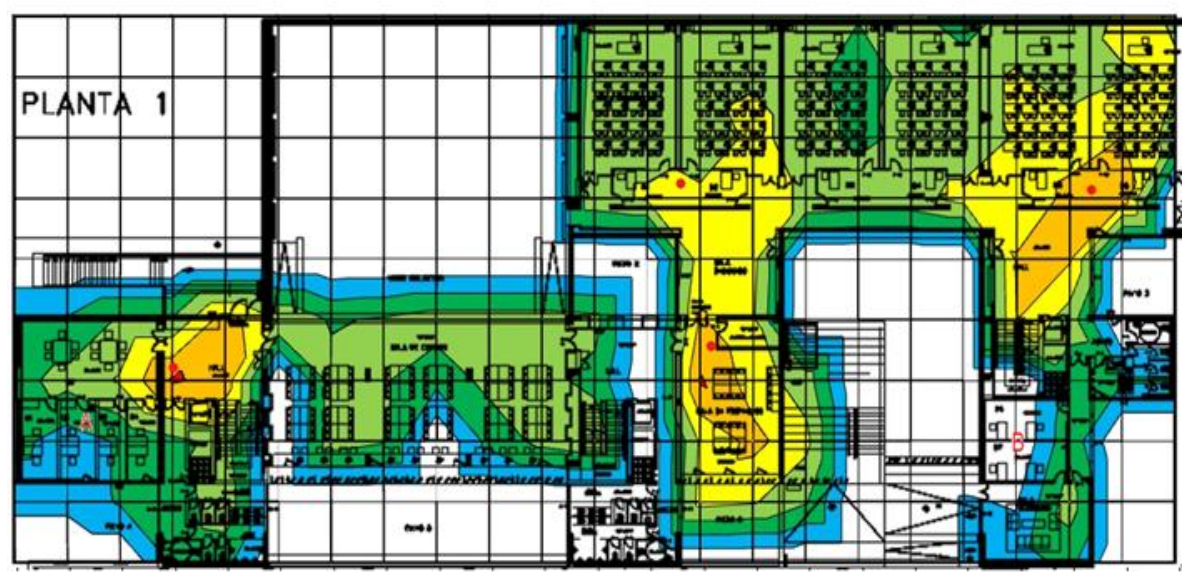

Figure 5.8. Radio coverage map of the first floor for UPVNET.

Figure 5.9 shows the UPVNET2G wireless network signal strengths on the first floor. We can see that the classroom on the left side is not well covered because of the position of the AP. It is located on the right side of the wall. The offices from the bottom right also have very poor coverage, because they are very close to the stairs, which generate significant signal attenuation. 


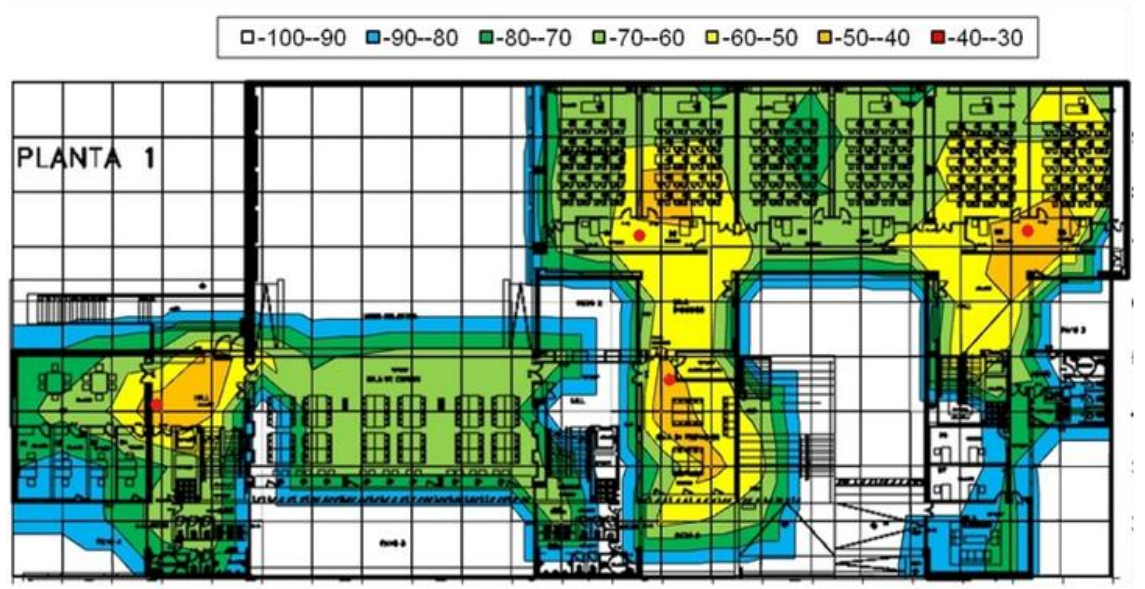

Figure 5.9. Radio coverage map of the first floor for UPVNET2G

Figure 5.10 shows the EDUROAM signal strengths on the first floor. In this case, we can see the same effect as in the other cases, but moreover there are tables in the study area (center of the picture) with low signal strength (lower than $-90 \mathrm{dBm}$ ).

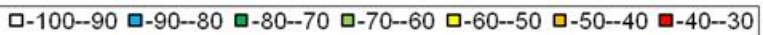

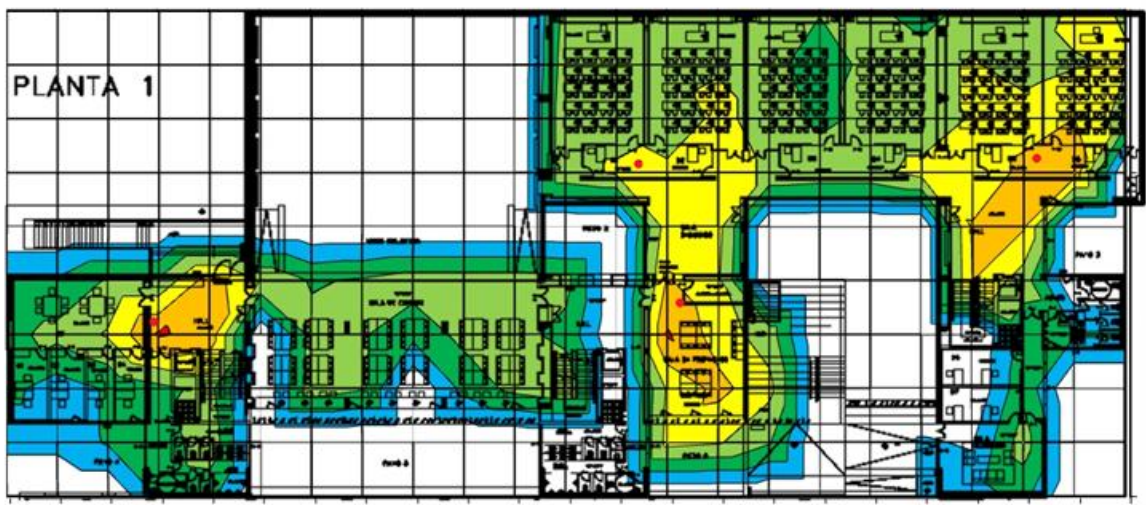

Figure 5.10. Radio coverage map of the first floor for EDUROAM

\section{Second floor.}

This subsection shows the signal strengths measured on the second floor. The floor is covered by 4 APs. Figure 5.11 shows the signal strengths for UPVNET wireless network on the second floor. The highest signal level is provided by the AP located at the professors offices zone (central zone of the image), which provides signal levels lower than $-60 \mathrm{dBm}$. Moreover, the AP located in the hall of the two computer rooms (top right of the Figure 5.8), covers virtually the entire rooms, registering levels of $-70 \mathrm{dBm}$ in the teacher's desk. The APs located at the central-left and the bottom-left areas of the library, have signal levels around $-60 \mathrm{dBm}$, except at the areas near to the outer walls where values of-70dBm have been registered. 


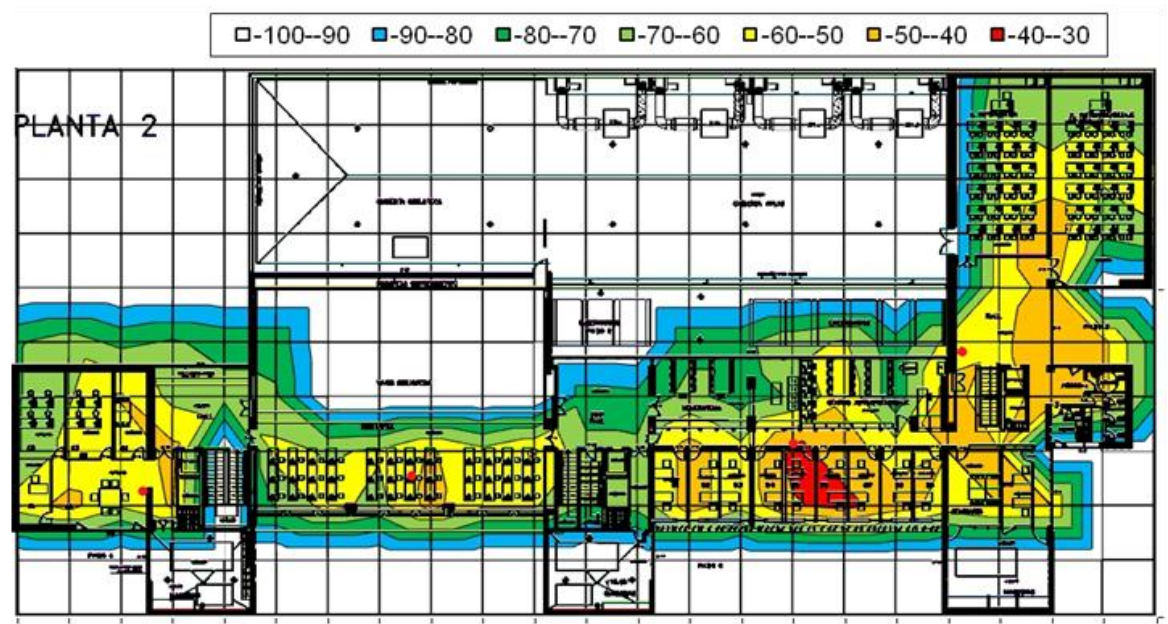

Figure 5.11. Radio coverage map of the second floor for UPVNET

Figure 5.12 shows the signal strengths from the UPVNET2G network on the second floor. In this case, the signal is propagated with levels above $-60 \mathrm{dBm}$, practically in both computer rooms (top right of the image). In contrast, the professor offices (central zone of the image) register levels close to $-50 \mathrm{dBm}$. Finally, the area of journals and audiovisual resources of the library (bottom - left of the image) presents levels around $-60 \mathrm{dBm}$, showing levels around to $-50 \mathrm{dBm}$ in the area near to the AP.

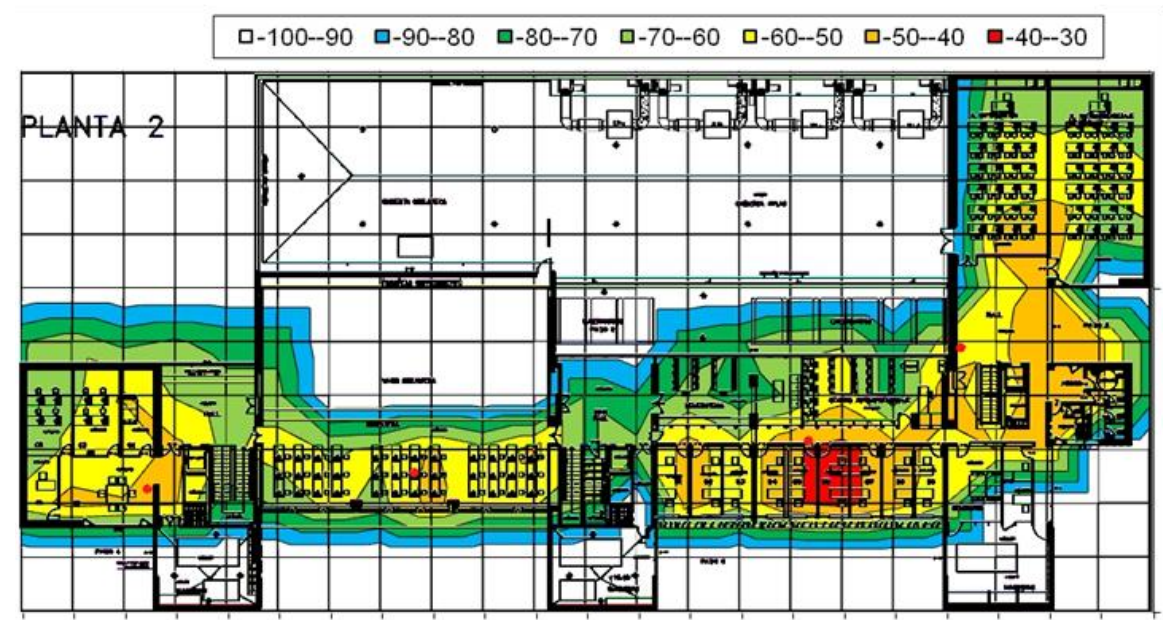

Figure 5.12. Radio coverage map of the second floor for UPVNET2G

Figure 5.13 shows the signal strengths from EDUROAM wireless network on the second floor. The signal strength offered by the EDUROAM network is slightly lower than those ones shown for UPVNET and UPVNET2G networks. We can see that there are more areas with signal levels close to $-70 \mathrm{dBm}$. This happens in the computer rooms (top right of the image) and in the library (bottom left of the image). Most of these areas are zones near to walls or walkways, which are not usually used as workplaces. 


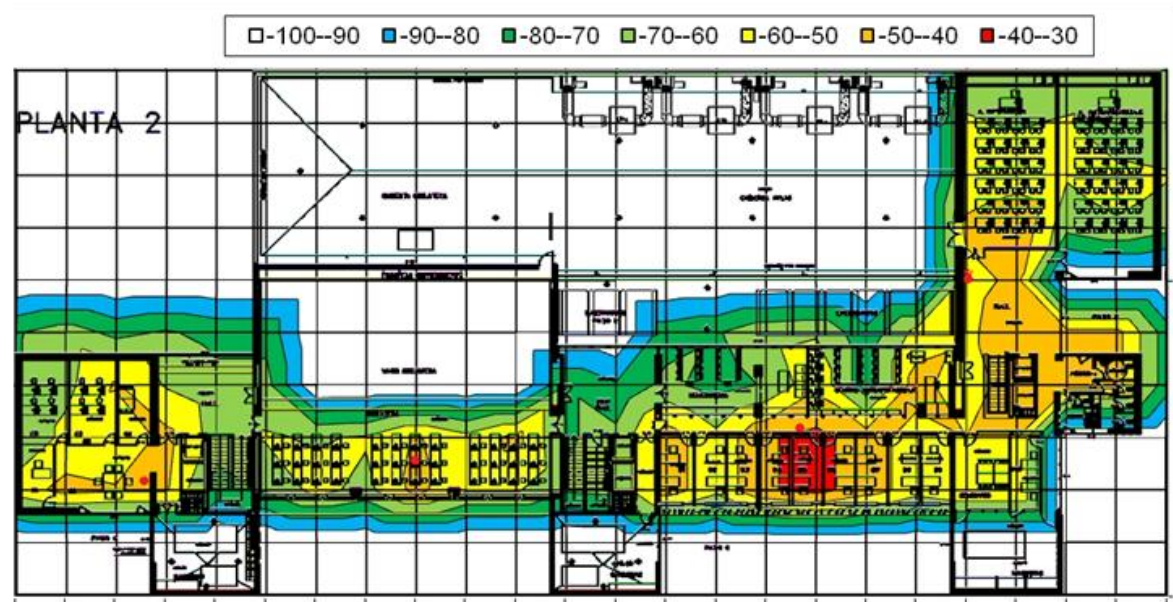

Figure 5.13. Signal strength on the ground floor.

We can conclude that the signal is correctly broadcasted through the entire floor and their signal strength levels are enough acceptable to cover the working places.

After analyzing all the radio coverage images, it is easy to see that the behavior of the wireless signal in each floor is quite similar, only small variations have been registered. In addition to this, we have checked that the received signal strength is very low from bathrooms and toilets. This is because the amount of water pipes and copper tubes in the walls affects to the propagation path of the wireless signals attenuating them. We have also found low signal strength levels in the stairwells. The stairs usually are made of metal framework and a foundation which avoids a correct propagation of the signal.

\subsubsection{Comparative study of three wireless signals}

In this section we compare the three received wireless signals from each available wireless network on the same plant. Figure 5.14 shows the three signals at the ground floor. UPVNET2G provides better signal strength levels than UPVNET and EDUROAM.

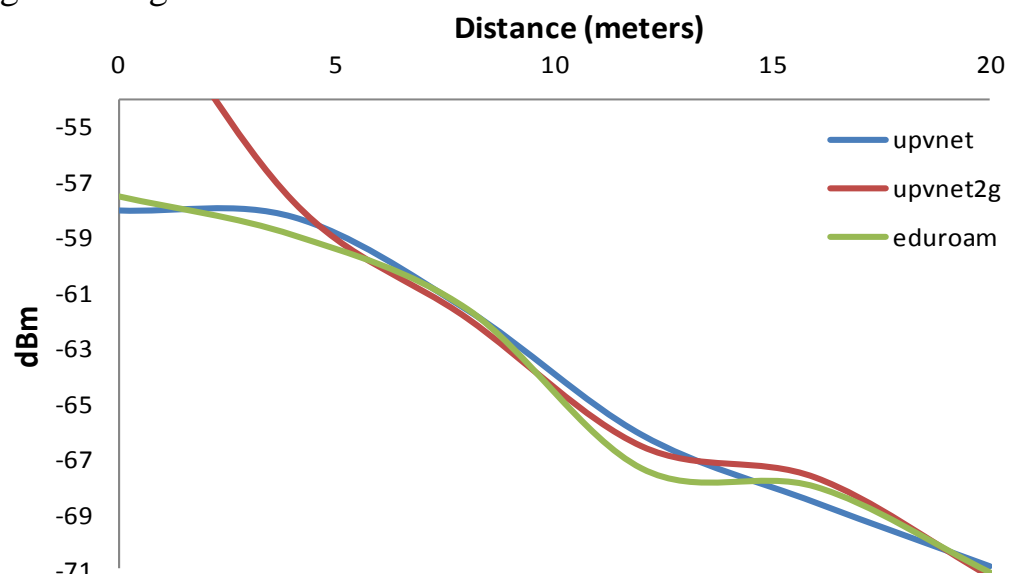

Figure 5.14. Signal strength on the first floor. 
Signal strengths from the first floor are shown in Figure 5.15. UPVNET2G is the network which reaches the highest signal strength. UPVNET and EDUROAM show similar behaviors although there are some locations where the received signal from EDUROAM network is better.

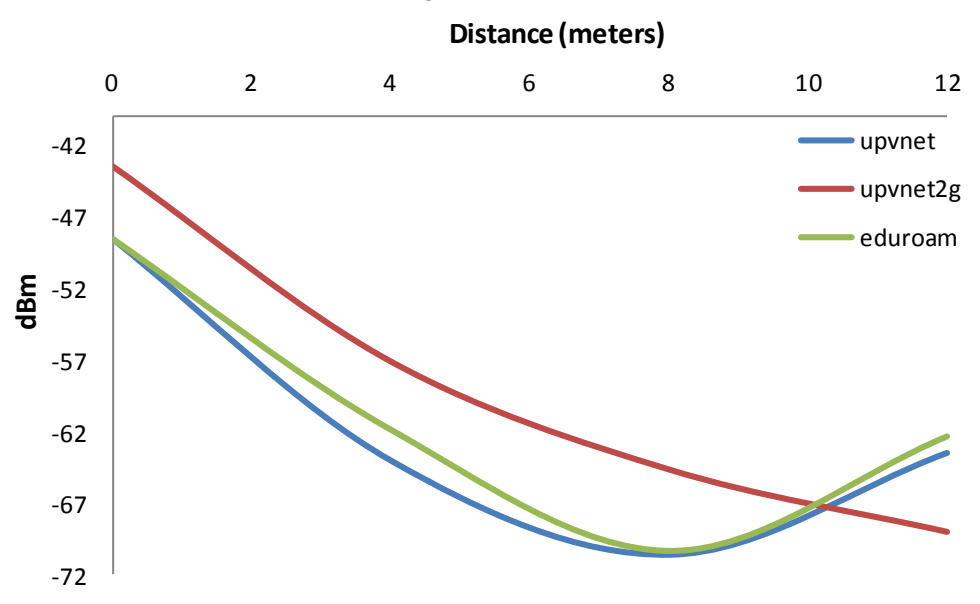

Figure 5.15. Signal strength on the first floor.

Figure 5.16 shows the behavior of signal strength on the second floor. UPVNET2G and EDUROAM show the same behavior from 3 meters to around 10 meters, but from 0 to 3 meters and from 10 meters to 12 meters, the signal strength from EDUROAM network is better. The lowest signal strength is always performed by UPVNET network. Keeping in mind all graphs, it is easy to conclude that according to signal strength, the best wireless network is UPVNET2G. Furthermore, we observe that the ground floor presents generally better signal strengths than in the other two floors.

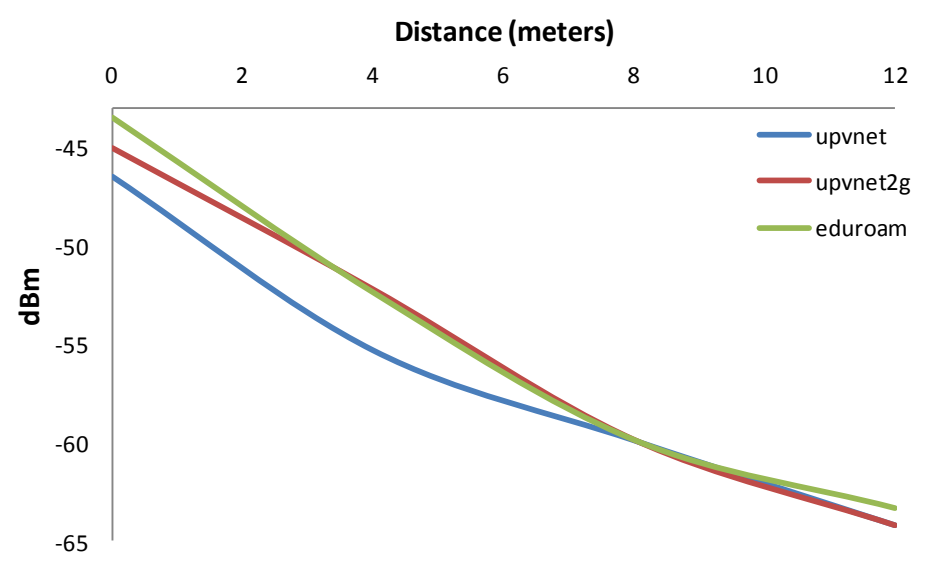

Figure 5.16. Signal strength on the second floor.

\section{Analytical study for three signals}

After analyzing the above figures, we can estimate the behavior of the wireless signals in indoor environments.

Therefore, this section shows how the signal strength varies depending on the distance from the AP. In the previous section, we have shown the signal strength per floor and per SSID (Figures 5.14, 


\section{Chapter 5: Wireless Sensor Development for Monitoring in Indoor Environments}

5.15 and 5.16). In this section, we are going to work with the average value of all APs (per floor) and the mean value recorded for the three signals in order to analyze and generalize the overall network behavior since all APs used in the network are equal and the three signals are provided by the same AP. The mathematical equation is calculated from the tendency line of each graph.

The analytical study is performed for three networks (UPVNET, UPVNET2G and EDUROAM) again. In order to draw each one of these graphs, we have estimated the average value of the three signals provided by each wireless network.

Figure 5.17 shows the average value of the signal strength depending on the distance from the AP on the ground floor.

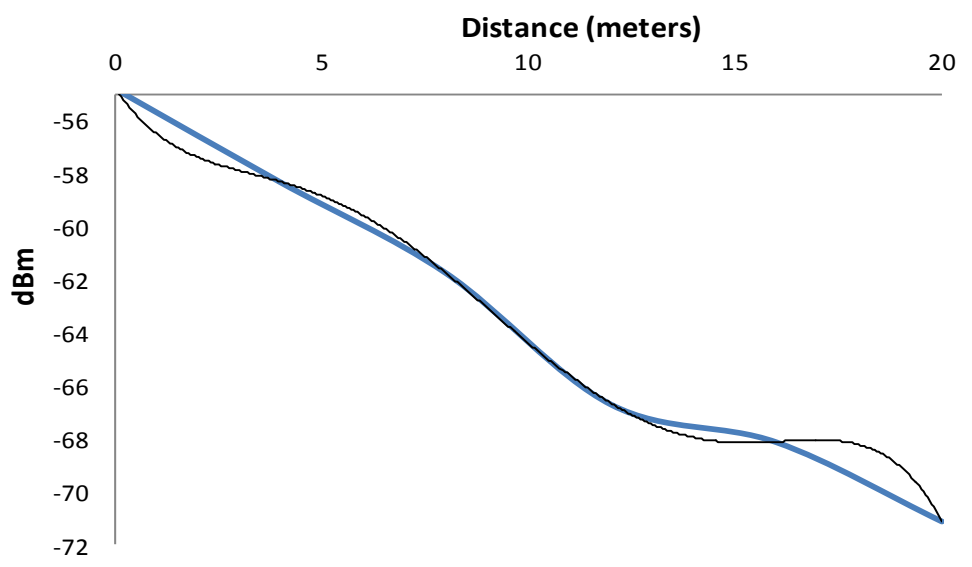

Figure 5.17. Average signal strength on the ground floor

Expression 5.1 shows the equation for the trend line (black line in Figure 5.17) from our measurements. As we can see, it is a fifth-order polynomial equation, with a correlation coefficient $\left(\mathrm{R}^{2}\right)$ equal to 1 . However, we can appreciate a slight difference between them in positions close to 34 meters, and further away than 17 meters from the APs.

$$
\mathrm{Y}=-0.0001 \mathrm{x}^{5}+0.0066 \mathrm{x}^{4}-0.1078 \mathrm{x}^{3}+0.6889 \mathrm{x}^{2}-2.3012 \mathrm{x}-54.75
$$

Where $Y$ represents the average value of the received signal strength in $\mathrm{dBm}$ and $\mathrm{X}$ is the distance in meters from the AP.

Figure 5.18 shows the average signal strength provided by the APs located on the first floor as a function of the distance from the APs. In positions further than 8 meters from the APs, both graphs vary very few between them, although the rest of the graph is identical. Equation 5.2 shows the expression for the trend line (black line in Figure 5.18) from our measurements.

The behavior of wireless signals based on the distance is described by a cubic polynomial with a correlation coefficient $\left(\mathrm{R}^{2}\right)$ equal to 1 .

$$
Y=-0.0117 x^{3}+0.0665 x^{2}-3.9909 x-46.83
$$

Where $\mathrm{Y}$ is the signal level in $\mathrm{dBm}$ and $\mathrm{X}$ is the distance in meters from the AP.

Figure 5.19 provides the behavior of the signal strength on the second floor. 


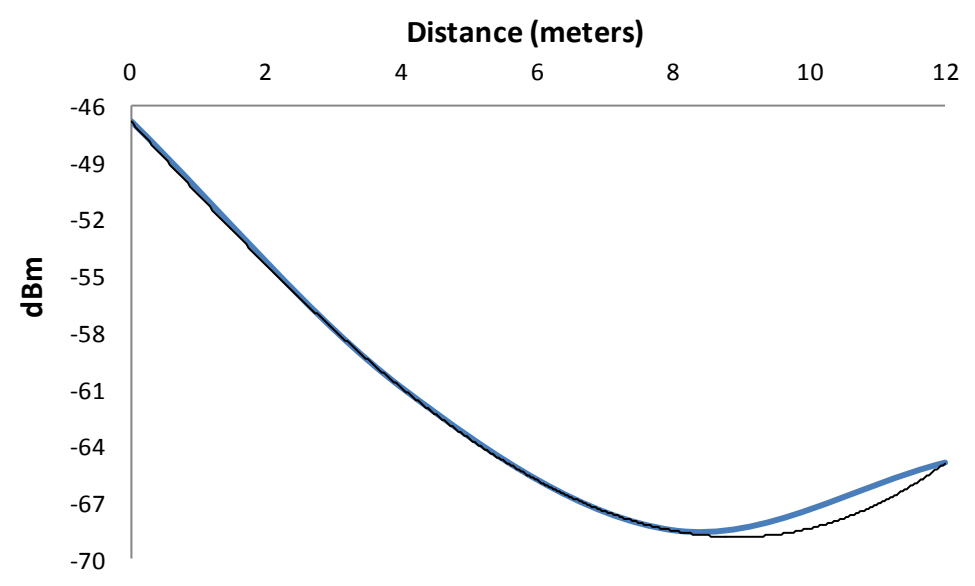

Figure 5.18. Average signal strength on the first floor

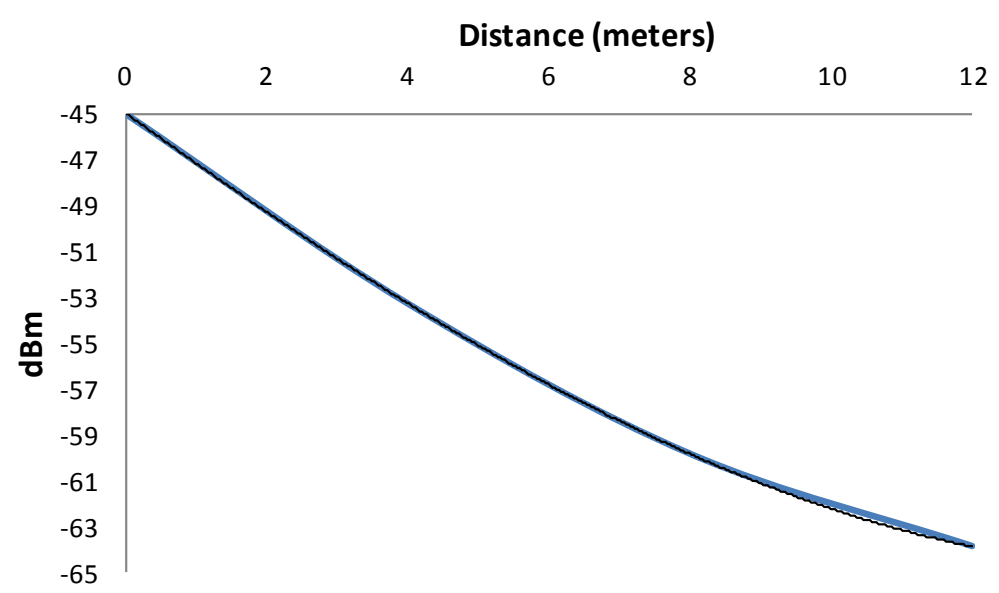

Figure 5.19. Average signal strength on the second floor

Equation 5.3 shows the trend line (black line in Figure 5.19) from our measurements. In this case equation 3 is a third-order polynomial equation with a correlation coefficient $\left(\mathrm{R}^{2}\right)$ equal to 1 . As its correlation coefficient shows, both graphs have a nearly perfect match.

$$
Y=0.0021 x^{3}+0.0292 x^{2}-2.2229 x-45
$$

Where $\mathrm{Y}$ is the average signal value in $\mathrm{dBm}$ and $\mathrm{X}$ is the distance in meters from the AP.

\section{WLAN redesign in CRAI}

Designing WLANs which main focus is to provide the best service using the available resources efficiently requires careful planning. WLANs can be as small as a home network or as large as a network of a company with complex distributions and several buildings. Before installing a WLAN, it is essential to have the technical information about network devices and a well-defined plan for the development process. The usual sequence of steps in the APs location process is as follows:

1) Firstly, an AP is placed in each corner of each floor of the building, and it is measured the maximum coverage area for each one of them. 
2) From these measurements, it is easy to determine the most suitable place for the APs taking into account that it is only needed $15 \%$ of overlapping area.

This is the most reliable process in order to find out the best location for the APs. However, it is very tedious, impractical and it implies too much time for the networks designers. Moreover, sometimes it is unfeasible for example in big buildings due to the number of measurements needed to make an accurate decision about the best potential locations or in buildings where it is not possible to gain access to take measurements [342].

We are going to relocate APs and redesign a WLAN already installed, because as we have seen in measurement results section, there are some areas where wireless coverage is very poor with wireless signal strength lower than $-70 \mathrm{dbm}$. So, in this section we propose several changes to improve the network infrastructure and to guarantee the greatest coverage in such areas. Moreover, wireless channels used by APs are also redefined in order to reduce the interference between them.

\section{WLAN Plan}

In order to relocate the APs, we have taken into account the structure and distribution of the building. This is so, because there are some manufacturing materials which attenuate wireless signals significantly, for instance metal or wood. So, there are environments such as bathrooms or large fitted wardrobes where APs must not be close to.

According to the results and coverage maps shown in Section 5.6, we decided to resign our network in order to improve its performance. The new APs placement is shown in Figure 5.20. Thus, several APs have been relocated on the ground floor.

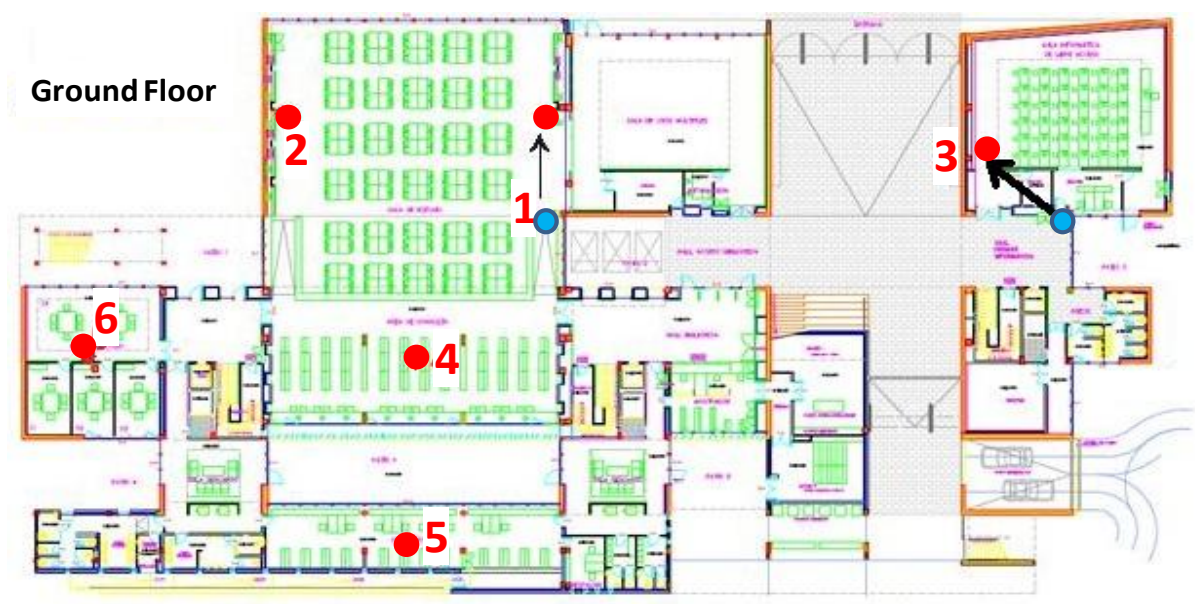

Figure 5.20. Relocation of APs on the ground floor

Firstly, the AP placed in the study room (point 1) is moved to the window and a new AP is added just in the opposite wall (point 2). This relocation has involved a better service for students who connect to the Internet in the study room because wireless coverage has been increased. Secondly, the AP located at the hall of the free access computer room is moved to beside wall in the same room. Its current position provided very low signal strength to this room. The estimated signal strengths in the ground floor are shown in Figure 5.21. 

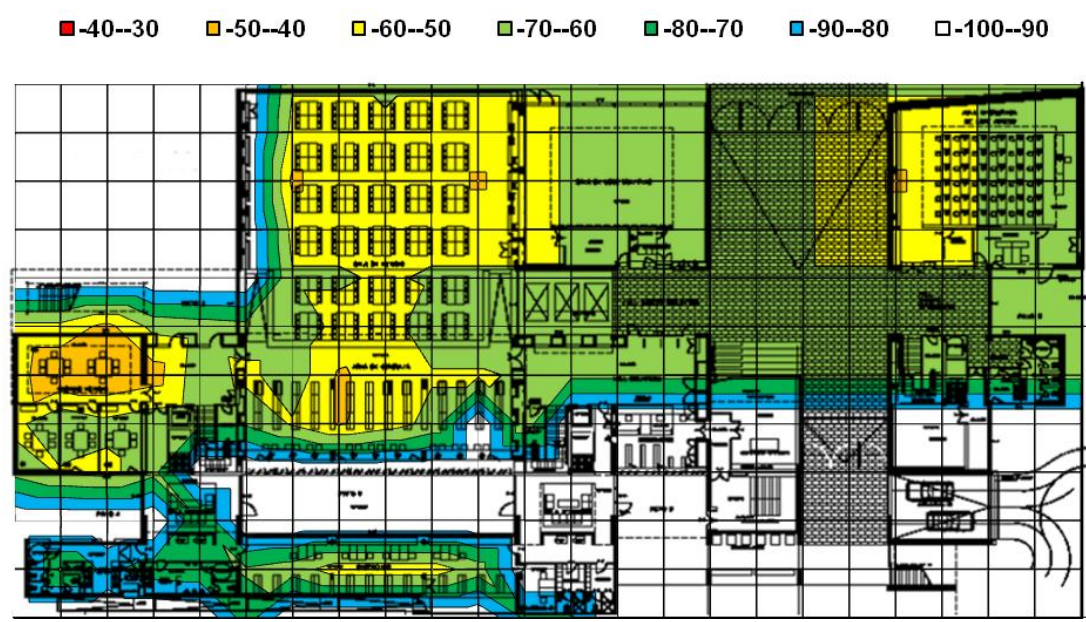

Figure 5.21. Estimated signal strength on the ground floor for the proposed AP relocation.

Moreover, on the first floor an AP (point 1) has been also relocated and two new APs have been added. The AP located in the hall on the left side (point 1) is moved to the door of the bookable study rooms. In this way, wireless signals pass through fewer walls and consequently they suffer less attenuation. The AP in point 2 is added in the center of the study room. This is a room where many students often go to work with their computers. As we have seen in the coverage maps on the first floor (section 4), some areas of the computer rooms do not have enough signal level, so we decided to add another AP in point 3 in order to improve the coverage. Figure 5.22 shows the new APs location.

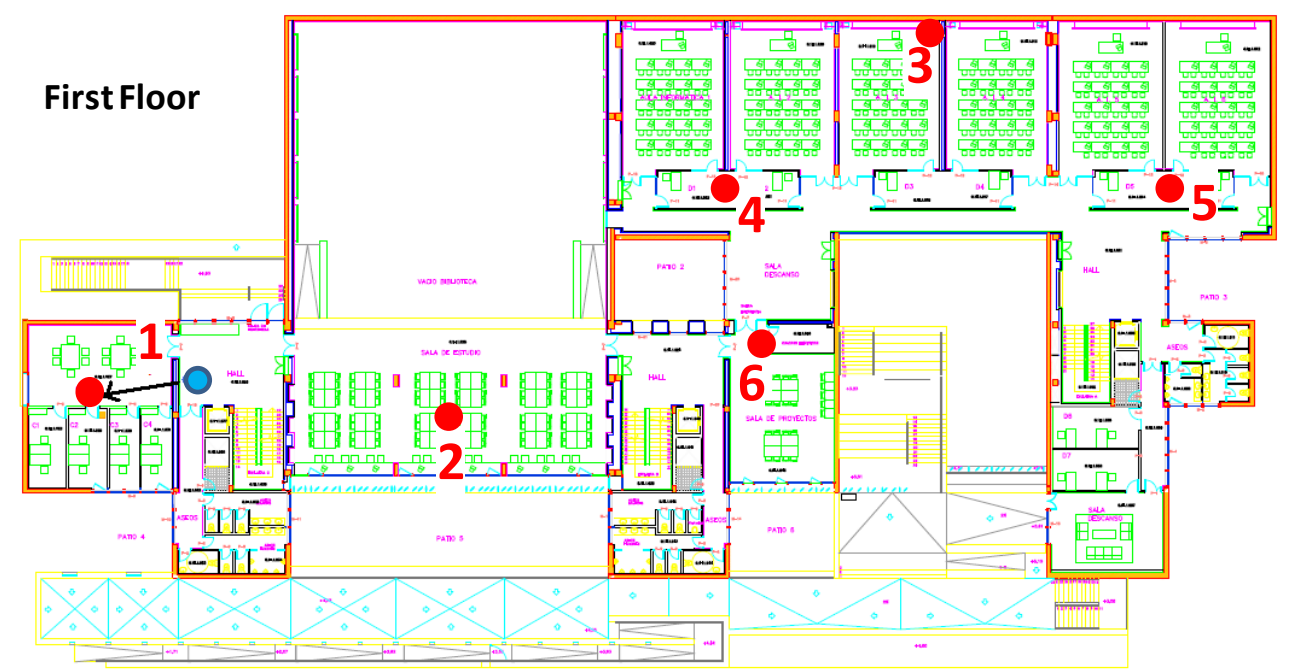

Figure 5.22. Relocation of APs on the first floor

The estimated signal strengths in the first floor are shown in Figure 5.23. In both cases where APs have been relocated, we can observe that the signal strengths have been improved. 
$\square-100--90 \quad \square-90--80 \quad \square-80--70 \quad \square-70--60 \quad \square-60--50 \quad \square-50-40 \quad \square-40--30$

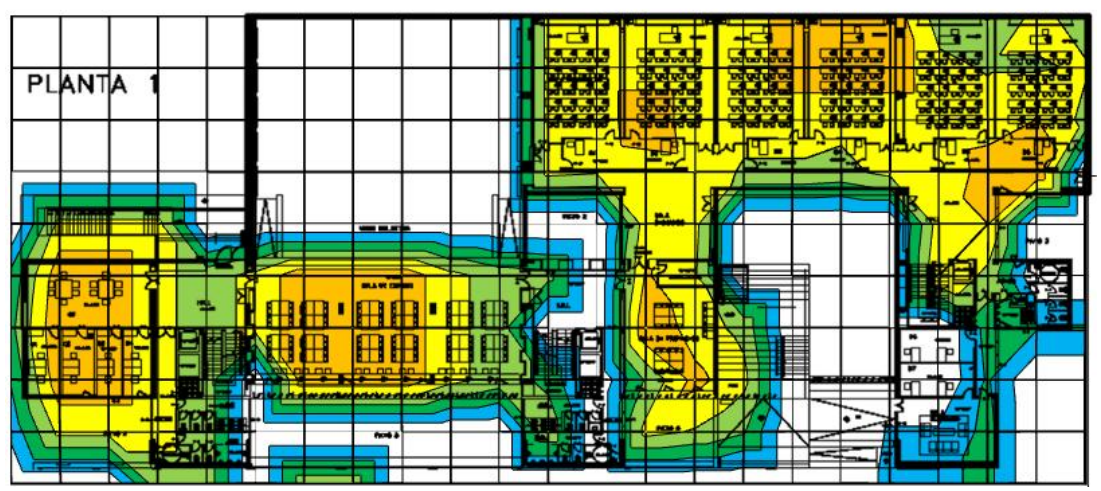

Figure 5.23. Estimated signal strength on the first floor for the proposed AP relocation.

In contrast, we have not changed anything on the second place since we have checked that the signal strengths are good enough. Figure 5.24 shows the position of the APs on the second floor.

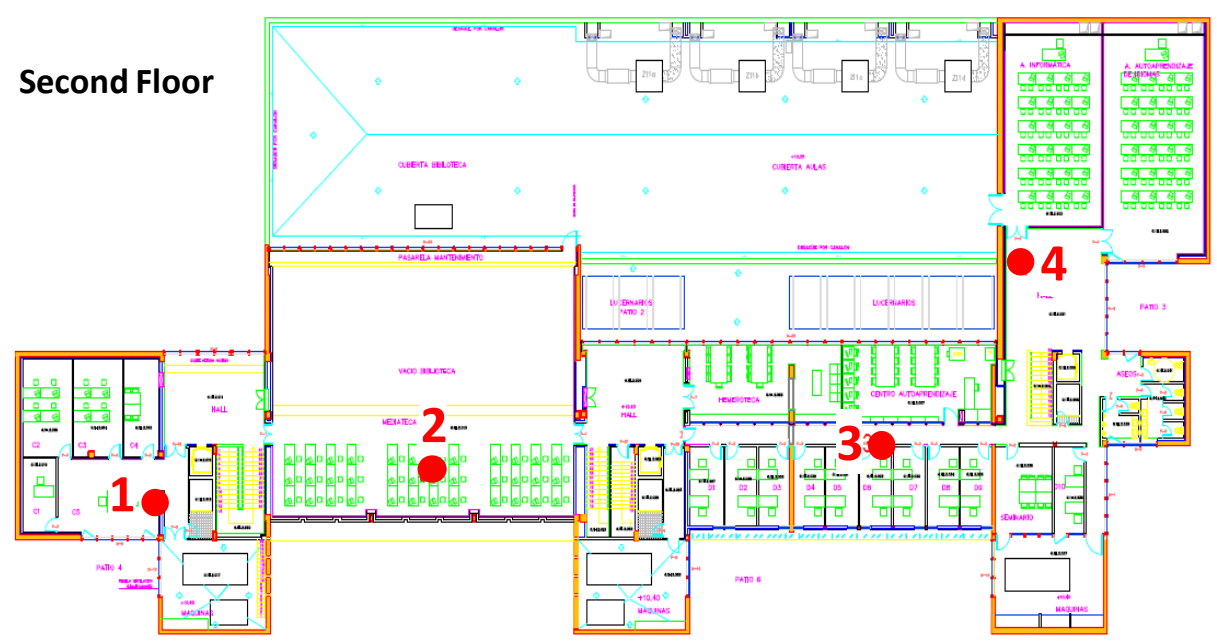

Figure 5.24. Relocation of APs on the second floor

\section{Channel assignment}

IEEE $802.11 \mathrm{~b}$ and IEEE $802.11 \mathrm{~g}$ standards define up to 14 channels available for wireless devices. But each country or geographic area applies their own restrictions regarding to the number of available channels. The channels are not completely independent because each channel overlaps and causes interference to the nearest four channels. Signal bandwidth $(22 \mathrm{MHz})$ is greater than the distance between consecutive channels $(5 \mathrm{MHz})$. For this reason, a gap of at least 5 channels is needed to avoid interference between adjacent cells. Using a gap of 5 channels means reaching a difference of $25 \mathrm{MHz}$. Channels 1, 6 and 11 are usually the most used but the use of channels 1, 5, 9 and 13 in European domains is not bad for the performance of networks [82].

Figure 5.25 shows the channel distribution from 2.412 to $2.484 \mathrm{GHz}$ for the 5 main regulatory domains. In our case we use the regulatory domain of Europe, Middle East and Africa (EMEA) [98]. 


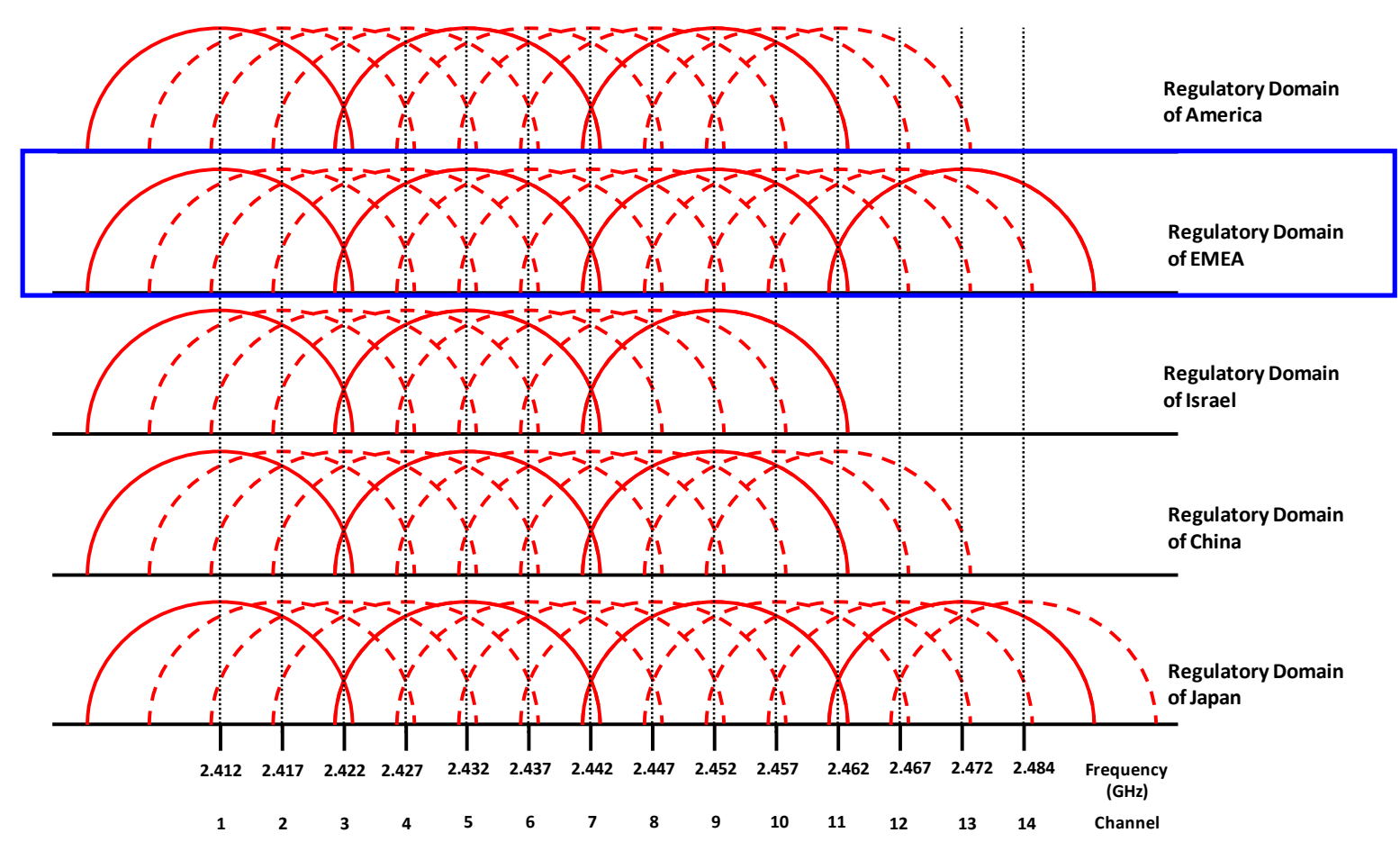

Figure 5.25. Channels in the frequency band of $2.4 \mathrm{GHz}$

When new APs have been added to the network, we should define a new channel assignment for the network in order to avoid interference between the already installed APs and the new ones.

Thus, our proposal of channel assignment according to our relocation and considering one more AP for the ground floor is shown in Table 5.2. Moreover, it can be compared with the current distribution channel in which the APs 1 and 3 share the same channel (channel 11) and the same happens with APs 4 and 5 in channel 1. This can make that these devices are interfering to each other. In our proposed channel assignment, these interferences will not occur.

Table 5.2. Channel distribution for the ground floor

\begin{tabular}{|l|l|l|}
\hline Access Point & Proposed Channel & Current Channel \\
\hline 1 & 1 & 11 \\
\hline 2 & 5 & AP nonexistent \\
\hline 3 & 13 & 11 \\
\hline 4 & 9 & 1 \\
\hline 5 & 13 & 1 \\
\hline 6 & 1 & 5 \\
\hline
\end{tabular}

In the same way, Table 5.3 shows the channel assignment for the first floor before and after relocating the APs and adding two more. We can see that the APs 1, 4 and 6, was initially working on channel 5, while in our proposed channel assignment the interferences between devices are insignificant. 
Table 5.3. Channel distribution for the first floor

\begin{tabular}{|l|l|l|}
\hline Access Point & Proposed Channel & Current Channel \\
\hline 1 & 5 & 5 \\
\hline 2 & 1 & AP nonexistent \\
\hline 3 & 5 & AP nonexistent \\
\hline 4 & 9 & 5 \\
\hline 5 & 1 & 8 \\
\hline 6 & 13 & 5 \\
\hline
\end{tabular}

Finally, Table 5.4 shows the channel assignment for the second floor where no change was needed. In this case, we change the channel allocation, only to not interfere with the devices of the lower floor.

Table 5.4. Channel distribution for the second floor

\begin{tabular}{|l|l|l|}
\hline Access Point & Proposed Channel & Current Channel \\
\hline 1 & 1 & 5 \\
\hline 2 & 5 & 1 \\
\hline 3 & 6 & 7 \\
\hline 4 & 13 & 1 \\
\hline
\end{tabular}

As three tables show, the current channel assignment is made so that the devices do not interfere with the devices of the same floor. However, with the proposed channel scheme in this paper, interferences between floors are also avoided.

\subsubsection{Measurements in Scenario 2: Garage}

We performed the measurements in a flat with a $91 \mathrm{~m}^{2}$ area. It had a length of $12.5 \mathrm{~m}$., a width of $6.68 \mathrm{~m}$., and a height of $2.30 \mathrm{~m}$. This scenario has been chosen because it is made of very common building materials (like a regular house). Figure 28 shows the plane of the build. It has rectangular base, divided into two parts by a wall of $9 \mathrm{~cm}$ of thick: the garage (left) and the kitchen (right). The staircase is made of bricks with high consistency, prepared to hold a fire. All these walls have a layer of plaster and paint on both sides. The bathroom is made of $9 \mathrm{~cm}$ hollow bricks. These walls are covered by ceramic tiles. The external walls are double with a thermic and $5 \mathrm{~cm}$ of acoustic insulation polystyrene.

In order to perform this work, we draw a grid in the building floor. It allows us to take measurements of all devices in the same place. Figure 5.26 shows the APs placement (in green AP1 and AP2, which have been used for the interference test bench). Their placements have been decided randomly in order to avoid having equidistant placements. In red we can see the AP used for the coverage measurements test bench. Each AP has been located in the stairwell, at a height of $50 \mathrm{~cm}$. The signal power levels received at each measure point is collected by a laptop running the application software InSSIDer. The signal strength has been captured with a WUSB600N wireless 


\section{Sandra Sendra Compte}

card for all computers in order to avoid adding some sort of error when taking the measurements (due to its sensitivity or antenna orientation). With this AP distribution, we have measured the wireless coverage offered by each device, working on various wireless technologies and different wireless devices. These signal values depend mainly, on the losses suffered due to the walls traversed and the multipath effect. These measurements let us estimate the average values of the power received, taking into account the distance to the AP. Then, we have sought a mathematical approach in order to characterize the signal behavior in order to easily determine the best place to locate the wireless sensors. In order to verify that the approach was correct, we used the correlation coefficient $\mathrm{R}^{2}$. It is a criterion parameter that represents the percentage of variance justified by the independent variable. It can be interpreted as the square of the Pearson correlation coefficient between the dependent and independent variables. If all values are represented in the regression line, the value of $R^{2}$ is 1 , and if there is no linear relationship between dependent and independent variables, the $\mathrm{R}^{2}$ value is 0 . It is a measure of the linear relationship between two variables. As its value is greater, the line adjust to the data is better and the discrepancy resulted from the replacement of the values observed by the predicted is lower.

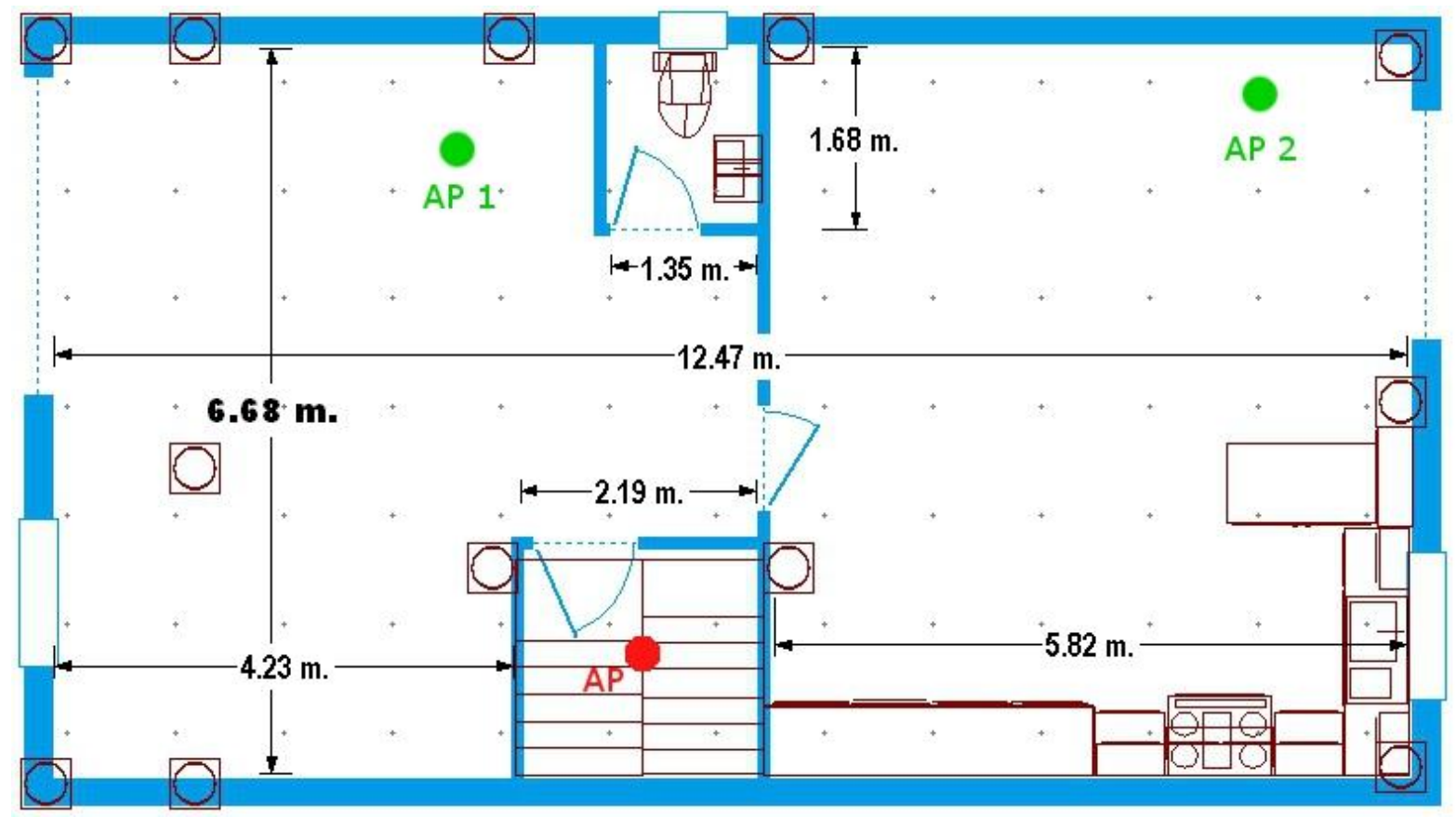

Figure 5.26. Scenario and APs placement

\subsubsection{Coverage measurements for garage}

In order to establish the correct position of a set of sensor nodes, we should characterize the behavior of the wireless signals. It is important to consider that phenomena such as multi-path effect, interference from other IEEE 802.11 sources or the variation of signal-to-noise ratio and transmit bitrate can directly affect the signal strength measurements [343]. These effects are more evident in smaller spaces. However, all our measurements were performed under the same environmental conditions and consequently all measures are affected by these phenomena in the same way. 


\section{Chapter 5: Wireless Sensor Development for Monitoring in Indoor Environments}

This section shows the IEEE 802.11 radio coverage measurements. All of them have been performed for the variants IEEE $802.11 \mathrm{~b} / \mathrm{g} / \mathrm{n}$ working at $2.4 \mathrm{GHz}$, IEEE $802.11 \mathrm{n}$ working at $5 \mathrm{GHz}$ and IEEE 802.11a working at $5 \mathrm{GHz}$. The measurements, which have been performed on each device, have been grouped by wireless technology, in order to obtain an average value of the signal strength values and determine the performance of each technology in indoor environments. In order to extract the equations that model the behavior of each technology, we have used a polynomial interpolation based on divided difference method of Newton.

\section{Analysis of coverage by device}

Figure 5.27 shows the level of coverage obtained with Linksys WRT320N when it is configured to work only in 802.11a. As the figure shows, the best coverage is located in the stairwell. The signal is propagated out of the walls of the stairwell to the outer walls. Then, there signal strength is quickly decreased with some low peaks in the coverage area.

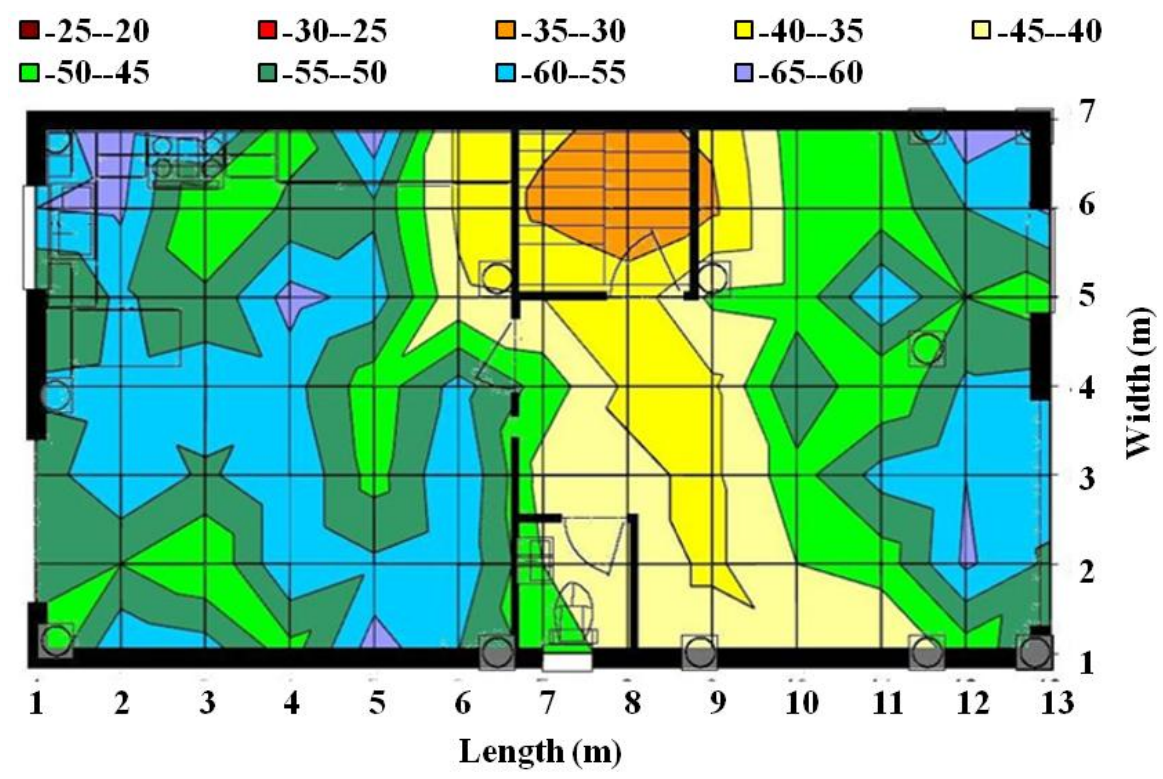

Figure 5.27. Coverage to Linksys WRT320N in 802.11a.

Figure 5.28 shows the level of coverage obtained with Linksys WRT320N configured to work only in $802.11 \mathrm{~b}$. In this case, the best coverage is located in the staircase, but the signal is decreased quickly as it is propagated to the garage. The kitchen area has a lower signal level than the garage.

Figure 5.29 shows the level of coverage obtained for the Linksys WRT320N configured to work only in $802.11 \mathrm{~g}$. As we can see, it coverage when it is working in IEEE $802.11 \mathrm{~b}$ is better than in IEEE $802.11 \mathrm{~g}$. It is even more significant in closest distances. 


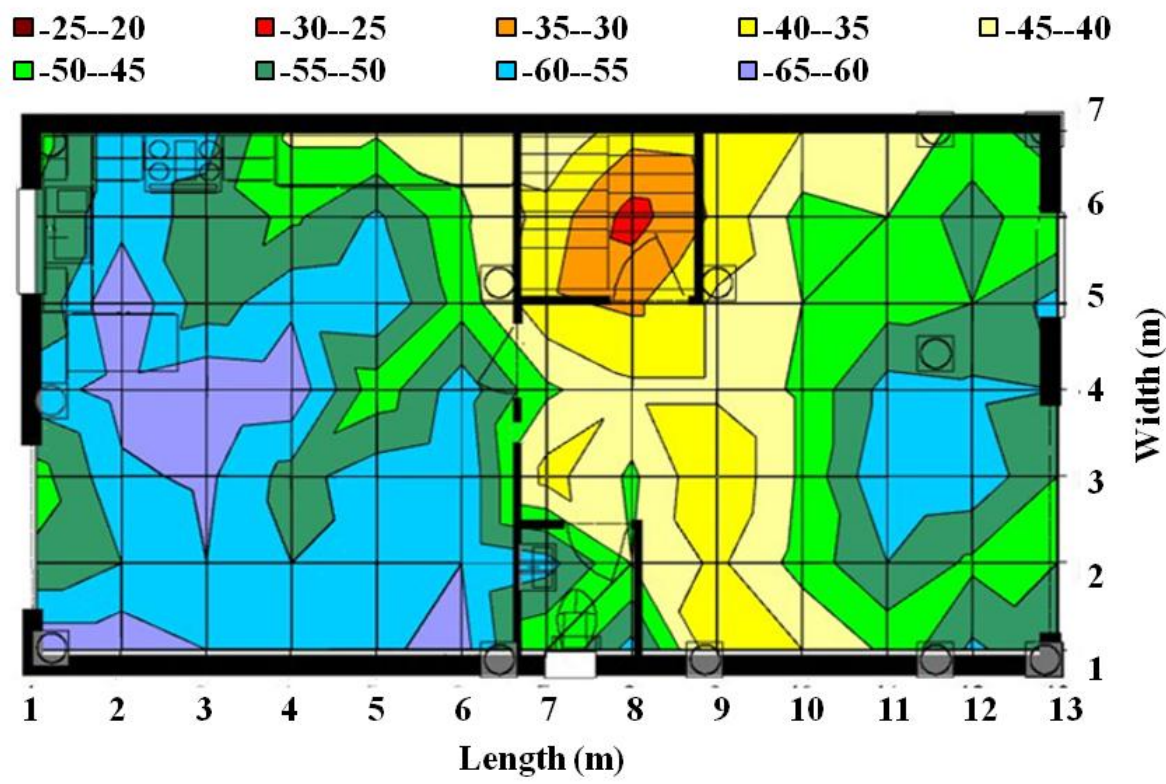

Figure 5.28. Coverage to Linksys WRT320N in 802.11 b.

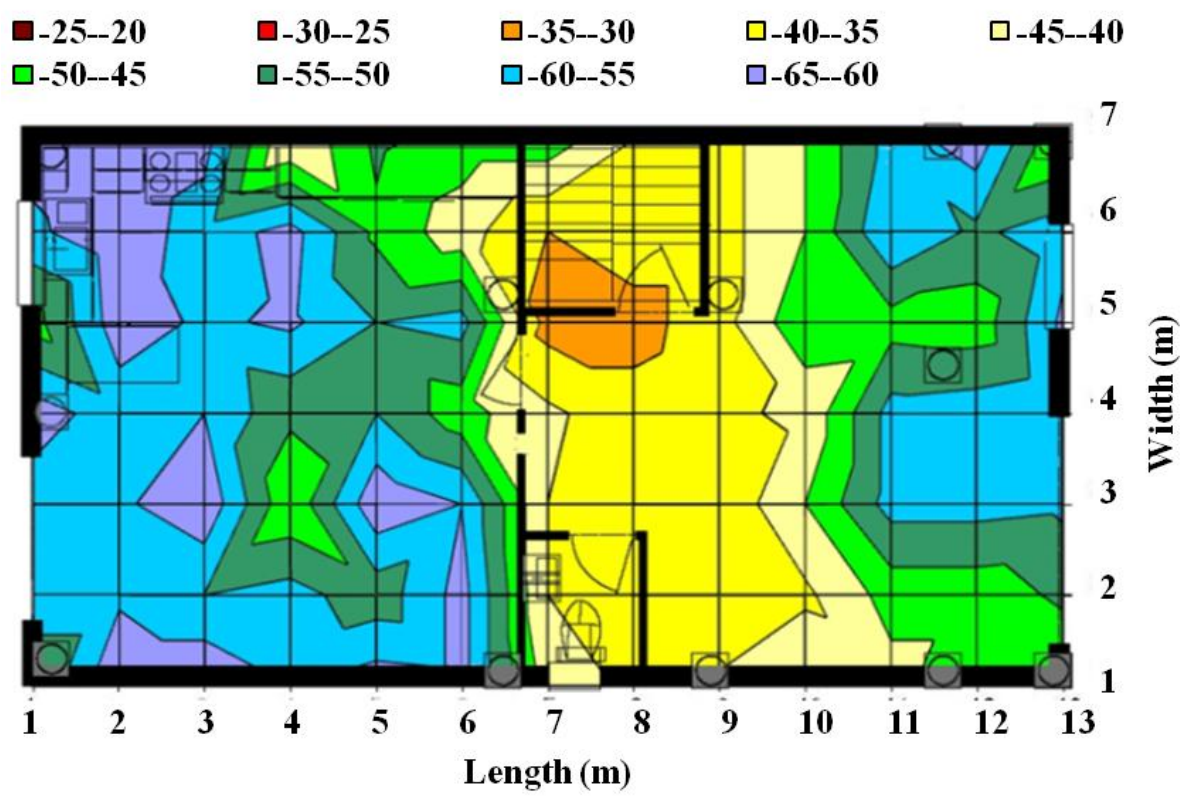

Figure 5.29. Coverage to Linksys WRT320N in $802.11 \mathrm{~g}$.

Figure 5.30 shows the level of coverage obtained with the Linksys WRT320N configured to work only in $802.11 \mathrm{n}$. Although there is high signal strength close to the access point, there are suddenly low values in the coverage area.

Figure 5.31 shows the level of coverage obtained with the Dlink DWL-2000AP configured to work only in $802.11 \mathrm{~b}$. This device presents the highest signal levels in almost all the garage surface, and the kitchen's area. 


$\begin{array}{lllll}\square-25--20 & \square-30--25 & \square-35--30 & \square-40--35 & \square-45--40 \\ \square-50--45 & \square-55--50 & \square-60--55 & \square-65--60 & \end{array}$

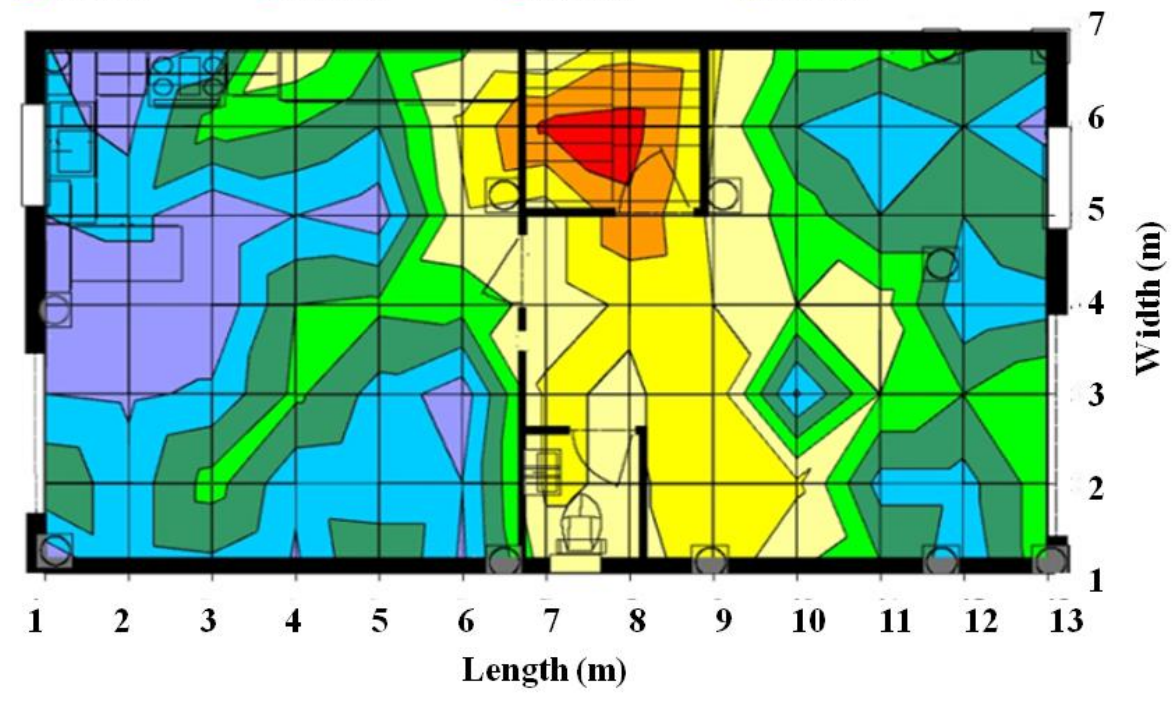

Figure 5.30. Coverage to Linksys WRT320N in $802.11 \mathrm{n}$.

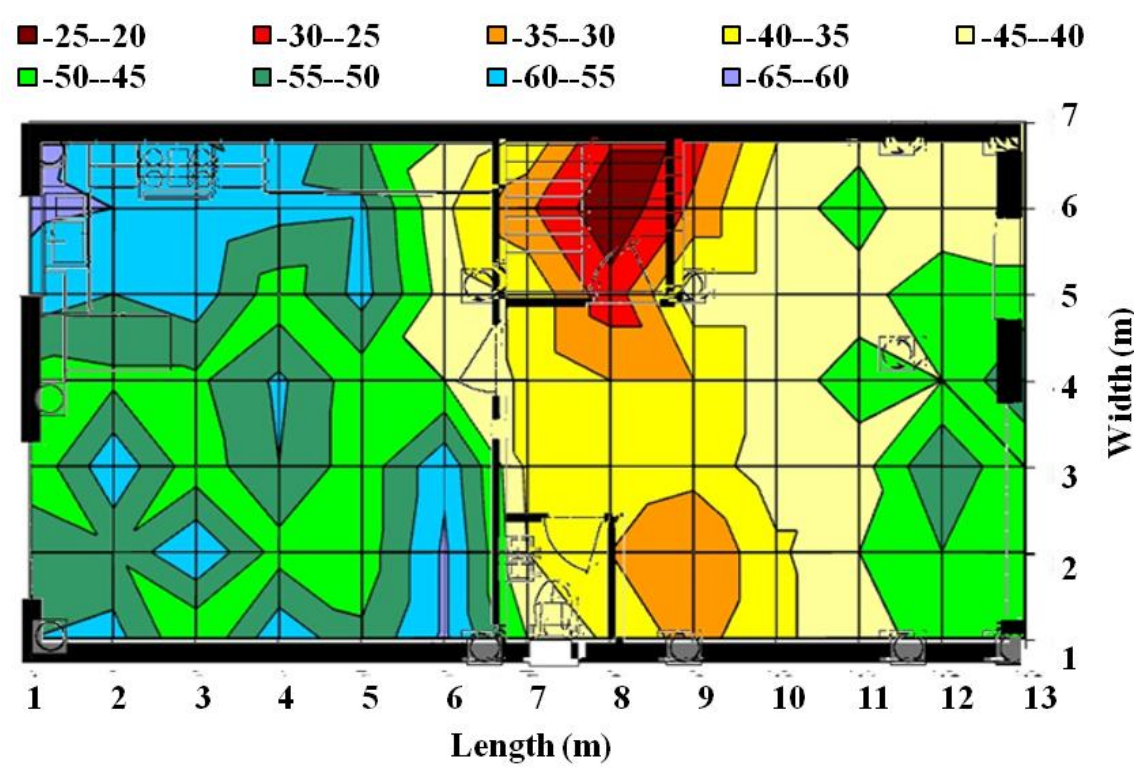

Figure 5.31. Coverage to Dlink DWL-2000AP in 802.11b.

Figure 5.32 shows the level of coverage obtained with the Cisco Aironet 1130AG configured to work only in $802.11 \mathrm{~g}$. This device is the one that presents the lowest signal level. This may be due to the antenna radiation direction (we place all devices in the same position, independently of the placement of the antenna inside of them).

Figure 5.33 shows the level of coverage obtained with the Linksys WRT54GL configured to work only in 802.11 b. It has been the device that provides higher signal strength in the coverage area. 


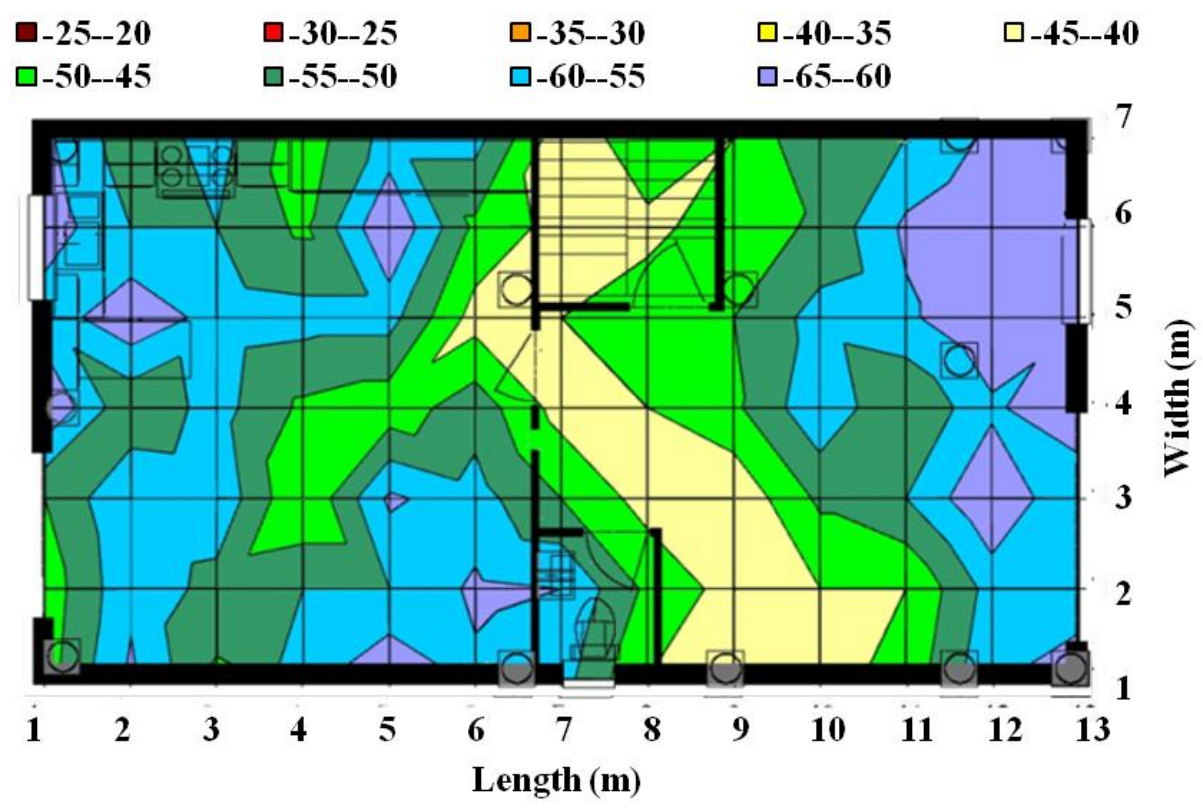

Figure 5.32. Coverage to Cisco Aironet $1130 \mathrm{AG}$ in $802.11 \mathrm{~g}$.

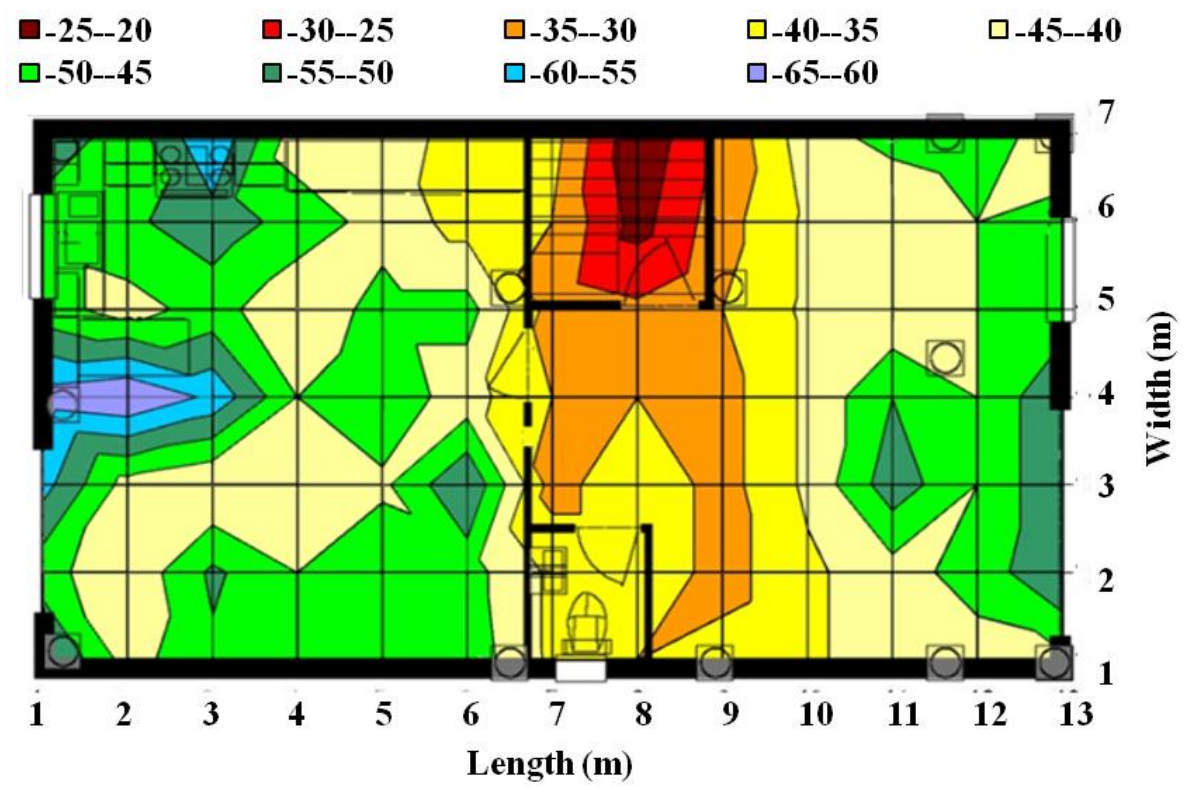

Figure 5.33. Coverage to Linksys WRT54GL in 802.11 b.

\section{Coverage analysis}

In this subsection, measurements are grouped by wireless technology. Figure 5.34 shows obtained mean coverage when the devices are configured to work at IEEE 802.11a. The best coverage is located in the stairwell. The signal is propagated out of the walls of the stairwell to the outer walls. Then, the signal strength is quickly decreased with some low peaks in the coverage area. This variant 
presents the lowest signal level. This may be due to the antenna radiation direction (we place all the devices in the same position, independently of the placement of the antenna inside of them).

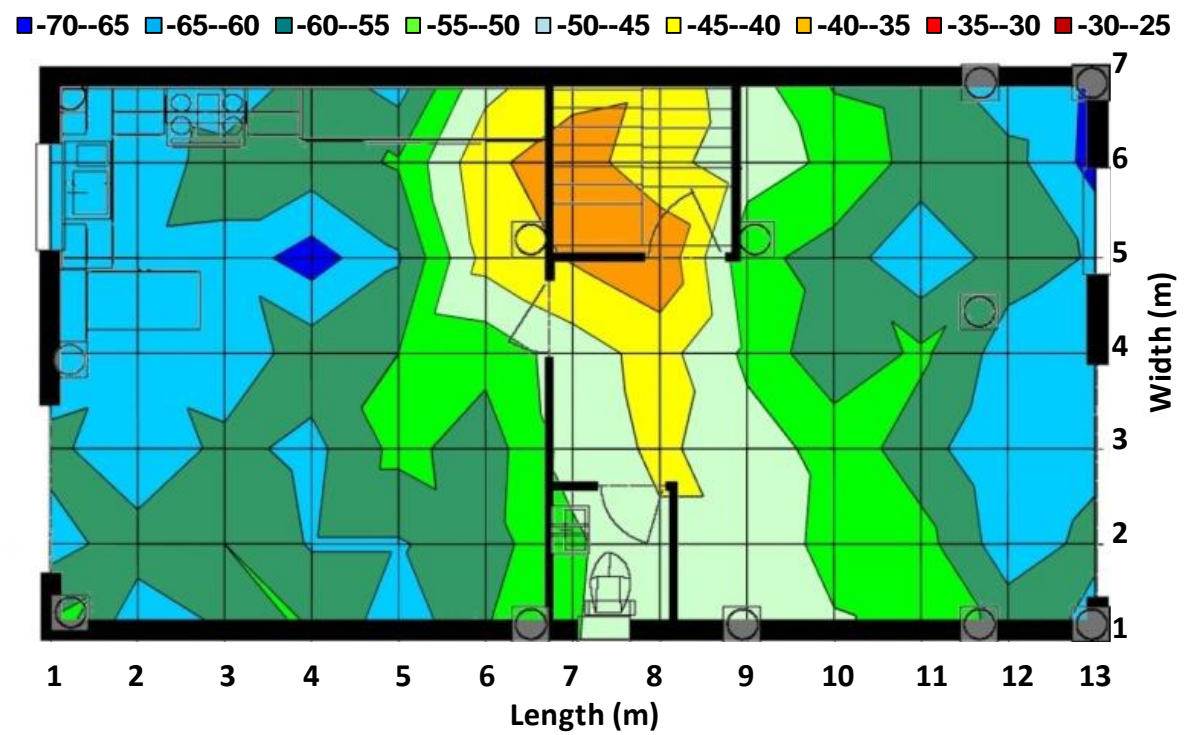

Figure 5.34. Average radio coverage of IEEE 802.11a

Figure 5.35 shows the obtained radio coverage when devices are configured to work at IEEE 802.11 b. In this case, the best coverage is located in the staircase, but the signal is decreased quickly as it is propagated outside of the place. This standard variant presents the highest signal levels in almost all the location. There are low and high values in the kitchen area.

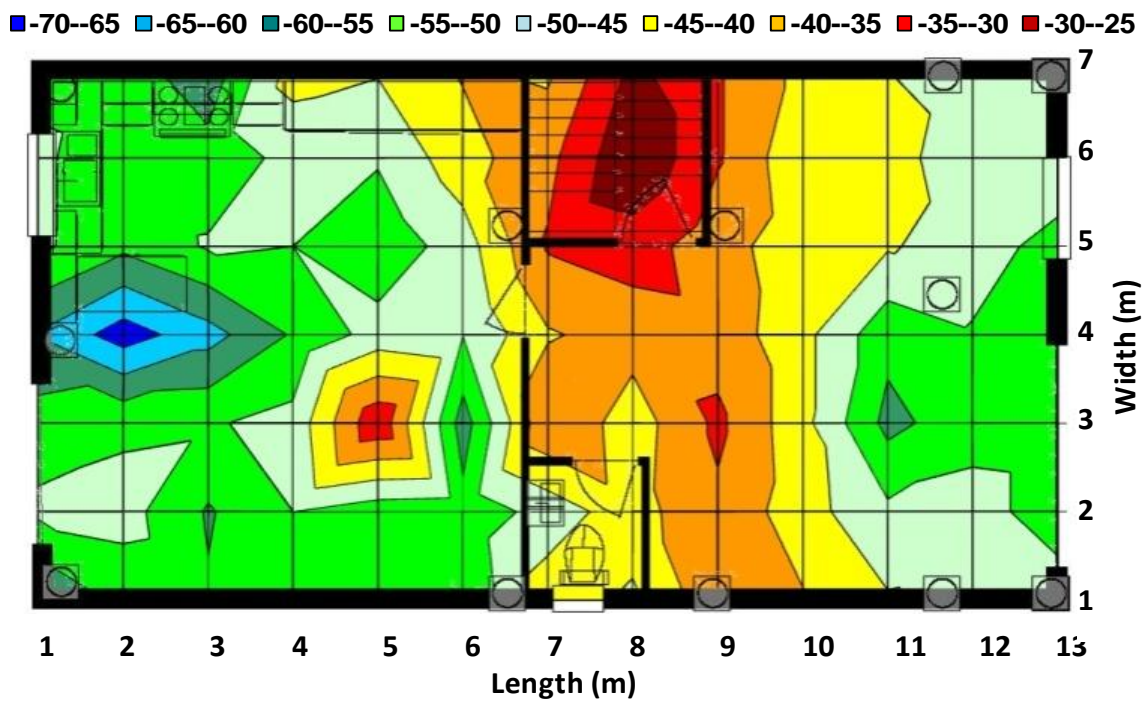

Figure 5.35. Mean radio coverage of IEEE $802.11 \mathrm{~b}$

Figure 5.36 shows the obtained radio coverage for IEEE $802.11 \mathrm{~g}$. The radio coverage of a device working at IEEE $802.11 \mathrm{~b}$ is better than at IEEE 802.11g. However, this variant seems to be more stable in terms of coverage when we move away from the AP. 


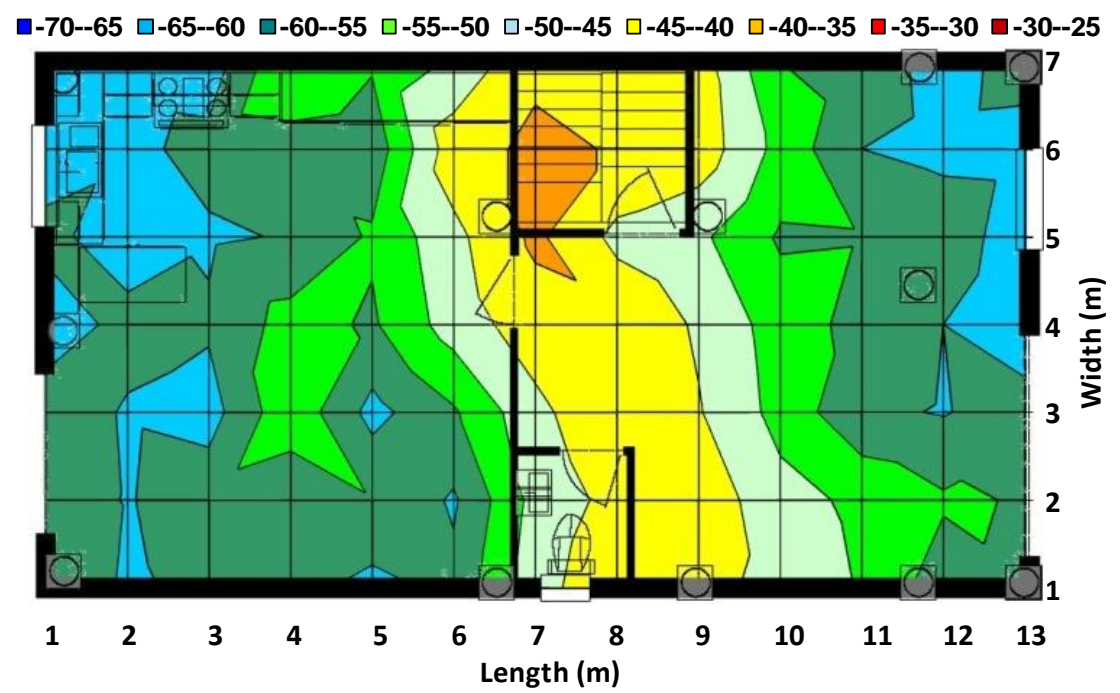

Figure 5.36. Average radio coverage of IEEE $802.11 \mathrm{~g}$

Figure 5.37 shows the obtained radio coverage when the devices are working at IEEE 802.11n. Although it presents one of the highest signal strengths in zones close to the access point, we can see that it offers the greatest attenuation in the kitchen than in the rest of the area with low values zones.

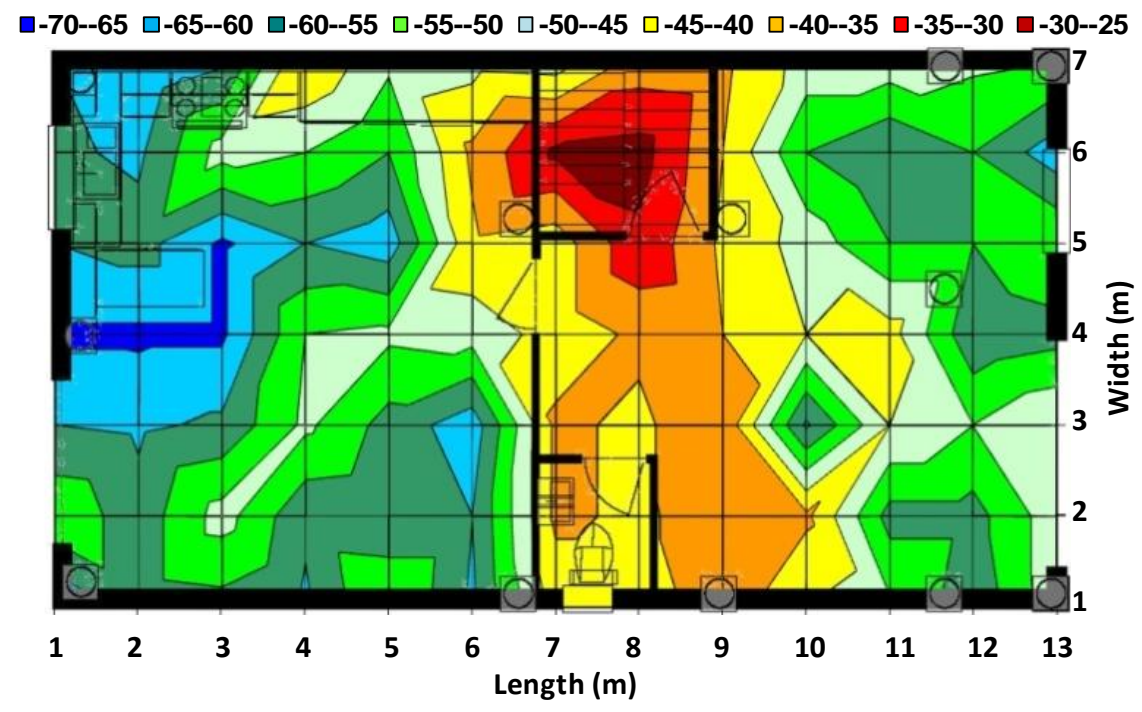

Figure 5.37. Mean radio coverage of IEEE $802.11 \mathrm{n}$

Figure 5.38 shows the signal strength in function of the distance from the AP for the four IEEE 802.11 variants. 


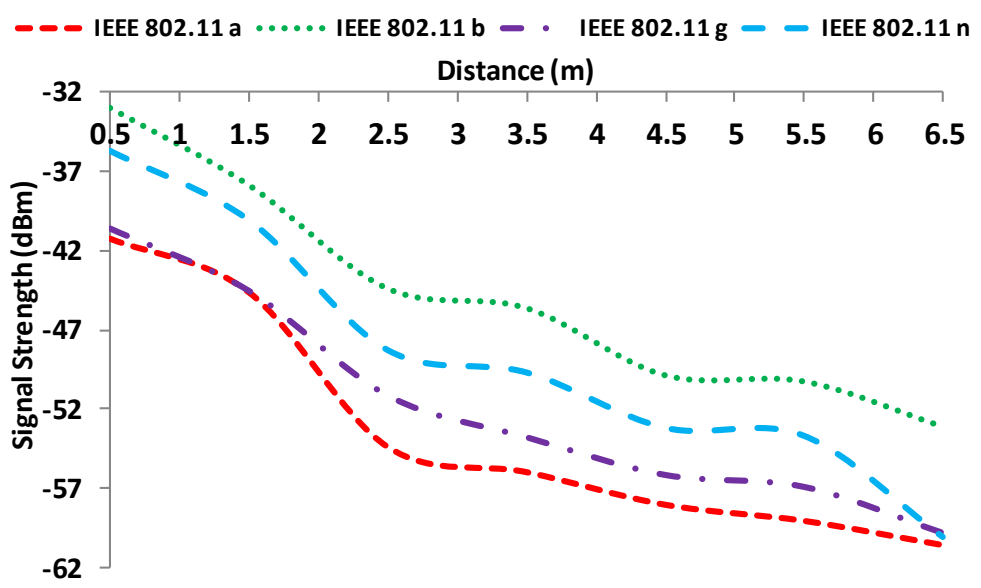

Figure 5.38. Lost signal strength for IEEE $802.11 \mathrm{a} / \mathrm{b} / \mathrm{g} / \mathrm{n}$ for garage.

Although each wireless technology presents different values compared with other signals, all of them show similar behavior. We can distinguish several stages where the signal varies differently.

On the one hand, IEEE 802.11a (red line) is a linear behavior at distances up to $1.5 \mathrm{~m}$, which does not decrease more than $1.30 \mathrm{dBm} / \mathrm{m}$. It would be a good place to put a sensor. At distances of between $1.5 \mathrm{~m}$. and $2.5 \mathrm{~m}$., there is a drop of approximately $10 \mathrm{dBm} / \mathrm{m}$. This area is not preferred to place the sensor, taking into account the high signal loss variation. The best distances to place a sensor would be at $3.5 \mathrm{~m}$., which has almost the same signal strength than at $2.5 \mathrm{~m}$. If we analyze the behavior of the signal, it follows Equation 5.4 with a value of correlation coefficient $\mathrm{R}^{2}=0.9999$, where $S$ is the received signal strength in $\mathrm{dBm}$ and $\mathrm{x}$ is the distance to the AP.

$$
S=-0,0645 \mathrm{x}^{6}+1,4384 \mathrm{x}^{5}-12,575 \mathrm{x}^{4}+54,142 \mathrm{x}^{3}-116,69 \mathrm{x}^{2}+106,77 \mathrm{x}-71,49
$$

The signal, working under IEEE $802.11 \mathrm{~b}$ variant (green line), decreases $5 \mathrm{dBm} / \mathrm{m}$ and it alternates with areas of constant signal strength. When we are working with this variant, the best position is in the areas where the signal is almost constant. This happens between $2.5 \mathrm{~m}$. and $3.5 \mathrm{~m}$. (the preferred place will be at $3.5 \mathrm{~m}$.), and between $4.5 \mathrm{~m}$. and $5.5 \mathrm{~m}$. (the preferred place will be at $5.5 \mathrm{~m}$.). In this case, this graph can be approximated by Equation 5.5 with a value of correlation coefficient $\mathrm{R}^{2}=0.9999$.

$$
S=-0,0816 \mathrm{x}^{6}+1,7228 \mathrm{x}^{5}-14,112 \mathrm{x}^{4}+56,343 \mathrm{x}^{3}-111,79 \mathrm{x}^{2}+94,047 x-58,29
$$

The signal of IEEE $802.11 \mathrm{~g}$ variant decreases approximately $3.3 \mathrm{dBm} / \mathrm{m}$, alternating with areas where it is up to $5 \mathrm{dBm} / \mathrm{m}$. Using this variant, we should place sensors at greater distances than the previous variants. We have seen that the optimum place is between $4.5 \mathrm{~m}$. and $5.5 \mathrm{~m}$. The function that better fits this graph is a polynomial of degree 6 (see Equation 5.6) with a value of correlation coefficient $\mathrm{R}^{2}=0.9999$.

$$
S=-0,0356 x^{6}+0,7695 x^{5}-6,5329 x^{4}+27,428 x^{3}-57,836 x^{2}+49,863 x-54,183
$$




\section{Sandra Sendra Compte}

The behavior of IEEE $802.11 \mathrm{n}$ is similar to IEEE $802.11 \mathrm{~b}$ variant. It has a first stage area where the signal decreases less than $2 \mathrm{dBm} / \mathrm{m}$, followed by a stage (between 1.5 and $2.5 \mathrm{~m}$ ) which decreases about $10 \mathrm{dBm} / \mathrm{m}$. When we are working with IEEE $802.11 \mathrm{n}$, we can place sensors at $3.5 \mathrm{~m}$. (because it has almost the signal strength than at $2.5 \mathrm{~m}$ ). But if we need to place a sensor at distances greater than $4.5 \mathrm{~m}$, the optimum would be $5.5 \mathrm{~m}$. The function that approximates this behavior is a polynomial of degree 6 (see Equation 5.7) with a value of correlation coefficient $R^{2}=0.9999$.

$$
S=-0,0853 \mathrm{x}^{6}+1,8136 \mathrm{x}^{5}-15,063 \mathrm{x}^{4}+61,394 \mathrm{x}^{3}-125,16 \mathrm{x}^{2}+108,97 x-65,736
$$

We can characterize the behavior of the wireless signals in this flat for all IEEE 802.11 variants as a polynomial function of degree 6 . All have 2 areas of almost constant signal strength (one between $2.5 \mathrm{~m}$. and $3.5 \mathrm{~m}$. and another one between $4.5 \mathrm{~m}$. and $5.5 \mathrm{~m}$.). We have seen that IEEE 802.11a has higher signal loss than other variants working at $2.4 \mathrm{GHz}$, which proves that walls penetration provides poorer performance than with $2.4 \mathrm{GHz}$, so it is not recommended for indoor environments.

\subsubsection{Testbench description for interference measurements}

In this test bench, we have used four PCs and two APs of the same brand and model. Figure 5.25 shows the location of AP1 and AP2 (marked in green). These sites are chosen to ensure that there are walls between both APs. Figure 5.39 shows the topology. PCs are placed at a distance of approximately $1 \mathrm{~m}$. from the AP which is associated to.

In order to do these tests, we used the AP1 to do the measurements of the packet loss, throughput and bandwidth. Meanwhile AP2 is used as interference source, so we transmitted a large file between the computers associated to that AP.

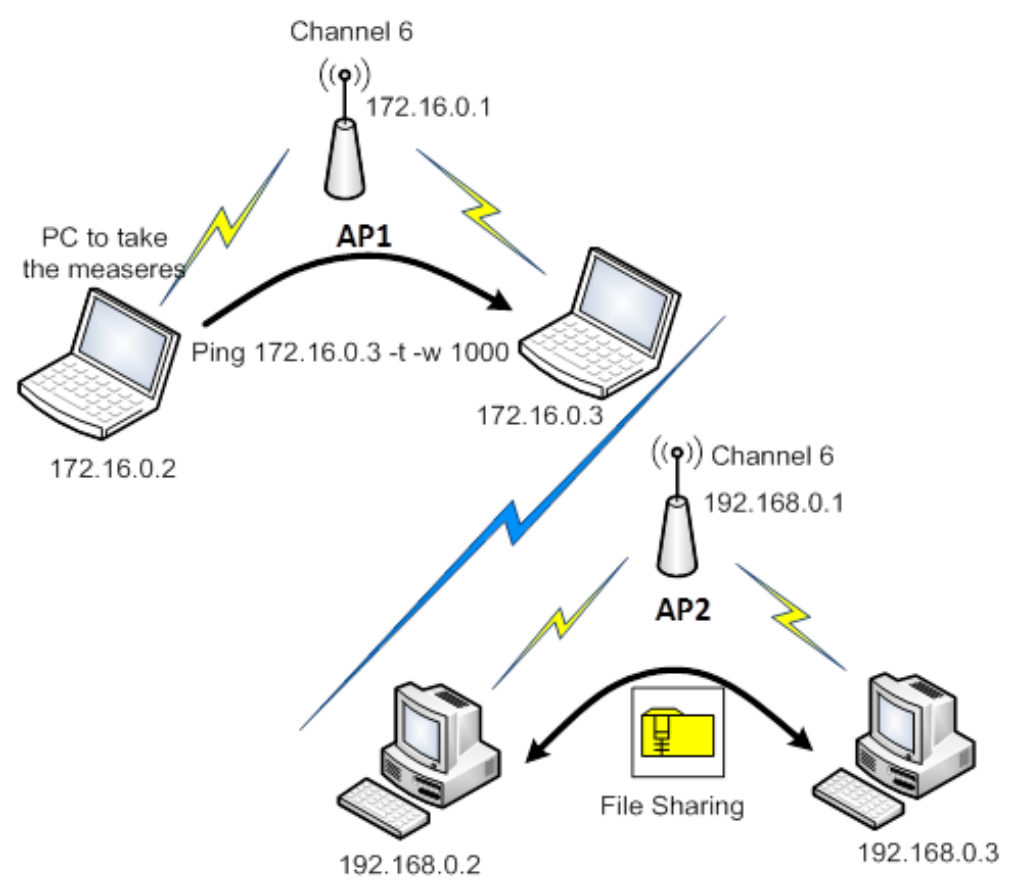

Figure 5.39. Network topology for interference measurements 


\section{Lost Packets}

In order to know the number of lost packets, an echo is sent between the PCs associated to AP1. We set the maximum time to 1000 milliseconds. After this time, the packet will be considered lost or wrong. We choose a small time, because it is a small network, without a large number of intermediate devices that may introduce delays. Measurements were taken during 3 minutes for each device. Packet loss tests have been done with all the devices presented in section 4, trying to discard any dependence with the used devices.

First, we used channel 6 for both wireless networks. Then, we configured different IP networks and tested the effect of working in neighboring channels (by setting one AP in channel 6 and setting the other AP different in neighboring channels).

Figure 5.40 shows a comparison of the number of lost packets (in \%) of all devices working at IEEE $802.11 \mathrm{~b} / \mathrm{g} / \mathrm{n}$. We can see that Linksys WRT320N device (using IEEE 802.11n), does not lose any packet, even when working in a busy channel. An interesting issue is that 2 devices working at IEEE 802.11g have constant losses regardless of the channel used for transmission. Moreover, other devices have very similar behavior. They present few losses when working at the same channel. This behavior is repeated when there is a frequency separation of approximately an even number of channels. If frequency separation is an odd number of channels, there is higher losses percentage.

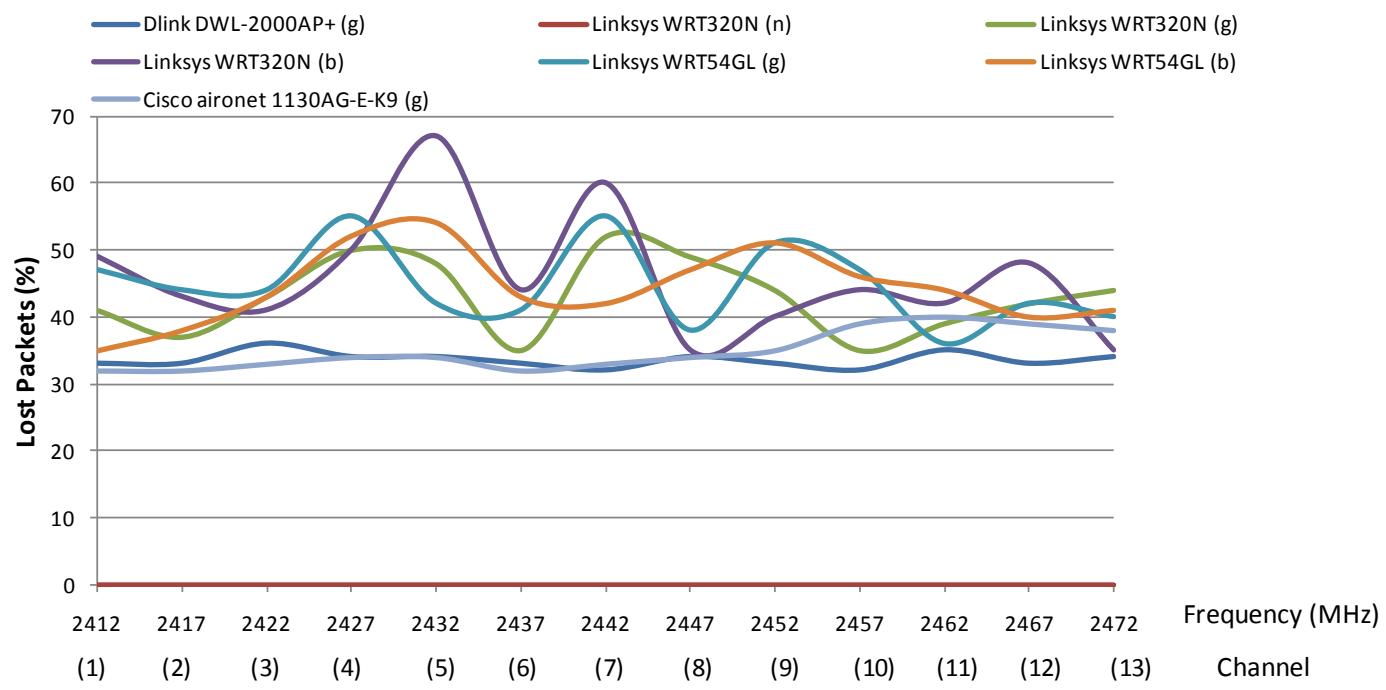

Figure 5.40. Lost Packets working at IEEE $802.11 \mathrm{~b} / \mathrm{g} / \mathrm{n}$

Looking at the graphs, when we use Linksys WRT320N working at IEEE $802.11 \mathrm{~b}$, we should set one device in channel 6 and other one on channel 8 or 4 , in order to minimize the number of lost packets.

Figure 5.41 shows the measurements of lost packets for interferences between channels for Linksys WRT320N at IEEE 802.11a. One AP was configured at channel 56, and we sequentially changed the configuration of the other to the 8 available channels. Packet losses reach a maximum of about $40 \%$. The device has an average level loss of $28 \%$ when working in the same channel as another device. There are high losses when there are APs working in channels that are separated by an odd number, and there are very few losses when frequency separation between channels is an even number. For example, when a device is set in channel 56, highest performance is obtained when 
the other device is set in channel $36,40,48$ or 64 , because the interference and the number of lost packets is minimized.

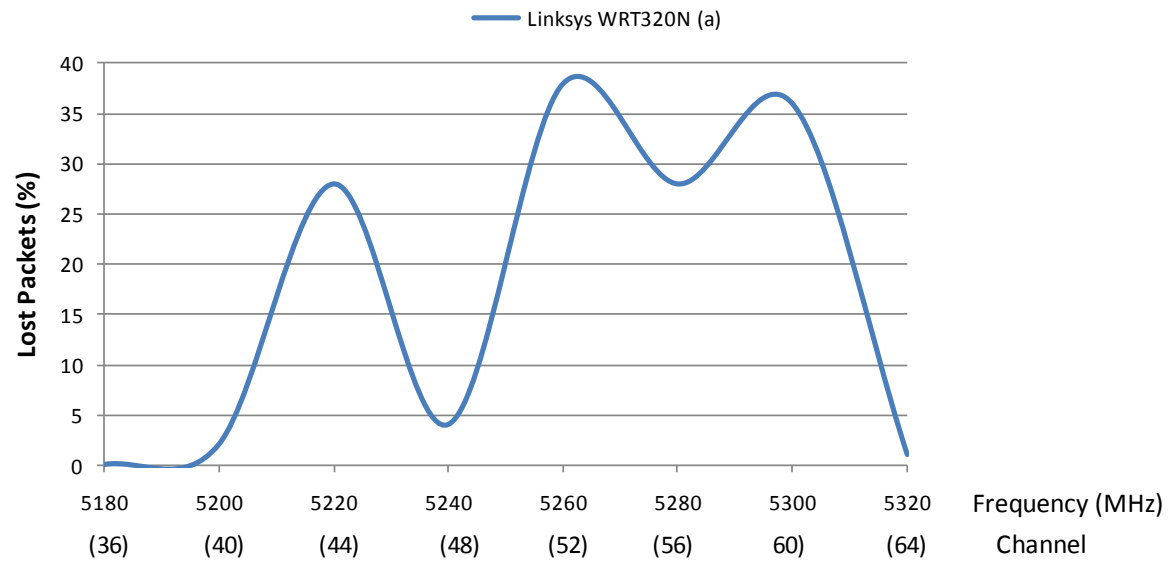

Figure 5.41. Lost Packets working at IEEE 802.11a

\section{Throughput and Bandwidth Consumption Measurements}

In order to measure the throughput offered by each device, we have performed the following test. First, 2 PCs were associated to AP2 and large files were transmitting consuming all available bandwidth in the network. Then, there were 2 PCs associated to AP1, that were transmitting a large file too and we used them to measure the throughput during this transmission. Net Meter captured the consumed bandwidth in one of these PCs. Measurements were carried out during 2 minutes. Results are shown in Figure 5.42.

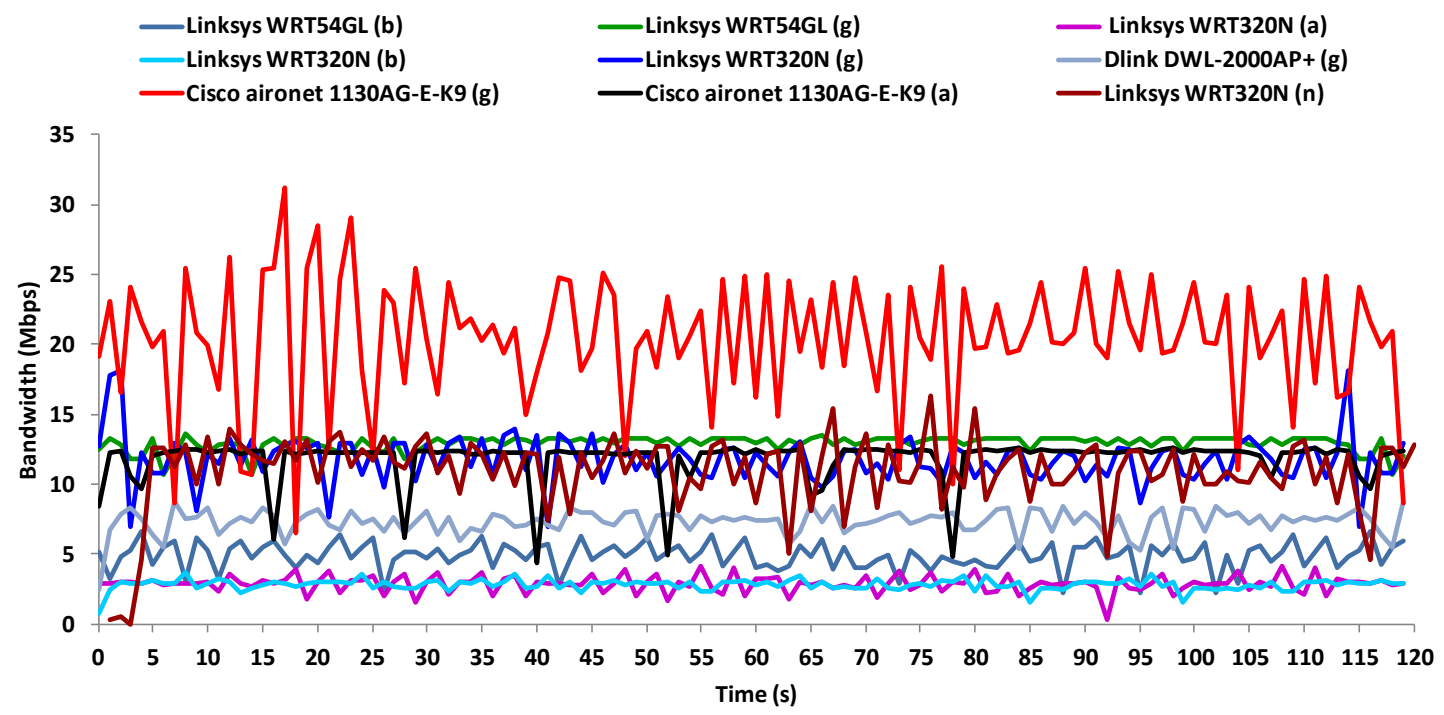

Figure 5.42. Bandwidth Consumption for all devices

Cisco Aironet presents more bandwidth when working at IEEE $802.11 \mathrm{~g}$ variant, but its signal seems to be less stable. It presents a big number of oscillations. Linksys WRT320N also has a high number of oscillations. Other variants have remained fairly stable, and have slight variations in the 
signal. These behaviors are mainly given due to the interference generated between APs and the multipath effect.

Finally, we divided the average bandwidth consumption by the theoretical bandwidth of the technology in order to know the \% of throughput consumption for each technology. The result of this estimation is shown in Figure 5.43. The device offering higher throughput was Linksys WRT54GL device, working at IEEE 802.11b, followed by Cisco Aironet working at IEEE 802.11g. The worst throughput was presented by Linksys WRT320N working at IEEE 802.11a, although Cisco Aironet, working at IEEE 802.11a shows a much higher throughput. In this case the interference highly affects the performance of the wireless network technology. IEEE 802.11b and IEEE 802.11g have higher throughput than IEEE 802.11a and IEEE 802.11n. We observe that interferences affect more to a device than another. For example, the throughput difference between Linksys WRT54GL is approximately double than the Linksys WRT320N.

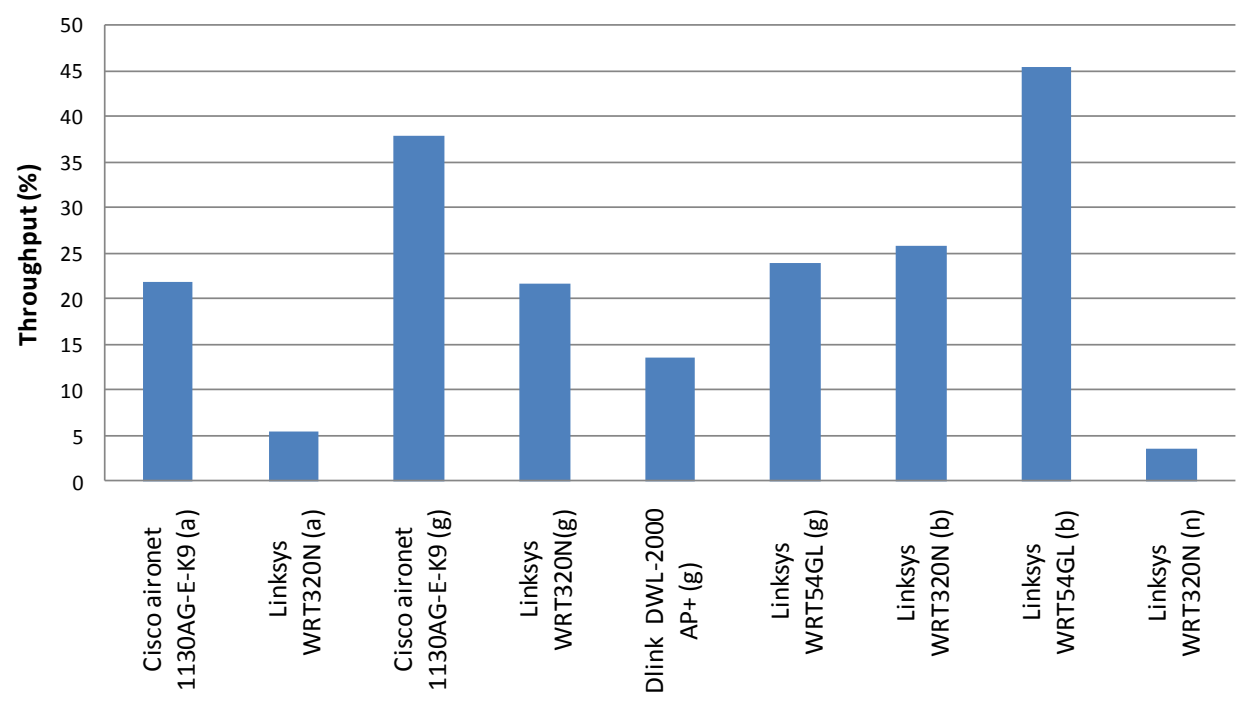

Figure 5.43. Throughput in $\%$ for all devices.

\subsubsection{Measurements Comparison for Both Scenarios and Sensor Placement in Indoor Environments.}

So far, we have analyzed the behavior of 4 variants of the IEEE 802.11 standard in two buildings of different sizes. For the garage, we have taken measures for the 4 variants. However, for the CRAI, we have only gathered measurements for a technology (IEEE 802.11g). So, first we will analyze the differences that this variant shows in both scenarios.

Figure 5.44 shows the behavior of the wireless signal working in IEEE $802.11 \mathrm{~g}$ as a function of the distance. The red line shows the average of the signal strength in the new scenario and the blue line shows the average of the signal strength in the CRAI building. The most important conclusion is that the behavior of the signal is similar in both cases. However the signal strength values are different in both cases. This fact might suggest that the building area can influence in the coverage that an AP might offer. It is also shown that there are two flat zones that maintain the signal level in 


\section{Sandra Sendra Compte}

both signals and they could be used for future applications. We think that this difference is given by the multipath effect. In the CRAI building the signal levels (through walls) are acceptable in ranges up to 18 meters (having around $-70 \mathrm{dBm}$ ). If we extend the graph of the results obtained for the new scenario presented in this section, we estimate that we can achieve signal strengths higher than $70 \mathrm{dBm}$ up to $13-14 \mathrm{~m}$.

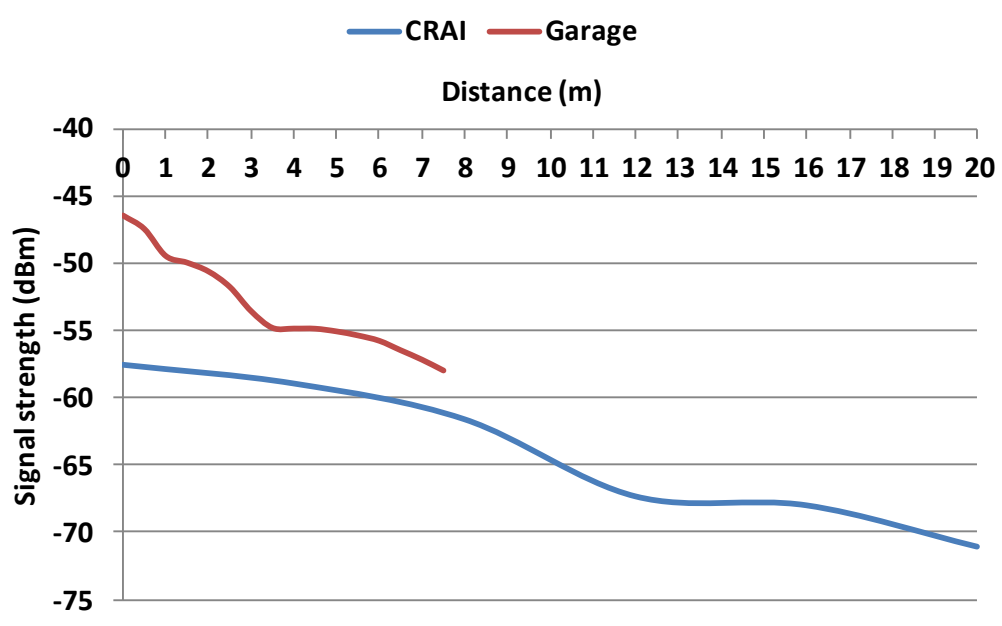

Figure 5.44. Signal strength for the IEEE $802.11 \mathrm{~g}$

Once we have analyzed the behavior and performance of 4 variants, we take the measurements performed in Section 5.3. Keeping in mind these observations, it is possible to estimate the behavior for the other variants. Figure 5.45 shows the behavior of IEEE $802.11 \mathrm{~g}$ wireless technology in the CRAI. It also shows the behavior estimation for IEEE 802.11a, IEEE 802.11b and IEEE 802.11n variants.

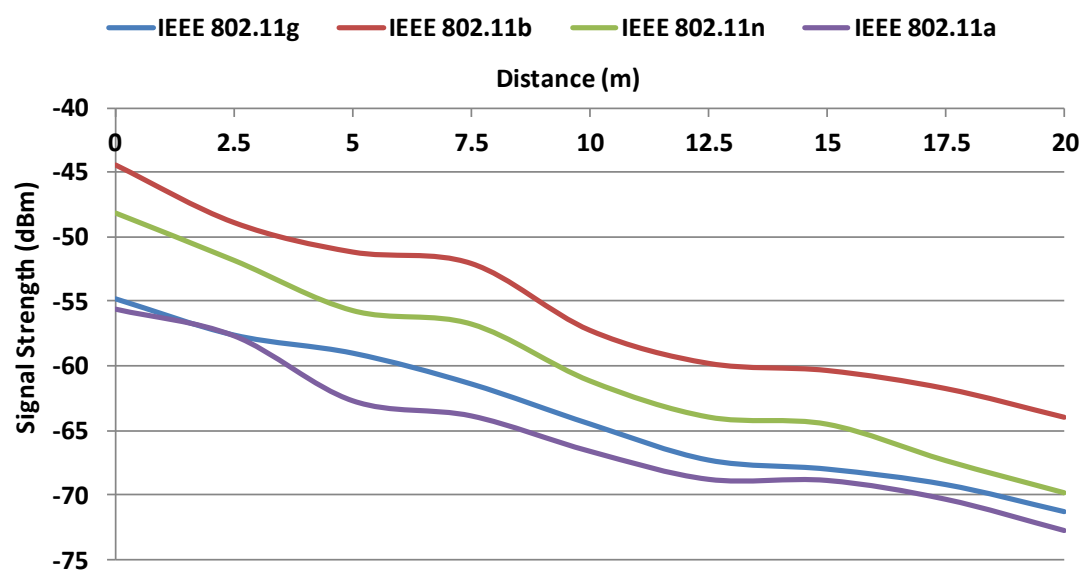

Figure 5.45. Behavior for four variants in CRAI.

The behavior of each technology can be modeled by a polynomial expression. Equations 5.8, 5.9, 5.10 and 5.11 model the behavior of wireless signals for IEEE 802.11a, IEEE 802.11b, IEEE 802.11g and IEEE $802.11 \mathrm{n}$ respectively.

$$
y=-10^{-5} x^{6}+0.0007 x^{5}-0.0161 x^{4}+0.1714 x^{3}-0.8429 x^{2}+0.2569 x-55.524
$$




$$
\begin{gathered}
y=10^{-5} x^{6}-0.0007 x^{5}+0.0204 x^{4}-0.2572 x^{3}+1.4851 x^{2}-4.3207 x-44.423 \\
y=-9 * 10^{-5} x^{5}+0.0043 x^{4}-0.0704 x^{3}+0.4455 x^{2}-1.8087 x-54.828 \\
y=-2 * 10^{-5} x^{6}+0.0009 x^{5}-0.0188 x^{4}+0.1728 x^{3}-0.6315 x^{2}+0.7628 x-48.16
\end{gathered}
$$

Is this section, we use the values obtained from both buildings to develop our design algorithm to know the best location to place the sensors. Our algorithm is developed to be used inside buildings and structures, containing walls and objects that generate reflections and refractions that modify the linear behavior of wireless signals (given in free space).

\subsubsection{Method for estimating the best position of wireless sensors in indoor environments.}

In WSN design and sensor nodes positioning, the first step is to determine the number of sensors that are needed to cover the sensing area. In our monitoring system, we consider an acceptable level of signal strength values higher than $-70 \mathrm{dBm}$. Using equations provided by Figures from 5.34, 5.35, 5.36 and 5.46, we can see that devices, with an output signal of $18 \mathrm{dBi}$, can cover approximately 7.5 m., while for the device used in CRAI, whose output power is $20 \mathrm{dBi}$, we can cover distances of about $17.5 \mathrm{~m}$. (and about $20 \mathrm{~m}$. for IEEE 802.11a variant).

From Figures 5.38 and 5.46, we can observe several behaviors:

- The signal strength value, at 1 meter from the AP, depends on the technology and the output power of the device. However, it also depends on the flat size. We have measured the behavior for the Cisco Aironet 1130AG (AIR-AP1131AG-E-K9), in the flat and the CRAI, and in both cases different values have been obtained (See Figure 5.45). The flat shows higher values of signal strength than for the CRAI.

- Both flat areas have a very similar behavior. Area grows when the coverage area of the node also increases. The length is 1 meter in the flat and 2.5 meters for the CRAI.

- The signal strength value in the flat areas can be calculated from the initial value registered in each case.

Following these observations, we propose an algorithm to estimate the behavior of wireless signals for indoor environments. These estimations allow us to find the best positions for the wireless sensor nodes.

\section{1st step: Determine the signal initial value at a distance of $1 m$ from the AP.}

This value can be obtained as a linear relationship which is in function of the plant area. When the area increases, the walls where the signal produces reflections and refractions generate destructive and constructive interferences. Equation (5.12) shows the relationship between the initial value of the signal at 1 meter from de AP depending on the area of the plant.

$$
V_{\text {ini }}=a \cdot S+c
$$

Where $S$ is the area of the plant and, $a$ and $c$ are coefficients that depend on the technology. Table 5.5 shows the linear relationship for the 4 standard variants. 
Table 5.5. Equations that estimate the initial value in our studied technologies.

\begin{tabular}{|l|l|}
\hline Technology & Equation \\
\hline IEEE 802.11a & $\mathrm{V}_{\text {ini_a }}=-0.0038 \mathrm{~S}-40.911$ \\
\hline IEEE $802.11 \mathrm{~b}$ & $\mathrm{~V}_{\text {ini_b }}=-0.0029 \mathrm{~S}-32.738$ \\
\hline IEEE $802.11 \mathrm{~g}$ & $\mathrm{~V}_{\text {ini_g }}=-0.0038 \mathrm{~S}-40.257$ \\
\hline IEEE $802.11 \mathrm{n}$ & $\mathrm{V}_{\text {ini_n }}=-0.0032 \mathrm{~S}-35.458$ \\
\hline
\end{tabular}

2nd step: Determine the value of the signal strength of the two stages where the signal strength does not vary significantly.

In this case, both values will be estimated as a linear relationship of the initial signal value and the maximum distance from the access point, where the signal strength is above $-70 \mathrm{dBm}$. The signal strength value of the first stage is calculated by Equation (5.13).

$$
V_{P 1}=V_{\text {ini }}+\Delta P o s 1=V_{\text {ini }}+\left[K \cdot \text { Dist }_{\text {Max_AP }}+C_{2}\right]=K \cdot \operatorname{Dist}_{\text {Max }_{A P}}+N
$$

Where, $\triangle P O s 1$ is the position in $\mathrm{m}$ of the first flat zone from the AP, Dist ${ }_{M a x_{-} A P}$ is the maximum distance from the AP (always with values greater than $-70 \mathrm{dBm}$ ), $C_{2}, K$ and $N$ are coefficients of the equation that depend on the technology. Table 5.6 shows the coefficient values for the equation of each technology.

Table 5.6. Equations estimating the signal strength of the first stage value.

\begin{tabular}{|l|l|}
\hline Technology & Equation \\
\hline IEEE 802.11a & $\mathrm{V}_{\mathrm{P} 1 \_\mathrm{a}}=-0.8571 \mathrm{x}-48.571$ \\
\hline IEEE 802.11b & $\mathrm{V}_{\mathrm{P} 1 \_\mathrm{b}}=-0.6667 \mathrm{x}-40$ \\
\hline IEEE 802.11g & $\mathrm{V}_{\mathrm{P} 1 \_\mathrm{g}}=-1.0952 \mathrm{x}-44.286$ \\
\hline IEEE 802.11n & $\mathrm{V}_{\mathrm{P} 1 \_\mathrm{n}}=-0.6667 \mathrm{x}-44$ \\
\hline
\end{tabular}

In order to estimate the second stage, we use the same procedure. Equation (5.14) shows the signal strength value of the second stage.

$$
V_{P 2}=V_{\text {ini }}+\Delta P o s 2=V_{\text {ini }}+\left[K_{2} \cdot \text { Dist }_{M a x \_A P}+C_{3}\right]
$$

Where, $\triangle P$ Pos 2 is the position in $\mathrm{m}$ of the second flat zone from the AP, Dist Max_AP $_{-}$has been previously defined, and $\mathrm{K}_{2}$ and $\mathrm{C}_{3}$ coefficients depend on the technology. Coefficient values for the equation of each technology are shown in Table 5.7. 
Table 5.7. Equations estimating the signal strength of the second stage.

\begin{tabular}{|l|l|}
\hline Technology & Equation \\
\hline IEEE 802.11a & $\mathrm{V}_{\mathrm{P} 2 \_^{\mathrm{a}}}=-0.9524 \mathrm{x}-42.857$ \\
\hline IEEE 802.11b & $\mathrm{V}_{\mathrm{P} 2 \mathrm{~b}}=-1.1429 \mathrm{x}-49.429$ \\
\hline IEEE 802.11g & $\mathrm{V}_{\mathrm{P} 2 \mathrm{~g}}=-1.1429 \mathrm{x}-47.429$ \\
\hline IEEE 802.11n & $\mathrm{V}_{\mathrm{P} 2 \_\mathrm{n}}=-0.7619 \mathrm{x}-48.286$ \\
\hline
\end{tabular}

\section{3rd step: Calculation of the length of the stages.}

Figures 5.38 and 5.46 show that the stages are approximately $1 \mathrm{~m}$. long in the flat, and $2.5 \mathrm{~m}$. long in the CRAI. This value is also related to the maximum distance from the AP, where the signal is greater than $-70 \mathrm{dBm}$. The size of the stage is the same in the 4 variants, and, therefore, we can calculate this value by Equation (5.15).

$$
Z=0.0952 \cdot \text { Dist }_{\text {Max_AP }}+0.2857
$$

Where $Z$ is the stage length in meters, and Dist $_{\text {Max_AP }}$ has been previously defined.

\section{4th step: calculation of the position of the stages.}

Figures 5.38 and 5.46 show the first stage in the flat placed at $2.5 \mathrm{~m}$. from the position of the AP. While in the CRAI it is placed at a distance of $4 \mathrm{~m}$. from the AP. In all IEEE 802.11 variants the size of this area is the same and, therefore, we can calculate its size using Equation (5.16).

$$
\text { Pos }_{1}=0.2381 \cdot \text { Dist }_{\text {Max_AP }}+0.7143
$$

Where $P o s_{1}$ the position of the first stage in meters from the AP, and Dist ${ }_{M a x \_A P}$ has been previously defined.

The second stage is located at $4.5 \mathrm{~m}$. from the position of the AP (in the flat case). While in the CRAI it is placed at a distance of $12 \mathrm{~m}$. from the AP. In all variants of the second stage is located in the same place. Thus, its position can be calculated using Equation (5.17).

$$
\text { Pos }_{2}=0.7143 \cdot \text { Dist }_{\text {Max_AP }}-0.8571
$$

Where $\mathrm{Pos}_{2}$ is the position of the first flat area in meters from the AP and Dist Max_AP $_{-}$has been previously defined.

Following these equations, we can estimate the behavior of a wireless device indoors of any area. Thus, it is possible to determine the best places for positioning the sensors.

In order to verify the advantages offered by our positioning system, we present an indoor case with $132 \mathrm{~m}^{2}$ area (it is like a regular office floor).

We use IEEE $802.11 \mathrm{~g}$ variant because it has good performance against interferences. AP has an output power of $17 \mathrm{dBm}$. Our experience shows that the maximum distance with signal strength greater than $-70 \mathrm{dBm}$ is approximately $7.5 \mathrm{~m}$. Then, we can determine that the signal strength to $1 \mathrm{~m}$ from the AP is $-40.75 \mathrm{dBm}$. The extension of both stages is 1 meter. The signal strength for the first 


\section{Sandra Sendra Compte}

stage is $-52.5 \mathrm{dBm}$ and it is located at $2.5 \mathrm{~m}$. from the AP, while the second stage is $4.5 \mathrm{~m}$ from the AP. Its value is $-56 \mathrm{dBm}$. Figure 5.46 shows the sensor distribution without using our method (orange dots) and using our method (purple dots). It also shows the estimated signal strength value. We can see that there is a $15 \%$ reduction of the number of sensors needed to cover the entire area of the floor, regardless of the staircase and bathrooms. This reduction of the number of sensors means to save costs buying devices. This also means network energy savings.
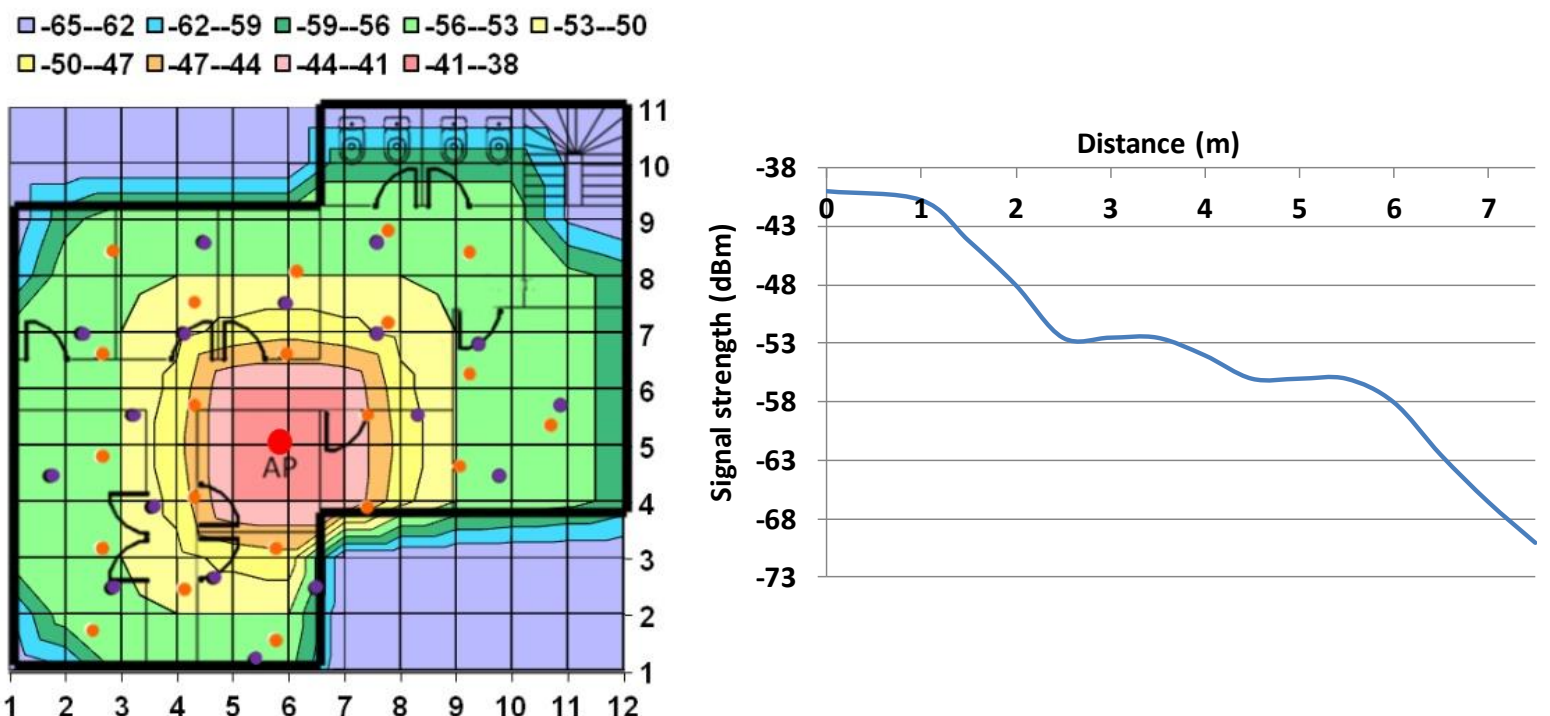

Figure 5.46. Sensor distribution in a building and the estimated values of signal strength.

\subsection{Conclusion}

When we need to monitor an area in indoor environments, it is important to know the signals behavior and the losses they can suffer. It is also important to know where to place the sensor to cover all area.

In this chapter, we have performed different test benches in order to characterize the behavior of wireless signals in indoor environments. Measurements have been carried out in two scenarios of different size and form. We have measured the signal strength of IEEE $802.11 \mathrm{a} / \mathrm{b} / \mathrm{g} / \mathrm{h}$ variants in order to know which the best location to place the sensors is. Moreover, we have compared the interferences between channels for each technology in order to determine the optimal channel distribution when designing WSN.

We have measured the signal strength of IEEE $802.11 \mathrm{a} / \mathrm{b} / \mathrm{g} / \mathrm{n}$ variants indoors in order to know which the best location to place the sensors is. Moreover, we have compared the interferences between channels for each technology in order to determine the optimal channel distribution when designing WSN.

Our results provide several conclusions. On the one hand, we have observed that the best technologies for indoor environments are IEEE 802.11b and IEEE 802.11n, while the worst ones have been IEEE 802.11g and IEEE 802.11a. But if we analyze the signal strength as a function of the 


\section{Chapter 5: Wireless Sensor Development for Monitoring in Indoor Environments}

distance from the AP, the one with highest signal strength in larger distances has been IEEE 802.11b and the worst ones have been IEEE 802.11g and IEEE 802.11n. We have observed for all variants that we can place sensors at $3.5 \mathrm{~m}$ or at $5.5 \mathrm{~m}$. of the AP, instead of at $2.5 \mathrm{~m}$. or at $4.5 \mathrm{~m}$. respectively, without decreasing significantly the signal strength. On the other hand, we have measured the interferences between neighboring channels for each variant. We see that we do not need to use a separation of 4 or 5 channels to avoid interferences. We have registered lower interferences when two APs are working in the same channel than if they are working at one channel of separation. Although, we have observed that the hardware used affects more to the packet loss than the used technology, it seems that IEEE $802.11 \mathrm{~b}$ and IEEE $802.11 \mathrm{~g}$ are better.

From measurements obtained in both scenarios, we have designed a method for estimating the signal strength in indoor environments. The extracted mathematical models have allowed us determine the best places for wireless sensors. Our results and estimations show us, that our method involves a reduction of $15 \%$ in the number of sensors needed to cover an area. Furthermore, the reduction in the number of sensors entails high economic savings as well as an important energy saving.

In a future work, we will improve our algorithm by adding intelligence (smart algorithms) in order to improve network performance. Our purpose is to adapt their operation to the environmental conditions. We would also like to extend our study to other buildings, with other construction materials.

Finally, work presented in this chapter has been published in the following references [168], [170], [171], [172] and [173] and [336]. 



\section{Chapter 6}

\section{Underwater Wireless Communications based on Electromagnetic Waves}

\subsection{Introduction}

Nowadays, there is an extensive research activity in underwater communications and underwater ad-hoc networks. On one hand, the main research lines are based on increasing the distance and bandwidth, and, on the other hand, the attempt to reduce the energy consumption of underwater devices, with the aim of increasing the network lifetime [157] [344]. Underwater communication research is primarily focused on the use of optical signals, EM signals and the propagation of acoustic and ultrasonic signals. Each technique has its own characteristics, with benefits and drawbacks, mainly due to the chemical characteristics [174] and physical constraints of the medium [114] [115].

Systems based on optical communication are able to reach very high propagation speed. However a strong backscattering is caused by suspended particles and they are affected by the turbidity of the water, so they are not good options for long distances.

Systems based on acoustic waves are not so sensitive to the particles suspended in the water and to the turbidity of the water. Moreover, they are the most used methods, since they are able to reach large distances (over $20 \mathrm{~km}$ [133]). Although acoustic communication is a proven technology, it presents some main drawbacks, like the low data rate $(0 \mathrm{~b} / \mathrm{s}$ to $20 \mathrm{~kb} / \mathrm{s})$, which is limited by some factors, such as low carrier frequency, strong reflections and attenuation when the communication is performed near the surface, as well as poor performance in turbid water, sensitivity to varying 
environmental characteristics, and the salinity or turbidity of the water. In acoustic and ultrasonic communications, researchers usually work on varying the type of modulation and communication protocol, in order to minimize the effects of reflections, and on achieving a communication data rate as high as possible.

When higher data rates are needed, we should make use of radio frequency (RF) methods, which are able to reach communication data rates of up to $100 \mathrm{Mb} / \mathrm{s}$ in very short distances, apart from presenting substantial immunity from the environmental features. EM waves, in the RF range, can also be a good option for underwater wireless communication systems. EM waves are less sensitive to reflection and refraction effects in shallow water than acoustic waves. In addition, suspended particles have very little effect on them. The speed of EM waves is higher than the acoustic ones, (150,000 times greater). The speed of an EM wave mainly depends on permeability $(\mu)$, permittivity $(\varepsilon)$, conductivity $(\sigma)$ and volume charge density $(\rho)$ [345]. These parameters change with the type of water and the electrical conductivity value associated with the medium often varies, thus the wave propagation speed and absorption coefficient, which are directly related to the working frequency, also vary. Conductivity presents different values for each case, seawater has a high conductivity average value, which is around $4 \mathrm{~S} / \mathrm{m}$ (obviously it changes with the salinity and physical properties of sea water), but in fresh water the typical value is $0.01 \mathrm{~S} / \mathrm{m}$ and drinking water presents a conductivity between 0.005 and $0.05 \mathrm{~S} / \mathrm{m}$. Moreover, the permittivity of seawater changes as a function of the frequency, the temperature and the salinity. In [346][347], authors provided a relationship model of this dependency in the water. Thus, the main problem for underwater communications based on EM waves is the high attenuation due to the conductivity of the water. This attenuation increases when the EM wave frequency increases. Hence, the higher frequencies will register greater signal losses.

In this chapter, we present the tests performed at different frequencies and modulations in order to measure several parameters such as minimum depth, distance between devices and signal transmission characteristics. We perform the practical study at $2.4 \mathrm{GHz}$ ISM frequency band, using devices compatible with IEEE 802.11 standard. In order to determine if there is any dependence between the wireless signal behavior and the frequency, data transfer rates or temperature, we will analyze the results of each case by using the analysis of variance (ANOVA).

We are going to use a two-way ANOVA, because we want to determine if the value of maximum distance depends on the data transfer rate and/or the working frequency. Both factors are analyzed separately as Rows (dependence with data transfer rate) and as columns (dependence with working frequency). For all analysis of variance, the significance level $\alpha=0.05$.

We have analyzed IEEE 802.15.4 standard [6] which also works on this frequency. A priori, we thought that due to the low-power consumption of IEEE 802.15.4, it would be better to use these devices as sensor nodes. However, our application needs data transfer rates higher than the ones offered by IEEE 802.14.5. For this reason, we decided to sacrifice a little power consumption in favor of improved data transfer rates. Table 6.1 shows a comparison of the maximum data transfer rates of both wireless technologies. 
Table 6.1. Comparison of different wireless standards.

\begin{tabular}{|c|c|c|}
\hline Standard & Frequency & Data Rate \\
\hline IEEE $802.11 \mathrm{~b}$ & $2.4 \mathrm{GHz}$ & $11 \mathrm{Mbps}$ \\
\hline IEEE $802.11 \mathrm{~g}$ & $2.4 \mathrm{GHz}$ & $54 \mathrm{Mbps}$ \\
\hline IEEE 802.15 .4 & $2.4 \mathrm{GHz}$ & $250 \mathrm{kbps}$ \\
\hline IEEE 802.15 .4 & $868 / 915 \mathrm{MHz}$ & $40 \mathrm{kbps}$ \\
\hline
\end{tabular}

These tests are performed in a swimming pool with fresh water. We set up an underwater pointto-point link between two antennae in a waterproof case. We also use two computers connected to each antenna in order to monitor the activity of the underwater point-to-point link. We have used the echo request and echo reply packets in order to perform our tests.

The rest of this chapter is structured as follow. Section 6.2 explains the scenario used in our test bench and the strategies used to take measurements. This section also shows the results of two practical studies in underwater environment. In Section 6.3, we show the best results and comparison with other studies. Finally, we will see the conclusion of this chapter.

\subsection{Signal Behavior of EM Waves in Fresh Water}

In this section, we show the scenario used to take our measurements. In addition, we explain the equipment and the software used in our tests. Finally, we describe the performed tests and the parameters used to perform our measurements.

We have performed two studies at different temperatures. In first study, we have analyzed the behavior of EM waves for temperatures of $16^{\circ} \mathrm{C}, 18^{\circ} \mathrm{C}, 20^{\circ} \mathrm{C}$ and $22^{\circ} \mathrm{C}$ for all modulations supported by IEEE 802.11 Standard meanwhile in the second study we have only performed the study for BPSK, QPSK and CCK modulations at $26^{\circ} \mathrm{C}$. The main reason of this division is because the results of OFDM al $26^{\circ} \mathrm{C}$ are very bad compared to the ones obtained at the rest of temperatures.

\subsubsection{Experimental Setup}

For the measurements, we have used a swimming pool with $32 \mathrm{~m}^{2}$ surface, with a length of 8 meters and 4 meters wide. The swimming pool depth ranges between $1.50 \mathrm{~m}$ and $1.80 \mathrm{~m}$. It is built with walls of bricks that are covered with small mosaic tiles. The size of this structure allows us to avoid any reflection on the walls, ground and water surface. In order to perform our measurements, we have used an ad hoc wireless connection between two laptops with two vertical monopole antennae placed inside the water. We used two HP pavilion dv6-6c13ss Intel Core i7 2670QM with 4GB RAM memory and the antennae had $2 \mathrm{dBi}$ gain.

We put each antenna in a sealed plastic box to make it both watertight and airtight. We used a pigtail of $3 \mathrm{~m}$. to connect each antenna with each laptop which was located outside the water. Antennae are placed under the water with enough depth to avoid any transmission to the open air. 
Measurements were taken in freshwater. The amount of chlorine and bromine dissolved in the water was fixed to $0.3 \mathrm{mg} / \mathrm{l}$. and $\mathrm{pH}$ value was 7.2 . Figure 6.1 shows a scheme of the scenario and the location of the devices inside the swimming pool.

In order to determine the minimum depth to ensure that the taken measures are valid and the signal does not spread out of the water, we have done the following process. We established an ad hoc wireless connection between both antennae outside the water. After that, we introduced one antenna progressively inside the water and checked it every $5 \mathrm{~cm}$. We stopped when we did not detect any signal with the antenna placed outside the water from the antenna located inside the water. We placed the antennae at $30 \mathrm{~cm}$. Then, we used that distance to make our tests. This test demonstrates that this depth is enough to ensure that the only signal received by the laptop is provided by the antenna placed inside the water.

In order to take the measurements, we have used a MS-DOS shell command that let us check the status of the network connection. Concretely we used the ping command which provides the round trip time (RTT) for each packet (see Figure 6.2).

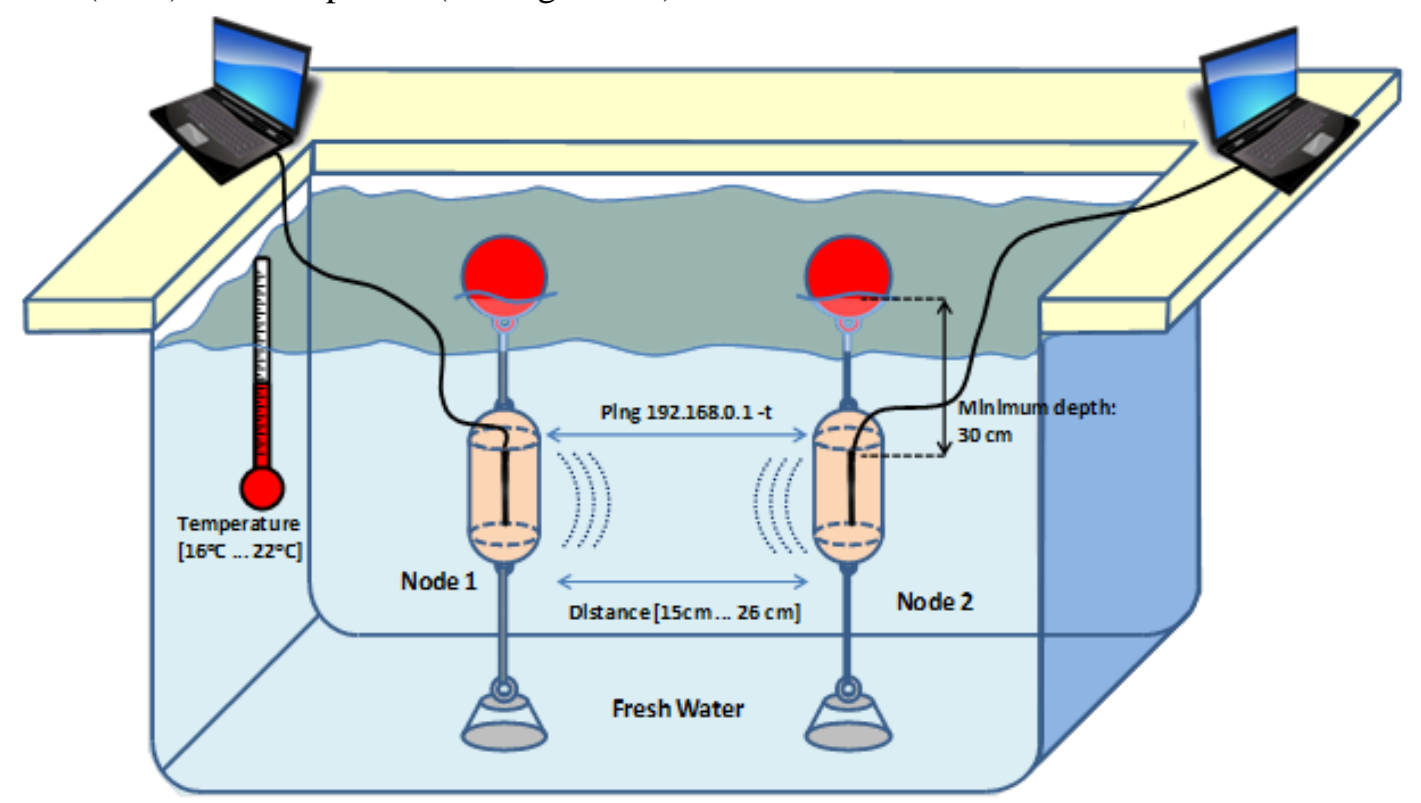

Figure 6.1. Swimming pool where measures have been taken

In our tests, we have analyzed the RTT between both devices as a function of the distance between them. We have also measured this parameter for different types of modulations and frequencies. RTT average has been estimated taking into account only the packets that performed the round-trip successfully. When a packet was not received or was received wrong, we assigned the value of 3,000 ms but this value was not taken into account in the RTT average estimation. We have used a threshold value of $3,000 \mathrm{~ms}$, because it is commonly used [348]. 


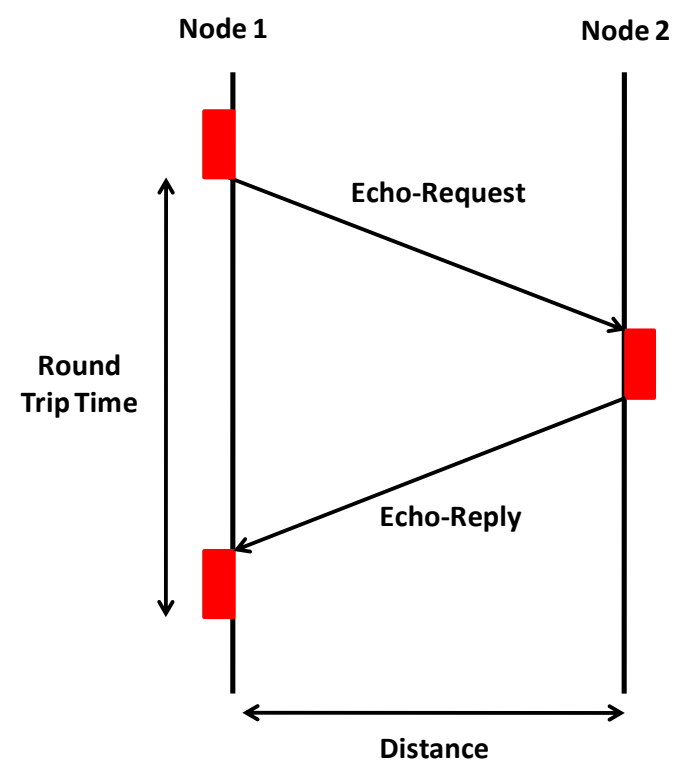

Figure 6.2. Packet flow diagram

Tests have performed at $2.4 \mathrm{GHz}$ frequency band, specified in the IEEE $802.11 \mathrm{~b} / \mathrm{g}$ standard (range between 2,412 $\mathrm{MHz}$ and 2,462 MHz). The modulation used in our test were BPSK at 1 Mbps, QPSK at $2 \mathrm{Mbps}$, CCK at 5.5 Mbps and $11 \mathrm{Mbps}$, and finally OFDM at $6 \mathrm{Mbps}, 9 \mathrm{Mbps}, 12$ Mbps, 18 Mbps, 22 Mbps, 24 Mbps, 36 Mbps, 48 Mbps, 54 Mbps.

We have performed all these tests for the temperatures of $16^{\circ} \mathrm{C}, 18^{\circ} \mathrm{C}, 20^{\circ} \mathrm{C}, 22^{\circ} \mathrm{C}$ and $26^{\circ} \mathrm{C}$.

Table 6.2. Modulations of IEEE $802.11 \mathrm{~b} / \mathrm{g}$ standard used in our tests.

\begin{tabular}{|l|l|l|l|l|}
\hline $\begin{array}{c}\text { Modulation or } \\
\text { scheme }\end{array}$ & Data transfer rate & $\begin{array}{c}\text { Modulation or } \\
\text { scheme }\end{array}$ & \multicolumn{2}{|c|}{ Data transfer rate } \\
\hline BPSK & $1 \mathrm{Mbps}$ & \multirow{4}{*}{ OFDM } & $6 \mathrm{Mbps}$ & $9 \mathrm{Mbps}$ \\
\hline QPSK & $2 \mathrm{Mbps}$ & & $12 \mathrm{Mbps}$ & $18 \mathrm{Mbps}$ \\
\hline \multirow{3}{*}{$\mathrm{CCK}$} & $5.5 \mathrm{Mbps}$ & & $22 \mathrm{Mbps}$ & $24 \mathrm{Mbps}$ \\
\cline { 2 - 2 } & \multirow{2}{*}{$11 \mathrm{Mbps}$} & & $36 \mathrm{Mbps}$ & $48 \mathrm{Mbps}$ \\
\cline { 5 - 5 } & & & $54 \mathrm{Mbps}$ \\
\hline
\end{tabular}

Finally, the maximum distance will be determined as the distance between antennae where the number of delivered packets without errors is higher than $50 \%$ at least. Each test has duration of 3 minutes. The average value of RTT for each case and its typical error are calculate with a minimum of 150 samples.

\subsubsection{First study: Measurements at $16{ }^{\circ} \mathrm{C}, 18{ }^{\circ} \mathrm{C}, 20{ }^{\circ} \mathrm{C}$ and $22{ }^{\circ} \mathrm{C}$}

This section shows the obtained results. Each subsection represents the maximum distance between the two antennae and the average RTT value for these distances as a function of the 
working frequency. The results are analyzed for each one of the defined temperatures in our tests.

\subsubsection{Measurements for $16{ }^{\circ} \mathrm{C}$}

At $16{ }^{\circ} \mathrm{C}$, we performed the test for the frequencies between $2,412 \mathrm{MHz}$ and 2,452 MHz. For higher frequency values, in this temperature, it was not possible to establish an underwater wireless link. Figure 6.3 shows the maximum distances for each data transfer rate as a function of the working frequency at $16{ }^{\circ} \mathrm{C}$.

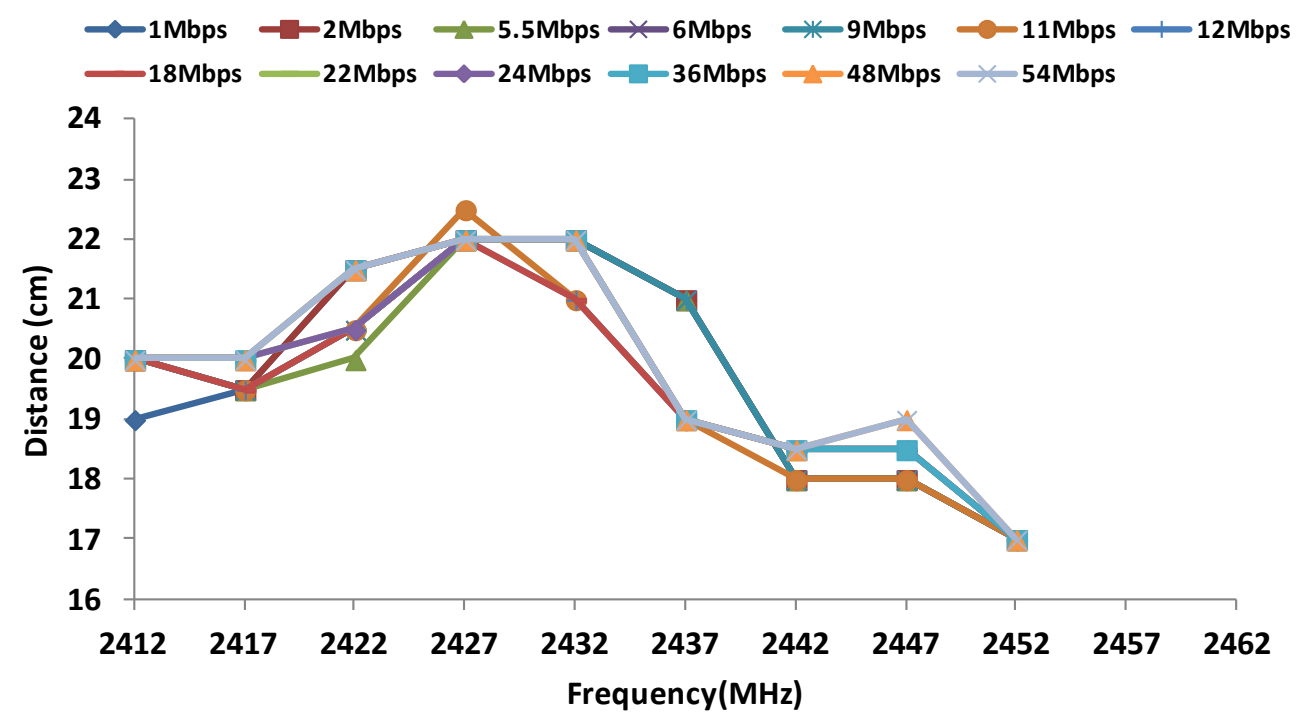

Figure 6.3. Maximum distances for data transfer rates at $16^{\circ} \mathrm{C}$.

The first significant feature is that all data transfer rates have similar behaviors. We can see that the greater distances for all data transfer rates are observed between the frequencies $2,427 \mathrm{MHz}$ and $2,432 \mathrm{MHz}$. The data transfer rate that shows greater distance $(22.5 \mathrm{~cm})$ is $11 \mathrm{Mbps}$, which uses CCK modulation scheme. However the data transfer rate of $1 \mathrm{Mbps}$ (which uses the BPSK modulation) and $2 \mathrm{Mbps}$ (which uses QPSK modulation) maintains distances between 21.5 and 22 $\mathrm{cm}$ in the frequency range between $2,422 \mathrm{MHz}$ and 2,432 MHz. Table 6.3 shows a summary of the statistical results for measurements at $16{ }^{\circ} \mathrm{C}$.

- Description of groups to be compared: Each Frequency and each data transfer rate at $16^{\circ} \mathrm{C}$

- Variable to be studied: maximum distance between antennae at $16^{\circ} \mathrm{C}$.

As Table 6.3 shows, the average value of each group (columns) is numerically distinct. We can focus our analysis on the dependence of maximum distance with working frequency and data transfer rate. Average values of rows seem to be very similar. This leads us to think that the variation in data transfer rates for a same frequency is not significant.

Table 6.4 shows the analysis of variance measurements at $16{ }^{\circ} \mathrm{C}$. 
Table 6.3. Sumary of statistical results for measurements at $16{ }^{\circ} \mathrm{C}$

\begin{tabular}{|c|c|c|c|c|}
\hline Summary & $N^{0}$ of data & Sum & Average & Variance \\
\hline Row 1 & 9 & 178 & 19.7777778 & 3.63194444 \\
\hline Row 2 & 9 & 179 & 19.8888889 & 3.54861111 \\
\hline Row 3 & 9 & 177.5 & 19.7222222 & 3.19444444 \\
\hline Row 4 & 9 & 178 & 19.7777778 & 3.25694444 \\
\hline Row 5 & 9 & 178 & 19.7777778 & 3.25694444 \\
\hline Row 6 & 9 & 175.5 & 19.5 & 2.9375 \\
\hline Row 7 & 9 & 176 & 19.5555556 & 2.27777778 \\
\hline Row 8 & 9 & 176 & 19.5555556 & 2.27777778 \\
\hline Row 9 & 9 & 177.5 & 19.7222222 & 2.75694444 \\
\hline Row 10 & 9 & 177.5 & 19.7222222 & 2.75694444 \\
\hline Row 11 & 9 & 178.5 & 19.8333333 & 3.0625 \\
\hline Row 12 & 9 & 179 & 19.8888889 & 2.92361111 \\
\hline Row 13 & 9 & 179 & 19.8888889 & 2.92361111 \\
\hline Column1 & 13 & 259 & 19.9230769 & 0.07692308 \\
\hline Column2 & 13 & 256 & 19.6923077 & 0.06410256 \\
\hline Column 3 & 13 & 271 & 20.8461538 & 0.30769231 \\
\hline Column4 & 13 & 286.5 & 22.0384615 & 0.01923077 \\
\hline Column5 & 13 & 283 & 21.7692308 & 0.19230769 \\
\hline Column6 & 13 & 257 & 19.7692308 & 1.02564103 \\
\hline Column7 & 13 & 237.5 & 18.2692308 & 0.06730769 \\
\hline Column8 & 13 & 238.5 & 18.3461538 & 0.14102564 \\
\hline Column9 & 13 & 221 & 17 & 0 \\
\hline
\end{tabular}

Table 6.4. ANOVA for measurements at $16{ }^{\circ} \mathrm{C}$

\begin{tabular}{|c|c|c|c|c|c|c|}
\hline $\begin{array}{c}\text { Origin of } \\
\text { variations }\end{array}$ & $\begin{array}{c}\text { Sum of } \\
\text { squares }\end{array}$ & $\begin{array}{c}\text { Degree of } \\
\text { Freedom }\end{array}$ & $\begin{array}{c}\text { Average } \\
\text { Squares }\end{array}$ & F & Probability & $\begin{array}{c}\text { Critical } \\
\text { Value for F }\end{array}$ \\
\hline Rows & 1.85470085 & 12 & 0.1545584 & 0.71074719 & 0.7375945 & 1.85440887 \\
\hline Columns & 289.568376 & 8 & 36.196047 & 166.449949 & $9.0723 \mathrm{E}-53$ & 2.03631902 \\
\hline Error & 20.8760684 & 96 & 0.21745905 & & & \\
\hline & & & & & & \\
\hline Total & 312.299145 & 116 & & & & \\
\hline
\end{tabular}

\section{Analysis of Rows:}

We formulate two hypotheses:

- $\mathrm{H} 0=$ maximum distance does not depend on the data transfer rate (null hypothesis)

- $\mathrm{H} 1=$ maximum distance depends on the data transfer rate (alternative hypothesis) 
We see that in the case of rows, the probability value $(0.7375945)$ is higher than the one defined for $\alpha$. Therefore, the variation of results is not significant. On the other hand, the value of $F(0.710)$ is lower than the critical value of $\mathrm{F}(1.85)$. For this reason, we discard the alternative hypothesis and take the null hypothesis. This means that the maximum distance for each frequency does not depend on the data transfer rate. The results do not show significant difference between groups (rows) due to variations in data transfer rates.

\section{Analysis of Columns:}

We formulate two hypotheses:

- $\mathrm{H} 0=$ maximum distance does not depend on the working frequency (null hypothesis).

- $\mathrm{H} 1$ = maximum distance depends on the working frequency (alternative hypothesis).

We see that in case of the columns, the value of the probability $\left(9.0723 * 10^{-53}\right)$ is lower than defined for $\alpha$. Therefore, the variation of the results is significant. Moreover, the value of F (166.44) is greater than the critical value of $\mathrm{F}$ (2.036). We can discard the null hypotheses for taking the alternative which means that the maximum distance between antennae at $16{ }^{\mathrm{a}} \mathrm{C}$ depends on the working frequency. There is a significant difference between groups (columns) which is due to the increase in the working frequency and not due to the random effect.

With this, we have checked that the maximum distance values are statistically different and they depend on the working frequency significantly. In addition, the effect of data transfer rate and consequently the modulations, is not significant to obtain the maximum distance.

Table 6.5 shows the RTT values and their typical errors in ms for maximum distances of each data transfer rate depending on the working frequency to the temperature of $16{ }^{\circ} \mathrm{C}$. Numbers in red indicate the best values of RTT in ms., for each data transfer rate. We can see that increasing the distance between antennas does not mean higher RTT values. We observe that the data transfer rate of $11 \mathrm{Mbps}$, operating at the frequency of 2,427 MHz, has a RTT value of $4.85 \mathrm{~ms}$. This is the most efficient combination of settings for this temperature.

\subsubsection{Measurements for $18{ }^{\circ} \mathrm{C}$}

We took measurements for frequencies between $2,412 \mathrm{MHz}$ and $2,452 \mathrm{MHz}$ at $18^{\circ} \mathrm{C}$. It was not possible to establish an underwater wireless link for higher frequency in this temperature. Figure 6.4 shows the maximum distance for each data transfer rate depending on the frequency, for a temperature of $18^{\circ} \mathrm{C}$.

Under these conditions of temperature, we observe that the maximum distance achieved was $22.5 \mathrm{~cm}$., for 2,437 MHz. The data transfer rate that reached this distance was $11 \mathrm{Mbps}$ (which uses the CCK modulation scheme), $12 \mathrm{Mbps}$ and $18 \mathrm{Mbps}$ (using OFDM). The data transfer rates of 22 Mbps, 24 Mbps, 36 Mbps, 48 Mbps and 54 Mbps (using OFDM modulation) reached the maximum distance of $21.5 \mathrm{~cm}$ at the frequency of $2,417 \mathrm{MHz}$. The rest of data transfer rates present maximum distances lower than $21 \mathrm{~cm}$. Table 6.6 shows a summary of the statistical results for measurements at $18{ }^{\circ} \mathrm{C}$. 
Table 6.5. Values of RTT in $\mathrm{ms}$ for $16^{\circ} \mathrm{C}$

\begin{tabular}{|c|c|c|c|c|c|c|c|c|c|c|c|}
\hline \multirow[b]{2}{*}{$\begin{array}{c}\text { Data } \\
\text { Transfer } \\
\text { Rates }\end{array}$} & \multicolumn{11}{|c|}{ Frequency (MHz) } \\
\hline & 2,412 & 2,417 & 2,422 & 2,427 & 2,432 & 2,437 & 2,442 & 2,447 & 2,452 & 2,457 & 2,462 \\
\hline 1 Mbps & \begin{tabular}{|c|}
$5.22 \pm$ \\
0.24 \\
\end{tabular} & \begin{tabular}{c|}
3.29 \\
\pm 0.06 \\
\end{tabular} & $\begin{array}{c}3.98 \pm \\
0.21 \\
\end{array}$ & \begin{tabular}{|c|}
$3.53 \pm$ \\
0.34 \\
\end{tabular} & \begin{tabular}{|c|}
$3.12 \pm$ \\
0.05 \\
\end{tabular} & $\begin{array}{c}2.21 \\
\pm 0.05 \\
\end{array}$ & $\begin{array}{c}3.00 \pm \\
0.00\end{array}$ & $\begin{array}{c}3.22 \pm \\
0.06\end{array}$ & \begin{tabular}{|c|}
$3.19 \pm$ \\
0.03 \\
\end{tabular} & $\mathrm{X}$ & $\mathrm{X}$ \\
\hline 2 Mbps & \begin{tabular}{|c|}
$6.75 \pm$ \\
0.46 \\
\end{tabular} & \begin{tabular}{|c|}
$3.02 \pm$ \\
0.07 \\
\end{tabular} & $\begin{array}{c}5.75 \pm \\
0.21 \\
\end{array}$ & \begin{tabular}{|c|}
$2.74 \pm$ \\
0.08 \\
\end{tabular} & \begin{tabular}{|c|}
$6.27 \pm$ \\
1.57 \\
\end{tabular} & $\begin{array}{c}2.95 \pm \\
0.06 \\
\end{array}$ & $\begin{array}{c}2.13 \pm \\
0.03 \\
\end{array}$ & $\begin{array}{c}3.66 \pm \\
0.11 \\
\end{array}$ & \begin{tabular}{|c|}
$4.08 \pm$ \\
0.46 \\
\end{tabular} & $X$ & $X$ \\
\hline $5.5 \mathrm{Mbps}$ & $\begin{array}{c}11.64 \pm \\
0.75 \\
\end{array}$ & \begin{tabular}{|c|}
$2.02 \pm$ \\
0.11 \\
\end{tabular} & $\begin{array}{c}2.56 \\
\pm 0.11 \\
\end{array}$ & \begin{tabular}{|c|}
$3.29 \pm$ \\
0.15 \\
\end{tabular} & \begin{tabular}{|c|}
$3.57 \pm$ \\
0.14 \\
\end{tabular} & $\begin{array}{c}.41 \pm \\
0.12 \\
\end{array}$ & $\begin{array}{c}1.18 \pm \\
0.03 \\
\end{array}$ & $\begin{array}{c}4.44 \pm \\
0.12 \\
\end{array}$ & \begin{tabular}{|c|}
$3.93 \pm$ \\
0.11 \\
\end{tabular} & $X$ & $X$ \\
\hline 6 Mbps & $\begin{array}{c}15.47 \pm \\
0.96\end{array}$ & $\begin{array}{c}4.12 \pm \\
0.12\end{array}$ & $\begin{array}{c}4.37 \pm \\
0.12\end{array}$ & $\begin{array}{c}4.28 \pm \\
0.11\end{array}$ & $\begin{array}{c}5.25 \pm \\
0.11 \\
\end{array}$ & $\begin{array}{c}7.08 \\
\pm 0.12 \\
\end{array}$ & $\begin{array}{c}1.08 \pm \\
0.02\end{array}$ & $\begin{array}{c}4.87 \pm \\
0.14\end{array}$ & \begin{tabular}{|c|}
$5.75 \pm$ \\
0.14
\end{tabular} & $X$ & $X$ \\
\hline 9 Mbps & \begin{tabular}{|c|}
$18.65 \pm$ \\
1.39 \\
\end{tabular} & \begin{tabular}{|c|}
$4.94 \pm$ \\
0.12 \\
\end{tabular} & $\begin{array}{c}5.06 \pm \\
0.10\end{array}$ & $\begin{array}{c}5.07 \pm \\
0.11 \\
\end{array}$ & \begin{tabular}{|c|}
6.25 \\
\pm 0.23 \\
\end{tabular} & \begin{tabular}{|c|}
$8.43 \pm$ \\
0.13 \\
\end{tabular} & $\begin{array}{c}1.11 \pm \\
0.04\end{array}$ & $\begin{array}{c}7.66 \pm \\
0.26\end{array}$ & \begin{tabular}{|c|}
$6.12 \pm$ \\
0.10
\end{tabular} & $\Lambda$ & $\Lambda$ \\
\hline 11 Mbps & $\begin{array}{c}41.07 \pm \\
4.38 \\
\end{array}$ & $\begin{array}{c}5.27 \pm \\
0.12 \\
\end{array}$ & $\begin{array}{c}5.59 \pm \\
0.36\end{array}$ & $\begin{array}{c}.85 \pm \\
0.15 \\
\end{array}$ & $\begin{array}{c}3.51 \pm \\
0.29 \\
\end{array}$ & $\begin{array}{c}3.59 \pm \\
0.11 \\
\end{array}$ & \begin{tabular}{|c|}
$1.58 \pm$ \\
0.04 \\
\end{tabular} & $\begin{array}{c}9.44 \pm \\
0.20\end{array}$ & \begin{tabular}{|c|}
$6.26 \pm$ \\
0.40 \\
\end{tabular} & 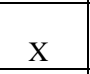 & 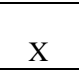 \\
\hline $12 \mathrm{Mbps}$ & $\begin{array}{c}22.56 \pm \\
2.04 \\
\end{array}$ & \begin{tabular}{|c|}
$6.43 \pm$ \\
0.14 \\
\end{tabular} & $\begin{array}{c}6.95 \pm \\
0.13 \\
\end{array}$ & \begin{tabular}{|c|}
$6.45 \pm$ \\
0.14 \\
\end{tabular} & $\begin{array}{c}5.00 \pm \\
0.07 \\
\end{array}$ & \begin{tabular}{|c|}
$7.27 \pm$ \\
0.19 \\
\end{tabular} & $\begin{array}{c}1.20 \pm \\
0.06\end{array}$ & $\begin{array}{c}5.37 \pm \\
0.22 \\
\end{array}$ & \begin{tabular}{|c|}
$8.09 \pm$ \\
0.36 \\
\end{tabular} & $\Lambda$ & 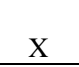 \\
\hline 18 Mbps & \begin{tabular}{|c|}
$5.34 \pm$ \\
0.20
\end{tabular} & $\begin{array}{c}3.25 \pm \\
0.10\end{array}$ & $\begin{array}{c}4.03 \pm \\
0.10\end{array}$ & \begin{tabular}{|c|}
$8.21 \pm$ \\
0.40
\end{tabular} & \begin{tabular}{|c|}
$40.90 \pm$ \\
2.93 \\
\end{tabular} & \begin{tabular}{|c|}
$3.64 \pm$ \\
0.12 \\
\end{tabular} & \begin{tabular}{c|}
$1.25 \pm$ \\
0.03 \\
\end{tabular} & $\begin{array}{c}5.36 \pm \\
0.22\end{array}$ & $\begin{array}{c}5.13 \\
\pm 0.11 \\
\end{array}$ & $\Lambda$ & 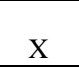 \\
\hline 22 Mbps & $\begin{array}{c}11.16 \pm \\
0.39\end{array}$ & $\begin{array}{c}14.97 \pm \\
0.51\end{array}$ & $\begin{array}{c}6.00 \pm \\
0.59\end{array}$ & \begin{tabular}{|c|}
$22.49 \pm$ \\
3.36
\end{tabular} & \begin{tabular}{|c|}
$39.17 \pm$ \\
1.42
\end{tabular} & \begin{tabular}{|c|}
$20.99 \pm$ \\
1.94
\end{tabular} & \begin{tabular}{|c|}
$1.58 \pm$ \\
0.04
\end{tabular} & $\begin{array}{c}5.07 \pm \\
0.13\end{array}$ & \begin{tabular}{|c|}
$5.48 \pm$ \\
0.14
\end{tabular} & 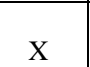 & 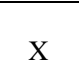 \\
\hline 24 Mbps & \begin{tabular}{|c|}
$6.69 \pm$ \\
0.14 \\
\end{tabular} & \begin{tabular}{|c|}
$7.33 \pm$ \\
0.23 \\
\end{tabular} & $\begin{array}{c}5.17 \pm \\
0.13\end{array}$ & \begin{tabular}{|c|}
$15.16 \pm$ \\
2.74 \\
\end{tabular} & \begin{tabular}{|c|}
$56.23 \pm$ \\
5.34 \\
\end{tabular} & \begin{tabular}{|c|}
$4.53 \pm$ \\
0.09 \\
\end{tabular} & \begin{tabular}{|c|}
$1.49 \pm$ \\
0.04 \\
\end{tabular} & $\begin{array}{c}4.68 \pm \\
0.11\end{array}$ & \begin{tabular}{|c|}
$7.49 \pm$ \\
0.15 \\
\end{tabular} & $\Lambda$ & $\Lambda$ \\
\hline 36 Mbps & \begin{tabular}{|c|}
$8.95 \pm$ \\
0.17 \\
\end{tabular} & \begin{tabular}{|c|}
$9.59 \pm$ \\
0.27 \\
\end{tabular} & $\begin{array}{c}7.81 \pm \\
0.19\end{array}$ & \begin{tabular}{|c|}
$8.04 \pm$ \\
0.49 \\
\end{tabular} & \begin{tabular}{|c|}
$77.92 \pm$ \\
2.47 \\
\end{tabular} & \begin{tabular}{|c|}
$6.08 \pm$ \\
0.17 \\
\end{tabular} & \begin{tabular}{|c|}
$2.13 \pm$ \\
0.03 \\
\end{tabular} & $\begin{array}{c}5.11 \pm \\
0.11 \\
\end{array}$ & $\begin{array}{c}10.44 \pm \\
0.34 \\
\end{array}$ & $X$ & $\Lambda$ \\
\hline 48 Mbps & $\begin{array}{c}8.81 \pm \\
0.17 \\
\end{array}$ & $\begin{array}{c}9.05 \pm \\
0.29 \\
\end{array}$ & $\begin{array}{c}9.45 \pm \\
0.22\end{array}$ & $\begin{array}{c}9.07 \pm \\
1.39 \\
\end{array}$ & $\begin{array}{r}126.72 \\
39.68 \\
\end{array}$ & \begin{tabular}{|c|}
$6.23 \pm$ \\
0.16
\end{tabular} & \begin{tabular}{|c|}
$2.61 \pm$ \\
0.04 \\
\end{tabular} & $\begin{array}{c}6.46 \pm \\
0.16\end{array}$ & $\begin{array}{c}10.29 \pm \\
0.22 \\
\end{array}$ & $X$ & $X$ \\
\hline 54 Mbps & \begin{tabular}{|c|}
$9.03 \pm$ \\
0.18 \\
\end{tabular} & \begin{tabular}{|c|}
$8.87 \pm$ \\
0.26 \\
\end{tabular} & $\begin{array}{c}9.67 \pm \\
0.23 \\
\end{array}$ & $\begin{array}{c}7.51 \pm \\
0.18 \\
\end{array}$ & $\begin{array}{c}9.35 \pm \\
0.23 \\
\end{array}$ & \begin{tabular}{|c|}
$7.04 \pm$ \\
0.13 \\
\end{tabular} & \begin{tabular}{|c|}
$3.20 \pm$ \\
0.05 \\
\end{tabular} & $\begin{array}{c}15.83 \pm \\
3.24 \\
\end{array}$ & \begin{tabular}{|c|}
$10.73 \pm$ \\
0.33 \\
\end{tabular} & $X$ & $X$ \\
\hline
\end{tabular}

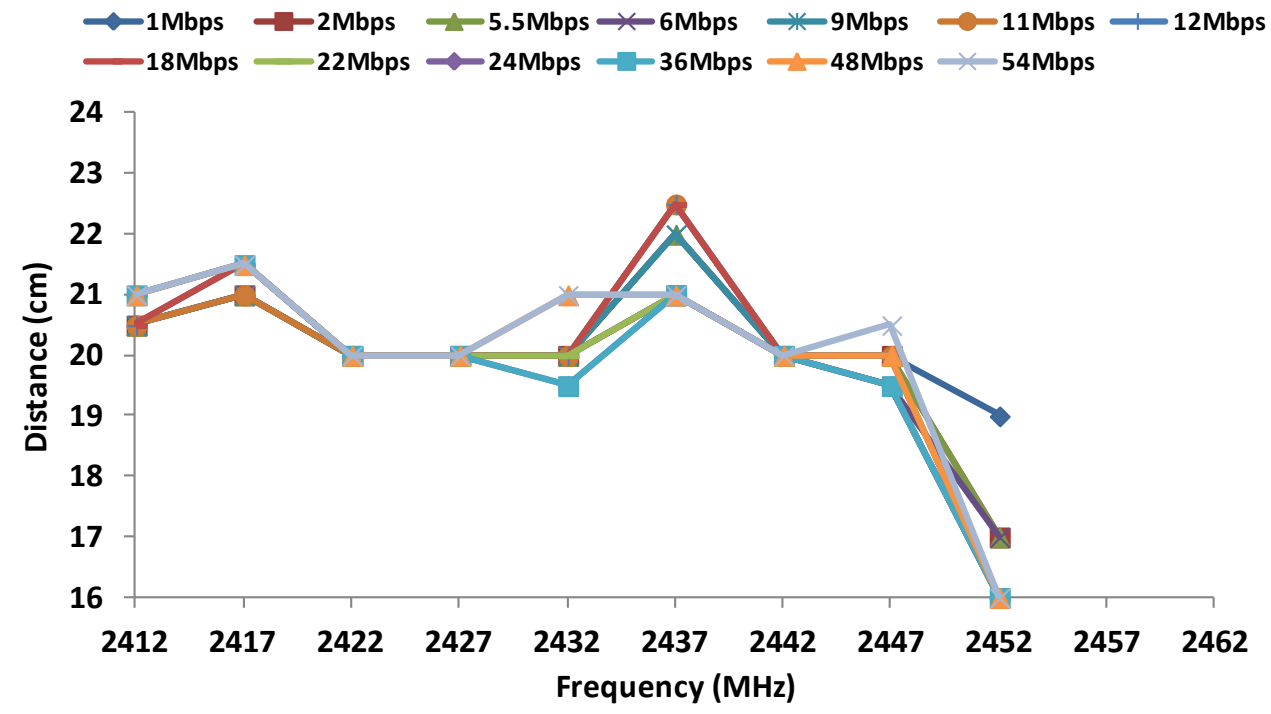

Figure 6.4. Maximum distances for data transfer rates at $18^{\circ} \mathrm{C}$. 
Table 6.6. Summary of statistical results for measurements at $18{ }^{\circ} \mathrm{C}$

\begin{tabular}{|c|c|c|c|c|}
\hline Summary & $N^{0}$ of data & Sum & Average & Variance \\
\hline Row 1 & 9 & 181.5 & 20.1666667 & 0.375 \\
\hline Row 2 & 9 & 179.5 & 19.9444444 & 1.40277778 \\
\hline Row 3 & 9 & 180.5 & 20.0555556 & 1.77777778 \\
\hline Row 4 & 9 & 180 & 20 & 1.8125 \\
\hline Row 5 & 9 & 179 & 19.8888889 & 2.67361111 \\
\hline Row 6 & 9 & 180 & 20 & 2.9375 \\
\hline Row 7 & 9 & 180.5 & 20.0555556 & 3.09027778 \\
\hline Row 8 & 9 & 180 & 20 & 3.125 \\
\hline Row 9 & 9 & 179 & 19.8888889 & 2.54861111 \\
\hline Row 10 & 9 & 178.5 & 19.8333333 & 2.5625 \\
\hline Row 11 & 9 & 178.5 & 19.8333333 & 2.5625 \\
\hline Row 12 & 9 & 180.5 & 20.0555556 & 2.65277778 \\
\hline Row 13 & 9 & 181 & 20.1111111 & 2.67361111 \\
\hline Column1 & 13 & 269 & 20.6923077 & 0.06410256 \\
\hline Column 2 & 13 & 276.5 & 21.2692308 & 0.06730769 \\
\hline Column3 & 13 & 260 & 20 & 0 \\
\hline Column4 & 13 & 260 & 20 & 0 \\
\hline Column5 & 13 & 261 & 20.0769231 & 0.20192308 \\
\hline Column6 & 13 & 280.5 & 21.5769231 & 0.45192308 \\
\hline Column7 & 13 & 260 & 20 & 0 \\
\hline Column8 & 13 & 257.5 & 19.8076923 & 0.10576923 \\
\hline Column9 & 13 & 214 & 16.4615385 & 0.76923077 \\
\hline
\end{tabular}

- Description of groups to be compared: Each Frequency and each data transfer rate at $18^{\circ} \mathrm{C}$

- Variable to be studied: maximum distance between antennae at $18^{\circ} \mathrm{C}$.

As Table 6.6 shows, the average value of each group (columns) is numerically distinct. We can focus our analysis on the dependence of maximum distance with working frequency and data transfer rate. Average values of rows seem to be very similar. This leads us to think that the variation in data transfer rates for a same frequency is not significant.

Table 6.7 shows the analysis of variance measurements at $18^{\circ} \mathrm{C}$.

For the same reasons explained in Section 6.2.2.1, we can conclude that the maximum distance depends significantly of the working frequency and the influence of data transfer rates is not significant.

Table 6.8 shows the RTT values and their typical errors in ms. of the maximum distances for all data transfer rates as a function of the working frequency at $18{ }^{\circ} \mathrm{C}$. Numbers in red indicate the best RTT values in ms., for each data transfer rate. Looking at the three data transfer rates which reach the maximum distances (11 Mbps, $12 \mathrm{Mbps}$ and $18 \mathrm{Mbps}$ ) we can see that the data rate that provides lowest RTT was $18 \mathrm{Mbps}$, with a value of $5.61 \mathrm{~ms}$. 
Chapter 6: Underwater Wireless Communications based on Electromagnetic Waves

Table 6.7. ANOVA for measurements at $18{ }^{\circ} \mathrm{C}$

\begin{tabular}{|c|c|c|c|c|c|c|}
\hline $\begin{array}{c}\text { Origin of } \\
\text { variations }\end{array}$ & $\begin{array}{c}\text { Sum of } \\
\text { squares }\end{array}$ & $\begin{array}{c}\text { Degree of } \\
\text { Freedom }\end{array}$ & $\begin{array}{c}\text { Average } \\
\text { Squares }\end{array}$ & F & Probability & $\begin{array}{c}\text { Critical Value } \\
\text { for F }\end{array}$ \\
\hline Rows & 1.17521368 & 12 & 0.09793447 & 0.50148165 & 0.90909075 & 1.85440887 \\
\hline Columns & 222.807692 & 8 & 27.8509615 & 142.613175 & $8.5592 \mathrm{E}-50$ & 2.03631902 \\
\hline Error & 18.7478632 & 96 & 0.19529024 & & & \\
\hline & & & & & & \\
\hline Total & 242.730769 & 116 & & & & \\
\hline
\end{tabular}

Table 6.8. Values of RTT in $\mathrm{ms}$ for $18^{\circ} \mathrm{C}$

\begin{tabular}{|c|c|c|c|c|c|c|c|c|c|c|c|}
\hline \multirow[b]{2}{*}{$\begin{array}{c}\text { Data } \\
\text { Transfer } \\
\text { Rates }\end{array}$} & \multicolumn{11}{|c|}{ Frequency (MHz) } \\
\hline & 2412 & 2417 & 2422 & 2427 & 2432 & 2437 & 2442 & 2447 & 2452 & 2457 & 2462 \\
\hline 1 Mbps & $\begin{array}{c}3.14 \pm \\
0.03\end{array}$ & $\begin{array}{c}4.19 \pm \\
0.12\end{array}$ & $\begin{array}{c}3.08 \pm \\
0.03\end{array}$ & $\begin{array}{c}4.86 \pm \\
0.24\end{array}$ & \begin{tabular}{|c|}
$5.01 \pm$ \\
0.31
\end{tabular} & $\begin{array}{c}3.30 \pm \\
0.05\end{array}$ & \begin{tabular}{|c|}
$5.29 \pm$ \\
1.69
\end{tabular} & $\begin{array}{c}3.31 \pm \\
0.09\end{array}$ & $\begin{array}{c}24.42 \pm \\
2.04\end{array}$ & $X$ & $X$ \\
\hline 2 Mbps & $\begin{array}{c}2.58 \pm \\
0.05\end{array}$ & $\begin{array}{c}4.01 \pm \\
0.32\end{array}$ & $\begin{array}{c}2.86 \pm \\
0.08\end{array}$ & $\begin{array}{l}8.91 \pm \\
0.55\end{array}$ & $\begin{array}{c}8.40 \pm \\
0.50\end{array}$ & \begin{tabular}{|c|}
$4.50 \pm$ \\
0.12
\end{tabular} & $\begin{array}{c}3.72 \pm \\
0.11\end{array}$ & \begin{tabular}{|c|}
$2.70 \pm$ \\
0.08
\end{tabular} & $\begin{array}{l}22.17 \pm \\
2.37\end{array}$ & X & $X$ \\
\hline $5.5 \mathrm{Mbps}$ & $\begin{array}{c}2.58 \pm \\
0.07\end{array}$ & \begin{tabular}{|c|}
$3.73 \pm$ \\
0.24
\end{tabular} & $\begin{array}{c}2.47 \pm \\
0.09\end{array}$ & $\begin{array}{c}14.16 \pm \\
0.73\end{array}$ & $\begin{array}{l}14.32 \pm \\
0.83\end{array}$ & \begin{tabular}{|c|}
$10.65 \pm$ \\
0.34
\end{tabular} & \begin{tabular}{|l|}
$7.78 \pm$ \\
0.42
\end{tabular} & $\begin{array}{c}4.87 \pm \\
0.35\end{array}$ & $\begin{array}{l}84.071 \\
\pm 4.21\end{array}$ & $X$ & $X$ \\
\hline $6 \mathrm{Mbps}$ & $\begin{array}{c}3.63 \pm \\
0.06\end{array}$ & \begin{tabular}{|c|}
$6.69 \pm$ \\
0.25
\end{tabular} & $\begin{array}{l}4.78 \pm \\
0.15\end{array}$ & $\begin{array}{c}10.39 \pm \\
0.18\end{array}$ & \begin{tabular}{|c|}
$9.71 \pm$ \\
0.13 \\
\end{tabular} & $\begin{array}{c}8.24 \pm \\
0.20\end{array}$ & $\begin{array}{c}10.14 \pm \\
0.32\end{array}$ & \begin{tabular}{|c|}
$3.22 \pm$ \\
0.06
\end{tabular} & $\begin{array}{c}1.00 \pm \\
0.00\end{array}$ & X & X \\
\hline 9 Mbps & $\begin{array}{c}4.43 \pm \\
0.08 \\
\end{array}$ & \begin{tabular}{|c|}
$7.93 \pm$ \\
0.31 \\
\end{tabular} & $\begin{array}{c}12.38 \pm \\
0.64\end{array}$ & $\begin{array}{c}11.24 \pm \\
0.17\end{array}$ & $\begin{array}{c}10.49 \pm \\
0.13\end{array}$ & $\begin{array}{c}10.39 \pm \\
0.15\end{array}$ & $\begin{array}{c}10.21 \pm \\
0.31\end{array}$ & \begin{tabular}{|c|}
$4.11 \pm$ \\
0.07 \\
\end{tabular} & $\begin{array}{c}1.00 \pm \\
0.00\end{array}$ & $X$ & $X$ \\
\hline 11 Mbps & $\begin{array}{c}3.60 \pm \\
0.09\end{array}$ & $\begin{array}{c}6.54 \pm \\
0.43\end{array}$ & $\begin{array}{c}24.74 \pm \\
4.97\end{array}$ & $\begin{array}{c}21.56 \pm \\
1.04\end{array}$ & $\begin{array}{c}17.25 \pm \\
0.56\end{array}$ & $\begin{array}{c}14.57 \pm \\
0.41\end{array}$ & \begin{tabular}{|c|}
$8.27 \pm$ \\
0.22
\end{tabular} & $\begin{array}{l}11.26 \pm \\
3.23\end{array}$ & $\begin{array}{c}1.00 \pm \\
0.00\end{array}$ & $X$ & X \\
\hline 12 Mbps & $\begin{array}{c}5.57 \pm \\
0.09\end{array}$ & $\begin{array}{c}11.48 \pm \\
1.33\end{array}$ & $\begin{array}{c}21.46 \pm \\
1.23\end{array}$ & $\begin{array}{c}11.61 \pm \\
0.27\end{array}$ & $\begin{array}{c}12.85 \pm \\
0.16\end{array}$ & $\begin{array}{c}13.14 \pm \\
0.74\end{array}$ & $\begin{array}{c}10.75 \pm \\
0.13\end{array}$ & \begin{tabular}{|c|}
$8.71 \pm$ \\
1.23
\end{tabular} & $\begin{array}{c}1.00 \pm \\
0.00\end{array}$ & $X$ & X \\
\hline 18 Mbps & $\begin{array}{c}2.84 \pm \\
0.05 \\
\end{array}$ & \begin{tabular}{|c|}
$4.82 \pm$ \\
0.08 \\
\end{tabular} & $\begin{array}{c}3.68 \pm \\
0.08\end{array}$ & $\begin{array}{c}5.16 \pm \\
0.14\end{array}$ & $\begin{array}{c}7.20 \pm \\
0.16\end{array}$ & $\begin{array}{c}5.61 \pm \\
0.24\end{array}$ & \begin{tabular}{|c}
$6.094 \pm$ \\
0.22 \\
\end{tabular} & \begin{tabular}{|c|}
$7.21 \pm$ \\
0.23 \\
\end{tabular} & $\begin{array}{c}1.00 \pm \\
0.00 \\
\end{array}$ & $X$ & $X$ \\
\hline 22 Mbps & $\begin{array}{c}14.56 \pm \\
0.94\end{array}$ & $\begin{array}{c}11.21 \pm \\
0.28\end{array}$ & $\begin{array}{c}10.84 \pm \\
0.81\end{array}$ & $\begin{array}{c}16.60 \pm \\
1.47\end{array}$ & $\begin{array}{c}19.40 \pm \\
0.94\end{array}$ & $\begin{array}{c}11.57 \pm \\
0.19\end{array}$ & \begin{tabular}{|c|}
$9.83 \pm$ \\
0.34
\end{tabular} & \begin{tabular}{|c|}
$7.07 \pm$ \\
0.36
\end{tabular} & $\begin{array}{c}1.00 \pm \\
0.00\end{array}$ & X & X \\
\hline 24 Mbps & $\begin{array}{c}7.53 \pm \\
0.24\end{array}$ & \begin{tabular}{|c|}
$5.77 \pm$ \\
0.11
\end{tabular} & $\begin{array}{c}9.94 \pm \\
0.14\end{array}$ & $\begin{array}{l}6.76 \pm \\
0.156\end{array}$ & \begin{tabular}{|c|}
$8.21 \pm$ \\
0.13
\end{tabular} & \begin{tabular}{|c|}
$7.53 \pm$ \\
0.11
\end{tabular} & $\begin{array}{l}7.71 \pm \\
0.10\end{array}$ & $\begin{array}{c}6.58 \pm \\
0.16\end{array}$ & $\begin{array}{c}1.00 \pm \\
0.00\end{array}$ & $X$ & $X$ \\
\hline $36 \mathrm{Mbps}$ & $\begin{array}{c}8.54 \pm \\
0.20\end{array}$ & $\begin{array}{c}7.64 \pm \\
0.18\end{array}$ & $\begin{array}{c}9.54 \pm \\
0.20\end{array}$ & $\begin{array}{c}9.06 \pm \\
0.19\end{array}$ & $\begin{array}{c}11.01 \pm \\
0.21\end{array}$ & $\begin{array}{c}10.00 \pm \\
0.17\end{array}$ & $\begin{array}{c}9.75 \pm \\
0.14\end{array}$ & $\begin{array}{l}7.97 \pm \\
0.175\end{array}$ & $\begin{array}{c}1.00 \pm \\
0.00\end{array}$ & $X$ & $X$ \\
\hline 48 Mbps & $\begin{array}{c}8.62 \pm \\
0.18\end{array}$ & $\begin{array}{c}7.49 \pm \\
0.15\end{array}$ & $\begin{array}{c}9.35 \pm \\
0.12\end{array}$ & $\begin{array}{c}9.02 \pm \\
0.17\end{array}$ & $\begin{array}{c}10.39 \pm \\
0.20\end{array}$ & $\begin{array}{c}7.74 \pm \\
0.16\end{array}$ & \begin{tabular}{|c|}
$6.81 \pm$ \\
0.71
\end{tabular} & $\begin{array}{c}15.23 \pm \\
2.31\end{array}$ & $\begin{array}{c}1.00 \pm \\
0.00\end{array}$ & $X$ & $X$ \\
\hline $54 \mathrm{Mbps}$ & $\begin{array}{c}8.93 \pm \\
0.21\end{array}$ & $\begin{array}{c}8.25 \pm \\
1.20\end{array}$ & $\begin{array}{c}10.03 \pm \\
0.62\end{array}$ & $\begin{array}{c}9.31 \pm \\
0.30\end{array}$ & $\begin{array}{c}9.67 \pm \\
1.86\end{array}$ & \begin{tabular}{|c}
$7.00 \pm$ \\
0.13
\end{tabular} & $\begin{array}{c}8.06 \pm \\
0.20\end{array}$ & $\begin{array}{c}7.98 \pm \\
0.17\end{array}$ & $\begin{array}{c}1.00 \pm \\
0.00\end{array}$ & X & X \\
\hline
\end{tabular}

The best combination of parameters at $18{ }^{\circ} \mathrm{C}$ is to work at $2,437 \mathrm{MHz}$, with $18 \mathrm{Mbps}$ data transfer rate (which is used OFDM modulation).

\subsubsection{Measurements for $20{ }^{\circ} \mathrm{C}$}

We were able to establish an underwater link for frequencies between $2,457 \mathrm{MHz}$ and $2,462 \mathrm{MHz}$ at $20{ }^{\circ} \mathrm{C}$. Figure 6.5 shows the maximum distances for each data transfer rate as a function of the working frequency at $20^{\circ} \mathrm{C}$. 


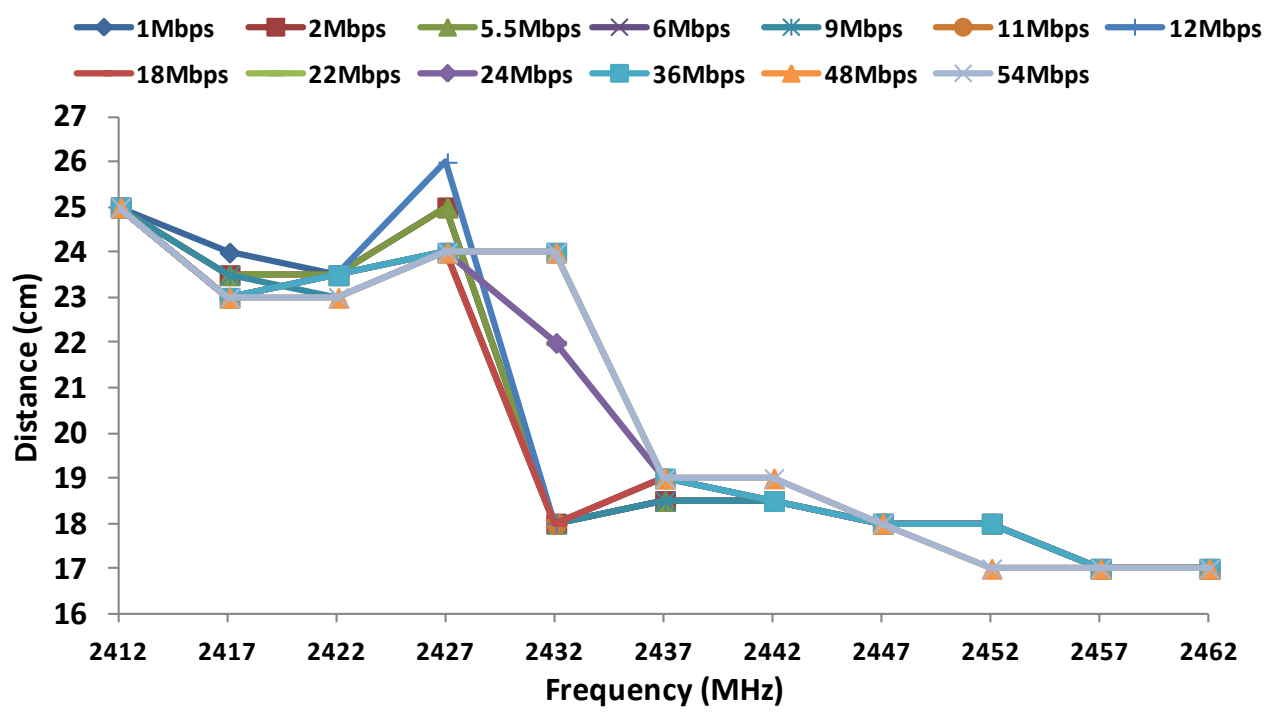

Figure 6.5. Maximum distances for data transfer rates at $20^{\circ} \mathrm{C}$.

As we can see, all data transfer rates have two frequencies which have greater distances between antennas. For 2,412 MHz, all data transfer rates reach a distance of $25 \mathrm{~cm}$. The following frequency to highlight is $2,427 \mathrm{MHz}$ where the data transfer rate of $12 \mathrm{Mbps}$ reaches a maximum distance of $26 \mathrm{~cm}$. For the same frequency, data rates of $1 \mathrm{Mbps}, 2 \mathrm{Mbps}$ and $5.5 \mathrm{Mbps}$ reached distances of 25 $\mathrm{cm}$. The other data transfer rates are kept below $24 \mathrm{~cm}$. We can also note that $36 \mathrm{Mbps}, 48 \mathrm{Mbps}$ and $54 \mathrm{Mbps}$ present their maximum distances at the frequency of $2,432 \mathrm{MHz}(24 \mathrm{~cm})$, while the remaining data transfer rates (except for $12 \mathrm{Mbps}$ ) offer very low distances $(18 \mathrm{~cm})$. Table 6.9 shows a summary of the statistical results for measurements at $20{ }^{\circ} \mathrm{C}$. In this case, the parameters to be analyzed are:

- Description of groups to be compared: Each Frequency and each data transfer rate at $20^{\circ} \mathrm{C}$

- Variable to be studied: maximum distance between antennae at $20^{\circ} \mathrm{C}$.

As Table 6.9 shows, the average value of each group (columns) is numerically distinct. We can focus our analysis on the dependence of maximum distance with working frequency and data transfer rate. Average values of rows seem to be very similar. This leads us to think that the variation in data transfer rates for a same frequency is not significant.

Table 6.10 shows the analysis of variance measurements at $20{ }^{\circ} \mathrm{C}$.

For the same reasons explained in Section 6.2.2.1, we can conclude that the maximum distance depends significantly of the working frequency and the influence of data transfer rates is not significant.

Table 6.11 shows the RTT values in ms. for the maximum distances for all data transfer rates as a function of working frequency at $20{ }^{\circ} \mathrm{C}$. Numbers in red indicate the best RTT values in ms., for each data transfer rate. On the one hand we can see that $12 \mathrm{Mbps}$ has a RTT value of $26.21 \mathrm{~ms}$ for distances of $26 \mathrm{~cm}$. Moreover, we see that data rates of $1 \mathrm{Mbps}, 2 \mathrm{Mbps}$ and $5.5 \mathrm{Mbps}$ record RTT values below $9 \mathrm{~ms}$ for 2,412 MHz, while for 2,427 MHz, their values are above $22 \mathrm{~ms}$. Both frequencies reach the same distances. 
Table 6.9. Summary of statistical results for measurements at $20^{\circ} \mathrm{C}$.

\begin{tabular}{|c|c|c|c|c|}
\hline Summary & $N^{0}$ of data & Sum & Average & Variance \\
\hline Row 1 & 11 & 222.5 & 20.2272727 & 11.2181818 \\
\hline Row 2 & 11 & 222 & 20.1818182 & 10.8636364 \\
\hline Row 3 & 11 & 222 & 20.1818182 & 10.8636364 \\
\hline Row 4 & 11 & 220.5 & 20.0454545 & 9.67272727 \\
\hline Row 5 & 11 & 220.5 & 20.0454545 & 9.67272727 \\
\hline Row 6 & 11 & 221 & 20.0909091 & 9.54090909 \\
\hline Row 7 & 11 & 223 & 20.2727273 & 11.4681818 \\
\hline Row 8 & 11 & 221 & 20.0909091 & 9.54090909 \\
\hline Row 9 & 11 & 225 & 20.4545455 & 9.32272727 \\
\hline Row 10 & 11 & 225 & 20.4545455 & 9.32272727 \\
\hline Row 11 & 11 & 227 & 20.6363636 & 10.3045455 \\
\hline Row 12 & 11 & 226 & 20.5454545 & 10.4727273 \\
\hline Row 13 & 11 & 226 & 20.5454545 & 10.4727273 \\
\hline Column 1 & 13 & 325 & 25 & 0 \\
\hline Column 2 & 13 & 301.5 & 23.1923077 & 0.10576923 \\
\hline Column3 & 13 & 304 & 23.3846154 & 0.04807692 \\
\hline Column 4 & 13 & 317 & 24.3846154 & 0.42307692 \\
\hline Column5 & 13 & 260 & 20 & 7.33333333 \\
\hline Column6 & 13 & 244.5 & 18.8076923 & 0.06410256 \\
\hline Column 7 & 13 & 241.5 & 18.5769231 & 0.03525641 \\
\hline Column 8 & 13 & 234 & 18 & 0 \\
\hline Column9 & 13 & 232 & 17.8461538 & 0.14102564 \\
\hline Column10 & 13 & 221 & 17 & 0 \\
\hline Column11 & 13 & 221 & 17 & 0 \\
\hline
\end{tabular}

Table 6.10. ANOVA for measurements at $20{ }^{\circ} \mathrm{C}$

\begin{tabular}{|c|c|c|c|c|c|c|}
\hline $\begin{array}{c}\text { Origin of } \\
\text { variations }\end{array}$ & $\begin{array}{c}\text { Sum of } \\
\text { squares }\end{array}$ & $\begin{array}{c}\text { Degree of } \\
\text { Freedom }\end{array}$ & $\begin{array}{c}\text { Average } \\
\text { Squares }\end{array}$ & F & Probability & $\begin{array}{c}\text { Critical } \\
\text { Value for F }\end{array}$ \\
\hline Rows & 5.84265734 & 12 & 0.48688811 & 0.6353129 & 0.808496131 & 1.83369528 \\
\hline Columns & 1235.3986 & 10 & 123.53986 & 161.200213 & $1.31338 \mathrm{E}-64$ & 1.91046106 \\
\hline Error & 91.965035 & 120 & 0.76637529 & & & \\
\hline & & & & & & \\
\hline Total & 1333.20629 & 142 & & & & \\
\hline
\end{tabular}


Table 6.11. Values of RTT in $\mathrm{ms}$ for $20^{\circ} \mathrm{C}$

\begin{tabular}{|c|c|c|c|c|c|c|c|c|c|c|c|}
\hline \multirow[b]{2}{*}{$\begin{array}{c}\text { Data } \\
\text { Transfer } \\
\text { Rates }\end{array}$} & \multicolumn{11}{|c|}{ Frequency (MHz) } \\
\hline & 2412 & 2417 & 2422 & 2427 & 2432 & 2437 & 2442 & 2447 & 2452 & 2457 & 2462 \\
\hline 1 Mbps & $\begin{array}{c}4.77 \\
\pm 0.37 \\
\end{array}$ & $\begin{array}{c}5.90 \pm \\
0.35 \\
\end{array}$ & $\begin{array}{c}3.05 \pm \\
0.02 \\
\end{array}$ & $\begin{array}{l}22.87 \\
\pm 2.52 \\
\end{array}$ & $\begin{array}{c}3.99 \\
\pm 0.34 \\
\end{array}$ & $\begin{array}{c}5.58 \\
\pm 0.56 \\
\end{array}$ & $\begin{array}{l}3.47 \\
\pm .07 \\
\end{array}$ & $\begin{array}{r}28.39 \\
\pm 3.01 \\
\end{array}$ & $\begin{array}{c}.00 \\
\pm 0.97 \\
\end{array}$ & $\begin{array}{c}3.00 \\
\pm 0.02 \\
\end{array}$ & $\begin{array}{c}3.12 \\
\pm 0.04 \\
\end{array}$ \\
\hline 2 Mbps & $\begin{array}{c}8.80 \\
\pm 0.54 \\
\end{array}$ & $\begin{array}{c}4.05 \pm \\
0.11 \\
\end{array}$ & $\begin{array}{l}55.38 \\
\pm 9.69 \\
\end{array}$ & $\begin{array}{l}30.41 \\
\pm 4.64 \\
\end{array}$ & $\begin{array}{c}6.45 \\
\pm 0.41 \\
\end{array}$ & $\begin{array}{r}4.60 \\
\pm 0.19 \\
\end{array}$ & $\begin{array}{c}5.57 \\
\pm 0.10 \\
\end{array}$ & $\begin{array}{l}20.73 \\
\pm 1.78 \\
\end{array}$ & $\begin{array}{c}3.73 \\
\pm 0.09 \\
\end{array}$ & $\begin{array}{c}2.08 \\
\pm 0.03 \\
\end{array}$ & $\begin{array}{c}2.28 \\
\pm 0.08 \\
\end{array}$ \\
\hline 5.5 Mbps & $\begin{array}{c}3.71 \\
\pm 1.43 \\
\end{array}$ & $\begin{array}{c}5.70 \pm \\
0.16 \\
\end{array}$ & $\begin{array}{r}17.08 \\
\pm 1.52 \\
\end{array}$ & $\begin{array}{c}66.61 \\
\pm 20.26 \\
\end{array}$ & $\begin{array}{r}9.10 \\
\pm 0.38 \\
\end{array}$ & $\begin{array}{c}5.53 \\
\pm 0.22 \\
\end{array}$ & $\begin{array}{c}8.57 \\
\pm 0.18 \\
\end{array}$ & $\begin{array}{l}20.56 \\
\pm 4.65 \\
\end{array}$ & $\begin{array}{c}6.22 \\
\pm 0.78 \\
\end{array}$ & $\begin{array}{c}1.77 \\
\pm 0.06 \\
\end{array}$ & $\begin{array}{r}2.09 \\
\pm 0.13 \\
\end{array}$ \\
\hline 6 Mbps & $\begin{array}{c}3.17 \\
\pm 0.06 \\
\end{array}$ & $\begin{array}{c}9.10 \pm \\
0.13\end{array}$ & $\begin{array}{l}20.10 \\
\pm 1.44 \\
\end{array}$ & $\begin{array}{l}17.65 \\
\pm 3.32 \\
\end{array}$ & $\begin{array}{c}9.39 \\
\pm 0.13 \\
\end{array}$ & $\begin{array}{c}8.16 \\
\pm 0.12 \\
\end{array}$ & $\begin{array}{c}8.71 \\
\pm 0.09 \\
\end{array}$ & $\begin{array}{l}21.88 \\
\pm 4.31 \\
\end{array}$ & $\begin{array}{c}6.19 \\
\pm 0.13 \\
\end{array}$ & $\begin{array}{c}1.07 \\
\pm 0.03 \\
\end{array}$ & $\begin{array}{r}1.44 \\
\pm 0.09 \\
\end{array}$ \\
\hline 9 Mbps & $\begin{array}{c}4.01 \\
\pm 0.06 \\
\end{array}$ & $\begin{array}{r}10.05 \\
\pm 0.13 \\
\end{array}$ & $\begin{array}{r}18.91 \\
\pm 1.14 \\
\end{array}$ & $\begin{array}{r}42.31 \\
\pm 30.78 \\
\end{array}$ & $\begin{array}{l}10.16 \\
\pm 0.17 \\
\end{array}$ & $\begin{array}{r}8.36 \\
\pm 0.15 \\
\end{array}$ & $\begin{array}{c}9.51 \\
\pm 0.10 \\
\end{array}$ & $\begin{array}{l}17.27 \\
\pm 1.09 \\
\end{array}$ & $\begin{array}{c}7.07 \\
\pm 0.15 \\
\end{array}$ & $\begin{array}{r}1.86 \\
\pm 0.08 \\
\end{array}$ & $\begin{array}{c}1.21 \\
\pm 0.06 \\
\end{array}$ \\
\hline 11 Mbps & $\begin{array}{c}3.47 \pm \\
0.07\end{array}$ & $\begin{array}{r}15.00 \\
\pm 0.61 \\
\end{array}$ & $\begin{array}{l}22.44 \\
\pm 1.65 \\
\end{array}$ & $\begin{array}{r}27.00 \\
\pm 30.87 \\
\end{array}$ & $\begin{array}{r}14.00 \\
\pm 0.49 \\
\end{array}$ & $\begin{array}{c}6.77 \\
\pm 0.17 \\
\end{array}$ & $\begin{array}{r}12.23 \\
\pm 0.22 \\
\end{array}$ & $\begin{array}{l}20.77 \\
\pm 1.87 \\
\end{array}$ & $\begin{array}{c}8.23 \\
\pm 0.30 \\
\end{array}$ & $\begin{array}{c}1.84 \\
\pm 0.04 \\
\end{array}$ & $\begin{array}{c}2.03 \\
\pm 0.08 \\
\end{array}$ \\
\hline 12 Mbps & $\begin{array}{c}5.35 \pm \\
0.08 \\
\end{array}$ & $\begin{array}{r}11.37 \\
\pm 0.11 \\
\end{array}$ & $\begin{array}{l}16.02 \\
\pm 0.80\end{array}$ & $\begin{array}{l}26.21 \\
\pm 3.64\end{array}$ & $\begin{array}{r}14.68 \\
\pm 2.47 \\
\end{array}$ & $\begin{array}{c}8.49 \\
\pm 0.16\end{array}$ & $\begin{array}{l}12.23 \\
\pm 0.32\end{array}$ & $\begin{array}{l}18.57 \\
\pm 1.09 \\
\end{array}$ & $\begin{array}{c}4.32 \\
\pm 0.10\end{array}$ & $\begin{array}{c}1.14 \\
\pm 0.06\end{array}$ & $\begin{array}{c}1.10 \\
\pm 0.05\end{array}$ \\
\hline 18 Mbps & $\begin{array}{c}4.10 \pm \\
0.14 \\
\end{array}$ & $\begin{array}{c}5.94 \pm \\
0.11 \\
\end{array}$ & $\begin{array}{r}3.76 \\
\pm 0.14 \\
\end{array}$ & $\begin{array}{c}5.85 \\
\pm 0.16 \\
\end{array}$ & $\begin{array}{r}7.26 \\
\pm 0.14 \\
\end{array}$ & $\begin{array}{r}4.58 \\
\pm 0.08 \\
\end{array}$ & $\begin{array}{r}5.74 \\
\pm 0.08 \\
\end{array}$ & $\begin{array}{r}9.75 \\
\pm 5.5 \\
\end{array}$ & $\begin{array}{c}4.32 \\
\pm 0.10 \\
\end{array}$ & $\begin{array}{r}1.05 \\
\pm 0.01 \\
\end{array}$ & $\begin{array}{r}1.30 \\
\pm 0.09 \\
\end{array}$ \\
\hline 22 Mbps & $\begin{array}{c}3.46 \pm \\
0.07\end{array}$ & $\begin{array}{l}15.35 \\
\pm 0.61 \\
\end{array}$ & $\begin{array}{c}4.98 \\
\pm 0.13 \\
\end{array}$ & $\begin{array}{l}18.22 \\
\pm 0.92\end{array}$ & $\begin{array}{l}33.85 \\
\pm 0.84 \\
\end{array}$ & $\begin{array}{c}8.08 \\
\pm 0.28\end{array}$ & $\begin{array}{c}9.94 \\
\pm 0.26\end{array}$ & $\begin{array}{c}4.94 \\
\pm 0.16 \\
\end{array}$ & $\begin{array}{c}7.74 \\
\pm 0.30 \\
\end{array}$ & $\begin{array}{c}1.99 \\
\pm 0.08 \\
\end{array}$ & $\begin{array}{c}1.96 \\
\pm 0.10 \\
\end{array}$ \\
\hline 24 Mbps & $\begin{array}{c}5.03 \pm \\
0.14 \\
\end{array}$ & $\begin{array}{c}7.38 \pm \\
0.14 \\
\end{array}$ & $\begin{array}{r}4.68 \\
\pm 0.17 \\
\end{array}$ & $\begin{array}{r}33.72 \\
\pm 7.82 \\
\end{array}$ & $\begin{array}{c}4.71 \\
\pm 0.11 \\
\end{array}$ & $\begin{array}{r}5.76 \\
\pm 0.11 \\
\end{array}$ & $\begin{array}{r}6.50 \\
\pm 0.11 \\
\end{array}$ & $\begin{array}{c}5.06 \\
\pm 0.15 \\
\end{array}$ & $\begin{array}{c}5.29 \\
\pm 0.13 \\
\end{array}$ & $\begin{array}{c}1.22 \\
\pm 0.07 \\
\end{array}$ & $\begin{array}{r}1.26 \\
\pm 0.06 \\
\end{array}$ \\
\hline 36 Mbps & $\begin{array}{c}6.25 \pm \\
0.21 \\
\end{array}$ & $\begin{array}{c}9.36 \pm \\
0.24 \\
\end{array}$ & $\begin{array}{r}5.45 \\
\pm 0.12 \\
\end{array}$ & $\begin{array}{l}10.66 \\
\pm 0.26 \\
\end{array}$ & $\begin{array}{r}11.34 \\
\pm 0.49 \\
\end{array}$ & $\begin{array}{r}8.00 \\
\pm 0.15 \\
\end{array}$ & $\begin{array}{c}8.77 \\
\pm 0.17 \\
\end{array}$ & $\begin{array}{c}6.04 \\
\pm 0.14 \\
\end{array}$ & $\begin{array}{r}6.39 \\
\pm 0.17 \\
\end{array}$ & $\begin{array}{r}1.60 \\
\pm 0.11 \\
\end{array}$ & $\begin{array}{r}1.47 \\
\pm 0.07 \\
\end{array}$ \\
\hline 48 Mbps & $\begin{array}{c}6.03 \pm \\
0.17 \\
\end{array}$ & $\begin{array}{c}9.86 \pm \\
0.34 \\
\end{array}$ & $\begin{array}{c}6.07 \\
\pm 0.28 \\
\end{array}$ & $\begin{array}{c}9.66 \\
\pm 0.24 \\
\end{array}$ & $\begin{array}{c}7.74 \\
\pm 0.20 \\
\end{array}$ & $\begin{array}{c}8.57 \\
\pm 0.18 \\
\end{array}$ & $\begin{array}{r}10.37 \\
\pm 0.26 \\
\end{array}$ & $\begin{array}{c}6.21 \\
\pm 0.17 \\
\end{array}$ & $\begin{array}{c}6.15 \\
\pm 0.18 \\
\end{array}$ & $\begin{array}{r}2.56 \\
\pm 0.11 \\
\end{array}$ & $\begin{array}{c}2.34 \\
\pm 0.06 \\
\end{array}$ \\
\hline 54 Mbps & $\begin{array}{c}7.32 \pm \\
0.22 \\
\end{array}$ & $\begin{array}{c}9.98 \pm \\
1.27 \\
\end{array}$ & $\begin{array}{c}6.90 \\
\pm 0.22 \\
\end{array}$ & $\begin{array}{c}9.58 \pm \\
0.32 \\
\end{array}$ & $\begin{array}{r}7.82 \\
\pm 0.17 \\
\end{array}$ & $\begin{array}{c}8.63 \\
\pm 0.16 \\
\end{array}$ & $\begin{array}{c}9.70 \\
\pm 0.17 \\
\end{array}$ & $\begin{array}{r}7.12 \\
\pm 0.14 \\
\end{array}$ & $\begin{array}{c}6.93 \\
\pm 0.16 \\
\end{array}$ & $\begin{array}{c}2.64 \\
\pm 0.06 \\
\end{array}$ & $\begin{array}{c}2.93 \\
\pm 0.06 \\
\end{array}$ \\
\hline
\end{tabular}

\subsubsection{Measurements for $22{ }^{\circ} \mathrm{C}$}

The highest frequency that allows establishing an underwater wireless link at $22{ }^{\circ} \mathrm{C}$ is 2,452 MHz.

Figure 6.6 shows the maximum distances for all data transfer rates depending on the working frequency.

These measures have been carried out at 22 degrees. Similar to the signals behavior to at $18{ }^{\circ} \mathrm{C}$, we can observe that two frequencies at $22{ }^{\circ} \mathrm{C}$ showed greater distances.

Firstly, we note that at $2,412 \mathrm{MHz}$, all data transfer rates can achieve a distance between antennae of $26 \mathrm{~cm}$. At 2,437 MHz we observed the second longest distance for all data transfer rates, with the exception of $1 \mathrm{Mbps}$ and $48 \mathrm{Mbps}$. The maximum distance between antennae was $25 \mathrm{~cm}$. Table 6.12 shows a summary of the statistical results for measurements at $22{ }^{\circ} \mathrm{C}$. 


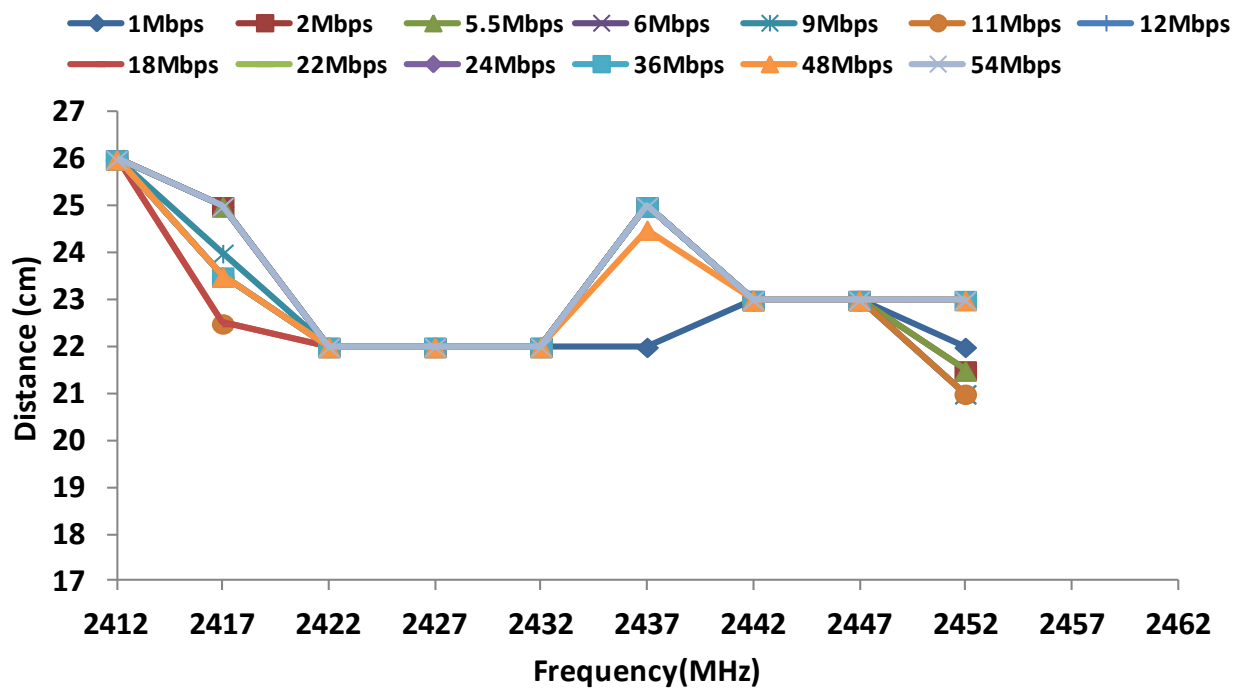

Figure 6.6. Maximum distances for data transfer rates at $22^{\circ} \mathrm{C}$.

Table 6.12. Summary of statistical results for measurements at $22{ }^{\circ} \mathrm{C}$.

\begin{tabular}{|c|c|c|c|c|}
\hline Summary & $N^{0}$ of data & Sum & Average & Variance \\
\hline Row 1 & 9 & 207 & 23 & 2.25 \\
\hline Row 2 & 9 & 209.5 & 23.2777778 & 2.69444444 \\
\hline Row 3 & 9 & 209.5 & 23.2777778 & 2.69444444 \\
\hline Row 4 & 9 & 209 & 23.2222222 & 2.94444444 \\
\hline Row 5 & 9 & 208 & 23.1111111 & 2.61111111 \\
\hline Row 6 & 9 & 206.5 & 22.9444444 & 2.52777778 \\
\hline Row 7 & 9 & 209.5 & 23.2777778 & 1.94444444 \\
\hline Row 8 & 9 & 208.5 & 23.1666667 & 2 \\
\hline Row 9 & 9 & 209.5 & 23.2777778 & 1.94444444 \\
\hline Row 10 & 9 & 209.5 & 23.2777778 & 1.94444444 \\
\hline Row 11 & 9 & 209.5 & 23.2777778 & 1.94444444 \\
\hline Column 1 & 13 & 338 & 26 & 0 \\
\hline Column2 & 13 & 311.5 & 23.9615385 & 0.89423077 \\
\hline Column3 & 13 & 286 & 22 & 0 \\
\hline Column4 & 13 & 286 & 22 & 0 \\
\hline Column5 & 13 & 286 & 22 & 0 \\
\hline Column6 & 13 & 321.5 & 24.7307692 & 0.69230769 \\
\hline Column 7 & 13 & 299 & 23 & 0 \\
\hline Column8 & 13 & 299 & 23 & 0 \\
\hline Column9 & 13 & 289 & 22.2307692 & 0.81730769 \\
\hline Column10 & 9 & 207 & 23 & 2.25 \\
\hline Column11 & 9 & 209.5 & 23.2777778 & 2.69444444 \\
\hline
\end{tabular}


- Description of groups to be compared: Each Frequency and each data transfer rate at $22^{\circ} \mathrm{C}$

- Variable to be studied: maximum distance between antennae at $20^{\circ} \mathrm{C}$.

As Table 6.12 shows, the average value of each group (columns) is numerically distinct. We can focus our analysis on the dependence of maximum distance with working frequency and data transfer rate. Average values of rows seem to be very similar. This leads us to think that the variation in data transfer rates for a same frequency is not significant.

Table 6.13 shows the analysis of variance measurements at $22{ }^{\circ} \mathrm{C}$.

Table 6.13. ANOVA for measurements at $22^{\circ} \mathrm{C}$

\begin{tabular}{|c|c|c|c|c|c|c|}
\hline $\begin{array}{c}\text { Origin of } \\
\text { variations }\end{array}$ & $\begin{array}{c}\text { Sum of } \\
\text { squares }\end{array}$ & $\begin{array}{c}\text { Degree of } \\
\text { Freedom }\end{array}$ & $\begin{array}{c}\text { Average } \\
\text { Squares }\end{array}$ & F & Probability & $\begin{array}{c}\text { Critical } \\
\text { Value for F }\end{array}$ \\
\hline Rows & 1.88034188 & 12 & 0.15669516 & 0.55784469 & 0.87039698 & 1.85440887 \\
\hline Columns & 209.311966 & 8 & 26.1639957 & 93.1454834 & $8.2962 \mathrm{E}-42$ & 2.03631902 \\
\hline Error & 26.965812 & 96 & 0.28089387 & & & \\
\hline & & & & & & \\
\hline Total & 238.15812 & 116 & & & & \\
\hline
\end{tabular}

For the same reasons explained in Section 6.2.2.1, we can conclude that the maximum distance depends significantly of the working frequency and the influence of data transfer rates is not significant. Table 6.14 shows the RTT values and their typical errors in ms of the maximum distances for all data transfer rates as a function of working frequency at $22{ }^{\circ} \mathrm{C}$. Numbers in red indicate the best values of RTT in ms., for each data transfer rate. As Figure 5 shows all data transfer rates record their greatest distance in the lowest frequency. However, we observed significant changes in RTT values. Firstly, 1 Mbps and 2 Mbps present the lowest values (about $4.75 \mathrm{~ms}$ ). Furthermore, the data rate of $22 \mathrm{Mbps}$ has a RTT values above $16.50 \mathrm{~ms}$, which is the highest value. At $22^{\circ} \mathrm{C}$, the best combination of parameters is selecting 2,412 $\mathrm{MHz}$ with data transfer rates of $1 \mathrm{Mbps}$ and $2 \mathrm{Mbps}$.

\subsubsection{Second Study: Performance Results at $26^{\circ} \mathrm{C}$}

This section shows the obtained results in the second test. Each subsection represents the maximum distance between the two antennae and the average RTT value for these distances as a function of the working frequency. The results are analyzed for $26^{\circ} \mathrm{C}$. In this case, the modulations used are BPSK at $1 \mathrm{Mbps}$, QPSK at $2 \mathrm{Mbps}, \mathrm{CCK}$ at 5.5 Mbps and $11 \mathrm{Mbps}$.

\subsubsection{Performance of BPSK modulation and analytical study for 1Mbps data transfer rate.}

Figure 6.7 shows the average RTT in milliseconds for 1 Mbps data transfer rate, when the BPSK modulation is used, as a function of the working frequency and the distance between the wireless sensor nodes. We observe that the highest variations occur between $15 \mathrm{~cm}$ and $18 \mathrm{~cm}$. The RTT value for $15 \mathrm{~cm}$ is close to $3 \mathrm{~ms}$, while the communication is lost for $18 \mathrm{~cm}$. The average RTT value for distances between $15 \mathrm{~cm}$ and $18 \mathrm{~cm}$ (at 2,412 MHz, 2,417 MHz, 2,422 MHz, 2,427 MHz and $2,432 \mathrm{MHz}$ ) is relatively small, around $20 \mathrm{~ms}$. But at 2,437 MHz the RTT value for $16 \mathrm{~cm}$ increases 
up to $500 \mathrm{~ms}$, while for $17 \mathrm{~cm}$ there are not registered packets.

Table 6.14. Values of RTT in $\mathrm{ms}$ for $22^{\circ} \mathrm{C}$

\begin{tabular}{|c|c|c|c|c|c|c|c|c|c|c|c|}
\hline \multirow[b]{2}{*}{$\begin{array}{c}\text { Data Transfer } \\
\text { Rates }\end{array}$} & \multicolumn{11}{|c|}{ Frequency (MHz) } \\
\hline & 2412 & 2417 & 2422 & 2427 & 2432 & 2437 & 2442 & 2447 & 2452 & 2457 & 2462 \\
\hline $1 \mathrm{Mbps}$ & $\begin{array}{l}4.89 \\
\pm 0.35 \\
\end{array}$ & $\begin{array}{l}20.12 \\
\pm 4.22 \\
\end{array}$ & $\begin{array}{l}3.01 \\
\pm 0.01 \\
\end{array}$ & $\begin{array}{l}3.21 \\
\pm 0.2 \\
\end{array}$ & $\begin{array}{l}3.03 \\
\pm 0.05 \\
\end{array}$ & $\begin{array}{l}3.13 \\
\pm 0.12 \\
\end{array}$ & $\begin{array}{l}3.03 \\
\pm 0.01 \\
\end{array}$ & $\begin{array}{l}3.05 \\
\pm 0.03 \\
\end{array}$ & $\begin{array}{l}3.83 \\
\pm 0.26 \\
\end{array}$ & $X$ & $\mathrm{X}$ \\
\hline 2 Mbps & $\begin{array}{l}4.73 \\
\pm 0.17 \\
\end{array}$ & $\begin{array}{l}13.66 \\
\pm 2.77 \\
\end{array}$ & $\begin{array}{l}2.17 \\
\pm 0.03\end{array}$ & $\begin{array}{l}2.88 \\
\pm 0.37\end{array}$ & $\begin{array}{l}2.19 \\
\pm 0.03\end{array}$ & $\begin{array}{l}2.19 \\
\pm 0.04\end{array}$ & $\begin{array}{l}3.01 \\
\pm 0.24\end{array}$ & $\begin{array}{l}9.73 \\
\pm 0.33\end{array}$ & $\begin{array}{l}12.00 \\
\pm 4.06\end{array}$ & $X$ & $\mathrm{X}$ \\
\hline 5.5 Mbps & $\begin{array}{l}6.86 \\
\pm 0.31\end{array}$ & $\begin{array}{l}18.35 \\
\pm 2.99\end{array}$ & $\begin{array}{l}1.17 \\
\pm 0.04\end{array}$ & $\begin{array}{l}1.82 \\
\pm 0.10\end{array}$ & $\begin{array}{l}1.93 \\
\pm 0.08\end{array}$ & $\begin{array}{l}1.10 \\
\pm 0.03\end{array}$ & $\begin{array}{l}4.22 \\
\pm 0.10\end{array}$ & $\begin{array}{l}5.55 \\
\pm 0.21\end{array}$ & $\begin{array}{l}8.82 \\
\pm 2.20\end{array}$ & $X$ & $X$ \\
\hline 6 Mbps & $\begin{array}{l}8.00 \\
\pm 0.43\end{array}$ & $\begin{array}{l}34.58 \\
\pm 6.26\end{array}$ & $\begin{array}{l}5.79 \\
\pm 0.41\end{array}$ & $\begin{array}{l}42.63 \\
\pm 0.83\end{array}$ & $\begin{array}{l}8.53 \\
\pm 1.21\end{array}$ & $\begin{array}{l}7.24 \\
\pm 0.11\end{array}$ & $\begin{array}{l}6.26 \\
\pm 0.14\end{array}$ & $\begin{array}{l}7.41 \\
\pm 0.12\end{array}$ & $\begin{array}{l}4.92 \\
\pm 0.31\end{array}$ & $X$ & $\mathrm{X}$ \\
\hline 9 Mbps & $\begin{array}{l}9.31 \\
\pm 0.40\end{array}$ & $\begin{array}{l}20.11 \\
\pm 1.90 \\
\end{array}$ & $\begin{array}{l}6.29 \\
\pm 0.31\end{array}$ & $\begin{array}{l}6.84 \\
\pm 0.34\end{array}$ & $\begin{array}{l}10.19 \\
\pm 1.01\end{array}$ & $\begin{array}{l}7.89 \\
\pm 0.15\end{array}$ & $\begin{array}{l}7.04 \\
\pm 0.14\end{array}$ & $\begin{array}{l}7.41 \\
\pm 0.12\end{array}$ & $\begin{array}{l}7.96 \\
\pm 0.40\end{array}$ & $X$ & $X$ \\
\hline 11 Mbps & $\begin{array}{r}14.72 \\
\pm 0.83 \\
\end{array}$ & $\begin{array}{l}7.87 \\
\pm 0.35 \\
\end{array}$ & $\begin{array}{l}3.33 \\
\pm 0.08\end{array}$ & $\begin{array}{l}4.93 \\
\pm 0.34 \\
\end{array}$ & $\begin{array}{l}4.29 \\
\pm 0.10 \\
\end{array}$ & $\begin{array}{l}2.47 \\
\pm 0.05\end{array}$ & $\begin{array}{l}7.83 \\
\pm 0.50 \\
\end{array}$ & $\begin{array}{l}9.84 \\
\pm 0.91 \\
\end{array}$ & $\begin{array}{l}14.76 \pm \\
2.64\end{array}$ & $X$ & $X$ \\
\hline 12 Mbps & $\begin{array}{r}11.75 \\
\pm 0.30 \\
\end{array}$ & $\begin{array}{l}13.15 \\
\pm 0.72 \\
\end{array}$ & $\begin{array}{l}9.17 \\
\pm 0.21 \\
\end{array}$ & $\begin{array}{l}7.99 \\
\pm 0.35 \\
\end{array}$ & $\begin{array}{l}14.42 \\
\pm 1.27 \\
\end{array}$ & $\begin{array}{l}9.51 \\
\pm 0.18 \\
\end{array}$ & $\begin{array}{l}8.38 \\
\pm 0.16 \\
\end{array}$ & $\begin{array}{l}10.68 \\
\pm 0.39 \\
\end{array}$ & $\begin{array}{l}4.21 \\
\pm 0.14 \\
\end{array}$ & $X$ & $\mathrm{X}$ \\
\hline 18 Mbps & $\begin{array}{l}10.38 \\
\pm 2.16 \\
\end{array}$ & $\begin{array}{l}3.48 \\
\pm 0.07\end{array}$ & $\begin{array}{l}5.53 \\
\pm 0.52\end{array}$ & $\begin{array}{l}4.24 \\
\pm 0.12\end{array}$ & $\begin{array}{l}3.97 \\
\pm 0.35\end{array}$ & $\begin{array}{l}9.53 \\
\pm 0.11\end{array}$ & $\begin{array}{l}4.94 \\
\pm 0.10\end{array}$ & $\begin{array}{l}5.96 \\
\pm 0.09\end{array}$ & $\begin{array}{l}3.85 \\
\pm 0.27\end{array}$ & $\mathrm{X}$ & $X$ \\
\hline 22 Mbps & $\begin{array}{l}16.51 \\
\pm 1.60\end{array}$ & $\begin{array}{l}10.93 \\
\pm 0.83\end{array}$ & $\begin{array}{l}8.77 \\
\pm 0.57\end{array}$ & $\begin{array}{l}6.84 \\
\pm 0.32\end{array}$ & $\begin{array}{l}4.59 \\
\pm 0.13\end{array}$ & $\begin{array}{l}6.93 \\
\pm 0.22\end{array}$ & $\begin{array}{l}6.49 \\
\pm 0.23\end{array}$ & $\begin{array}{l}8.37 \\
\pm 0.20\end{array}$ & $\begin{array}{l}5.46 \\
\pm 0.15\end{array}$ & $\mathrm{X}$ & $\mathrm{X}$ \\
\hline 24 Mbps & $\begin{array}{l}8.22 \\
\pm 0.28 \\
\end{array}$ & $\begin{array}{l}4.21 \\
\pm 0.08 \\
\end{array}$ & $\begin{array}{l}6.88 \\
\pm 0.33 \\
\end{array}$ & $\begin{array}{l}5.53 \\
\pm 0.18 \\
\end{array}$ & $\begin{array}{l}4.57 \\
\pm 0.13 \\
\end{array}$ & $\begin{array}{l}5.99 \\
\pm 0.12 \\
\end{array}$ & $\begin{array}{l}5.75 \\
\pm 0.10 \\
\end{array}$ & $\begin{array}{l}7.55 \\
\pm 0.29 \\
\end{array}$ & $\begin{array}{l}6.17 \\
\pm 0.15 \\
\end{array}$ & $X$ & $\mathrm{X}$ \\
\hline 36 Mbps & $\begin{array}{r}11.03 \\
\pm 0.44 \\
\end{array}$ & $\begin{array}{l}6.02 \\
\pm 0.46 \\
\end{array}$ & $\begin{array}{l}10.67 \\
\pm 0.48 \\
\end{array}$ & $\begin{array}{l}7.67 \\
\pm 0.25 \\
\end{array}$ & $\begin{array}{l}4.57 \\
\pm 0.37 \\
\end{array}$ & $\begin{array}{l}6.25 \\
\pm 0.14 \\
\end{array}$ & $\begin{array}{l}7.65 \\
\pm 0.16 \\
\end{array}$ & $\begin{array}{l}8.02 \\
\pm 0.22 \\
\end{array}$ & $\begin{array}{l}7.56 \\
\pm 0.19 \\
\end{array}$ & $X$ & $X$ \\
\hline 48 Mbps & $\begin{array}{l}10.93 \\
\pm 0.36\end{array}$ & $\begin{array}{l}5.34 \\
\pm 0.12\end{array}$ & $\begin{array}{l}7.55 \\
\pm 0.55\end{array}$ & $\begin{array}{l}7.40 \\
\pm 0.24\end{array}$ & $\begin{array}{l}5.66 \\
\pm 025\end{array}$ & $\begin{array}{l}9.65 \\
\pm 0.27\end{array}$ & $\begin{array}{l}7.51 \\
\pm 0.17\end{array}$ & $\begin{array}{l}7.33 \\
\pm 0.19\end{array}$ & $\begin{array}{l}8.17 \\
\pm 0.20\end{array}$ & $\mathrm{X}$ & $\mathrm{X}$ \\
\hline 54 Mbps & $\begin{array}{l}10.93 \\
\pm 0.32\end{array}$ & $\begin{array}{l}6.49 \\
\pm 0.09\end{array}$ & $\begin{array}{l}8.34 \\
\pm 0.34\end{array}$ & $\begin{array}{l}7.79 \\
\pm 0.21\end{array}$ & $\begin{array}{l}7.77 \\
\pm 0.35\end{array}$ & $\begin{array}{r}13.00 \\
\pm 2.73\end{array}$ & $\begin{array}{l}7.55 \\
\pm 0.18\end{array}$ & $\begin{array}{l}8.64 \\
\pm 0.16\end{array}$ & $\begin{array}{l}8.78 \\
\pm 0.40\end{array}$ & $\mathrm{X}$ & $\mathrm{X}$ \\
\hline
\end{tabular}

$\square 0-500 \square 500-1000 \square 1000-1500 \square 1500-2000 \square 2000-2500 \square 2500-3000$

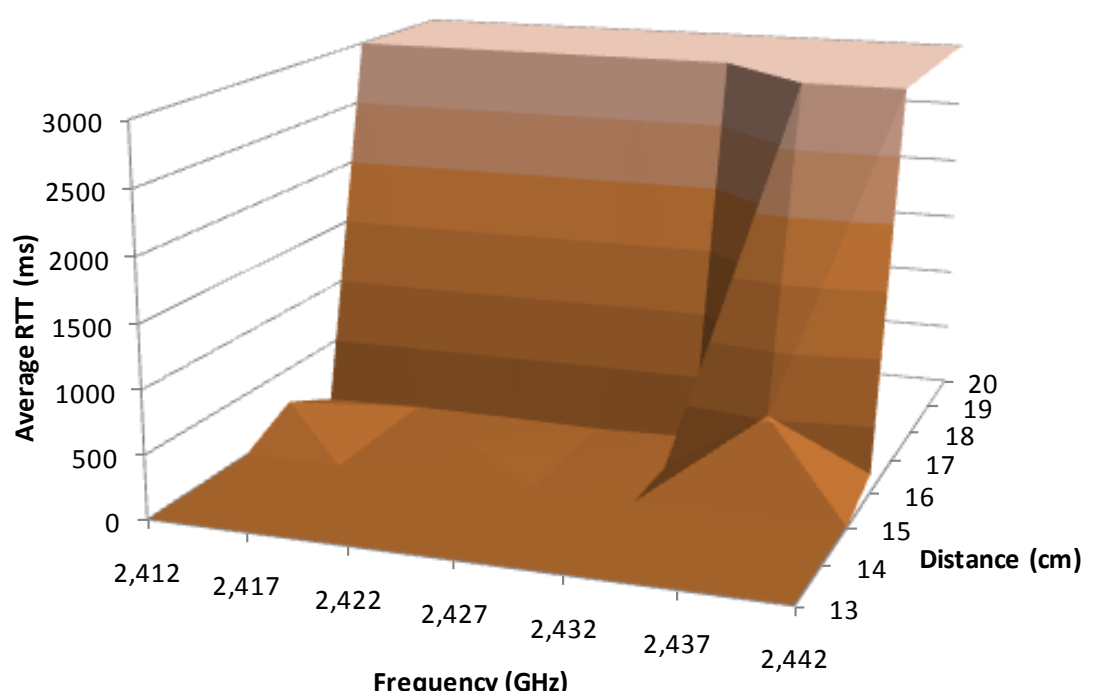

Figure 6.7. Average RTT and maximum distance for $1 \mathrm{Mbps}$ at $26^{\circ} \mathrm{C}$. 
Table 6.15 shows the analysis of variance of $1 \mathrm{Mbps}$ measurements at $26^{\circ} \mathrm{C}$.

Table 6.15. ANOVA for $1 \mathrm{Mbps}$ at $26^{\circ} \mathrm{C}$

\begin{tabular}{|c|c|c|c|c|c|c|}
\hline $\begin{array}{c}\text { Origin of } \\
\text { variations }\end{array}$ & $\begin{array}{c}\text { Sum of } \\
\text { squares }\end{array}$ & $\begin{array}{c}\text { Degree of } \\
\text { Freedom }\end{array}$ & $\begin{array}{c}\text { Average } \\
\text { Squares }\end{array}$ & F & Probability & $\begin{array}{c}\text { Critical } \\
\text { Value for F }\end{array}$ \\
\hline Rows & 106807534 & 7 & 15258219.1 & 58.0980224 & $1.4664 \mathrm{E}-19$ & 2.2370703 \\
\hline Columns & 1924465.18 & 6 & 320744.196 & 1.22128299 & 0.31472057 & 2.3239938 \\
\hline Error & 11030413.4 & 42 & 262628.89 & & & \\
\hline & & & & & & \\
\hline Total & 106807534 & 7 & 15258219.1 & 58.0980224 & $1.4664 \mathrm{E}-19$ & 2.2370703 \\
\hline
\end{tabular}

For the same reasons explained in Section 6.2.2.1, we can conclude that the maximum distance depends significantly of the working frequency and the influence of data transfer rates is not significant.

\subsubsection{Performance of QPSK modulation and analytical study for 2 Mbps data transfer rate}

Figure 6.8 shows the average RTT, in milliseconds, for 2 Mbps data transfer rate, when QPSK modulation is used, as a function of the working frequency work and the distance between wireless sensor nodes. The average RTT values for distances between $15 \mathrm{~cm}$ and $18 \mathrm{~cm}$ are kept below 500 ms for a frequency of $2,432 \mathrm{MHz}$, while at $2,437 \mathrm{MHz}$ the average RTT increases up to 1,000 ms when there is a distance of $16 \mathrm{~cm}$, and up to $3,000 \mathrm{~ms}$ when there is a distance of $17 \mathrm{~cm}$. We observe RTT average values around $3 \mathrm{~ms}$ for distances below $15 \mathrm{~cm}$, and for distances above $18 \mathrm{~cm}$ we obtained $3000 \mathrm{~ms}$. At 17 and $18 \mathrm{~cm}$, we receive very few packets. These present values of RTT near to $3,000 \mathrm{~ms}$

$\square 0-500 \square 500-1000 \square 1000-1500 \square 1500-2000 \square 2000-2500 \square 2500-3000$

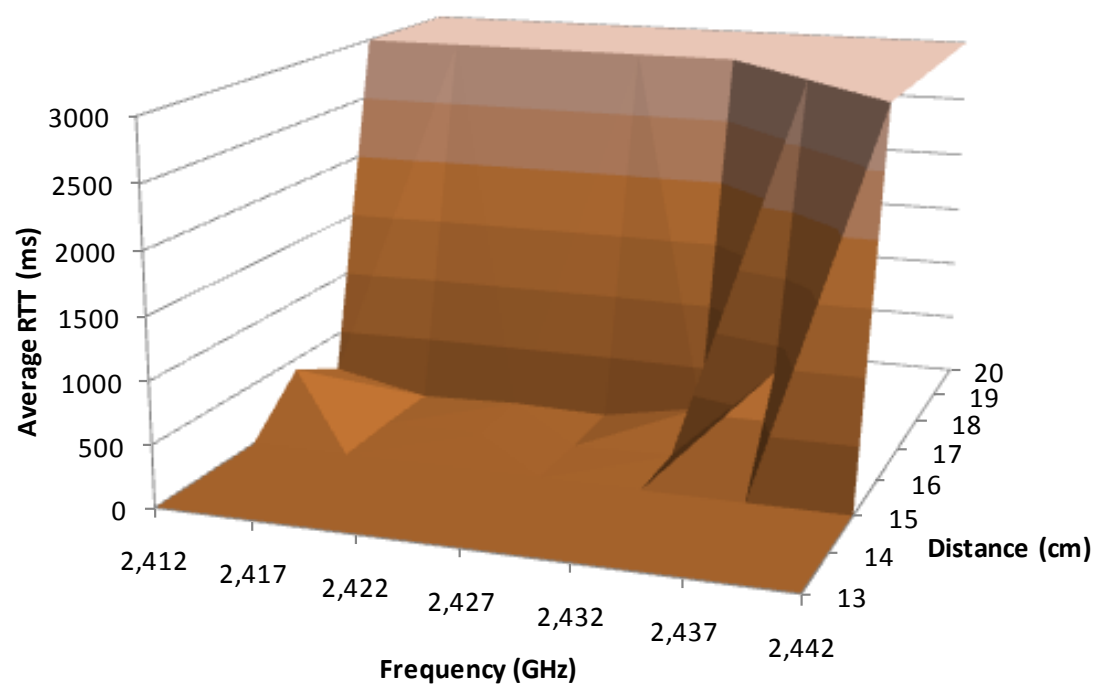

Figure 6.8. Average RTT and maximum distance for $2 \mathrm{Mbps}$ at $26^{\circ} \mathrm{C}$. 
Table 6.16 shows the analysis of variance of $2 \mathrm{Mbps}$ measurements at $26^{\circ} \mathrm{C}$.

Table 6.16. ANOVA for $2 \mathrm{Mbps}$ at $26^{\circ} \mathrm{C}$

\begin{tabular}{|c|c|c|c|c|c|c|}
\hline $\begin{array}{c}\text { Origin of } \\
\text { variations }\end{array}$ & $\begin{array}{c}\text { Sum of } \\
\text { squares }\end{array}$ & $\begin{array}{c}\text { Degree of } \\
\text { Freedom }\end{array}$ & $\begin{array}{c}\text { Average } \\
\text { Squares }\end{array}$ & F & Probability & $\begin{array}{c}\text { Critical } \\
\text { Value for F }\end{array}$ \\
\hline Rows & 100480369 & 7 & 14354338.4 & 40.1704344 & $1.3067 \mathrm{E}-16$ & 2.2370703 \\
\hline Columns & 4300487.5 & 6 & 716747.917 & 2.00580997 & 0.08629679 & 2.3239938 \\
\hline Error & 15008107.9 & 42 & 357335.903 & & & \\
\hline & & & & & & \\
\hline Total & 119788965 & 55 & & & & \\
\hline
\end{tabular}

For the same reasons explained in Section 6.2.2.1, we can conclude that the maximum distance depends significantly of the working frequency and the influence of data transfer rates is not significant.

\subsubsection{Performance of CCK modulation and analytical study for 5.5 Mbps data transfer rate}

Figure 6.9 shows the average RTT in milliseconds for 5.5 Mbps data transfer rate, when CCK modulation is used, as a function of the working frequency and the distance between devices. In this case, the RTT values for distances of $16 \mathrm{~cm}$ and $17 \mathrm{~cm}$ are kept below $500 \mathrm{~ms}$ for $2.432 \mathrm{GHz}$, while at $2.437 \mathrm{GHz}$ the RTT value increases up to $2,000 \mathrm{~ms}$ for $16 \mathrm{~cm}$, and near to $3,000 \mathrm{~ms}$ for $17 \mathrm{~cm}$. The biggest RTT variations are observed for distances between 15 and $18 \mathrm{~cm}$, the measurements obtained beyond this range remain quite stable, between $3 \mathrm{~ms}$ and $4 \mathrm{~ms}$ for distances below $15 \mathrm{~cm}$ and close to $3,000 \mathrm{~ms}$ for distances above $18 \mathrm{~cm}$.

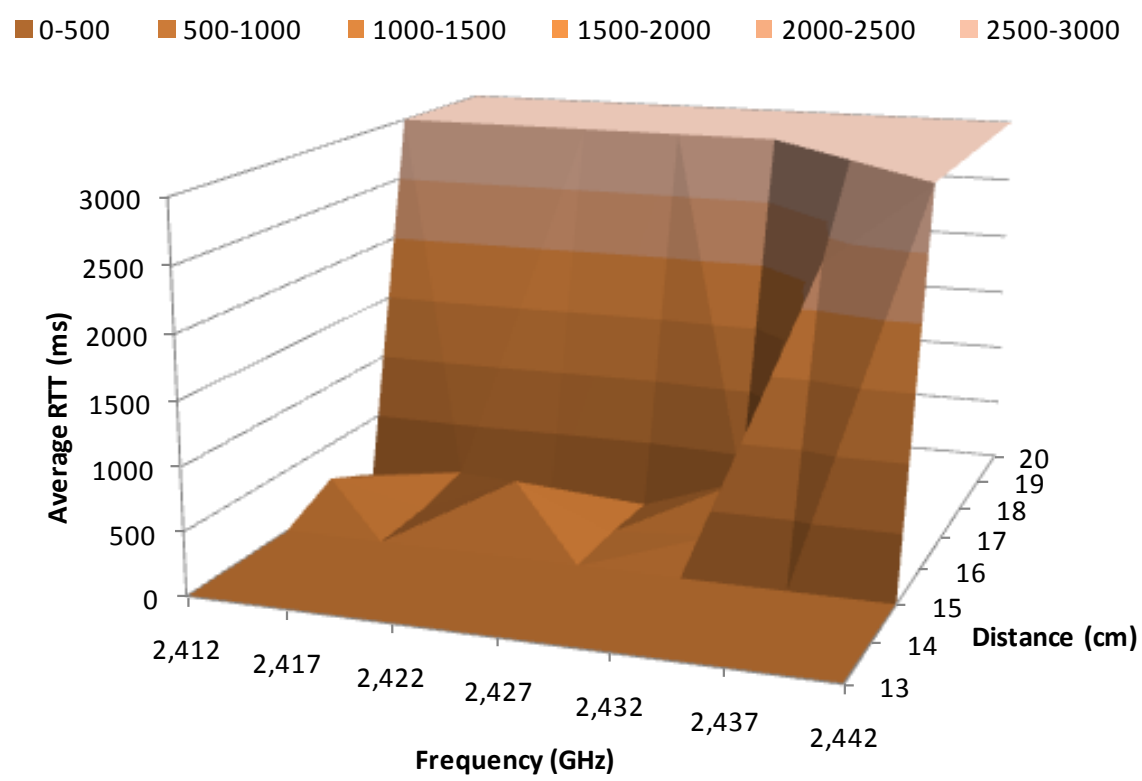

Figure 6.9. Average RTT and maximum distance for $5.5 \mathrm{Mbps}$ at $26^{\circ} \mathrm{C}$. 
Table 6.17 shows the analysis of variance of $5.5 \mathrm{Mbps}$ measurements at $26^{\circ} \mathrm{C}$.

Table 6.17. ANOVA for $5.5 \mathrm{Mbps}$ at $26^{\circ} \mathrm{C}$

\begin{tabular}{|c|c|c|c|c|c|c|}
\hline $\begin{array}{c}\text { Origin of } \\
\text { variations }\end{array}$ & $\begin{array}{c}\text { Sum of } \\
\text { squares }\end{array}$ & $\begin{array}{c}\text { Degree of } \\
\text { Freedom }\end{array}$ & $\begin{array}{c}\text { Average } \\
\text { Squares }\end{array}$ & F & Probability & $\begin{array}{c}\text { Critical } \\
\text { Value for F }\end{array}$ \\
\hline Rows & 112495732 & 7 & 16070818.8 & 48.1755146 & $8.2587 \mathrm{E}-20$ & 2.20323159 \\
\hline Columns & 5345097.71 & 7 & 763585.388 & 2.28900092 & 0.04231778 & 2.20323159 \\
\hline Error & 16345858 & 49 & 333588.939 & & & \\
\hline & & & & & & \\
\hline Total & 134186687 & 63 & & & & \\
\hline
\end{tabular}

For the same reasons explained in Section 6.2.2.1, we can conclude that the maximum distance depends significantly of the working frequency and the influence of data transfer rates is not significant.

\subsubsection{Performance of CCK modulation and analytical study for 11 Mbps data transfer rate}

Figure 6.10 shows the average RTT in milliseconds for $11 \mathrm{Mbps}$ data transfer rate, using CCK modulation, as a function of the working frequency and the distance between devices. The average RTT values obtained for $16 \mathrm{~cm}$ remain stable (between $400 \mathrm{~ms}$ and $600 \mathrm{~ms}$ ) at frequencies below 2,437 MHz, while at $2.442 \mathrm{GHz}$ we did not measure the RTT of any packet. In $17 \mathrm{~cm}$, the average RTT values are very low for $2.412 \mathrm{GHz}, 2.417 \mathrm{GHz}$ and $2.427 \mathrm{GHz}$, but at other frequencies it reached $3000 \mathrm{~ms}$. We obtained the same behavior than in the previous cases for distances below 15 $\mathrm{cm}$ and for distances above $18 \mathrm{~cm}$.

$$
\square 0-500 \square 500-1000 \square 1000-1500 \square 1500-2000 \square 2000-2500 \square 2500-3000
$$

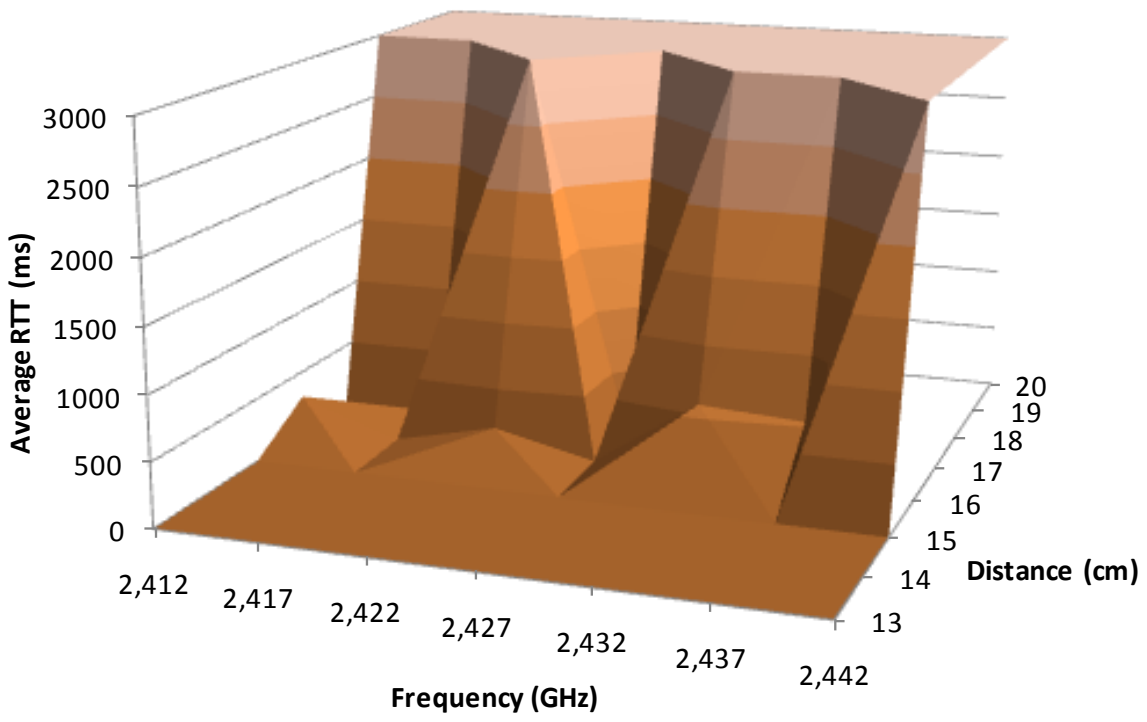

Figure 6.10. Average RTT and maximum distance for $11 \mathrm{Mbps}$ at $26^{\circ} \mathrm{C}$. 
Table 6.18 shows the analysis of variance of $5.5 \mathrm{Mbps}$ measurements at $26^{\circ} \mathrm{C}$.

Table 6.18. ANOVA for $11 \mathrm{Mbps}$ at $26^{\circ} \mathrm{C}$

\begin{tabular}{|c|c|c|c|c|c|c|}
\hline $\begin{array}{c}\text { Origin of } \\
\text { variations }\end{array}$ & $\begin{array}{c}\text { Sum of } \\
\text { squares }\end{array}$ & $\begin{array}{c}\text { Degree of } \\
\text { Freedom }\end{array}$ & $\begin{array}{c}\text { Average } \\
\text { Squares }\end{array}$ & F & Probability & $\begin{array}{c}\text { Critical } \\
\text { Value for F }\end{array}$ \\
\hline Rows & 113595680 & 7 & 16227954.2 & 44.3247445 & $4.7351 \mathrm{E}-19$ & 2.20323159 \\
\hline Columns & 3969516.86 & 7 & 567073.837 & 1.54889536 & 0.17331523 & 2.20323159 \\
\hline Error & 17939635.4 & 49 & 366115.009 & & & \\
\hline & & & & & & \\
\hline Total & 135504832 & 63 & & & & \\
\hline
\end{tabular}

For the same reasons explained in Section 6.2.2.1, we can conclude that the maximum distance depends significantly of the working frequency and the influence of data transfer rates is not significant.

\subsection{Summary of Best Results and Comparison with Other Studies}

Table 6.6 shows a summary of the best results for all tests. We can see that the best results are registered at $22{ }^{\circ} \mathrm{C}$. At this temperature the maximum achieved distance was $26 \mathrm{~cm}$ using $2412 \mathrm{MHz}$ for all data transfer rates. If we analyze the RTT values, we can see that the lowest value is registered for 2Mbps which uses QPSK modulation. Our measurements and tests have been carried out under controlled conditions and have been performed for four different temperatures. Our measurements let us to compare our results with the results obtained by other researchers.

On the one hand, the statement made by A.C. Balanis in [124] said that the absorption coefficient and the speed propagation were independent to the working frequency. Our results show that there is a clear dependency with the frequency. Moreover, at certain frequencies, the maximum distances are greater than others. We can also see that higher frequencies not always generate more signal deterioration and, consequently, shorter communication distances between devices. Throughout all graphics, we can see that using $2412 \mathrm{MHz}$ at $22{ }^{\circ} \mathrm{C}$, we obtain better RTT values and greater distances than the ones obtained for other data transfer rates. We can also observe that, the data transfer rates of $12 \mathrm{Mbps}$ at $20^{\circ} \mathrm{C}$, reaches a distance of $26 \mathrm{~cm}$, but the RTT value is $26.21 \mathrm{~ms}$. This value is six times greater than the RTT value offered by $2 \mathrm{Mbps}$ at $22{ }^{\circ} \mathrm{C}$.

On the other hand, the maximum obtained distance for our tests is $26 \mathrm{~cm}$. If we compare our values with the ones provided by the estimations of $X$. Che et al, we can observe that our results are less optimistic than the approximations estimated in [133].

Moreover, our results show a relationship between the type of modulation and the network performance. There is better performance in some data transfer rates than in others. For example, 6 Mbps and 24 Mbps (in Table 6.19) show RTT values lower than the values of the rest data transfer rates (using the same modulation). 
Table 6.19. Summary of best results

\begin{tabular}{|c|c|c|c|c|c|}
\hline $\begin{array}{c}\text { Data Transfer } \\
\text { Rates }\end{array}$ & $\begin{array}{c}\text { Best frequency } \\
(\mathbf{M H z})\end{array}$ & $\begin{array}{c}\text { Max. distance } \\
(\mathbf{c m})\end{array}$ & $\begin{array}{c}\text { Temperature } \\
\left({ }^{\circ} \mathbf{C}\right)\end{array}$ & RTT (ms) & Modulation \\
\hline $\mathbf{1} \mathbf{M b p s}$ & 2,412 & 26 & 22 & 4.89 & BPSK \\
\hline $\mathbf{2} \mathbf{M b p s}$ & 2,412 & 26 & 22 & 4.73 & QPSK \\
\hline $\mathbf{5 . 5} \mathbf{M b p s}$ & 2,412 & 26 & 22 & 6.86 & CCK \\
\hline $\mathbf{6} \mathbf{M b p s}$ & 2,412 & 26 & 22.00 & OFDM \\
\hline $\mathbf{9} \mathbf{M b p s}$ & 2,412 & 26 & 22 & 9.31 & OFDM \\
\hline $\mathbf{1 1} \mathbf{M b p s}$ & 2,412 & 26 & 22 & 11.75 & CCK \\
\hline $\mathbf{1 2} \mathbf{M b p s}$ & 2,412 & 26 & 22 & 10.38 & OFDM \\
\hline $\mathbf{1 8} \mathbf{M b p s}$ & 2,412 & 26 & 22 & 16.51 & OFDM \\
\hline $\mathbf{2 2} \mathbf{M b p s}$ & 2,412 & 26 & 22 & 8.22 & OFDM \\
\hline $\mathbf{2 4} \mathbf{M b p s}$ & 2,412 & 26 & 22 & 11.03 & OFDM \\
\hline $\mathbf{3 6} \mathbf{M b p s}$ & 2,412 & 26 & 22 & 10.93 & OFDM \\
\hline $\mathbf{4 8} \mathbf{M b p s}$ & 2,412 & 26 & 22 & 10.93 & OFDM \\
\hline $\mathbf{5 4} \mathbf{M b p s}$ & 2,412 & & 26 & 22 & 22 \\
\hline
\end{tabular}

It has been difficult to predict the obtained results because there are not previous references where authors analyze the behavior of EM waves at $2.4 \mathrm{GHz}$ ISM band. Neither, we have not found any work where compare the performance of these modulations considering the environmental temperature. We have only found some works where authors provided some of parameters we have measured. Table 6.20 compares other works with the results obtained in this Thesis. 
Table 6.20. Comparison of best results in several studies.

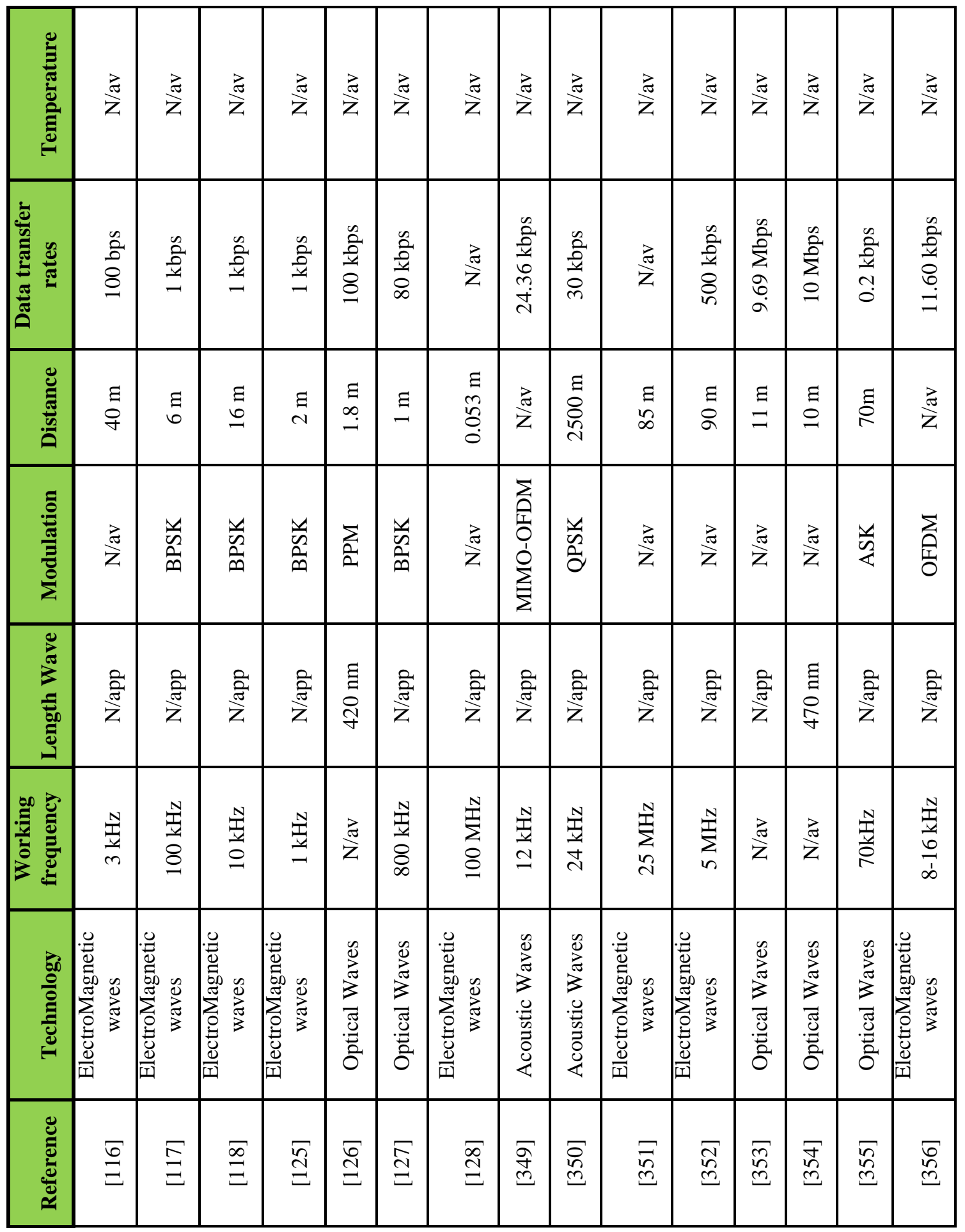

Note: N/app: Not applicable, N/av: Not available 


\begin{tabular}{|c|c|c|c|c|c|c|c|c|c|}
\hline & $\frac{\vec{\pi}}{z}$ & $\stackrel{\sim}{\sim}$ & $\stackrel{\sim}{\sim}$ & $\stackrel{\sim}{\sim}$ & $\stackrel{\sim}{\sim}$ & ป & ปี & ปิ & ป \\
\hline 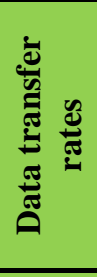 & $\begin{array}{l}\frac{0}{0} \\
\frac{0}{0} \\
0\end{array}$ & $\stackrel{\text { مे }}{\stackrel{0}{\Sigma}}$ & 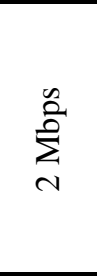 & $\begin{array}{l}\sum_{n}^{n} \\
i n \\
i n\end{array}$ & $\begin{array}{l}\stackrel{\tilde{\partial}}{\sum} \\
=\end{array}$ & 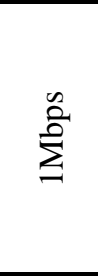 & $\sum_{i}^{\infty}$ & 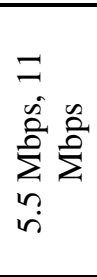 & 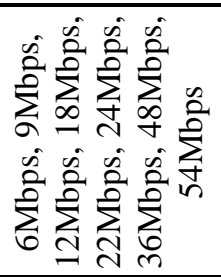 \\
\hline 这 & ఏ్ర & $\begin{array}{l}\Xi \\
\Xi \\
\end{array}$ & $\begin{array}{l}\Xi \\
\stackrel{\Xi}{0}\end{array}$ & $\begin{array}{l}\Xi \\
\Xi \\
\end{array}$ & $\begin{array}{l}\Xi \\
\Xi \\
\end{array}$ & 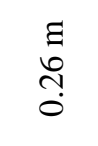 & 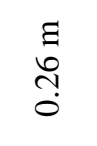 & 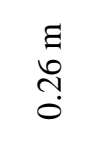 & 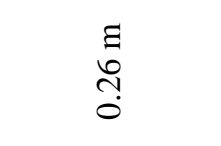 \\
\hline 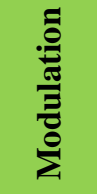 & $\frac{\vec{a}}{\mathrm{z}}$ & $\frac{v}{\sqrt[n]{n}}$ & $\begin{array}{l}\frac{v}{v} \\
\tilde{\sigma}\end{array}$ & 艺 & Uै & $\begin{array}{l}\frac{v}{\tilde{n}} \\
\frac{n}{n}\end{array}$ & $\begin{array}{l}\frac{1}{\tilde{2}} \\
\tilde{\sigma}\end{array}$ & U & 空 \\
\hline 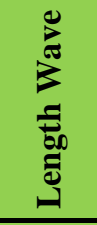 & $\frac{\stackrel{2}{\vec{z}}}{\mathrm{z}}$ & $\frac{\text { ह̂े }}{\text { z }}$ & $\frac{\text { â }}{\text { z }}$ & $\frac{\stackrel{2}{\text { ż }}}{\text { z }}$ & $\frac{\text { है }}{\text { zे }}$ & $\frac{\text { 空 }}{\mathrm{Z}}$ & $\frac{\stackrel{\Xi}{\tilde{z}}}{\mathrm{z}}$ & $\frac{\text { है }}{\text { zे }}$ & $\frac{\text { : }}{\text { ż }}$ \\
\hline 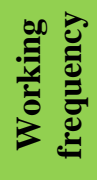 & $\frac{\vec{a}}{\bar{z}}$ & 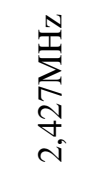 & $\begin{array}{l}\underset{N}{N} \\
\underset{N}{N} \\
\underset{i}{N}\end{array}$ & 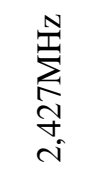 & 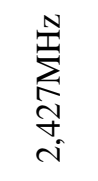 & $\begin{array}{l}\underset{i}{N} \\
\underset{i}{J} \\
\underset{i}{N}\end{array}$ & $\begin{array}{l}\underset{N}{N} \\
\underset{N}{J} \\
\text { i }\end{array}$ & $\begin{array}{l}\underset{N}{N} \\
\underset{N}{J} \\
\underset{i}{J}\end{array}$ & $\begin{array}{l}\underset{N}{N} \\
\underset{i}{J} \\
\underset{i}{J}\end{array}$ \\
\hline 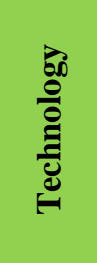 & 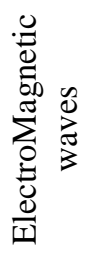 & 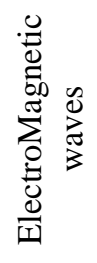 & 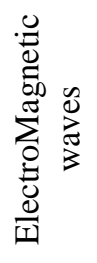 & 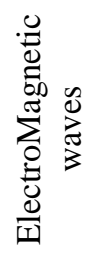 & 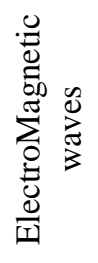 & 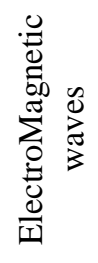 & 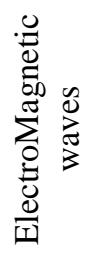 & 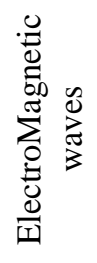 & 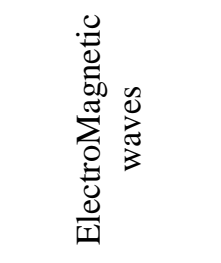 \\
\hline 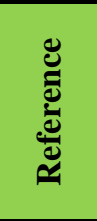 & $\begin{array}{l}\sqrt{n} \\
\approx \\
0\end{array}$ & 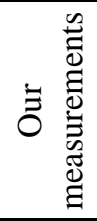 & 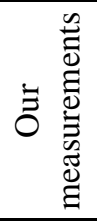 & 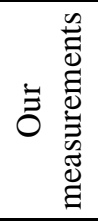 & 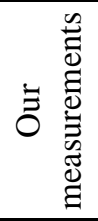 & 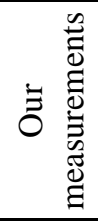 & 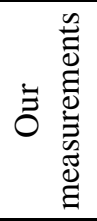 & 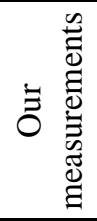 & 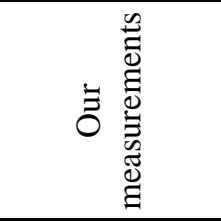 \\
\hline
\end{tabular}

Note: N/app: Not applicable, N/av: Not available 


\section{Chapter 6: Underwater Wireless Communications based on Electromagnetic Waves}

\subsection{Mathematical Model}

Finally, using data shown in Section 6.2, we can model the behavior of the signal as a function of the temperature, working frequency and modulation. The represented temperature range is from 16 to $27^{\circ} \mathrm{C}$ except to $\mathrm{CCK}$ modulation which only shows coherent values up to $22{ }^{\circ} \mathrm{C}$. For each modulation, we have represented all possible values which show coherent values.

The set of all our data can be represented as points of three coordinates $(f, t, d)$, where $f$ is frequency, $t$ is temperature and $d$ is the maximum distance between antennae. All points compose a surface $S$ in the space which is the image of a continuous application:

$$
\vec{r}: D \subset \mathfrak{R}^{2} \rightarrow \mathfrak{R}^{3}
$$

The set of points of the surface $S$ can be represented as:

$$
S=\left\{(f, t, d) \in \Re^{3}:(f, t, d)=\vec{r}(u, v)=\langle f(u, v), t(u, v), d(u, v)\rangle \operatorname{con}(u, v) \in D\right\}
$$

Where $u, v$ define the plane and $\vec{r}$ is a continuous vectorial function defined in $S$, i.e.:

$$
\vec{r}: S \rightarrow \mathfrak{R}^{3}
$$

When the pair $(u, v)$ takes all possible values, the vector $\vec{r}$ draws a surface $S$ in the space $\mathfrak{R}^{3}$. Given in mind these statements, we can define each point as the set of coordinates as follows:

$$
(f, t, d)=f \vec{\imath}+t \vec{\jmath}+F(f, t) \vec{k}
$$

Thus, we express the value of the maximum distance between antennas as a function of the operating frequency and the temperature of the medium.

$$
d=F(f, t)
$$

We have used Eureqa Formulize [358] to estimate the mathematical expression.

Firstly, we have used all obtained values to extract the mathematical model for underwater communications using BPSK modulation. Equation 6.1 relates the distance with the working frequency and the environmental temperature using BPSK modulation.

$$
d=669.9 * f+360.8 * t+0.393 * f * t^{3}-3341-8.921 * t^{2}-7.834 * f * t^{2}-0.004985 * f * t^{4}
$$

Where $d$ is the distance in cm., $f$ is the frequency in $\mathrm{GHz}$ and $t$ the temperature in ${ }^{\circ} \mathrm{C}$. This equation has a correlation coefficient of 0.8676 and an average absolute error of $1.036 \mathrm{~cm}$

Using Equation 6.1, we can estimate the maximum distances for BPSK modulation as a function of the working frequency and temperature (Figure 6.11). As we can see, the maximum distance is obtained for $23.5^{\circ} \mathrm{C}$ working at $2.412 \mathrm{GHz}$. In addition, we can see that for temperatures of $27^{\circ} \mathrm{C}$, the maximum reached distance is about $9 \mathrm{~cm}$, meanwhile for temperature around $16{ }^{\circ} \mathrm{C}$, the maximum distances is $21 \mathrm{~cm}$ for $2.412 \mathrm{GHz}$ and less than $19 \mathrm{~cm}$. for $2.457 \mathrm{GHz}$.

Equation 6.2 relates the distance with the working frequency and the environmental temperature using QPSK modulation.

$$
d=594.9 * f * f+335.9 * t+0.3719 * f * t^{3}-3024-8.426 * t^{2}-7.336 * f * t^{2}-0.00475 * f * t^{4}
$$

Where $d$ is the distance in cm., $f$ is the frequency in $\mathrm{GHz}$ and $\mathrm{t}$ the temperature in ${ }^{\circ} \mathrm{C}$. This 
equation has a correlation coefficient of 0.8831 and an average absolute error of $0.961 \mathrm{~cm}$.

Figure 6.12 shows the estimation of maximum distances for QPSK modulation as a function of the working frequency and temperature. These approximations are obtained from Equation 6.2. The maximum distance $(26 \mathrm{~cm})$ is obtained for $23.5^{\circ} \mathrm{C}$ working at $2.412 \mathrm{GHz}$. Furthermore, we can see that for temperatures of $27 \mathrm{~cm}$, the maximum reached distance is about $7 \mathrm{~cm}$. and for temperatures of $16^{\circ} \mathrm{C}$, we can obtain distances between $18,8 \mathrm{~cm}$ to 21,5 $\mathrm{cm}$.

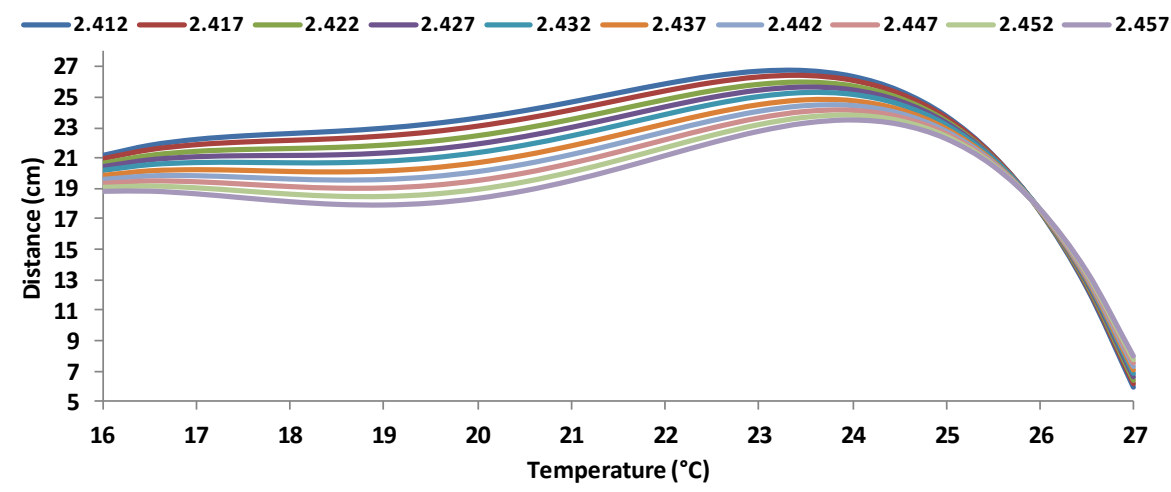

Figure 6.11. Estimated maximum distances for BPSK modulation.

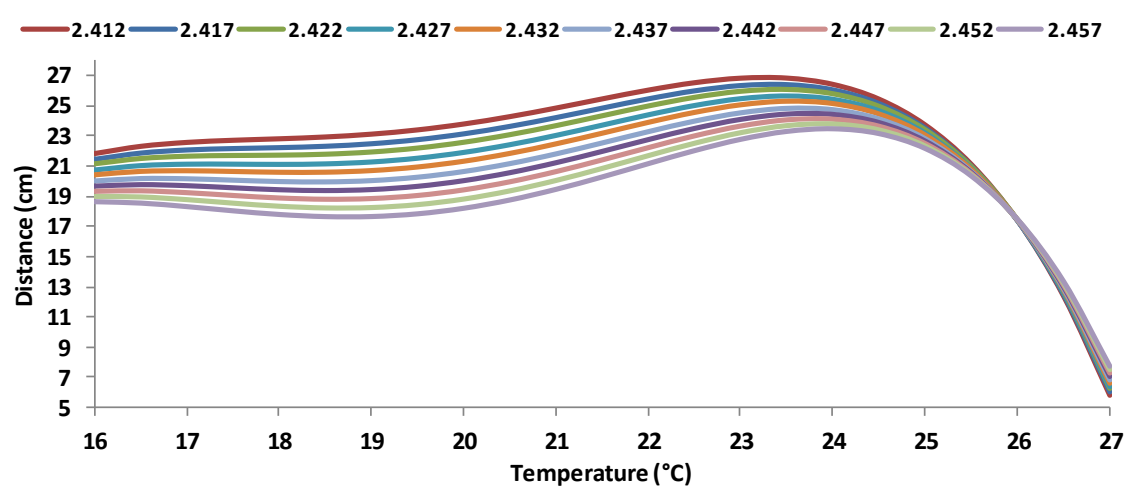

Figure 6.12. Estimated maximum distances for QPSK modulation.

Working with OFDM, we can estimate values between 16 to $22{ }^{\circ} \mathrm{C}$. Equation 6.3 relates the distance with the working frequency and the environmental temperature using QPSK modulation.

$$
\begin{array}{r}
d=226.5+0.1493 * t^{2}+0.3006 * t * \sin (0.04688-t-69.33 * f)+0.1493 * \sin (0.04688-t-69.33 f)^{2}+ \\
\sin (\tan (226.5-t-69.33 * f))-69.33 * f-1.979 * f *(t-\sin (0.04688-t-69.33 * f))(6.3)
\end{array}
$$

Where $d$ is the distance in cm., $f$ is the frequency in $\mathrm{GHz}$ and $\mathrm{t}$ the temperature in ${ }^{\circ} \mathrm{C}$. This equation has a correlation coefficient of 0.9238 and an average absolute error of $0.63 \mathrm{~cm}$.

Figure 6.13 shows the estimation of maximum distances for OFDM modulation as a function of the working frequency and temperature. These approximations are obtained from Equation 6.3. The maximum distance $(26 \mathrm{~cm})$ is obtained for $21^{\circ} \mathrm{C}$ working at $2,412 \mathrm{MHz}$. We can also see that for temperatures of $22{ }^{\circ} \mathrm{C}$, the maximum reached distance is about $25 \mathrm{~cm}$. and for temperatures of $16^{\circ} \mathrm{C}$, we can obtain distances between $15 \mathrm{~cm}$ to $21 \mathrm{~cm}$.

Finally, CCK transmission scheme can be modeled by Equation 6.4 which relates the distance 


\section{Chapter 6: Underwater Wireless Communications based on Electromagnetic Waves}

with the working frequency and the environmental temperature.

$$
d=1538 * f+4.057 * f * t^{2}+0.003264 * t^{4}-2981-7.857 * t-0.2975 * t^{3}-9.28 * f^{3} * t
$$

Where $d$ is the distance in $\mathrm{cm} ., f$ is the frequency in $\mathrm{GHz}$ and $\mathrm{t}$ the temperature in ${ }^{\circ} \mathrm{C}$. This equation has a correlation coefficient of 0.8635 and an average absolute error of $1.16 \mathrm{~cm}$.

Figure 6.14 shows the estimation of maximum distances for CCK transmission scheme as a function of the working frequency and temperature. These approximations are obtained from Equation 6.4. The maximum distance $(26 \mathrm{~cm})$ is obtained for $22.5^{\circ} \mathrm{C}$ working at $2,412 \mathrm{MHz}$. In addition, we can see that for temperatures of $27{ }^{\circ} \mathrm{C}$, the maximum reached distance is between $10 \mathrm{~cm}$ to $15 \mathrm{~cm}$. and for temperatures of $16^{\circ} \mathrm{C}$, we can obtain distances between $17 \mathrm{~cm}$ to $22 \mathrm{~cm}$.

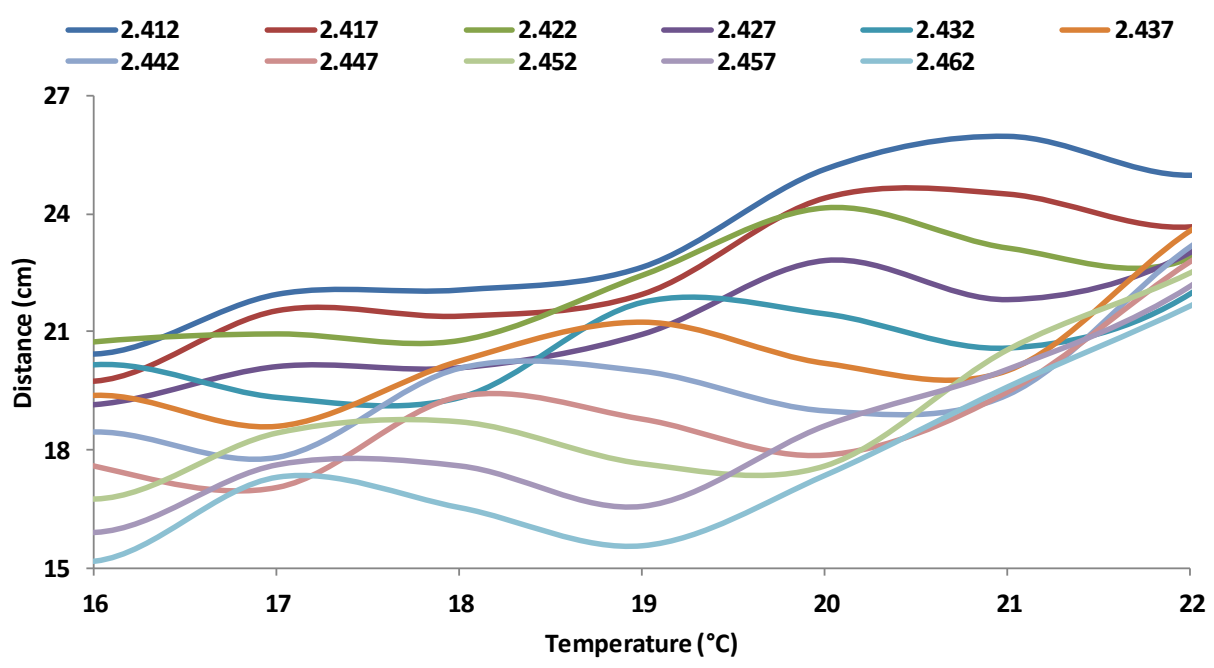

Figure 6.13. Estimated maximum distances for OFDM modulation.

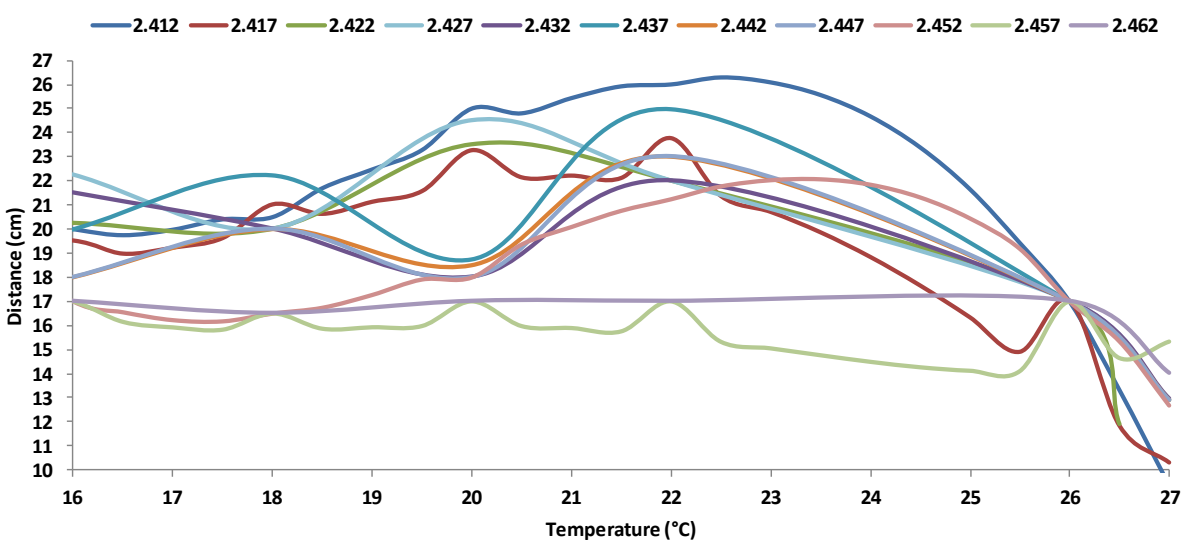

Figure 6.14. Estimated maximum distances for CCK transmission scheme. 


\subsection{Conclusion}

Underwater Networks presents several application fields such as monitoring applications, as oceanographic data collection, pollution monitoring/detection, and off-shore oil/gas field monitoring. They can also be used for exploration applications such as submarine detection, loss treasure discovery, and hurricane disaster recovery [114] [178].

In this chapter we have analyzed the behavior of EM signals in underwater environments, for $16^{\circ} \mathrm{C}, 18^{\circ} \mathrm{C}, 20^{\circ} \mathrm{C}, 22^{\circ} \mathrm{C}$ and $26^{\circ} \mathrm{C}$ and its dependence with the working frequency. Previous published works showed that the EM waves behavior has no relationship with the working frequency in freshwater. However, we have observed that there is an obvious relationship with the working frequency, type of modulation used and the environmental temperature. In addition, we have added a comparative of BPSK, QPSK, CCK and OFDM schemes for several temperatures. This type of analysis has not been performed in previous studies. We have compared our result with theoretical estimations and we have checked that these values were more optimistic than those obtained on the real environment. Moreover, we have seen that the temperature of the water affects the distance. For this reason, we have modeled the value of maximum distance between devices as a parametric function of the working frequency and the temperature of medium.

Although our proposal provides short communication distances in underwater wireless sensor networks, we can use it for precision monitoring such as ecosystems contaminated by invasive plants (especially in ponds where there are some poisonous plants that can contaminate the water) or hazardous waste (e.g. in swamps, the quality of the water is different depending on the season because the water may contain some organic material that may be affected when it is warmer because the $\mathrm{pH}$ is different). In both cases the water cannot be used for human consumption, but, in some cases, it can be used by industries to run their plants and supply the water cooling system. Moreover, our proposal can be used to control the pollution of the water, which may come from industries and nearby roads, accurately.

Another application is for communicating with some parts of the neutrino telescope [359]. The neutrino telescope is an underwater structure located at the bottom of the Mediterranean Sea. Researchers are seeking ways to connect a hydrophone, for the positioning system of different parts of this structure. Until now, they have been using cables and penetrators, to connect the different parts. These pieces have a high economic cost. Using wireless communications, we would be reducing the cost of this material and would avoid the critical connections that can propagate a fault (or leak) through the system. Finally, the fact, that the distances between the devices are extremely small (practically in contact), means that the depth of this infrastructure is not a problem for wireless transmission of information. There are other applications such as, military applications, marine monitoring and even industrial applications such as marine fish farms [178], to reduce the deposition of organic waste on the seabed and to fight against environmental contamination

The measurements shown provide several benefits. On one hand, the use of IEEE 802.11 standard is cheap (IEEE 802.11 devices are very cheap nowadays) and, on the other hand, it provides high data transfer rates, for the inclusion of all types of sensed data, even images.

Finally, work presented in this chapter has been published in the following references [174], [175], [176], [177] and [360]. 


\section{Chapter 7}

\section{Application of WSN in Marine Environments}

\subsection{Introduction}

Aquaculture is defined as an activity aimed at producing and fattening aquatic organisms such as fish, mussels, oysters or other shellfish and vegetables. It is also defined as the breeding under controlled conditions of species that are developed in the aquatic medium. The most developed cultivations are the edible species belonging to the molluscs, crustaceans and fish groups. These three groups and the production of algae constitute the fourth biggest group of aquaculture activity. Although, marine aquaculture originates a smaller environmental impact compared with other productive sectors and coastal activities, some impact is produced. These effects should be identified to eliminate and/or minimize them.

When marine fish farm viability is studied, marine biologists should make an orographic and hydrodynamic environmental evaluation of the farm cages mounting area [361]. Many water parameters should be measured: $\mathrm{pH}$, Salinity, Temperature, dissolved Oxygen $\left(\mathrm{dO}_{2}\right)$ the transparency, the suspended solids (SS), Ammonium, Nitrates, the Total Nitrogen (NT) or match soluble reagent (PSR), among other [362] [363]. Other parameters such as Total Organic Carbon (TOC), soluble phosphorus, soluble total nitrogen, redox potential or grain-size composition give us information of the physic-chemical characteristics of the sediments. This information is used to select the most appropriate place for the cages and the most appropriate environmental measuring 
system. In open sea facilities, nutritious concentrations in silts tend to increase and they are usually located at the address of the predominant current, outside the polygon of cultivation [361].

One of the main issues in aquaculture is the high cost of the lost food when the fish are fed. Furthermore, this wasted food is deposited in the seabed and generates an environmental impact on the surrounding area. It causes failure to uphold aquaculture legislation.

When the fish are fed in marine fish cages, it is difficult to control the point of cessation. The lost food in the cages is close to $8.26 \%$, of the total food. Bearing in mind that food expenses represent almost $60 \%$ of the total costs of marine farm exploitation, the food that gets lost in the feeding process of these facilities should be controlled [364]. This undesired waste is dispersed in the pelagic system and deposited on the seabed [365]. It causes some impact on the benthic habitats, and can affect to sensitive communities such as seagrass meadows or rocky reefs, and it also reduces the economic benefits of the fish farm because the wastage of the uneaten food.

As Figure 7.1 shows, the most important producer of fish from aquaculture is Asia with approximately twenty times more than America or Europe. If we analyze the European total production of fish from aquaculture, we can see that Spain is one of the primary European aquaculture producers in terms of volume (See Figure 7.2) [366]. Moreover, in recent years, Latin America, and especially Chile, has emerged as an aquaculture power [367]. This has meant that many of the studies and researches are written in the Spanish language.

The aquaculture is the fastest growing food sector. The production has doubled since 1987 and in 2040 the global demand for fish will again be doubled. In 2001, nearly of $30 \%$ of seafood consumed came from farms, although it is estimated that this value will be multiplied by 7 in the next 25 years to keep global fish consumption. For this reason, aquaculture should be understood as a way to offset the collapse of fish wild populations.

Seeing these values and the estimation of production, we think it is necessary to improve the current methods in order to reduce the environmental impact of the farms in the surrounding areas and the economic losses due to food waste.

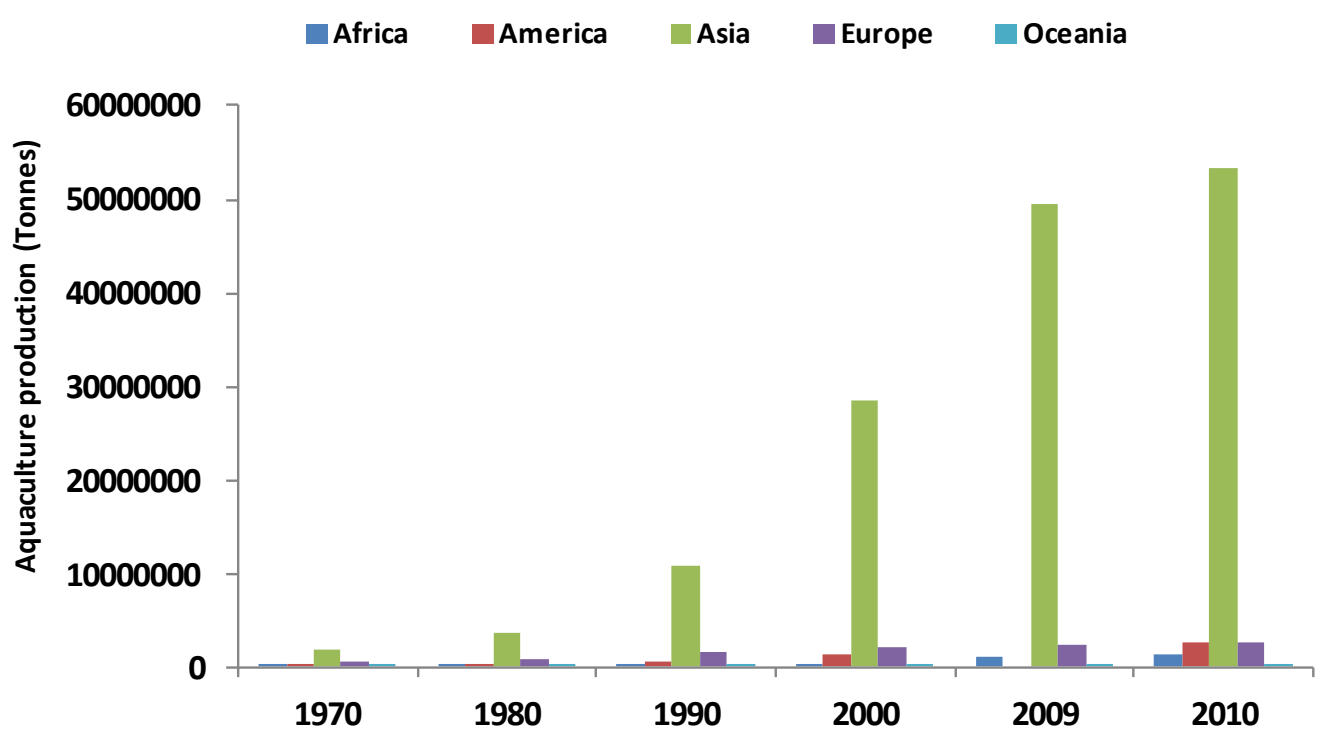

Figure 7.1. Total production of fish from aquaculture in the world. 


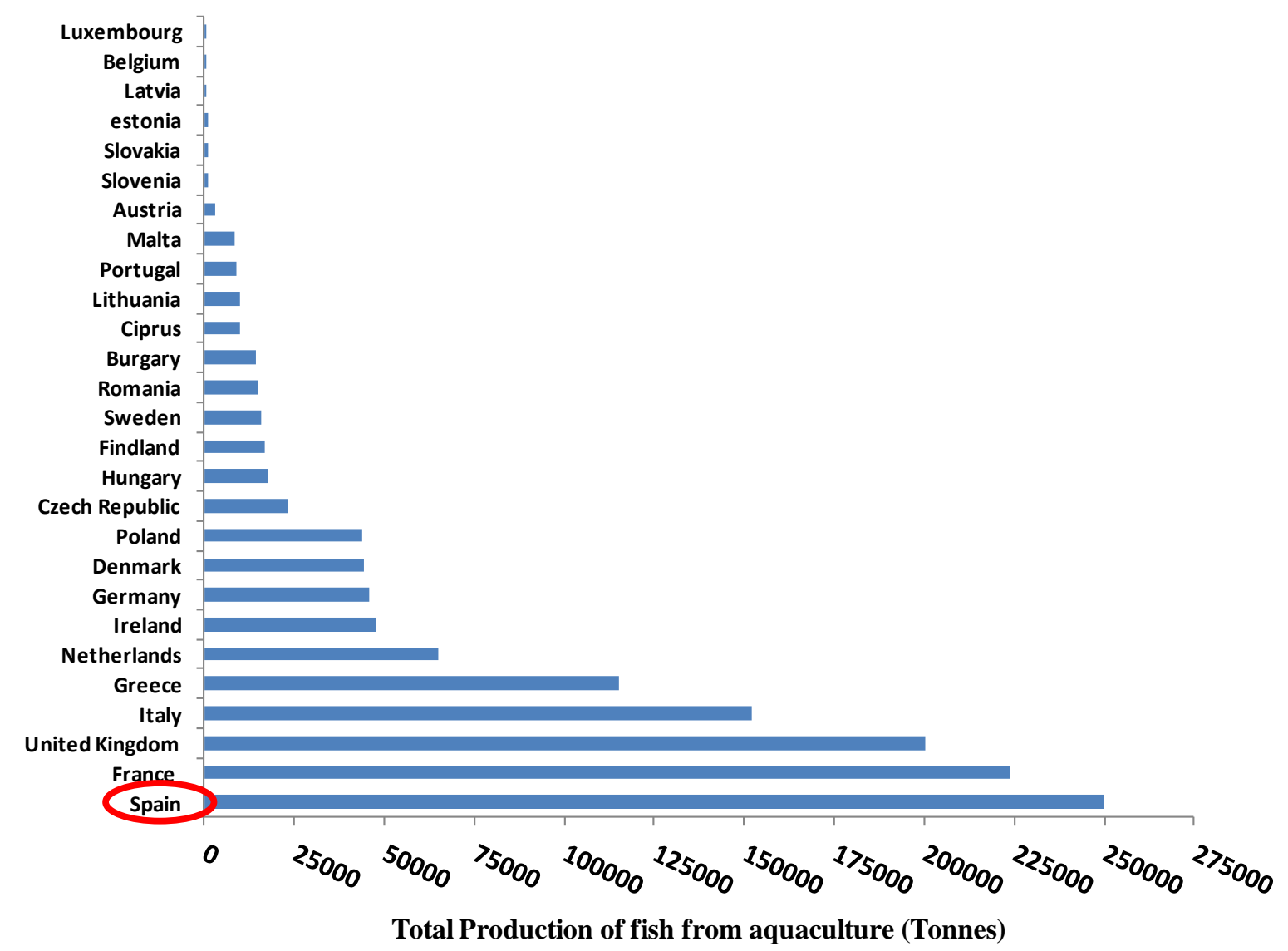

Figure 7.2. European total production of fish from aquaculture in 2010 .

In this chapter, we are going to show the proposal of a wireless sensor to deposition monitoring. In addition, we are going to present a low cost turbidity sensor which helps us to control de quality of water. To understand the deployment of these sensors, firstly we will see the main problems that fish farms managers have. We will also see the seabed depositions estimation. After presenting our proposal, we will show the estimation of the number of sensor nodes we need. Finally, we will see a simulation of the amount of food we should use in regular procedure and with our proposal. Last Section shows the conclusion of this chapter.

\subsection{Problem Description}

One of the main problems to be considered in several marine related activities, such as the marine fish farms and their surroundings, is the amount of uneaten feed and the faecal waste generated by the fish and deposited on the seabed under the cages. The wastage accumulation in the seabed causes notorious changes in the silt chemistry of the nearby farm areas. It impacts the fauna and flora, and the whole sustainability of the system.

Nowadays, the feeding process is carried out distributing the food by hand or impelled canyons by air. It can also be used crafts with fixed pipes in each cage impelled by air compressors can be 
used or by self-demand troughs. The control of the exact moment when the food begins to fall to the seabed is currently done by a camera which is introduced and removed from the water every time the fish should eat. Scuba-divers are also in charge of this task. Sometimes the conditions of the water are not always good and suitable for immersions, but fishes must be fed all year round.

The maintenance of equipment and the own feeding process have associated high economic costs.

The currently mathematical formulations can estimate the amount of food in the tank by knowing the number of fish in the cage and supposing that all the fish in the cage have the same weight and size. But in most cases, there are some groups of fishes which present different behaviour. Some ones usually acquire dominant aptitude and tend to eat more. However, others may acquire a submissive attitude and eat less or even after the dominant fish have eaten. So, we will probably have groups infra and overfed.

Additionally, we have observed that fishes usually adopt particular behaviours in function of the environmental conditions. It may happen that the fishes do not eat the quantities envisaged due small changes in water temperature, so we will be wasting food. According to some studies, fishes have certain behaviour patterns that can help us to know the level of satiation of them. When the shoal is hungry, they increase their physical activity and swim faster and near to the surface and due to be on cage, fishes usually swim following the perimeter of the cage. Therefore, our system should consider this increase in speed and depending on these results, we will obtain several results. With the set of sensors, the system will be able to discriminate between whether the fish are hungry or if they are just frightened by a predator which prowls around [178].

Therefore, a significant advance in aquaculture, from the standpoint of feeding control, would be the real-time monitoring of the activity of the fish and the detection of the fall of uneaten feed.

For this, we want to develop some sensors to measure these parameters. The set of these sensors can help us to interact with the feed dispensing system. This system will control the amount of food per unit of time to drop into the water.

Some of these sensors, we can buy it directly (although it has a very high cost), but others do not exist and we would need to develop them. Some of the most important sensors to install in a fish cage are the following ones:

- Sensors that sense the cage water column temperature, dissolved oxygen, and turbidly: The species usually eat more when temperature is higher and grow best between some specific temperature ranges. In addition, fishes do not feed well at low oxygen concentration. These parameters and the water turbidity are limiting factors in fish growth.

- Sensors that senses in specific places the water current and salinity: It is important to define the water current in order to calibrate the measures of speed sensors and to define the uneaten food spread. A very abrupt salinity change may mean the death of many fish. Salinity changes should not be too high.

- Sensors that measure the fish speed: In most species, the speed of the fish is directly related to their hunger. If the fishes are moving quickly, it is because they are hungry (but it could also be caused by the appearance of a depredator near the cage).

- Sensors that let us know when the feed begins to fall on the seabed and its quantity: One of the main issues to take into account in sustainability in marine fish farms is how faecal 
pellets and uneaten feeds are spread under the cages. These depositions are settled some distance from the farm implying an adversely impact in the coastal environment.

- Sensors to detect and follow the water pollution of the water column in the cage surroundings: The quality of water is one of the most important parameter related to the fish growth. If water would contain heavy metals, these fish would be unfit for human consumption. The dumping of other substances may cause massive death of fishes inside the cage.

Figure 7.3 shows a fish cage with the most important sensors to improve the sustainability of a marine fish farm.

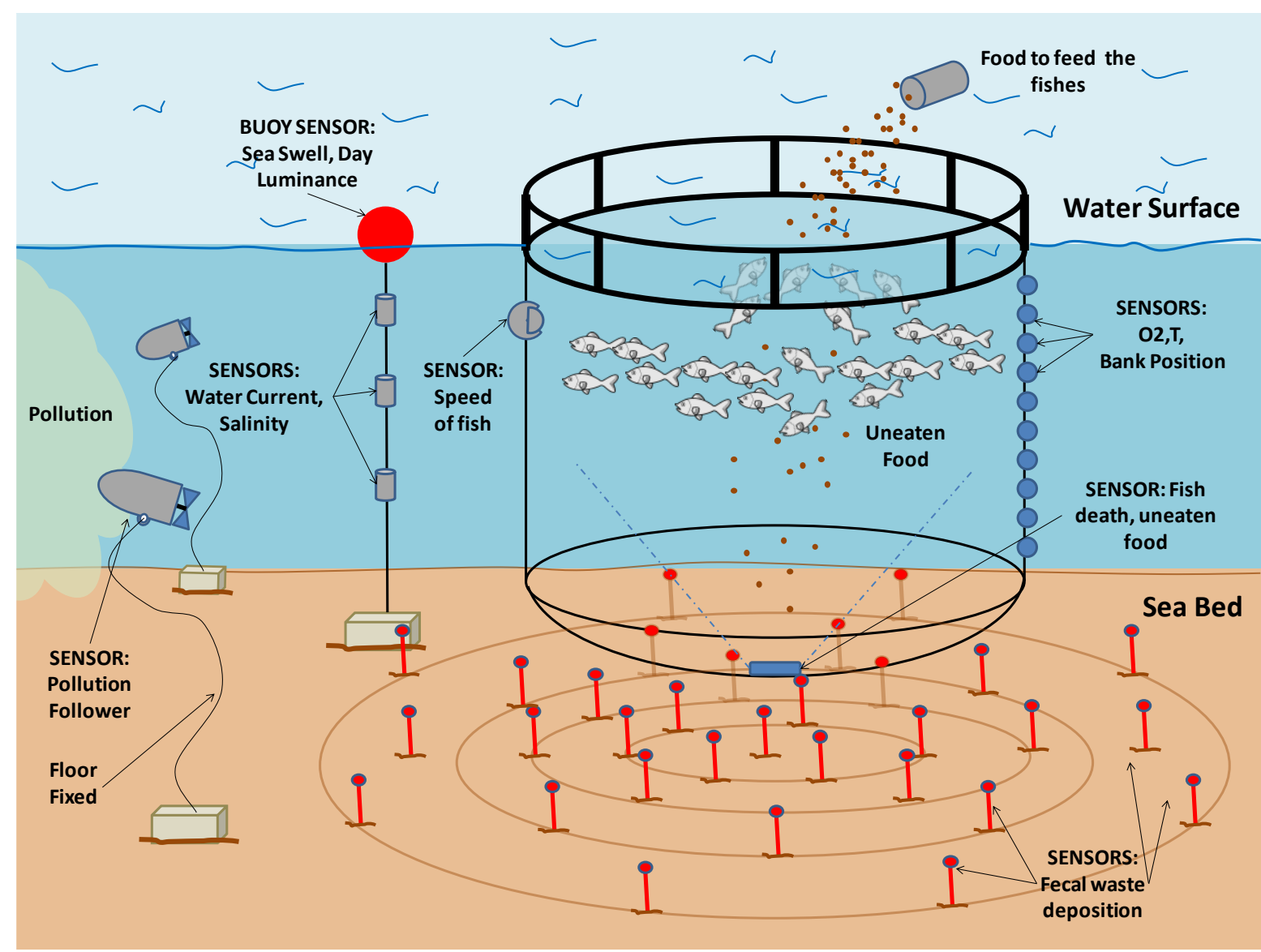

Figure 7.3. Marine fish cage with a sensor network for monitoring the fishes activity

Considering all of these considerations, it is evident that a monitoring and control sensor system for fish feeding in marine fish farms is justified. An adequate feeding distribution system will be essential to assure a homogeneous growth of the fish cultivation [368]. Moreover, the system will be able to detect and warn special cases such as temperatures out of the regular values for a specific season, which will allow us to see the effects of the climate change. 


\subsection{Seabed Depositions Estimation}

As we have read at the beginning of this chapter, one of the most important problems in aquaculture activity is how to control the amount of uneaten feeds which is spread under de cages and surrounding areas. Due to the underwater currents this food can be settled some distance from the farm implying an adversely impact in the coastal environment. This Section shows the analytical estimation of deposition in seabed in terms of studied dispersions models, current speeds and settling speed as a function of the particle features.

We can find several studies about the factors influencing the sedimentation and accumulation of organic material under and near the fish cages [369][370][371]. We are interested on their distribution area for determining the best sensor distribution along the seabed surface. To do the estimations of waste deposition, we should take into account the following parameters:

- Biomass of the fish

- Metabolic rates of the fish

- Settling rates of excess fish feed

- Settling rates of fecal pellets

- Feeding rates

- Amount of excess (waste) feed

- Consumption of waste feed by other species

- Rate of decay of organic particles on the bottom

- Sinking velocity of the particles

- Velocity and direction of the current

- Depth-varying currents

- Water depth

It is also important to estimate the concentration or the amount of different elements like carbon, nitrates and phosphates generated from wasted food and feces. The physic-chemical characterization of the water can be used to estimate the pollution level in an area. Stigebrandt et al. present a dispersion model [372][373] which gives us the average value of carbon emission from the cages. Equation (7.1) represents the mean carbon emission from cages in $\mathrm{g} /\left(\mathrm{m}^{2} \cdot\right.$ Day).

$$
\mathrm{F}=\frac{1}{2} \cdot \frac{\mathrm{T}_{\mathrm{P}}}{\Delta \mathrm{f}}\left(\mathrm{FCR}-\mathrm{FCR}_{\mathrm{t}}+0.1\right)
$$

Where, FCRt is the theoretical feed conversion ratio; FCR is defined as the factual feed conversion ratio; TP is the Total Proteins and $\Delta \mathrm{f}$ is the total area of the cage.

According to [372], it is possible to consider that FCR - FCRt $=0.3$. This means that the waste feed is equals to $0.3 \mathrm{~kg}$ per each $\mathrm{kg}$ of produced fish. For out calculation, we have assumed that $\mathrm{Tp}$ can be a value between 20 and 30 (depending of the fish species) [374], and that the size of cage is between 6 and $25 \mathrm{~m}$ of diameter. Figure 7.4 shows the estimated carbon emission in function of the cage size and de value of TP 


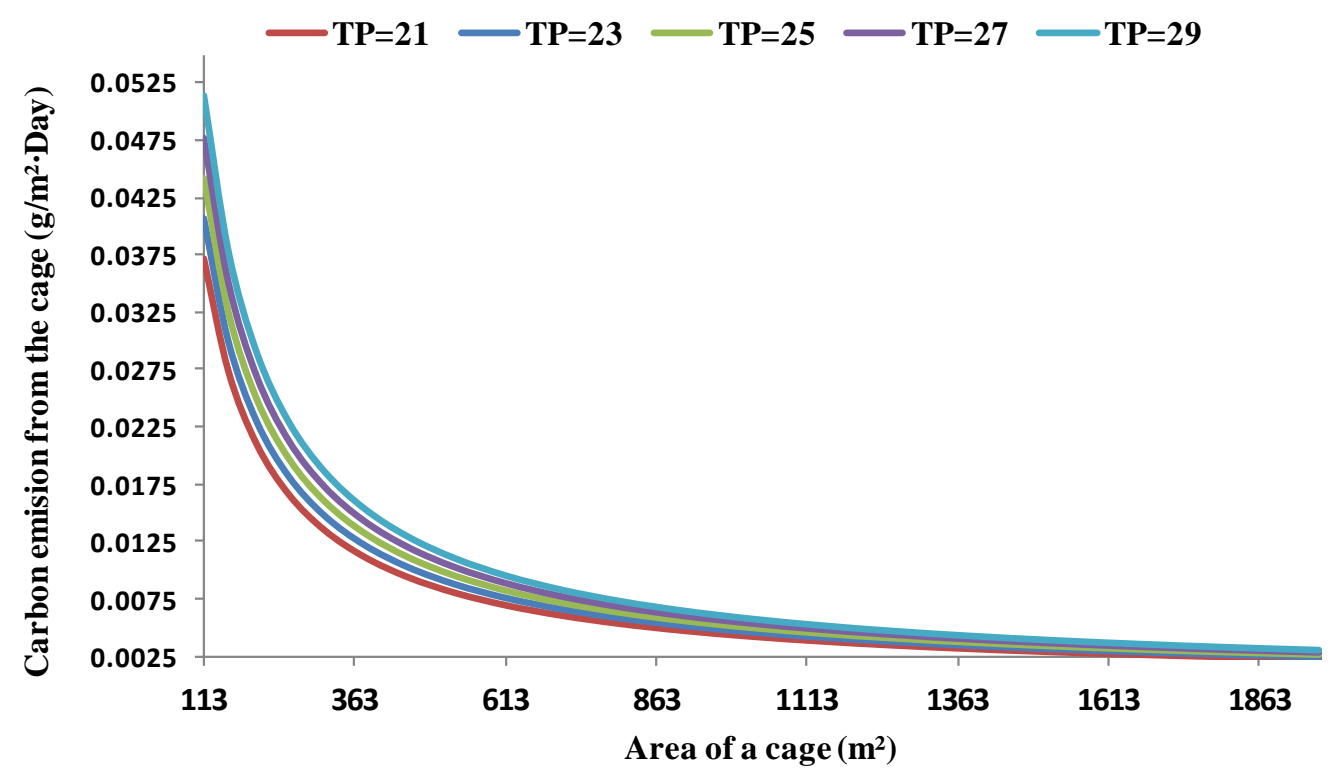

Figure 7.4. Carbon emision from the cage

Considering the measurements gathered in reference [372], it is possible to estimate analytically the sedimentation coefficient $S$ (expressed in Svedbergs) as a function of the distance, for a sinking speed per sinking time equal to $15(\delta \mathrm{T}=15)$. This estimation is shown in Equation 7.2 (it has a correlation coefficient of 0.9974$)$.

$$
S=-1 \cdot 10^{-7} r^{4}+1 \cdot 10^{-5} r^{3}-0.0004 r^{2}+0,0012 r+0,077
$$

Where $\mathrm{S}$ represents de sediments in svedbergs and $\mathrm{r}$ de distance from de center of cage.

Figure 7.5 shows the depositions of a marine fish farm with 6 cages, each one with 15 meters of radius, that are placed in the positions $(20,20),(60,20),(100,20),(60,30),(60,70)$ and $(60,110)$ for $\delta \mathrm{T}=15$.

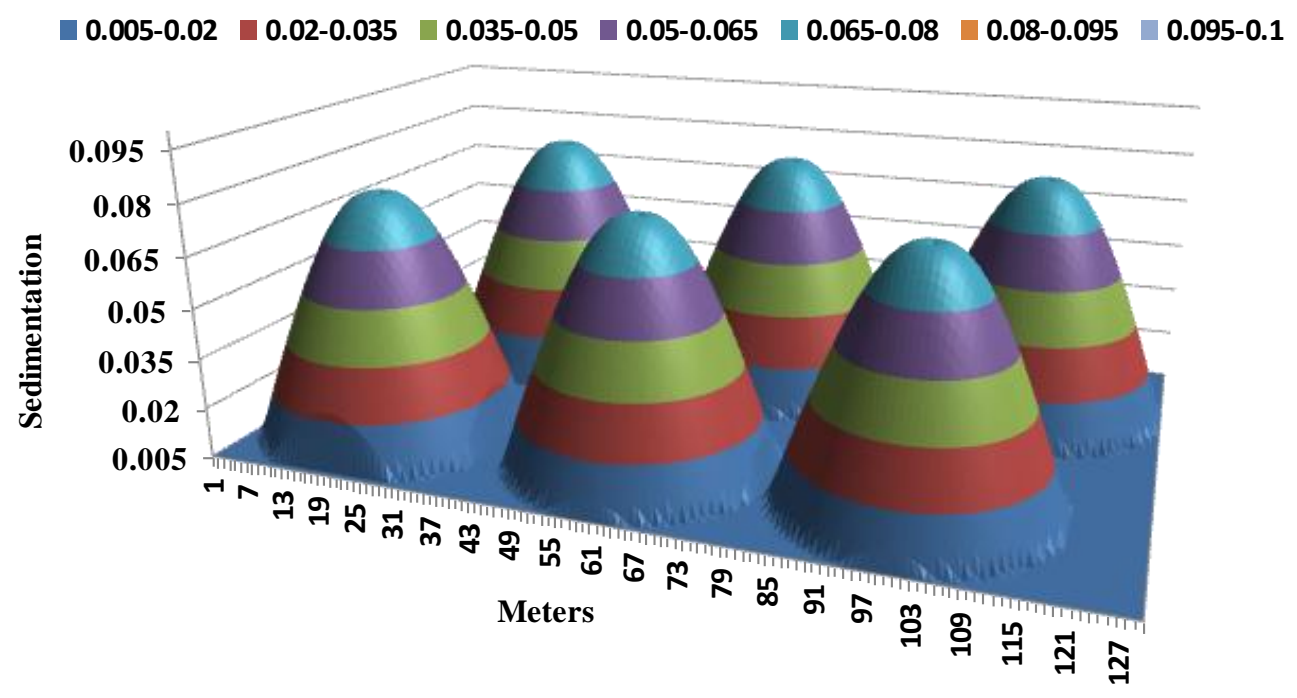

Figure 7.5. Sedimentation for $\delta T=15$, in a fish farm with 6 cages. 


\subsection{Wireless Sensor Node for Depositions Monitoring}

In this section we present the proposal of a sensor node for deposition monitoring. We explain its operation and its mobility. Finally, we will do an estimation of the amount of sensors we will need to cover an area

\subsubsection{Sensor Nodes Operation}

Ultrasonic sensor works using the "pulse-echo" principle combined with triangulation techniques. Sound waves can be irradiated only when there is a medium. This medium can be, in the case of ultrasound, a gas, a fluid or a rigid material. Ultrasonic sensors are normally used with atmospheric pressure. When the control unit sends a digital pulse, the electronic circuit excites the aluminum membrane with rectangular pulses in the resonant frequency to generate vibrations typical of $300 \mu \mathrm{s}$ (although this value depends on the application and the kind of transducer). Then, it emits ultrasonic waves which are reflected with the obstacle. They, in turn, vibrate a stabilized membrane. The piezoceramics convert the vibrations into an analog electrical signal, which is amplified by the sensor and converted into a digital signal. Figure 7.6 shows the operation diagram of a common ultrasonic sensor.

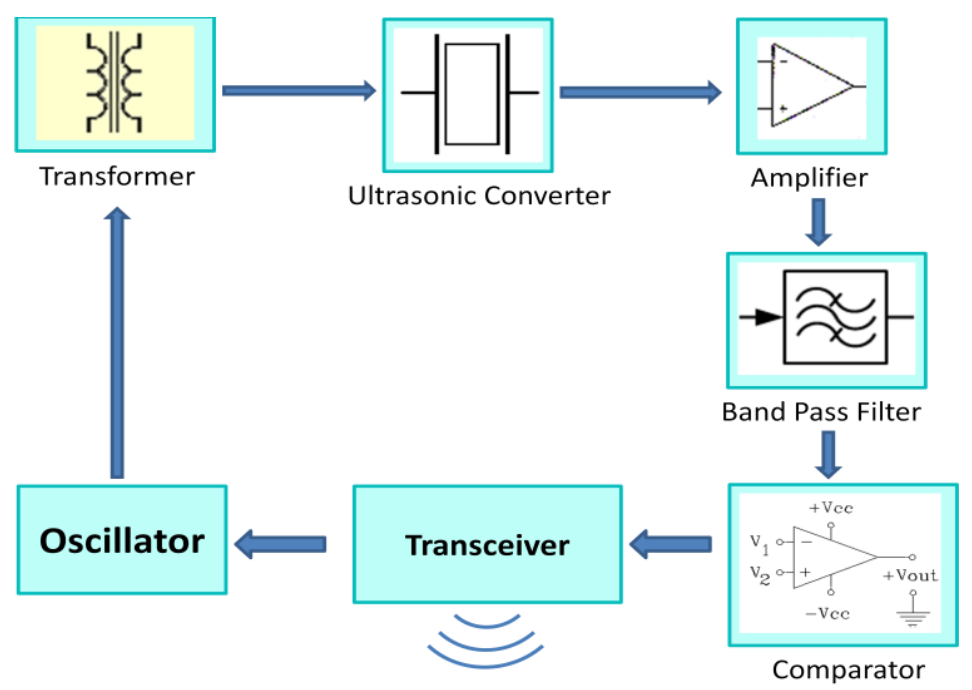

Figure 7.6. Operation diagram of an ultrasonic sensor

Communication between sensor nodes can be performed using acoustic hardware built-in to sensor modules [375].

In order to cover a large area (as wide as possible), the sensor detection angle should be big in the horizontal plane. On the contrary, it should have a small angle in the vertical plane in order to avoid disturbing reflections from other obstacles. Figure 7.7 shows the radiation patterns in the vertical plane (blue) and in horizontal plane (orange) for the UM18-X111X sensor series (manufactured by Sickusa [376]). The sensor is placed in $(0,0)$. These sensor models are able to locate objects at distances between $30 \mathrm{~mm}$ to $250 \mathrm{~mm}$, with a resolution of $0.36 \mathrm{~mm}$. If this sensor is fixed in a place, without movement, it is able to cover a measuring area of about $120 \mathrm{~mm}$. 


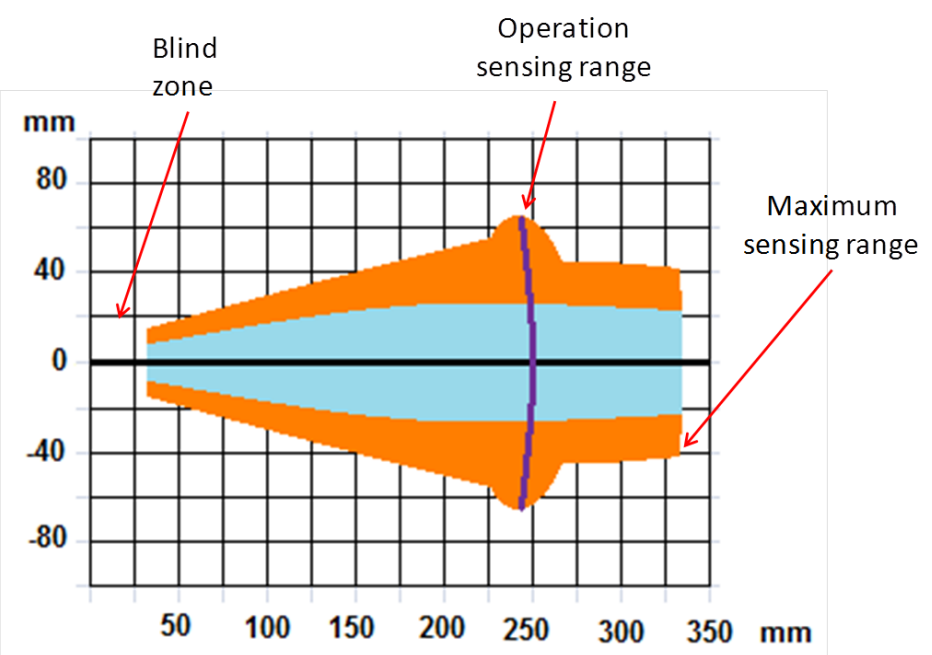

Figure 7.7. Radiation diagram for UM18-X111X sensor series

\subsubsection{Sensor Mobility Model.}

Some studies have demonstrated that controlled mobility can be exploited practically [377]. So, in this section we are going to study how we can take advantage of the sensor mobility. Because our sensors are held to an inverted pendulum system, which offers greater mobility, the measured area of each device is much higher. Each one of the sensors is anchored to the ground with a brace of 20 $\mathrm{cm}$. Therefore, each sensor would be able to cover an area of approximately $1.32 \mathrm{~m}^{2}$, forming a circle with a diameter of $52 \mathrm{~cm}$, as it is shown in Figure 7.8. In order to set the maximum sensing area, we defined the maximum slope that it may suffer due to ocean currents. It is approximately 40 degrees, although this value may be different depending on the location of the marine fish farm.

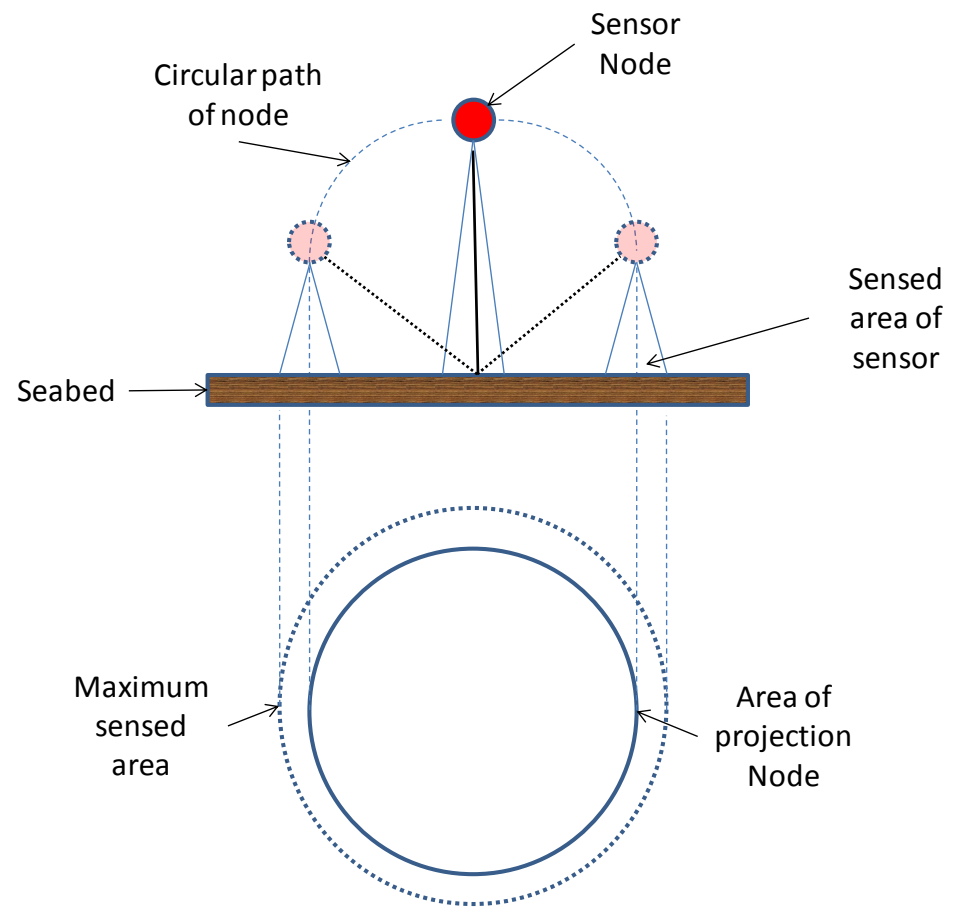

Figure 7.8. Maximun sensed area by one sensor 
In order to model the movement of our sensor node, we use basic principles of body dynamics. The system performs a series of movements that can be decomposed into two parts. In this section we discuss the motion in the $\mathrm{XZ}$ plane, mainly due to the action of the water flow, considering only its magnitude and the motion in the XY plane, where the node describes a circle of radius $\mathrm{R}$ (the the position of the node on the XY plane is also related to the direction of water flow). Thus, we have to estimate the equation of the position of the node in a three-dimensional space. Figure 7.9 shows the explained situation. We have used a Cartesian axis system.

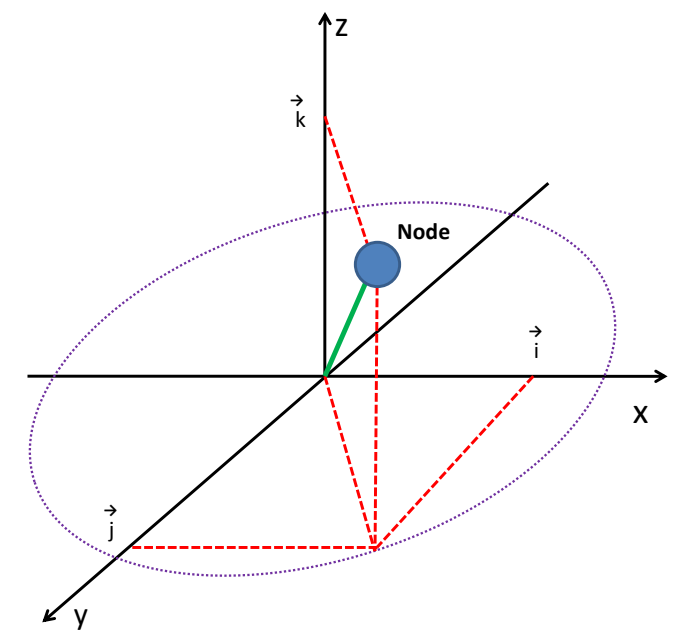

Figure 7.9. Wireless Sensor node movement

From the point of view of the vertical plane motion, we used Archimedes' Principle, but taking into account that the sensor node floats (it is inside a ball with air), but it is fixed to a point on the seabed. Our system has the mass $m$ of the sensor, secured by a straight wire of length $l$ to the seabed. The wire has a tenseness $T$ on the mass that prevents the sensor to float. Figure 7.10 shows this situation. In order to define the equation of the sensor movement in the vertical plane, the second Newton's law is used, which states that the system remains in balance, if the water upward force is equal to the total weight of the node and the tenseness of the wire. Some easy estimations let as find the angle of the wire of the sensor ball $(\theta)$ respect to the vertical line position. It is shown in Equation 7.4.

$$
\theta=\sin ^{-1}\left(\frac{m}{V_{\text {node }} \cdot g \cdot\left(\rho_{f}-\rho_{s}\right)} \cdot \frac{\partial v_{\text {water }}}{\partial T}\right)
$$

Thus, for the $\mathrm{P}_{\mathrm{X}, \mathrm{Z}}$ plane analysis, we can draw our node position on the $\mathrm{P}_{\mathrm{Z}}$ axis following equation 7.5 .

$$
P_{Z}=l \cdot \cos \left(\sin ^{-1}\left(\frac{m}{V_{\text {node }} \cdot g \cdot\left(\rho_{f}-\rho_{s}\right)} \cdot \frac{\partial v_{\text {water }}}{\partial T}\right)\right) \vec{k}
$$

Where $\rho_{s}$ is the density of the sensor node (the ball), $\rho_{f}$ is the fluid density, $V_{\text {node }}$ is the sensor node volume, $g$ is the acceleration due to earth gravity, $m$ is the sensor node mass, and finally $v_{\text {water }}$ represents the seawater flow.

From the point of view of the horizontal plane motion, the sensor node draws a circle of radius $R$ because of the water flow direction and the wire. Where $=l \cdot \sin (\theta)$, the wire has length $l$ and the 
angle $\theta$ is estimated by Equation 7.4. Figure 7.11 and 7.12 show the status of the sensor node and the parameters involved in its movement. Thus, for the $\mathrm{P}_{\mathrm{X}, \mathrm{Y}}$ plane axis, assuming a flow direction with angle $\alpha$, we obtain Equation 7.6 (all parameters have been previously defined for equation 7.4).

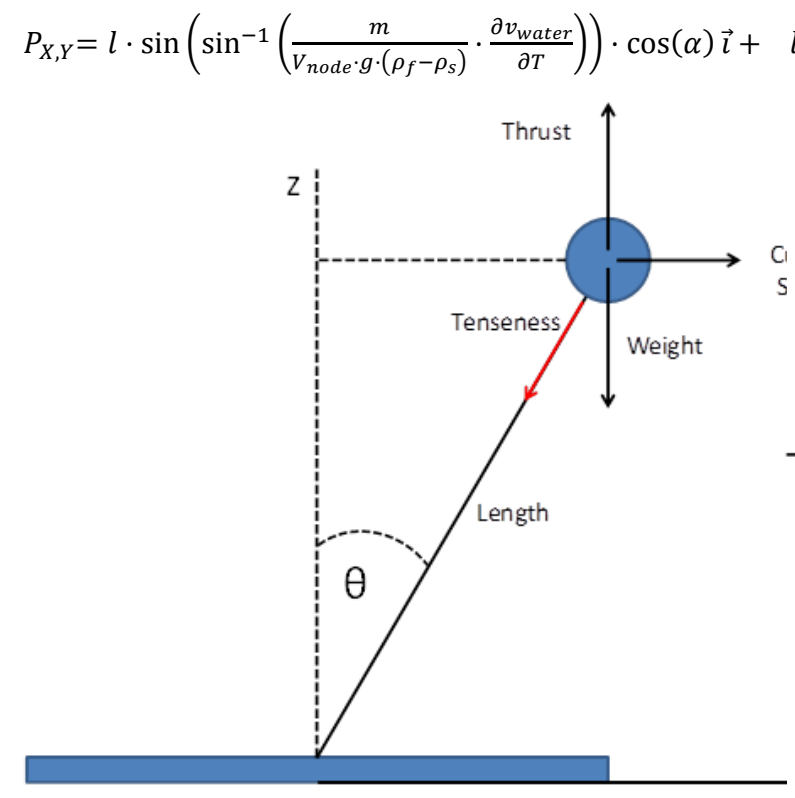

Figure 7.11. Movement in the vertical plane

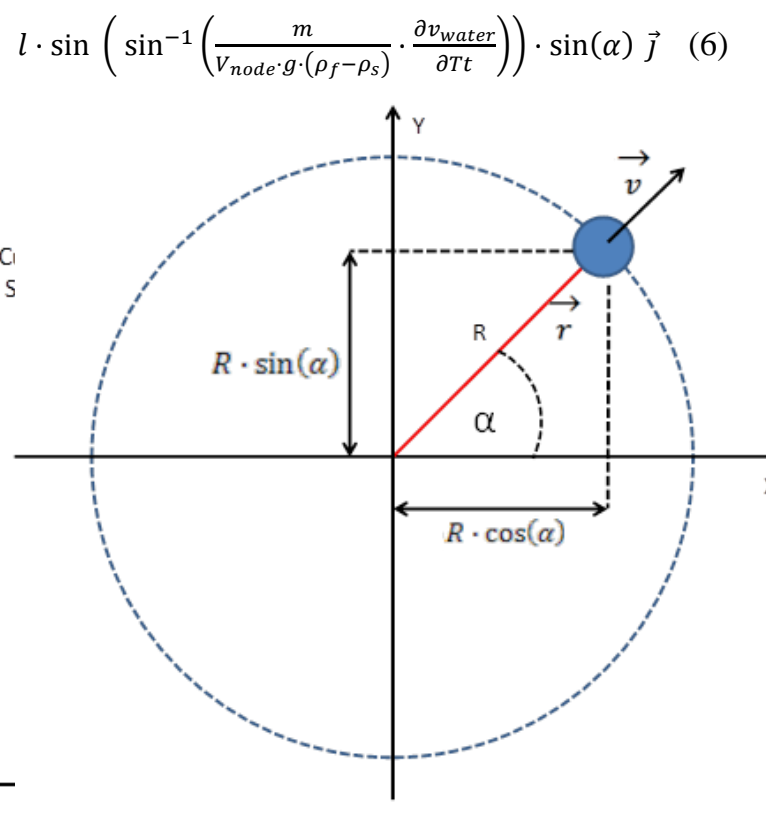

Figure 7.12. Movement in the Horizontal plane

The three-dimensional movement of the sensor node is given by the sum of the vertical plane and the horizontal plane. Expression 7.7 shows the sensor node movement in the three axes.

$$
\begin{gathered}
P_{X, Y, Z}=l \cdot \sin \left(\sin ^{-1}\left(\frac{m}{V_{\text {node }} \cdot g \cdot\left(\rho_{f}-\rho_{S}\right)} \cdot \frac{\partial v_{\text {water }}}{\partial T}\right)\right) \cdot \cos (\alpha) \vec{\imath}+l \cdot \sin \left(\sin ^{-1}\left(\frac{m}{V_{\text {node }} \cdot g \cdot\left(\rho_{f}-\rho_{S}\right)} \cdot \frac{\partial v_{\text {water }}}{\partial T}\right)\right) \\
\sin (\alpha) \vec{\jmath}+l \cdot \cos \left(\sin ^{-1}\left(\frac{m}{V_{\text {node }} \cdot g \cdot\left(\rho_{f}-\rho_{s}\right)} \cdot \frac{\partial v_{\text {water }}}{\partial T}\right)\right) \vec{k}
\end{gathered}
$$

Where, all parameters have been previously defined.

\subsubsection{Sensor Nodes Distribution}

We have seen in Figure 7.5 that there are more sediment concentrated in the center of each cage and this value decreases when the distance from the center of the cage increases. When the variations of faeces and pellets deposition and sedimentation are lower, we can decrease the number of sensors. Sensors that are closest to the center of the cage have to send more number of messages about the sedimentation information due to the constant change of the depositions than the ones located in the outer zones of the cages. Bearing in mind all this information, we have estimated the density of sensors needed as a function of the distance to the center of each cage. Figure 7.13 shows the sensor density for $\delta \mathrm{T}=15$. 


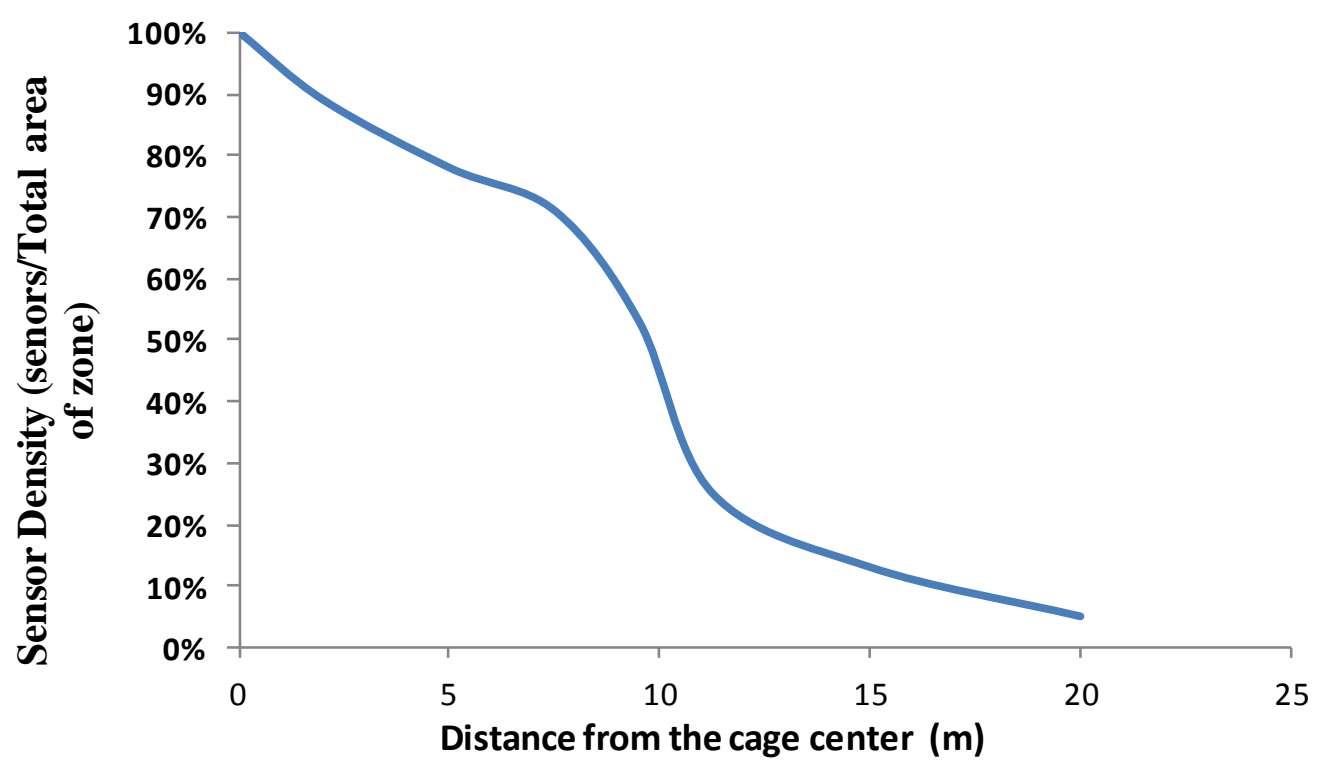

Figure 7.13. Sensor density as a function of distance to the center of the cage

Table 7.1 shows the number of sensors and the sensors density for $\delta \mathrm{T}=15$. These estimations are based on the area to be covered for each zone specified in Figure7.5. The sensor density estimation for each zone is proportional to the deposition level in each zone. We have estimated it bearing in mind that the maximum number is registered in the center of the cage. The sensors are distributed uniformly in each zone. As we can see, the distribution of sensors up to $10 \mathrm{~m}$. is fairly uniform. From $10 \mathrm{~m}$. in advance, the number of sensors decreases considerably because the amount of depositions observed in this area is quite smaller, and therefore the variation of this parameter is also lower.

Table 7.1. Density of sensors in each area

\begin{tabular}{|l|l|l|l|}
\hline \multicolumn{3}{|c|}{$\delta \mathbf{T}=\mathbf{1 5}$} & Sensors density \\
\hline Distance from the cage center $(\mathbf{m})$ & Resultant area $\left(\mathbf{m}^{\mathbf{2}}\right)$ & Number of sensors & $100 \%$ \\
\hline 1 & 3.14 & 3 & $89 \%$ \\
\hline 2 & 12.56 & 8 & $78 \%$ \\
\hline 5 & 78.5 & 46 & $71 \%$ \\
\hline 7.5 & 176.625 & 95 & $53 \%$ \\
\hline 9.5 & 283.385 & 114 & $25 \%$ \\
\hline 11.25 & 397.40625 & 75 & $13 \%$ \\
\hline 15 & 706.5 & 70 & $5 \%$ \\
\hline 20 & 1256 & 48 & 5 \\
\hline
\end{tabular}




\subsection{Low Cost Turbidity Sensor}

Nowadays, it is important to have wide knowledge in underwater parameters because large changes can be important in some areas such as aquaculture, pollution monitoring, sewage treatment or oceanography.

The Water Framework Directive [378] proposes to measure some physicochemical parameters like temperature, dissolved oxygen or salinity, among others, to evaluate the quality of all water bodies (including marine water till one nautical mile from the coast).

In lasts years, the most common methods to analyze the water and to define these variables were sampling in-situ and the subsequent analysis at laboratories. The use of sensors to perform this kind of measurements is becoming increasingly common because these methods can reduce the analysis cost of the physicochemical parameters. The use of sensor nodes can be a way to eliminate the manual process of sampling because the measurements are performed automatically in the environment (river, lake, fish farm...) by the sensor nodes. The use of sensors reduces costs avoiding going to the field for taking samples. When measurements and analysis are performed directly in the medium by using sensors, the sampling process is enhanced and we are sure that the number of samples is representative.

The lifetime of a physical sensor is longer than a chemical sensor. The chemical methods used at laboratories use reagents consumed during the analyses. Physical sensors also let us measure in real time. If we use several sensors forming a network along a river, it is possible to have measures along any type of environment such as a river bed or in the surrounding area of a marine fish farm. For these reasons, the use of sensor networks is growing day by day and now, it is easy to find many examples [161].

The turbidity is defined as the decrease of transparency of a water solution caused by the presence of dissolved or suspended particles. These particles reflected, scatter and attenuate the light [379].

Turbidity measurements are important for environmental monitoring and management. In some cases, the sampling process is complicated and it may alter the environmental conditions. The alteration of a sample can make it not being a representative sample, so it should not be taken into account. Turbidity is expressed in nephelometric turbidity units (NTU).

Turbidity values can vary by changes in the composition of the solids and some dissolved substances in water. In the seas, oceans and rivers those substances can have different origins and different effects. On one hand, the solids can come from the erosion of the emerged zones which provide nutrients to the water. On the other hand, the solids can come from different industrial effluents and in this case they can be dangerous for the environment. The suspended solids can also have a biological origin. They can be different type of microorganisms like phytoplankton, zooplankton or organic particles matter. Consequently, turbidity can indicate a wide range of situations (pollution, eutrophication or the increase of solids in the water mass).

The sediments in suspension can cause several environmental damages such as benthic smothering, irritation of fish gills and the transport of absorbed contaminants. These sediments also produce an attenuation of the light penetration and this affects to the photosynthesis process [380]. The light dispersion can be used to measure the turbidity at specific wavelength and at specific angle, usually $90^{\circ}$ [379]. 
In this Section, we propose the design of a low cost turbidity sensor. In addition we have performed a set of measurements in sea water for several turbidity levels. Finally, we have performed a verification process to ensure that our system is working correctly.

\subsubsection{Our Proposal}

Our system is based on the use of an infrared LED as a source of emission and a photodiode as a receiver. Both elements are disposed at an angle of $180^{\circ}$ and they are faced so that the photodiode can capture maximum infrared light from the LED. The infrared LED and the photodiode are placed at a distance of $4 \mathrm{~cm}$.

TSUS5400 is an infrared emitting diode using GaAs technology molded in a blue-gray tinted plastic package. This infrared diode has a peak wavelength of $950 \mathrm{~nm}$ and its angle of half intensity is $22^{\circ}$.

S186P is a high speed and high sensitive photodiode in a plastic package. It is an IR filter, spectrally matched to GaAs or GaAs on GaAlAs IR emitters ( $\geq 900 \mathrm{~nm}$ ). The large active area combined with a flat case gives a high sensitivity at a wide viewing angle. The angle of half sensitivity is $\pm 65^{\circ}$. S186P is covered by a plastic case with IR filter $(950 \mathrm{~mm})$ and it is suitable for near infrared radiation.

The transmitter circuit is powered by a voltage of $5 \mathrm{~V}$ while the photodiode is supplied with a voltage of $15 \mathrm{~V}$. To achieve these values, we use two voltage regulators of LM78XX series, where $\mathrm{XX}$ is 05 , for the transmitter circuit, and 15 for the receiver circuit. This type of regulatory permits a maximum output current of $1 \mathrm{~A}$.

In our system, the water turbidity is proportional to the potential difference registered in the resistance R3. To process the voltage values recorded in the receiver circuit a microcontroller is used. This element is responsible for taking the data and performs the necessary calculations to estimate the turbidity value of the analyzed solution. The model we have used is the PIC18F2525 which is fed at $5 \mathrm{~V}$. We can use the microcontroller to display information via a LCD display or to send the information through the RS232 port to a personal computer. Figure 7.13 shows the schematic of our turbidity sensor.
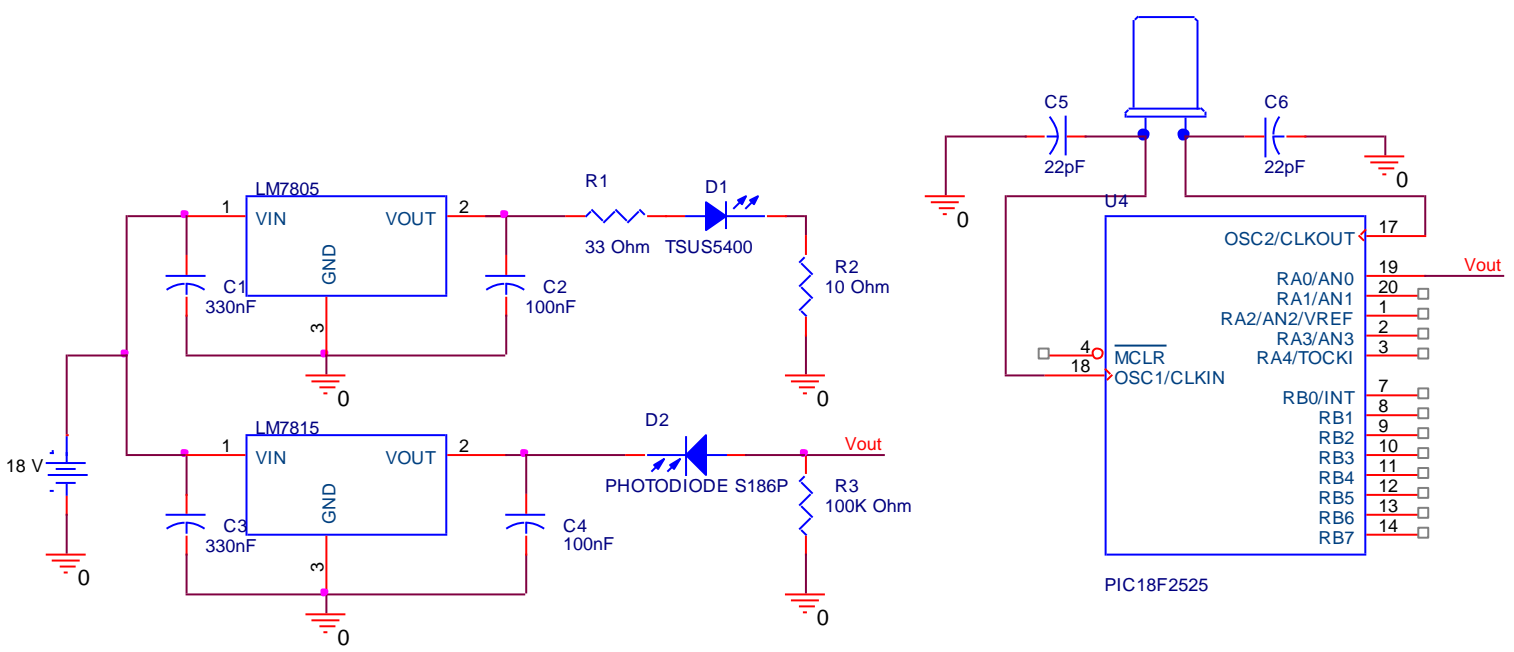

Figure 7.13. Esquematic of our low cost turbidity sensor. 


\subsubsection{Cost of Our Turbidity Sensor}

The electronic components used in our turbidity sensor can be easily found. We have asked for their prize in a Spanish electronic distributor. The prizes of the main components are shown in Table 7.2. As Table 7.2 shows, the prize of the needed electronic components is very low.

Table 7.2. Price of main components

\begin{tabular}{|c|l|}
\hline Component & Prize of components(in $€$ ) \\
\hline Voltage regulator LM7805 & 0.80 \\
\hline Voltage regulator LM7815 & 0.86 \\
\hline IR LED TSUS5400 & 0.176 \\
\hline Photodiode S186P & 0.94 \\
\hline PIC 18F2525 & 5.71 \\
\hline All Capacitors & 2 \\
\hline Oscilator & 2.18 \\
\hline
\end{tabular}

\subsubsection{Test Bench and Measurement Results}

Our main problem when taking the turbidity measurements was to determine the turbidity level of each solution test probe. These values were necessary in order to estimate the analytical model of our system. This section explains the process carried out to take the measurements and the calibration process of our system.

\subsubsection{Used elements}

On the one hand, we employed a calibrated turbidimeter to know the accurate value of turbidity of each test probe to be measured. The used device is Turbidimeter Hach 2100N. This turbidimeter can take measurements in two modes (ratio mode and non-ratio mode). We have used the non-ratio mode, which measures the turbidity by using a detector placed at 90 degrees.

All measures have been performed for seawater. The salinity of the sea water depends on the geographic position. Our samples were taken from the east coast of the Mediterranean Sea (Spain). The average water salinity ranges from 36 to 38 grams per liter. $\mathrm{pH}$ is 8.07 and its temperature is $21.1^{\circ} \mathrm{C}$.

Regarding to the elements used to generate the dissolutions with different turbidities, we have used a mixture of clay and silt. The silt is a loose material with a grain size between fine sand and clay. It is incoherent classic sediment transported in suspension by rivers. The particle size of our sample is between $0.002 \mathrm{~mm}$ and $0.06 \mathrm{~mm}$.

The glass containers used in our test had a volume of $30 \mathrm{ml}$.

First, took four calibration samples in the turbidimeter Hach 2100 N. Then, we took 10 samples with turbidities. Next, we performed more samples at lower concentrations than at higher ones because lower concentrations are most usual. The concentration samples we prepared are shown in Table 7.3. 
Table 7.3. Samples, their concentration and turbidity

\begin{tabular}{|l|l|l|l|}
\hline \multirow{2}{*}{ Sample } & \multicolumn{3}{|c|}{ Samples and Their Concentration } \\
\cline { 2 - 4 } & $\begin{array}{c}\text { Amount of clay } \\
\text { and silt added }(\mathbf{g})\end{array}$ & $\begin{array}{c}\text { Concentration } \\
(\mathbf{m g} / \mathbf{l})\end{array}$ & \multicolumn{1}{c|}{$\begin{array}{c}\text { Turbidity } \\
\text { (NTU) }\end{array}$} \\
\hline 1 & 0 & 0 & 0.072 \\
\hline 2 & 0.00109 & 36.33 & 23.7 \\
\hline 3 & 0.00301 & 100.33 & 50.8 \\
\hline 4 & 0.00331 & 110.44 & 60.1 \\
\hline 5 & 0.00526 & 175.33 & 97.2 \\
\hline 6 & 0.00696 & 232.10 & 123 \\
\hline 7 & 0.00782 & 260.66 & 142 \\
\hline 8 & 0.00980 & 326.66 & 171 \\
\hline 9 & 0.01821 & 607 & 385 \\
\hline 10 & 0.0453 & 1512.59 & 785 \\
\hline
\end{tabular}

Figure 7.14 shows the relationship between the amount of solute and the concentration of the test probe.

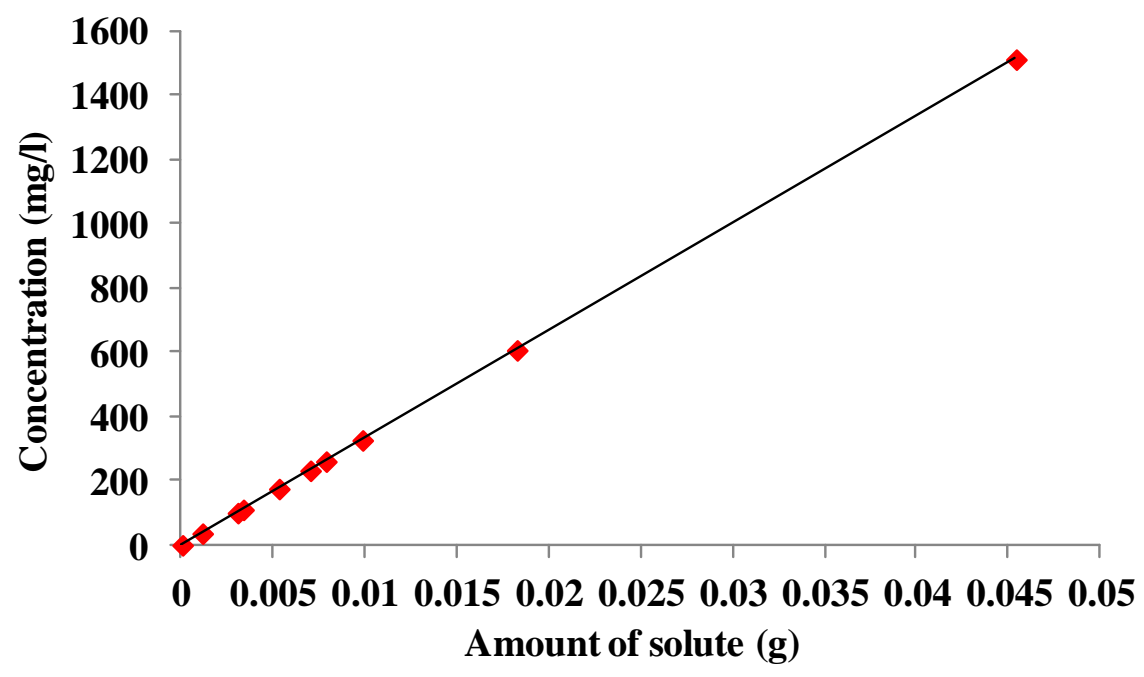

Figure 7.14. Value of dissolution turbidity as a function of the dissolution concentration.

We can extract the relationship between both values using Equation 7.8 with a correlation coefficient of 0.9999. Because the independent term is extremely small value, we can approximate this relationship without take into account the independent term.

$$
C=33.333 \cdot S+10^{-13} \cong 33.333 \cdot S
$$

Where $\mathrm{C}$ represents the concentration of the dissolution in $\mathrm{mg} / \mathrm{l}$ and $\mathrm{S}$ represents the amount of solute in $\mathrm{g}$. .

Each prepared sample has a turbidity value. Figure 7.15 shows the relationship between the concentration of silt-clay in seawater and the water turbidity value. 


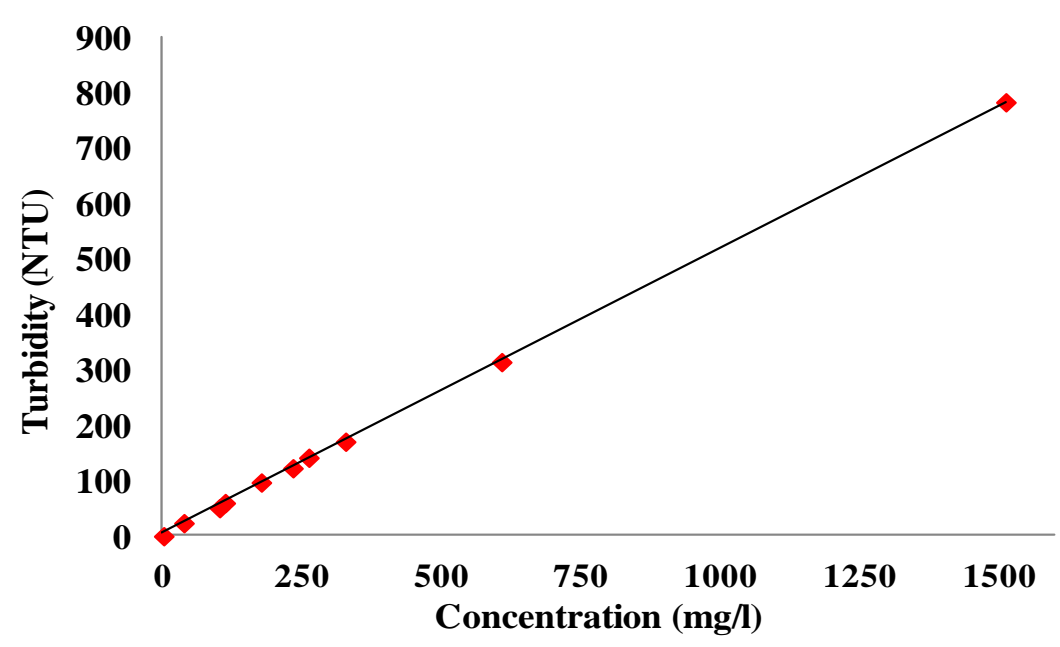

Figure 7.15. Value of dissolution concentration as a function of the amount of solute.

Equation 7.9 shows the relationship between both parameters with a correlation coefficient of 0.9999.

$$
T=0.517 \cdot C+3.0027
$$

Where, T represents the turbidity in NTU and $\mathrm{C}$ is the dissolution concentration in $\mathrm{mg} / \mathrm{l}$.

Finally, we can express the turbidity as a function of the amount of clay-silt dissolved combining Equation 7.8 and Equation 7.9. It is given by Equation 7.10.

$$
T=17233.161 \cdot S+3.0027
$$

Where, $\mathrm{T}$ represents the turbidity in NTU and $\mathrm{S}$, the amount of solute in $\mathrm{g}$.

\subsubsection{Obtained results}

After measuring the turbidity of each sample, we placed each one in our system in order to measure their voltage values.

Figure 7.16 shows the gathered output voltage value for each value of turbidity. The prepared samples are shown in red while the standard samples are shown in blue. We have also add, in black, our mathematical model extracted by Equation 7.11. We can see that the behavior of our system is not linear. Its behavior can be approximated to a linear function up to turbidity values of 300 NTU. But for higher turbidity values, the behavior is closer to a curve function.

Using these 14 samples, we have estimated the analytical expression that models the behavior of our turbidity sensor. Our system is modeled following Equation 7.11, where, $\mathrm{x}$ represents the independent term and $y$ the represents the dependent value.

$$
y=a+\frac{b}{(c+x+d \cdot \sin (e+f \cdot x)+g * \cos (h+i \cdot \sin (j+k \cdot x)))}
$$

We used Eureqa Formulize [358] to estimate the analytical model. Equation 7.12 relates the turbidity with the output voltage of our system.

$$
y=0.9767+\frac{1550}{(353.4+x+31.69 \cdot \sin (5.435+2.004 \cdot x)+19.34 * \cos (6.141+18.85 \cdot \sin (5.443+2.004 \cdot x)))}
$$




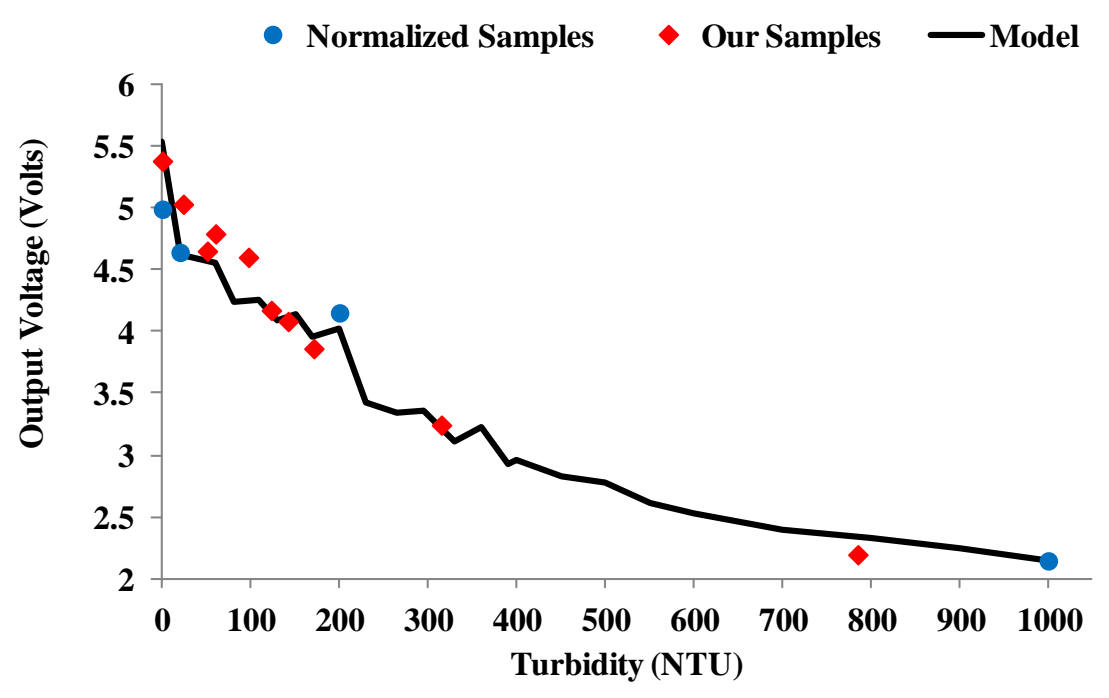

Figure 7.16. Output voltage as a function of the turbidity value and our mathematical model.

Finally, in order to check the accuracy of our system, we represent the output voltage value predicted by our model versus the value of output voltage obtained by our system. Figure 7.17 shows this relation. As Figure18 shows, most points remain in the black line which indicates a perfect match.

From Equation 7.12 and Figure 7.17, we observe that our equation has a correlation coefficient of 0.99897 and its maximum error is 0.1 Volts.

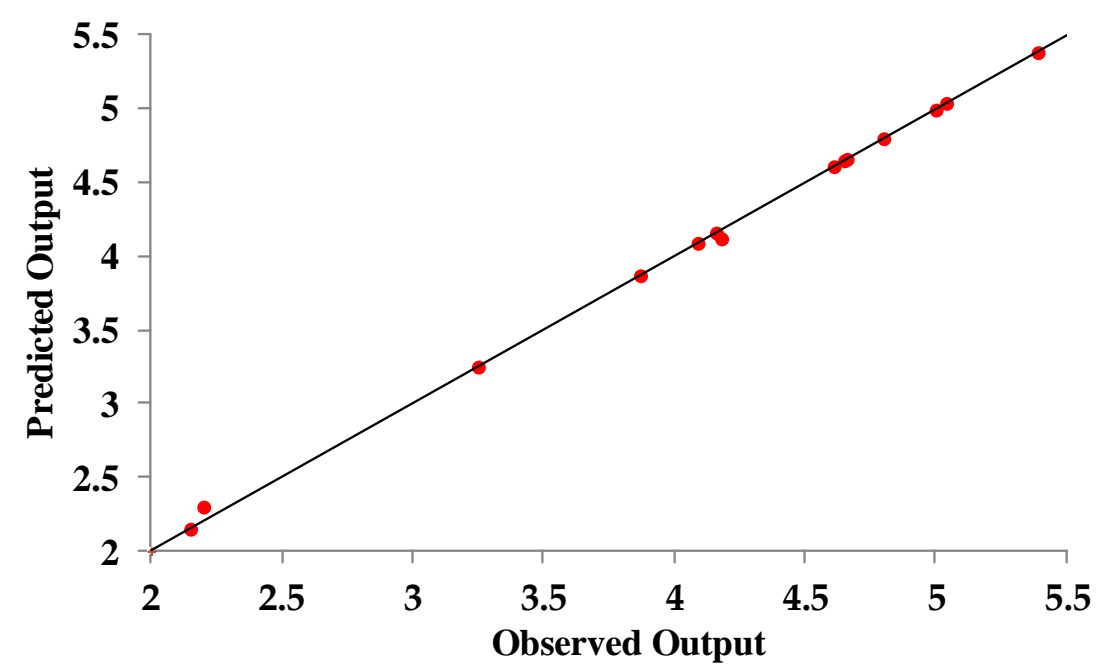

Figure 7.17. Observed output vs. Predicted output

\subsubsection{Verification}

In order to test the accuracy of our system, we have compared the values obtained by the turbidimeter Hach $2100 \mathrm{~N}$ and the values obtained by our turbidity sensor.

In the verification test, we have chosen low concentration samples, which means low turbidity. In these situations it is more difficult to measure accurately the turbidity. Commercial Turbidimeters 
have also problems in low turbidities. In order to perform this step, we have prepared four samples with unknown concentrations. The output voltages obtained for each sample are shown in Table 7.4.

Table 7.4. Unknown samples.

\begin{tabular}{|c|c|c|c|c|}
\hline \multirow{2}{*}{ Samples } & \multicolumn{4}{|c|}{ Samples to verify } \\
\cline { 2 - 5 } & $\boldsymbol{1}$ & $\mathbf{2}$ & $\mathbf{3}$ & $\mathbf{4}$ \\
\hline Output Votlage & $4.67 \mathrm{~V}$ & $4.82 \mathrm{~V}$ & $4.80 \mathrm{~V}$ & $4.33 \mathrm{~V}$ \\
\hline
\end{tabular}

Estimating the turbidity values by using Equation 7.12, we have introduced our samples (in blue) in Figure 7.18. It shows the behavior of our system.

In order to compare the estimated turbidity values with the measured values and the error for the four samples, we provide Table 7.5.

Table 7.5. Verification results.

\begin{tabular}{|l|c|l|r|r|}
\hline \multirow{2}{*}{\multicolumn{1}{|c|}{ Results }} & \multicolumn{4}{c|}{ Samples to verify } \\
\cline { 2 - 5 } & $\boldsymbol{1}$ & $\mathbf{2}$ & $\mathbf{3}$ & \multicolumn{4}{|c|}{} \\
\hline Turbidity obtained & 73.66 & 55.36 & 57.80 & 115.2 \\
\hline Turbidity (Hach 2100N) & 70 & 54.8 & 59 & 129 \\
\hline Error in Turbidity (\%) & 5.22 & 1.03 & 2.03 & 10.75 \\
\hline
\end{tabular}

The biggest error in turbidity is registered for the fourth sample $(10.75 \%)$ meanwhile the samples with lower turbidity present an error of $1.03 \%$.

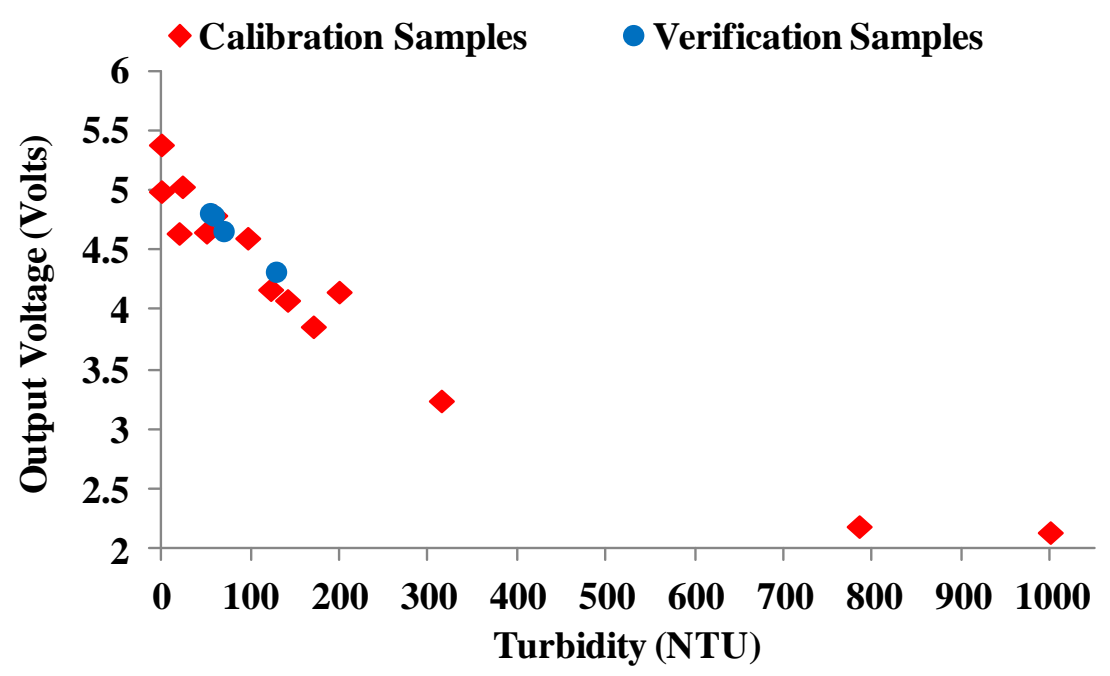

Figure 7.18. Output voltage value for the verification samples

\subsection{Fish Feeding Simulation}

Finally, we are going to show a simulation where we compare the amount of food that is thrown into the water in a regular process and the estimation of amount of food thrown into the water, if our 
system was used. We will also see the amount of pellets which are deposited on the seabed for both systems.

A regular feed dispenser can feed from just a few grams of pellets every second up to 200-300 grams per second. On-growing fish farm systems are delivered with the capacity up to $3 \mathrm{~kg}$ per second. They can feed up to 45 tons each hour. The bigger on-growing systems can dispense between $10 \mathrm{~kg}$ and 3 tons every hour [381].

In order to simulate our system, we have supposed two marine fish cage (biomass of 2.800 kilograms inside) in the Mediterranean zone. The first one contains Sparus aurata and the second cage contains Dicentrarchus labrax. We simulate two situations. The first one is the process of fish feeding in summer season with a temperature range of $24.5^{\circ} \mathrm{C}-26.5^{\circ} \mathrm{C}$. The second situation is the process of fish feeding during winter season with a temperature range on $12^{\circ} \mathrm{C}-13^{\circ} \mathrm{C}$. We have compared the process feeding for our system and for a regular procedure.

To do it, we have used Octave [382] which is a high-level language created for numerical computations.

We should note that the fishes are fed during 75 minutes in summer while in winter, this process takes 45 minutes.

Figure 7.19 shows the system performance for regular procedure during the seasons of summer and winter for both the Sparus aurata and the Dicentrarchus labrax. In a regular procedure in summer season, the Sparus aurata eats more than in winter season. In summer, they get to eat 3 tons of pellets each time (approximately 667 grams per second) while in winter, the Sparus aurata needs to eat approximately 500 kilograms (about 185 grams per second). In summer, the Dicentrarchus labrax usually eats a mean of 2.5 tons of pellets (approximately 556 grams per second) meanwhile this specie eats around 600 Kilograms each time (approximately 222 grams per second). As we can see all feeding processes are similar and they maintain these values during all process.

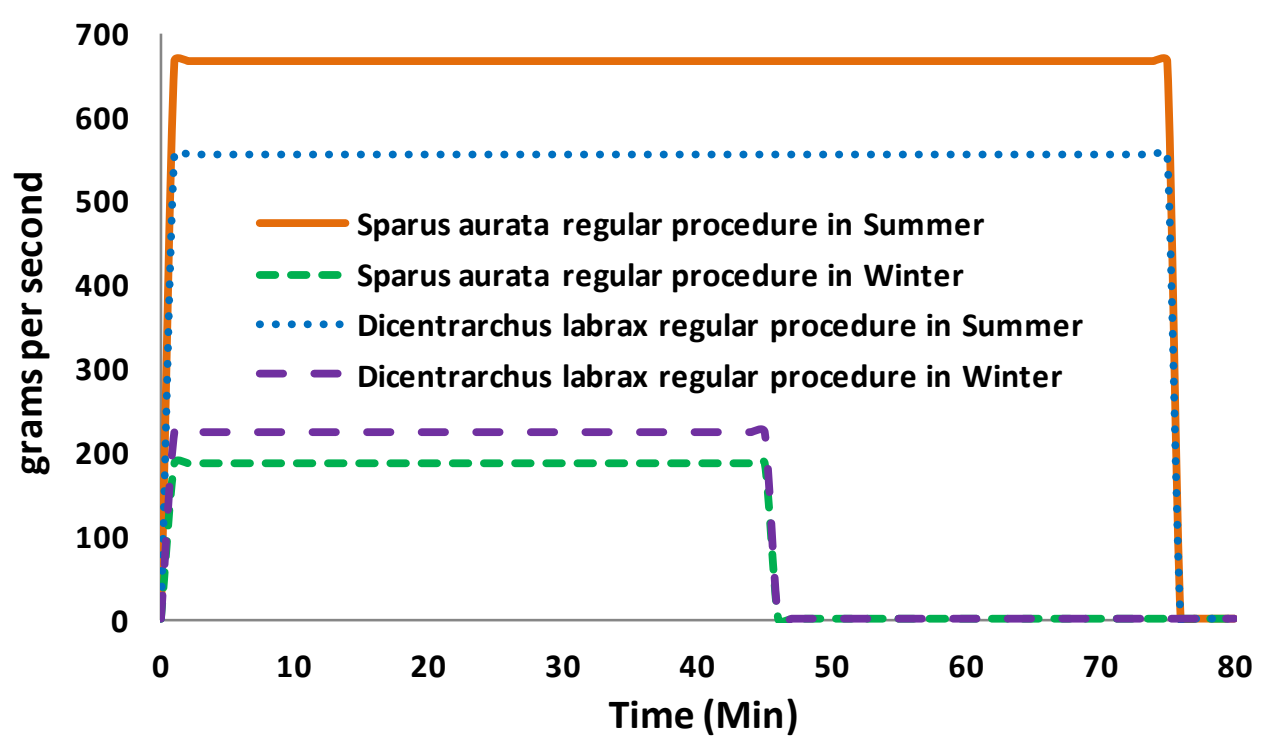

Figure 7.19. System performance for regular procedure in winter and summer.

Figure 7.20 shows the system performance for our system during the seasons of summer and winter for both the Sparus aurata and the Dicentrarchus labrax. In our case, the amount of pellets 
per second is the same for both seasons. We can see that the behaviour is the identical for the four cases. In summer season, both the Sparus aurata and the Dicentrarchus labrax eat constantly during 65 minute. In this moment, our system detects enough biomass moved to the bottom (although there are some fishes satisfied before). Our system changes its dispensing speed to a slower speed. This behavior is also repeated at 72 minutes. Finally at 79 minutes our system stops the dispenser

During the winter season, both species eat fewer amounts of pellets. The system behaviour is the same as the one explained before. At 30 minutes the system detects enough biomass moved to the bottom, so, our system changes its dispensing speed to a slower speed. This behaviour is also repeated at 38 minutes. Finally, at 45 minutes our system stops the dispenser.

We can see that our feeding process changes the feeding speed of the dispenser as the fishes are satisfied. This implies the saving of more food.

Pellets can be found in many different sizes; from tiny ones (around $2 \mathrm{~mm}$ in diameter) to big ones (around $28 \mathrm{~mm}$ of diameter). Both Sparus aurata and Dicentrarchus labrax usually eat $6 \mathrm{~mm}-$ $6.5 \mathrm{~mm}$ pellets when they have a weight of 500 grams. A regular pellet with this size has a mean weight of 0.5 grams.

Figure 7.21 shows the number of pellets that have fallen to the seabed for both species during a regular procedure management feeding process (using a table with time values or observing that there are no hungry fishes on the surface) during summer and winter seasons. We can see that the most amounts on pellets are concentrated at the end of the feeding process.

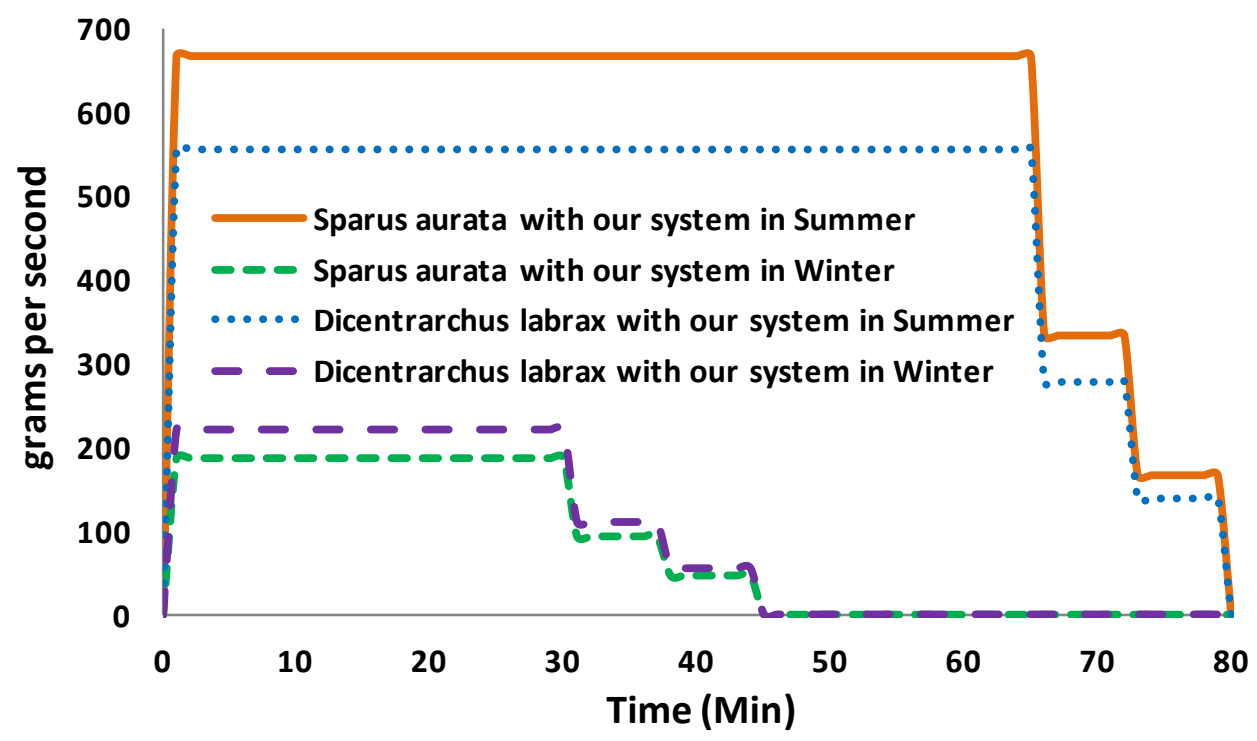

Figure 7.20. System performance for our system in winter and summer. 


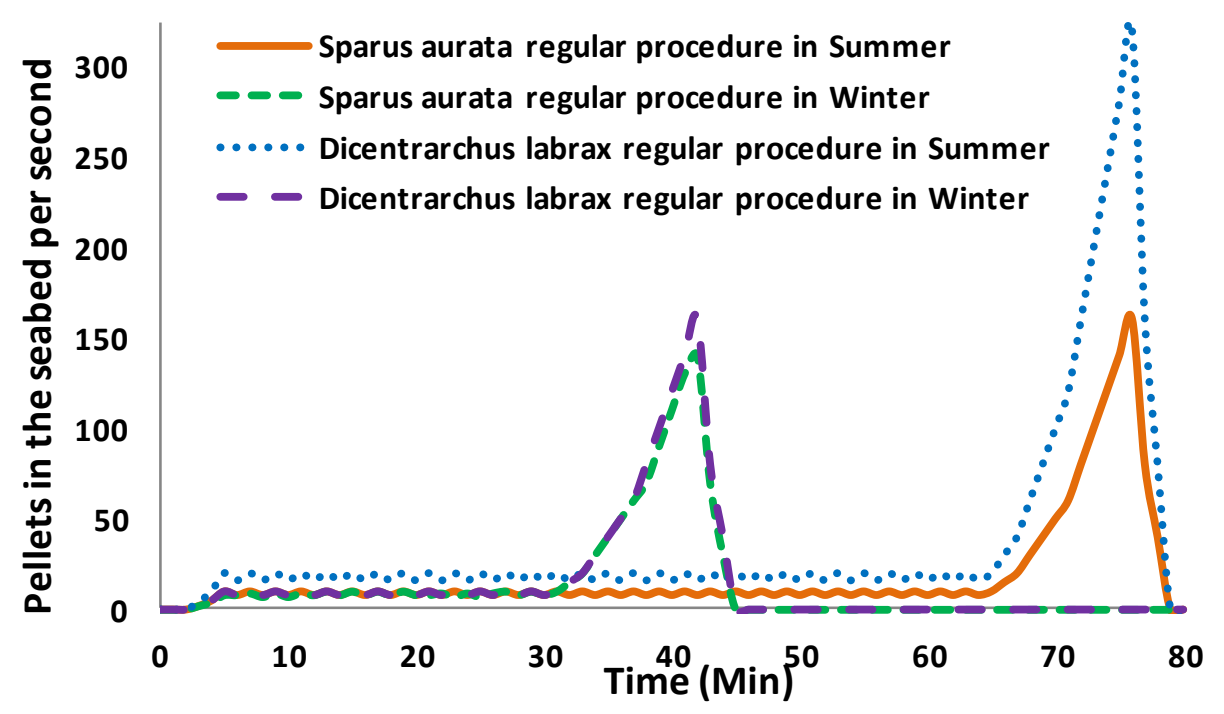

Figure 7.21. Pellets in the seabed for a regular procedure in winter and summer.

Figure 7.22 shows the number of pellets which have fallen to the seabed for both species during our automatic management feeding process in summer and winter seasons (using a table with time values or observing that there are no hungry fishes on the surface). We can compare the behaviour of our system with the behaviour of a regular process (Figure 7.21) and it is easy to see that fewer pellets fell to the seabed in our proposal. We can see that at the end of the feeding process the difference of the number of pellets in the seabed is substantially lower.

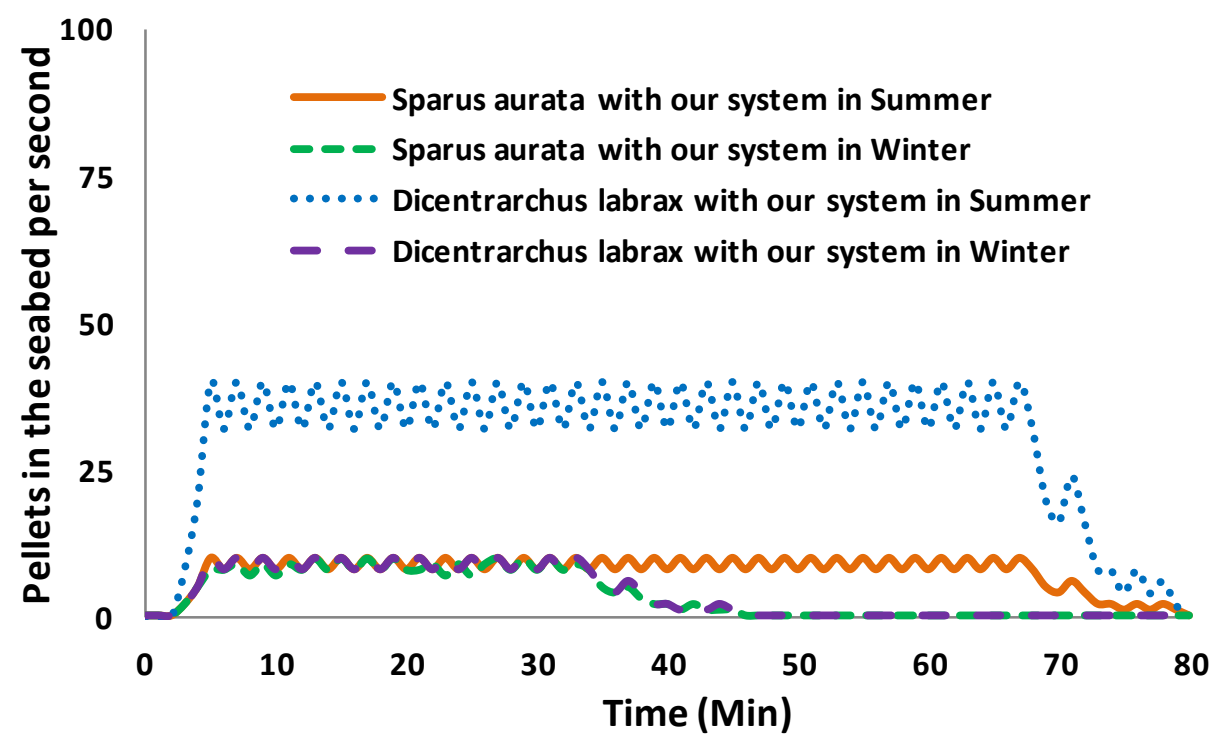

Figure 7.22. Pellets in the seabed for our system in winter and summer. 


\subsection{Conclusion}

In this chapter, we have seen that one of the most important problems in regards to sustainability in marine fish farms is the economical losses and environmental impact generated by the faecal pellets and uneaten feeds sprawling under the cages.

In this chapter, we have presented a system in order to monitor and control the process of fish feeding in marine fish farms. Our system is based on group of several sensors which control parameters such as depth under the cage, the mean current speed, the current direction, the settling speed, the position of each cage and the physic-chemical characterization of the water and water pollution, among others.

Our simulation shows that the system reduces the problem of wasted food, its economic losses and, furthermore, decreases the environmental impact. Moreover, the number of people controlling the feeding process is reduced because it is controlled automatically.

In addition, this chapter shows the deployment of two low cost oceanographic sensors. The first ones are based on an optical sensor and it is used to detect the amount of depositions in the seabed. The second sensor is a turbidity sensor which can help us to determine the quality of water. The most notable characteristic of both developments is its low manufacturing cost. While commercial sensors for oceanography can cost more than $2000-3000 €$, we are developing systems with much lower costs.

We can conclude that the development of low cost sensors can be possible and their application to the marine fish farms suppose a big economical cost regarding to the maintenance costs and a reduction on the environmental impact on surrounding areas.

Now, we are developing other oceanographic sensors taking in to account several energy constrictions to deploy them in a real fish farm. Future works will show the difference between our estimations and the real measurements using our sensors.

Finally, work presented in this chapter has been published in the following references [178], [179] and [180]. 


\section{Chapter 8}

\section{Conclusion}

\subsection{Introduction}

The implementation of a sensor network must always be preceded by an analysis of the problems presented in the environment. This process will give us the information about the sensing type we should perform the kind of devices we want to develop and the performance which can be obtained depending on the type of technology we use.

As we have seen in this dissertation, the scope of the WSN is very wide and sensor nodes share many characteristics. The first is that when we are working in natural environments the main problem we have, is the limitations of energy. This problem can be treated from different points of view. Maybe, the most important one is the hardware used to implement the node. We can also reduce the energy consumption of network using groups-based topology networks where the amount of routing information between groups and nodes is much lower than the information exchanged on a regular ad-hoc topology. Finally, the choice of an optimized communication protocols that take into account the amount of power remaining in the node to route the information another way, could help us to reduce the global energy consumption.

Because this kind of network uses wireless communication technologies, once defined the environment where the node will work and the type of device used, it is important to determine the transmission loss due to vegetation, reflections, refractions, hostility of medium, etc. This will give us an idea of the number of nodes we need to monitor an area and the type of design for our network. This will also define the most appropriate technology.

Finally, we should implement our proposals and verify its operation. 
This chapter presents the main conclusions of this Thesis and presents possible future research lines.

Because at the end of each chapter, we have presented the summaries and main conclusion about the proposals and measurements performed in each chapter, in this chapter, we are going to present these conclusions from a wider and global perspective.

\subsection{Conclusion and Contributions}

Throughout this dissertation we have seen the improvements provided by each of our proposals and deployments. In this section we are going to show a summary about every contribution performed on behalf this Doctoral Thesis.

In this dissertation, we have presented the main features and requirements of a wireless sensor node and a selection of the most used commercial wireless nodes. We have also presented the main issue which can be found in nodes and have defined the analytical expression of energy consumption in wireless nodes in function of the amount of information transmitted.

We have checked that network topologies based on group made fewer packet retransmissions, compared to the traffic generated in a network using common protocols such as AODV, DSDV or DRS. This reduction of packets is translated in to a considerable energy savings.

Regarding to WSN for environment monitoring, we have been seen that it is possible to develop several kinds of networks to apply them to any environment. We only need to specify the problem and the measurable parameters.

We would like also highlight the practical studies of the wireless signal behavior of IEEE $802.11 \mathrm{a} / \mathrm{b} / \mathrm{g} / \mathrm{n}$. From the results of the scenarios analyzed we have proposed a new method for the positioning of wireless sensor nodes, which reduces in $15 \%$ the number of nodes required to cover an area. Unlike the already existing models, our method only requires as input parameters, the size of the building and the transmission power of access point. Reducing the number of sensor nodes is important when we want to completely cover a given area. The smaller the number of nodes, the lower energy and economic costs will have the network.

Related to underwater environment, we have analyzed the performance of underwater communications in fresh water and its dependence on the working frequency and temperature. As we have seen at the end of Chapter 6, several previous works had performed erroneous theoretical estimations in regards to this type of communication. So far, we are working with distances of 26 $\mathrm{cm}$, but we are investigating ways to increase this distance. The main findings after performing all these tests it is possible to extract a combination of transmission parameters as a function of temperature.

Finally, we have proposed the use of a network of groups of sensors for monitoring and controlling the process of fish feeding. Just as people, fishes change their activity according to their level of satiety. Therefore, it seemed obvious to link the amount of food thrown to the fish with the activity and behavior of these.

The latest figures of Chapter 7 show that using our sensor network, we could reduce food thrown to the cages in a $23 \%$ compared to the food used in a regular procedure. Further, we would reduce by almost $10 \%$ the amount of food deposited in the seabed. 
Regarding the development of sensors, as we have seen in the case of turbidity sensor, the implementation of a sensor is not as expensive as we think. With a careful design, we can develop sensors for a few tens of Euros while its market price can exceed several thousands of Euros.

From the work performed in this Thesis, we have published 28 papers. All of them are directly or partially related to the presented work. Seven of these publications have been presented in international conferences and four of them are chapters in research books. The rest belong to international journals publications where eleven of them have Impact Factor (JCR) of ISI Thomson.

Last contributions and participations have been related to the inclusion of network security mechanisms [383]. As a future work, we would like to add the most suitable security mechanisms for each network in order to prevent any attack to the network and data corruption.

\subsection{Future Work and Research Lines}

Following the ideas and proposals presented in this dissertation, we can propose different futures projects to continue this investigation. Because underwater wireless communications based on the EM waves are still poorly investigated, most of these new ideas would be for underwater applications.

The first improvement we would like to provide to our developments, especially for underwater sensors (although it is applicable to other devices) is the use of the energy harvesting from other sources to provide energy to our devices. In this way, we would increase the network lifetime reducing the maintenance costs of devices.

Given in mind that underwater freshwater communications depend on several factors, such as temperature, operating frequency and transfer rates, we would like to design and develop an intelligent node. The node would be able to adapt its transmission conditions depending on water conditions. It would be able to switch between the transmission parameters and communicate their state to its neighboring nodes, to optimize the transmission between them. With the development of this node, we would also like to design a more efficient antenna underwater environment. We have not found any model that meets these characteristics. Finally, we would like to move all these tests at seawater, because the final goal it to apply these as if the aim is the application of marine farms.

Finally, we continue working on the development of low cost efficient sensor nodes for rural and underwater environments. We believe that, just as we have done with the turbidimeter, we can design and develop multiple devices with much lower costs than current ones without reducing their accuracy.

We are confident that the use of these sensors will provide significant cost savings and improved sustainability for marine farms. 


\section{Bibliography}

[1] J. Yick, B. Mukherjee, D. Ghosal, "Wireless sensor network survey". Compututer Networks 2008, Vol. 52, Pp. 2292-2330.

[2] D. S. Nyce, Linear position sensors: Theory and applications. New Jersey: Wiley-Interscience John Wiley \& Sons, Inc. (2004)

[3] J. Lloret, M. Garcia, J. Tomás, F. Boronat, "GBP-WAHSN: a group-based protocol for large wireless ad hoc and sensor networks". Journal of Computer Science Technology, 2008, Vol. 23, Pp. 461-480.

[4] M. Garcia, D. Bri, F. Boronat, J. Lloret, "A new neighbor selection strategy for group-based wireless sensor networks", In The Fourth International Conference on Networking and Services (ICNS 2008), Gosier, Guadalupe, March 16-21, 2008.

[5] IEEE Std 802.15.1 (2005) IEEE Standard for Information technology - Telecommunications and information exchange between systems - Local and metropolitan area networks - Specific requirements. - Part 15.1: Wireless medium access control (MAC) and physical layer (PHY) specifications for wireless personal area networks (WPANs). Pp. 0_1-580. Institute of Electrical and Electronics Engineers, Inc. New York, USA.

[6] IEEE Std 802.15.4 (2006) IEEE Standard for Information technology - Telecommunications and information exchange between systems - Local and metropolitan area networks - Specific requirements Part 15.4: Wireless Medium Access Control (MAC) and Physical Layer (PHY) Specifications for LowRate Wireless Personal Area Networks (WPANs). Pp. 0_1-305. Institute of Electrical and Electronics Engineers, Inc. New York, USA.

[7] IEEE Std 802.11 (2007) IEEE Standard for Information technology - Telecommunications and information exchange between systems - Local and metropolitan area networks - Specific requirements Part 11: Wireless LAN Medium Access Control (MAC) and Physical Layer (PHY) Specifications. Pp.11184. Institute of Electrical and Electronics Engineers, Inc. New York, USA.

[8] M. Garcia Pineda, PhD Thesis:"A group-based architecture and protocol for wireless sensor networks". Universidad Politécnica de Valencia, January 2013.

[9] J. Tomas, J. Lloret, D. Bri and S. Sendra, "Sensors and their Application for Disabled and Elderly People", Handbook of Research on Personal Autonomy Technologies and Disability Informatics, IGI Global. Pp. 311-330. 2011.

[10] J. Fraden, "Handbook of modern sensor: Physics, designs, and applications". 3rd ed. New York: Springer Science. (2003)

[11] L. K. Baxter, “Capacitive sensors: Designs and applications”. New York: IEEE Press. (1997).

[12] Cisco Report. Server power calculator analysis: Cisco UCS power calculator and HP power advisor. (2011)

[13] M. Kakemizu, A. Chugo, "Approaches to Green Networks". Fujitsu Scientific and Technical Journal. Vol. 45, Iss. 4, pp. $398-403$. (2009)

[14] A.P. Bianzino, C. Chaudet, D. Rossi, J-L. Rougier, “A Survey of Green Networking Research”. IEEE Communications Surveys \& Tutorials, vol.14, no.1, pp.3-20 (2012)

[15] V. Raghunathan, S.Ganeriwal and M.Srivastava, "Emerging techniques for long lived wireless sensor networks," IEEE Communications Magazine, vol.44, no.4, pp. 108- 114, April 2006

[16] N. A. Pantazis and D. D. Vergados, "A survey on power control issues in wireless sensor networks", Journal IEEE Communications Surveys and Tutorials, Vol. 9, Pp. 86-107, 2007

[17] S. Saxena, S. Mishra, A. Kumar and D. S. Chauhan, "Efficient Power Utilization Techniques for Wireless Sensor Networks-A Survey", International Journal on Computer Science and Engineering, vol.:3, Issue:2, Pp. 905-925, February 2011

[18] K. Akkaya and M. Younis," A survey on routing protocols for wireless sensor networks", Ad Hoc Networks, Vol. 3, Issue 3, Pp. 325-349, May 2005 
[19] C. E. Jones, K. M. Sivalingam, P. Agrawal and J. C. Chen, "A Survey of Energy Efficient Network Protocols for Wireless Networks" Journal Wireless Networks archive Vol. 7, Issue 4, pp 343-358. (2001)

[20] E. Ferro, F. and Fotorti, F. "Bluetooth and Wi-Fi wireless protocols: A survey and a comparison" . IEEE Wireless Communications, vol. 12, no.1, pp. 12-16, 2005.

[21] L.M. Feeney, and M. Nilsson, "Investigating the Energy Consumption of a Wireless Network Interface in an Ad Hoc Networking Environment", Proceedings of the 20th Annual Joint Conference of the IEEE Computer and Communications Societies (IEEE INFOCOM 2001). Anchorage, Alaska, USA, 22 - 26 April, Vol.3, pp. 1548-1557. IEEE Computer Society Press. Washington, USA.

[22] Y-C., Tseng, C-S. Hsu, and T-Y. Hsieh, "Power-saving protocols for IEEE 802.11-based multi-hop ad hoc networks", Proceedings of the 21st Annual Joint Conference of the IEEE Computer and Communications Societies (IEEE INFOCOM 2002), New York, NY, USA, 23-27 June, pp. 200-209. IEEE Computer Society Press. Washington, USA.

[23] G. Razzano, and A. Pietrabissa, "An Efficient Power Saving Mechanism for Wireless LAN", Proceedings of the Eighth IEEE International Symposium on Computers and Communication (ISCC'03), Kemer, Antalya, Turkey, 30 June-3 July, Vol.2, pp. 705- 709. IEEE Computer Society Press. Washington, USA.

[24] S. S. Meiyappan, G. Frederiks, S. and Hahn, S. "Dynamic Power Save Techniques for Next Generation WLAN Systems". Proceedings of the 38th Southeastern Symposium on System Theory (SSST), Cookeville, Tennessee, USA, 5-7 March, pp. 508-512, 2006. IEEE Computer Society Press. Washington, USA.

[25] X. Pérez, and D. Camps, "A Protocol Enhancement for IEEE 802.11 Distributed Power Saving Mechanisms No Data Acknowledgement". Proceedings of the 16th IST Mobile and Wireless Communications Summit, Budapest, Hungary, 1-5 July, 2007, pp. 1-7. IEEE Computer Society Press. Washington, USA.

[26] E-S. Jung, and N. H Vaidya, "An Energy Efficient MAC Protocol for Wireless LANs”, Proceedings of the 21st Annual Joint Conference of the IEEE Computer and Communications Societies (IEEE INFOCOM 2002), New York, NY, USA, 23-27 June, 2002, Vol.3, pp.1756- 1764. IEEE Computer Society Press. Washington, USA.

[27] S. Jayashree, B.S. Manoj, and C.S.R. Murthy, "A battery aware medium access control (BAMAC) protocol for Ad-hoc wireless network", Proceedings of the 15th IEEE International Symposium on Personal, Indoor and Mobile Radio Communications (PIMRC 2004), Barcelona, Spain, 5-8 September, 2004, Vol. 2, Pp. 995-999. IEEE Computer Society Press. Washington, USA.

[28] O. Younis, and S. Fahmy, "Distributed Clustering in Ad-hoc Sensor Networks: A Hybrid, EnergyEfficient Approach", Proceedings of the 23rd Annual Joint Conference of the IEEE Computer and Communications Societies (IEEE INFOCOM 2004). Hong Kong, China, 7-11 March, 2004, Vol.1, pp: 640-652. IEEE Computer Society Press. Washington, USA.

[29] G. Halkes, T. V. Dam, and K. Langendoen. Comparing energy-saving MAC protocols for wireless sensor networks. ACM Mobile Networks and Applications, Vol. 10, Issue: 5, Pp.783-791, 2005.

[30] MODIS Web Page. http://modis.gsfc.nasa.gov (accessed October 29, 2009).

[31] Z. Li, S. Nadon, J. Cihlar, "Satellite-based detection of Canadian boreal forest fires: development and application of the algorithm". International. Journal of Remote Sensing, Vol. 21, 3057-3069, (2000)

[32] T. Antoine-Santoni, J.-F. Santucci, E. De Gentili, X.Silvani, F. Morandini, "Performance of a protected wireless sensor network in a fire. Analysis of fire spread and data transmission". Sensors. Journal, Vol.9, Pp. 5878-5893, 2009.

[33] D. M. Doolin, N. Sitar, "Wireless sensors for wildfire monitoring". In Smart Structures and Materials 2005: Sensors and Smart Structures Technologies for Civil, Mechanical, and Aerospace Systems, San Diego, CA, USA, May 7, 2005.

[34] K. Sha, W. Shi, O. Watkins, "Using wireless sensor networks for fire rescue applications: requirements and challenges". In Proceedings of IEEE International Conference on Electro/information Technology, East Lansing, MI, USA, May 7-10, 2006; pp. 239-244. 
[35] B. Son, Y. Her, J. Kim, "A design and implementation of forest-fires surveillance system based on wireless sensor networks for South Korea Mountains". International Journal of Computer Science and Network (IJCSNS), Vol. 6, Pp.124-130, 2006.

[36] M. Hefeeda, M. Bagheri, "Forest fire modeling and early detection using wireless sensor networks". Ad Hoc Sensor Wireless. Networks, Vol. 7, Pp.169-224. 2009

[37] C. Hartung, R. Han, C. Seielstad, S. Holbrook, "FireWxNet: a multitiered portable wireless system for monitoring weather conditions in wildland fire environments". In ACM, 4th International Conference on Mobile Systems, Applications and Services, Uppsala, Sweden, June 19-22, 2006.

[38] E.M. Garcia, M.A. Serna, A. Bermúdez, R. Casado, "Simulating a WSN-based wildfire fighting support system" In Proceedings of the IEEE International Workshop on Modeling, Analysis and Simulation of Sensor Networks (MASSN-08), held in conjunction with the IEEE International Symposium on Parallel and Distributed Processing and Applications (ISPA 2008), Sydney, Australia, December 10-12, 2008.

[39] A. Bermúdez, R. Casado, E.M. García, A. Gomez, F.J. Quiles, J.R. Ruiz-Gallardo, "Empleo de una red de sensores en el reajuste de modelos de comportamiento del fuego en incendios forestales". $4^{\mathrm{a}}$ Conferencia Internacional sobre Incendios Forestales. Wildfire 2007, Sevilla, Spain, May 13-17, 2007.

[40] J.I. Huircan, J. Bustos, C. Muñoz, G. Vivallo, V. Barrigaz, G. Donoso and, M. Toneatti. "TICs y Ganadería: Manejo Electrónico de Ganado". In proceedings of international workshop, 3rd Encuentro Informática y Gestión (EIG2009), Temuco (Chile), Decembre 3-4, 2009.

[41] J. Hwang and, H. Yoe, "Study of the Ubiquitous Hog Farm System Using Wireless Sensor Networks for Environmental Monitoring and Facilities Control". Sensors. Vol. 10, Pp 10752-10777. December, 2010.

[42] A.I.C. Arce, A.R.B. Tech, A.C.S. Silva and, E.J.X. Costa, "Wireless sensor networks for bovine herd monitoring". Archivos de zootecnia. Vol. 58, No. 222, Pp 253-263. May, 2008.

[43] J. Quiza, T. Castelblanco and, D. González, "Sistema Web de Identificación de Ganado Bovino y Monitoreo en Tiempo Real de su Temperatura y Frecuencia Cardiaca Usando Redes de Sensores Inalámbricos". ENGI Revista Electrónica De La Facultad De Ingeniería. Vol. 1, No. 1, 2012.

[44] L.C. Hicks, W.S. Hicks W.S., R.A. Bucklin, J.K. Shearer, D.R. Bray, P. Soto and, V. Carvalho, "Comparison of Methods of Measuring Deep Body Temperatures of Dairy Cows". ASAE Proceeding of the 26th International Symposium, May 21-23, 2001, Louisville, Kentucku, (USA). Núm. 701P0201, Pp 432-438.

[45] A. Martinez, S. Schoenig, D. Andresen, S. Warren, "Ingestible Pill for Heart Rate and Core Temperature Measurement in Cattle". In proceedings of 28th Annual International Conference of the IEEE Engineering in Medicine and Biology Society (EMBS'06), August 30 - September 3, 2006, New York City, New York ( USA). Pp 3190-3193.

[46] L. Nagll, R. Schmitzl, S. Warren, T.S. Hildreth, H. Ericksonand, D. Andresen, "Wearable Sensor System for Wireless State-of-Health Determination in Cattle". In proceeding on the 25th Annual International Conference of the IEEE Emgimeers in Medicine and Biology Society, September 17-21, 2003. Cancun, (Mexico), Pp 3012-3015.

[47] EFE Agency. Online press article (August 11, 2012), "Que viene el lobo: las ovejas ya avisan a través de mensajes de texto".Available at: http://www.20minutos.es/noticia/1560855/0/ovejas/sms/lobo/ [Last access 15/05/2013].

[48] Y.R. Chen, K. Chao, and M.S. Kim, "Future trends of machine vision technology for agricultural applications". Computers and Electronics in Agriculture, 2002, Vol. 36, (2-3), Pp.173-191.

[49] T. Baidyk, E. Kussul, O. Makeyev, and A. Vega, "Limited receptive area neural classifier based image recognition in micromechanics and agriculture", International Journal of applied mathematics and informatics, Vol. 2, Issue 3, Pp. 96-103, 2008.

[50] C.-C. Yang, S.O. Prasher, J-A. Landry, H.S. Ramaswamy, A. DiTommaso, "Application of artificial neural networks in image recognition and classification of crop and weeds", Canadian Agricultural Engineering, Vol 42, Issue 3, Pp 147-152, 2000.

[51] C.-C. Yang, S.O. Prasher, J-A. Landry, J. Perret, and H.S. Ramaswamy, "Recognition of weeds with image processing and their use with fuzzy logic for precision farming", Canadian Agricultural Engineering, Vol 42, Issue 4, Pp 195-200, 2000 
[52] D. G. Sena, F.A. C. Pimto, D.M. Queiroz, and P. A. Viana, "Algoritmo de processamento de imagens para controle localizado de pragas na cultura do milho", 2nd International Symposium on Precision Agriculture, 12-14 June, 2002, Viçosa- Minas Gerais (Brazil).

[53] P.E. Cruvinel, E.R. Minatel, M.L. Mucheroni, S.R. Vieira, and S. Crestana, "An automatic method based on image processing for measurements of drop size distribution from agricultural sprinklers" Simpósio Brasileiro de Computação Gráfica e Processamento de Imagens (SIBGRAPI), 1996, vol 9, Pp.39-46, Caxambú- Minas Gerais (Brazil) .

[54] A. Macedo-Cruz, G. Pajares, M. Santos and I. Villegas-Romero, "Assessing the Current State of Oat after Frost Damage (Avena sativa L.) Based on Digital Image Sensors", Sensors, Vol. 11, Issue 6, Pp. 6015-6036, 2011.

[55] P. Kulkarni, D. Ganesan, P. Shenoy and Q. Lu, "SensEye: A Multitier Camera Sensor Network", Proceedings of the 13th annual ACM international conference on Multimedia MM'05, November 6-11, 2005, Singapore.

[56] L. Liu, H. Ma and X. Zhang, "Coverage analysis for target localization in camera sensor networks", Wireless Communications and Mobile Computing. Vol. 12, issue 14, p. 1239-1250, 2012.

[57] C. Istin, D. Pescaru, H. Ciocarlie, D. Curiac, A. Doboli, "Reliable Field of View Coverage in VideoCamera based Wireless Networks for Traffic Management Applications", IEEE Symposium on Signal Processing and Information Technology ISSPIT 2008, Sarajevo, Bosnia-Herzegovina, December 16-19, 2008, pp. 63-68

[58] W. S. Jang, and M. J. Skibniewski, "Wireless sensor technologies for automated tracking and monitoring of construction materials utilizing zigbee networks". ASCE Construction Research Congress: "The Global Construction Community". 6-8 May 2007, Grand Bahama Island, USA.

[59] N. Kurata, S. Saruwatari, and H. Morikawa, "Ubiquitous Structural Monitoring using Wireless Sensor Networks", International Symposium on Intelligent Signal Processing and Communications 2006 (ISPACS '06), 12-15 December, 2006, Yonago (Japan)

[60] S. Kim, S. Pakzad, D.E. Culler, J. Demmel, G. Fenves, S. Glaser, and M. Turon, "Health Monitoring of Civil Infrastructures Using Wireless Sensor Networks", IPSN 2007, Proceedings of the 6th International Conference on Information Processing in Sensor Networks. Cambridge, MA, April 2007.

[61] M.J. Whelan, M.V. Gangone, K.D. Janoyan, and R. Jha, "Wireless vibration monitoring for damage detection of highway bridges", In Proceedings of Smart sensor phenomena, technology, networks, and systems 2008 (SPIE 2008), 10-12 March 2008, San Diego, California (USA).

[62] G. Sun, G. Qiao, and B. Xu, "Corrosion Monitoring Sensor Networks with Energy Harvesting”, IEEE Sensors, Vol.11, No. 6, pp.1476-1477, 2011.

[63] C. Kim, H. Kim, J. Ryu, and C. Kim, "Ubiquitous sensor network for construction material monitoring" Journal of Construction Engineering and Management,.Vol.137, No. 2, pp.158-165, 2010.

[64] N. Lane, E. Miluzzo, H. Lu, D. Peebles, T. Choudhury, A. Campbell, A survey of mobile phone sensing, IEEE Communications Magazine, 2010, Vol. 48, Issue 9, pp. 140-150.

[65] C. Muldoon, G. OHare, M. OGrady, Collaborative agent tuning: Performance enhancement on mobile devices Engineering Societies in the Agents World VI, Lecture Notes in Computer Science, Volume 3963/2006, 2006, pp. 241-258.

[66] H. Turner, J. White, C. Thompson, K. Zienkiewicz, S. Campbell, D.C. Schmidt, Building Mobile Sensor Networks Using Smartphones and Web Services: Ramifications and Development Challenges, Handbook of Research on Mobility and Computing, Hershey, PA. 2009. Available: http://lsrg.cs.wustl.edu/ schmidt/PDF/new-ww-mobile-computing.pdf. [Last access 15/05/2013]

[67] A. Kansal, M. Goraczko, F. Zhao, Building a sensor network of mobile phones, 6th International Conference on Information Processing in Sensor Networks. Cambridge, Massachusetts, USA, April 24 27, 2007 pp. 547-548.

[68] I. Plaza, L. Martín, S. Martin, C. Medrano, Mobile applications in an aging society: Status and trends, Journal of Systems and Software, 2011, Vol 84, Issue 11, pp 1977-1988. 
[69] L. Camarinha-Matos, H. Afsarmanesh, Telecare: Collaborative virtual elderly support communities, 1st Workshop on Tele-Care and Collaborative Virtual Communities in Elderly Care, Porto, Portugal, 13 April, 2004.

[70] B. Chen, D. Pompili, Transmission of Patient Vital Signs Using Wireless Body Area Networks, Journal of Mobile Networks and Applications, 2011, Vol. 16, Issue 6, pp. 663-682.

[71] K.E. Thomas, J.A. Stevens, K. Sarmiento, M.M. Wald, Fall-related traumatic brain injury deaths and hospitalizations among older adults_-United States, 2005, Journal of Safety Research, 2008, vol. 39, Issue 3, pp. $269-272$.

[72] J. Dai, X. Bai, Z. Yang, Z. Shen, D. Xuan, "Mobile phone-based pervasive fall detection,” Personal and ubiquitous computing, 2010, Vol. 14, Issue 7, pp. 633-643.

[73] P. Martin, M. A. Sánchez, L. Álvarez, V. Alonso, J. Bajo, Multiagent system for detecting elderly people falls through mobile devices, International Symposium on Ambient Intelligence (ISAmI'11), Salamanca (Spain) 6-8 April 2011.

[74] P.N. Fahmi, V. Viet, C. Deok-Jai, "Semi-supervised fall detection algorithm using fall indicators in smartphone," Proceedings of the 6th International Conference on Ubiquitous Information Management and Communication, 2012, pp. 122.

[75] M. Sánchez, P. Martín, L. Álvarez, V. Alonso, C. Zato, A. Pedrero, J. Bajo, A New Adaptive Algorithm for Detecting Falls through Mobile Devices, Trends in Practical Applications of Agents and Multiagent Systems, 2011, pp. 17--24.

[76] M. Fahim, I. Fatima, S. Lee, and Y.K. Lee, Daily Life Activity Tracking Application for Smart Homes using Android Smartphone, 14th International Conference on Advanced Communication Technology, Yongin, South Korea, 19-22 February 2012 , pp. $241-245$.

[77] B. Kaluža, V. Mirchevska, E. Dovgan, M. Luštrek, M. Gams, An agent-based approach to care in independent living, Ambient Intelligence, Lecture Notes in Computer Science, 2010, vol. 6439, pp. 177186.

[78] A. Costa, G. Barbosa, T. Melo, P. Novais, Using mobile systems to monitor an ambulatory patient, in International Symposium on Distributed Computing and Artificial Intelligence, Advances in Intelligent and Soft Computing, 2011, vol. 91, pp. 337-344.

[79] R. Olfati-Saber, J. Fax, R. Murray, Consensus and cooperation in networked multi-agent systems, Proceedings of the IEEE, 2007, vol. 95, Issue 1, pp. 215-233.

[80] A. Arcelus, M. H. Jones, R. Goubran, F. Knoefel, Integration of smart home technologies in a health monitoring system for the elderly, 21st International Conference on Advanced Information Networking and Applications Workshops, 2007, Vol. 2, pp. 820--825.

[81] N. García, "Modelo de cobertura en redes inalámbricas basado en radiosidad por refinamiento progresivo", PhD. Thesis. Computer Science Department, University of Oviedo, Spain, March 2006.

[82] J. Lloret, J. J. López, and G. Ramos,'Wireless LAN Deployment in Large Extension Areas: The Case of a University Campus", In proceedings of the International Conference on Communication Systems and Networks 2003, Benalmádena, Málaga (España), September 8-10, 2003.

[83] J. Lloret, M. Garcia, F. Boronat and J. Tomás, "The Development of Two Systems for Indoor Wireless Sensors Self-location", Ad Hoc \& Sensor Wireless Networks: An International Journal, VOL: 8, Issue: 3-4, Pp. 235-258, June 2009.

[84] G. Foschini and M. Gans, "On Limits of Wireless Communications in Fading Environments when Using Multiple Antennas”, Wireless Personal Communications, vol. 6, no. 3, pp. 311-335, Mar. 1998.

[85] M. A. Maddah-ali and A. S. Motahari, "Communication over mimo x channels: Interference alignment, decomposition, and performance analysis”, IEEE Transactions on Information Theory. , Vol. 54, p.p. 3457 - 3470, August 2008.

[86] H. Xu, D. Chizhik, H. Huang, and R. Valenzuela, “A generalized space-time multiple-input multipleoutput (MIMO) channel model,” IEEE Trans. Wireless Commun., Vol. 3, 966-975, May 2004.

[87] W. Weichselberger, M. Herdin, H. Ozcelik and E. Bonek, "A stochastic MIMO channel model with joint correlation of both link ends", IEEE Trans. Wireless Commun., Vol. 5, 90-100, Jan. 2006. 
[88] IEEE 802.11-03/940r4: TGn Channel Models. IEEE. [Online]. Available: IEEE ftp://ieee:wireless@ftp.802wirelessworld.com/11/03/11-03-0940-02-000n-tgn-channel-models.doc [[Last access 15/05/2013]

[89] J. Medbo and P. Schramm, "Channel models for hiperlan/2 in different indoor scenarios", ETSI BRAN 3ERI085B. ETSI UMTS 30.03 V3.2.0, March 1998.

[90] E. Amaldi, A. Capone, M. Cesana, F. Malucelli, F. Palazzo, WLAN Coverage Planning: Optimization Models and Algorithms, in proceedings of the IEEE Vehicular Technology Conference (VTC-Spring 2004), 17-19 May 2004, Volume: 4, Page(s): 2219-2223

[91] A. Sandeep, Y. Shreyas, S. Seth, R. Agarwal, and G. Sadashivappa. "Wireless Network Visualization and Indoor Empirical Propagation Model for a Campus WI-FI Network", World Academy of Science, Engineering and Technology, vol. 42, pp.730-734, 2008.

[92] A. Eisenblatter, H.F. Geerdes, I. Siomina, "Integrated Access Point Placement and Channel Assignment for Wireless LANs in an Indoor Office Environment", IEEE International Symposium on World of Wireless, Mobile and Multimedia Networks (WoWMoM 2007), pp.1-10, Helsinki, Finland, June 18-21, 2007.

[93] M. Kamenetsky, and M. Unbehaun. "Coverage Planning for Outdoor Wireless LAN Systems", International Zurich Seminar on Broadband Communications, Access, Transmission, Networking, pp. 49-1 - 49-6, Zurich, Switzerland, February 19-21, 2002

[94] E. Amaldi, A. Capone, M. Cesana, and F. Malucelli, "Optimizing WLAN Radio Coverage", Procedings of the IEEE International Conference on Communications, vol. 1, pp. 180-184. June 20-24, 2004.

[95] E. Amaldi, A. Capone, M. Cesana, L. Fratta and F. Malucelli, "Algorithms for WLAN Coverage Planning", Wireless systems and mobility in next generation Internet, Springer Berlin / Heidelberg, vol. 3427/2005, pp. 52-65, 2005.

[96] K. Kaemarungsi and P. Krishnamurthy, "Properties of Indoor Received Signal Strength for WLAN Location Fingerprinting". In proceedings of The First Annual International Conference on Mobile and Ubiquitous Systems: Networking and Services 2004 (MOBIQUITOUS 2004), pp. 14-23, Boston, Massachusetts, USA, August 22-26, 2004.

[97] J. Lloret, J.J. López, C. Turró, and S. Flores, “A Fast Design Model for Indoor Radio Coverage in the 2.4 GHz Wireless LAN", 1st International Symposium on Wireless Communication Systems 2004 (ISWCS'04), Port Louis (Mauricio Island), September 20-22, 2004.

[98] J. Lloret and J.J. López, "Despliegue de Redes WLAN de Gran Extensión, el Caso de la Universidad Politécnica de Valencia", XVIII Simposium Nacional de la Unión Científica Internacional de Radio, A Coruña, Spain, September 10-12, 2003.

[99] J.N. Davies, V. Grout and R. Picking, "Prediction of Wireless Network Signal Strength within a Building", 7th International Network Conference (INC 2008), Plymouth, UK, 8-10 July 2008

[100] C.Prommak, J. Kabara, D. Tipper and, C. Charnsripinyo, "Next generation wireless LAN system design", In proceedings of Military communications conference (MILCOM 2002). Anaheim, California, October 7-10, 2002.

[101] I. Broustis, K. Papagiannaki, S.V. Krishnamurthy, M. Faloutsos, V.P. Mhatre, "Measurement-Driven Guidelines for 802.11 WLAN Design," The IEEE/ACM Transactions on Networking, vol.18, no.3, pp.722-735.

[102] D. Niculescu, "Interference map for 802.11 networks". In Proceedings of the 7th ACM SIGCOMM Conference on Internet Measurement IMC '07. San Diego, California, USA, October 24 - 26, 2007.

[103] P. Fuxjager, D. Valerio, F. Ricciato, "The myth of non-overlapping channels: interference measurements in IEEE 802.11". 4th Annual Conference on Wireless On demand Network Systems and Services (IEEE/IFIP WONS'07). Oberguyrgl, Austria, Pp. 1-8. Jan. 2007.

[104] J. Padhye, S. Agarwal, V. Padmanaban, L. Qiu, A. Rao, and B. Zill, "Estimation of Link Interference in Static Multi-Hop Wireless Networks," In proceedings of the 5th Conference on Internet Measurement 2005, Berkeley, California, USA, October 19-21, 2005. 
[105] J. Jun, P. Peddabachagari, and M. Sichitiu. "Theoretical Maximum Throughput of IEEE 802.11 and its Applications". In Proceedings of the IEEE International Symposium on Network Computing and Applications, pages 249-257, Cambridge, MA, April 2003.

[106] B. Bing, "Measured performance of the ieee 802.11 wireless LAN," in proceedings 26th Conference on Local Computer Networks, Lowell, Massachusetts, USA, 17-20 October, 1999.

[107] M. Peng, H. Chen, Y. Xiao, S. Ozdemir, A.V. Vasilakos, and J. Wu, "Impacts of sensor node distributions on coverage in sensor networks". Journal of Parallel and Distributed Computing, Vol.71, No.12, pp.1578-1591, 2011.

[108] R. Mulligan, and H.M. Ammari, "Coverage in Wireless Sensor Networks: A Survey". Network Protocols and Algorithms. Vol. 2, No. 2, pp. 27-53, 2011.

[109] S. Martínez, and F. Bullo, "Optimal sensor placement and motion coordination for target tracking", Automatica Vol.42, No. 4, pp. 661-668, 2006.

[110] A. Redondi, M. Tagliasacchi, M. Cesaría, L. Borsani, P. Tarrío Alonso, and F. Salice, F. "LAURA: LocAlization and Ubiquitous monitoRing of pAtients for health care support". In proceedings of the IEEE 21st International Symposium on Personal, Indoor and Mobile Radio Communications Workshops, Estambu (Turkey), September 26-29, 2010.

[111] E. Coca, and V. Popa, "Wireless sensor location systems comparative performances evaluation", In proceedings of the IEEE 18th International Symposium for Design and Technology in Electronic Packaging (SIITME), Alba Iulia (Romania), 25-28 Oct. 2012

[112] X. Li, X., H. Shi, and Y. Shang, "Sensor network localisation based on sorted RSSI quantisation". International Journal of Ad Hoc and Ubiquitous Computing, Vol.1,No.4, pp.222-229, 2009.

[113] S.S Dhillon, and K. Chakrabarty, "Sensor placement for effective coverage and surveillance in distributed sensor networks", In Proceedings of. IEEE Wireless Commununication Network Conference, pp. 1609-1614, 2003.

[114] L. Liu, S. Zhou, J-H Cui. "Prospects and problems of wireless communications for underwater sensor networks". Wireless Communications and Mobile Computing - Special Issue on Underwater Sensor Networks. Vol. 8, no. 8, pp. 977-994, 2008.

[115] M. Stojanovic, "Underwater acoustic communication," Wiley Encyclopedia of Electrical and Electronics Engineering, John Wiley \& Sons, pp. 688-698, 1998.

[116] D.E Chaitanya, C.V Sridevi, G.S.B Rao, "Path loss analysis of underwater communication systems", IEEE Students' Technology Symposium (TechSym 2011), Kharagpur (India), pp.65-70, Jan. 2011.

[117] A. Sehgal,I. Tumar, J. Schonwalder, "Variability of available capacity due to the effects of depth and temperature in the underwater acoustic communication channel", OCEANS 2009 - Bremen, Germany, pp.1-6, May 2009.

[118] S. Arnon, "Underwater optical wireless communication network", Opt. Eng. 49, 015001. Jan 15, 2010; doi:10.1117/1.3280288

[119] M.J. Buckingham, "Ocean-acoustic propagation models", J. Acoustique, 3, pp. 223-287, June 1992.

[120] M. A. Ainslie, J. G.McColm, "A simplified formula for viscous and chemical absorption in sea water," Journal of the Acoustical Society of America, vol. 103, no. 3, pp. 1671-1672, 1998.

[121] J. Preisig, "Acoustic propagation considerations for underwater acoustic communications network development," in Proceedings of First ACM International Workshop on Underwater Networks (WUWNet), Los Angeles, CA, Sep. 2006.

[122] M. I. Mishchenko, L. D. Travis, A. A. Lacis, "Scattering, absorption, and emission of light by small particles", Cambridge University, New York, 2002.

[123] R. G. Newton, "Scattering theory of waves and particles", 2nd edition, Springer-Verlag, New York, 2002.

[124] C.A. Balanis, "Advanced Engineering Electromagnetics". John Wiley \& Sons, New York, NY, 1989. 
[125] I. Wells, A. Davies, X. Che, P. Kear, G. Dickers, X. Gong, and M. Rhodes, "Node Pattern Simulation of an Undersea Sensor NEtwork using RF Electromagnetic Communications". Ultra Modern Telecommunications \& Workshops, St. Petersburg (Russia), 12-14 Oct. 2009.

[126] M.R. Frater, M.J. Ryan, and R.M. Dunbar, "Electromagnetic Communications within Swarms of Autonomous Underwater Vehicles". In Proc. ACM WUWNet 2006, Los Angeles, California, (USA), Sep. 25, 2006, pages 64-70.

[127] D. Anguita, D. Brizzolara, and G. Parodi, "Optical Communication for Underwater Wireless Sensor Networks: a VHDL-implementation of a Physical Layer 802.15.4 Compatible". IEEE OCEANS 2009. May 2009. Europe, pp. 1-2.

[128] N. Nowsheen, C. Benson, and M. Frater, "A High Data-Rate, Software-Defined Underwater Acoustic Modem" IEEE OCEANS 2010, Sept. 2010, pp 1-5.

[129] N. Nowsheen, C. Benson, C. and M. Frater, "Design of a high frequency FPGA acoustic modem for underwater communication". IEEE OCEANS 2010. May 2010. Sydney, pp. 1-6.

[130] S. Jiang, and S. Georgakopoulos, "Electromagnetic Wave Propagation into Fresh Water". Journal of Electromagnetic Analysis and Applications 2011, Vol.3, Issue.07, Pp. 261-266.

[131] T. Melodia, H. Kulhandjian, L. C. Kuo, and E. Demirors, "Advances in Underwater Acoustic Networking. Mobile Ad Hoc Networking: Cutting Edge Directions, Second Edition, 2013, Pp.804-852.

[132] J. Partan, J. Kurose and, B.N. Levine, "A survey of practical issues in underwater networks". ACM SIGMOBILE Mobile Computing and Communications Review 2007, Vol. 11, Issue. 4, Pp. 23-33.

[133] X. Che, I. Wells, G. Dickers, P. Kear, and X. Gong, "Re-evaluation of RF electromagnetic communication in underwater sensor networks". IEEE Communications Magazine 2010, Vol. 48, Issue. 12, Рp.:143-151.

[134] A.A. Abdou A. A., A. Shaw, A. Mason, A. Al-Shamma'a, J. Cullen and S. Wylie, "Electromagnetic (EM) wave propagation for the development of an underwater Wireless Sensor Network (WSN)", IEEE Sensors. October 28-31, 2011. Limerick, Ireland

[135] O. M. Perez, R. G. Goss, T. C. Telfer, and L.M. del Campo Barquin, "Water quality requirements for marine fish cage site selection in Tenerife (Canary Islands): predictive modelling and analysis using GIS”. Aquaculture, 224 (1-4), 51-68., 2003.

[136] U. Neumeier U., P.L. Friend,. U. Gangelhof, J. Lunding, M. Lundkvist, A. Bergamasco, C.L. Amos, and M. Flindt. "The influence of fish feed pellets on the stability of seabed sediment: A laboratory flume investigation. Estuarine", Coastal and Shelf Science, Vol. 75, Issue. 3, Pp.347-357, 2007.

[137] S. Porrello, P. Tomassetti, L. Manzueto, M.G. Finoia, E. Persia, I. Mercatali and P. Stipa, "The influence of marine cages on the sediment chemistry in the Western Mediterranean Sea". Aquaculture, Vol. 249, Pp. 145- 158, 2005.

[138] E. Mantzavrakos, M. Kornaros, G. Lyberatos and P. Kaspiris. "Impacts of a marine fish farm in Argolikos Gulf (Greece) on the water column and the sediment". Desalination, Vol. 210, Pp.110-124, 2007.

[139] M. J. Añón. "Un sistema informático para optimizar la alimentación de los peces", R+D OTT-CSIC Valencia. February 25, 2008. Available at:http://www.ott.csic.es/rdcsic/rdcsicesp/rdal28esp.htm

[140] C.J. Cromey, T.D. Thomas and K.D. Black, "DEPOMOD - Modeling the deposition and biological effects of waste solids from marinecage farms", Aquaculture, 214, pp 211-239, 2002

[141] J.G. Ferreira, A.J.S. Hawkins and S.B. Bricker, "Management of productivity, environmental effects and profitability of shellfish aquaculture - the Farm Aquaculture Resource Management (FARM) model”, Aquaculture 264, pp. 160-174, 2007

[142] O. M. Pérez, T. C. Telfer, M. C. M. Beveridge and L. G. Ross, "Geographical information systems (GIS) as a simple tool to aid modelling of particulate waste distribution at marine fish cage sites". Estuarine, Coastal and Shelf Science, 54, pp. 761-768, 2002

[143] R.A. Corner, A.J. Brooker, T.C. Telfer and L.G. Ross, "A fully integrated GIS-based model of particulate waste distribution from marine fishcage sites", Aquaculture 258, pp 299-311, 2006 
[144] F. Huettmann, "Towards a Marine Environmental Information System (MEnvIS) for the Northwest Atlantic: Experiences and suggestions from a multi-disciplinary GIS conservation", WSEAS Transactions on Biology and Biomedicine. Volume 1, Issue 1, January 2004.

[145] W.D. McCausland, E. Mente, G.J. Pierce and I. Theodossiou, "A simulation model of sustainability of coastal communities: Aquaculture, fishing, environment and labour markets", Ecological Modelling, 193 (3-4), pp. 271-294, 2006

[146] G. Lloret Mauri and J. Lloret Mauri, "Simulator Software for Marine Fish Farms Sustainability", WSEAS TRANSACTIONS on ENVIRONMENT and DEVELOPMENT. Volume 3, Issue 12, pp. 214222, December 2007

[147] A. C. Ziegler, "Issues related to use of turbidity measurements as a surrogate for suspended sediment". Turbidity and Other Sediment Surrogates Workshop, Reno, Nevada, USA, April 30 - May 2, 2002.

[148] R. J. Davies-Colley and, D. G. Smith,"Turbidity suspended sediment, and water clarity: a review" Journal of the American Water Resources Association Vol. 37, No. 5 (Oct. 2001) pp. 1085-1101.

[149] E. A. Steel and, S. Neuhausser, "A comparison of methods for measuring visual water clarity", Journal of the North American Benthological Society Vol. 21, No. 2 (Jun., 2002), pp. 326-335

[150] D. G. Smith., R. J. Davies-Colley, J. Knoeff and , G. W. J. Slot, "Optical Characteristics of New Zealand Rivers in Relation to Flow", Journal of the American Water Resources Association Vol. 33, No. 2 (April 1997), pp. 301-312.

[151] A. F. Bin Omar and, M. Z. Bin MatJafri, "Turbidimeter design and analysis: a review on optical fiber sensors for the measurement of water turbidity”, Sensors Vol. 9, No 10 (2009), pp. 8311-8335.

[152] M. J. Sadar, "Turbidity Science". Technical Information Series, Booklet 11; Hach Company: Loveland, USA, 1998

[153] D. H. Schoellhamer and, S. A. Wright "Continuous measurement of suspended-sediment discharge in rivers by use of optical backscatterance sensors" Workshop on Erosion and Sediment Transport Measurement in Rivers: Technological and Methodological Advances, Oslo, Norway, 19-21 (June 2002).

[154] T. S. Melis, D. J. Topping and, D. M. Rubin, "Testing laser-based sensors for continuous in situ monitoring of suspended sediment in the Colorado River, Arizona", Workshop on Erosion and Sediment Transport Measurement in Rivers: Technological and Methodological Advances, Oslo, Norway, 19-21 June 2002.

[155] J. R. Gray, T.S. Melis, E. Patiño, M.C. Larsen, D.J. Topping, P.P. Rasmussen, C. Figueroa-Alamo, "US Geological Survey research on surrogate measurements for suspended sediment" 1st Interagency Conference on Research in Watersheds Benson, Arizona, USA, 27-30 Oct. 2003, pp. 95-100.

[156] H. Chanson, M. Takeuchi and, M. Trevethan "Using turbidity and acoustic backscatter intensity as surrogate measures of suspended sediment concentration in a small subtropical estuary" Journal of Environmental Management, Vol. 88, No 4 (2008), pp. 1406-1416.

[157] S. Sendra, J. Lloret, M. García, and J.F. Toledo, "Power Saving and Energy Optimization Techniques for Wireless Sensor Neworks". Journal of communications, Vol. 6, issue. 6,Pp. 439-459.

[158] J. Lloret, S. Sendra, H. Coll, and M. Garcia, "Saving energy in wireless local area sensor networks". The Computer Journal,Vol. 53, Issue. 10, Pp. 1658-1673, 2010.

[159] S. Andrade, S. Sendra, E. Granell, and J. Lloret, "Green Networking and Communications" in Book Towards green networks using optimized network devices, CRC Press, Taylor and Francis. 2013

[160] S. Andrade, E. Ruiz-Sanchez, S. Sendra, and J. Lloret, "Power Consumption in Network Devices, based on the Routing Protocol Used" in Proceedings of The Second International Conference on Green Communications and Networking (GreeNets 2012). Gandia, Valencia (España), October,24 - 26, 2012.

[161] M. Garcia, D. Bri, S. Sendra, and J. Lloret,"Practical deployments of wireless sensor networks: A survey". International Journal on Advances in Networks and Services, Vol. 3, Issues. 1 and 2, Pp.170$185,2010$. 
[162] J. Tomas, J. Lloret, D. Bri, and S. Sendra, S. "Sensors and their Application for Disabled and Elderly People. Handbook of Research on Personal Autonomy Technologies and Disability Informatics, Vol 1, Chapter 22.,2010

[163] J. Lloret, M. Garcia, D. Bri, and S. Sendra, "A wireless sensor network deployment for rural and forest fire detection and verification. Sensors, Vol. 9, Issue 11, Pp.8722-8747, 2009

[164] J. Lloret, I. Bosch, S. Sendra, and A. Serrano, "A wireless sensor network for vineyard monitoring that uses image processing. Sensors, Vol.11, Issue 6, Pp. 6165-6196, 2011

[165] S. Sendra, J. Lloret, A. T. Lloret and J. J. P. C. Rodrigues, "A Wireless Sensor Network to Detect the Degeneration of Cement used in Construction", International Journal of Ad Hoc and Ubiquitous Computing (IJAHUC) ACCEPTED and in Press process. 2013 Available at: $\mathrm{http}: / / \mathrm{w} w \mathrm{w}$. inderscience.com/info/ingeneral/forthcoming.php?jcode=ijahuc

[166] S. Sendra, E. Granell, J. Lloret, and J. J. P. C. Rodrigues, ”Smart Collaborative Mobile System for Taking Care of Disabled and Elderly People" International Journal of Mobile Networks and Applications. S.I: "Wireless Technology for Pervasive Healthcare". ACCEPTED and in Press process. 2013 Available at: http://www.inderscience.com/info/ingeneral/forthcoming.php?jcode=ijahuc

[167] F. Llario, S. Sendra, L. Parra, J. Lloret, "Detection and Protection of the Attacks to the Sheep and Goats Using an Intelligent Wireless Sensor Network", 3rd IEEE Smart Communication Protocols \& Algorithms 2013 - Summer, Budapest (Hungria), 9 - 13 de Junio, 2013

[168] S. Sendra, P. Fernandez, C. Turro, and J. Lloret, "IEEE $802.11 \mathrm{a} / \mathrm{b} / \mathrm{g} / \mathrm{n}$ Indoor Coverage and Performance Comparison". In 6th International Conference on Wireless and Mobile Communications (ICWMC 2010). September 20-25, 2010 - Valencia, Spain

[169] D. Bri, H. Coll, S. Sendra, and J. Lloret, Providing Outdoor and Indoor Ubiquity with WLANs", in Handbook of Research on Mobility and Computing: Evolving Technologies and Ubiquitous Impacts, Chapter 70, Pp. 1155-1168, IGI Global, 2011.

[170] S. Sendra, M. Garcia, C. Turro, C., and J. Lloret," WLAN IEEE $802.11 \mathrm{a} / \mathrm{b} / \mathrm{g} / \mathrm{n}$ Indoor Coverage and Interference Performance Study". International Journal On Advances in Networks and Services, Vol. 4, Issue 1 and 2, Pp. 209-222., 2011.

[171] S. Sendra, L. Ferrando, J. Lloret, and A. Canovas, "Indoor IEEE 802.11 g Radio Coverage Study". In The Sixth International Conference on Digital Society (ICDS 2012), January 30 - February 4, 2012, Valencia, Spain.

[172] S. Sendra, J. Lloret, C. Turró, J Aguiar, "IEEE 802.11a/b/g/n Short Scale Indoor Wireless Sensor Placement", International Journal of Ad Hoc and Ubiquitous Computing (IJAHUC,) ACCEPTED and in Press process. 2013 Available at: http://www.inderscience.com/info/ingeneral/forthcoming.php?jcode=ijahuc [Last Access: 15/05/2013]

[173] S. Sendra, D. Bri, E. Granell, J. Lloret, "IEEE 802.11g Radio Coverage Study for Indoor Wireless Network Redesign", International Journal On Advances in Intelligent Systems, VOL: 5 Issue: 3\&4. Pp. 518-532, 2012

[174] M. Garcia, S. Sendra, M. Atenas, and J. Lloret,."Underwater wireless ad-hoc networks: A survey". Mobile Ad hoc Networks: Current Status and Future Trends, Chapter 14. Pp. 379-411. 2011

[175] S. Sendra, J.V. Lamparero, J. Lloret, and M. Ardid, "Underwater communications in wireless sensor networks using WLAN at 2.4 Ghz". In IEEE 8th International Conference on Mobile Adhoc and Sensor Systems (MASS), 2011 (pp. 892-897).

[176] J. Lloret, S. Sendra, M. Ardid and J. J. P. C. Rodrigues, "Underwater Wireless Sensor Communications at 2.4 GHz ISM Frequency Band”. Sensors. VOL: 12 Issue: 4. Pp. 4237-4264. 2011.

[177] S. Sendra, J.V. Lamparero, J. Lloret, and M. Ardid, "Study of the optimum frequency at $2.4 \mathrm{GHz}$ ISM band for underwater wireless ad hoc communications". In Ad-hoc, Mobile, and Wireless Networks (pp. 260-273). Springer Berlin Heidelberg. (2012).

[178] M. Garcia, S. Sendra, G. Lloret, and J. Lloret, "Monitoring and Control Sensor System for Fish Feeding in Marine Fish Farms”, IET Communications. Vol. 5 Issue. 12, Pp. 1682-1690, 2011. 
[179] J. Lloret, M. Garcia, S. Sendra, G. Lloret, "An Underwater Wireless Group-Based Sensor Network for Marine Fish Farms Sustainability Monitoring", Telecommunication Systems, Springer-Verlag Berlin Heidelberg. ACCEPTED and in Press process.

[180] J. Lloret, S. Sendra, M. Garcia, G. Lloret, "Group-based Underwater Wireless Sensor Network for Marine Fish Farms", IEEE Global Communications Conference (IEEE Globecomm 2011), Houston, Texas, USA. December, 5 - 9, 2011.

[181] M. Hempstead, M. J. Lyons, D. Brooks, and G-Y Wei, "Survey of Hardware Systems for Wireless Sensor Networks", Journal of Low Power Electronics, Vol.4, pp.1-10, 2008.

[182] V. Raghunathan, C.Schurgers, S. Park and M.B. Srivastava, "Energy-aware wireless microsensor networks," Journal of IEEE Signal Processing Magazine, , vol.19, no.2, pp.40-50, Mar 2002

[183] M.A.M. Vieira, C.N. Coelho, D.C. da Silva, J.M. da Mata, "Survey on wireless sensor network devices,", Emerging Technologies and Factory Automation (ETFA '03).Vol.1, no., pp. 537- 544, 16-19 Sept. 2003

[184] Y. Xu, J. Heidemann, and D. Estrin, "Geography-informed energy conservation for ad hoc routing". 7th annual international conference on Mobile computing and networking, July 16-21, 2001, Rome, Italy. pp. 70-84

[185] 185 R. Amirtharajah, S. Meringer, J. O. Mur-Miranda, A. Chandrakasan and J. Lang, "A Micropower Programmable DSP Powered using a MEMSbased Vibration-to-Electric Energy Converter," 5th IEEE Symposium on Computers and Communications (ISCC 2000). Vol. 43, pp. 362 363, February, 2000.

[186] M. Bhardwaj, T. Garnett, A. P. Chandrakasan. "Upper bounds on the lifetime of sensor networks". IEEE International Conference on ICC 2001., vol.3, no., pp.785-790 vol.3, Helsinki, Finland, June 1115,2001

[187] WiPort (2008). Available at: http://www.lantronix.com/pdf/WiPort_PB.pdf [Last Access: $15 / 05 / 2013]$

[188] Matchport b/g (2007). Available at: http://www.lantronix.com/pdf/MatchPort_PB.pdf [Last Access: $15 / 05 / 2013]$

[189] Matchport b/g Pro (2008). Available at: http://www.lantronix.com/pdf/MatchPort-bg-Pro_PB.pdf [Last Access: 15/05/2013]

[190] S103 WLAN Compact Serial Module specifications (2007), Available at:http://www.rfsolutions.co.uk/acatalog/DS-S103.pdf [Last Access: 15/05/2013]

[191] Nano WiReach specifications (2009), Available at: http://www.connectone.com/media/upload/Nano_WiReach_PB.pdf [Last Access: 15/05/2013]

[192] Mini Socket iWiFi, specifications (2009), Available at: http://www.connectone.com/media/upload/Mini_Socket_iWiFi_DS.pdf [Last Access: 15/05/2013]

[193] RN-134 specifications (2009), Available at: http://www.rovingnetworks.com/documents/rn-134ds.pdf [Last Access: 15/05/2013]

[194] RN-131G specifications (2009), Available at: http://www.rovingnetworks.com/documents/rn-131ds.pdf

[195] RN-111B specifications (2009), Available at: http://www.rovingnetworks.com/documents/rn-111bds.pdf [Last Access: 15/05/2013]

[196] RCM4400W RabbitCore module specifications (2009), Available at: http://www.rabbit.com/products/RCM4400W/\#description [Last Access: 15/05/2013]

[197] Connect Wi-ME specifications (2009), Available at: http://ftp1.digi.com/support/documentation/90000897_G.pdf [Last Access: 15/05/2013]

[198] ConnectCore Wi-9C specifications (2007), Available at: http://ftp1.digi.com/support/documentation/connectcore_wi9c_eu_doc.pdf [Last Access: 15/05/2013]

[199] Linear (2001) Technology LTC3440 Data Sheet, http://www.linear.com/pc/downloadDocument.do?navId=H0,C1,C1003,C1042,C1116,P2123,D3314

[Last Access: 15/05/2013] 
[200] Y. Yao and J. Gehrke, "The cougar approach to in-network query processing in sensor networks", Special Interest Group on Management Of Data (SIGMOD) 2002, Vol 31, No 3, September 2002

[201] A. Wang, and A. Chandrakasan "Energy-efficient DSPs for wireless sensor networks", IEEE Signal Processing Magazine., Vol. 19, No. 4, pp. 68-78, 2002.

[202] M.n. Halgamuge, M. Zukerman, K. Ramamohanarao, and H.L. Vu, H. L. "An Estimation of Sensor Energy Consumption”. Progress in Electromagnetics Research B, Vol. 12, pp. 259-295, 2009.

[203] Y. Sankarasubramaniam, I. F. Akyildiz and S. W. McLaughlin, "Energy efficiency based packet size optimization in wireless sensor networks", First IEEE Workshop on Sensor Network Protocols and Applications (SNPA 2003). Anchorage, Alaska, USA, May 2003

[204] M. Cardei, M. T. Thai, Yingshu Li and Weili Wu, "Energy-efficient target coverage in wireless sensor networks,". 24th Annual Joint Conference of the IEEE Computer and Communications Societies (INFOCOM 2005), Pp. 1976- 1984, vol. 3, 13-17 March 2005.

[205] A. Sinha, A. Chandrakasan, "Dynamic power management in wireless sensor networks," IEEE Design \& Test of Computers, vol.18, no.2, pp.62-74, Mar/Apr 2001

[206] C. Schurgers, V. Tsiatsis, S. Ganeriwal and M. Srivastava, “Optimizing Sensor Networks in the Energy-Latency-Density Design Space”, IEEE Transactions on Mobile Computing, Vol. 1, No. 1, pp. 70-80.

[207] S. S. Meiyappan, G. Frederiks and S. Hahn. Dynamic Power Save Techniques for Next Generation WLAN Systems. Proceedings of the 38th Southeastern Symposium on System Theory (SSST), Cookeville, Tennessee, USA, 5-7 March 2006, pp. 508-512.

[208] B. McFarland, and M.Wong “The Family Dynamics of 802.11”, ACM Queue, Volume 1, Issue 3. Pp. 28-38, 2003.

[209] J.N. Al-Karaki, A.E. Kamal, "Routing techniques in wireless sensor networks: a survey", IEEE Wireless Communications, vol.11, no.6, pp. 6- 28, Dec. 2004.

[210] J. Lach, D. Evans, J. McCune, and J. Brandon. "Power efficient adaptable wireless sensor networks". In International Conference on Military and Aerospace Programmable Logic Devices (MAPLD), Washington, D.C. (USA), September 9-11, 2003

[211] I. F. Akyildiz, W. Su, Y. Sankarasubramaniam and E. Cayirci, "Wireless sensor networks: a survey", Computer Networks, Vol. 38, Issue 4, Pages 393-422, March 2002.

[212] J. Lloret, C. Palau, F. Boronat, J. Tomas, "Improving Networks Using Group-based Topologies". Computer Communications Vol. 31, Issue 14, Pp.3438-3450, 2008.

[213] M. Garcia, and J. Lloret, “A Cooperative Group-Based Sensor Network for Environmental Monitoring”. In: Luo, Y. (ed.) CDVE 2009. LNCS, vol. 5738, pp. 276-279. Springer, Heidelberg, 2009.

[214] R. Kannan, R. Kalidindi, S. S. Iyengar, Vijay Kumar. "Energy and rate based MAC protocol for wireless sensor networks. Special section on sensor network technology and sensor data management", Vol. 32 , Issue 4, Pp. 60-65, December 2003.

[215] Y-C. Tseng, C-S. Hsu y T-Y Hsieh,. Power-saving protocols for IEEE 802.11-based multi-hop ad hoc networks, 21st Annual Joint Conference of the IEEE Computer and Communications Societies (IEEE INFOCOM 2002), New York, NY, USA, June 23-27, Pp. 200-209.

[216] V. Kanodia, A. Sabharwal, B. Sadeghi and E. Knightly, "Ordered packet scheduling in wireless ad hoc networks: mechanisms and performance analysis", In proceedings of The Third ACM International Symposium on Mobile Ad Hoc Networking and Computing, 9-11 June 2002, Lausanne, Switzerland.

[217] S. Jayashree, B. S. Manoj y C.S. R. Murthy. A battery aware medium access control (BAMAC) protocol for Ad-hoc wireless network. 15th IEEE International Symposium on Personal, Indoor and Mobile Radio Communications (PIMRC 2004), Barcelona (Spain), 5-8 September, Vol. 2, Pp. 995-999.

[218] G. Razzano y A. Pietrabissa. An Efficient Power Saving Mechanism for Wireless LAN, Proceedings of the Eighth IEEE International Symposium on Computers and Communication (ISCC'03), Kemer , Antalya, Turkey. June 30 - july 3, Pp. 705- 709. 
[219] E-S. Jung and N. H. Vaidya. An Energy Efficient MAC Protocol for Wireless LANs, Proceedings of the 21st Annual Joint Conference of the IEEE Computer and Communications Societies (IEEE INFOCOM 2002), New York, NY, USA, June 23-27, 2002, Vol.3, pp.1756- 1764.

[220] P. Karn, "MACA - a new channel access method for packet radio". Proceedings of the 9th Computer Networking Conference ARRL/CRRL Amateur Radio. Pp. 134- 140. September 22, 1990. London, Ontario Canada.

[221] V. Bhargavan, A. Demers, S. Shenker, L. Zhang, "MACAW: a media access protocol for wireless LAN's". ACM SIGCOMM Computer Communication Review. Volume 24, Issue 4, pp. 212-225. October, 1994.

[222] S. Singh and C.S. Raghavendra, PAMAS: Power aware multi-access protocol with signalling for ad hoc networks, ACM SIGCOMM Computer Communication Review, Volume 28 Issue 3, July 1998.

[223] Wei Ye; J. Heidemann, D. Estrin, "An energy-efficient MAC protocol for wireless sensor networks," 21st Annual Joint Conference of the IEEE Computer and Communications Societies. INFOCOM 2002. vol.3, pp. 1567- 1576, 2002

[224] C. L. Fullmer and J. J. Garcia-Luna-Aceves, "Floor Acquisition Multiple Access (FAMA) for packet-radio networks", ACM SIGCOMM, Cambridge MA, August 28-September 1, 1995.

[225] S. Jagadeesan, B. S. Manoj and C.S.R. Murthy, "Interleaved carrier sense multiple access: an efficient MAC protocol for ad hoc wireless networks," IEEE International Conference on Communications, 2003. Anchorage, Alaska, 11-15 May 2003, Pp. 1124- 1128.

[226] F. Talucci, M. Gerla and L. Fratta, "MACA-BI (MACA By Invitation)-a receiver oriented access protocol for wireless multihop networks," 8th IEEE International Symposium on Personal, Indoor and Mobile Radio Communications (PIMRC '97). 1-4 Sep 1997, Helsinki, Finland. vol.2, pp.435-439

[227] C.-K. Toh, V. Vassiliou, G. Guichal and C.-H. Shih, "MARCH: a medium access control protocol for multihop wireless ad hoc networks". 21st Century Military Communications Conference (MILCOM 2000), 22 - 25 October 2000, Los Angeles, CA, USA, no., pp.512-516.

[228] Z. Yang and J.J Garcia-Luna-Aceves, "Hop-reservation multiple access (HRMA) for ad-hoc networks". 8th Annual Joint Conference of the IEEE Computer and Communications Societies. March 21-25, 1999, New York, NY, USA, pp.194-201.

[229] G. Holland, N. Vaidya and P. Bahl, "A rate-adaptive MAC protocol for multi-Hop wireless networks", 7th annual int. conference on Mobile computing and networking, July 16-21, 2001, Rome, Italy.

[230] R. C. Shah and J. Rabaey, "Energy Aware Routing for Low Energy Ad Hoc Sensor Networks", IEEE Wireless Communications and Networking Conference (WCNC), March 17-21, 2002, Orlando, FL.

[231] W. Heinzelman, A. Chandrakasan and H. Balakrishnan, "Energy-Efficient Communication Protocol for Wireless Microsensor Networks," 33rd Hawaii International Conference on System Sciences (HICSS '00), 4-7 January, 2000, Maui, Hawaii.

[232] O. Younis and S. Fahmy. Distributed Clustering in Ad-hoc Sensor Networks: A Hybrid, EnergyEfficient Approach, 23rd Annual Joint Conference of the IEEE Computer and Communications Societies (IEEE INFOCOM 2004). Hong Kong, China, 7-11 de Marzo, Vol.1, pp: 640-652. IEEE Computer Society Press. Washington, USA.

[233] Q. Li, J. Aslam and D. Rus. "Hierarchical power-aware routing in sensor networks" DIMACS Workshop on Pervasive Networking. 21 May 2001, Piscataway (USA).

[234] S. Lindsey, C. Raghavendra, "PEGASIS: Power-Efficient Gathering in Sensor Information Systems", IEEE Aerospace Conference 2002, Vol. 3, Big Sky, Montana, 9-16 March 2002. Pp. 11251130.

[235] A. Savvides, C-C Han, aind M. Srivastava,"Dynamic fine-grained localization in Ad-Hoc networks of sensors," Proceedings of the Seventh ACM Annual International Conference on Mobile Computing and Networking (MobiCom), July 16-21, 2001, Rome, Italy. pp. 166-179.

[236] V. Rodoplu and T. H. Meng, "Minimum Energy Mobile Wireless Networks", IEEE Journal Selected Areas in Communications, Vol. 17, n. 8, August 1999, Pp. 1333-1344. 
[237] L. Li and J. Y Halpern, "Minimum energy mobile wireless networks revisited," IEEE International Conference on Communications (ICC'01), Helsinki, Finland, 11-15 June. 2001.

[238] A. Manjeshwar and D. P. Agarwal, "TEEN: a routing protocol for enhanced efficiency in wireless sensor networks," In 1st International Workshop on Parallel and Distributed Computing Issues in Wireless Networks and Mobile Computing, April 23-27 2001, San Francisco, California, USA

[239] A. Manjeshwar and D. P. Agarwal, "APTEEN: A hybrid protocol for efficient routing and comprehensive information retrieval in wireless sensor networks", 16th International Parallel and Distributed Processing Symposium (IPDPS 2002), 15-19 April 2002, Fort Lauderdale, FL, USA, pp. 195-202.

[240] N. Sadagopan, B. Krishnamachari, A. Helmy, "The ACQUIRE mechanism for efficient querying in sensor networks". 1st IEEE International Workshop on Sensor Network Protocols and Applications, 2003, Pp. 149- 155, 11 May 2003

[241] M. Chu, H. Haussecker, and F. Zhao, "Scalable Information-Driven Sensor Querying and Routing for ad hoc Heterogeneous Sensor Networks," The International Journal of High Performance Computing Applications, Vol. 16, No. 3, Pp. 293-313, August 2002.

[242] Y. Yu, D. Estrin, and R. Govindan, "Geographical and Energy-Aware Routing: A Recursive Data Dissemination Protocol for Wireless Sensor Networks," UCLA Computer Science Department Technical Report, UCLA-CSD TR-01-0023, May 2001.

[243] K. Sohrabi, J. Gao, V. Ailawadhi, and G. J. Pottie, "Protocols for self-organization of a wireless sensor network," IEEE Personal Communications, Vol. 7, No. 5, pp. 16-27, October 2000.

[244] T. Hea, J. A. Stankovica, C. Lub and T. Abdelzahera, "SPEED: A stateless protocol for real-time communication in sensor networks", in the Proceedings of International Conference on Distributed Computing Systems, 19-22 May 2003, Providence, RI, USA.

[245] C. Intanagonwiwat, R. Govindan and D. Estrin, "Directed diffusion: A scalable and robust communication paradigm for sensor networks", 6th Annual ACM/IEEE international conference on Mobile computing and networking, 6-11 August, Boston, MA, USA, 2000.

[246] D. Braginsky and D. Estrin, "Rumor Routing Algorithm for Sensor Networks," 1st ACM international workshop on Wireless sensor networks and applications, 28 September, 2002. Atlanta, GA, USA.

[247] L. Subramanian and R. H. Katz, "An Architecture for Building Self Configurable Systems," IEEE/ACM Workshop on Mobile Ad Hoc Networking and Computing, Boston, MA, August 2000.

[248] L. Shu, Y. Zhang, L. Yang, Y. Wang, M. Hauswirth, N. Xiong, "TPGF: Geographic Routing in Wireless Multimedia Sensor Networks". In Springer Journal of Telecommunication Systems (JTS), Vol. 44, Issue1-2, 2010

[249] Z. Yuan, L. Wang, Lei Shu, T. Hara, Z. Qin. A Balanced Energy Consumption Sleep Scheduling Algorithm in Wireless Sensor Networks. In the 7th International Wireless Communications \& Mobile Computing Conference (IWCMC 2011), Istanbul, Turkey, July 5-8, 2011

[250] C. Bianco, F. Cucchietti, G. Griffa, "Energy consumption trends in the next generation access network - a telco perspective". 29th International Telecommunications Energy Conference (INTELEC 2007), Sept. 30 - Oct. 4, Rome, Italy, 2007.

[251] S. Haller, S. Karnouskos, C. Schroth, "The internet of things in an enterprise context," Lecture Notes in Computer Science, 2009, Volume 5468/2009, pp. 14-28, 2009.

[252] Y. Zhang, P. Chowdhury, M. Tornatore, B. Mukherjee, "Energy Efficiency in Telecom Optical Networks," IEEE Communications Surveys \& Tutorials, vol. 12, n.4, pp. 44 -458, 2010.

[253] Datasheet of Router Allied AR410. Available at Allied Telesis web site: http://www.alliedtelesis.com/media/fount/datasheet/AR410Series_Datasheet_RevQ.pdf [Last Access: $15 / 05 / 2013]$

[254] Datasheet of Router Cisco 1800. Available at Cisco web site: http://www.cisco.com/en/US/prod/collateral/routers/ps5853/product_data_sheet0900aecd8016a59b.pdf [Last Access: 15/05/2013] 
[255] W. Fisher, M. Suchara, J. Rexford, "Greening Backbone Networks: Reducing Energy Consumption by Shutting Off Cables in Bundled Links". In proceedings of the first ACM SIGCOMM workshop on Green networking, Aug. 30, New Delhi, India, 2010.

[256] N. González, L. Moran, J.M. Angioleti, J. A. Varela, "Green IT. Chapter 4. Green Telecom Networks". eKISS n⿳82.Internal publication of Telefónica. (2009).

[257] H. Galperin, "Wireless Networks and Rural Development: Opportunities for Latin America", Information Technologies and International Development, vol. 2, no. 3, pp. 47--56, 2005.

[258] M. Segal, "Improving lifetime of wireless sensor networks", Network Protocols and Algorithms, Vol 1, No 2, Pp. 48-60, 2009.

[259] Datasheet of Access Point Cisco Linksys WRT320N-EZ. Available at Cisco web site: http://homesupport.cisco.com/es-eu/support/routers/WRT320N/download [Last Access: 15/05/2013]

[260] Datasheet of Access Point Cisco Systems AIR-AP1131AG-E-K9. Available at Cisco web site: http://www.cisco.com/en/US/docs/wireless/access_point/1130/installation/guide/1130hig_book.pdf [Last Access: 15/05/2013]

[261] Datasheet of Access Point Cisco Systems WRT54GL. Available at Cisco web site: http://home.cisco.com/es-eu/products/routers/WRT54GL [Last Access: 15/05/2013]

[262] Datasheet of Access Point D-link DWL-2000AP+. Available at D-link web site: ftp://ftp.dlink.de/dwl/dwl-2000applus/documentation/DWL-2000applus_man_en_040401.pdf [Last Access: 15/05/2013]

[263] Datasheet of Access Point Avaya AP-I. Available at Avaya web site: http://downloads.avaya.com/css/P8/documents/003702825 [Last Access: 15/05/2013]

[264] Datasheet of Access Point Ovislink WX-1590. Available at web site: http://www.ferimex.com/download/Manual/WX-1590/WX-1590L-en.pdf [Last Access: 15/05/2013]

[265] M. Garcia, S, Sendra, J. Lloret, and R. Laquesta, "Saving Energy with Cooperative Group-based Wireless Sensor Networks", Lecture Notes in Computer Science. Vol.6240, Pp. 231-238, 2010. Springer-Verlag Berlin Heidelberg.

[266] M. Garcia, S, Sendra, J. Lloret, and R. Laquesta," Saving Energy and Improving Communications using Cooperative Group-based Wireless Sensor Networks", Telecommunication Systems, Vol. 57, Issue1-2, September/October 2014. Springer-Verlag Berlin Heidelberg.

[267] J. Lloret, J. Tomas, M. Garcia, A. Canovas, "A hybrid stochastic approach for self-location of wireless sensors in indoor environments". Sensors Vol. 9, issue 5, Pp.3695-3712, 2009.

[268] D. Bri, H. Coll, M. Garcia, J. Lloret, "A multisensor proposal for wireless sensor networks". In The Second International Conference on Sensor Technologies and Applications, SENSORCOMM 2008, Cap Esterel, France, August 25-31, 2008.

[269] J.S. Seybold, "Introduction to RF propagation”. John Wiley: New York, NY, USA, 2005.

[270] J.C Giacomin, and F.H. Vasconcelos, "Wireless sensor network as a measurement tool in precision agriculture". In XVIII IMEKO World Congress. Metrology for a Sustainable Development. Rio de Janeiro, Brazil, September 17-22, 2006.

[271] P. Barsocchi, G. Oligeri, and F. Potortì, "Transmission range and frame error process in rural area Wi-Fi networks". CNR-ISTI Technical Report: ISTI-2006-TR-43, June 2006.

[272] A. Luntovskyy, D. Gütter, A. Schill, and U. Winkler, "Design Particularities for Wireless Networks". In Proceedings of IEEE CriMiCo-05, Sevastopol, Ucrania, September 16, 2005; pp.955-958.

[273] Recommendation UIT-R P.838-3. Specific attenuation model for rain for use in prediction methods. http://www.itu.int/rec/R-REC-P.838/en [Last access 15/05/2013]

[274] Recommendation ITU-R P.833-4. Attenuation in vegetation. http://www.itu.int/md/R03-SG03-C0017/en [Last access 15/05/2013]

[275] J. Lloret, P.V. Mauri, J.M. Jimenez, J.R. Diaz, "802.11g WLANs Design for Rural Environments Video-surveillance". In International Conference on Digital Telecommunications ICDT'06, Cap Esterel, French Riviera, France, August 26-31, 2006. 
[276] Recommendation UIT-R PN.837-5. Characteristics of precipitation for propagation modeling. http://www.itu.int/md/R07-WP3J-C-0014/en [Last access 15/05/2013]

[277] P. Asadoorian, and L. Pesce, Linksys WRT54g Ultimate Hacking. Syngress: Burlington, MA, USA, 2007.

[278] Cisco Aironet 350 Series Wireless Bridge Datasheet. http://www.cisco.com/warp/public/cc/pd/witc/ao350ap/prodlit/a350m_ds.pdf [Last access 15/05/2013]

[279] Linksys WRT54GL router Web site. http://www.linksysbycisco.com/US/en/products/WRT54GL [Last access 15/05/2013]

[280] Renovables Futura S.L. Website. http://www.renovablesfutura.com/fotovoltaica/index.php?name=News\&file=article \&sid=3 [Last access $15 / 05 / 2013]$

[281] Sunnetworks, S.L website. http://www.sun-networks.org/html/__radiacion_solar.html [Last access 15/05/2013].

[282] The Family Dynamics of 802.11. Queue 2003, 1, 28-38.

[283] Press release by the International Organization of Vine and Wine (OIV). Balance de la OIV sobre la situación vitivinícola mundial en 2009. Available at: http://news.reseauconcept.net/images/oiv_es/Client/Communique_Stats_Tbilissi_ES.pdf [Last access 15/05/2013]

[284] A. Carbonneau, "Éléments de la conduite du vignoble favorisant la protection du terroir et l'expression de ses vins". XXVème Congrès Mondial de la Vigne et du Vin de l'OIV, June 19-23, 2000, Paris (France).

[285] Directive 68/193/CEE of the European council, http://eurlex.europa.eu/LexUriServ/LexUriServ.do?uri=CELEX:31968L0193:ES:NOT [Last access 15/05/2013].

[286] Directive of the European Parliament and of the Council COM (2010)359 final, http://www.europarl.europa.eu/meetdocs/2009_2014/documents/com/com_com\%282010\%290359_/co m_com\%282010\%290359_en.pdf [Last access 15/05/2013]

[287] New South Wales Acts As Made Legislation, Vine and Vegetation Diseases Act 1901 No 14, http://www.legislation.nsw.gov.au/sessionalview/sessional/act/1901-14.pdf [Last access 15/05/2013].

[288] Biosecurity Act 1993, 1993 No 95, Reprint as at 7 July 2010, New Zealand Legislation Acts, http://www.legislation.govt.nz/act/public/1993/0095/latest/DLM314623.html[Last access 15/05/2013].

[289] D.O. Adams, K.E. Franke, and P. Christensen, "Elevated putrescine levels in grapevine leaves that display symptoms of potassium deficiency". American Journal of Enology and Viticulture.Vol. 41, Pp.121-125,1990

[290] A.C. Goheen, J. A. Cook, "Leafroll (red-leaf or rougeau) and its effects on vine growth, fruit quality, and yields". American Journal of Enology and Viticulture, Vol 10, Pp 173-181. 1959

[291] P. Rodríguez, "Plagas y enfermedades de la vid en canarias". Sección de Sanidad Vegetal, Dirección general de producción agraria. 3rd Edition. 1996.

[292] W.D. Gubler, K. Baumgartner, G.T. Browne, A. Eskalen, S. Rooney Latham, E. Petit, and L.A. Bayramian, "Root diseases of grapevine in California and their control". Australas Plant Pathol. 2004. Vol 33, Pp. 157-165.

[293] R.N. Van Den Driessche, "Estimating potential response to fertilizer based on tree tissue and litter analysis", In: Proceedings of Forest Fertilization Conference, Contribution No. 40, College of Forest Resources, University of Washington, Seattle, WA. 1979. Pp. 214-220.

[294] G.P. Martelli, "Graft-transmissible Diseases of Grapevines. Handbook for Detection and Diagnosis". FAO Publication Division, 1993. Rome, Italy.

[295] R.N. Van Den Driessche, "Prediction of mineral nutrient status of trees by foliar analysis". Botanical Review. Vol. 40, Pp. 347-394, 1974.

[296] C. Anand, S. Sadistap, S. Bindal, B.A. Botre, and K.S.N. Rao, "Wireless multi-sensor embedded system for Agro-industrial monitoring and control", International Journal on Advances in Networks and Services, Vol. 3, Issue. 1 - 2, Pp 1-10, 2010 
[297] D. Di Palma, L. Bencini, G. Collodi, G. Manes, F. Chiti, R. Fantacci, and A. Manes, "Distributed Monitoring Systems for Agriculture based on Wireless Sensor Network Technology", International Journal on Advances in Networks and Services, , Vol. 3,Issue 1-2, Pp 11-21, 2010

[298] D. Sunding, and D. Zilberman, "The Agricultural Innovation Process: Research and Technology Adoption in a Changing Agricultural Industry", in: B. Gardner and G.C. Rausser, eds., Handbook of Agricultural and Resource Economics (North-Holland, Amsterdam), forthcoming. 2000.

[299] J. Lloret, P.V. Mauri, M. Garcia, and A. J. Ferrer, "Designing WLANS for Video Transmission in Rural Environments for Agriculture and Environmental Researches and Educational Purposes", WSEAS Transactions on Communications, Issue 11, Volume 5, Pp. 2064-2070, November 2006.

[300] M. Garcia, H. Coll, D. Bri, and J. Lloret, "Using MANET protocols in Wireless Sensor and Actor Networks", The Second International Conference on Sensor Technologies and Applications (SENSORCOMM 2008), Cap Esterel, (France), August 25-31, 2008.

[301] Recommendation UIT-R P.838-3. Specific attenuation model for rain for use in prediction methods. http://www.itu.int/rec/R-REC-P.838/en [Last access 15/05/2013].

[302] Recommendation UIT-R PN.837-5. Characteristics of precipitation for propagation modeling. http://www.itu.int/md/R07-WP3J-C-0014/en [Last access 15/05/2013]..

[303] L. Liu, H. Ma and X. Zhang, "Coverage analysis for target localization in camera sensor networks", Wireless Communications and Mobile Computing. Vol.14 Issue. 14, Pp.1239-1250, 2012.

[304] Y. Zou, and K. Chakrabarty, "Sensor deployment and target localization based on virtual forces" Twenty-Second Annual Joint Conference of the IEEE Computer and Communications Societies. IEEE Vol. 2, 30 March-3 April 2003 Page(s):1293 - 1303 vol.2

[305] X. Shen, J. Chen, Z. Wang, and Y. Sun, "Grid Scan: A Simple and Effective Approach for Coverage Issue in Wireless Sensor Networks". IEEE International Communications Conference, June 2006 Volume: 8, pp.: 3480-3484.

[306] F. Aurenhammer, Voronoi diagrams - a survey of a fundamental geometric data structure, ACM Computing Surveys 23 (4) (1991) 345-406.

[307] Circular Segment Wikipedia. At http://en.wikipedia.org/wiki/Circular_segment

[308] E.S. Biagioni, and G. Sasaki, "Wireless Sensor Placement for Reliable and Efficient Data Collection", Proceeding of the 36th Hawaii International Conference on System Science. January 6-9, 2003, Big Island, HI, USA.

[309] OpenWRT website. Available at: http://openwrt.org/ [Last access 15/05/2013]

[310] Open WRT List of supported devices. Available at: http://wiki.openwrt.org/toh/start [Last access $15 / 05 / 2013]$

[311] Atheros AR7161 information, Atheros website. Available at: http://www.atheros.com/networking/brand.php?brand=4\&product=68 [Last access 15/05/2013]

[312] Hercules Classic Webcam information in Hercules website. Available at: http://www.hercules.com/es/webcam/bdd/p/17/hercules-classic-webcam/ [Last access 15/05/2013]

[313] QuickCam information in Logitech website. Available at: http://logitech-esemea.custhelp.com/app/answers/detail/a_id/1253/section/troubleshoot/crid/435/lt_product_id/269/tabs/1, 3,2,5/cl/es,es/kw/ [Last access 15/05/2013]

[314] Creative WebCam NX Pro information in Creative website. Available at: http://en.europe.creative.com/products/productarchive. asp?category $=218 \&$ subcategory $=219 \&$ product $=6$ $28 \&$ nav $=1 \&$ listby $=$ [Last access 15/05/2013]

[315] Creative WebCam Instant information in Creative website. Available at: http://es.creative.com/products/productarchive.asp?category=269\&subcategory=293\&product=10410\&n av $=1 \&$ listby $=$ [Last access 15/05/2013]

[316] A4tech PKS-635K information in a4tech website. Available at: http://www.a4tech.de/?q=node/166 [Last access 15/05/2013]

[317] ASAJA (Asociación Agraria de Jovenes Agricultores), Online press article (September 4, 2012), "ASAJA Castilla y León apunta que el lobo avanza a "su libre albedrío" sin que el Plan de Gestión 
consiga sus objetivos de hacerlo compatible con la ganadería". Available at: http://www.asaja.com/publicaciones/asaja_castilla_y_leon_apunta_que_el_lobo_avanza_a_\%E2\%80\%9 Csu_libre_albedrio\%E2\%80\%9D_sin_que_el_plan_de_gestion_consiga_sus_objetivos_de_hacerlo_com patible_con_la_ganaderia_738. [Last access 15/05/2013].

[318] Principado de Asturias Government. Online press article (October 9, 2012): "El principado propone autorizar controles poblacionales del lobo hasta finales de 2013". Gobierno del Principado de Asturias. Available http://www.asturias.es/portal/site/webasturias/menuitem.6282925f26d862bcbc2b3510f2300030/?vgnext oid=f1cce3d1ad74a310VgnVCM10000097030a0aRCRD\&i18n.http.lang=es [Last access 15/05/2013].

[319] Ministère de l'Ecologie, du Développement Durable, des Transports et du Logement; Ministère de l'Agriculture, de l'Alimentation, de la Pêche, de la Ruralité et de l'Aménagement du Territoire.Wolf in France, in the french context of and traditional livestock farming. Available at: http://www.rdbrmctravaux.com/loup/spip.php?rubrique90 [Last access 15/05/2013]

[320] M. O. B.Yassien, M. Kh. Salayma, W. E. Mardini and, Y. M. Khamayseh, "Fibonacci Backoff Algorithm for IEEE 802.15.4/ZigBee", Network Protocols and Algorithms, Vol 4, No 3 (2012). Pp. 6278.

[321] C. Martín, D. Villa, O. Aceña, F.J. Villanueva, F. Moyaand, J.C. López, "Plataforma de alto nivel para el desarrollo de sensores y actuadores de bajo coste en entornos inteligentes". Laboratorio ARCO, Escuela Superior de Informática, Universidad de Castilla-La Mancha. Available at: https://arco.esi.uclm.es/public/papers/2011-Ei3-cleto.martin.pdf. [Last Access: 15/05/2013].

[322] Datasheet Waspmote. Available at Libelium Comunicaciones Distribuidas S.L. web site: http://www.libelium.com/documentation/waspmote/waspmote-datasheet_eng.pdf . [Last access $15 / 05 / 2013]$

[323] Datasheet AP Ezport-101. Available at Passport Networks inc. Web site: http://passportnetworks.com/download/EZport_um.pdf[Last access 15/05/2013]

[324] J. Singh, C. W. F. Yu, J. T. Kim, "Building Pathology, Investigation of Sick Buildings -Toxic Moulds", Indoor and Built Environment', Vol. 19, No. 1, Pp. 40-47, 2010.

[325] P. Montoya, A. Garcia, and F. Morán, "Hormigón Armado", Gustavo Gili, S.A. 2nd Edition, Barcelona, Spain. 2010

[326] V. Martínez, R. Cebrián, J.A. Férriz, J. Muñoz, R. Prado, G. Furest, and S. Spairani, "Introducción a los materiales de construcción”, Publisher: Ramón Torres Gosálvez, San Vicente, Spain. 2011.

[327] D. Bri, M. Garcia, J. Lloret, and P. Dini, "Real deployments of wireless sensor networks, Third International Conference on Sensor Technologies and Applications", In proceedings of The Third International Conference on Sensor Technologies and Applications (SENSORCOMM 2009), June 1823, 2009 - Athens/Vouliagmeni, Greece.

[328] T.O. Olwal, B.J. Van Wyk, N., Ntlatlapa, K. Djouani, P. Siarry, and Y. Hamam, "Dynamic Power Control for Wireless Backbone Mesh Networks: a Survey", Network Protocols and Algorithms, Vol. 2, No. 1, pp.1-44, 2011.

[329] A. Pal, "Localization algorithms in wireless sensor networks: Current approaches and future challenges". Network Protocols and Algorithms, Vol.2, No.1, pp. 45-73, 2010

[330] C. Huang, and R.H. Cheng, "Localised sensing strategies for point coverage in directional Wireless Sensor Networks". International Journal of Ad Hoc and Ubiquitous Computing, Vol.8, No.1, pp. 125134, 2011.

[331] T. Clausen, and P. Jaquet, (2003) 'RFC 3626 - Optimized Link State Routing Protocol (OLSR)'. October 2003. Available at: http://www.faqs.org/rfcs/rfc3626.html[Last access 15/05/2013].

[332] Sol LM870 mobile phone features. Available at: http://es.made-inchina.com/co_runrise/product_Dual-SIM-Card-Dual-Standby-GPS-Temperature-UV-Sensor-

Pedometer-Sunrise-LM870-Mobile-Phone_hesighyiy.html.

[333] STLM20 temperature sensor features. Datashhet available at: http://www.st.com/internet/com/TECHNICAL_RESOURCES/TECHNICAL_LITERATURE/DATASH EET/CD00119601.pdf. [Last access 15/05/2013]. 
[334] Matlab Website. Available at: www.mathworks.com/products/matlab.

[335] S.P. Kuo, Y.C. Tseng, F.J. Wu, and C.Y. Lin, "A probabilistic signal-strength-based evaluation methodology for sensor network deployment". International Journal of Ad Hoc and Ubiquitous Computing, Vol.1, No. 1, pp.3-12., 2005.

[336] S. Sendra, L. Ferrando, J. Lloret and, A. Canovas, “Indoor IEEE 802.11g Radio Coverage Study”. In proceedings of the Sixth International Conference on Digital Society (ICDS 2012), pp. 121-126, Valencia (Spain), January 30 - February 4, 2012.

[337] H.Hashemi, "The indoor radio propagation channel",in Proceedings of the IEEE, vol.81, no.7, pp.943-967., 1993.

[338] D. Molkdar, "Review on radio propagation into and within buildings", IEE Proceedings - Part H: Microwaves, Antennas \& Propagation, Vol.138, No.1, pp. 61- 73, 1991.

[339] N. Pérez , C. Pabón , J.R. Uzcátegui and E. Malaver,"Nuevo modelo de propagación para redes WLAN operando en $2.4 \mathrm{Ghz}$, en ambientes interiores". TÉLÉMATIQUE 2010, Vol.9, Issue: 3, Pp.1-22.

[340] InSSIDer website, at http://www.metageek.net/products/inssider [Last access 15/05/2013].

[341] Net Meter website, at http://www.hootech.com/NetMeter/ [Last access 15/05/2013].

[342] W. Stallings, "Data and Computer Communications", (7th ed.), Prentice Hall PTR, 2004.

[343] D. Aguayo, J. Bicket, S. Biswas, G. Judd, and R. Morris, "Link-level measurements from an 802.11 b mesh network". In Proceedings of the 2004 conference on Applications, technologies, architectures, and protocols for computer communications (SIGCOMM '04). Portland (Oregon-USA), August 30September 3, 2004.

[344] I. F. Akyildiz, D. Pompili, and T. Melodia, "Challenges for efficient communication in underwater acoustic sensor networks"., ACM Sigbed Review, Vol. 1, Issue. 2. Jul. 2004

[345] U. Chakraborty, T. Tewary, R.P. Chatterjee, "Exploiting the loss-frequency relationship using RF communication in Underwater communication networks". In 4th International Conference on Computers and Devices for Communication, CODEC 2009, Kolkata, India, December 14-16, 2009.

[346] H.J. Liebe, G.A. Hufford, and T. Manabe, "A model for the complex permittivity of water at frequencies below $1 \mathrm{THz}$ ". International Journal of Infrared and Millimeter Waves. April 1991. Vol.12, no.7, pp. 659-675.

[347] R. Somaraju, J. Trumpf, "Frequency, Temperature and Salinity Variation of the Permittivity of Seawater". IEEE Transactions Antennas and Propagation, Nov. 2006, Vol.54, Issue. 11, Pp.3441-3448.

[348] Z. Wang, A. Zeitoun, S. Jamin, "Challenges and Lessons Learned in Measuring Path RTT for Proximity-Based Applications". In 6th workshop on Passive and Active Measurement 2003. San Diego, CA, USA.

[349] Li, B.; Zhou, S.; Stojanovic, M.; Freitag, L.; Huang, J. and Willett, P., "MIMO-OFDM over an underwater acoustic channel". In Proceedings of MTS/IEEE OCEANS conference, Vancouver, BC, Canada, 2007. Sept. 29 - Oct. 4.

[350] M. Stojanovic, "Low Complexity OFDM Detector for Underwater Acoustic Channels". In Proccedings of MTS/IEEE OCEANS conference, Boston, Massachusetts, USA .2006, 18-21 Sept

[351] A.I. Al-Shamma'a, A. Shaw, and S. Saman, "Propagation of electromagnetic waves at $\mathrm{MHz}$ frequencies through seawater". Transactions on IEEE Antennas and Propagation, Nov. 2004. vol.52, no.11, pp. 2843- 2849.

[352] A. Shaw, A.I. Al-Shamma'a, S.R. Wylie, and D. Toal, "Experimental Investigations of Electromagnetic Wave Propagation in Seawater", 36th European Microwave Conference, 2006., Vol., Pp.572-575, 10-15 Sept.

[353] G. Baiden, and Y. Bissiri, "High bandwidth optical networking for underwater untethered telerobotic operation", In Proccedings of MTS/IEEE OCEANS conference, Vancouver, BC, Canada, 2007. Sept. 29 - Oct. 4.

[354] N. Farr, A.D. Chave, L. Freitag, J. Preisig, S.N. White, D. Yoerger, and F. Sonnichsen, "Optical Modem Technology for Seafloor Observatories". In Proceedings of MTS/IEEE OCEANS conference, Boston, Massachusetts, USA. 2006, 18-21 Sept. 
[355] T-H. Won, and S-J. Park, "Design and Implementation of an Omni-Directional Underwater Acoustic Micro-Modem Based on a Low-Power Micro-Controller Unit”. In Sensors Journal, Vol. 12 Issue 2 , Pp.2309-2323, 2012.

[356] B.C. Kim, and I.T. Lu "Parameter study of OFDM underwater communications system". In OCEANS 2000 MTS/IEEE Conference and Exhibition. September 11-14, 2000. Providence, Rhode Island-The Ocean State.

[357] U.M. Cella, R. Johnstone, and N. Shuley "Electromagnetic wave wireless communication in shallow water coastal environment: theoretical analysis and experimental results". In Proceedings of the Fourth ACM International Workshop on UnderWater Networks. November 3, 2009, Berkeley, California, USA

[358] Eureqa Formulize web site. Available at: http://formulize.nutonian.com [Last access 15/05/2013].

[359] M. Ardid, “ANTARES: An Underwater Network of Sensors for Neutrino Astronomy and Deep-Sea Research", Ad Hoc \& Sensor Wireless Networks. 2009. 8, pp. 21-34.

[360] S. Sendra, J. Lloret, J.J.P.C. Rodrigues, and J.M. Aguiar, "Underwater Wireless Communications in Freshwater at $2.4 \mathrm{GHz}$ ", IEEE Communications Letters, IN PRESS

[361] J.C.Macías, J. Collado, C. Álamo, M. Escalona, and E. García, "Seguimiento ambiental para instalaciones de acuicultura marina", Boletín Instituto Español de Oceanografía. Vol. 21, Issue 1-4, P.p. 57-66, 2005

[362] E. Martí, C. M. Martí, R. Martínez, M. Paches, and S. Falco, "El programa de vigilancia ambiental de piscifactorías en jaulas flotantes”. Boletin Instituto Español de Oceanografía Vol. 21,Issue. 1-4, Pp. 67-73, 2005

[363] O. Ahn, R.J. Petrell, and P.J. Harrison, “Ammonium and nitrate uptake by Laminaria saccharina and Nereocystis luetkeana originating from a salmon sea cage farm”, Journal of Applied Phycology Vol.10, Pp. 333-340, 1998

[364] M.V. Goulão, C.A.P. Andrade, N.M.A. Gouveia, J.R.J. Gomes, V.M.F.A. Timóteo, and F. Soares, "Evaluación de pérdidas de piensos en una piscifactoría en mar abierto y su uso en modelos del crecimiento de peces de cultivo y de la ración diaria", AquaTIC no 13, May 2001. Available at: http://www.revistaaquatic.com/aquatic/art.asp?t=h\&c=113, [Last access 15/05/2013].

[365] R. Riera, P. Sanchez-Jerez, M. Rodríguez, O. Monterroso and E. Ramos. ”Long-term monitoring of fish farms: Application of Nematode/Copepod index to oligotrophic conditions. Marine Pollution Bulletin Vol.64, Pp. 844-850, 2012.

[366] B. Basurco, and G. Larrazabal, "Marine Fish Farming in Spain”, Cahiers Options Méditerranéennes, Vol. 30, Pp. 45-56, 2000.

[367] P. Carvajal, “Acuicultura Latinoamericana: el poder de un gigante”, Industry Reports, IntraFish Media AS, January 20, $2006 . \quad$ Available at: http://www.intrafish.no/global/industryreports/article99314.ece, [Last access 15/05/2013].

[368] FAO Animal Production and Health Manual, Section 4: On-farm production and use of feed and feed ingredients, Manual of Good Practices for the Feed Industry, No. 9, 2010.

[369] R.J. Gowen, N.B. Bradbury and J. R. Brown, "The use of simple models in assessing two of the interactions between fish-farming and the marine environment". Aquaculture-A Biotechnology in Progress. European Aquaculture Society, Pp.1071-1080. ,1989

[370] H. Ackerfors and M. Ennel "Discharge of nutrients from Swedish fish farming to adjacent sea areas". Ambio, Vol. 19, Pp.28-35, 1990.

[371] P.K. Hansen, K. Pittman, and A. Ervik, "Organic waste from marine fish farms-effects on the seabed”. Marine Aquaculture and Environment, Pp. 105-119, 1991

[372] A. Stigebrandt, J. Aure, A. Ervik, and P. K. Hansen, "Regulating the local environmental impact of intensive marine fish farming: III. A model for estimation of the holding capacity in the ModellingOngrowing fish farm-Monitoring system", Aquaculture, Vol. 234, Issues 1-4, Pp. 239-261, 2004.

[373] A. Stigebrandt, and J. Aure, "A model for critical loads beneath fish farms", Fisken and Havet Institute of Marine Research, Vol. 26, Pp. 1-27, 1995 
[374] R. Atanasova, L. Hadajinikolova, and L. Nikolova, "Investigations on the biochemical composition of carp fish (Cyprinidae) blood al conditions of organic acuaculture". Bulgarian Journal of Agricultural Science, Vol.14, Issue. 2, Pp.117-120,2008.

[375] R. Jurdak, A. G. Ruzzelli, G. M. P. O’Hare, and C. Videira, "Mote-based underwater sensor networks: opportunities, challenges, and guidelines", Telecommunication Systems, Vol. 37, Issue. 1-3, Pp. 37-47, 2008.

[376] Industrial sensors catalogue with UM18-X111X specifications. In Sickusa web site. Available at: http://www.sick.com/us/en-

us/home/products/product_portfolio/industrial_sensors/Pages/industrial_sensors.aspx._Accessed 3 September 2012.

[377] E. Natalizio, and V. Loscrí, "Controlled mobility in mobile sensor networks: advantages, issues and challenges", Telecommunication Systems, 19 August 2011. DOI: 10.1007/s11235-011-9561-X

[378] DIRECTIVE 2000/60/EC OF THE EUROPEAN PARLIAMENT AND OF THE COUNCIL of 23 October 2000 establishing a framework for Community action in the field of water policy. Official Journal of the European Communities. Available at: http://faolex.fao.org/cgibin/faolex.exe?rec_id=017501\&database=FAOLEX\&search_type=link\&table=result\&lang=eng\&format _name=@ERALL [Last access 15/05/2013].

[379] Directive, S. E. A. (2001). Directive 2001/42/EC of the European Parliament and of the Council of 27 June 2001 on the assessment of the effects of certain plans and programmes on the environment (OJ L 197, 21.7. 2001, pp. 30-37). Official Journal L, 197(21/07), 0030-0037.

[380] A. C. Ziegler, "Issues related to use of turbidity measurements as a surrogate for suspended sediment". Turbidity and Other Sediment Surrogates Workshop, Reno, Nevada, USA, April 30 - May 2, 2002.

[381] Y. Liu, and X. Ge, "Underwater Blue-Green Laser Sensor Network: Challenges and Approaches. WSEAS Transactions on Communications, Vol.5, Issue 6, Pp.421-425., 2006.

[382] Y. J. Jeong, S-Y.Shin, S-H. Park, C-H. Kim, "PBA: A New MAC Mechanism for Efficient Wireless Communication in Underwater Acoustic Sensor Network". WSEAS Transactions on Communications. Vol. 6, Issue 3, Pp.401-407, 2007

[383] D. M. Monteiro, J. J. P. C. Rodrigues, J. Lloret, and S. Sendra, "A hybrid NFC-Bluetooth secure protocol for Credit Transfer among mobile phones", Security and Communication Networks, 2013. Available at: http://onlinelibrary.wiley.com/journal/10.1002/(ISSN)1939-0122/earlyview [Last access 7/06/2013]. 